Stefan Lorenz

Der Zusammenhang von Arbeitsgestaltung und Erwerbsleben unter besonderer Berücksichtigung der Erwerbstätigkeit von Frauen und Alteren 
Stefan Lorenz

\section{Der Zusammenhang von Arbeitsgestaltung und Erwerbsleben unter besonderer Berücksichtigung der Erwerbstätigkeit von Frauen und Älteren}

Dieses Buch behandelt die Frage, wie mit Blick auf die Veränderungen des Arbeitsmarktes die Erwerbstätigkeit von Frauen und Älteren erleichtert werden kann. Die demographische Entwicklung führt dazu, daß das durchschnittliche Alter der Arbeitskräfte steigt und sich das Arbeitskräftepotential zukünftig verkleinern wird. Andererseits verschieben sich die Arbeitsanforderungen durch die Nutzung moderner Produktions- und Organisationsmethoden. In einer mikroökonomisch und arbeitswissenschaftlich ausgerichteten Analyse werden Maßnahmen der Arbeitsgestaltung und der Personalentwicklung entworfen und beurteilt, die zur Erhöhung der Frauenerwerbstätigkeit und zur Verlängerung der Lebensarbeitszeit beitragen können.

Stefan Lorenz wurde 1965 in Wiesbaden geboren. Von 1986 bis 1992 studierte er Wirtschaftsingenieurwesen der technischen Fachrichtung Maschinenbau an der Technischen Hochschule Darmstadt. Diese Arbeit führte 1996 zur Promotion an der Technischen Hochschule Darmstadt. 
Der Zusammenhang von Arbeitsgestaltung und Erwerbsleben unter besonderer Berücksichtigung der Erwerbstätigkeit von Frauen und Älteren 


\section{Sozialökonomische Schriften \\ Herausgegeben von Bert Rürup}

Band 13

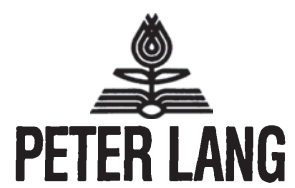

Frankfurt am Main - Berlin - Bern - New York · Paris · Wien 


\section{Stefan Lorenz}

\section{Der Zusammenhang von Arbeitsgestaltung und Erwerbsleben unter besonderer Berücksichtigung der Erwerbstätigkeit von Frauen und Älteren}

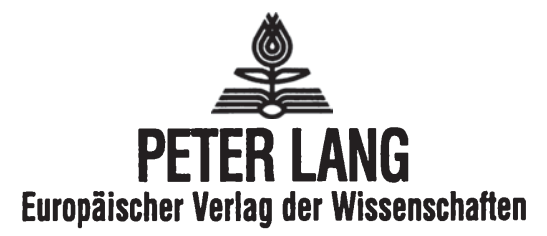


Die Deutsche Bibliothek - CIP-Einheitsaufnahme

Lorenz, Stefan:

Der Zusammenhang von Arbeitsgestaltung und Erwerbsleben unter besonderer Berücksichtigung der Erwerbstätigkeit von Frauen und Älteren / Stefan Lorenz. - Frankfurt am Main ; Berlin ; Bern ; New York ; Paris ; Wien : Lang, 1997

(Sozialökonomische Studien ; Bd. 13)

Zugl.: Darmstadt, Techn. Hochsch., Diss., 1996

ISBN 3-631-30701-2

Open Access: The online version of this publication is published on www.peterlang.com and www.econstor.eu under the international Creative Commons License CC-BY 4.0. Learn more on how you can use and share this work: http://creativecommons. org/licenses/by/4.0.

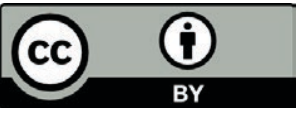

This book is available Open Access thanks to the kind support of ZBW - Leibniz-Informationszentrum Wirtschaft.

NE: GT

\author{
D 17 \\ ISSN 0172-1747 \\ ISBN 3-631-30701-2 \\ ISBN 978-3-631-75027-8 (eBook) \\ (C) Peter Lang GmbH \\ Europäischer Verlag der Wissenschaften \\ Frankfurt am Main 1997 \\ Alle Rechte vorbehalten.
}

Das Werk einschließlich aller seiner Teile ist urheberrechtlich geschützt. Jede Verwertung außerhalb der engen Grenzen des

Urheberrechtsgesetzes ist ohne Zustimmung des Verlages unzulässig und strafbar. Das gilt insbesondere für

Vervielfältigungen, Übersetzungen, Mikroverfilmungen und die Einspeicherung und Verarbeitung in elektronischen Systemen.

Printed in Germany 123467 


\section{Geleitwort}

Kennzeichnend für die Finanzierung des deutschen Systems der sozialen Sicherung ist seine Lohnzentrierung; denn die Einnahmen sind für alle Sozialversicherungen eine Funktion der Entwicklung der versicherungspflichtigen Entgelte multipliziert mit den jeweiligen Beitragssätzen.

Auf der Leistungs-, d.h. Ausgabenseite dominieren

- in der gesetzlichen Krankenversicherung und der Pflegeversicherung das Sachleistungs- bzw. Erstattungsprinzip während

- bei der Arbeitslosen-, Unfall- und Rentenversicherung die Leistungen eine Lebensstandardsicherungsfunktion erfüllen sollen und sich damit der Höhe und Struktur nach an den Löhnen orientieren.

Diese Lohnzentrierung ist es, die Arbeitsmarktprobleme via Beitragsausfälle zu Finanzproblemen dieses Systems macht und sie impliziert, daß das finanzielle Gleichgewicht dieses Systems gleichermaßen von

- einer demographisch bedingten Verschlechterung des Verhältnisses von Beitragszahlern und Leistungsempfängern gestört wird, als auch von einer steigenden und lang anhaltenden Arbeitslosigkeit und

- einer zunehmenden Substitution von Arbeitsverträgen durch Werkverträge bzw. beitragspflichtigen Arbeitnehmern durch geringfügig Beschäftigte.

Kennzeichnend für die seit längerem laufende Rentendiskussion ist - ohne die in der demographischen Entwicklung angelegten Probleme negieren zu wollen - eine Überzeichnung der aus der Bevölkerungsentwicklung resultierenden Probleme bei gleichzeitiger Vernachlässigung sowohl der Risiken als auch der Chancen der ökonomischen Entwicklung. Vor diesem Hintergrund stellt sich Herr Lorenz die Frage, ob und wie es möglich ist, durch

- eine arbeitsmarktorientierte Zuwanderung

- eine Verkürzung der Ausbildungszeiten

- eine Verschiebung des Renteneintrittsalters und damit eine Erhöhung der Erwerbstätigkeit der Älteren sowie

- eine generelle Steigerung der Erwerbstätigkeit der Frauen

längerfristig trotz eines und aus einem irreversibel schrumpfenden Erwerbspersonenpotential die Zahl der - zumindest relativ zur Entwicklung der Gesamtbevölkerung - aktiv Erwerbstätigen zu steigern. 
Sehr verdienstvoll ist es, daß der Verfasser bei seiner betont einzelwirtschaftlich fundierten Analyse dieses gesamtwirtschaftlichen Problems von den einschlägigen Kenntnissen der Arbeitswissenschaft ausgeht. In der vorliegenden Analyse geht es mithin zunächst um eine zukunftsbezogene Abschätzung insbesondere der Verwendung moderner Produktions- und Organisationsmethoden in der Wirkung auf die verschiedenen Möglichkeiten der Erhöhung der Erwerbsbeteiligung von Frauen und Älteren. Hierbei untersucht der Verfasser nicht die derzeit beobachtbaren konkreten Einzeltätigkeiten sondern versucht zukunftsorientierte Aussagen zu typischen bzw. durchschnittlichen künftigen Arbeitssystemen zu formulieren.

Im wesentlichen sind es die vier Fragenkomplexe, die in dieser Arbeit zu beantworten versucht werden:

- Welche gruppentypischen Eigenschaften haben die potentiellen Erwerbspersonen, und inwiefern wirken sich diese auf die Möglichkeit einer Erwerbstätigkeit aus?

- Welche gruppenspezifischen Problem bestehen hinsichtlich einer erhöhten Ausschöpfung des Erwerbspersonenpotentials?

- Wie wird die Arbeit im Produktions- und Bürobereich künftig organisiert sein, und wie ändern sich typische Arbeitsaufgaben bzw. Anforderungsprofile?

- Wie können durch Maßnahmen in den Unternehmen Hemmnisse, die der Aufnahme der Erwerbstätigkeit entgegenstehen, beseitigt und Fehlbeanspruchungen während der Erwerbstätigkeit, die ggf. zu einem frühzeitigen Ausscheiden aus dem Erwerbsleben führen können, abgemildert werden?

Da diese Fragestellungen gleichermaßen analytisch anspruchsvoll wie politisch hoch relevant sind, kann man der fundierten Arbeit von Herrn Lorenz nur viele kritische Leser wünschen.

Bert Rürup 


\section{Inhaltsverzeichnis}

0. Einleitung

A. Die Problemstellung und ihre Behandlung 1

B. Aufbau der Arbeit 5

I. Zukünftige Probleme auf dem Arbeitsmarkt und Lösungsoptionen aus mikroökonomischer Perspektive

A. Die prognostizierte Entwicklung auf dem Arbeitsmarkt 9

$\begin{array}{ll}\text { 1. Angebotsseite } & 10\end{array}$

a) Demographische Entwicklung 10

b) Entwicklung der Erwerbsquoten 14

2. Nachfrageseite 19

B. Defizite des zukünftigen Arbeitskräfteangebots 21

1. Quantitative Defizite 21

2. Qualitative Defizite 23

C. Optionen zur Erreichung einer verbesserten Situation 25

D. Analytisch-methodische Vorgehensweise $\quad 35$

E. Systematisierung möglicher Maßnahmen 39

1. Arbeitsgestaltung 41

2. Personalentwicklung 43

F. Arbeitssystembezogene und wirtschaftliche Kriterien 45

II. Arbeitspersonen - Arbeitsaufgaben - Arbeitssysteme : Spezifische Problemlagen als Ansatzpunkte und bestimmende Faktoren unternehmensbezogener Maßnahmen

A. Arbeitspersonen und ihre Eigenschaften $\quad 50$

1. Frauen und Erwerbstätigkeit 53

a) Kennzeichen der Erwerbstätigkeit von Frauen 53 
b) Beschäftigungsrelevante Eigenschaften von Frauen

(1) Fähigkeiten $\quad 57$

(2) Kenntnisse und Fertigkeiten $\quad 60$

(3) Verfügungsfreiheiten 64

c) Auswirkungen auf die Erwerbstätigkeit von Frauen 67

2. Ältere Personen und Erwerbstätigkeit 71

a) Kennzeichen der Erwerbstätigkeit älterer Personen 71

b) Beschäftigungsrelevante Eigenschaften älterer $\begin{array}{ll}\text { Personen } & 78\end{array}$

(1) Fähigkeiten $\quad 80$

(2) Kenntnisse und Fertigkeiten 83

(3) Verfügungsfreiheiten $\quad 85$

c) Auswirkungen auf die Erwerbstätigkeit älterer Personen $\quad 86$

3. Zwischenfazit $\quad 88$

B. Arbeitsaufgaben und Anforderungen $\quad 91$

1. Leitvorstellungen moderner Produktionsund Organisationsmethoden 95

a) CIM-Konzept $\quad 97$

(1) Veränderungen in der Produktion 99

(2) Veränderungen im Bürobereich 103

(3) Arbeitsaufgabenbezogene Auswirkungen 107

b) Lean Production 110

(1) Entwicklung und Ausrichtung des Konzeptes 110

(2) Umfeld-, unternehmensorganisationsund personalbezogene Vorstellungen $\quad 114$

2. Auswirkungen der zunehmenden Umsetzung der Methoden $\begin{array}{ll}\text { auf die Tätigkeitsanforderungen } & 118\end{array}$

3. Zwischenfazit 132

$\begin{array}{ll}\text { C. Arbeitssysteme und Beanspruchungen } & 134\end{array}$

1. Kennzeichen zukünftiger Arbeitssysteme 134

2. Beanspruchungsentwicklung und Ableitung von Maßnahmen 136

a) Maßnahmen für Frauen $\quad 137$

b) Maßnahmen für Ältere $\quad 141$

3. Unternehmensbezogene Maßnahmen im Überblick 145 


\section{Unternehmensbezogene Maßnahmen als} mikroökonomische Lösungsbeiträge

A. Handlungsträger und Interessen 149

B. Gestaltung und Beurteilung der Maßnahmen 155

1. Raster zur Betrachtung der Maßnahmen 155

a) Gestaltungsformen und -grundsätze 156

b) Wirtschaftliche Beurteilung 158

c) Möglichkeiten und Bedingungen der Umsetzung 160

2. Maßnahmen der Arbeitsgestaltung 161

a) Aufgabenbearbeitung 161

(1) Erfahrungswissen 161

(2) Gruppenarbeit 170

(3) Heimarbeit 177

b) Arbeitszeitkonzepte 182

c) Arbeitsschutz 194

d) Arbeitshilfen und Schonarbeit 199

3. Maßnahmen der Personalentwicklung 206

a) Weiterbildung 206

b) Frauenförderung 218

C. Integrierende Gesamtbetrachtung der behandelten Optionen 225

1. Widersprüche und Zielkonflikte 226

2. Kostenrelationen 228

3. Potentialabschätzung 231

a) Erhöhung der Erwerbsquote der Frauen 232

b) Erhöhung der Erwerbsquote älterer Personen 236

c) Auswirkungen auf die gesetzliche Rentenversicherung 240

D. Zeitliche Aspekte angesichts der demographischen Entwicklung 243

IV. SchluBbetrachtung 
Stefan Lorenz - 978-3-631-75027-8

Downloaded from PubFactory at 01/11/2019 09:06:41AM

via free access 


\section{Abbildungsverzeichnis}

Kapitel 0

Abb. 1: Übersicht des Aufbaus vorliegender Arbeit

\section{Kapitel I}

Abb. 2: Bestimmungsfaktoren der beiden Seiten des Arbeitsmarktes

Abb. 3: Entwicklung der Bevőlkerung in Deutschland, achte koordinierte

Bevölkerungsvorausberechnung (Quelle: SOMMER, 1994, 501)

Abb. 4: Rückgang der Gesamtbevölkerung und der Bevölkerung im Alter von 20 bis 60 Jahren in Deutschland, achte koordinierte Bevőlkerungsvorausberechnung (vgl. SOMMER, 1994, 501)

Abb. 5: Altenquotient in der Bundesrepublik / alte Bundeslănder und in der DDR / neue Bundesländer (Quelle: Bundesministerium für Familie und Senioren, 1994, nach BUSLEI, 1995, 18)

Abb. 6: Erwerbsbeteiligung deutscher Frauen (Quelle: SCHULZ/KIRNER, 1994, 80ff.)

Abb. 7: Anteil der Frauen an der Erwerbsbevölkerung und Erwerbsquote, 1991 (Quelle: EUROSTAT, 1993)

Abb. 8: Erwerbsbeteiligung deutscher Mănner (Quelle: Statistisches Bundesamt und Berechnungen des DIW nach SCHULZ/KIRNER, 1994, 86)

Abb. 9: Erwerbsbeteiligung der 60jährigen und ălteren Bevölkerung in Privathaushalten, 1991 (Quelle: Eurostat, Erhebung über Arbeitskrăfte 1991 nach STATISTISCHES BUNDESAMT, 1994a)

Abb. 10: Entwicklung der Qualifikationsanforderungen (vgl. eine entsprechende Darstellung in INSTITUT DER DEUTSCHEN WIRTSCHAFT, 1994, 5)

Abb. 11: Einflüsse auf den Arbeitsmarkt und resultierende Situationen

Abb. 12: Modell der menschlichen Arbeit

Abb. 13: Beziehungsmodell der menschlichen Arbeit und problematische Situationsausprägungen

Abb. 14: Systematik unternehmensbezogener Maßnahmen

Abb. 15: Dimensionen der Arbeitsgestaltung

Abb. 16: Aufgabenbereiche der Personalentwicklung

Abb. 17: Arbeitssystembezogene und wirtschaftliche Kriterien 
Kapitel II

Abb. 18: Beschăftigungsrelevante Eigenschaften der Arbeitspersonen

Abb. 19: Personen der Altersklasse von 20 bis unter 60 Jahren, Stand: April 1994

(Quelle: STATISTISCHES BUNDESAMT, MIKROZENSUS und

eigene Zusammenfassung)

Abb. 20: Erwerbsquoten nach Geschlecht und Gebiet, Stand: April 1994, Mikrozensus (Quelle: CORNELSEN, 1995)

Abb. 21: Erwerbsquoten deutscher Frauen nach Altersgruppen und Familienstand im zeitlichen Vergleich (Quelle: FRANZ, 1994, 21)

Abb. 22: Familienstandabhăngige Erwerbsquoten der Frauen nach Altersklassen, Stand: April 1993 (Quelle: STATISTISCHES BUNDESAMT, MIKROZENSUS)

Abb. 23: Verteilung der Schulabschlüsse nach Geschlecht im Alter von 15 bis unter 30 Jahren (Quelle: Mikrozensus 1993 - freiwillige Angaben, STATISTISCHES BUNDESAMT, 1994c)

Abb. 24: Anteil der Weiterbildungsteilnahme nach höchstem AusbildungsabschluB (Quelle: BIBB/IAB-Erhebung 1991/92 nach BERUFSBILDUNGSBERICHT, 1994, 125)

Abb. 25: Eintritte in die AFG-geforderte Weiterbildung 1992 nach Geschlecht (Quelle: Bundesanstalt für Arbeit nach BERUFSBILDUNGSBERICHT, 1994, 127)

Abb. 26: Anteile der Voll- und Teilzeitarbeit in den neuen und alten Bundesländern, Stand: April 1994 (Quelle: STATISTISCHES BUNDESAMT, MIKROZENSUS)

Abb. 27: Von Erwerbslosen gesuchte Tătigkeitsformen, Stand: April 1994 (Quelle: STATISTISCHES BUNDESAMT, MIKROZENSUS)

Abb. 28: Anteil der Teilzeitbeschaftigten, 1991 (Quelle: EUROSTAT, 1994)

Abb. 29: Gründe für die Suche von ausschlieblich männlichen Arbeitspersonen (Quelle: ENGELBRCH/KRAFT, 1992, 20)

Abb. 30: Lebenserwartung bei der Geburt und im Alter von 60 Jahren (Quelle: STATISTISCHES BUNDESAMT, JAHBÜCHER; Eurostat Bevolkerungsstatistik 1993 nach STATISTISCHES BUNDESAMT, 1994a)

Abb. 31: Erwerbsqoten deutscher Männer nach Altersgruppen im zeitlichen Vergleich (Quelle: FRANZ, 1994, 21)

Abb. 32: Mittleres Rentenzugangsalter (Quelle: VDR, 1993)

Abb. 33: Mittleres BU-/EU-Rentenzugangsalter (Quelle: VDR, 1993)

Abb. 34: Gesetzliche Rentenversicherung, Gliederung des Rentenzugangs 1993 wegen verminderter Erwerbsfahigkeit und wegen Alters nach Geschlecht für das frühere Bundesgebiet (abl) und das Gebiet Neue Lănder und Berlin-Ost (nbl) (Quelle: VDR, 1993) 
Abb. 36: Das Defizit- und das Kompetenzmodell des Alters im Überblick

Abb. 37: Verteilung der Schulabschlüsse (Quelle: Mikrozensus 1993 - freiwillige Angaben, STATISTISCHES BUNDESAMT, 1994c und eigene Zusammenfassung)

Abb. 38: Zeitaufwand für die berufliche Weiterbildung nach Alter und Gebiet je Teilnehmer/in und je Person der Gesamtgruppe (Quelle: Berichtssystem Weiterbildung, 1991 nach BERUFSBILDUNGSBERICHT, 1994, 126)

Abb. 39: Arbeitsmarktbezogene Kennzeichen für die Erwerbstătigkeit von Frauen

Abb. 40: Arbeitsmarktbezogene Kennzeichen für die Erwerbstătigkeit von Älteren

Abb. 41: Auf das Unternehmen einflußnehmende Disziplinen

Abb. 42: Einflußfaktoren, Wettbewerbsmerkmale und Wettbewerbsdimensionen

Abb. 43: Erwartungen und Ziele des Einsatzes moderner Methoden

Abb. 44: Konzepte der automatisierten Fertigung (Quelle: CRONJÄGER, 1990, 78)

Abb. 45: Direkte und indirekte Funktionen im Produktionsbereich

Abb. 46: Bestandteile eines CIM-Systems (in Anlehnung an CRONJÄGER, 1990, 9 und 112)

Abb. 47: Leistungsmerkmale der Montagewerke, Großserienhersteller, 1989 (Durchschnitt der Fabriken je Region) (Quelle: WOMACK u.a., 1991, 97)

Abb. 48: Merkmale des Arbeitskrăfteeinsatzes der Montagewerke, Großserienhersteller, 1989 (Durchschnitt der Fabriken je Region) (Quelle: WOMACK u.a., 1991, 97)

Abb. 49: Umfeld-, unternehmensorganisations- und personalbezogene Vorstellungen des Lean-Konzeptes

Abb. 50: Anforderungen als Einflubfaktor der Arbeitsgestaltung

Abb. 51: Unternehmensbezogene Maßnahmen zur Erhőhung der Erwerbsbeteiligung 146

Abb. 52: Beeinflussung und Gestaltung der Arbeitssysteme

\section{Kapitel III}

Abb. 53: Mikrookonomische und staatliche Anreizmechanismen

Abb. 54: Grundlegende arbeitswissenschaftliche Vorstellungen und Ziele

Abb. 55: Ziele und Bestimmungsfaktoren unternehmensbezogener Maßnahmen

Abb. 56: Schwerpunkte technischer Entwicklungen für die Unterstützung erfahrungsgeleiteter Arbeit in der Werkstatt (Quelle: LENNARTZ, 1993, 75)

Abb. 57: Zielbeeinflussung durch die Umsetzung der MaBnahme Erfahrungswissen

Abb. 58: Idealtypische Vorstellungen zur Gestaltung und Wirkung der Gruppenarbeit

Abb. 59: Einordnung der Gruppenarbeit in arbeits- bzw. produktionsund organisationsbezogene Vorstellungen 
Abb. 60: Zielbeeinflussung durch die Umsetzung der Maßnahme Gruppenarbeit 176

Abb. 61: Zielbeeinflussung durch die Umsetzung der Maßnahme Heimarbeit

Abb. 62: Motive für den Wunsch nach Teilzeitarbeit

(Quelle: SCHULZE BUSCHOFF, 1994, nach SOEP-West 1990)

Abb. 63: Zielbeeinflussung durch die Umsetzung der Arbeitszeitkonzepte

Abb. 64: Anteile mittelständischer Unternehmen verschiedener Branchen, die

Teilzeitstellen aus den angegebenen Gründen anbieten (Quelle:

Creditreform nach INSTITUT DER DEUTSCHEN WIRTSCHAFT, 1994)

Abb. 65: Zielbeeinflussung durch die Umsetzung der MaBnahme Arbeitsschutz

Abb. 66: Zielbeeinflussung durch die Umsetzung der Maßnahme Arbeitshilfen

Abb. 67: Prozentuale Teilnahmequoten verschiedener Gruppen von Erwerbstătigen an Maßnahmen der beruflichen Weiterbildung in den alten Bundesländern Ergebnisse einer Kontrastgruppenanalyse (Berichtssystem Weiterbildung, BSW 1991), (Quelle: STRAKA/KUWAN, 1994)

Abb. 68: Zielbeeinflussung durch die Umsetzung der MaBnahme Weiterbildung

Abb. 69: Beispiele für Vereinbarungen zur Frauenforderung

Abb. 70: Zielbeeinflussung durch die Umsetzung der Maßnahme Frauenforderung

Abb. 71: Bildung zweier Maßnahmenbündel zur Erhőhung der Erwerbsbeteiligung von Frauen

Abb. 72: Erwerbstătigkeit von Mănnern und Frauen im Alter von 25 bis 55 Jahren im Jahre 1993

Abb. 73: Geschătzte zukünftig erreichbare Ausweitung der Zahl der erwerbstătigen Frauen

Abb. 74: Beitrăge der Maßnahmen zur Ausweitung der Erwerbsbeteiligung von Frauen jüngerer und mittlerer Altersklassen

Abb. 75: Bildung zweier Maßnahmenbündel zur Erhöhung der Erwerbsbeteiligung Älterer

Abb. 76: Erwerbstătigkeit und Rentenzugang Älterer im Jahre 1993

Abb. 77: Geschătzte zukünftige Rentenzugănge Älterer unter Beibehaltung der Erwerbsquoten und Rentenzugangsanteile von 1993

Abb. 78: Geschătzte zukünftig mőgliche Erhøhung der Zahl ălterer Erwerbspersonen

Abb. 79: Geschătzte zukünftig erreichbare Erwerbsquoten Älterer

Abb. 80: Beitrăge der Maßnahmen zur Ausweitung der Erwerbsbeteiligung Älterer

Abb. 81: Verănderung des zukünftigen Belastungsanstiegs durch die Ausschőpfung der aufgezeigten Potentiale

Abb. 82: Möglichkeit einer zeitlichen Systematisierung

Abb. 83: Zeitliche Einordnung der Maßnahmenumsetzung und ihrer Wirkungen 
Kapitel IV

Abb. 84: Zusammenfassende Einschătzung der Beeinflussung wesentlicher

Unternehmensziele durch die Umsetzung der Arbeitsgestaltungs-

und Personalentwicklungsmaßnahmen 
Stefan Lorenz - 978-3-631-75027-8

Downloaded from PubFactory at 01/11/2019 09:06:41AM

via free access 


\section{Einleitung}

\section{A. Die Problemstellung und ihre Behandlung}

Die Problemstellung, mit der sich die vorliegende Arbeit beschäftigt, läßt sich kurz gefaßt in folgende Frage kleiden:

Was kann in den Unternehmen arbeitsgestalterisch getan werden, um die zukünftige Erwerbsbeteiligung von Frauen und Älteren zu erhöhen?

Diese Frage ergibt sich aus verschiedenen langfristig feststellbaren, arbeitsmarktbezogenen Dysfunktionalitäten. Die Betrachtung und Einschätzung der bisherigen und der prognostizierten Entwicklungen der Erwerbsbeteiligung und der Erwerbsarbeit decken zukünftig entstehende und sich verschärfende Problemlagen auf, die eine erhebliche ökonomische Relevanz besitzen. Ansätze zur Lösung dieser Probleme können makro- oder mikroökonomisch geprägt sein. Die vorgelegte Arbeit ist mikroökonomisch ausgerichtet und konzentriert sich auf die im Bereich der Unternehmen umzusetzenden Maßnahmen, zur Minderung der mit dem Angebot an Arbeitskräften verbundenen Probleme. Hierzu wird der Zusammenhang von Arbeitsgestaltung und Erwerbsleben beleuchtet und insbesondere die Erwerbstätigkeit von Frauen und Älteren berücksichtigt. Einleitend kann die Problemstellung anhand der folgenden Punkte entwickelt werden:

- Das durchschnittliche Renteneintrittsalter und die Erwerbsbeteiligung höherer Altersgruppen gingen in den vergangenen Jahren deutlich zurück. Betrachtet man die Erwerbsquoten, die die Anteile von erwerbstätigen und erwerbslosen Personen an der Bevölkerung insgesamt oder einer bestimmten Gruppe darstellen, nach Altersklassen getrennt, so wird dieser Rückgang deutlich. Er zeigt sich beispielsweise bei einem Vergleich der Erwerbsquoten von Frauen und Männern im Alter von 60 bis 65 Jahren von 1970 und von 1994 für das frühere Bundesgebiet. Die Erwerbsquote der Frauen ging in diesem Zeitraum von ca. $23 \%$ auf ca. $12 \%$ und die der Männer von ca. $75 \%$ auf ca. $33 \%$ zurück (vgl. STATISTISCHES BUNDESAMT, JAHRBUCH; CORNELSEN, 1995).

- Weiterhin kann festgestellt werden, daß die Erwerbsquoten der Frauen in der Bundesrepublik verglichen mit denen der Männer und je nach Wahl der betrachteten Länder auch im internationalen Vergleich relativ niedrig sind. So betrug die Gesamterwerbsquote der Männer in Deutschland im April 1994 
$58,3 \%$ und die der Frauen 41,1\%. Dabei lag sie bei den Frauen in den neuen Bundesländern mit $48,3 \%$ deutlich höher als im früheren Bundesgebiet mit 39,4\% (vgl. CORNELSEN, 1995). Die Erwerbsbeteiligung zwischen Männern und Frauen ist demnach noch immer sehr unterschiedlich obwohl die Erwerbsquoten der Frauen in der Vergangenheit besonders in den mittleren Altersklassen deutlich angestiegen sind. So erhöhten sich beispielsweise bezogen auf das frühere Bundesgebiet von 1970 bis 1994 die Erwerbsquoten von Frauen der Altersgruppen von 30 bis 45 Jahren um über die Hälfte (auf je nach Altersklasse 69-74\%), während die der Männer dieser Altersgruppen leicht zurückgegangen sind (je nach Altersklasse auf 96-97\%).

- Gesamtwirtschaftlich stellen die relativ niedrige Erwerbsbeteiligung von älteren Frauen und Männern sowie der Frauen insgesamt eine Vergeudung von Humankapital dar. Zur Vermeidung von Beeinträchtigungen bzw. zur Förderung der wirtschaftlichen Entwicklung müssen für diese Probleme mikro- und makroökonomische Lösungen entwickelt und Maßnahmen abgeleitet werden.

- Für die Senkung des faktischen Renteneintrittsalters können verschiedene Gründe angeführt werden, die auf eine gewisse Übereinstimmung der Interessenlagen von Arbeitgebern, Arbeitnehmern und Staat verweisen. In der Situation der Arbeitslosigkeit besteht gerade gegenüber der Ausgliederung von Älteren aus der Erwerbstätigkeit ein vergleichsweise geringer Widerstand. Verbunden mit der Vermeidung von Neueinstellungen ermöglicht sie eine einfache Anpassung der Beschäftigung. Die jugendzentrierte Personalpolitik der Unternehmen erleichtert darüber hinaus die Aktualisierung der Qualifikation der Beschäftigten solange ausreichend qualifizierter bzw. qualifizierbarer Nachwuchs vorhanden ist und senkt wegen der tendenziell höheren Entlohnung Älterer die Personalkosten. Der (vorzeitige) Renteneintritt bietet den Arbeitnehmern verglichen mit der Arbeitslosigkeit einen anerkannten Status und ermöglicht es, beruflichen Anforderungen und Diskriminierungen auszuweichen.

- Als Gründe für die niedrigere Erwerbstätigkeit der Frauen können neben der traditionellen geschlechtsspezifischen Arbeitsteilung besonders die ungenügende familienbezogene Infrastruktur und die herrschende Arbeitslosigkeit genannt werden. Die geringere Beteiligung der Frauen an der Erwerbsarbeit bedeutet eine Entlastung der erwerbstätigen (Ehe-) Männer von außerberuflichen Arbeiten und Verpflichtungen, die, zumindest solange genügend Arbeitskräfte vorhanden sind, aus Sicht der Unternehmen günstig beurteilt werden kann. 
Darüber hinaus mindern geringere Erwerbsquoten der Frauen das Arbeitskräfteangebot, so daß die Arbeitslosenquote bei bestehender Arbeitslosigkeit verringert wird. Auch wenn sich die Erwerbsbeteiligung der Frauen in der Vergangenheit erhöht hat, fehlen ausreichend wirksam werdende Anreize zur Angleichung der geschlechtsspezifischen Erwerbsquoten. Weiterhin bestehen unternehmensbezogene Vorbehalte gegenüber der Beschäftigung von Frauen.

- Die Erwerbstätigkeit von Älteren und Frauen und die sie beeinflussenden Faktoren gewinnen durch die zukünftigen Veränderungen von Angebot und Nachfrage auf dem Arbeitsmarkt an Bedeutung. Bezüglich des Arbeitskräfteangebots muß insbesondere auf den demographischen Wandel verwiesen werden, der zur Schrumpfung und Alterung der Bevölkerung führt und erhebliche Verschiebungen in der Zusammensetzung des Erwerbspersonenpotentials und einen möglichen sich verbreitenden Mangel an Arbeitskräften nach sich zieht. Hinsichtlich der zukünftigen Nachfrage nach Arbeitskräften sind der Strukturwandel und die Veränderungen der Organisations- und Produktionsmethoden in den Unternehmen wesentlich. Bei der Betrachtung der zukünftigen Arbeitsmarktsituation zeichnen sich aufgrund dieser Entwicklungen quantitative und qualitative Defizite des Arbeitskräfteangebots ab. Diesen Defiziten können zur Kompensation mehrere Optionen gegenübergestellt werden, die zu ihrer Realisierung die Ergreifung verschiedener makro- und mikroökonomischer Maßnahmen erfordern.

Vorliegende Arbeit behandelt die Optionen, die sich auf die Erhöhung der Erwerbsbeteiligung von Frauen und von Älteren richten, zumal diese Gruppen besondere arbeitsmarktbezogene Probleme haben und wachsende Anteile am Erwerbspersonenpotential stellen. Die Arbeit konzentriert sich auf die Bestimmung und Beurteilung innerhalb der Unternehmen umsetzbarer mikroökonomischer Maßnahmen zur einzelwirtschaftlichen Sicherung des den Unternehmen zur Verfügung stehenden Humankaptials und zur gesamtwirtschaftlich sinnvollen Vermeidung der Humankapitalvergeudung.

Die mikroökonomischen Maßnahmen sind auf eine Änderung der jugendzentrierten Personalpolitik der Unternehmen und eine Verbesserung der Erwerbschancen der Frauen gerichtet. Sie müssen sich aus Unternehmenssicht besonders an zwei miteinander verknüpften Zielen orientieren. Zum einen muß dem prognostizierten demographischen Wandel hinsichtlich seiner quantitativen und qualitativen Auswirkungen auf das Arbeitskräfteangebot begegnet werden. Zum zweiten müssen die 
personellen Voraussetzungen der im Interesse der Wettbewerbsfähigkeit der Unternehmen einzuführenden Organisations- und Produktionsmethoden gesichert werden.

Zur Ableitung und Gestaltung unternehmensbezogener Maßnahmen soll der Zusammenhang von Arbeitsgestaltung und Erwerbstätigkeit herausgearbeitet werden. Von entscheidender Bedeutung hierfür sind die Bedürfnisse der Beschäftigten und die Ziele der Unternehmen. Die entsprechend der spezifischen Problemlagen entwickelten Maßnahmen werden anschließend besonders aus Sicht der Arbeitgeber hinsichtlich ihrer Gestaltungsformen, ihrer wirtschaftlichen Auswirkungen und ihrer Umsetzungsbedingungen betrachtet werden.

Zumindest kurz- und mittelfristig sind in gesamtwirtschaftlichem Interesse staatliche Anreize sinnvoll, um eine rechtzeitige Umsetzung unternehmensbezogener Maßnahmen zu ermöglichen, da in der Situation der Arbeitslosigkeit einzelwirtschaftlich noch in zu geringem Maße Anreize zur Erhöhung der Erwerbsbeteiligung von Älteren und Frauen bestehen. Die zukünftig aufgrund des demographischen Wandels und der Verschiebungen der qualitativen Arbeitskräftenachfrage entstehenden möglichen Defizite können andererseits die Chancen für eine Änderung der arbeitsbezogenen Handlungs- und Gestaltungsweisen in den Unternehmen erhöhen. Ziele wie altersgerechte Arbeitsplätze und umfangreiche Belastungsanpassungen, die bisher primär im Interesse der Humanisierung der Arbeit sowie Ziele der Verbesserung der Erwerbschancen der Frauen und der Vereinbarkeit von Familie und Beruf, die bisher primär im Interesse der geschlechtlichen Gleichberechtigung erhoben wurden, können aufgrund der sich abzeichnenden Defizite des Arbeitskräfteangebots zusätzlich ökonomisch an Bedeutung gewinnen, so daß die Umsetzungschancen von Maßnahmen zur Erreichung dieser wichtigen gesellschaftlichen Ziele zukünftig steigen können.

Vorhandene Studien diskutieren die Gestaltung der Arbeit insbesondere mit dem Ziel der "Humanisierung des Arbeitslebens" (Titel des 1974 begonnenen Forschungsprogramms der Bundesregierung, das 1989 durch das Programm "Arbeit und Technik" abgelöst wurde) und behandeln beispielsweise die Verteilung der Tätigkeiten zwischen Arbeitspersonen und Maschinen. Ausgangspunkt vieler Studien sind die Arbeitsaufgaben unter Berücksichtigung der Fähigkeiten durchschnittlicher Arbeitspersonen. Seltener Beachtung finden gruppentypische Eigenschaften, Eigenschaftsveränderungen und außerberufliche Anforderungen. Ausgehend von den Arbeitspersonen werden diese Punkte in vorliegender Arbeit ergänzend betont, um die Möglichkeiten der Fortsetzung bzw. Aufnahme einer 
Erwerbstätigkeit gruppenbezogen zu erweitern. Entsprechend dieser unterschiedlichen Perspektive stehen hier an Stelle von Aussagen zu bestimmten Berufsgruppen Aussagen zu bestimmten Arbeitspersonengruppen, nämlich zu Frauen und Älteren, im Vordergrund. Im Gegensatz zu arbeitsanalytischen Studien und Empfehlungen sollen hier keine einzelnen, konkreten Arbeitsaufgaben oder Arbeitsplätze gestaltet werden, sondern unternehmensbezogene Maßnahmen bestimmt und beurteilt werden, die eine breitere Gültigkeit aufweisen und sich an den modernen Organisations- und Produktionsmethoden sowie den gruppenspezifischen Bedürfnissen von Frauen und Älteren orientieren. Die Konzeption bewegt sich daher zwischen der Gestaltung spezieller Einzellösungen und der Behandlung allgemeiner Lösungsvorschläge, die sich einseitig an den Zielen der Steigerung der technisch-wirtschaftlichen Effizienz oder der Humanisierung orientieren.

\section{B. Aufbau der Arbeit}

In Kapitel I wird die grundlegende Problemstellung umrissen, indem die zukünftige Arbeitsmarktsituation betrachtet und die voraussichtlichen Defizite festgestellt werden. Nach der Zusammenstellung denkbarer Optionen zur Verbesserung der zukünftigen Situation wird die mikroökonomische Auswahl der auf Frauen und Ältere gerichteten Optionen begründet. Anschließend werden die analytischmethodische Vorgehensweise im Rahmen dieser Arbeit und eine Systematisierung möglicher unternehmensbezogener Maßnahmen dargestellt, bevor Kriterien zur Ableitung und Beurteilung geeigneter Maßnahmen angeführt werden.

Die gegenwärtige Situation und die sich abzeichnende Entwicklung hinsichtlich der Bedürfnisse der Beschäftigten und der Ziele der Unternehmen werden in Kapitel II vertiefend analysiert. Die Betrachtung von Arbeitspersonen, Arbeitsaufgaben und Arbeitssystemen erfolgt zur Offenlegung spezifischer Problemlagen, die Ansatzpunkte und Bedingungen für die unternehmensbezogenen Maßnahmen darstellen. Der damit verdeutlichte Zusammenhang von Arbeitsgestaltung sowie Personalentwicklung und Erwerbstätigkeit bildet die Grundlage für die mikroökonomische Beurteilung der Maßnahmen.

Die Gestaltungsformen und die Wirkungen des Einsatzes der Maßnahmen sowie die relevanten Kosten- und Nutzengrößen werden in Kapitel III vornehmlich aus Sicht der Arbeitgeber erörtert. Hierzu werden die einzelnen Maßnahmen konkretisiert beschrieben und beurteilt. Anschließend erfolgt eine integrierende Betrachtung der 
Maßnahmen mit Blick auf ihren Beitrag zur Realisierung der ausgewählten Optionen. Dabei müssen Widersprüche und Zielkonflikte angesprochen und quantitative Lösungsbeiträge abgeschätzt werden. Schließlich erfolgt eine zeitliche Einordnung der Maßnahmen aus einzelwirtschaftlicher Sicht.

Aufgrund der durchgeführten Analyse wird in Kapitel IV die als notwendig erachtete Entwicklung arbeitsbezogener Handlungs- und Gestaltungsweisen in einer Schlußbetrachtung zusammenfassend dargestellt.

Die folgende Abbildung stellt den Aufbau der Arbeit in einer Übersicht dar. 


\begin{tabular}{|ll|}
\hline 0. & Einleitung \\
\hline A. & Die Problemstellung und ihre Behandlung \\
\hline B. & Aufbau der Arbeit \\
\hline
\end{tabular}

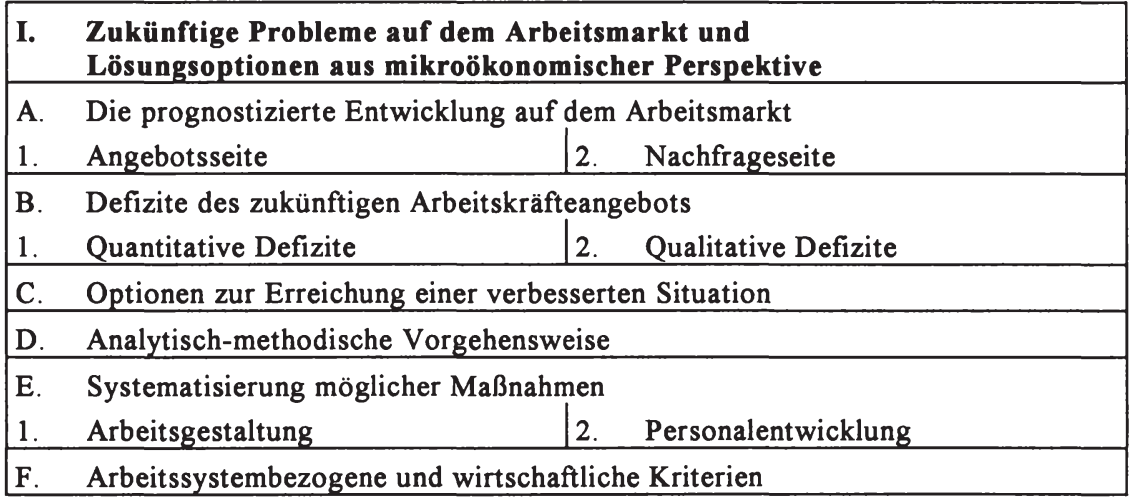

II. Arbeitspersonen - Arbeitsaufgaben - Arbeitssysteme : Spezifische Problemlagen als Ansatzpunkte und bestimmende Faktoren unternehmensbezogener Maßnahmen

A. Arbeitspersonen und ihre Eigenschaften

\begin{tabular}{l|l} 
1. Frauen und Erwerbstätigkeit & 2. $\begin{array}{l}\text { Ältere Personen und Erwerbs- } \\
\text { tätigkeit }\end{array}$
\end{tabular}

B. Arbeitsaufgaben und Anforderungen
\begin{tabular}{l|l} 
1. Leitvorstellungen moderner & 2. Auswirkungen der zunehmenden
\end{tabular} Produktions- und Organisations- $\quad$ Umsetzung der Methoden auf die methoden Tätigkeitsanforderungen

C. Arbeitssysteme und Beanspruchungen
1. Kennzeichen zukünftiger Arbeits- $\quad$ 2. Beanspruchungsentwicklung und systeme Ableitung von Maßnahmen

III. Unternehmensbezogene Maßnahmen als mikroökonomische Lösungsbeiträge

A. Handlungsträger und Interessen

B. Gestaltung und Beurteilung der Maßnahmen

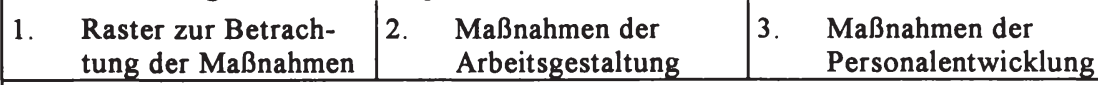

C. Integrierende Gesamtbetrachtung der behandelten Optionen

D. Zeitliche Aspekte angesichts der demographischen Entwicklung

\section{Schlußbetrachtung}

Abb. 1: Übersicht des Aufbaus vorliegender Arbeit 
Stefan Lorenz - 978-3-631-75027-8

Downloaded from PubFactory at 01/11/2019 09:06:41AM

via free access 


\section{Zukünftige Probleme auf dem Arbeitsmarkt und Lösungsoptionen aus mikroökonomischer Perspektive}

\section{A. Die prognostizierte Entwicklung auf dem Arbeitsmarkt}

Die menschliche Arbeit wird als Produktionsfaktor unter technisch-organisatorischen Bedingungen zur Erreichung bestimmter Zielsetzungen verwendet. $\mathrm{Ihr}$ Leistungsbeitrag ist hierbei besonders von der individuellen physischen und psychischen Leistungsfähigkeit sowie der Bereitschaft diese einzusetzen abhängig. Die Verrichtung von Arbeit gegen Zahlung eines Entgelts bedeutet die Herausbildung der Erwerbsarbeit und begründet die Institution des Arbeitsmarktes. Zur Darstellung der Entwicklung auf dem Arbeitsmarkt können die beiden Marktseiten, das Angebot an Arbeitskräften und die Nachfrage nach Arbeitskräften zur Besetzung betrieblicher Arbeitsplätze, betrachtet werden, um wesentliche Merkmale der prognostizierten Arbeitsmarktsituation und die hiermit zusammenhängenden Defizite zu erkennen. Die folgende Abbildung nennt miteinander verknüpfte Wirkfaktoren der beiden Marktseiten.

\begin{tabular}{|c|c|}
\hline \multicolumn{2}{|c|}{ Die Seiten des Arbeitsmarktes } \\
\hline Angebotsseite & $\begin{array}{r}\text { Nachfrageseite } \\
\end{array}$ \\
\hline $\begin{array}{l}\text { Das Arbeitskräfteangebot wird quantitativ und } \\
\text { hinsichtlich seiner Zusammensetzung durch } \\
\text { das Erwerbspersonenpotential und damit } \\
\text { durch die demographische Entwicklung } \\
\text { sowie } \\
\text { die gruppenspezifischen Erwerbsquoten } \\
\text { aufgrund der Entwicklung der Erwerbs- } \\
\text { neigungen bestimmt. }\end{array}$ & $\begin{array}{l}\text { Die Nachfrage nach Arbeitskräften wird } \\
\text { quantitativ und qualitativ durch } \\
\text { die Entwicklung der Produktivităt und des } \\
\text { Wirtschaftswachstums sowie } \\
\text { die Verănderungen der mit den Tătig- } \\
\text { keiten verbundenen Anforderungen } \\
\text { aufgrund der Entwicklung der angewen- } \\
\text { deten Produktions- und Organisations- } \\
\text { methoden bestimmt. }\end{array}$ \\
\hline
\end{tabular}

Abb. 2: Bestimmungsfaktoren der beiden Seiten des Arbeitsmarktes

Die folgenden Betrachtungen beziehen sich auf den gesamtdeutschen Arbeitsmarkt. Gleichwohl wird vereinzelt auf mikroökonomisch relevant erscheinende Unterschiede zwischen der Erwerbsarbeit in den alten und den neuen Bundesländern verwiesen. Insgesamt kann zumindest langfristig und für die herauszuarbeitenden Bestimmungsgrößen der unternehmensbezogenen Maßnahmen von einem Abbau noch bestehender Unterschiede ausgegangen werden. 


\section{Angebotsseite}

Quantitative und strukturbezogene Veränderungen des Arbeitskräfteangebots beruhen auf demographischen und erwerbsquotenbezogenen Entwicklungstendenzen.

\section{a) Demographische Entwicklung}

Die Bevölkerungsentwicklung wird durch Fertilitäts-, Mortalitäts- und Wanderungsraten bestimmt. Während sich die natürlichen Veränderungen aufgrund ihrer zumindest zeitweisen Konstanz und der Langfristigkeit ihrer Auswirkungen recht genau prognostizieren lassen, bestehen besondere Prognoseunsicherheiten hinsichtlich der Einschätzung der u.a. politisch bestimmten Höhe der Zuwanderung (vgl. beispielsweise SCHULZ, 1993; SOMMER, 1992 und 1994; VON ROTHKIRCH, 1993). Allerdings läßt sich feststellen, daß die politisch umsetzbare Zuwanderung nicht so hoch sein wird, als daß sie die Auswirkungen der natürlichen demographischen Veränderungen kompensieren könnte (vgl. die Simulationsrechnungen von STEINMANN, 1993; die Modellrechnungen der Bundesforschungsanstalt für Landeskunde und Raumordnung, BUCHER/KOCKS, 1991 und eine Zusammenfassung entsprechender Studien für verschiedene EG-Länder, COLEMAN, 1992, 450ff.).

Die seit langem nicht bestandserhaltende Geburtenrate und die gestiegene Lebenserwartung (vgl. beispielsweise MIEGEL/WAHL, 1993, 84ff; HÖHN/HULLEN, 1993 und DINKEL, 1992) führen in Deutschland zur Schrumpfung und Alterung der Bevölkerung. Diese Phänomene verschärfen vorhandene und bewirken zusätzliche gesellschaftliche Probleme. Dabei ist der Prozeß der demographischen Alterung, der durch die Zuwanderung lediglich zeitlich verzögert wird, von größerer Bedeutung als der zu erwartende Rückgang der Bevölkerungszahl. Der demographische Entwicklungsprozeß verläuft in den übrigen europäischen Staaten (mit Ausnahme Irlands) ähnlich, wenngleich er in Deutschland besonders stark ausgeprägt ist (vgl. HOF, 1993; BIRG, 1989). In Deutschland entwickelte Lösungen für die mit dem demographischen Wandel zusammenhängenden Probleme können daher Modellfunktion für andere Gesellschaften erlangen (vgl. MIEGEL/WAHL, 1993, 93ff.).

Zur Darstellung der demographischen Entwicklung können die Ergebnisse der achten koordinierten Bevölkerungsvorausberechnung des Statistischen Bundesamtes und der Statistischen Landesämter herangezogen werden (vgl. SOMMER, 
1994). Die Vorausberechnung bezieht sich mit dem Basisjahr 1992 auf den Zeitraum 1993 bis 2040 und geht aufgrund der unwägbaren Höhe der Außenwanderung von drei Varianten aus. Der Modellrechnung wurden verschiedene Annahmen zugrundegelegt, die für das frühere Bundesgebiet und die neuen Länder einschließlich des Ostteils Berlins zunächst voneinander abweichen. Für die alten Länder wird mit einer konstanten Geburtenziffer des Jahres 1992 gerechnet. Für die neuen Länder wird eine weitere Abnahme und ab 1996 ein allmähliches Wiederansteigen der Geburtenhäufigkeit angenommen, so daß sich die Geburtenziffer je nach Annahme der neuen Länder spätestens im Jahr 2010 an das frühere Bundesgebiet angeglichen haben wird. Hinsichtlich der Sterblichkeit wird für die alten Länder bis zum Jahr 2000 von einer um über 1,5 Jahre steigenden Lebenserwartung ausgegangen. Der Angleichungsproze $B$ an die für die alten Länder verwendeten Werte wird in den neuen Ländern als zumindest bis 2030 dauernd angenommen. Bezüglich der Binnenwanderung wird ein allmählicher Rückgang und ab 2010 ein Ausgleich erwartet. Für die Außenwanderung der deutschen Bevölkerung wird angenommen, daß die Zuzüge zunächst konstant hoch bleiben, zum Ende des Jahrzehnts absinken und ab 2011 ausgeglichen sind. Für das Ausmaß der Wanderung der Ausländer werden drei Varianten berechnet. Der angenomme Zuwanderungsüberschuß beträgt ab dem Jahr 2000 jährlich 100.000 (in Variante 1), 200.000 (in Variante 2) bzw. 300.000 (in Variante 3). Die Berechnung dieser unterschiedlichen Varianten erfolgt aufgrund der angesprochenen besonderen Unsicherheit der zukünftigen Entwicklung der Zuwanderungshöhe und der entsprechenden Schwankungen in der Vergangenheit. Das Ergebnis der Vorausberechnung soll damit ein breites Spektrum möglicher Entwicklungen darstellen.

Im Ergebnis zeigt die Vorausberechnung, daß die Bevölkerungszahl im Jahr 2040 je nach Variante bei 67,6 bis 77,1 Millionen und damit zwischen $16,5 \%$ und 4,8\% unter dem Ausgangswert von 81 Millionen Ende 1992 liegen wird. Dieser Rückgang der Zahl der in Deutschland lebenden Personen müßte ohne die Zuwanderung noch deutlich höher prognostiziert werden. Ökonomisch problematischer als dieser unterschiedlich hohe Rückgang der Bevölkerungszahl ist allerdings die erhebliche Veränderung des Altersaufbaus der Bevölkerung. Die Zahl der unter 20jährigen wird etwa ab 1998 ständig abnehmen; ihr Anteil an der Bevölkerung geht von 21,5\% 1992 auf ca. 16\% im Jahr 2040 zurück. Die Zahl der 20- bis 60jährigen bewegt sich je nach Variante unterschiedlich; ihr Anteil an der Bevölkerung geht nach allen Varianten von 58,1\% 1992 auf ca. 50\% im Jahr 2040 zurück. Die Zahl der über 60jährigen wird bis 2031 zunehmen und erst danach 
zurückgehen; ihr Anteil an der schrumpfenden Bevölkerung wird von 20,4\% 1992 je nach Variante auf 33 bis 35\% 2040 deutlich steigen.

Zur Illustration der tendenziellen Entwicklung enthält folgende Tabelle neben den altersgruppenbezogenen Gesamtzahlen und des jeweiligen prozentualen Anteils an der gesamten Bevölkerung auch den Jugendquotienten (Anteil der Bevölkerung im Alter von unter 20 Jahren an der Bevölkerung im Alter von 20 bis unter 60 Jahren), den Altenquotienten (Anteil der Bevölkerung im Alter ab 60 Jahren an der Bevölkerung im Alter von 20 bis unter 60 Jahren) und den Gesamtquotienten (Anteil der Bevölkerung im Alter von unter 20 und ab 60 Jahren an der Bevölkerung im Alter von 20 bis unter 60 Jahren).

\begin{tabular}{|c|c|c|c|c|c|c|c|c|c|c|}
\hline \multirow{2}{*}{$\begin{array}{c}\text { Ende } \\
\text { des } \\
\text { Jahres }\end{array}$} & \multirow{2}{*}{$\begin{array}{c}\text { Ins- } \\
\text { ressmt } \\
\text { In } 1.000 \\
\end{array}$} & \multicolumn{2}{|c|}{ unter 20 Jahren } & \multicolumn{2}{|c|}{ 20-60 Jahre } & \multicolumn{2}{|c|}{$\begin{array}{r}60 \text { Jahre } \\
\text { und glter } \\
\end{array}$} & \multirow{2}{*}{$\begin{array}{l}\text { Jugend- } \\
\text { quotlent }\end{array}$} & \multirow{2}{*}{$\begin{array}{c}\text { Alten- } \\
\text { quotient }\end{array}$} & \multirow{2}{*}{$\begin{array}{l}\text { Gesamt- } \\
\text { quotient }\end{array}$} \\
\hline & & in 1.000 & in $\%$ & in 1.000 & In $\%$ & In 1.000 & in $\%$ & & & \\
\hline \multicolumn{11}{|c|}{ Basisbevőlkerung der Modellrechnungen } \\
\hline 1992 & $80.974,6$ & $17.402,8$ & 21,5 & $47.072,8$ & 58,1 & $16.499,0$ & 20,4 & 37,0 & 35,0 & 72,0 \\
\hline \multicolumn{11}{|c|}{ Variante 1} \\
\hline 2000 & $83.347,4$ & $17.661,4$ & 21,2 & $46.387,0$ & 55,7 & $19.299,1$ & 23,2 & 38,1 & 41,6 & 79,7 \\
\hline 2010 & $81.960,3$ & $15.169,6$ & 18,5 & $46.096,0$ & 56,2 & $20.694,6$ & 25,2 & 32,9 & 44,9 & 77,8 \\
\hline 2020 & $78.581,0$ & $13.355,8$ & 17,0 & $42.554,5$ & 54,2 & $22.670,7$ & 28,9 & 31,4 & 53,3 & 84,7 \\
\hline 2030 & $73.677,3$ & $12.287,9$ & 16,7 & $35.870,6$ & 48,7 & $25.518,9$ & 34,6 & 34,3 & 71,1 & 105,4 \\
\hline 2040 & $67.580,2$ & $10.758,6$ & 15,9 & $33.198,0$ & 49,1 & $23.623,5$ & 35,0 & 32,4 & 71,2 & 103,6 \\
\hline \multicolumn{11}{|c|}{ Variante 2} \\
\hline 2000 & $83.740,5$ & $17.756,9$ & 21,2 & $46.662,1$ & 55,7 & $19.321,5$ & 23,1 & 38,1 & 41,4 & 79,5 \\
\hline 2010 & $83.433,0$ & $15.504,9$ & 18,6 & $47.129,1$ & 56,5 & $20.799,0$ & 24,9 & 32,9 & 44,1 & 77,0 \\
\hline 2020 & $81.183,4$ & $13.911,6$ & 17,1 & $44.356,4$ & 54,6 & $22.915,3$ & 28,2 & 31,4 & 51,7 & 83,0 \\
\hline 2030 & $77.413,5$ & $13.019,8$ & 16,8 & $38.370,2$ & 49,6 & $26.023,4$ & 33,6 & 33,9 & 67,8 & 101,8 \\
\hline 2040 & $72.413,0$ & $11.649,5$ & 16,1 & $36.217,1$ & 50,0 & $24.546,4$ & 33,9 & 32,2 & 67,8 & 99,9 \\
\hline \multicolumn{11}{|c|}{ Variante 3} \\
\hline 2000 & $84.133,4$ & $17.850,1$ & 21,2 & $46.937,8$ & 55,8 & $19.345,5$ & 23,0 & 38,0 & 41,2 & 79,2 \\
\hline 2010 & $84.894,8$ & $15.823,9$ & 18,6 & $48.163,7$ & 56,7 & $20.907,2$ & 24,6 & 32,9 & 43,4 & 76,3 \\
\hline 2020 & $83.748,0$ & $14.424,9$ & 17,2 & $46.157,5$ & 55,1 & $23.165,6$ & 27,7 & 31,3 & 50,2 & 81,4 \\
\hline 2030 & $81.072,1$ & $13.683,6$ & 16,9 & $40.852,7$ & 50,4 & $26.535,7$ & 32,7 & 33,5 & 65,0 & 98,4 \\
\hline 2040 & $77.115,4$ & $12.450,6$ & 16,1 & $39.187,9$ & 50,8 & $25.476,9$ & 33,0 & 31,8 & 65,0 & 96,8 \\
\hline
\end{tabular}

Abb. 3: Entwicklung der Bevolkerung in Deutschland, achte koordinierte Bevoblkerungsvorausberechnung (Quelle: SOMMER, 1994, 501) 
In vorliegender Arbeit findet der demographische Wandel hinsichtlich seiner Auswirkungen auf die Zahl und die Zusammensetzung der Erwerbstätigen im Produktions- und Dienstleistungsbereich Beachtung. Ökonomisch wird sonst häufig das Potential älterer Menschen mit Blick auf ihre Konsumaktivitäten, die alterungsbedingte Wandlung der Konsumnachfrage und die relative oder sogar absolute Senkung der Konsumgüternachfrage infolge des Bevölkerungsrückgangs behandelt (vgl. beispielsweise SCHNEIDER, 1992 und OTT, 1980).

Die Ergebnisse der achten koordinierten Bevölkerungsvorausberechnung machen wesentliche Auswirkungen der demographischen Entwicklung auf das vorhandene Erwerbstätigenpotential deutlich. Die Verkleinerung der Bevölkerungsgruppe der unter 20jährigen verringert das auf dem Arbeitsmarkt zur Verfügung stehende Nachwuchspotential. Der sinkende Anteil der Bevölkerung im erwerbsfähigen Alter von 20 bis 60 Jahren verringert das derzeit hauptsächlich genutzte Erwerbspersonenpotential. Durch den Bevölkerungsrückgang und den Prozeß der Alterung, sinkt bis 2040 die Bevölkerung im Alter derzeit durchschnittlicher Erwerbstätigkeit deutlich und während dieser Zeitspanne zudem um 12 bis 13\%-Punkte stärker als die Gesamtbevölkerungszahl, wie folgende Abbildung zeigt.

\begin{tabular}{|ccccc|}
\hline \multicolumn{5}{c|}{ Rückgang der Bevolkerung von 1992 bis 2040 } \\
\hline \multicolumn{2}{c}{ Insgesamt } & \multicolumn{2}{c|}{$20-60$ Jahre } \\
\hline Variante 1 & 13,4 Mio. & $16,5 \%$ & 13,9 Mio. & $29,5 \%$ \\
Variante 2 & 8,6 Mio. & $10,6 \%$ & 10,9 Mio. & $23,1 \%$ \\
Variante 3 & 3,9 Mio. & $4,8 \%$ & 7,9 Mio. & $16,8 \%$ \\
\hline
\end{tabular}

Abb. 4: Rückgang der Gesamtbevollkerung und der Bevolkerung im Alter von 20 bis 60 Jahren in Deutschland, achte koordinierte Bevolkerungsvorausberechnung (vgl. SOMMER, 1994, 501)

Zusätzlich muß der steigende Anteil der über 60jährigen und damit auch der steigende Anteil der Personen, die einen beachtlichen Lebensabschnitt nach ihrer Pensionierung verbringen, Beachtung finden. Da sich zudem die Personengruppe im Alter von 20 bis 60 Jahren verkleinert, steigt der Altenquotient deutlich an und beträgt je nach Variante im Jahr 2030 wie auch im Jahr 2040 zwischen 65 und $71 \%$. Zur Einordnung dieser Veränderung kann die Entwicklung des Altenquotienten seit 1950 betrachtet werden. Die geburtenstarken Jahrgänge von 1955 bis 1965 wirken seit Ende der 70er Jahre verringernd auf den Altenquotienten. Für die zuletzt deutlichere Senkung des Quotienten in der DDR bzw. in den neuen Ländern sind höhere Geburtenraten in den 70er Jahren sowie die niedrigere Lebenserwartung verantwortlich (vgl. BUSLEI, 1995). 


\begin{tabular}{|c|c|c|}
\hline \multirow{2}{*}{ Jahr } & \multicolumn{2}{|c|}{$\begin{array}{c}\text { Die Entwicklung des Altenquotienten } \\
\text { in Deutschland seit 1950 }\end{array}$} \\
\cline { 2 - 3 } & alte Bundeslander & neue Bundesländer \\
\hline 1950 & 26,0 & 30,9 \\
1955 & 28,2 & 36,4 \\
1960 & 29,9 & 40,9 \\
1965 & 34,8 & 44,9 \\
1970 & 39,0 & 48,5 \\
1975 & 40,2 & 45,5 \\
1980 & 36,9 & 37,5 \\
1985 & 37,2 & 34,1 \\
1990 & 36,5 & 34,5 \\
1991 & 36,2 & 34,9 \\
\hline
\end{tabular}

Abb. 5: Altenquotient in der Bundesrepublik / alte Bundeslănder und in der DDR / neue Bundesländer (Quelle: Bundesministerium für Familie und Senioren, 1994, nach BUSLEI, 1995, 18)

Die in allen Varianten der Bevölkerungsvorausberechnung zu erwartende deutliche Verschiebung im Altersaufbau der Bevölkerung wirft vielfältige Probleme auf. Bezogen auf den Arbeitsmarkt muß in ökonomischer Sicht im Interesse einer positiven wirtschaftlichen Entwicklung, wie insbesondere

- zur Sicherung des wirtschaftlichen Wohlstandes der Gesamtbevölkerung,

- zum Erhalt der Fähigkeit, internationalen Zahlungsverpflichtungen nach zukommen und

- zur Sicherung der Finanzierung der gesetzlichen Rentenversicherung

das zukünftig vorhandene Erwerbspersonenpotential erweitert und möglichst hoch ausgeschöpft werden.

\section{b) Entwicklung der Erwerbsquoten}

Neben den demographischen Änderungen ist die Entwicklung der Erwerbsbeteiligung relevant für die zukünftige Zahl und Zusammensetzung des Erwerbspersonenpotentials. Die Veränderungen der Erwerbsquoten bestimmter Personengruppen, wie der Frauen und Älteren, lassen sich allerdings schwieriger vorhersagen als demographische Entwicklungen, da sie beispielsweise von der Arbeitsmarktsituation, der Haushalts- und Familienstruktur sowie gesellschaftlich vermittelten Einstellungs- und Verhaltensweisen abhängig sind. Andererseits können sie aufgrund ihrer geringeren Determiniertheit besser beeinflußt werden als der demographische Wandel. 
Die durchschnittliche Erwerbsquote der Personen im Alter von 15 bis 64 Jahren betrug 1991 in den alten Bundesländern $71 \%$ und in den neuen Bundesländern $85 \%$. Dieser Unterschied wird sich voraussichtlich zukünftig abbauen, so daß für das Jahr 2010 Erwerbsquoten von 74 bzw. 76\% prognostiziert werden (vgl. VON ROTHKIRCH, 1993, 180). Im folgenden sollen einige aus verschiedenen Studien zusammengestellte Zahlen den Stand und die Entwicklung des geschlechts- und altersspezifischen Erwerbsverhaltens illustrieren.

In den alten Bundesländern ist die Erwerbsbeteiligung der Frauen seit Mitte der 70er Jahre deutlich angestiegen (vgl. KLAUDER, 1992a). So erhöhte sich die Erwerbsquote der 15-65jährigen Frauen von 46,4\% 1975 auf 55,0\% 1993 (STATISTISCHES BUNDESAMT, MIKROZENSUS). Der Anstieg der weiblichen Erwerbsbeteiligung wird sich voraussichtlich auch zukünftig fortsetzen. Zum Vergleich kann die Situation in den neuen Bundesländern betrachtet werden. In der DDR war die Erwerbsbeteiligung der Frauen, insbesondere aufgrund systembedingter Arbeitskräfteknappheit, deutlich höher als in der alten Bundesrepublik (vgl. WENDT, 1992; ENGELBRECH, 1992b). Vielfältige Anpassungsvorgänge und Anpassungsschwierigkeiten sowie z.B. die erweiterten Möglichkeiten eine frei gewählte höhere Qualifizierung zu erreichen, und die Verringerung der Bedeutung kollektiver Kinderbetreuung lassen die Erwerbsquoten der Frauen in vielen Altersgruppen sinken. Aufgrund der ausgeprägten beruflichen Orientierung und der individuellen wirtschaftlichen Notwendigkeit kann allerdings erwartet werden, daß sich das Erwerbsverhalten der Frauen in den neuen Bundesländern auch zukünftig von der Situation in den alten Bundesländern unterscheiden wird (vgl. SCHULZ/KIRNER, 1994, 79ff. und ähnlich HOLST/SCHUPP, 1994, 153ff.).

Nach Vorausschätzungen des DIW (vgl. SCHULZ/KIRNER, 1994, 80ff.) ergeben sich zukünftig für Frauen in den alten und den neuen Bundesländern die in Abbildung 6 angeführten Erwerbsquoten als Anteile der erwerbstätigen und erwerbslosen Personen je Altersklasse.

Die besonderen Verschiebungen der Erwerbsquoten der über 55jährigen in den neuen Bundesländern erklären sich durch zeitlich befristete Regelungen zur Zahlung von Altersübergangsgeld. Auffällig ist weiterhin der erwartete Anstieg der Erwerbsquoten bei Frauen der mittleren Altersgruppen in den alten Bundesländern und die steigende Erwerbsbeteiligung von älteren Frauen, die zumindest teilweise auf sich erhöhende Anteile von Teilzeitarbeit zurückzuführen ist. Im übrigen 
erwartet HOF (vgl. 1993, 75) eine ähnliche Erhöhung der Erwerbsbeteiligung der Frauen für das durchschnittliche Erwerbsverhalten in den EG-Staaten.

\begin{tabular}{|c|c|c|c|c|c|c|}
\hline \multirow[t]{2}{*}{$\begin{array}{c}\text { Alter von } \\
\text {... bis unter } \\
\text {... Jahren }\end{array}$} & \multicolumn{3}{|c|}{$\begin{array}{l}\text { Erwerbsquoten deutscher } \\
\text { Frauen in den alten } \\
\text { Bundeslandern (in \%) }\end{array}$} & \multicolumn{3}{|c|}{$\begin{array}{l}\text { Erwerbsquoten deutscher } \\
\text { Frauen in den neuen } \\
\text { Bundeslændern (in \%) }\end{array}$} \\
\hline & 1992 & 2000 & 2010 & 1992 & 2000 & 2010 \\
\hline $15-20$ & 35,5 & 33,0 & 33,1 & 54,0 & 43,0 & 33,1 \\
\hline $20-25$ & 76,7 & 77,5 & 78,2 & 85,5 & 82,0 & 78,2 \\
\hline $25-30$ & 73,7 & 77,7 & 80,7 & 95,0 & 90,0 & 80,7 \\
\hline $30-35$ & 65,9 & 71,3 & 73,7 & 93,0 & 90,0 & 78,7 \\
\hline $35-40$ & 66,3 & 72,2 & 76,9 & 96,0 & 92,0 & 90,0 \\
\hline $40-45$ & 67,7 & 72,8 & 80,9 & 94,5 & 92,0 & 90,0 \\
\hline $45-50$ & 63,0 & 66,8 & 74,8 & 94,5 & 92,0 & 92,0 \\
\hline $50-55$ & 51,8 & 51,8 & 57,0 & 86,0 & 87,0 & 88,0 \\
\hline $55-60$ & 41,8 & 38,9 & 42,6 & 20,0 & 60,0 & 70,0 \\
\hline $60-65$ & 10,3 & 10,1 & 15,4 & 3,0 & 5,0 & 15,4 \\
\hline $65-70$ & 3,0 & 3,0 & 2,3 & 0,0 & 0,0 & 2,0 \\
\hline $15-65$ & 57,0 & 57,3 & 61,6 & 73,7 & 72,3 & 75,1 \\
\hline
\end{tabular}

Abb. 6: Erwerbsbeteiligung deutscher Frauen (Quelle: SCHULZ/KIRNER, 1994, 80ff.)

Im internationalen Vergleich ist die Erwerbsbeteiligung der Frauen in Schweden besonders hoch. So betrug die Erwerbsquote der 20 bis 64jährigen Frauen 1991 $84,9 \%$ gegenüber $90,1 \%$ der Männer dieser Altersgruppe, ohne daß dementsprechend bereits die geschlechtsspezifische berufliche Segregation aufgehoben wäre (vgl. HOEM, 1992). Innerhalb der Europäischen Union sind die Erwerbsquoten in Dänemark und im Vereinigten Königreich höher als in Deutschland (die nachfolgenden Zahlen beziehen sich auf die alten Bundesländer) (vgl. zur Entwicklung der Quoten bis 1990 beispielsweise KNAUTH, 1992).

\begin{tabular}{|l|c|cccccccccccc|}
\hline $\begin{array}{l}\text { Erwerbstätigkeit von } \\
\text { Frauen }\end{array}$ & $\begin{array}{c}\text { EUR } \\
\text { 12 }\end{array}$ & B & DK & D & GR & E & F & IRL & I & L & NL & P & UK \\
\hline $\begin{array}{l}\text { Anteil der Frauen } \\
\text { an der E rwerbs- } \\
\text { bevölkerung (\%) } \\
\text { Erwerbsquote der } \\
\text { 20- bis 59jährigen } \\
\text { Frauen (\%) }\end{array}$ & 41,0 & 40,0 & 46,6 & 42,5 & 35,8 & 35,3 & 44,3 & 34,1 & 37,1 & 35,8 & 39,5 & 43,8 & 43,2 \\
\hline
\end{tabular}

Abb. 7: Anteil der Frauen an der Erwerbsbevölkerung und Erwerbsquote, 1991

(Quelle: EUROSTAT, 1993)

Für eine relative Einordnung der zuvor für Frauen genannten Erwerbsquoten und zur Illustration der altersabhängigen und zukünftigen Entwicklung des Erwerbsverhaltens der Männer werden nachfolgend tatsächliche und prognostizierte Erwerbsquoten der Männer nach Altersklassen wiedergegeben. 


\begin{tabular}{|c|c|c|c|}
\hline \multirow{2}{*}{$\begin{array}{l}\text { Alter von } \\
\text {... bis unter } \\
\text {... Jahren }\end{array}$} & \multicolumn{3}{|c|}{ Erwerbsquoten deutscher Mănner (in \%) } \\
\hline & $\begin{array}{c}\text { Mikrozensus } \\
1991 \\
\end{array}$ & $\begin{array}{c}\text { Projektionsjahr } \\
2000\end{array}$ & $\begin{array}{c}\text { Projektionsjahr } \\
2010\end{array}$ \\
\hline $15-20$ & 45,2 & 36,5 & 36,6 \\
\hline $20-25$ & 79,7 & 76,8 & 76,8 \\
\hline $25-30$ & 88,8 & 87,9 & 87,4 \\
\hline $30-35$ & 96,9 & 96,7 & 96,7 \\
\hline $35-40$ & 98,1 & 97,8 & 97,9 \\
\hline $40-45$ & 97,8 & 97,5 & 97,5 \\
\hline $45-50$ & 96,7 & 96,9 & 96,8 \\
\hline $50-55$ & 93,5 & 93,5 & 93,1 \\
\hline $55-60$ & 79,5 & 82,1 & 84,3 \\
\hline $60-65$ & 33,0 & 37,9 & 66,7 \\
\hline $15-65$ & 83,0 & 82,0 & 85,1 \\
\hline
\end{tabular}

Abb. 8: Erwerbsbeteiligung deutscher Mănner (Quelle: Statistisches Bundesamt und Berechnungen des DIW nach SCHULZ/KIRNER, 1994, 86)

Die Erwerbsquote sinkt mit steigendem Alter deutlich. Betrachtet man rückblickend die Entwicklung der Erwerbsquoten der 60- bis 64jährigen Männer der letzten dreißig Jahre, so ergibt sich für Deutschland (ebenso wie für andere westliche Länder) ein deutlicher Rückgang der Erwerbsbeteiligung von fast $80 \%$ Mitte der 60 er Jahre auf etwa 33\% Mitte der 90er Jahre, so daß das Alter von 65 Jahren nicht mehr als 'normale' Altersgrenze bezeichnet werden kann. Für Frauen lassen sich im zeitlichen Vergleich zwei sich überlagernde Trends feststellen. Aufgrund der steigenden Erwerbsbeteiligung der Frauen beginnt der Ausstiegsprozeß auf einem immer höheren Erwerbsquotenniveau. Von diesem höheren Niveau beginnend erfolgt der Rückgang der Erwerbsbeteiligung aufgrund des Trends zum früheren Ausstieg schneller (vgl. KOHLI, 1992, 238ff.). Die prognostizierte Entwicklung weist dagegen für die höheren Altersgruppen für Männer und Frauen zukünftig deutlich steigende Erwerbsquoten aus. Allerdings sind vielfältige makro- und mikroökonomische Maßnahmen erforderlich, damit die hiermit zum Ausdruck kommende Erhöhung des gesetzlichen Renteneintrittsalter zu einer Verlängerung der qualifizierten Erwerbstätigkeit führen kann und nicht $\mathrm{zu}$ wachsender Arbeitslosigkeit älterer Arbeitnehmer beiträgt (vgl. BÄCKER/NAEGELE, 1993a, $143 \mathrm{ff}$.).

Vergleicht man die Erwerbsquoten von 1989 im früheren Bundesgebiet mit denen der ehemaligen DDR nach Altersgruppen und Geschlecht, so erkennt man die dort deutlich höhere Erwerbsbeteiligung der Frauen und Älteren. Die Erwerbsquoten der Frauen in der DDR erreichten mit ca. 90\% die Höhe der Erwerbsbeteiligung der Männer in den westlichen Ländern. Bezogen auf Ältere betrug beispielsweise die Erwerbsquote der 65 bis 69jährigen Männer 23\% gegenüber $7 \%$ im früheren 
Bundesgebiet, bei den 60 bis 64jährigen Frauen betrugen die Erwerbsquoten 28 gegenüber $11 \%$ und bei den 65 bis 69jährigen Frauen 10 gegenüber $3 \%$ (vgl. ENGELBRECH, 1992b, 47; SCHMIDTKE/SCHWITZER, 1993, 28).

Im Vergleich der EU-Staaten ist die Erwerbsbeteiligung Älterer in Deutschland (alte Bundesländer) unterdurchschnittlich. Besonders hoch sind die Erwerbsquoten in Portugal, Irland, Griechenland, Dänemark und im Vereinigten Königreich, während sie in Belgien, Luxemburg und Frankreich ebenfalls sehr niedrig sind.

\begin{tabular}{|c|c|c|c|c|c|c|c|c|c|c|c|c|c|}
\hline $\begin{array}{l}\text { Erwerbsbeteiligung } \\
\text { Älterer }\end{array}$ & $\begin{array}{c}\text { EUR } \\
12 \\
\end{array}$ & B & DK & D & GR & E & $\mathbf{F}$ & IRL & I & $\mathbf{L}$ & NL & $\mathbf{P}$ & UK \\
\hline $\begin{array}{l}\text { Erwerbsquote der } \\
\text { Männer (\%) }\end{array}$ & 16,1 & 6,9 & 21,0 & 15,0 & 22,1 & 17,2 & 6,6 & 27,9 & 17,2 & 6,7 & 11,2 & 33,2 & 21,3 \\
\hline $\begin{array}{l}\text { Erwerbsquote der } \\
\text { Frauen }(\%)\end{array}$ & 5,3 & 1,2 & 8,5 & 4,2 & 7,4 & 5,2 & 4,0 & 6,0 & 4,3 & 2,3 & 2,9 & 13,9 & 7,8 \\
\hline $\begin{array}{l}\text { Erwerbsquote } \\
\text { insgesamt }(\%)\end{array}$ & 9,8 & 3,7 & 13,7 & 8,5 & 14,2 & 10,4 & 5,1 & 15,9 & 9,8 & 5,4 & 6,5 & 22,0 & 13,5 \\
\hline
\end{tabular}

Abb. 9: Erwerbsbeteiligung der 60jăhrigen und ălteren Bevőlkerung in Privathaushalten, 1991 (Quelle: Eurostat, Erhebung über Arbeitskrăfte 1991 nach STATISTISCHES BUNDESAMT, 1994a)

Der altersbezogene Rückgang der Erwerbsbeteiligung ist in Deutschland und den Niederlanden besonders steil und findet vor dem Erreichen des gesetzlichen Renteneintrittsalters statt, so daß dieses von geringer Bedeutung für die Erwerbsbeteiligung Älterer ist (vgl. auch zur Entwicklung in den übrigen EGStaaten KNAUTH, 1993, 220ff.).

Neben den hier zur demographischen und erwerbsquotenbezogenen Entwicklung in Deutschland dargestellten Studien gibt es weitere Vorausberechnungen, die aufgrund abweichender Annahmen $\mathrm{zu}$ anderen Bevölkerungszahlen und zu unterschiedlichen Altersstrukturen führen (vgl. eine Gegenüberstellung verschiedener aktueller Berechnungen BUSLEI, 1995). Insbesondere ergibt sich aus den Annahmen ein unterschiedlich hoher quantitativer Rückgang des Arbeitskräfteangebots; der allerdings übereinstimmend etwa ab 2010 deutlich wirksam werden wird (vgl. beispielsweise auch HOF, 1993; HOF, 1992; THON, 1991; RÜRUP/SESSELMEIER, 1993a). Zur Erörterung differenziert wirkender mikroökonomisch relevanter Maßnahmen ist neben der Gesamtzahl der potentiell zur Verfügung stehenden Erwerbspersonen besonders die Zusammensetzung des Erwerbspersonenpotentials bedeutsam. Betrachtet man daher die Struktur des zukünftigen Arbeitskräfteangebots, so läßt sich festhalten, daß die Anteile der weiblichen sowie der ausländischen Arbeitspersonen und insgesamt der älteren 
Erwerbstätigen deutlich steigen werden, während der Anteil der aktuell qualifizierten und jüngeren Personen deutlich zurückgehen wird (trotz unterschiedlicher Annahmen wird diese Entwicklungstendenz in zahlreichen Studien übereinstimmend prognostiziert, vgl. beispielsweise die Projektion des Instituts für Arbeitsmarkt- und Berufsforschung der Bundesanstalt für Arbeit (IAB), THON, 1991; KLAUDER, 1992c, 33f. sowie den PROGNOS Deutschland-Report, VON ROTHKIRCH, 1993, 181 und auch die DIW-Berechnungen, SCHULZ/KIRNER, 1994).

\section{Nachfrageseite}

Die Nachfrage nach Arbeitskräften wird wesentlich durch die Entwicklung des internationalen und nationalen Wirtschaftswachstums sowie des technischen und organisatorischen Fortschritts beeinflußt. Die hiermit zusammenhängenden Größen Produktivität, Einkommen, Arbeitszeit und die Art der Anforderungen bestimmen die Nachfrageseite des Arbeitsmarktes quantitativ und qualitativ.

Eine makroökonomische Betrachtung der Beschäftigung kann sich auf die Verschiebung der Beschäftigung zwischen den Sektoren richten. Der ökonomischtechnische Strukturwandel führt zur Verschiebung der Beschäftigung von Produktions-, Handels- und 'einfachen' Dienstleistungstätigkeiten hin zu 'komplexen' Dienstleistungstätigkeiten (vgl. PROGNOS, 1989 und ähnlich bereits PROGNOS, 1985). Während die Beschäftigung im Bereich der Dienstleistungen voraussichtlich deutlich zunehmen wird, verläuft die Beschäftigungsentwicklung in der öffentlichen Verwaltung, im Handel und besonders in verschiedenen Branchen der Industrie sehr unterschiedlich. Allerdings kann betont werden, daß der sich ausweitende Bereich 'komplexer' Dienstleistungen wesentlich auf die Unterstützung der hochentwickelten Produktion gerichtet ist und insofern von Entwicklungen im industriellen Bereich abhängig ist (vgl. z.B. KLAUDER, 1992c, 33).

Ein Hilfsmittel zur Voraussage der Entwicklungstendenz der Nachfrage nach Arbeitskräften ist die Beschäftigungsschwelle, die die Höhe des Wirtschaftswachstums angibt, ab der sich die Zahl der Beschäftigten erhöht. Die Beschäftigungsschwelle ist in den vergangenen 30 Jahren stetig gesunken (vgl. RÜRUP/SESSELMEIER, 1993a, 39f. und 1993c, 7); sie ging von 3,7\% im Zeitraum 1960-73 auf 2,2\% 1973-80 und auf 0,7\% 1980-92 zurück (vgl. HOF, 1994). Die Senkung der Beschäftigungsschwelle läßt sich durch die Verkürzung der 
Arbeitszeit und die Verschiebung der Quelle des Wirtschaftswachstums vom sekundären in den tertiären Sektor, der eine geringere Arbeitsproduktivität aufweist, begründen. Da zumindest der zweite Einflußfaktor erhalten bleibt, kann auch für die kommenden Jahre angenommen werden, daß ein angestrebtes Wirtschaftswachstum zur Erhöhung der Nachfrage auf dem Arbeitsmarkt beiträgt.

Mikroökonomisch kann die Beschäftigung hinsichtlich der Verschiebungen der Tätigkeitsinhalte bzw. der mit den Tätigkeiten verbundenen Anforderungen betrachtet werden. Verschiebungen der Beschäftigung ergeben sich insbesondere von ausführenden zu planenden und dispositiven Tätigkeiten (vgl. TESSARING, 1991; PROGNOS, 1989 und ähnlich bereits PROGNOS, 1985). Die PROGNOSProjektion besagt, daß produktionsorientierte Arbeitsplätze verlorengehen werden. Die Zahl der Stellen mit den Funktionen "Gewinnen und Herstellen" geht stark zurück, während die Zahl solcher mit der Funktion "Reparieren" in etwa gleich bleibt und die Anzahl der Stellen mit den Funktionen "Maschinen einrichten/einstellen/warten" zunimmt, allerdings voraussichtlich nicht einmal die Hälfte des sonstigen Rückgangs ausgleichen kann. Im Bürobereich verlieren einfachere Tätigkeiten gegenüber spezifischen Tätigkeiten bzw. solchen mit Führungsaufgaben relativ an Bedeutung. Anders systematisiert gehen Arbeitsplätze im Bereich der "primären" Dienstleistungen (Handel, Büro, Allgemeine Dienste) verloren, während solche im Bereich der "sekundären" Dienstleistungen (Forschung und Entwicklung; Organisation und Management; Betreuen, Beraten, Lehren u.a.) stark zunehmen. Diese bis 2010 prognostizierte Zunahme der "sekundären" Dienstleistungsstellen überwiegt den Rückgang der Zahl der Erwerbstätigen im produktionsorientierten Bereich, im "primären" Dienstleistungsbereich und der Auszubildenden deutlich.

Bezogen auf die Anforderungsprofile wird ein deutlicher Rückgang einfacher Tätigkeiten, eine leichte Zunahme mittelqualifizierter und eine starke Zunahme höherqualifizierter Tätigkeiten prognostiziert (vgl. PROGNOS, 1989, 252 und ähnlich PROGNOS, 1990, 437ff.). Entsprechend wird es immer weniger Arbeitsplätze für Unausgebildete, einen etwa gleichbleibenden Bedarf an Personen mit Lehrabschluß und einen steigenden Bedarf an Hochschulabsolventen (besonders FH-Absolventen und je nach Ausrichtung auch Universitätsabsolventen) geben. Der Bedarf richtet sich zunehmend auf flexible, mobile, breit und fachübergreifend ausgebildete, beruflich und sozial hoch qualifizierte Arbeitskräfte, die aufgrund der dynamischen Wissensentwicklung zudem aktuell qualifiziert sein müssen (vgl. KLAUDER, 1992c, 30ff.). Zur Illustration der tendenziellen Entwicklung zeigt die folgende Abbildung eine Gegenüberstellung der Qualifikationsstruktur der 
Erwerbstätigen von 1976, 1991 und Vorausschätzungen für 2010 nach einem IAB/PROGNOS-Gutachten und einem von WEIBHUHN/WAHSE im Auftrag der Bund-Länder-Kommission für Bildungsplanung und Forschungsförderung (BLK) erstellten Gutachten.

\begin{tabular}{|c|c|c|c|c|c|c|}
\hline \multirow[b]{2}{*}{$\begin{array}{c}\text { Angaben } \\
\text { in } 1.000 \\
\text { in } \%\end{array}$} & \multirow[t]{2}{*}{1976} & \multirow[t]{2}{*}{1991} & \multicolumn{4}{|c|}{ Projektion 2010} \\
\hline & & & $\begin{array}{c}\text { Tessari } \\
\text { Variante } \\
\text { a } \\
\end{array}$ & $\begin{array}{l}\text { g, IAB } \\
\text { Variante } \\
\text { C } \\
\end{array}$ & $\begin{array}{c}\text { Weibhuh } \\
\text { BI } \\
\text { Variante } \\
\text { a } \\
\end{array}$ & $\begin{array}{l}\text { /Wahse, } \\
\mathbf{K} \\
\text { Variante } \\
\quad \text { d }\end{array}$ \\
\hline ohne AusbildungsabschluB & $\begin{array}{c}8.612 \\
34,9\end{array}$ & $\begin{array}{l}5.601 \\
20,2\end{array}$ & $\begin{array}{c}3.266 \\
11,7\end{array}$ & $\begin{array}{c}2.712 \\
9,7\end{array}$ & $\begin{array}{c}3.657 \\
13,1\end{array}$ & $\begin{array}{c}4.223 \\
13,2\end{array}$ \\
\hline Lehre, Berufsfachschule & $\begin{array}{c}12.678 \\
51,3\end{array}$ & $\begin{array}{c}16.352 \\
59,1\end{array}$ & $\begin{array}{c}17.884 \\
63,8\end{array}$ & $\begin{array}{c}17.545 \\
62,6 \\
\end{array}$ & 20.183 & 23.065 \\
\hline $\begin{array}{l}\text { Fach-, Meister-, } \\
\text { Technikerschule }\end{array}$ & $\begin{array}{c}1.609 \\
6,5\end{array}$ & $\begin{array}{c}2.325 \\
8,4\end{array}$ & $\begin{array}{c}2.641 \\
9,4\end{array}$ & $\begin{array}{c}2.733 \\
9,8\end{array}$ & 72,3 & 72,2 \\
\hline Fachhochschule & $\begin{array}{l}543 \\
2,2 \\
\end{array}$ & $\begin{array}{c}1.127 \\
4,1 \\
\end{array}$ & $\begin{array}{c}1.434 \\
5,1 \\
\end{array}$ & $\begin{array}{c}1.599 \\
5,7\end{array}$ & $\begin{array}{c}1.593 \\
5,7\end{array}$ & $\begin{array}{c}1.816 \\
5,7\end{array}$ \\
\hline Universităt & $\begin{array}{c}1.263 \\
5,1\end{array}$ & $\begin{array}{c}2.257 \\
8,2\end{array}$ & $\begin{array}{c}2.802 \\
10,0\end{array}$ & $\begin{array}{c}3.433 \\
12,2\end{array}$ & $\begin{array}{c}2.483 \\
8,9\end{array}$ & $\begin{array}{c}2.842 \\
8,9\end{array}$ \\
\hline
\end{tabular}

Abb. 10: Entwicklung der Qualifikationsanforderungen (vgl. eine entsprechende Darstellung in INSTITUT DER DEUTSCHEN WIRTSCHAFT, 1994, 5)

Der Einsatz neuer Techniken sowie moderner Produktions- und Organisationsmethoden führt $\mathrm{zu}$ den prognostizierten Verschiebungen der Arbeitskräftenachfrage, konkretere Aussagen zu den sich hieraus ergebenden Veränderungen der Arbeit in den Unternehmen werden in Kapitel II entwickelt.

\section{B. Defizite des zukünftigen Arbeitskräfteangebots}

Führt man die Entwicklungstendenzen der Angebots- und der Nachfrageseite des Arbeitsmarktes zusammen, so ergeben sich miteinander verwobene quantitative und qualitative Defizite des Arbeitskräfteangebots (vgl. z.B. KLAUDER, 1992c, 30ff.). Auf diese Defizite muß sich die Nachfrageseite einstellen, damit in den Unternehmen rechtzeitig Maßnahmen zur Sicherung des Humankapitals entwickelt und umgesetzt werden.

\section{Quantitative Defizite}

Die derzeitige Situation auf dem Arbeitsmarkt ist durch hohe Arbeitslosigkeit bei gleichzeitig bereichsweise vorhandenem Mangel an Auszubildenden und Facharbeitern gekennzeichnet (vgl. beispielsweise JAGODA, 1993). Aufgrund der 
voraussichtlichen Entwicklung des Wirtschaftswachstums und der Produktivität sowie des durch die Zuwanderung und der steigenden Erwerbsbeteiligung der Frauen im Westen Deutschlands zunächst noch zunehmenden Erwerbspersonenpotentials muß auch in den nächsten Jahren mit anhaltender Arbeitslosigkeit gerechnet werden (vgl. z.B. VON ROTHKIRCH, 1993, 182f.). Trotz der Arbeitslosigkeit fehlen immer stärker fachlich qualifizierte Arbeitskräfte. Tendenziell wird sich zunächst ein Mangel an jüngeren und ein Überschuß an älteren Arbeitskräften zeigen (vgl. KLAUDER, 1992b, 11ff.). Dieser Nachwuchsmangel wird längerfristig zu einem stark zurückgehenden Arbeitskräfteangebot und damit zu breiten quantitativen Defiziten führen, die eine Erhöhung der durchschnittlichen Erwerbsquoten notwendig machen. Bei konstanten Erwerbsquoten stößt nach einer Modellrechnung des Verbandes Deutscher Rentenversicherungsträger (VDR) die Beschäftigtenentwicklung ab 2009 an die Grenzen des Arbeitskräfteangebots, so daß das volkswirtschaftliche Wachstum durch das Arbeitskräfteangebot begrenzt wird. Eine Erhöhung der Erwerbsquoten verschiebt den Zeitpunkt an dem das Arbeitskräfteangebot zum Engpaß wird nach hinten (vgl. GRÜTZ u.a., 1993). Jedenfalls müssen sich die Arbeitgeber auf eine veränderte Zusammensetzung des Erwerbspersonenpotentials einstellen. Zukünftig werden mehr ältere, weibliche und zugewanderte und weniger jüngere Personen beschäftigt werden.

Die Diskrepanz zwischen der gegenwärtigen und mittelfristigen Situation des dominierenden Mangels an Arbeitsplätzen bei gleichzeitigem Fachkräftemangel und der längerfristigen Situation des verbreiteten Mangels insbesondere an jüngeren Arbeitskräften ist ein entscheidendes Problem bei der Betrachtung des Arbeitsmarktes. Die hieraus entstehenden Konflikte erschweren die Konzeption notwendiger mikro- und makropolitischer Anpassungsmaßnahmen, da die Arbeitslosigkeit andere Handlungsweisen nahelegt als ein sich verstärkender Arbeitskräftemangel. Allerdings wird es, insbesondere aufgrund der nachfolgend betrachteten qualitativen Defizite, trotz des sich abzeichnenden Arbeitskräftemangels, weiterhin Arbeitslosigkeit geben.

Aufgrund dieser vielfältigen und sich verschiebenden Problemstellungen sind differenziert wirkende Maßnahmen notwendig, die zudem reversibel zu gestalten sind. Kurz- und mittelfristig muß die Arbeitslosigkeit bekämpft und gleichzeitig langfristig das Arbeitskräfteangebot gesichert werden. In der allgemeinen Ausrichtung übereinstimmend müssen hierzu die Beschäftigungschancen und die Eignung der Arbeitskräfte gesichert und verbessert werden. 


\section{Qualitative Defizite}

Die demographische Entwicklung führt zur Verringerung des Anteils jüngerer und zur Erhöhung des Anteils älterer Erwerbspersonen. Andererseits muß aufgrund der voraussichtlichen Entwicklung der Arbeitsaufgaben und der Anforderungsverschiebungen, z.B. wegen der häufiger auftretenden Einführung neuer Technologien, mit einer zunehmenden Nachfrage nach aktuell qualifizierten Arbeitskräften gerechnet werden. Die Erhöhung der Anforderungen lassen erhebliche Qualifikationsdefizite der Arbeitskräfte befürchten, die durch die veränderte Zusammensetzung des Erwerbspersonenpotentials (abnehmender Umfang des aktuell qualifizierten Nachwuchses) voraussichtlich noch verschärft werden. Für unqualifizierte und nicht qualifizierungsfähige Personen wird das Risiko der Arbeitslosigkeit zukünftig weiter steigen.

Tendenziell werden sich zukünftig verstärkt ein Mangel an Fachkräften, ein Überschuß an Personen ohne Ausbildung und eine (allerdings von der Fachrichtung abhängige) zum Ausgleich neigende Beschäftigungssituation bei Akademikern zeigen (vgl. KLAUDER, 1992b, $11 \mathrm{ff}$; TESSARING, 1991, 55). Der Fachkräftemangel und der sich auf der Ebene der Facharbeiter verringernde Nachwuchs resultieren u.a. aus dem Trend $\mathrm{zu}$ höheren Bildungsabschlüssen sowie aus Einkommens- und Aufstiegsnachteilen; dies führt $\mathrm{zu}$ einer zunehmenden Gefährdung eines traditionellen Standortvorteils Deutschlands (vgl. KLAUDER, 1992c, 34; JAGODA, 1993; TESSARING, 1993). Entsprechend stellt sich die Aufgabe, die Ausübung der Berufe, die im anspruchsvollen dualen Ausbildungssystem erlernt werden, attraktiver zu gestalten, indem z.B. die Aufstiegs- und Weiterbildungsmöglichkeiten - besonders aber auch die Arbeitsbedingungen, der Status und das Einkommen der Fachkräfte - verbessert und eine qualifikationsgerechter Einsatz gefördert werden (vgl. VOLKHOLZ, 1992; TESSARING, 1993).

Die beschriebenen quantitativen und qualitativen Defizite führen in den Unternehmen zu wachsenden Schwierigkeiten qualifizierte Arbeitskräfte in ausreichender Zahl zu gewinnen. Der Faktor Arbeit wird in Teilbereichen zum Engpaßfaktor werden und die Löhne und Lohnnebenkosten werden defizitbedingt steigen. Weiterhin werden die Schwierigkeiten die personellen Voraussetzungen des Wirtschaftens in den Unternehmen sicherzustellen dazu führen, daß die Umstrukturierung der Unternehmen im Sinne moderner Produktions- und Organisationsmethoden zur Gewährleistung der Innovationsfähigkeit und zur Nutzung mitarbeiterorientierter Rationalisierungsstrategien verzögert bzw. 
verhindert wird. Die Schrumpfung und Alterung des Erwerbspersonenpotentials können insofern zu einer Beeinträchtigung der Wettbewerbsfähigkeit und zu einer angebotsseitigen Minderung des Wirtschaftswachstums führen.

Folgende Abbildung stellt die bisher behandelten Zusammenhänge als schematisches Modell dar:

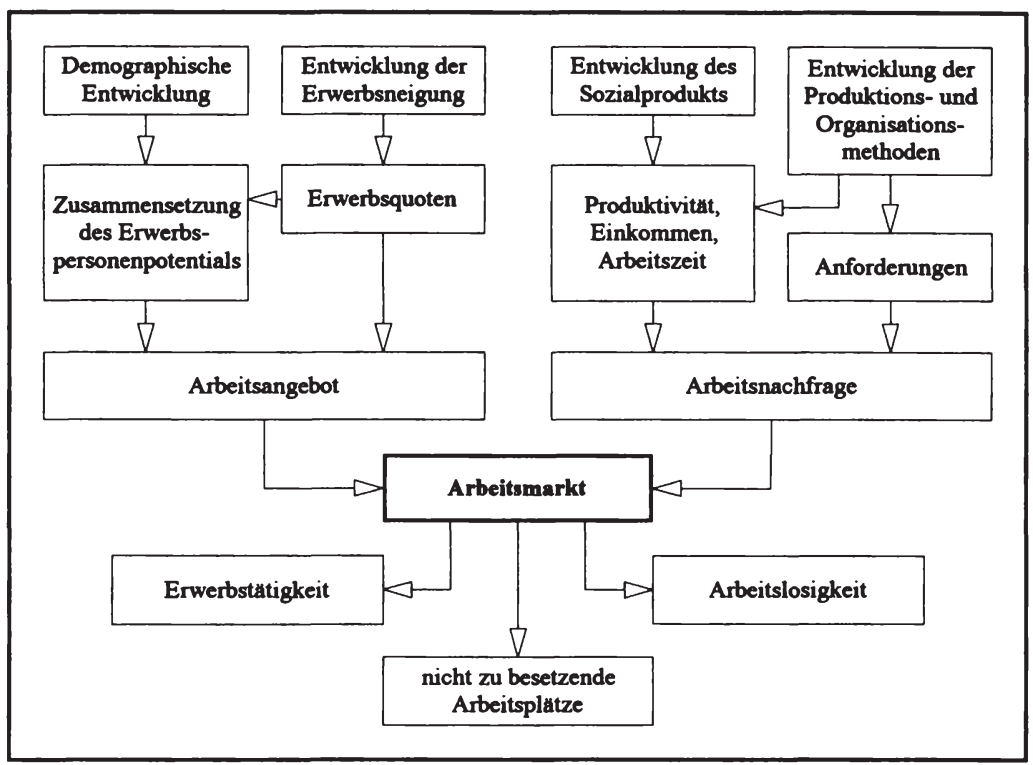

Abb. 11: Einflüsse auf den Arbeitsmarkt und resultierende Situationen

Einerseits wird es zukünftig schwieriger die angebotenen Arbeitsplätze mit geeigneten Arbeitspersonen zu besetzen, andererseits herrscht gleichzeitig Arbeitslosigkeit. Angesichts dieser prognostizierten Situation muß die Frage gestellt werden, was in den Unternehmen zur Verbesserung der zukünftigen Situation, d.h. zur Minderung der prognostizierten Defizite, getan werden kann. Eine verbesserte zukünftige Situation kann durch eine zumindest nicht verringerte Erwerbstätigenzahl und einen ausgeglicheneren Arbeitsmarkt charakterisiert werden, so daß das Arbeitskräfteangebot keinen Engpaßfaktor darstellt und die Wettbewerbsfähigkeit der Unternehmen und das Wirtschaftswachstum nicht beeinträchtigt werden. Zur Erreichung einer in diesem Sinne verbesserten zukünftigen Situation ist es notwendig, die makro- und mikropolitischen 
Rahmenbedingungen so zu gestalten, daß das Erwerbspersonenpotential erweitert und ausgeschöpft werden kann. Wesentliche Voraussetzung hierfür sind ein hoher Beschäftigungsstand, der Abbau 'vermeidbarer' Arbeitslosigkeit und der adäquate Einsatz der Arbeitspersonen.

Die Änderungen der Alterszusammensetzung in der Bevölkerung und der Beschäftigungsstruktur bieten somit auch die Chance, durch gestalterische Maßnahmen, die sich auf die Phasen Ausbildung, Weiterbildung, Arbeit, Erwerbstätigkeitsunterbrechung und Ruhestand beziehen können, starre Strukturen aufzubrechen und die Arbeitswelt zu modernisieren. Da Arbeit nicht nur zweckgerichtete Tätigkeit und Mittel zum Einkommenserwerb ist, sondern auch ein Mittel zur Entfaltung und Entwicklung der Persönlichkeit darstellt, ist eine frühzeitige und breite Diskussion denkbarer Handlungsweisen notwendig und sinnvoll.

\section{Optionen zur Erreichung einer verbesserten Situation}

Beabsichtigt man angesichts der prognostizierbaren Defizite gesamtwirtschaftlich unerwünschte Handlungsweisen wie Produktionsverlagerungen und verstärkte Mitarbeiterabwerbungsstrategien zu vermeiden, so müssen Optionen zur Milderung der zu erwartenden Defizite entwickelt werden (vgl. RÜRUP/SESSELMEIER, 1993a, 38ff. und 1993c, 6ff.). Diese Optionen richten sich entweder auf die Senkung der Arbeitskräftenachfrage, d.h. den Abbau von Arbeitsplätzen und die Freisetzung von Arbeitskräften durch die Steigerung der Arbeitsproduktivität, oder auf die Ausweitung des Arbeitskräfteangebots durch eine Erhöhung der Beschäftigung verschiedener Personengruppen. Männer mittleren Alters bilden diesbezüglich keine eigene Zielgruppe, da ihre Erwerbsquote bereits so hoch ist, daß sie nicht in relevantem Maße gesteigert werden kann. Die Optionen

- Steigerung der Arbeitsproduktivität

- Verlängerung der durchschnittlich geleisteten Arbeitszeit

- Einwanderung ausländischer Arbeitspersonen

- Erhöhung der Erwerbsquote jüngerer Personen

- Erhöhung der Erwerbsquote der Frauen

- Erhöhung der Erwerbsquote älterer Personen 
werden im folgenden, vornehmlich aus der mikroökonomischen Perspektive der Unternehmen, betrachtet.

\section{- Steigerung der Arbeitsproduktivität}

Ein gesamtwirtschaftliches Maß für die Arbeitsproduktivität läßt sich ermitteln, indem das Bruttosozialprodukt durch die Zahl der Erwerbstätigen dividiert wird. Demnach muß zu einer Einsparung von Arbeitsplätzen die Arbeitsproduktivität stärker steigen als das Wirtschaftswachstum. Vorliegende empirische Daten zeigen allerdings zum einen den Rückgang des Wirtschaftswachstums und des Produktivitätsanstiegs und zum anderen die stetige Absenkung der Beschäftigungsschwelle als der Wachstumsrate, ab der das Wirtschaftswachstum zu Beschäftigungszuwächsen führt. Das Ziel, die Produktivität dauerhaft stärker zu erhöhen als die Wirtschaft wächst, erscheint daher nicht erreichbar (vgl. RÜRUP/SESSELMEIER, 1993a, 39f. und 1993c, 7). Die Steigerung der Produktivität wird auch zukünftig beispielsweise durch die wachsende Bedeutung des personalintensiver arbeitenden Dienstleistungsund Handelssektors beeinträchtigt werden. Eine parallele Entwicklung von Produktivität und Sozialprodukt oder ein im Vergleich zum Wirtschaftswachstum niedrigerer Produktivitätszuwachs prognostiziert bis zum Jahr 2040 auch PROGNOS (vgl. STANDFEST, 1995). Der Produktivitätsanstieg wird demzufolge die demographisch bedingten quantitativen Defizite des Arbeitskräfteangebots nicht ausgleichen können.

In einer mikroökonomisch orientierten Betrachtungsweise müssen zusätzlich folgende Vorbehalte gegenüber dieser Option angeführt werden:

- Da Produktivitätssteigerungen 'ohnehin' international im Interesse der Wettbewerbsfähigkeit angestrebt und erreicht werden, verbleiben keine 'zusätzlichen' bisher ungenutzte Möglichkeiten zur technisch-organisatorischen Poduktivitätssteigerung, die eigens aufgrund der demographischen Entwicklung eingesetzt werden könnten.

- Maßnahmen zur technischen Produktivitätssteigerung substituieren Arbeit durch Kapital, um den Rückgang des Arbeitskräfteangebots auszugleichen. Die Steigerung der technischen Produktivität ist zweifellos notwendig, sie ist aber nicht die wichtigste Option zur Behebung qualitativer und quantitativer Defizite des Arbeitskräfteangebots. Dies wird deutlich, wenn man moderne Produktionsund Organisationsmethoden betrachtet, die im Interesse der Steigerung der 
Wettbewerbsfähigkeit zukünftig verstärkt in deutschen Unternehmen umgesetzt werden sollen. Diese Methoden richten sich insbesondere darauf, die Leistungspotentiale der Arbeitspersonen besser zu nutzen. Würde man nun angesichts der demographischen Entwicklung in den Unternehmen lediglich technische Produktivitätssteigerungen anstreben und (aufgrund der Defizite des Arbeitskräfteangebots verstärkt) auf Arbeitspersonen verzichten, so könnte sich dies negativ auf die Entwicklung der Wettbewerbsfähigkeit der Unternehmen auswirken.

- Andererseits kann in neoklassischer Sichtweise angenommen werden, daß die Entlohnung des Faktors Arbeit relativ zu den Kapitalkosten steigen wird, so daß sich zukünftig die Investitionen in Sachkapital erhöhen dürften und der Einsatz neuerer Maschinen und Anlagen produktivitätssteigernd wirkt (vgl. beispielsweise SIEGMUND, 1994, 105ff.).

- Allerdings werden zur Steigerung der Produktivität gerade spezialisierte und aktuell qualifizierte Arbeitskräfte benötigt, und diese werden aufgrund der demographischen Entwicklung knapp, so daß das zukünftige Arbeitskräfteangebot ins Zentrum der Überlegungen rückt.

\section{- Verlängerung der durchschnittlich geleisteten Arbeitszeit}

Eine Verlängerung der durchschnittlichen wöchentlichen oder jährlichen Arbeitszeit wäre eine Möglichkeit zur Kompensation eines zukünftigen Arbeitskräftemangels. Hierzu müßten die Tarifpartner entsprechende Vereinbarungen zur Arbeitszeitverlängerung treffen. Solche sind allerdings bei anhaltender Arbeitslosigkeit aufgrund der Haltung der Gewerkschaften nicht zu erwarten (vgl. beispielsweise LANG, 1993) und von den Beschäftigten im allgemeinen nicht erwünscht. Die zukünftig mögliche höhere Verbreitung der Teilzeitarbeit, die insbesondere auch zur Realisierung der auf Frauen und Ältere gerichteten Optionen anzustreben ist, wird die Chancen der Erhöhung der durchschnittlich geleisteten Arbeitszeit zusätzlich verringern. Würden zukünftig Arbeitszeitverlängerungen vereinbart werden, so ließen sie sich relativ kurzfristig umsetzen, sofern zugleich unternehmensspezifische Anpassungsmöglichkeiten vereinbart und genutzt würden.

Weiterhin muß betont werden, daß eine Arbeitszeitverlängerung lediglich einen quantitativen, zudem langfristig zum Ausgleich des Arbeitskräfterückgangs kaum 
ausreichenden Effekt bietet. Die prognostizierten qualitativen Defizite könnten langfristig hierdurch kaum behoben werden, zumal die Veränderungen der Arbeitszeit den Verschiebungen der qualitativen Arbeitskräftenachfrage entsprechen müßten und somit ein qualifikationsabhängiges zunehmendes arbeitszeitliches und einkommensbezogenes Auseinanderdriften der verschiedenen Beschäftigtengruppen akzeptiert werden müßte. Damit besteht die Gefahr der zusätzlichen Benachteiligung solcher Personen, die arbeitsmarktbezogen bereits Wettbewerbsnachteile aufweisen, da ihre Arbeitsleistung eben in geringerem Maße nachgefragt wird bzw. sie die gestellten zeitlichen Anforderungen einer Erwerbstätigkeit nicht erfüllen können.

Da relevante Arbeitszeitverlängerungen nicht zu erwarten sind und die Produktivitätsfortschritte nicht ausreichen dürften, um langfristig den Rückgang des Arbeitskräftepotentials und besonders die qualitativen Defizite auszugleichen, müssen die anderen, auf besondere Erwerbspersonengruppen gerichtete Optionen betrachtet werden.

\section{- Einwanderung ausländischer Arbeitspersonen}

Die Anwerbung spezifisch qualifizierter ausländischer Arbeitskräfte ist, insbesondere angesichts der in nahezu allen Industrienationen gleichgerichteten demographischen Entwicklung, zunächst ein Suchproblem. Anwerbungsbereite Arbeitskräfte werden zukünftig nur in geringer Zahl in westeuropäischen Staaten gefunden werden können. Zum einen zeichnet sich dort eine ähnliche Arbeitskräfteentwicklung $a b$, wie sie für Deutschland angesprochen wurde, auch wenn der demographisch bedingte Einwanderungsbedarf in den anderen Staaten wesentlich niedriger ist als in Deutschland (vgl. SCHMID/CHRUSCZ, 1994). Zum anderen bauen sich zumindest zwischen den EU-Staaten die Unterschiede hinsichtlich der wirtschaftlichen Entwicklung und der Einkommenshöhe ab, so daß sich die Wanderungsmotivation abschwächen wird (vgl. WERNER, 1990; PROGNOS, 1990, 74ff; VON ROTHKIRCH, 1993). Die Zuwanderung wird daher eher aus Herkunftsländern erfolgen, die sich hinsichtlich der kulturellen Traditionen und Lebensweisen sowie des Ausbildungsniveaus deutlich von Deutschland unterscheiden. Durch die Migration können infolgedessen größere Probleme auftreten, die z.B. das psychische Wohlbefinden, die Gesundheit und Leistungsfähigkeit der zuwandernden Arbeitspersonen beeinträchtigen können (vgl. BRANDENBURG u.a., 1989). 
Zur Realisierung dieser Option müssen vielfältige gesellschaftliche, politische sowie makroökonomische Entscheidungen getroffen und Bedingungen erfüllt werden, die von einer Anerkennung der faktischen Einwanderung der ausländischen Arbeitnehmer ausgehen. Es muß ein verbreitetes gesellschaftlich positives Interesse an der Einwanderung ausländischer Arbeitspersonen und ihrem Leistungsbeitrag bestehen (hinsichtlich der Wirkungen der Einwanderung auf den Arbeitsmarkt sowie auf das staatliche Einnahmen- und Ausgabensystem, vgl. RÜRUP/SESSELMEIER, $1993 \mathrm{~b}$ und 1994). Weiterhin müssen im gesellschaftlichen Konsens bestimmte Infrastruktur-, Integrations- und Einbürgerungsbemühungen erfolgen (vgl. beispielsweise VOGEL, 1994), die zunächst zur Lösung der bereits bestehenden Probleme der in der Vergangenheit zugewanderten Personen beitragen (vgl. BOOSNÜNNING, 1990). Schließlich wäre eine an Beruf, Alter und Qualifikation orientierte, selektive Einwanderungspolitik zu einer am Arbeitskräftebedarf orientierten Steuerung der Zuwanderung die geeignete Rahmenbedingung dieser Option (vgl. GUSY, 1994).

Ausgehend von der prognostizierten demographischen Entwicklung wird die Zuwanderung zur Sicherung des Arbeitskräfteangebots zumindest langfristig als notwendig erachtet und trotz der anhaltenden und mittelfristig dann erhöhten Arbeitslosigkeit eine "gewisse Vorsorgehaltung" im Sinne einer kontinuierlichen Einwanderung empfohlen (HOF, 1993, 70; vgl. auch HOF, 1992; BUTTLER, 1993). Andererseits erscheint die Zuwanderung aufgrund der in Europa verbreiteten Arbeitslosigkeit und der vorhandenen stillen Reserve kurz- und mittelfristig arbeitsmarktpolitisch unnötig und problematisch (vgl. COLEMAN, 1992). Übereinstimmend wird auf die Notwendigkeit von Integrationsbemühungen verwiesen und betont, daß auch eine sehr hohe Zuwanderung die Alterung und Schrumpfung der Bevölkerung nicht aufhalten, sondern nur verzögern kann (vgl. auch STEINMANN, 1993; DINKEL/LEBOK, 1993 und BUCHER/KOCKS, 1991). Die demographisch bedingten Defizite des Arbeitskräfteangebots sind, auch aufgrund der voraussichtlich durchschnittlich niedrigeren Qualifikation dieser Personengruppe, weder quantitativ noch qualitativ allein durch Zuwanderung zu vermeiden. Außerdem muß betont werden, daß eine kurz- und mittelfristig erhöhte Zuwanderung die Lösung langfristiger Arbeitsmarktprobleme erschwert, da der Druck auf die Unternehmen zur Bereitstellung qualifizierter Arbeitsplätze für Frauen und Ältere gemindert wird.

Falls es den Unternehmen gelingen würde, qualifizierte Arbeitskräfte in ausreichender Zahl zu finden und zur Einwanderung zu bewegen, so wäre deren 
Beschäftigung mit relativ geringem arbeitsgestalterischen Aufwand möglich. Diese auf die Steigerung der Zuwanderung gerichtete Option wird unternehmensbezogen wegen der einzelwirtschaftlichen Umsetzungsschwierigkeiten, der fehlenden Einbindung in ein entsprechend gestaltetes politisches Umfeld und des vergleichsweise geringeren Bezugs zu mikroökonomischen Handlungsweisen im Rahmen dieser Arbeit nicht behandelt.

\section{- Erhöhung der Erwerbsquote jüngerer Personen}

Der Rückgang des Anteils der Jüngeren an der Bevölkerung ist ein integraler Bestimmungsgrund der angeführten Defizite des Arbeitskräfteangebots. Insofern kann die Realisierung dieser Option nur einen geringen Lösungsbeitrag leisten.

Da die Arbeitsmarktprobleme Jugendlicher aufgrund ihrer sinkenden Zahl zukünftig an Bedeutung verlieren werden und die Unternehmen in einen Wettbewerb um Auszubildende treten werden, würde die Erhöhung der Erwerbsbeteiligung Jüngerer eine Verkürzung der Ausbildungs- und Studienzeiten erfordern. Die wesentlichen Maßnahmen zur Verkürzung der Ausbildungs- und Studienzeiten müssen allerdings auf der Makroebene von staatlicher Seite konzipiert und durchgesetzt werden, so $\mathrm{da} \beta$ diese Option nicht durch unternehmensbezogene Maßnahmen realisierbar ist. Aufgrund der zukünftig steigenden qualifikatorischen Anforderungen der Arbeitsaufgaben erscheint es zudem fraglich, ob es sinnvoll ist die Ausbildungs- und Studienzeiten deutlich zu senken. Zur Sicherung ihrer beruflichen Chancen in der an Dynamik zunehmenden Arbeitswelt werden außerdem immer mehr Jugendliche einen höheren Bildungsabschluß anstreben und somit später erwerbstätig werden.

Da die Männer der mittleren Altersklassen bereits derzeit fast alle erwerbstätig sind und sich der Anteil der jüngeren Personen am Erwerbstätigenpotential verringern wird, richten sich die Betrachtungen im folgenden auf Frauen und Ältere. Zur Kompensation der zukünftigen Entwicklung des Arbeitskräfteangebots erscheint eine Erhöhung der im internationalen Vergleich unterdurchschnittlichen Quote der Erwerbstätigkeit der Frauen und der Älteren notwendig. 


\section{- Erhöhung der Erwerbsquote der Frauen}

Wie die angeführten Erwerbsquoten der Frauen zeigen, besteht die Möglichkeit einer Ausweitung der Frauenerwerbsbeteiligung. Zur Realisierung dieser Option müssen makro- und mikroökonomische Maßnahmen die Möglichkeiten, einer Erwerbstätigkeit nachzugehen, verbessern und die Attraktivität der Arbeitsplätze für Frauen erhöhen. Dabei ist die Erhöhung der kontinuierlichen Beschäftigung ebenso anzustreben wie die Erleichterung der Reintegration nach einer familienbedingten Unterbrechung der Erwerbstätigkeit. So können eine familienfreundlichere Gestaltung der Arbeit und der Qualifikationsmöglichkeiten sowie die Verbesserung der auf die Kinderbetreuung gerichteten Infrastruktur dazu beitragen, daß mehr Frauen ganztags oder Teilzeit arbeiten werden. Der seit geraumer Zeit anhaltende Trend der steigenden Erwerbsbeteiligung der Frauen, muß im langfristigen gesamtwirtschaftlichen Interesse unterstützt und durch Maßnahmen zur Erleichterung der Belastungssituation erwerbstätiger Frauen begleitet werden.

Daß Frauen aufgrund des noch herrschenden Überangebots von Arbeitskräften besonders in den östlichen Bundesländern aus der Arbeitswelt verdrängt werden, ist langfristig kontraproduktiv, da Frauen zuküntig dringend als qualifizierte und erfahrene Arbeitskräfte benötigt werden. Erfolgt der Ausschluß aus dem Arbeitsprozeß über nicht stattfindende Wiedereingliederungsmaßnahmen nach der Geburt und Erziehung eines Kindes, so wirkt sich dies zudem fertilitätsmindernd aus.

Allerdings muß betont werden, daß auch die Realisierung dieser Option nur zu einer zeitlichen Verschiebung des demographischen Alterungsprozesses der Erwerbspersonen führt, da die Altersstruktur der Frauen der der Gesamtbevölkerung entspricht (vgl. RÜRUP/SESSELMEIER, 1993a, 45f.).

\section{- Erhöhung der Erwerbsquote älterer Personen}

Wie die altersabhängige Entwicklung der Erwerbsquoten zeigen, ist eine Erhöhung der Erwerbsbeteiligung Älterer möglich und insbesondere angesichts der gestiegenen Lebenserwartung auch sinnvoll. In der Vergangenheit sank das durchschnittliche Alter beim Ausscheiden aus der Erwerbstätigkeit deutlich. Die Phase der Berufstätigkeit, als wirtschaftlich besonders produktive Phase verkürzte sich, während sich die Phasen der Ausbildung und des Ruhestands verlängert haben. Diese Verschiebung des lebenszyklischen Verhältnisses von Ausbildung, Arbeit und 
Ruhestand muß sich aufgrund des demographischen Wandels zukünftig ändern. Der bisherige Trend muß umgekehrt werden. Eine Erhöhung des tatsächlichen durchschnittlichen Renteneintrittsalters ist u.a. abhängig von einer erfolgreichen Senkung der Zahl der Frühverrentungen und der Verbesserung der Möglichkeiten Älterer, eine qualifizierte Tätigkeit auszuführen. Hierzu kann eine 'altersgerechte' Gestaltung der Arbeitswelt beitragen.

Makroökonomisch besonders problematisch erscheint der geringer werdende Anteil der Personen im erwerbsfähigen Alter und die damit zusammenhängende Entwicklung des für die Rentenfinanzierung bedeutsamen Rentnerquotienten (Verhältnis der Zahl der Rentenempfänger zu der der Beitragszahler). Die Rentenreform 92 richtet sich auf die stufenweise Heraufsetzung der Altersgrenzen, die zu einer Verlängerung der Lebensarbeitszeit beitragen soll. Die Rentenreform zielt darauf, eine Flexibilisierung des Rentenalters auch in Richtung eines späteren Rentenbezugs zu erreichen und damit zur Erhöhung der geleisteten Beiträge und zur Senkung der Ausgaben der Rentenversicherung beizutragen. Bezogen auf den Beitragssatz zur Rentenversicherung wird pro Jahr der Erhöhung des durchschnittlichen Renteneintrittsalters für den Zeitraum zu dem der Rentnerquotient am ungünstigsten ausgeprägt ist eine Beitragssatzminderung von 1,5 bis $3 \%$ geschätzt (vgl. SCHMÄHL, 1993). Eine Senkung bzw. die Minderung eines Anstiegs der Beiträge erfolgt auch im Interesse der Unternehmen, da die Sozialversicherungsbeiträge die Lohnkosten erhöhen. Allerdings muß darauf verwiesen werden, daß die Heraufsetzung des Renteneintrittsalters (gerade bei bestehender Arbeitslosigkeit und vorhandenen Qualifikationsnachteilen Älterer) die Situation der arbeitsmarktbezogen Benachteiligten zusätzlich verschlechtert, da ihr Risiko arbeitslos zu werden steigt (vgl. BÄCKER/NAEGELE, 1993a, 144f.; VOLKHOLZ, 1992, 16).

Die sinkende Zahl der jüngeren und die steigende Zahl der älteren Arbeitnehmer läßt Befürchtungen hinsichtlich der langfristigen Sicherung der Arbeitsproduktivität entstehen (vgl. SIEGMUND, 1994), die sich insbesondere auf die langsamere Verbreitung neuen Wissens und neuer Methoden, eine abnehmende Leistungsfähigkeit und die durchschnittlich steigenden Arbeitskosten beziehen. Der Staat, die Unternehmen und die Tarifvertragsparteien müssen vorsorgend Maßnahmen ergreifen, die es den Unternehmen ermöglichen, die vorhandenen Aufgaben in der Zukunft mit älteren Arbeitnehmern als bisher zu bewältigen, ohne diese zu überlasten. Die Unternehmen betreiben eine stark jugendzentrierte Personalpolitik (so erfolgen beispielsweise knapp $\mathbf{8 0 \%}$ der Einstellungen bis zum 35 . Lebensjahr und mittlere bzw. ältere Jahrgänge nehmen weniger an Fort- und Weiterbildungs- 
maßnahmen teil), die sich zukünftig auch durch den Einsatz arbeitsgestalterischer Maßnahmen ändern muß (vgl. beispielsweise VOLKHOLZ, 1992; BERUFSBILDUNGSBERICHT, 1994, 124ff.). Allerdings besteht auch diesbezüglich der arbeitsmarktpolitische Zielkonflikt. Einerseits ist bei bestehender Arbeitslosigkeit ein frühes Ausscheiden aus der Erwerbstätigkeit erwünscht, so daß in den vergangenen Jahren zur Entlastung des Arbeitsmarktes, besonders auch in den neuen Bundesländern, Ältere in die Frühverrentung entlassen wurden. Andererseits werden ab der Jahrtausendwende mehr Erwerbstätige über 50 als unter 30 Jahren sein (vgl. THON, 1991, 710) und demographisch bedingt wird im gesamtwirtschaftlichen Interesse die Lebensarbeitszeit gesetzgeberisch verlängert und damit mittelfristig die Umkehr der Tendenz zu früherem Renteneintritt angestrebt, so daß in den Unternehmen eine Änderung der jugendzentrierten Personalpolitik bereits heute zu unterstützen wäre (vgl. BÄCKER, 1993; BÄCKER/NAEGELE, 1993a; VOLKHOLZ, 1992).

Die Erhöhung der Erwerbsbeteiligung Älterer würde z.B. hinsichtlich der Finanzierung der gesetzlichen Rentenversicherung, der Sicherung des Arbeitskräfteangebots und des betrieblichen Humankapitals zur Lösung demographisch bedingter Probleme beitragen. Hierzu müssen Maßnahmen entwickelt und umgesetzt werden, die zu einer altersgerechten bzw. an einer verlängerten oder anders verteilten Lebensarbeitszeit orientierten Gestaltung der Arbeitswelt beitragen.

Die derzeit vergleichsweise geringere Einbindung der Frauen und Älteren in das Erwerbsleben kann auf gruppenspezifische Problemlagen, Kennzeichen der Arbeitsaufgaben und Arbeitsbedingungen sowie gegenseitige Anpassungsprobleme zurückgeführt werden, die durch unternehmensbezogene Maßnahmen zu beeinflussen sind. Die Entwicklung und Beurteilung unternehmensbezogener Maßnahmen zur Realisierung der auf Frauen und Ältere gerichteten Optionen ist das Ziel vorliegender Arbeit. Neben den mikroökonomischen Maßnahmen zur Bereitstellung adäquater Arbeitsplätze in den Unternehmen müssen makroökonomische Maßnahmen zur Gestaltung fördernder Rahmenbedingungen ergriffen werden, damit die beiden auf Frauen und Ältere gerichteten Optionen realisiert werden können und die Gegensätze der Entscheidungen nach einzelwirtschaftlicher und gesamtwirtschaftlicher Rationalität gemindert werden. Zur Verbesserung der prognostizierten Situation muß eine Abstimmung der Mikro- und Makropolitik gelingen. Die mikroökonomischen Maßnahmen sind vorwiegend durch die Arbeitgeber, die Arbeitnehmer und ihre jeweiligen Vertreter zu gestalten bzw. 
umzusetzen, während sie im gesamtwirtschaftlichen Interesse insbesondere von staatlichem Handeln im Sinne der makroökonomischen Maßnahmen begleitet werden müssen. Zur Sicherung des Arbeitskräfteangebots können, ähnlich wie zum Abbau der Arbeitslosigkeit, kooperative Ansätze unter Beteiligung der genannten Akteure verfolgt werden (vgl. beispielsweise RÜRUP/SESSELMEIER, 1989), die auch zur Förderung einer breiten, langfristigen Umsetzung der mikroökonomischen Maßnahmen in den Unternehmen beitragen können.

Bezüglich der Dilemmasituation von Arbeitslosigkeit und Arbeitskräftemangel kann ca. bis zum Jahre 2010 ein allmählicher Rückgang der Arbeitslosigkeit erwartet werden, zudem können die Bemühungen zur Kompensation der quantitativen und qualitativen Defizite durch eine Erhöhung der Erwerbsbeteiligung der Älteren und der Frauen auch einen Beitrag zur Behebung der Wettbewerbsnachteile arbeitsmarktbezogener Problemgruppen darstellen. Betrachtet man nämlich das Profil der Arbeitslosen, so kann man feststellen, daß neben einer fehlenden Berufsausbildung (47\%) die Unterbrechung der Erwerbstätigkeit (30\%), gesundheitliche Beeinträchtigungen (29\%) sowie ein Alter von über 55 Jahren $(20 \%)$ vorherrschende Kennzeichen der Arbeitslosen sind (die Prozentangaben beziehen sich auf die Arbeitslosen in den alten Bundesländern, Stand September 1991, nach HAGEDORN, 1992, 274). Unabhängig von den Prognosen zum Arbeitskräfteangebot sind Überlegungen zur Erleichterung der Erwerbstätigkeit von Frauen und Älteren demnach sinnvoll, da ihre Beschäftigungssituation hinsichtlich Arbeitslosigkeit, Verdienst, beruflicher Stellung und Entwicklungschancen vergleichsweise ungünstig ist und diese beiden Personengruppen ohne wirksame unterstützende makro- sowie mikroökonomische Maßnahmen auch zukünftig zu den beschäftigungsbezogenen Problemgruppen zählen werden (vgl. PROGNOS, 1990, 447ff.). Die Betrachtung der beiden Optionen besitzt demnach einen hohen eigenen Wert, da die Maßnahmen zu ihrer Realisierung die entscheidungs- und verhaltensbezogenen Freiheitsgrade der betroffenen Personen erhöhen. Außerdem erscheint es angesichts der Auswirkungen längerer Erwerbslosigkeit auf die soziale Sicherung im Alter und der finanziellen Situation alleinstehender bzw. alleinerziehender Frauen sinnvoll, die Möglichkeiten der Erwerbstätigkeit von Frauen und Älteren zu verbessern. 


\section{Analytisch-methodische Vorgehensweise}

Zur Erhöhung der Erwerbsbeteiligung von Frauen und Älteren müssen die Arbeiten in den Unternehmen zukünftig so gestaltet werden, daß sie in höherem Maße den Bedürfnissen dieser Personengruppen entsprechen. Das Aufzeigen von Gestaltungsmöglichkeiten zur Verbesserung der Beschäftigung von Frauen und Älteren erfordert eine arbeitsbezogene Analyse, die hier mit Hilfe des erweiterten Belastungs-Beanspruchungs-Konzepts (vgl. auch zu folgendem ROHMERT, 1984) erfolgen soll. Zur Verdeutlichung der methodischen Vorgehensweise zur Analyse der relevanten arbeitsbezogenen Entwicklungslinien werden zunächst die für die Zusammenhänge wesentlichen Begriffe umrissen.

Die Arbeitnehmerinnen und Arbeitnehmer werden im folgenden häufig als Arbeitspersonen bezeichnet, um eine einfache geschlechtsneutrale Bezeichnung zu verwenden und um (z.B. deutlicher als mit der Bezeichnung Arbeitskraft) auf die zu beachtenden spezifischen Eigenschaften der die Arbeit durchführenden Personen, zu verweisen. Die Eigenschaften der Arbeitspersonen, wie z.B. körperliche oder qualifikatorische Voraussetzungen, beeinflussen ihre Eignung für bestimmte Tätigkeiten bzw. Arbeitsplätze. Die von Arbeitspersonen durchzuführenden Tätigkeiten werden als Arbeitsaufgaben bezeichnet (vgl. beispielsweise REFA, 1991a, 151). Mit den Arbeitsaufgaben sowie den Ausführungs- und Umgebungsbedingungen sind verschiedene Anforderungen verbunden, die zusammengesetzt exogen auf die Arbeitspersonen einwirken und mit dem umfassenden Begriff Belastungen bezeichnet werden. Die zweckgerichtete Durchführung der Arbeitsaufgaben durch hierfür geeignete Arbeitspersonen geschieht besonders unter Verwendung technischer Sachmittel in sozio-technischen Systemen, die man als Arbeitssysteme (Mensch-Maschine-Arbeitsumwelt-Systeme) bezeichnet (vgl. SCHWERES, 1980). Diese Arbeitssysteme sind verschieden organisiert und beinhalten u.U. auch zur Erfüllung gleicher Aufgaben beispielsweise verschiedene Arbeitsmethoden und -objekte. Für die folgende Analyse werden als Systemelemente die Arbeitspersonen (personengruppenbezogen oder individuell) und die Arbeitsaufgaben (in einem umfassenden technisch-organisatorischen und sozialen Sinn) unterschieden. Arbeitsaufgaben beziehen dabei insbesondere Merkmale des Arbeitsablaufs, Ausführungs- und Umgebungsbedingungen sowie, zur Erfassung der Beziehungen innerhalb und außerhalb des Arbeitssystems, die Organisation mit ein (vgl. WOBBE, 1980). 
In einem Arbeitssystem führen Belastungen zu Auswirkungen der Arbeit auf den Menschen. Diese Auswirkungen werden Beanspruchungen genannt. Die Beanspruchungen können den physischen und psychischen Funktionen der Arbeitsperson angemessen sein oder als Fehlbeanspruchungen über- bzw. unterfordernd wirken. Objektiv gleiche Belastungen können abhängig von den jeweiligen Eigenschaften sowie den Handlungs- und Verarbeitungsweisen der Arbeitspersonen inter- und intraindividuell unterschiedliche Beanspruchungen hervorrufen. Betrachtet man die Belastungen zunächst als gegeben, so können die Eigenschaften, Leistungen und Beanspruchungen durch verschiedene Anpassungsvorgänge verändert werden. Positive Anpassungsvorgänge (z.B. Übung) mindern die Beanspruchungen bzw. verbessern die Leistungen, während negative Anpassungsvorgänge (z.B. Ermüdung) die Beanspruchungen erhöhen bzw. die Leistungen verschlechtern. Bei der Verwendung des Belastungs-BeanspruchungsKonzepts zur Analyse konkreter Tätigkeiten ergeben sich beispielsweise Schwierigkeiten hinsichtlich der ergonomischen Superposition vorhandener Teilbelastungen. Außerdem können Belastungen häufig direkt (z.B. bei Überwachungstätigkeiten) nicht nachgewiesen werden, so da $B$ allenfalls physiologisch oder biochemisch meßbare Belastungsauswirkungen Hinweise auf die Dauer und Höhe vorhandener Belastungen geben (vgl. ROHMERT, 1984, 196f.). Folgende Abbildung, die noch erweitert wird, stellt die Begriffe in einem graphischen Zusammenhang dar.

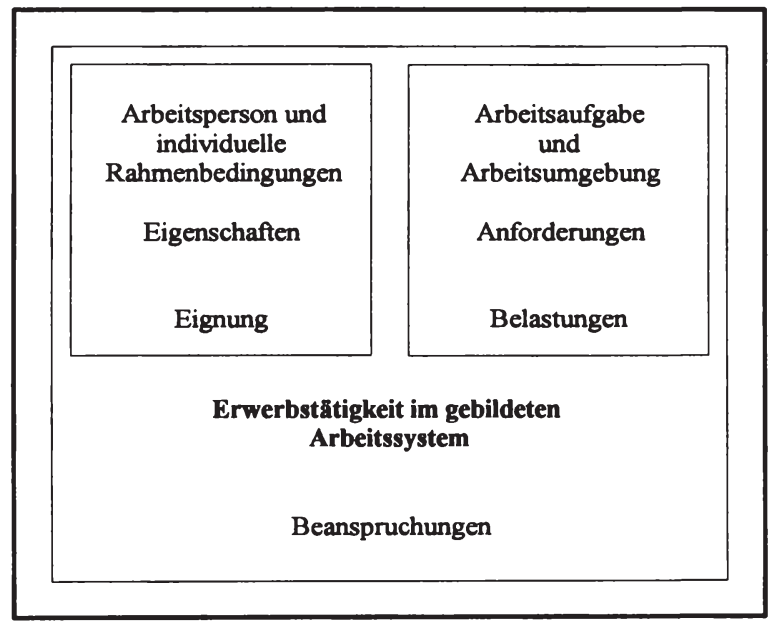

Abb. 12: Modell der menschlichen Arbeit 
Im Rahmen dieser Arbeit geht es zunächst um eine zukunftsbezogene Abschätzung verbreitet wirkender Bestimmungsgrößen wie die Verwendung moderner Produktions- und Organisationsmethoden und anschließend um den Entwurf sowie die Beurteilung unternehmensbezogener Maßnahmen zur Erhöhung der Erwerbsbeteiligung von Frauen und Älteren. Statt einer Analyse derzeit beobachtbarer konkreter Einzeltätigkeiten werden im folgenden Aussagen zu typischen bzw. durchschnittlichen zukünftigen Arbeitssystemen gemacht. Entsprechend werden Arbeitssysteme nicht als statische Systeme betrachtet. Vielmehr wird versucht, die Dynamik der Arbeitssysteme zu beachten, indem Kennzeichen des technischorganisatorischen und gesellschaftlichen Wandels, mögliche Gestaltungs- und Problemlösungsprozesse sowie wesentliche gestaltungsbezogene Interessenkonstellationen beleuchtet werden. Die Argumentation und die weitere Gliederung der Arbeit sind hierfür an folgenden Leitfragen ausgerichtet:

\section{Arbeitsperson}

Welche gruppentypischen Eigenschaften haben die potentiellen Erwerbspersonen und inwiefern wirken sich diese auf die Möglichkeiten einer Erwerbstätigkeit aus? Welche gruppenspezifischen Probleme bestehen hinsichtlich einer erhöhten Ausschöpfung des Erwerbspersonenpotentials? (Teil II. A.)

\section{Arbeitsaufgabe}

Wie wird die Arbeit im Produktions- und Bürobereich zukünftig organisiert sein? Wie ändern sich typische Arbeitsaufgaben sowie Arbeitsbedingungen und welche veränderten Anforderungsprofile resultieren daraus für die Erwerbstätigen? (Teil II. B.)

\section{Arbeitssystem}

Welche Hindernisse bestehen für die Aufnahme einer Erwerbstätigkeit? Welche typischen Beanspruchungen, insbesondere Fehlbeanspruchungen, treten (gruppenbezogen) während der Erwerbstätigkeit auf? (Teil II. C.)

Die Kombination personengruppenspezifischer Eigenschaften und beruflicher Anforderungen läßt bestimmte 'resultierende' Problemfelder erkennen. Für die Sicherung des Arbeitskräfteangebots problematische Situationen ergeben sich bei Fehlbeanspruchungen (Über- und Unterforderung während der Erwerbstätigkeit) 
und bei fehlender Eignung (ausbleibende Erwerbstätigkeit). Die identifizierten Probleme weisen auf Ansatzpunkte für unternehmensbezogene Maßnahmen zur Verbesserung der Ausschöpfung des Erwerbspersonenpotentials und zur Erhöhung der Sozialverträglichkeit der Arbeit, gerade auch im Interesse der Funktionsfähigkeit und Wirtschaftlichkeit der Arbeitssysteme (vgl. z.B. VDI, 1989, 10f.).

\section{Mikroökonomische Lösungen in den Unternehmen}

Wie können durch Maßnahmen in den Unternehmen Hemmnisse, die der Aufnahme einer Erwerbstätigkeit entgegenstehen, beseitigt werden und Fehlbeanspruchungen während der Erwerbstätigkeit, die u.U. zu einem frühzeitigen Ausscheiden aus dem Erwerbsleben führen können, gemindert werden? Wie sind diese Maßnahmen mit Blick auf die erörterten Problemlagen zu gestalten und aus Sicht der Arbeitgeber zu beurteilen? (Teil II. C. und Kapitel III)

Folgende Abbildung verdeutlicht den Zusammenhang dieser Fragen, ohne eine vollständige Erfassung anzustreben. So wird z.B. vereinfachend die Möglichkeit, daß trotz vorhandener Eignung keine Erwerbstätigkeit ausgeübt wird bzw. werden kann, nicht betrachtet. Entsprechend wird besonders die Situation der arbeitslosen Personen, die sonst zu den Erwerbstätigen zu zählen sind, und die der Hausarbeit leistenden oder Rente beziehenden Personen, die nicht zu den Erwerbstätigen zu zählen sind, nicht differenzierend dargestellt. Die punktierten Linien der Abbildung umgrenzen die in den Teilen II. A., B. und C. behandelten Aspekte. Die aufgeführten Gliederungen der Eigenschaften und Anforderungen werden in den jeweiligen Abschnitten dargestellt. 


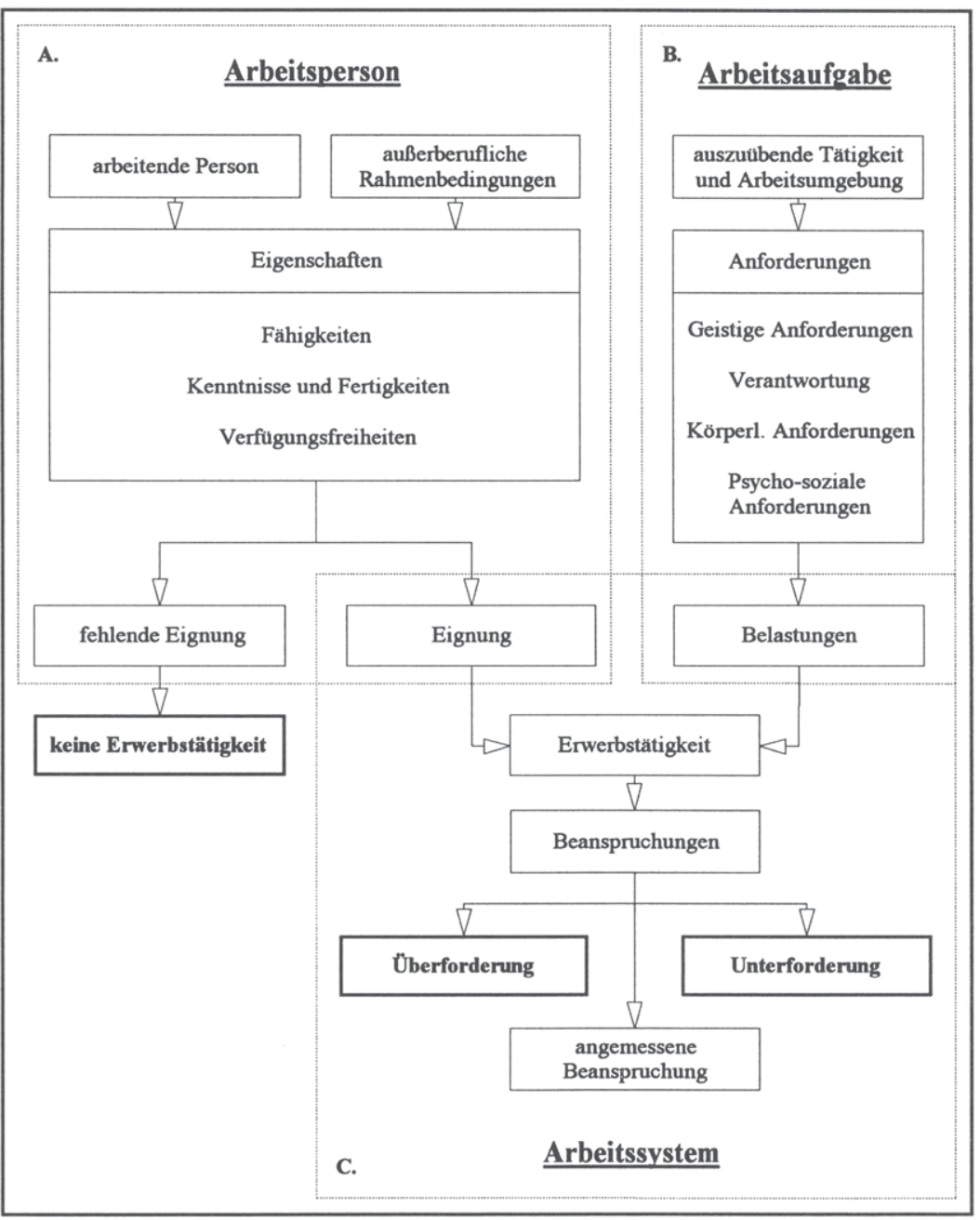

Abb. 13: Beziehungsmodell der menschlichen Arbeit und problematische Situationsausprägungen

\section{E. Systematisierung möglicher Maßnahmen}

Im Interesse ihrer Wettbewerbsfähigkeit müssen die Unternehmen dem demographisch bedingten Wandel des Arbeitskräfteangebots begegnen und die 
personellen Voraussetzungen ihres Wirtschaftens sowie der Einführung moderner Organisations- und Produktionsmethoden sichern. Hierzu müssen sie gestaltend auf die Arbeitsaufgaben und Anforderungen sowie die Eigenschaften der Arbeitspersonen einwirken und die Arbeitssysteme beeinflussen, verändern und anpassen. Eine erfolgreiche Umsetzung entsprechender Maßnahmen hat positive Effekte für die Unternehmen, da das ihnen zur Verfügung stehende Humankapital gesichert wird, und für die Arbeitspersonen, deren Möglichkeiten eine Erwerbstätigkeit aufzunehmen bzw. fortzusetzen, erweitert werden.

Die in den Unternehmen erforderlichen Maßnahmen richten sich auf die gegenseitige Anpassung von Mensch und Arbeit (vgl. beispielsweise ZÜLCH, 1992b, 246; REFA, 1985a, 161 und VDI, REFA, 1983, 111) und können gemäß der folgenden Abbildung systematisiert werden.

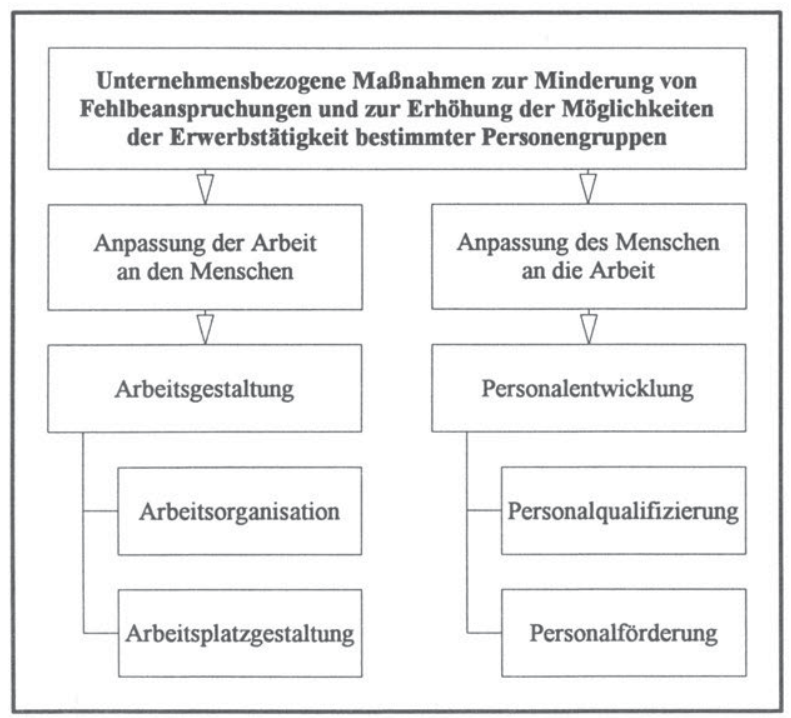

Abb. 14: Systematik unternehmensbezogener Maßnahmen

Die Arbeitsgestaltung ist arbeitsaufgabenbezogen und bezieht sich vornehmlich auf die Anpassung der Belastungssituation, während die Personalentwicklung arbeitspersonenbezogen wirkt und primär auf die Anpassung der Eignung gerichtet ist. Daneben können Arbeitsgestaltung und Personalentwicklung arbeitssystem- 
bezogen eine Verbesserung der Beanspruchungssituation erreichen. Die beiden Gestaltungsbereiche werden im weiteren näher betrachtet.

\section{Arbeitsgestaltung}

Die Arbeitsgestaltung bezieht sich auf die den Menschen betreffenden Arbeitsbedingungen innerhalb eines Arbeitssystems. Ihre Objekte können das Produkt eines Arbeitssystems (ein Erzeugnis oder eine Dienstleistung) und der Prozeß zur Erstellung des Produktes von der Gestaltung der organisatorischen Maßnahmen bis zur Arbeitsplatzgestaltung sein. Die Arbeitsorganisation beschäftigt sich primär mit der Gestaltung der Aufgaben von Abteilungen und Gruppen, während sich die Arbeitsplatzgestaltung auf die Verrichtung von Tätigkeiten einer Person bzw. an einer Stelle richtet (vgl. ZÜLCH, 1992a, 136f. und 1992b, 243). Wichtige Dimensionen der Arbeitsgestaltung, d.h. der Arbeitsorganisation und Arbeitsplatzgestaltung, werden im folgenden betrachtet:

Arbeitsorganisation kann als "systematische Gliederung und Gestaltung des Arbeitsablaufes nach aufgabenmäßigen, inhaltlichen und zeitlichen Gesichtspunkten" aufgefaßt werden (vgl. REFA, 1985a, 27). Andere Differenzierungen sind möglich, so werden z.B. statt der inhaltlichen die räumlichen Gesichtspunkte des Arbeitsprozesses oder neben der Gestaltung der Arbeitszeit insbesondere die Struktur der Aufgaben und die Bedeutung sozialer Prozesse betont (vgl. KLEINBECK, 1993). Jedenfalls bestimmt die Arbeitsorganisation einen wesentlichen Teil der mit der Durchführung der Arbeitsaufgaben verbundenen Anforderungen und der resultierenden Beanspruchungen der Arbeitsperson. Zur Entwicklung arbeitsorganisatorischer Aussagen über diesen Zusammenhang werden Fragen der Ablauforganisation durch die Analyse von betrieblichen Aufgaben und Fragen der Aufbauorganisation durch deren Synthese behandelt (vgl. ZÜLCH, 1992a, 135). Für eine Darstellung des Gestaltungsfeldes der Arbeitsorganisation werden hier die durch die Arbeitsorganisation betroffenen Dimensionen mit Aufgabenstellung, Arbeitsbedingung und Arbeitszeit bezeichnet.

- Die Dimension Aufgabenstellung bezieht sich auf die Bestimmung der Arbeitsaufgaben im Sinne der Festlegung der personellen und maschinellen Funktionen sowie der Zuordnung der Aufgaben an ein Arbeitssystem oder mehrere Arbeitssysteme, die verschiedene Arbeitsmethoden vollständig oder nur teilweise beherrschen. Neben der Struktur der Aufgaben werden die Arbeitsteilung, 
Formen der Zusammenarbeit, der Arbeitsablauf, die Information, Verantwortungsbereiche und aufgabenbezogene Kontrollmöglichkeiten sowie der zeitliche Arbeitsaufwand in Abhängigkeit von der betrieblichen Organisation (z.B. Durchlaufzeiten, Taktzeiten) festgelegt.

- Arbeitsbedingungen betreffen die räumliche Anordnung der Arbeitsplätze, die Einwirkungen der Arbeitsumgebung, den sozialen Bezug der Arbeitsaufgaben und ihrer Durchführung. Die Bezüge zwischen individuellen Arbeitspersonen und Gruppen sowie auch Kommunikationsmöglichkeiten und -formen werden gestaltet. Gruppendienliches Verhalten und Arbeitsmotivation können z.B. durch entsprechendes Führungsverhalten, durch Information, Qualifizierung und Partizipation gefördert werden. Daneben sind die betriebliche Kontrollstruktur, das Entlohnungssystem und die Bemessungsgrundlage der Entlohnung festzulegen.

- Die Dimension Arbeitszeit betrifft die zeitliche Regelung und den zeitlichen Ablauf der Arbeitsvorgänge, die Festlegung bzw. die Flexibilität der individuellen und betriebsweiten Arbeitszeit sowie der Pausenregelung.

Arbeitsplatzgestaltung betrifft den räumlichen Bereich der Arbeitsdurchführung und z.B. die Gestaltung der Arbeitsmittel und Umgebungseinflüsse sowie der Arbeitsmethoden und -verfahren. Exemplarisch wird hier die Arbeitsplatzgestaltung hinsichtlich ihrer anthropometrischen und physiologischen Ausrichtung (als Teilgebiete der ergonomischen Arbeitsplatzgestaltung nach REFA, 1985b, 142ff.) betrachtet.

- Eine anthropometrische Arbeitsgestaltung dient zur förmlichen und räumlichen Anpassung der Arbeitsplatzbestandteile und der technischen Erzeugnisse entsprechend der menschlichen Gestalt und der auszuführenden Arbeitsaufgabe. Die Gestaltung bezieht sich auf die statischen Komponenten des Arbeitssystems, sie erleichtert beispielsweise die Bedienung von Maschinen und vergrößert die Toleranzen gegenüber Fehlbedienungen, so daß der Umgang sicherer wird.

- Die physiologische Arbeitsgestaltung bezieht sich nicht nur auf den Arbeitsplatz, sondern auch auf die Arbeitsmittel, die Methoden, die Abläufe und die Arbeitsumgebung. Vereinfachend wird sie hier allerdings der Arbeitsplatzgestaltung zugeordnet, da sie Maßnahmen zum Schutz des Individuums behandelt, im Gegensatz $\mathrm{zu}$ den eher kollektiv wirkenden Vorstellungen der Arbeits- 
organisation. Die physiologische Arbeitsgestaltung richtet sich auf die Anpassung der technischen Umwelt hinsichtlich der Form und der Dauer der Belastung an die Arbeitsperson als dynamische Komponente, sie betrifft u.a. vorbeugende Gesundheitsmaßnahmen sowie Fragen der Arbeitssicherheit und des Arbeitsschutzes.

Die einzelnen Dimensionen der Arbeitsgestaltung lassen sich hinsichtlich ihrer Zielobjekte und Auswirkungen nicht strikt voneinander trennen, vielmehr überlagern sie sich und sind wechselseitig voneinander abhängig. Außerdem können zusätzlich z.B. die Dimensionen der informations- und bewegungstechnischen Arbeitsgestaltung abgegrenzt werden. Die folgende Abbildung soll trotz dieser Einschränkungen einen nicht auf Vollständigkeit gerichteten zusammenfassenden Überblick über die hier relevant erscheinenden Dimensionen der Arbeitsgestaltung geben.

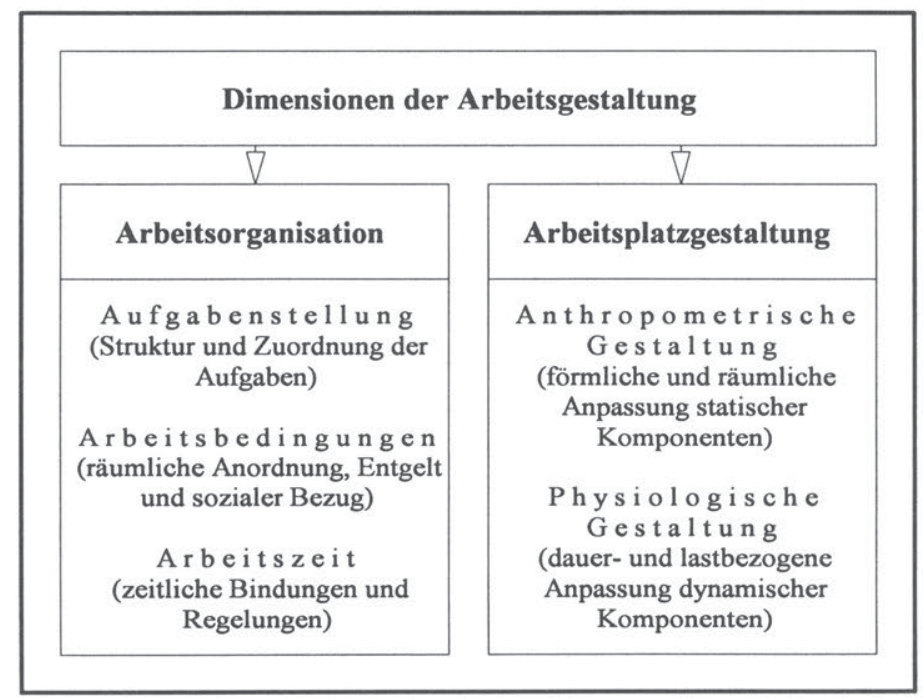

Abb. 15: Dimensionen der Arbeitsgestaltung

\section{Personalentwicklung}

Die Personalentwicklung versucht unter Beachtung vorhandener Fähigkeiten, Kenntnisse und Fertigkeiten, die Arbeitspersonen systematisch zu qualifizieren und 
zu fördern. Personalentwicklungsmaßnahmen tragen zur Anpassung von Kompetenzen und Anforderungen und damit zur Vermeidung von Überforderungs- und Unterforderungssituationen bei (vgl. HOLLING/LIEPMANN, 1993). Die Personalentwicklungsplanung ist neben der Bedarfs- und Einsatzplanung ein Teilbereich der Personalplanung (vgl. SONNTAG u.a., 1992, 51). Hier werden die Aufgabenbereiche der Personalqualifizierung und der Personalförderung unterschieden.

Personalqualifizierung betrifft die Sicherung des individuellen Arbeitsvermögens. Die arbeitsbezogene Qualifikation läßt sich in funktionale Qualifikationen und funktionsübergreifende bzw. extrafunktionale Qualifikationen einteilen (vgl. beispielsweise NEUBERGER, 1985, 114ff.; BULLINGER, 1992, $216 \mathrm{ff}$.). Funktionale Qualifikationen betreffen Kenntnisse und Fertigkeiten, die prozeßgebunden, technisch sowie tätigkeitsabhängig sind und zur Erfüllung der Arbeitsaufgaben benötigt werden. Extrafunktionale Qualifikationen betreffen vielseitig verwendbare Fertigkeiten und Orientierungen, die prozeßungebunden, metatechnisch, tätigkeitsunabhängig und funktionsübergreifend sind und sich auch auf organisatorische sowie soziale Zusammenhänge beziehen. Die verschiedenen Qualifikationsanforderungen können zudem unterschiedlichen Kompetenzbereichen zugeordnet werden. Die Fachkompetenz betrifft vorwiegend Anforderungen an funktionale, z.B. sensumotorische Fertigkeiten, die Methodenkompetenz bezieht sich z.B. auf mentale Anforderungen an Denkvermögen und Problemlösungsfähigkeit, während die Sozialkompetenz z.B. die Fähigkeit zur Zusammenarbeit angesichts gestellter Kommunikations- und Kooperationsanforderungen beinhaltet. Ergänzend kann auf die Lernkompetenz als Fähigkeit zur selbständigen Qualifizierung verwiesen werden (vgl. BULLINGER, 1992, 219f.). Als Qualifizierungsarten im Rahmen der Personalqualifizierung können Aus- und Weiterbildung, Unterweisung und Einarbeitung sowie die Qualifizierung während der Arbeitsdurchführung unterschieden werden. Die ausgewählten bzw. im Unternehmen angebotenen außer- und innerbetrieblichen Qualifizierungsmaßnahmen können sich an alle Beschäftigten oder an bestimmte Beschäftigtengruppen richten.

Die Personalförderung trägt zur Unterstützung der Entfaltung der Arbeitspersonen im Interesse der Leistungssteigerung und ihres adäquaten Einsatzes bei. Förderungsmaßnahmen können individuell und gruppenbezogen erfolgen. Der Personaleinsatz, im Sinne der quantitativen und qualitativen Zuordnung von Arbeitspersonen und Arbeitsaufgaben bzw. Arbeitsplätzen, ist somit an betrieblichen und individuellen Interessen und Bedürfnissen ausgerichtet. Aktivitäten sind insbeson- 
dere im Falle von Umsetzungen, Unterbrechungen und Neueinstellungen aber auch kontinuierlich zur Sicherung des zukünftig verfügbaren Humankapitals erforderlich.

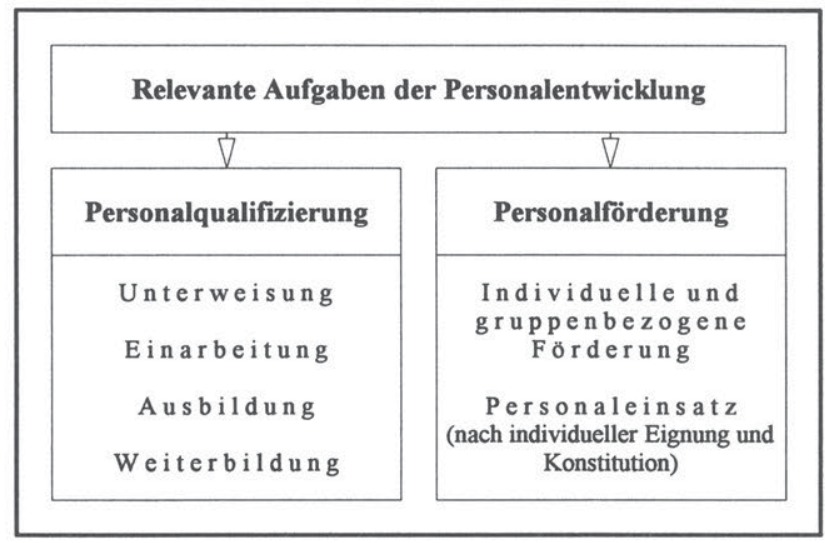

Abb. 16: Aufgabenbereiche der Personalentwicklung

\section{F. Arbeitssystembezogene und wirtschaftliche Kriterien}

Die Kriterien dienen im Rahmen dieser Arbeit der Ableitung und der Beurteilung unternehmensbezogener Maßnahmen, die im Interesse der Realisierung der zu betrachtenden Optionen zu ergreifen sind. Für diese Zwecke werden zwei Kriteriengruppen gebildet. Die arbeitssystembezogenen Kriterien werden, wie bei der Beschreibung der Vorgehensweise dargestellt, in Kapitel II hinsichtlich ihrer Veränderungen eingeschätzt, sie dienen der Einordnung der menschlichen Arbeit und der Ableitung relevanter Maßnahmen. Die wirtschaftlichen Kriterien drücken Kosten sowie Leistungen bzw. Nutzen aus, die den betrieblichen Zielgrößen entsprechen. Die wirtschaftlichen Kriterien bestimmen die Wahl und Gestaltung von Produktions- und Organisationsmethoden in den Unternehmen, so daß sie die Gestaltung der Arbeitssysteme beeinflussen (vgl. Kapitel II) und sie dienen in Kapitel III der Beurteilung der Maßnahmen aus Sicht der Arbeitgeber.

Arbeitssystembezogene Kriterien sind nicht monetär quantifizierbar. Sie richten sich auf die Einschätzung der menschlichen Arbeit, d.h. auf die Arbeitspersonen und Arbeitsaufgaben, die das Arbeitssystem kennzeichnen und damit auf 
- Eigenschaften der Arbeitspersonen,

- sich wandelnde Arbeitsanforderungen und Belastungen,

- Beanspruchungen und ihre Grenzen,

- Notwendigkeiten und Möglichkeiten der Arbeitsgestaltung und Personalentwicklung,

- individuelle und betriebssoziale Folgen und Voraussetzungen sowie

- Handlungs-, Entfaltungs- und Entwicklungsmöglichkeiten.

Die arbeitssystembezogenen Kriterien müssen zur Klärung des dynamischen Zusammenhangs von Arbeitsgestaltung sowie Personalentwicklung und Erwerbstätigkeit betrachtet werden, um hieraus Maßnahmen zur Verbesserung der Situation der Erwerbstätigkeit von Frauen und Älteren ableiten und zugleich wesentliche Bestimmungsfaktoren dieser Maßnahmen erkennen zu können.

Wirtschaftliche Kriterien beziehen sich auf die maßnahmenspezifischen Kosten und Nutzen. Während die Kostengrößen zumindest prinzipiell monetär quantifizierbar sind, lassen sich die Nutzengrößen vielfach nicht oder zumindest nicht monetär quantifizieren.

Kostengrößen:

- arbeitsplatzbezogene Investitionskosten

- Implementationskosten

- Personalaufwand

- personenbezogene Qualifizierungskosten

Nutzengrößen:

- Einsparung von Kosten

- Verbesserung der Qualität

- Erhöhung von Flexibilität und Innovationsfähigkeit

- Reduzierung des Zeitaufwandes

Die wirtschaftlichen Kriterien müssen aus Sicht der Arbeitgeber zur Beurteilung unternehmensbezogener Entscheidungs- und Handlungsweisen verwendet werden, wie auch ihre spätere Ableitung aus wettbewerblichen Überlegungen in Teil II. B. verdeutlichen wird. Vor ihrer praktischen Umsetzung müssen die arbeitssystem- 
bezogen geeignet erscheinenden Maßnahmen bezüglich des Verhältnisses von Kosten und Nutzen beurteilt werden. In folgender Abbildung sind die beiden Kriteriengruppen und ihre jeweiligen Verwendungsweisen zusammengestellt.

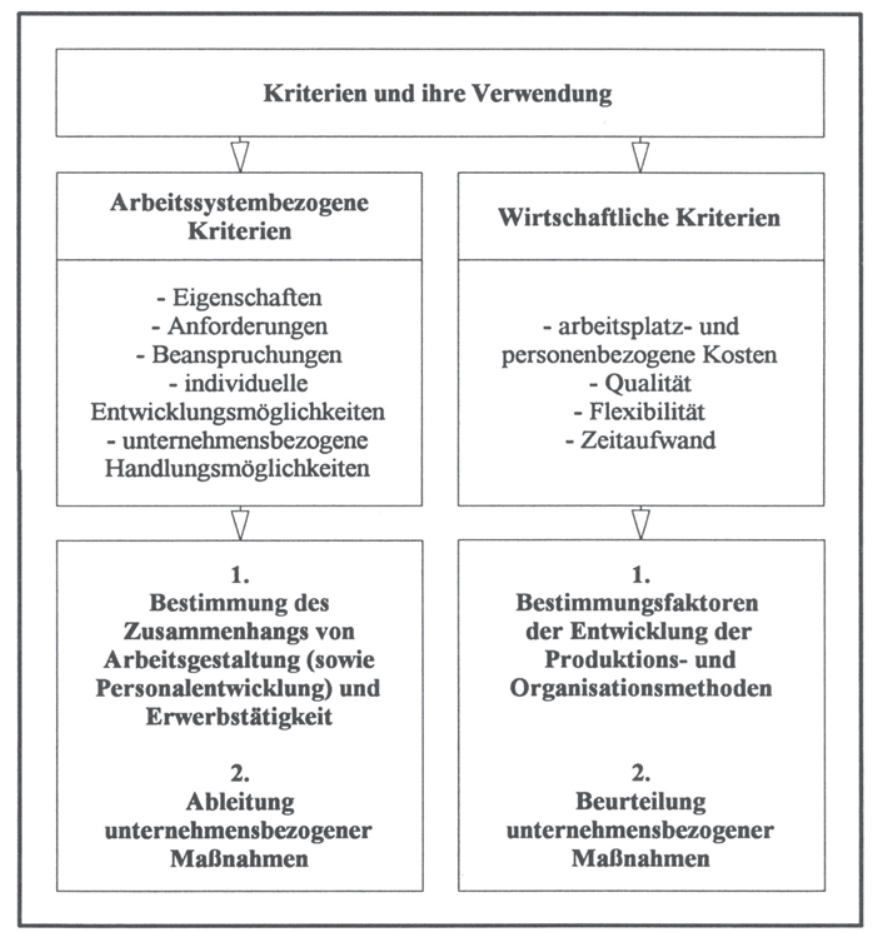

Abb. 17: Arbeitssystembezogene und wirtschaftliche Kriterien 
Stefan Lorenz - 978-3-631-75027-8

Downloaded from PubFactory at 01/11/2019 09:06:41AM

via free access 


\section{Arbeitspersonen - Arbeitsaufgaben - Arbeitssysteme: Spezifische Problemlagen als Ansatzpunkte und bestimmende Faktoren unternehmensbezogener Maßnahmen}

Im folgenden werden unternehmensbezogene Maßnahmen bestimmt, die eine Realisierung der auf Frauen und Ältere gerichteten Optionen unterstützen können. Zur Herausarbeitung der gruppenspezifischen Problemlagen werden in diesem Kapitel

- Arbeitspersonen und ihre Eigenschaften (Abschnitt II. A.)

- Arbeitsaufgaben und Anforderungen (Abschnitt II. B.)

- Arbeitssysteme und Beanspruchungen (Abschnitt II. C.)

hinsichtlich ihrer jeweiligen typischen Ausprägung und ihrer tendenziellen Entwicklung betrachtet. Zunächst werden die Eigenschaften der Arbeitspersonen und die Anforderungen der Arbeitsaufgaben systematisch getrennt erfaßt. Ihre gegenseitige Abhängigkeit und wechselseitige Beeinflussung kommen besonders bei der Behandlung der Arbeitssysteme und der sich ergebenden Beanspruchungen zum Ausdruck.

Die Aufnahme, Wiederaufnahme oder Weiterführung einer Erwerbstätigkeit kann dadurch verhindert werden, daß Arbeitspersonen und Arbeitsaufgaben, d.h. die Eigenschaften der Arbeitspersonen und die Anforderungen der Arbeitsaufgaben nicht genügend zueinander passen. Entweder finden bestimmte Personen beispielsweise aufgrund der Einstellungspolitik der Unternehmen keine Aufnahme in für sie in mancher Hinsicht geeignet erscheinende Arbeitssysteme oder ihre Beschäftigung kann in realisierten Arbeitssystemen durch außerberufliche Anforderungen oder durch (gegebenenfalls bereits lang andauernde) Fehlbeanspruchungen erschwert bzw. unmöglich gemacht werden. Die Entwicklung der Arbeitspersonen und der Arbeitsaufgaben beeinflussen die gegenseitige Anpassung, so daß die Chancen zur Ausschöpfung des Arbeitspotentials bestimmter Personengruppen verbessert oder verschlechtert werden. Die Änderungen der Arbeitsaufgaben und Anforderungen können insofern bereits bestehende arbeitsmarktbezogene Wettbewerbsnachteile von Frauen und Älteren zusätzlich verschärfen. Im folgenden finden insbesondere die auf die gegenseitige Anpassung negativ wirkenden Änderungen der Eigenschaften der Arbeitspersonen sowie der Anforderungen der Arbeitsaufgaben Beachtung, da sich hieraus Ansatzpunkte für unternehmensbezogene Maßnahmen 
zur Förderung der Erwerbstätigkeit von Frauen und Älteren ergeben. Die Betrachtungen verdeutlichen außerdem die Wirkungsweise der Maßnahmen bezüglich der Bedürfnisse der Arbeitspersonen einerseits und der Unternehmensziele andererseits. Hierauf basierend werden die Maßnahmen in Kapitel III im einzelnen dargestellt und vornehmlich aus Sicht der Arbeitgeber beurteilt.

\section{A. Arbeitspersonen und ihre Eigenschaften}

Die Betrachtung der Erwerbstätigkeit und diesbezüglich wesentlicher Eigenschaften von Frauen und Älteren soll dazu beitragen, Möglichkeiten zur besseren Ausschöpfung des Erwerbspersonenpotentials aufzuzeigen und Diskriminierungen aufgrund des Alters oder des Geschlechts zu überwinden. Die zu analysierenden arbeitsgestalterischen Maßnahmen zielen auf die Bedürfnisse bestimmter Gruppen von Arbeitspersonen und nicht auf individuelle Bedürfnisse (vgl. BOKRANZ/LANDAU, 1991, 306), wenngleich letztere im praktischen Einzelfall Beachtung finden müssen. Die Darstellung der beschäftigungsrelevanten Eigenschaften von Frauen und von Älteren wird deutlich machen, welche Hindernisse hinsichtlich der Ausweitung ihrer Erwerbsbeteiligung bestehen.

Die Einordnung der typisierten Eigenschaften erfolgt z.T. relativ zu den Eigenschaften von Männern, die noch nicht zur Gruppe der Älteren gezählt und die regelmäßig als 'Durchschnittserwerbstätige' angesehen werden. Da diese Abgrenzung der älteren und weiblichen Arbeitspersonen weithin bestimmend erscheint und die Problemlagen häufig alters- oder geschlechtsspezifisch zu begreifen sind, wird sie hier zur Entwicklung und Systematisierung der Handlungsnotwendigkeiten genutzt, obwohl die Anteile älterer und weiblicher Erwerbspersonen zukünftig steigen werden und die Vorstellung eines solchen Durchschnittserwerbstätigen an Bedeutung verliert (vgl. zur Beziehung der beiden "Ungleichheitsdimensionen" Geschlecht und Alter KOHLI, 1992, 247ff.).

Untersucht man nicht die Eigenschaften von individuellen Personen, sondern von Mitgliedern einer Gruppe, um zu allgemeineren, über-individuell gültigen Aussagen zu gelangen, so muß man sich mit gruppentypischen bzw. durchschnittlichen Eigenschaften befassen und entsprechende individuelle Abweichungen akzeptieren. Die gesellschaftliche Position einer Person, wie beispielsweise ihre Wettbewerbsfähigkeit am Arbeitsmarkt, wird sowohl durch objektiv feststellbare als auch durch lediglich vermutete Eigenschaften bestimmt (vgl. beispielsweise LUCZAK, 1993, 
36ff.). Problematisch ist es im besonderen, wenn nicht verallgemeinerbare Eigenschaften allen Mitgliedern einer Gruppe zugeschrieben werden und es aufgrund einer nicht ausreichend hohen Überprüfung bestehender Einschätzungen $\mathrm{zu}$ einer Verstetigung von Vorurteilen kommt, die ihrerseits beispielsweise $\mathrm{zu}$ geschlechts- oder altersspezifischen Benachteiligungen hinsichtlich der Erwerbstätigkeit führen. Weiterhin kann festgestellt werden, daß die Möglichkeit besteht, daß die Fremdeinschätzung einer Personengruppe von deren Mitgliedern als Selbsteinschätzung übernommen wird, so daß die Auswirkungen der Vorurteile noch verstärkt werden.

Im folgenden wird von einer prinzipiellen Gleichwertigkeit des Arbeitspotentials aller Arbeitspersonengruppen ausgegangen. Allerdings führt beispielsweise die geschlechtsspezifische Arbeitsteilung dazu, daß an Frauen häufig 'zusätzliche' außerberufliche Anforderungen gestellt werden, so daß im Falle ihrer Erwerbstätigkeit Belastungen durch Arbeit, Haushalt und Familie bestehen. Dies muß beachtet werden, um Maßnahmen zur Erhöhung der Erwerbsbeteiligung der Frauen entwickeln zu können. Ähnlich wie gegenüber Frauen existieren gegenüber Älteren sozialvermittelte Erwartungshaltungen, die unter anderem die berufsbezogene Motivation älterer Arbeitspersonen beeinträchtigen können. Die Betrachtungen sollen zu einer möglichst objektiven Einschätzung der Eigenschaften der potentiellen Erwerbspersonen und ihrer Situation am Arbeitsmarkt beitragen. Sie erfolgen weniger im Interesse einer dem Individuum angemessenen Gerechtigkeit, als im Interesse einer gesellschaftlich nützlichen Ausschöpfung persönlicher Potentiale. Entsprechend werden beispielsweise bezogen auf Frauen nicht die zu ihrer Emanzipation (im Sinne der Befreiung "aus rechtlicher, ökonomischer, sozialer und politischer Fremdbestimmung", BAUS, 1991, 213) notwendigen gesellschaftsverändernden Maßnahmen behandelt, sondern die unternehmensbezogenen Maßnahmen zur Anpassung der Arbeitssysteme, um die Möglichkeiten der Aufnahme oder Fortsetzung einer Erwerbstätigkeit der Frauen zu verbessern. Die verknüpfende Betrachtung der Erwerbsarbeit und außerberuflicher Lebensbereiche und -anforderungen geschieht in bisherigen Studien selten (vgl. BORN 1994, 209) und dient hier der Ableitung notwendiger Maßnahmen.

Die nachfolgende Abbildung zeigt die Einteilung der zu betrachtenden beschäftigungsrelevanten Eigenschaften potentieller Arbeitspersonen (für eine verwandte aber abweichende Systematisierung vgl. ROHMERT, 1984, 195f.). Im Falle der Eignung kann eine Erwerbstätigkeit ausgeübt werden, so daß es zur Bildung von Arbeitssystemen unter Beteiligung der jeweiligen Person kommen kann. 


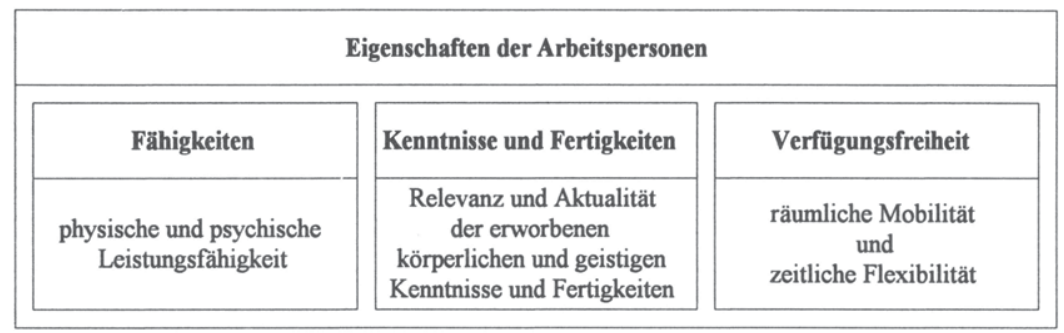

Abb. 18: Beschäftigungsrelevante Eigenschaften der Arbeitspersonen

Die Bezeichnung Eigenschaften wird hier als Oberbegriff für die in den Personengruppen begründeten Unterschiede verwendet. Die Eigenschaften werden während der Arbeitsdurchführung durch die Anforderungen bzw. die auf den Menschen wirkenden Belastungen beansprucht. Dabei werden Fähigkeiten im Sinne individueller physischer und psychischer Leistungsfähigkeiten verstanden, die die Grundfunktionen des Menschen betreffen. Demgegenüber werden konkrete Kenntnisse und Fertigkeiten durch die menschlichen Grundfunktionen und Voraussetzungen sowie den Gestaltungszustand der Arbeit beeinflußt und können beispielsweise durch Training oder Übung entwickelt werden. Erworbene Kenntnisse betreffen als 'Wissen' die Verwertung kognitiver Fähigkeiten, während sich die erworbenen Fertigkeiten als 'Können' primär auf die beherrschten Handlungskompetenzen beziehen. Kenntnisse und Fertigkeiten werden für die Arbeit vermittelt und bleiben durch entsprechend förderliche Arbeit erhalten und können durch solche Arbeit weiterentwickelt werden. Die Verfügungsfreiheit bezieht sich auf die räumliche Mobilität und zeitliche Flexibilität. Behandelt werden die Möglichkeiten der potentiellen Arbeitsperson über die Dauer, die zeitliche Lage und den Ort ihres Arbeitseinsatzes zu entscheiden und damit berufliche Anforderungen zu erfüllen. Einschränkungen der Verfügungsfreiheit ergeben sich häufig aus den persönlichen Lebensumständen, d.h. den außerberuflichen Anforderungen. Die Bezeichnung Verfügungsfreiheit wird anstelle der Bezeichnung Verfügbarkeit benutzt, um zu unterstreichen, daß die insbesondere durch außerberufliche Bedingungen eingeschränkten Entscheidungs- und Handlungsmöglichkeiten der Arbeitspersonen betrachtet werden und nicht die Bestimmungsmöglichkeiten der Arbeitgeber, im Sinne des Verfügungsrechts über die Arbeitskraft. 


\section{Frauen und Erwerbstätigkeit}

\section{a) Kennzeichen der Erwerbstă tigkeit von Frauen}

Anhand der Bevölkerungszahlen und der Zahlen zur Erwerbstätigkeit von Männern und Frauen beispielsweise im Alter von 20 bis unter 60 Jahren in Deutschland, kann festgestellt werden, daß rechnerisch eine stärkere Erwerbsbeteiligung der Frauen einen wesentlichen Beitrag zur erweiterten Ausschöpfung des Erwerbspersonenpotentials darstellen kann.

\begin{tabular}{|ccc|}
\hline $\begin{array}{c}\text { Bevölkerung } \\
\text { in 1000 }\end{array}$ & $\begin{array}{c}\text { Erwerbspersonen } \\
\text { in 1000 }\end{array}$ & $\begin{array}{c}\text { Erwerbsquote } \\
\text { in \% }\end{array}$ \\
\hline 23.711 & $\begin{array}{c}\text { Männlich } \\
21.364 \\
\text { Weiblich } \\
16.221\end{array}$ & 90,1 \\
22.932 & $\begin{array}{c}\text { Insgesamt } \\
37.583\end{array}$ & 70,7 \\
& 46.642 & 80,6 \\
\hline
\end{tabular}

Abb. 19: Personen der Altersklasse von 20 bis unter 60 Jahren, Stand: April 1994 (Quelle: STATISTISCHES BUNDESAMT, MIKROZENSUS und eigene Zusammenfassung)

Differenziert man die Erwerbsquoten nach dem Gebiet, so ist erkennbar, daß die Erwerbsbeteiligung der Frauen in den neuen Bundesländern deutlich höher ist als in den alten Bundesländern. Zur Erleichterung der zukünftigen Realisierung der auf Frauen gerichteten Option müßten daher Erwerbsbeteiligung und Erwerbsmotivation der Frauen in den neuen Bundesländern stabilisiert und zunächst gerade in den alten Bundesländern gefördert werden.

\begin{tabular}{|c|c|c|}
\hline \multicolumn{3}{|c|}{ Erwerbsquoten der Bevölkerung im Alter von 15 bis unter 65 Jahren (in \%) } \\
\hline & Männer & Frauen \\
\hline Deutschland & 81,3 & 62,7 \\
& & 60,0 \\
Früheres Bundesgebiet & 81,8 & 73,8 \\
Neue Länder und Ostteil Berlins & 79,2 & \\
\hline
\end{tabular}

Abb. 20: Erwerbsquoten nach Geschlecht und Gebiet, Stand: April 1994, Mikrozensus (Quelle: CORNELSEN, 1995)

Zur Einordnung der genannten Erwerbsquoten kann die Entwicklung der Erwerbsbeteiligung der Frauen im zeitlichen Verlauf betrachtet werden. Die folgende Abbildung zeigt die Erwerbsquoten nach Altersklassen und Familienstand seit 1960. 


\begin{tabular}{|c|c|c|c|c|c|c|c|c|}
\hline \multirow{3}{*}{$\begin{array}{c}\text { Erwerbs- } \\
\text { quoten (in \%) } \\
\text { Altersgruppe }\end{array}$} & \multicolumn{8}{|c|}{ Frauen } \\
\hline & \multicolumn{4}{|c|}{ ledig } & \multicolumn{4}{|c|}{ verheiratet } \\
\hline & 1960 & 1970 & 1980 & 1991 & 1960 & 1970 & 1980 & 1991 \\
\hline $15-20$ & 85 & 63 & 41 & 35 & 65 & 60 & 62 & 62 \\
\hline $20-25$ & 92 & 84 & 77 & 77 & 51 & 53 & 64 & 69 \\
\hline 25-30 & 93 & 87 & 85 & 85 & 39 & 41 & 55 & 63 \\
\hline 30-35 & 91 & 88 & 88 & 91 & 37 & 39 & 51 & 60 \\
\hline $35-40$ & 88 & 88 & 91 & 92 & 37 & 40 & 50 & 65 \\
\hline $40-45$ & 87 & 90 & 88 & 90 & 37 & 42 & 50 & 68 \\
\hline 45-50 & 83 & 89 & 88 & 88 & 34 & 41 & 47 & 64 \\
\hline $50-55$ & 78 & 84 & 87 & 84 & 30 & 37 & 41 & 54 \\
\hline $55-60$ & 69 & 75 & 77 & 75 & 25 & 29 & 32 & 40 \\
\hline $60-65$ & 39 & 41 & 26 & 19 & 16 & 17 & 11 & 11 \\
\hline $65+$ & 14 & 11 & 7 & 5 & 9 & 7 & 3 & 3 \\
\hline $15-65$ & 85 & 74 & 61 & 69 & 34 & 38 & 45 & 54 \\
\hline
\end{tabular}

Abb. 21: Erwerbsquoten deutscher Frauen nach Altersgruppen und Familienstand im zeitlichen Vergleich (Quelle: FRANZ, 1994, 21)

Für die Erwerbsquoten der Frauen lassen sich drei Entwicklungstendenzen hervorheben:

- Zunächst ist der Rückgang der Erwerbsbeteiligung lediger Frauen jüngerer Altersklassen, aufgrund der durchschnittlichen Verlängerung der Schul- und Ausbildungsphase, erkennbar.

- Weiterhin zeigt sich der Anstieg der Erwerbsquoten verheirateter Frauen auf ein Niveau, das in den mittleren Altersklassen allerdings zwischen 22 und 35\%Punkten unter den Erwerbsquoten lediger Frauen liegt.

- Schließlich wird der starke Rückgang der Erwerbsquoten der über 60jährigen deutlich. Differenziert man die Zahlen zur Erwerbstätigkeit von Männern und Frauen nach Altersklassen, wie in Kapitel I für die Erwerbsquoten geschehen, so zeigt sich zudem, daß Frauen früher als Männer aus dem Erwerbsleben ausscheiden. Die mit der Tendenz zum früheren Austritt aus der Erwerbstätigkeit zusammenhängenden altersspezifischen Fragen werden im Abschnitt über ältere Personen behandelt.

Die Zahlen machen deutlich, daß besonders verheiratete Frauen aufgrund ihrer niedrigeren Erwerbsquoten stärker an der Erwerbstätigkeit beteiligt werden können. Der Trend zu ihrer höheren Erwerbsbeteiligung muß sich hierfür fortsetzen. Der Zusammenhang von Familienstand und Erwerbsquote ergibt sich aus verschiedenen Gründen. So ist es für ledige Frauen regelmäßig notwendig, ihren Lebensunterhalt selbst zu verdienen. Verheiratete Frauen können dagegen von ihren 
Ehemännern finanziell versorgt werden und erhalten durch die Ehe eine abgeleitete Altersversorgung ohne eigene Erwerbstätigkeit. Außerdem bestehen für verheiratete Frauen, besonders aufgrund der verbreiteten geschlechtsspezifischen Arbeitsteilung, Schwierigkeiten, Erwerbstätigkeit und Familienarbeit zu vereinbaren. Für die Entwicklung der Erwerbsbeteiligung der Frauen kann das gestiegene Scheidungsrisiko als ein weiterer Bestimmungsfaktor aufgefaßt werden, da sich hierdurch tendenziell die Bereitschaft verheirateter Frauen zur Aufnahme einer Erwerbstätigkeit erhöhen dürfte. Weiterhin kann das Scheidungsrisiko das Ausbildungs- und Erwerbsverhalten der Frauen bereits vor der Ehe so beeinflussen, daß insbesondere durch höhere Humankapitalinvestitionen auch die Chancen für eine spätere Erwerbstätigkeit verbessert werden (vgl. entsprechende theoretische Überlegungen von DIEKMANN, 1994). Folgende Abbildung zeigt die nach dem Familienstand untergliederten Zahlen zur Erwerbstätigkeit verschiedener Altersklassen. Der 'Spielraum' für eine Ausweitung der Erwerbsbeteiligung gerade verheirateter Frauen wird so besonders deutlich.

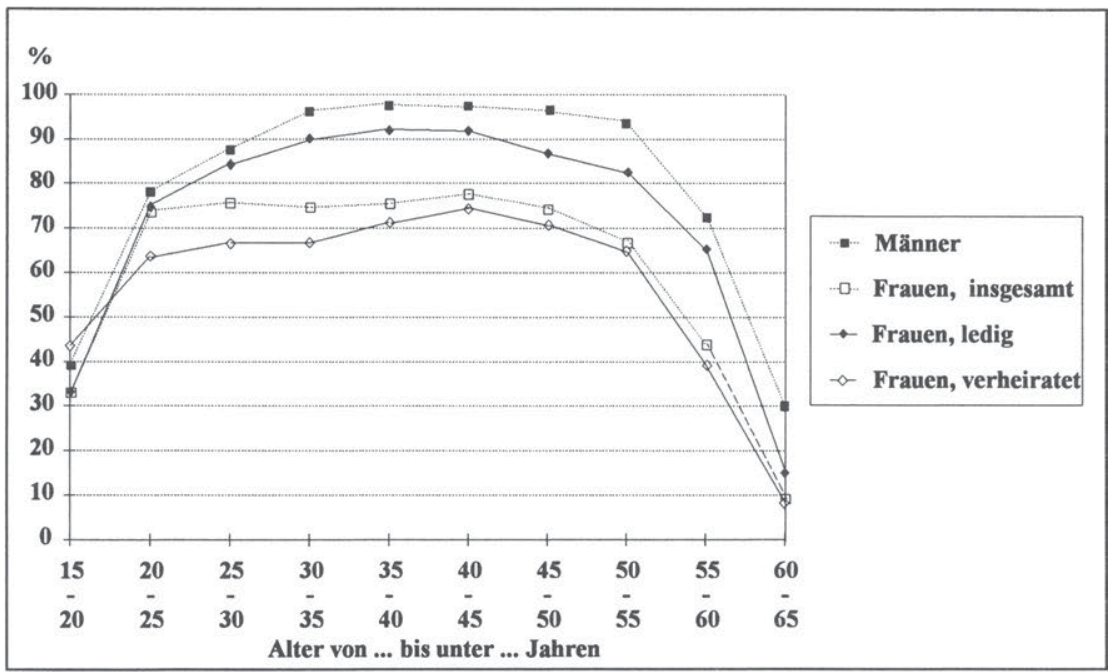

Abb. 22: Familienstandabhängige Erwerbsquoten der Frauen nach Altersklassen, Stand: April 1993 (Quelle: STATISTISCHES BUNDESAMT, MIKROZENSUS)

Der Familienstand hat einen deutlichen Einfluß auf das Ausmaß der Erwerbstätigkeit der Frauen. Dies ist auch daran erkennbar, daß die Erwerbsquoten bereits bei Frauen ohne Kinder unterschiedlich hoch sind. So sind beispielsweise in der Altersklasse von 25 bis unter 65 Jahren (mit der Wahl von 25 Jahren als untere 
Grenze dürfte der wesentliche Teil der Ausbildungszeiten ausgegrenzt sein) ca. 48\% der verheirateten und mit ihrem Mann zusammenlebenden Frauen erwerbstätig, aber 66\% aller alleinstehenden Frauen (Stand April 1993, STATISTISCHES BUNDESAMT, MIKROZENSUS und eigene Zusammenfassung).

Betrachtet man die Erwerbsquoten der Frauen mit Kindern, so zeigt sich, daß viele Frauen nach der Geburt eines Kindes ihre Berufstätigkeit unterbrechen. Diese Unterbrechungsphase war in den alten Bundesländern weiter verbreitet als in der ehemaligen DDR. Jedenfalls steigt mit wachsender Kinderzahl der Anteil der Frauen, die ihre Erwerbstätigkeit unterbrechen, d.h. ihre Erwerbsquote fällt (vgl. beispielsweise SCHULZ/KIRNER, 1994), und mit zunehmendem Alter der Kinder steigt die Erwerbsquote der Frauen wieder an.

Diese Überlegungen und Zahlen deuten an wie differenziert die Position von Frauen auf dem Arbeitsmarkt betrachtet werden kann, und sie zeigen, daß berufsbezogene Vorbehalte, die von einem traditionellen Modell der Familie und der geschlechtsspezifischen Arbeitsteilung ausgehen, die Vielfalt der bestehenden Situationen verdecken und gegenüber der Gesamtheit der Frauen wenig zutreffend sind. Da verheiratete Frauen im Vergleich zu ledigen bzw. verwitweten oder geschiedenen Frauen eine deutlich niedrigere Erwerbsquote haben, sind im Interesse einer höheren Erwerbsbeteiligung der Frauen besonders solche Hindernisse zu betrachten, die der Erwerbstätigkeit verheirateter Frauen entgegenstehen. Da sich das Erwerbsverhalten von Frauen ohne Kinder, beispielsweise aufgrund des zunehmenden Qualifikationsniveaus und der damit durchschnittlich steigenden Erwerbsmotivation, zukünftig voraussichtlich dem der Männer annähern wird, müssen insbesondere die Erwerbsmöglichkeiten von Frauen mit Kindern verbessert werden, um das Arbeitskräfteangebot zu sichern, Qualifikationen zu erhalten und damit die Vergeudung von Humankapital zu mindern.

Die Höhe der möglichen Ausweitung der Frauenerwerbsbeteiligung hängt von mikroökonomischen Maßnahmen und der Entwicklung der außerberuflichen Anforderungen, von der zukünftigen Vermögens- bzw. Einkommenssituation der privaten Haushalte sowie der Gestaltung der gesellschaftlichen und makroökonomischen Bedingungen ab. Angesichts des zukünftig wachsenden Anteils alter Personen an der Gesamtbevölkerung und der geringeren Zahl der Kinder sollte mit Blick auf die außerberuflichen Anforderungen die Bedeutung der häuslichen Pflege alter, behinderter oder kranker Familienmitglieder nicht unterschätzt werden. Die verschiedenen hieraus resultierenden Belastungen dürften hinsichtlich der 
zukünftigen Erhöhung der Erwerbsbeteiligung der Frauen einschränkend wirken. Negative Auswirkungen der Pflege auf die Erwerbstätigkeit können besonders aufgrund vielfältiger zeitlicher Beeinträchtigungen, beispielsweise in einer Minderung der Zuverlässigkeit, der Konzentrations- und Leistungsfähigkeit sowie der Teilnahmemöglichkeiten an Maßnahmen der beruflichen Weiterbildung bestehen (vgl. für eine Zusammenstellung der in verschiedenen Studien festgestellten Auswirkungen beispielsweise CULLEN/CLARKIN, 1994, 6f. und JANI-LE BRIS, 1994, 3f.).

\section{b) Beschăftigungsrelevante Eigenschaften von Frauen}

\section{(1) Fähigkeiten}

Zur Differenzierung der Fähigkeiten lassen sich besonders arbeitsmedizinische und arbeitsphysiologische Erkenntnisse anführen. Hinsichtlich der physischen Fähigkeiten treten Unterschiede zwischen den Geschlechtern vor allem bei der energetischen Leistungsfähigkeit auf. Frauen haben durchschnittlich eine geringere Muskelmasse und einen höheren Körperfettgehalt als Männer. "Die relative Muskelmasse erreicht im Durchschnitt $85 \%$, die Muskelkraft $70 \%$ und die maximale Sauerstoffaufnahme $60 \%$ der männlichen. Die Kraft der oberen Extremitäten und der Stammuskulatur liegt mit $56 \%$ bzw. $64 \%$ noch deutlich niedriger" (KONIETZKO, 1989). Die weibliche Leistung beträgt bei Schwerarbeit nur ca. $75 \%$ der männlichen, u.a. wegen einer ungünstigeren Herz-Kreislauf-Situation und einer geringeren Lungenkapazität. Da das Schlagvolumen bei Frauen kleiner ist erfolgt eine Anpassung durch eine Erhöhung der Frequenz der Leistung, die Pulsfrequenz ist bei gleicher Belastung höher. Außerdem ist die maximale Sauerstoffaufnahmefähigkeit geringer (vgl. KONIETZKO, 1989; BORNEFF, 1973, $301 \mathrm{ff}$.). Für die Möglichkeit eine bestimmte berufliche Tätigkeit auszuführen sind die Dauerleistungsgrenzwerte wichtig. Die Dauerleistungsgrenze ist für Frauen niedriger als für Männer. Daher werden arbeitsgestaltende Maßnahmen zur systematischen Anpassung des Arbeitsplatzes, der Arbeitsumgebung und der Arbeitsorganisation an die arbeitenden Frauen empfohlen, um zu vermeiden, daß die Frauen zu hoch beansprucht werden bzw. die Arbeit niedriger bewertet oder geringer entlohnt wird. Andererseits wird darauf verwiesen, daß die Annahme "Frauen seien 'arbeitsschutzbedürftiger' als Männer" die geschlechtsspezifische Arbeitsteilung unterstützt (vgl. LUCZAK, 1993, 44). Festzuhalten bleibt, daß die Spannweite der meisten Meßgrößen erheblich ist und sich entsprechend große 
Überschneidungsbereiche weiblicher und männlicher Meßwerte ergeben. Die gruppenbezogenen Unterschiede der physischen Fähigkeiten können bezogen auf die einzelne Arbeitsperson erheblich relativiert werden.

Besonders zu beachten ist die Situation der Schwangerschaft. Allgemein sind schützende gesetzliche Regelungen gerechtfertigt, falls für die Mitglieder einer bestimmten Gruppe, wie beispielsweise werdende Mütter, besonders hohe individuelle Risiken bestehen (vgl. BOLLE/SCHNEIDER, 1988, 154). Entsprechend sind Arbeitsschutzvorschriften für gebärfähige Frauen sinnvoll, wenn die Arbeit den Fötus im Falle einer noch nicht erkannten oder erkennbaren Schwangerschaft gefährden kann (vgl. PFARR, 1991, 677). Die gesetzlichen Regelungen sollen gesundheitliche Schädigungen während der Beschäftigung vermeiden und einen Verlust des Arbeitsplatzes infolge der Schwangerschaft verhindern. Das Mutterschutzgesetz bestimmt weiterhin ein Beschäftigungsverbot von 6 Wochen vor und 8 bzw. bei Früh-oder Mehrlingsgeburten 12 Wochen nach der Entbindung. Während dieser Schutzfrist zahlt die Krankenkasse den Versicherten Mutterschaftsgeld; den Differenzbetrag zum Nettolohn trägt der Arbeitgeber. Die Kosten für die Pauschalzahlung während des Erziehungsurlaubs werden dagegen aus staatlichen Mitteln gedeckt. So unverzichtbar der Mutterschutz aus arbeitsmedizinischer Sicht ist, so diskutierbar ist seine Ausgestaltung, insbesondere die Modalitäten seiner Finanzierung, hinsichtlich der Auswirkungen auf die Chancen von Frauen, qualifikationsgerecht erwerbstätig zu sein.

Bereits die 'potentielle Mutterschaft' von Frauen und die traditionell damit verbundene Rollenverteilung (beispielsweise bezüglich der Inanspruchnahme des Erziehungsurlaubs) bewirken, abgesehen von allen entsprechenden Arbeitsschutzmaßnahmen für die Zeit der Schwangerschaft, daß sie aus Sicht der Unternehmen als unsicherere Arbeitspersonen angesehen werden. Damit haben Frauen berufliche Wettbewerbsnachteile gegenüber Männern, die sich auch in geringeren Ausbildungs-, Weiterbildungs- und Aufstiegschancen widerspiegeln können. Bezüglich der Menstruation gibt es keine eindeutigen Beweise für eine gesetzmäßige Beeinflussung der körperlichen Leistungsfähigkeit (vgl. KONIETZKO, 1989, 2; ULMER, 1985, 55f.). Einschränkend bemerkt hierzu BORNEFF (1973, 308f.): "Die funktionellen, periodenabhängigen Störungen haben arbeitsmedizinisch erhebliche Bedeutung, wenn sie durch Schmerzen, schwankende Stimmungslage, gesteigerte effektive bzw. ineffektive Antriebshaftigkeit zu Konflikten mit der Umwelt führen bzw. Leistungsreduzierung oder Arbeitsausfall eintreten". Zu einer menstruationsbedingten Verminderung der Leistungsfähigkeit kann es besonders am Tage vor und 
an drei Tagen nach Mensesbeginn kommen. Weiterhin können während des Klimakteriums physische und psychische Beschwerden auftreten, die die Leistungsfähigkeit von Frauen beeinträchtigen können.

Die vorhandenen physischen Geschlechtsunterschiede sind, abgesehen von der Situation der Schwangerschaft, im wesentlichen quantitativer Art. "Das bedeutet, daß die Frau zwar im Kollektiv gegenüber dem Mann benachteiligt ist, im Einzelfall beim Vorliegen entsprechender anatomischer und physiologischer Voraussetzungen ihm durchaus gleichwertig oder überlegen sein kann" (KONIETZKO, 1989).

Hinsichtlich der psychischen Fähigkeiten läßt sich feststellen, daß es bezogen auf den Gesamtwert der Intelligenz keine meßbaren geschlechtsspezifischen Unterschiede gibt. Allerdings treten in Einzelbereichen verschiedene Fähigkeiten hervor. Frauen haben im sprachlichen Bereich und Männer bezogen auf räumliches Vorstellungsvermögen sowie im mathematischen Bereich höhere Begabungen (vgl. LUCZAK, 1993, 88f.). Diese Unterschiede könnten eine kulturspezifische Erscheinung sein und auf unterschiedlichen Erfahrungen beruhen. Für das Ertragen psychisch und physiologisch wirkender Monotoniezustände können jedenfalls statt geschlechtsspezifischer Unterschiede eher unterschiedliche Rollenerwartungen wichtig sein.

Für die Aktivierung des Potentials psychischer Fähigkeiten sind vielfältige äußere Bedingungen wichtig, so sind im besonderen Sozialisationseffekte bedeutsam, auch wenn sie nicht isoliert nachgewiesen werden können. Häufig werden besondere Eigenschaften von Frauen hervorgehoben, deren Entstehung durch eine frauentypische Sozialisation oder auch durch neurobiologische und -psychologische Unterschiede begründet werden. So wird ein frauenspezifisches Arbeitsvermögen als Kreativitätspotential bezeichnet, die Team- und Kooperationsfähigkeit sowie das Denken in komplexen Zusammenhängen wird bezogen auf Frauen besonders betont (vgl. ESTOR, 1991, 321f.). Frauen erscheinen somit für die Umsetzung moderner Mitarbeiterführungs- und Motivationskonzepte besonders geeignet. Fähigkeit zum Zuhören und zum Zeigen menschlicher Besorgtheit, Gefühle und Intuition sind Eigenschaften die Frauen zugeschrieben werden und die traditionell für die Arbeit im sozialen, familiären und privaten Bereich benötigt werden. Da in den Unternehmen zunehmend sozialintegrative Führungsqualitäten gefordert werden, können Frauen für Managementtätigkeiten diesbezüglich zukünftig besonders geeignet erscheinen. Allerdings steht ein breiterer tatsächlicher Einsatz von Frauen in Führungspositionen noch aus (vgl. HOETS, 1993). Obwohl Frauen 
derzeit die sozialen Kompetenzen aufgrund außerberuflicher Anforderungen und vermittelter Erwartungshaltungen durchschnittlich stärker zu entwickeln scheinen, muß besonders im Interesse einer höheren und gleichberechtigteren Erwerbsbeteiligung von Frauen betont werden, daß Männer soziale Kompetenzen ebenso erwerben und sich im besonderen stärker an familienbezogenen Arbeiten beteiligen können. In diesem Sinne ist die geschlechtsspezifische Arbeitsteilung nicht natürlich begründet und Arbeiten, die beispielsweise soziale Fähigkeiten erfordern, können bzw. müssen nicht nur von Frauen durchgeführt werden. Entsprechend kann auch das Konzept des "weiblichen Arbeitsvermögens", das seit den siebziger Jahren die geschlechtsspezifische Segmentation des Arbeitsmarktes erklären soll, relativiert werden. Angesichts der männlichen Dominanz im Arbeitsleben besteht die Gefahr, "daß sich das tradierte geschlechtsspezifische Meinungsbild weiter stabilisiert und Einschätzungen zum weiblichen Arbeitsvermögen einseitig von den männlichen Teilnehmern am Erwerbsleben geprägt werden" (ENGELBRECH, 1991a, 535).

Geschlechtsspezifisch verteilte, außerberufliche Anforderungen schränken das langfristig für die Arbeit nutzbare Potential psychischer Fähigkeiten vieler Frauen ein. Aufgrund der vielfältigen Belastungen durch Beruf, Haushalt, Erziehung und/oder Pflege kann es zur psychischen und physischen Überforderung kommen. Andererseits kann die wechselweise miteinander verbundene Zufriedenheit mit den verschiedenen Tätigkeiten und die vielfältige Stimulation durch unterschiedliche Anforderungen betont werden (vgl. MÜLLER-DAEHN/FOOKEN, 1992, 85).

\section{(2) Kenntnisse und Fertigkeiten}

Die Entfaltung menschlicher Fähigkeiten erfolgt wesentlich während des Durchlaufens 'formaler' Qualifikationsphasen sowie durch die Ausführung qualifizierender (bzw. umfassender: persönlichkeitsförderlicher) Tätigkeiten. Die ausgebildeten Fertigkeiten bleiben durch ihre Nutzung während der Erwerbstätigkeit erhalten und entwickeln sich fort. Strebt man für verschiedene Personengruppen Chancengleichheit hinsichtlich der Entfaltung ihrer Fähigkeiten an, so müssen entsprechende Hindernisse für die Wahl und Ausübung beruflicher Tätigkeiten erkannt und abgebaut werden. In diesem Sinn sollten Frauen nicht zur Wahl zwischen Familie und beruflicher Tätigkeit gezwungen werden. Im folgenden werden als Indikatoren für die erworbenen Kenntnisse und Fertigkeiten die durchlaufene Ausbildung, Weiterbildung und die berufliche Einsatzform betrachtet. Bei jeder festzustellenden bzw. noch anzustrebenden Verbesserung der Situation von Frauen im Bereich der 
berufsbezogenen Bildung bleibt festzuhalten, daß hieraus nicht automatisch verbesserte berufliche Chancen erwachsen und nur ein auf verschiedene Eigenschaften und Barrieren gerichtetes Maßnahmenbündel die Erwerbstätigkeit von Frauen erhöhen kann.

Bezogen auf die allgemeine Schulausbildung besteht heute kein geschlechtlicher Unterschied mehr; unter 30 jährige Frauen sind gleichaltrigen Männern sogar überlegen.

\begin{tabular}{|c|c|c|}
\hline \multicolumn{2}{|c|}{ Allgemeine Schulabschlüsse (in \%) } \\
\hline Volksschul-/HauptschulabschluB & Frauen & Mănner \\
\hline AbschluB der polytechnischen Oberschule & 28 & 37 \\
Realschul- oder gleichwertiger AbschluB & 14 & 14 \\
Fachhochschul- oder Hochschulreife & 32 & 22 \\
\hline
\end{tabular}

Abb. 23: Verteilung der Schulabschlüsse nach Geschlecht im Alter von 15 bis unter 30 Jahren (Quelle: Mikrozensus 1993 - freiwillige Angaben, STATISTISCHES BUNDESAMT, 1994c)

Bei der beruflichen Ausbildung sind die Unterschiede in der Verteilung ebenfalls gering. Allerdings existiert eine deutliche geschlechtsspezifische Segmentierung, beispielsweise hinsichtlich der gewählten Ausbildungsberufe und Studienfächer, die wesentlich durch gesellschaftliche Erwartungshaltungen und sozialisationsbedingte Verhaltensweisen bestimmt sein dürfte. So haben nach einer IAB-Befragung im kaufmännisch/verwaltenden Bereich nur die Hälfte und im gewerblich/technischen Bereich nur ein Viertel der Betriebe gleichzeitig weibliche und männliche Auszubildende (vgl. ENGELBRECH/KRAFT, 1992). Frauen konzentrieren sich auf nur wenige nicht-technische Ausbildungsberufe, so besetzten $1992 \quad 54,3 \%$ der weiblichen Auszubildenden die zehn für Frauen bedeutendsten Ausbildungsberufe, bei den männlichen Auszubildenden waren es dagegen nur $39,5 \%$ (STATISTISCHES BUNDESAMT, 1993). Zu den Ausbildungsberufen zählen Büro-, Verwaltungs- und personenbezogenen Dienstleistungsberufen (z.B. Arzthelferin, Zahnarzthelferin und Friseurin). Mit den überwiegend von Frauen durchlaufenen Ausbildungen zu Assistenten- oder Helferinnenberufen sind vielfach keine bzw. geringere berufliche Entwicklungsmöglichkeiten gegeben, so daß sie Sackgassenqualifikationen darstellen (vgl. ENGELBRECH, 1991a). Männer wählen statt dessen eher sachbezogene, gewerblich-technische oder naturwissenschaftliche Berufe, die zumindest unter aufstiegs- und verwertungsbezogenen Gesichtspunkten für die Zukunft aussichtsreicher zu beurteilen sind. Weiterhin erlernen Frauen häufig Schulberufe, statt Berufsausbildungen des dualen Systems zu durchlaufen, 
die eine weitaus engere Bindung an Erwerbs- und Aufstiegsmöglichkeiten bieten (vgl. RETTKE, 1992, 87ff.). Der in einer schulischen Ausbildung erworbene Abschluß stellt zudem häufig lediglich die Vorstufe einer weiteren Berufsausbildung dar (vgl. ENGELBRECH, 1991a und 1992a).

Neben der horizontalen Segregation durch das Durchlaufen unterschiedlicher Berufsausbildungen existiert eine vertikale Segregation, die die Form des beruflichen Einsatzes nach der Ausbildung betrifft. So üben Frauen auch in Berufen, in denen Frauen und Männern gleichermaßen ausgebildet werden, häufiger geringerwertige Tätigkeiten aus. Nach einer vom IAB durchgeführten Befragung setzt ein Viertel der Betriebe Männer und Frauen im gleichen Beruf nach Ausbildungsabschluß zumindest teilweise anders ein. Die unterschiedliche Entlohnung kann daher nicht nur auf die unterschiedliche Berufswahl, sondern auch auf den unterschiedlichen Einsatz nach der Ausbildung zurückgeführt werden (vgl. ENGELBRECH/KRAFT, 1992; ENGELBRECH, 1992a).

Insgesamt müssen die Ausbildung von Frauen in zukunftsorientierten Berufe und ihre Chancen während des Übergangs von der Ausbildung zur Beschäftigung gefördert werden (vgl. BOLLE/SCHNEIDER, 1988, 149f.), zumal aufgrund der gestiegenen Humankapitalinvestitionen der Frauen eine weiter zunehmende Berufsorientierung $\mathrm{zu}$ erwarten ist. Während der Bildungsabschluß für die Erwerbsquote der Männer keine entscheidende Bedeutung hat, steigt die Erwerbsbeteiligung der Frauen mit dem Niveau des Bildungsabschlusses (vgl. SCHWARZ, 1990, 364ff.). Es scheint verständlich, daß die Motivation eine längere Ausbildungsphase zu durchlaufen, mit derjenigen erwerbstätig zu sein wechselseitig verknüpft ist, so müssen auch für Frauen die während der Ausbildung erworbenen Kenntnisse und Fertigkeiten für die berufliche Tätigkeit und das innerbetriebliche Weiterkommen verwertbar werden. Da das durchschnittliche Qualifikationsniveau bei jüngeren Frauen höher ist als bei älteren wird es sich zukünftig auf die gesamte weibliche Bevölkerung ausweiten. Entsprechend dürfte die Motivation von Frauen, einer Erwerbstätigkeit nachzugehen, weiter steigen (vgl. LAUTERBACH, 1991; LAUTERBACH u.a. 1994). Allerdings kann festgehalten werden, daß die eingangs geschilderten Einflüsse des Familienstandes und der Kinderzahl auf die Erwerbsquote den Einfluß des Bildungsabschlusses überlagern (vgl. SCHWARZ, 1990).

Maßnahmen der beruflichen Bildung, die der Aktualisierung und Erweiterung der in vorausgegangenen Bildungsphasen erworbenen Kenntnisse und Fertigkeiten dienen, werden der beruflichen Weiterbildung zugeordnet. Ein wichtiger Faktor für das 
Ausmaß der Beteiligung an Weiterbildungsmaßnahmen ist die individuelle Vorbildung. Je höher der Ausbildungsabschluß ist, desto höher ist die Beteiligung an der beruflichen Weiterbildung. Durch Weiterbildung wird daher tendenziell der Qualifikationsunterschied zwischen qualifizierten und weniger qualifizierten Erwerbspersonen noch verstärkt; vorhandene Ungleichgewichte nehmen zu. So zeigt die folgende Abbildung die prozentuale Teilnahme an Maßnahmen der beruflichen Weiterbildung insgesamt nach einer BIBB/IAB-Erhebung 1991/92 in den fünf Jahren vor dem Befragungszeitpunkt:

\begin{tabular}{|l|c|c|c|c|}
\hline $\begin{array}{l}\text { Weiterbildungs- } \\
\text { teilnahme (in \%) }\end{array}$ & $\begin{array}{c}\text { ohne Berufs- } \\
\text { ausbildung }\end{array}$ & nur Lehre & Fachschule & Hochschule \\
\hline $\begin{array}{l}\text { Erwerbstătige } \\
\text { alte Lănder }\end{array}$ & 37 & 59 & 84 & 93 \\
\hline $\begin{array}{l}\text { Erwerbstătige } \\
\text { neue Lănder }\end{array}$ & 44 & 70 & 90 & 98 \\
\hline
\end{tabular}

Abb. 24: Anteil der Weiterbildungsteilnahme nach höchstem AusbildungsabschluB (Quelle: BIBB/IAB-Erhebung 1991/92 nach BERUFSBILDUNGSBERICHT, 1994, 125)

Bezüglich der betrieblichen Weiterbildung können verschiedene Sparten unterschieden werden. Neben der Einarbeitung und Umschulung sind die beiden Formen der Fortbildung, nämlich die Anpassungsfortbildung (zur Anpassung der Qualifikation an technische oder arbeitsorganisatorische Notwendigkeiten) und die Aufstiegsfortbildung (zur Förderung von Fach- und Führungskräften) zu nennen. Die Bundesanstalt für Arbeit fördert die berufliche Fortbildung, die Umschulung und die betriebliche Einarbeitung (AFG-geförderte Weiterbildung). Bereits ohne Einbeziehung der arbeitslosen Frauen, sind Frauen an der beruflichen Fortbildung geringer und an der Umschulung stärker beteiligt als Männer; bezieht man alle Personen mit ein, vergrößert sich das Ungleichgewicht weiter (vgl. BOLLE/SCHNEIDER, 1988, 133 und LÖWENBERGER, 1988, 192). Die Teilnahme an der AFG-Weiterbildung nach Geschlecht zeigt folgende Abbildung:

\begin{tabular}{|ll|c|c|c|}
\hline \multicolumn{4}{|c|}{ Teilnahme an der AFG-geforderten Weiterbildung (in \%) } \\
\hline & & Fortbildung & Umschulung & Einarbeitung \\
\hline \multirow{2}{*}{ alte Lănder: } & Frauen & 42 & 51 & 36 \\
& Mănner & 81 & 14 & 5 \\
\hline neue Lănder: & Frauen & 66 & 65 & 38 \\
& Mănner & 66 & 21 & 13 \\
\hline
\end{tabular}

Abb. 25: Eintritte in die AFG-geförderte Weiterbildung 1992 nach Geschlecht (Quelle: Bundesanstalt für Arbeit nach BERUFSBILDUNGSBERICHT, 1994, 127)

Frauen nennen besonders zwei Hinderungsgründe für ihre Teilnahme an Weiterbildungsmaßnahmen (BOLLE/SCHNEIDER, 1988, 123ff., 238). Zum einen fühlen sie 
sich schlecht informiert und im Vergleich zu Männern weniger unterstützt, zum zweiten können sie aufgrund ihrer Haus- und Familienarbeit weniger Zeit als Männer aufwenden. (vgl. hierzu auch MAIER, 1992, 378). Die erste Begründung findet Bestätigung darin, daß bei Männern häufiger als bei Frauen der Betrieb den Anstoß für die Weiterbildung gibt (vgl. ENGELBRECH, 1991a).

\section{(3) Verfügungsfreiheiten}

Die räumliche und zeitliche Verfügungsfreiheiten sind abhängig von den persönlichen Lebensumständen und werden wesentlich durch außerberufliche Anforderungen und Rahmenbedingungen eingeschränkt. Besonders qualifizierte Tätigkeiten erfordern eine hohe räumliche Mobilität und zeitliche Flexibilität, die für Frauen durchschnittlich schwerer als für ihre männlichen Kollegen erreichbar sind, da sie in deutlich höherem Ausmaß zusätzlich mit Haushalts- und Familienarbeit belastet sind (vgl. STATISTISCHES BUNDESAMT, 1994b, 11ff.). Die zeitliche Gesamtbelastung durch Familien- und Erwerbsarbeit ist für vollzeit- und teilzeiterwerbstätige Frauen insbesondere dann wesentlich höher als die der Männer, wenn ein Kleinkind vorhanden ist (vgl. SCHULZ, 1991). Aus Sicht der Unternehmen ist die zeitliche Verfügbarkeit von Frauen entsprechend geringer als die der Männer. Solange Männer Haushalts- und Familienarbeit in deutlich geringerem Maße leisten, bleiben Frauen im Wettbewerb bezüglich der Nutzung beruflicher Chancen benachteiligt. Zumal das üblicherweise herrschende Einkommensdifferential in der Ehe dafür sorgt, daß vorrangig die beruflichen Anforderungen, die den Männern gestellt werden, erfüllt werden, so daß neben der zeitlichen Flexibilität auch die räumlichen Mobilität der Frauen eingeschränkt ist.

Als Normalarbeitsverhältnis wird im allgemeinen die (vor allem für Männer typische) unbefristete und kontinuierliche Vollzeitarbeit verstanden. Auf sie ist das System der Absicherung und die gesellschaftliche Anerkennung ausgerichtet (vgl. $\mathrm{zu}$ den positiven Aspekten und den dysfunktionalen Wirkungen des Normalarbeitsverhältnisses beispielsweise GAUGLER, 1988, 16ff.). Während erwerbstätige Frauen häufig teilzeitbeschäftigt sind, wird die Teilzeitbeschäftigung für Männer kaum akzeptiert (vgl. STRÜMPEL, 1988, 38f.). Betrachtet man das geschlechtsspezifische Verhältnis der Vollzeit- und Teilzeittätigkeit der abhängig Beschäftigten, so ist es sinnvoll zwischen dem früheren Bundesgebiet sowie den neuen Ländern und Berlin-Ost zu unterscheiden: 


\begin{tabular}{|c|c|c|}
\hline $\begin{array}{c}\text { geleistete Arbeitszeiten } \\
\text { (in \%) }\end{array}$ & Vollzeit & Teilzeit \\
\hline Früheres Bundesgebiet & 97,1 & 2,9 \\
Männer & 63,4 & 36,6 \\
Frauen & 97,5 & 2,5 \\
Neue Länder und Berlin-0st & 79,6 & 20,4 \\
\hline Männer & Frauen &
\end{tabular}

Abb. 26: Anteile der Voll- und Teilzeitarbeit in den neuen und alten Bundesländern, Stand: April 1994 (Quelle: STATISTISCHES BUNDESAMT, MIKROZENSUS)

Auch die von Erwerbslosen gesuchte Art der Tätigkeit verdeutlicht die Unterschiede zwischen den Geschlechtern sowie den alten und neuen Ländern. Die Verteilung der gesuchten Tätigkeiten wird beeinflußt durch die wirtschaftlichen Notwendigkeiten, die gesellschaftlichen Normvorstellungen und die vorhandenen individuellen Wunschvorstellungen:

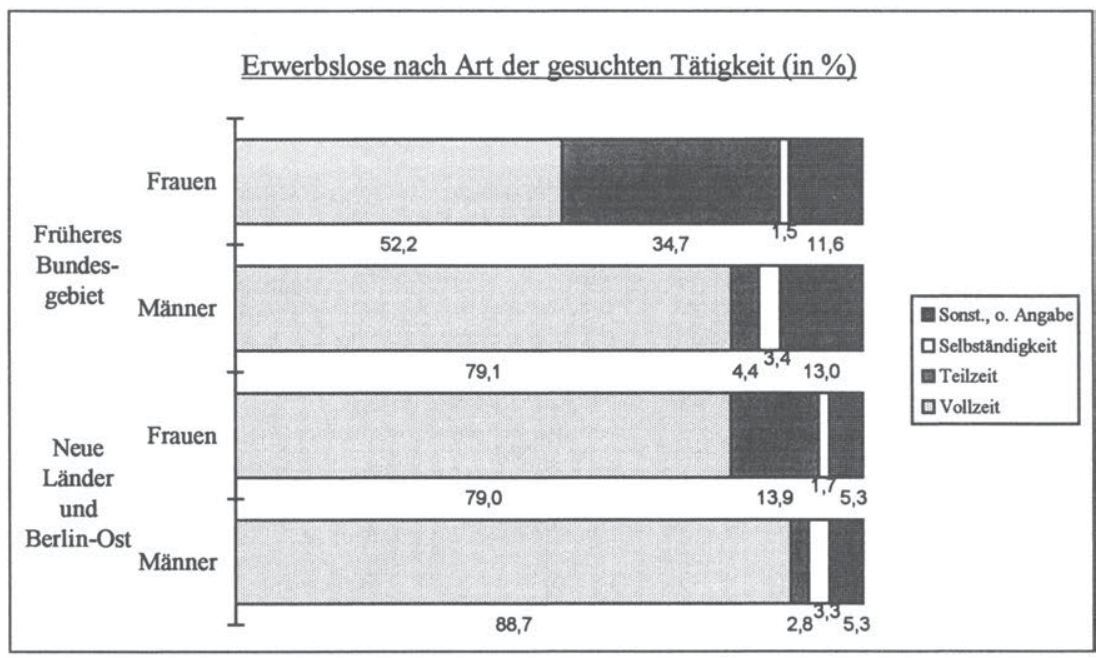

Abb. 27: Von Erwerbslosen gesuchte Tätigkeitsformen, Stand: April 1994 (Quelle: STATISTISCHES BUNDESAMT, MIKROZENSUS)

Ähnlich wie bei den Zahlen für die abhängig Beschäftigten fällt auf, daß der Anteil der Frauen, die eine Teilzeittätigkeit bevorzugen, im früheren Bundesgebiet mehr als doppelt so hoch ist wie in den neuen Ländern. Prozentual sind deutlich weniger Männer teilzeitbeschäftigt, als erwerbslose Männer eine Teilzeittätigkeiten suchen. Allerdings hat auch bei den gesuchten Tätigkeiten Teilzeit für Männer einen weitaus geringeren Stellenwert als für Frauen. 
Teilzeitarbeit ist entsprechend weitaus häufiger in Wirtschaftszweigen zu finden, in denen die Frauenbeschäftigung überdurchschnittlich hoch ist. Teilzeitarbeit ist insbesondere im Bereich des Handels stark verbreitet (so waren 1991 43,3\% der im Handel beschäftigten Frauen teilzeitbeschäftigt) und ist im öffentlichen Dienst häufiger anzutreffen als in der Industrie (z.B. waren 1991 im verarbeitenden Gewerbe nur 23,9\% der Arbeitnehmerinnen teilzeitbeschäftigt).

Bezogen auf die insgesamt geleistete Arbeitszeit läßt sich feststellen, daß die in der Vergangenheit zu beobachtende Erhöhung der Erwerbsbeteiligung der Frauen zum Teil durch die Ausweitung der Teilzeitarbeit ausgeglichen wurde (dies läßt sich auch für Großbritannien und die Niederlande zeigen, vgl. DALE/JOSHI, 1992; PLANTENGA, 1992). Der Anstieg der Zahl der beschäftigten Frauen überkompensierte jedoch diese Tendenz zur Teilzeitarbeit, so daß der Anteil der Frauen am Arbeitsvolumen (vgl. KLAUDER, 1992a, 18f.) zunahm.

Vergleicht man die Bedeutung der Teilzeitarbeit für die Beschäftigung der Frauen in den alten Bundesländern mit der in anderen EU-Staaten, so fallt auf, daß Teilzeitarbeit für alle Altersklassen in Großbritannien und Dänemark stärker und in den Niederlanden wesentlich stärker verbreitet ist (vgl. EUROSTAT, 1994; KNAUTH, 1992). Die Anteile der Männer mit Teilzeitbeschäftigung sind in der EU, mit Ausnahme der bereits bezüglich der höheren Teilzeitbeschäftigung von Frauen genannten Staaten sehr gering.

\begin{tabular}{|l|c|cccccccccccc|}
\hline Teilzeitbeschäftigte & $\begin{array}{c}\text { EUR } \\
\mathbf{1 2}\end{array}$ & B & DK & D & GR & E & F & IRL & I & L & NL & P & UK \\
\hline $\begin{array}{l}\text { Anteil an den } \\
\text { erwerbstätigen } \\
\begin{array}{l}\text { Frauen (\%) } \\
\text { Anteil an den } \\
\text { erwerbstätigen } \\
\text { Mannern (\%) }\end{array}\end{array}$ & 29,4 & 30,5 & 38,4 & 34,0 & 4,9 & 10,6 & 23,6 & 17,2 & 9,7 & 18,2 & 58,5 & 6,5 & 43,3 \\
\hline
\end{tabular}

Abb. 28: Anteil der Teilzeitbeschăftigten, 1991 (Quelle: EUROSTAT, 1994)

Die anforderungerechte Ausführung der beruflichen Tätigkeit setzt häufig eine Entlastung von Haus- und Familienarbeit voraus. Frauen, die die überkommene geschlechtsspezifische Rollenzuweisungen und die erhöhten Einschränkung der Flexibilität und Mobilität überwinden wollen, verzichten daher auf Ehe und Kinder, um qualifizierte Berufe ausüben zu können. Dies ist sicher kein Modell, dessen weitere Verbreitung gesellschaftlich gefördert werden sollte, zumal die emotionale Sicherung im privaten Bereiche positiv auf die Leistungen im beruflichen Bereich wirken dürfte. Entsprechend sind Maßnahmen zur Erleichterung der Vereinbarkeit 
von Familie und Beruf (für Frauen und Männer) zukünftig unerläßlich, um die Erwerbstätigkeit der Frauen quantitativ und qualitativ zu fördern, zumal als sicher gelten kann, daß die zukünftig durchschnittlich höher qualifizierten Frauen eine höhere Erwerbsmotivation aufweisen werden. Insgesamt dürften gerade Frauen in Führungspositionen eine Vorbildfunktion erfüllen und damit einen Beitrag zum Abbau traditioneller Vorstellungen und zur Aufhebung geschlechtsspezifisch unterschiedlicher Einsatzweisen leisten (vgl. NERGE/STAHMANN, 1991).

\section{c) Auswirkungen auf die Erwerbstätigkeit von Frauen}

Die Berufstätigkeit wird von den meisten Frauen nicht als vorübergehende Lebensphase betrachtet, vielmehr streben sie eine dauerhafte Erwerbstätigkeit an (vgl. BOLLE/SCHNEIDER, 1988, 107). Dies ist insbesondere angesichts der von ihnen getragenen Humankapitalinvestitionen auch ökonomisch rational. Frauen arbeiten aus den gleichen Gründen wie Männer, insbesondere finanzielle, soziale und kommunikative (vgl. ENGELBRECH, 1987). Die Erwerbstätigkeit kann zur Zufriedenheit beitragen sowie die ökonomische Unabhängigkeit der Frauen erhöhen, zumal die Ehe als Institution einer gesicherten Versorgung für Frauen zunehmend an Bedeutung verliert. Die betrachteten Eigenschaften haben allerdings Auswirkungen auf die Erwerbstätigkeit von Frauen, die zu geschlechtsspezifischen Unterschieden, beispielsweise bezüglich der Arbeitslosigkeit, des Arbeitsentgelts und der sozialrechtlichen Sicherung der Beschäftigungsverhältnisse, führen.

$\mathrm{Da}$ die (zugeschriebene) physische Kraft eine wichtige Rolle bei der Zuordnung der Tätigkeiten spielt, zeigen beispielsweise die Ergebnisse einer Betriebsbefragung, bei der $59 \%$ der gewerblich/technischen und $48 \%$ der kaufmännisch/verwaltenden Betriebe die erforderliche körperliche Kraft als bedeutendsten Grund für unterschiedliche Tätigkeiten von Männern und Frauen nach der Ausbildung nennen (vgl. ENGELBRECH/KRAFT, 1992, 18). Weitere Gründe sind (bei der Möglichkeit von Mehrfachnennungen) die geschlechtsspezifisch andere Arbeitsplatzwahl (40\% im gewerblich/technischen Bereich bzw. $42 \%$ im kaufmännisch/verwaltenden Bereich) und die geringere Betriebszugehörigkeit $(37 \% \mathrm{bzw}$. 43\%). Körperliche Belastungen sind demnach noch immer ein Hauptgrund für die geschlechtsspezifische Abgrenzung, obwohl fraglich ist, inwieweit die körperliche Leistungsfähigkeit während der Tätigkeit tatsächlich ausgeschöpft wird und Frauen für die Ausführung der meisten Tätigkeiten ausreichend 'stark' sein dürften. Arbeitgeber, die die unterschiedliche Höhe der physischen Leistungsfähigkeit als 
Grund für geschlechtsdifferente Arbeits- und Aufstiegschancen anführen, scheinen die geschlechtlichen Unterschiede überzubewerten, die Unterschiede innerhalb der Gruppe der Frauen (wie auch der Männer) zu vernachlässigen und die Möglichkeiten der Arbeitsgestaltung zu unterschätzen. Neben der geschlechtsspezifischen beruflichen Einsatzweise nach der Ausbildung suchen die Betriebe für viele Tätigkeiten bewußt nur männliche Mitarbeiter. Die Hauptgründe, die hierfür in einer Befragung des IAB angegebenen wurden, unterscheiden sich in ihrer Gewichtung je nach der zu erfüllenden Tätigkeit, wie folgende Abbildung zeigt.

\begin{tabular}{|c|c|c|c|}
\hline \multicolumn{5}{|c|}{$\begin{array}{c}\text { Angegebener Hauptgrund für die Suche von ausschließlich } \\
\text { männlichen Mitarbeitern für verschiedene Tätigkeiten (in \%) }\end{array}$} \\
\hline & $\begin{array}{c}\text { Hilfsarbeiter- } \\
\text { tätigkeit }\end{array}$ & $\begin{array}{c}\text { Facharbeiter- } \\
\text { tätigkeit }\end{array}$ & $\begin{array}{c}\text { einfache Ange- } \\
\text { stelltentätigkeit }\end{array}$ \\
\hline $\begin{array}{c}\text { geringere körperliche } \\
\text { Belastbarkeit von Frauen }\end{array}$ & 63 & 45 & 51 \\
\hline gesetzliche Bestimmungen & 26 & 21 & 9 \\
\hline $\begin{array}{c}\text { geeignete Frauen werden } \\
\text { nicht gefunden }\end{array}$ & 8 & 22 & 36 \\
\hline
\end{tabular}

Abb. 29: Gründe für die Suche von ausschließlich männlichen Arbeitspersonen

(Quelle: ENGELBRCH/KRAFT, 1992, 20)

Auffällig ist, daß für alle drei Tätigkeitsgruppen die körperliche Belastbarkeit als Hauptgrund gegen eine Beschäftigung von Frauen genannt wird und, daß für einfache Angestelltentätigkeiten häufig angegeben wird, daß keine geeignete Frau gefunden werden kann. Befragungsergebnisse dieser Art sollten allerdings nicht überbewertet werden, da die (vermeintlich) als legitim betrachteten Gründe einer geschlechtsspezifischen Selektion bevorzugt genannt werden dürften.

Eine schlechtere Ausbildung kann zukünftig immer weniger ein Grund für geschlechtsspezifische, frauendiskriminierende Personalrekrutierung sein. Allerdings sorgen die geschlechtsspezifische horizontale (eingeschränkte Berufswahl und Teilzeitarbeit) und vertikale Segregation (niedrigere Positionen und geringerer Verdienst auch im Falle gleicher Qualifikation) für eine Spaltung des Arbeitsmarktes. Zum Erhalt und zur Verbesserung der Erwerbschancen muß eine Dequalifizierung, beispielsweise durch eine längere Ausübung ausbildungsinadäquater Tätigkeiten oder Unterbrechungsphasen vermieden werden.

Da für beruflich qualifizierte Frauen, besonders wenn sie außerhalb 'frauentypischer' Berufen arbeiten bzw. arbeiten möchten, die (tatsächliche oder zukünftig mögliche) Diskontinuität ihrer Erwerbstätigkeit zum Problem wird und einen besonderen 
Wettbewerbsnachteil gegenüber erwerbstätigen Männern darstellt, wird nachfolgend auf die familienbedingte Unterbrechung der Erwerbstätigkeit eingegangen werden.

Das traditionelle Drei-Phasen-Modell der von Frauen zu leistenden Arbeit (vgl. MYRDAL/KLEIN, 1971) umfaßt in der ersten Phase die Ausbildung mit anschlieBender Erwerbstätigkeit, die zweite Phase bezeichnet die familienbedingte Unterbrechung der Erwerbstätigkeit, und die dritte Phase erfaßt schließlich die Wiederaufnahme der Erwerbstätigkeit. Auch wenn die Funktionsfähigkeit und die Gültigkeit dieses Drei-Phasen-Modells umstritten sind, verweist es auf verbreitete Problemlagen, die die Erwerbstätigkeit von Frauen erschweren. Die Entscheidung von Frauen, ihre Erwerbstätigkeit aufzugeben, zu unterbrechen oder einzuschränken, wird regelmäßig nicht frei getroffen, sondern beeinflußt durch gesellschaftliche Zwänge, Vorurteile und Erwartungen, die Frauen und Männer als rechtfertigende Sachargumente wahrnehmen. Wann und wie lange Frauen ihre Erwerbstätigkeit unterbrechen, bestimmt sich aus äußeren Notwendigkeiten und entspricht seltener einer freien Wahl. Der häufigste Grund für die Unterbrechung der Erwerbstätigkeit ist die Geburt eines Kindes. Neben der durchschnittlichen beruflichen Benachteiligungen ist die Mehrfachbelastung durch Haushalt/Familie und Beruf demnach entscheidend für die diskontinuierliche Erwerbstätigkeit von Frauen. Grundsätzlich sollte daher bei der Gestaltung der Berufsanforderungen von einer Einheit von Berufs- und Hausarbeit (nicht nur für Frauen, sondern auch für Männer) ausgegangen werden, falls man eine verbesserte Integration der Frauen in das Erwerbsleben anstrebt und erreichen möchte, daß Frauen ihre Eigenschaften in gleichem Maße beruflich einsetzen können wie erwerbstätige Männer. Allerdings ist die strukturelle Ungleichheit von Frauen und Männern auf dem Arbeitsmarkt nur zu ändern, wenn die traditionelle geschlechtsspezifische Arbeitsteilung überwunden werden kann.

Eine Unterbrechungsphase verhindert die kontinuierliche berufliche Entwicklung und birgt das Risiko der beruflichen Dequalifizierung, da die berufsbezogene soziale Integration und Möglichkeiten zur Weiterbildung verloren gehen, so daß sich Schwierigkeiten beim Wiedereinstieg ergeben können. Die Möglichkeiten nach einer Unterbrechung die zuvor ausgeübte Berufstätigkeit wieder aufzunehmen, verringern sich mit wachsender Dauer der Erwerbspause. Entsprechend sind die Unterbrechungsphasen im zeitlichen Vergleich kürzer geworden, gleichzeitig hat sich das Unterbrechungsrisiko verringert (vgl. LAUTERBACH u.a. 1994). Jüngere und besonders besser ausgebildete Frauen versuchen die Unterbrechungsphase zu verkürzen und Berufstätigkeit mit Haus- und Familienarbeit, ohne (außer- 
ordentliche) Mehrbelastung, zeitlich nebeneinander zu vereinbaren. Die längere Unterbrechungsphase niedriger qualifizierter Frauen ist ein Grund für ihre niedrigeren Erwerbsquoten. Wie durch die unterschiedlich ausgeprägte Teilnahme an Weiterbildungsmaßnahmen entwickeln sich durch die unterschiedlich langen Unterbrechungsphasen die beruflichen Chancen verschieden qualifizierte Personengruppen auseinander.

Verschiedene Motive führen dazu, daß Frauen die Erwerbstätigkeit wieder aufnehmen. Neben dem Beitrag zum Lebensunterhalt der Familie sind die Freude am Beruf und das vermittelte Gefühl der Unabhängigkeit und Selbständigkeit wichtig (vgl. ENGELBRECH, 1987). Weiterhin werden eigene Rentenansprüche erworben, die angesichts der gestiegenen Ehescheidungsraten an Bedeutung gewinnen. Allerdings bestehen Schwierigkeiten nach einer Unterbrechung eine Tätigkeit zu finden, die nach Inhalt und Entlohnung dem ursprünglichen Tätigkeitsniveau und den erworbenen Qualifikation entspricht (vgl. MÜLLER-DAEHN/ FOOKEN, 1992, 84f.). Befristete Beschäftigungen oder Teilzeitbeschäftigungsverhältnisse, die Frauen nach einer Unterbrechung annehmen weil sich keine anderen Tätigkeiten finden oder die Versorgung der Kinder bzw. pflegebedürftiger Personen keine Vollerwerbstätigkeit zuläßt, wirken sich negativ auf die Rentenansprüche aus. Aus Sicht der Unternehmen rechtfertigt die diskontinuierliche Erwerbstätigkeit den Einsatz von Frauen für geringer qualifizierte und entlohnte Tätigkeiten.

Die bisherigen Betrachtungen zeigen, daß die entscheidenden Bestimmungsfaktoren für die Erwerbsbeteiligung der Frauen die Art und Höhe der Qualifikation, die Anzahl der Kinder sowie insbesondere die Möglichkeiten Beruf und Familie zu vereinbaren sind (vgl. zu entsprechenden Feststellungen auch LAUTERBACH u.a. 1994). Unterschiedliche Modelle des Lebenslaufs zeigen Formen wie Frauen berufliche und familiäre Tätigkeiten miteinander koordinieren. Die sozial ermöglichte und gesellschaftlich (für Frauen und Männer) als selbstverständlich anerkannte Vereinbarkeit der Arbeit in beiden Bereichen ist das zentrale Problem der höheren Ausschöpfung des weiblichen Erwerbspersonenpotentials. Fragen der Vereinbarkeit richten sich insbesondere auf eine partnerschaftliche Arbeitsteilung in der Familiesowie auf eine gesellschaftliche Unterstützung bei der Bewältigung der Familienaufgaben und beim Wiedereintritt in den Beruf. Die Umsetzung entsprechender Maßnahmen muß - zur Sicherung des Arbeitskräfteangebots - in den Unternehmen angestrebt werden. 


\section{2. Ältere Personen und Erwerbstätigkeit}

\section{a) Kennzeichen der Erwerbstä tigkeit älterer Personen}

Die demographische Entwicklung hinsichtlich der Verlängerung der Lebenserwartung und die Entstehung der Begrenzung der Lebensarbeitszeit durch die an Bedeutung gewinnenden Altersgrenzen der Alterssicherungssysteme bewirkten die Herausbildung einer gesellschaftlich breiten Gruppe nichterwerbstätiger älterer Personen. Durch die Verallgemeinerung der Altersphase als Lebensphase nach der Erwerbsarbeit, ergibt sich eine Dreiteilung des Lebens in eine Ausbildungs-, eine Erwerbs- und eine Ruhestandsphase. Die Ruhestandsphase verbreitete sich erst in diesem Jahrhundert und bestimmt heute die gesellschaftlich vermittelte Vorstellung des Alters maßgeblich (vgl. KOHLI, 1992, 238f.).

Die Option der Erhöhung der Erwerbsbeteiligung Älterer richtet sich auf Personen bis zum Alter von 65 Jahren und zielt auf die Heraufsetzung des durchschnittlichen Berufsaustrittsalters. Zur Abgrenzung der Gruppe der älteren Erwerbstätigen gibt es verschiedene Definitionsversuche. So können beispielsweise aufgrund einer Untersuchung des IAB bereits über 50jährige hauptsächlich aufgrund der Kriterien Dauer der Arbeitslosigkeit und Erwerbsminderung als 'ältere' Erwerbspersonen definiert werden (vgl. HOFFMANN, 1993, 315). Ohne hier auf weitere abgrenzende Definitionen einzugehen läßt sich festhalten, daß die Zuordnung zur Gruppe der älteren Erwerbstätigen nicht allein durch das Lebensalter bestimmt wird. Sie ist unter anderem von der ausgeübten Tätigkeit, der Branche sowie unternehmens-, betriebs- und geschlechtsspezifischen Vorstellungen abhängig. Weiterhin wird die Abgrenzung von den Arbeitsmarktbedingungen und den sich wandelnden gesellschaftlichen Vorstellungen beeinflußt. Ältere Erwerbstätige sind entsprechend keine homogene Gruppe und die individuellen, biographischen und schichtspezifischen Unterschiede können schwerer wiegen, als die Gemeinsamkeiten aufgrund des Alters und der Erwerbstätigkeit (vgl. GÖCKENJAN, 1993). Trotzdem dient das Alter auch in vorliegender Arbeit als Kriterium der Gruppenbildung, da sich an ihm viele arbeitsmarktrelevante Regelungen sowie die Handlungsweisen der Arbeitnehmer und Arbeitgeber orientieren. Im praktischen Einzelfall sollten allerdings die konkreten individuellen Eigenschaften der Arbeitsperson die Beurteilung und Gestaltung der Beschäftigung bestimmen.

Günstigere Lebensbedingungen und eine verbesserte Gesundheitsversorgung führten in der Vergangenheit zu einer deutlichen Erhöhung der Lebenserwartung 
(vgl. beispielsweise ENQUETE-KOMMISSION, 1994, 26f.). Neben der Verringerung der Säuglingssterblichkeit konnte die Alterssterblichkeit in ein höheres Alter verschoben werden.

\begin{tabular}{|l|c|cc|cc|}
\hline \multicolumn{7}{|c|}{ Durchschnittliche Lebenserwartung (in Jahren) } \\
\hline & & \multicolumn{2}{c|}{ Männer } & \multicolumn{2}{c|}{ Frauen } \\
& Jahr & Neugeborene & 60jährige & Neugeborene & 60jährige \\
\hline früheres & $\mathbf{1 9 5 0}$ & 64,6 & 16,2 & 68,5 & 17,5 \\
Bundesgebiet & $\mathbf{1 9 7 0}$ & 67,4 & 15,3 & 73,8 & 19,1 \\
& $\mathbf{1 9 9 1}$ & 72,9 & 18,0 & 79,3 & 22,4 \\
\hline 12 EU-Länder & $\mathbf{1 9 9 1}$ & 72,8 & 18,2 & 79,4 & 22,5 \\
\hline
\end{tabular}

Abb. 30: Lebenserwartung bei der Geburt und im Alter von 60 Jahren (Quelle:

STATISTISCHES BUNDESAMT, JAHBÜCHER; Eurostat Bevölkerungsstatistik 1993 nach STATISTISCHES BUNDESAMT, 1994a)

Während sich durchschnittlich die Lebenserwartung erhöhte, stieg das Berufseintrittsalter und sank das Alter bei Austritt aus der Erwerbstätigkeit. So zeigt sich in der historischen Entwicklung ein deutlicher Rückgang der Erwerbsquoten niedriger und höherer Altersgruppen, der sich durch längere Ausbildungs- und früher beginnende Ruhestandszeiten erklärt. Folgende Abbildung stellt dies anhand der Erwerbsquoten der Männer von 1960 bis 1991 dar.

\begin{tabular}{|c|cccc|}
\hline Erwerbsquoten (in \%) & \multicolumn{4}{|c|}{ Männer } \\
\hline Altersgruppe & $\mathbf{1 9 6 0}$ & $\mathbf{1 9 7 0}$ & $\mathbf{1 9 8 0}$ & $\mathbf{1 9 9 1}$ \\
\hline $\mathbf{1 5 - 2 0}$ & $\mathbf{8 7}$ & 65 & 48 & 41 \\
$\mathbf{2 0 - 2 5}$ & 91 & 86 & $\mathbf{8 2}$ & $\mathbf{7 8}$ \\
$\mathbf{2 5 - 3 0}$ & 98 & 94 & 90 & $\mathbf{8 7}$ \\
\hline $\mathbf{3 0 - 3 5}$ & 99 & 99 & 97 & 96 \\
$\mathbf{3 5 - 4 0}$ & 98 & 99 & 98 & 98 \\
$\mathbf{4 0 - 4 5}$ & 97 & 98 & 98 & 97 \\
$\mathbf{4 5 - 5 0}$ & 96 & 97 & 97 & 96 \\
$\mathbf{5 0 - 5 5}$ & 94 & 94 & 93 & 93 \\
\hline $\mathbf{5 5 - 6 0}$ & $\mathbf{8 8}$ & 88 & 82 & 34 \\
$\mathbf{6 0 - 6 5}$ & 67 & 69 & 44 & 6 \\
$\mathbf{6 5 +}$ & 21 & 18 & 7 & $\mathbf{8 2}$ \\
\hline $\mathbf{1 5 - 6 5}$ & 92 & 89 & 83 & 34 \\
\hline
\end{tabular}

Abb. 31: Erwerbsquoten deutscher Männer nach Altersgruppen im zeitlichen Vergleich (Quelle: FRANZ, 1994, 21)

Für die Erwerbsbeteiligung höherer Altersgruppen ist die Entwicklung bei den Frauen ähnlich, allerdings wird sie durch den angesprochenen Trend der Erhöhung der Erwerbsbeteiligung der Frauen überlagert.

Die Verlängerung der Lebenserwartung und die Verkürzung der Lebensarbeitszeit stellen zusammen eine paradoxe Entwicklung dar. Die Verkürzung der 'produktiven' 
Tätigkeitsphase bedeutet, daß während des verkürzten Berufslebens die längeren Phasen der Ausbildung und Rente mitfinanziert werden müssen. Dagegen müßte aufgrund der Verlängerung der Lebenserwartung und der alterswissenschaftlichen Erkenntnisse bzgl. "der Erhaltung der geistigen und teilweise auch physischen Potenzen - der Zeitpunkt für das endgültige Ausscheiden aus dem Erwerbsleben heute wohl eher bei 75 Jahren liegen" (STRAKA u.a., 1990).

Das Ziel der Umkehr des Trends zum früheren Ausscheiden aus der Erwerbstätigkeit zur Minderung zukünftiger quantitativer Defizite des Arbeitskräfteangebots gewinnt aufgrund dieser Überlegungen an Bedeutung. Mit dem Rentenreformgesetz 1992 ist eine Anhebung der Altersgrenzen zum Bezug der ungekürzten Altersrente auf 65 festgelegt worden. Der angestrebte spätere Rentenbeginn und die damit zusammenhängende Kürzung der Ausgaben durch die Verringerung der Rentenbezugsdauer und der Zahl der Rentner einerseits sowie die Erhöhung der Einnahmen durch die Zunahme der Beitragszahler und der Beitragszeiten andererseits sollen die finanzielle Situation der gesetzlichen Rentenversicherung verbessern. Da nicht allein das gesetzliche Renteneintrittsalter für das tatsächliche Ende der Erwerbstätigkeit von Bedeutung ist, erfordert die Beurteilung der Möglichkeit der Verlängerung der Erwerbstätigkeit die Beachtung der relevanten Interessenlagen die wesentlich durch die Arbeitsmarktbedingungen bestimmt werden. Neben den betrieblichen Beschäftigungsbedingungen sind die individuellen gesundheitlichen und qualifikatorischen Voraussetzungen entscheidend für den Zeitpunkt des Berufsaustritts (vgl. beispielsweise BÄCKER/NAEGELE, 1993b). Bisher förderte eine allseitige Übereinkunft zwischen Arbeitgebern, Arbeitnehmern und dem Staat das frühzeitige Ausscheiden aus der Erwerbstätigkeit. So wird die frühzeitige Beendigung der Erwerbstätigkeit auch dadurch gestützt, daß die Senkung der Arbeitslosenzahl durch das Ausscheiden Älterer politisch erwünscht ist, obwohl ein frühes Ausscheiden aus dem Arbeitsmarkt aufgrund der unzureichenden Nutzung vorhandenen Humankapitals gesamtwirtschaftlich negativ $\mathrm{zu}$ beurteilen ist. Aufgrund der die Finanzierung betreffenden Interessenkonflikte und der voraussichtlichen Defizite des Arbeitskräfteangebots, werden staatliche und sollten unternehmensbezogene Handlungsweisen zunehmend auf eine Trendumkehr gerichtet sein. Die folgenden Überlegungen sollen eine arbeitspersonenbezogene Einschätzung der Verlängerung der Lebensarbeitszeit ermöglichen.

Neben der bisherigen Verkürzung der Lebensarbeitszeit kann hinsichtlich der Dauer der Lebensarbeitszeit betont werden, daß sich die allgemeine Gültigkeit des Renteneintritts verwischt. Statt der Einhaltung der Regelaltersgrenze gibt es 
verschiedene 'überbrückende' Phasen (mit Lohnersatzfunktionen) zwischen der Erwerbstätigkeit und dem gesetzlichen Renteneintritt, wie beispielsweise Phasen der Arbeitslosigkeit, der Frühverrentung wegen Erwerbsunfähigkeit oder des Vorruhestands. Die Arbeitnehmer und Arbeitgeber beeinflussen das durchschnittliche Renteneintrittsalter, indem sie in gewissen Grenzen entscheiden, wann der einzelne seine Erwerbstätigkeit beendet. Dabei gibt es kein einheitliches Bild der Motive und Strategien. Neben den Präferenzen älterer Personen, die heute häufig auf den Vorruhestand bei geringen Einkommenseinbußen gerichtet sind, ist das individuelle Handeln insbesondere abhängig von den jeweiligen Eigenschaften, Anforderungen und Möglichkeiten, so daß es auch durch die betriebliche Personalpolitik beeinflußt wird.

Für den Rückgang der Erwerbsbeteiligung Älterer gibt es viele Gründe (vgl. AMANN, 1993; NAEGELE, 1992, 242ff.; BÄCKER/NAEGELE, 1993b, 224ff.) von denen einige hervorgehoben werden können:

- Die (bisher) relativ gute materiellen Versorgung im Falle des vorgezogenen Ausscheidens,

- die Betroffenheit oder Gefährdung älterer Erwerbspersonen durch Arbeitslosigkeit und der demgegenüber nicht als diskriminierend empfundene Status des Ruhestandes,

- die vergleichsweise konfliktarme und sozialverträgliche Form der personalpolitischen Anpassungs- und Abbaustrategie, die Entlassungen vermeidet und Umschichtungen erleichtert,

- der Druck auf Ältere in den einzelnen Betrieben und auf dem gesamten Arbeitsmarkt aufgrund herrschender Arbeitslosigkeit und entsprechender gesellschaftlicher Wertvorstellungen,

- die Altersdiskriminierung in den einzelnen Betrieben und auf dem gesamten Arbeitsmarkt aufgrund verbreiteter Vorstellungen über ältere Arbeitspersonen,

- die tatsächliche oder vermeintliche Minderung der physischen bzw. psychischen Leistungsfähigkeit, die Verschlechterung der Gesundheit, die durchschnittlich vergleichsweise niedrigere Qualifikation,

- die Unzufriedenheit mit der beruflichen Tätigkeit und den Arbeitsbedingungen, der Sackgassencharakter der beruflichen Laufbahn, die Benachteiligung hinsichtlich Weiterbildung und Aufstieg, die Ängste vor neuen Anforderungen,

- die individuellen Präferenzen älterer Arbeitspersonen, positive Erwartungen und Hoffnungen für die Zeit nach dem Ausscheiden aus dem Erwerbsleben. 
Statt des vollständigen Ausscheidens aus dem Erwerbsleben mit unterschiedlich hohen Einkommenseinbußen sind für ältere Arbeitspersonen verschiedene Alternativen denkbar. So kann die Beschäftigung am gleichen Arbeitsplatz durch die Verbesserung der Arbeitsplatzbedingungen, der Anforderungen und der Umgebungsbedingungen ermöglicht werden. Weiterhin könnte eine Verkürzung der Arbeitszeit, die Inanspruchnahme einer Teilrente und das stufenweise Ausscheiden eine Weiterbeschäftigung am alten Arbeitsplatz erlauben. Schließlich könnte die Erwerbstätigkeit durch einen Wechsel an einen geeigneteren Arbeitsplatz fortgesetzt werden. Die Nutzung dieser Möglichkeiten setzt allerdings die Aufgabe der jugendzentrierten Personalpolitik der Unternehmen, die bisher ein bestimmender Faktor des Trends zum immer frühzeitigeren Ausscheiden aus der Erwerbstätigkeit ist, voraus.

Das tatsächliche mittlere Zugangsalter der Rentenempfänger liegt deutlich unterhalb der angestrebten Altersgrenze von 65 Jahren, wie die folgende Abbildung nach Geschlecht, Versicherungszweig und Gebiet zeigt.

\begin{tabular}{|l|cc|cc|}
\hline $\begin{array}{l}\text { Rentenzugangs- } \\
\text { alter (in Jahren) }\end{array}$ & \multicolumn{2}{|c|}{$\begin{array}{c}\text { Arbeiterrentenversicherung } \\
\text { insgesamt }\end{array}$} & \multicolumn{2}{c|}{$\begin{array}{c}\text { Angestelltenversicherung } \\
\text { insgesamt }\end{array}$} \\
\cline { 2 - 5 } $\mathbf{1 9 9 3}$ & Männer & Frauen & Männer & Frauen \\
\hline $\begin{array}{l}\text { alte } \\
\text { Bundesländer } \\
\text { neue } \\
\text { Bundesländer }\end{array}$ & 59,65 & 62,31 & 60,94 & 59,97 \\
& 59,84 & 58,02 & 62,49 & 57,14 \\
\hline
\end{tabular}

Abb. 32: Mittleres Rentenzugangsalter (Quelle: VDR, 1993)

Das durchschnittliche Berufsaustrittsalter ist noch niedriger als diese Angaben, da die Erwerbstätigkeit, beispielsweise aufgrund von Arbeitslosigkeit oder aus familiären Gründen, zum Teil erheblich vor dem Rentenbezug beendet wird.

Das mittlere Rentenzugangsalter wegen Berufs- und Erwerbsunfähigkeit (BU/EU) liegt noch einmal deutlich niedriger, wie folgende Abbildung zeigt:

\begin{tabular}{|c|c|c|c|c|}
\hline \multirow{2}{*}{$\begin{array}{l}\text { Rentenzugangs- } \\
\text { alter (in Jahren) } \\
1993\end{array}$} & \multicolumn{2}{|c|}{$\begin{array}{c}\text { Arbeiterrentenversicherung } \\
\text { BU-/EU-Renten }\end{array}$} & \multicolumn{2}{|c|}{$\begin{array}{c}\text { Angestelltenversicherung } \\
\text { BU-/EU-Renten }\end{array}$} \\
\hline & Männer & Frauen & Männer & Frauen \\
\hline $\begin{array}{l}\text { alte } \\
\text { Bundesländer } \\
\text { neue } \\
\text { Bundesländer }\end{array}$ & $\begin{array}{l}54,11 \\
48,80\end{array}$ & 53,25 & 53,72 & 50,82 \\
\hline
\end{tabular}

Abb. 33: Mittleres BU-/EU-Rentenzugangsalter (Quelle: VDR, 1993) 
Betrachtet man die Anteile verschiedener Rentenarten, so wird deutlich wie hoch der Anteil der Berufs- und Erwerbsunfähigkeitsrenten ist. Eine Verlängerung der Lebensarbeitszeit setzt daher den Abbau der Bedeutung diese Austrittsgrundes voraus.

\begin{tabular}{|c|c|c|c|c|c|}
\hline \multirow{2}{*}{$\begin{array}{c}\text { in } 1.000 \\
\text { in } \%\end{array}$} & \multicolumn{2}{|c|}{ Männer } & \multicolumn{2}{|c|}{ Frauen } & \multirow{2}{*}{ Insgesamt } \\
\hline & abl & nbl & abl & nbl & \\
\hline \multirow[t]{2}{*}{ BU-Renten } & 26.498 & 1.405 & 4.304 & 1.100 & 33.307 \\
\hline & $6 \%$ & $1 \%$ & $1 \%$ & $1 \%$ & $3 \%$ \\
\hline \multirow[t]{2}{*}{ EU-Renten } & 116.207 & 23.919 & 64.210 & 31.054 & 235.390 \\
\hline & $26 \%$ & $20 \%$ & $17 \%$ & $25 \%$ & $22 \%$ \\
\hline \multirow{2}{*}{$\begin{array}{l}\text { BU-/EU-Renten } \\
\text { insgesamt }\end{array}$} & 142.705 & 25.324 & 68.514 & 32.154 & 268.697 \\
\hline & $32 \%$ & $21 \%$ & $18 \%$ & $26 \%$ & $\mathbf{2 5} \%$ \\
\hline \multirow[t]{2}{*}{ Altersrenten ab $60 \mathrm{Lj}$. } & 119.232 & 23.743 & 110.936 & 88.127 & 342.038 \\
\hline & $27 \%$ & $19 \%$ & $30 \%$ & $71 \%$ & $32 \%$ \\
\hline \multirow[t]{2}{*}{ Altersrenten ab $63 \mathrm{Lj}$. } & 79.573 & 15.525 & 12.038 & 12 & 107.148 \\
\hline & $18 \%$ & $13 \%$ & $3 \%$ & $0 \%$ & $10 \%$ \\
\hline \multirow{2}{*}{ Altersrenten ab $65 \mathbf{L j}$. } & 95.594 & 57.810 & 181.415 & 3.815 & 338.634 \\
\hline & $22 \%$ & $47 \%$ & $49 \%$ & $3 \%$ & $32 \%$ \\
\hline \multirow[t]{2}{*}{ Altersrenten insgesamt } & 294.399 & 97.078 & 304.389 & 91.954 & 787.820 \\
\hline & $67 \%$ & $79 \%$ & $82 \%$ & $74 \%$ & $\mathbf{7 4} \%$ \\
\hline \multirow[t]{2}{*}{ Renten an Bergleute } & 2750 & 21 & 67 & 6 & 2844 \\
\hline & $1 \%$ & $0 \%$ & $0 \%$ & $0 \%$ & $0 \%$ \\
\hline \multirow[t]{2}{*}{ Rentenzugang } & 439.854 & 122.423 & 372.970 & 124.114 & 1.059 .361 \\
\hline & $100 \%$ & $100 \%$ & $100 \%$ & $100 \%$ & $100 \%$ \\
\hline
\end{tabular}

Abb. 34: Gesetzliche Rentenversicherung, Gliederung des Rentenzugangs 1993 wegen verminderter Erwerbsfähigkeit und wegen Alters nach Geschlecht für das frühere Bundesgebiet (abl) und das Gebiet Neue Länder und Berlin-Ost (nbl) (Quelle: VDR, 1993)

Vergleicht man die Daten für den Rentenzugang in der Angestelltenversicherung der alten und der neuen Bundesländer für 1993, so erkennt man, daß der Anteil der BU-/EU-Renten in den neuen Bundesländern (23\%) fast so hoch ist wie in den alten Bundesländern, wo diese Renten etwa ein Viertel des Rentenzugangs (26\%) ausmachen. Allerdings bestehen deutliche Unterschiede zwischen den Geschlechtern. So ist der Anteil der BU-/EU-Renten bei den Frauen in den neuen Bundesländern wesentlich höher als bei den Männern. Dies kann zum Teil damit begründet werden, daß Frauen häufiger von Arbeitslosigkeit betroffen sind (vgl. bezogen auf den Versichertenrentenzugang der BfA OHSMANN/STOLZ, 1994, 414).

Die Formen des vorzeitigen Ausscheidens aus dem Erwerbsleben haben arbeitsmarktpolitische, betriebs- und gesundheitsbedingte Gründe. Trotz dieser verschiedenen Gründe kann betont werden, daß Vorruhestandsregelungen ein arbeitsmarktpolitisches Mittel sind und keine Maßnahme zur Humanisierung des Arbeitslebens. Diese müßten statt dessen beispielsweise auf die Förderung der Fähigkeiten zur Ausübung einer Erwerbstätigkeit und auf die Vermeidung von Fehlbeanspruchungen 
gerichtet sein. Falls zukünftig solche Maßnahmen nicht im erforderlichen Maße umgesetzt werden, ist zu befürchten, daß die gesetzliche Erhöhung des Renteneintrittsalters eine verstärkte Inanspruchnahme von Erwerbsunfähigkeitsrenten zur Folge haben wird (vgl. BÄCKER/NAEGELE, 1993b).

Die Höhe der möglichen Ausweitung der Erwerbstätigenzahl durch die Verlängerung der Erwerbstätigkeit der zukünftig Älteren ist vor allem aus Gesundheits- und Wohlstandsgründen schwer vorhersagbar. Neben der Ausführbarkeit und Erträglichkeit gewinnen die Ebenen der Zumutbarkeit und Zufriedenheit für die Bewertung der Arbeit an Bedeutung (vgl. für Angaben zu diesen Bewertungsebenen ROHMERT, 1972, 8ff.), da die Attraktivität der Arbeit zum ökonomischen Kalkül wird (vgl. VOLKHOLZ, 1992, 11). Denn trotz der gestiegenen Lebenserwartung sowie der prognostizierten besseren körperlichen und geistigen Verfassung der zukünftig Älteren ist nicht sichergestellt, daß die Bereitschaft, später aus dem Berufsleben auszusteigen, wächst. Die prognostizierte Entwicklung der Vermögensund Einkommenssituation Älterer deutet darauf hin, daß das Vermögen dieser Haushalte überdurchschnittlich steigen und infolgedessen das Vermögenseinkommen deutlich an Bedeutung gewinnen wird (vgl. LANG, 1994; ENQUETEKOMMISSION, 1994). Weiterhin kann betont werden, daß bei steigender Erbschaftshöhe die Erbschaften immer älteren Personen zufallen werden. Allerdings wird die Verteilung der Vermögen und der Vermögenseinkommen voraussichtlich noch ungleichmäßiger werden, als sie es bereits heute sind. Für die meisten Haushalte werden die Einkommen aus den Systemen der Regel- und Zusatzsicherung ihre überragende Bedeutung behalten. Andererseits wird für einen größer werdenden Teil der Älteren eine Erhöhung der Rentenanwartschaften zukünftig an Bedeutung verlieren, so daß das Arbeitsentgelt künftig weniger Erwerbstätige dazu motivieren wird, länger zu arbeiten. Soweit die abnehmende Erwerbsbeteiligung ein Zeichen der Prosperität ist und nicht mit 'Zwangspensionierungen' oder gesundheitlichen Problemen zu begründen ist, werden unternehmensbezogene Maßnahmen zur durchschnittlichen Erhöhung der Erwerbsbeteiligung wenig nutzen. Aufgrund ihrer finanziellen Absicherung werden gerade gut qualifizierte Erwerbspersonen weiterhin relativ früh aus dem Erwerbsleben ausscheiden, während niedriger qualifizierte und entlohnte Personen länger arbeiten müssen (vgl. hierzu auch GIDION, 1992, 211). Eine solche Entwicklung trägt nicht zur angestrebten Minderung der qualitativen Defizite bei. Zur Ausschöpfung des Erwerbspersonenpotentials der Älteren wird daher eine Erhöhung der Attraktivität der Arbeitsaufgaben und Arbeitsbedingungen immer notwendiger. 


\section{b) Beschäftigungsrelevante Eigenschaften älterer Personen}

Wenn die durchschnittlichen beschäftigungsrelevanten Eigenschaften älterer Personen betrachtet werden, so bieten sich Vergleiche mit anderen Altersgruppen an, um Entwicklungslinien erkennbar zu machen. Auch für ältere Personen muß dabei die hohe interindividuelle Variabilität der Eigenschaften betont werden. Für die Entwicklung von Maßnahmen, die in den Unternehmen ergriffen werden können, um die Erwerbsbeteiligung älterer Personen zu erhöhen, müssen die Besonderheiten der Eigenschaften dieser Erwerbspersonengruppe Beachtung finden. Neben veränderten Interessenlagen sind mögliche qualifikatorische bzw. physische und psychische Vor- und Nachteile zu betrachten. Sofern die Veränderungen von Eigenschaften (als 'Einschränkungen') negativ auf die Möglichkeiten der Erwerbstätigkeit wirken oder Fehlbeanspruchungen zur Folge haben, stellen sie Ansatzpunkte für Maßnahmen dar.

Ältere Arbeitnehmer können nicht 'grundsätzlich' als leistungsgemindert angesehen werden, denn die mit dem Alter durchschnittlich einhergehenden Veränderungen stellen keine einseitige Verbesserung oder Verschlechterung der Eigenschaften dar, sondern qualitative Änderungen. Entsprechend sind die historisch vorherrschenden Defekt- und Disusemodelle, die von einem mehr oder weniger unabänderlichen altersabhängigen Verfall der körperlichen und geistigen Fähigkeiten ausgehen, durch ein Kompetenzmodell des Alters zu ergänzen, wie folgendes Schema der gerontologischen Modelle des Alters zeigt (vgl. OLBRICH, 1988; LEHR, 1988).

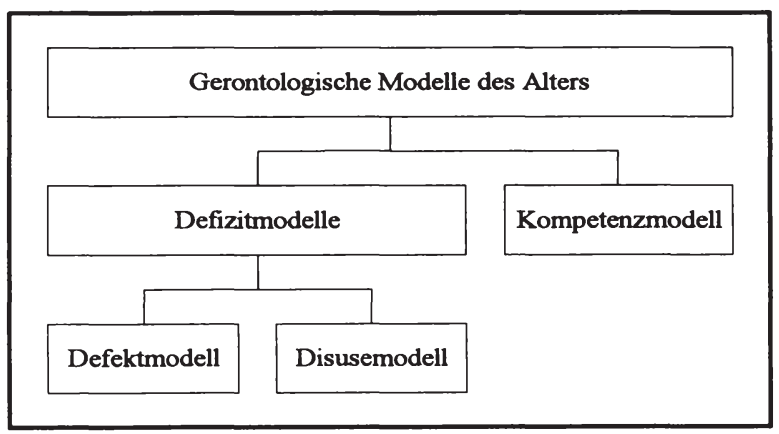

Abb. 35: Gerontologische Modelle des Alters 
Das Defizitmodell des Alters in Form des Defektmodells sieht das Auftreten von Defiziten durch den Alterungsprozeß festgelegt. Das Disusemodell begründet den altersbegleitenden Abbau von Fähigkeiten mit ihrer fehlenden Verwendung. Das Kompetenzmodell fordert demgegenüber eine differenziertere Betrachtung und verweist auf die Eigenständigkeit, die Vielfalt und den Wandel individueller, altersund umgebungsspezifischer Kompetenzen. Der Begriff der Kompetenz wird in der Wissenschaft vielfältig verwendet (vgl. OLBRICH, 1988; RUPPRECHT u.a., 1991). Vereinfacht ausgedrückt beziehen sich Kompetenzen auf die Möglichkeit der Erfüllung von Aufgaben. Kompetenzen lassen sich in jeder Entwicklungsperiode für einzelne Personen mit ihren individuellen Ressourcen, Potentialen und mit Bezug auf die Anforderungen der jeweiligen Lebenssituation und der Umwelt beschreiben. Zur Beeinflussung der Kompetenz müssen entsprechend situative und persönliche Variablen Beachtung finden. Ihr Zusammenwirken muß zur Erhaltung und zur Förderung der Kompetenzen von Älteren verbessert werden. Neben einer präventiv ausgerichteten Förderung der Möglichkeiten zur Entwicklung und zur Nutzung der Potentiale des Alters müssen die Möglichkeiten zur Anpassung an und zur Verarbeitung von Belastungen und Einschränkungen erweitert werden. Die folgende Abbildung stellt einige Kennzeichen der Defizitmodelle und des Kompetenzmodells einander gegenüber.

\begin{tabular}{|c|c|}
\hline Defizitmodelle & Kompetenzmodell \\
\hline $\begin{array}{l}\text { - Konzentration auf Einschrănkungen und } \\
\text { Defizite. Einseitige Betrachtung des } \\
\text { Alterns als Abbau korperlicher und } \\
\text { geistiger Făhigkeiten. } \\
\text { Problem: Pessimistische Sichtweise, die } \\
\text { die Aktivităt allseitig behindert. } \\
\text { Durchschnittsbezogener Vergleich mit } \\
\text { Personen jüngerer Altersklassen. } \\
\text { Betonung der Passivităt, der Verein- } \\
\text { samung, der Pflegebedürftigkeit und der } \\
\text { Schicksalhaftigkeit des Abbaus der Făhig- } \\
\text { keiten. } \\
\text { Die verwendeten Querschnittsunter- } \\
\text { suchungen kőnnen die Entwicklungs- } \\
\text { prozesse der Leistungsfahigkeit kaum er- } \\
\text { fassen. } \\
\text { Konzentration auf biologische Deter- } \\
\text { minanten. } \\
\text { Unterscheidung von Jugend und Alter mit } \\
\text { positiven und negativen Wertungen. }\end{array}$ & $\begin{array}{l}\text { Konzentration auf Möglichkeiten und } \\
\text { Stärken. Betrachtung des Alters als } \\
\text { eigenen Lebensabschnitt mit spezifischen } \\
\text { Făhigkeiten und Einschränkungen. } \\
\text { Problem: Allzu optimistische Sichtweise, } \\
\text { die vorhandene Schwierigkeiten verdeckt. } \\
\text { - Weitgehender Verzicht auf Vergleiche mit } \\
\text { anderen Altersgruppen und Normierungen. } \\
\text { Betonung der Regenerationsfahigkeit, des } \\
\text { Trainings, der Rehabilitation und der } \\
\text { Beeinflußbarkeit der individuellen Ent- } \\
\text { wicklung. } \\
\text { In Langzeitstudien werden Mănner und } \\
\text { Frauen von der Lebensmitte bis ins Alter } \\
\text { regelmaßig untersucht. } \\
\text { Differenzierte Betrachtung, Beachtung } \\
\text { sozialer und psychologischer Faktoren. } \\
\text { Unterschiede zwischen den Individuen und } \\
\text { zwischen 'Generationen', i.S. historisch } \\
\text { verschiedener Altersgruppen. }\end{array}$ \\
\hline
\end{tabular}

Abb. 36: Das Defizit- und das Kompetenzmodell des Alters im Überblick 
Die verschiedenen Modelle des Alters und Alterns zeigen unterschiedliche Perspektiven und unterstützen zunehmend differenzierte Betrachtungen der Eigenschaftsentwicklung, die den vielfältigen Situationen, Lebenslagen und Lebensbedingungen entsprechen (vgl. zu weiteren Theorieansätzen NAEGELE, 1992, 367ff.). So wichtig eine differenzierte und auf das Individuum bezogene Beurteilung der Arbeitssituation ist, können auch die folgenden Überlegungen allerdings nicht auf Verallgemeinerungen verzichten, um Aussagen über durchschnittliche Eigenschaften älterer Arbeitspersonen machen zu können. Die hieraus abzuleitenden Maßnahmen müssen dann bei ihrer Umsetzung an die individuellen Eigenschaften der Arbeitspersonen und die Besonderheiten der Unternehmen angepaßt werden.

\section{(1) Fähigkeiten}

Bezüglich der altersabhängigen Veränderung der körperlichen Leistungsfähigkeit kann zunächst die bereits ab einem Alter von 20 Jahren beginnende Verminderung des Grundumsatzes, der sich als umfassende Kenngröße der Vitalität auffassen läßt, hervorgehoben werden. Im einzelnen sinken mit dem Alter beispielsweise die maximal aufzubietende Muskelkraft, die Handgeschicklichkeit, die Bewegungsgenauigkeit, die Leistungsfähigkeit der Sinnesorgane, die maximale Herzschlagfrequenz, die Lungenventilation, die Vitalkapazität und die Geschwindigkeit der Bewegungen (vgl. LAURIG, 1990, 64ff.). Da andererseits der Leistungs-Puls-Index als $\mathrm{Maß}$ der körperlichen Dauerleistungsfähigkeit meist bis ins hohe Alter hinein konstant bleibt und die berufliche Erfahrung entstehende Leistungsminderungen zum Teil kompensieren kann, wirkt sich die körperliche Alterung nicht zwangsläufig auf die Erwerbstätigkeit aus, da z.B. auch die verbleibende körperliche Kraft zur Durchführung der meisten Tätigkeiten ausreichen wird.

Änderungen menschlicher Fähigkeiten können beispielsweise altersbedingt sein oder durch eine ausgeübte Erwerbstätigkeit hervorgerufen werden. Hinsichtlich des Zusammenhangs von Arbeit und Krankheit lassen sich Erkrankungen in drei Gruppen einteilen (vgl. BRANDENBURG, 1987, 154ff.). Für Berufskrankheiten sind direkte Ursache-Wirkungs-Beziehungen bekannt, an der Entstehung arbeitsbeeinflußter Erkrankungen ist die Arbeit nur als ein möglicher Faktor beteiligt und für allgemeine Krankheiten läßt sich keine Beziehung zur Arbeit bestimmen. Ärztlich festgestellte schwerwiegende Gesundheitsbeeinträchtigungen führen zur Berufs- und Erwerbsunfähigkeit, deren Anstieg ein wichtiger Faktor des Abbaus der 
Erwerbstätigkeit älterer Personen ist (vgl. NAEGELE, 1992, 75ff.). Die Daten zur Berufsunfahigkeit zeigen, daß die Arbeitspersonen insbesondere wegen Beschwerden am Bewegungsapparat sowie Herz- und Kreislauferkrankungen ausscheiden. Ein häufiger Grund für Frühinvalidität und die wichtigste Todesursache in westlichen Industrieländern ist die Arteriosklerose. Ursache dieser Krankheit kann berufsbedingte ständige nervliche Überlastung sein; andere Ursachen, wie falsche Ernährung, Bluthochdruck, Rauchen und Bewegungsmangel lassen sich allerdings weniger überzeugend mit der Erwerbstätigkeit begründen. Neben den Faktoren Arbeit und Beruf beeinflussen die außerberuflichen Lebensumstände, die Umweltbedingungen, die individuellen Eigenschaften, Verhaltensweisen und Einstellungen der Arbeitspersonen, die sozioökonomische Lage, die Arbeitsmarktbedingungen, die versicherungsrechtlichen Bedingungen und die sozialgerichtliche Rechtsprechung die Wahrscheinlichkeit einer Frühverrentung. Aufgrund des wachsenden Anteils Älterer am Erwerbspersonenpotential wird zukünftig voraussichtlich auch der Anteil kranker und berufs- bzw. erwerbsunfähiger Personen steigen. Gegenläufig können der durchschnittlich verbesserte Gesundheitszustand und die erhöhte Nutzung präventiv wirkender Maßnahmen zum Gesundheits- und Arbeitsschutz wirken.

Bezogen auf die Entwicklung der psychischen Fähigkeiten suggerieren ältere Studien, daß bezüglich der Intelligenz von einem allgemeinen altersbedingten Nachlassen ausgegangen werden muß. Allerdings verdeutlicht eine differenzierte Betrachtung, daß die geistige Leistungsfähigkeit häufig bis ins hohe Lebensalter hinein erhalten bleibt. Während nämlich die in Tests gemessenen elementaren Intelligenzleistungen abnehmen, steigt mit dem Alter das Erfahrungswissen, so daß die Gesamtintelligenz weiter zunehmen kann (vgl. LUCZAK, 1993). Verbreitet ist die Unterscheidung von 'flüssiger' und 'kristallisierter' Intelligenz. Die flüssige Intelligenz ist bedeutsam für die Verarbeitung neuer Information, die Fähigkeit Vergleiche anzustellen und Beziehungen herzustellen. Sie bestimmt entsprechend die Kombinationsfähigkeit, die Fähigkeit zur Neuorientierung und die Geschwindigkeit mit der dies geschieht. Die kristallisierte Intelligenz beruht demgegenüber auf dem Sprachverständnis, dem angesammelten Wissen und der Erfahrung. Sie ist daher bedeutsam für die (erfahrungsgeleitete) Lösung komplexer Probleme. Die kristallisierte Intelligenz ist unter anderem abhängig von den Kriterien Persönlichkeit, Bildung, Berufszugehörigkeit und Milieu. Während die flüssige Intelligenz mit dem Alter langsam abnimmt, kann die trainierbare kristallisierte Intelligenz bis ins Alter relativ stabil erhalten bleiben oder sogar zunehmen. 
Die Bewertung dieser Veränderungen der Intelligenz hinsichtlich der Erwerbstätigkeit Älterer ist davon abhängig, wie bedeutend die Erfahrung einerseits und die rasche Bewältigung insbesondere technischer Neuerungen andererseits für die jeweilige Tätigkeit eingeschätzt werden. Die Abnahme der flüssigen Intelligenz ist relevant, weil die Fähigkeit, sich gleichzeitig auf verschiedene Dinge zu konzentrieren abnimmt und entsprechend die Umweltkomplexität für ältere Personen durchschnittlich niedriger sein sollte als für jüngere. Weiterhin von Bedeutung ist, daß der Anteil der erfahrungsabhängigen Intelligenz mit dem Alter zu Lasten der erfahrungsunabhängigen zunimmt. $\mathrm{Da}$ die erfahrungsunabhängige Intelligenz wichtig für die Verarbeitung neuer Information in ungewohnter Form und höherer Komplexität ist, ergeben sich für Ältere größere Probleme beim anfänglichen Umgang mit neuen Techniken (vgl. STRAKA, 1990). Die Verarbeitungsgeschwindigkeit des Gehirns geht mit zunehmendem Alter zurück, so daß sich die Reaktionszeit verschlechtert und Ältere nicht unter Zeitdruck arbeiten sollten. Größere Anforderungen an die Aufmerksamkeit und Konzentration sollten, besonders wenn sie mit hoher Bewegungsgeschwindigkeit verbunden sind, an ältere Arbeitspersonen nicht gestellt werden.

Die große interindividuelle Variabilität der Leistungsfähigkeit älterer Personen gleichen Alters deutet darauf hin, daß Veränderungen der Leistungsfähigkeit nicht altersspezifisch sind und beeinflußt werden können (vgl. beispielsweise BALTES/BALTES, 1992, 14ff.). So sind die intellektuellen Leistungen im geringeren Maße vom Alter abhängig, als vom Verhalten und Erleben während des Lebensverlaufs. Lebenslange Anregungen scheinen die geistige Beweglichkeit im Alter zu erhöhen. Weitere Einflußfaktoren sind unter anderem Bildung, Beruf, Schichtzugehörigkeit, allgemeine Zufriedenheit, persönliche Einstellungen und Charaktereigenschaften. Entsprechend hat lebenslanges Lernen, das berufliche und sonstige Training geistiger Fähigkeiten positive Auswirkungen auf die Leistungsfähigkeit im Alter. Weiterhin werden dadurch das Selbstvertrauen Älterer und ihre Motivation zur Erwerbstätigkeit erhöht.

Es gibt vielfältige altersbedingte Änderungen in der Struktur des Leistungsvermögens. Einzelne Fähigkeiten, wie Kraft, Beweglichkeit, physisch-psychische Belastbarkeit, Flexibilität und Merkfähigkeit, nehmen ab, andere, wie Urteilsvermögen und Erfahrung, nehmen zu während weitere, wie Aufmerksamkeit, Urteilsvermögen und Verantwortungsbewußtsein, unverändert bleiben. Dieser Leistungswandel beeinträchtigt besonders die beruflichen Chancen jener Arbeitnehmer, die belastungsintensive Tätigkeiten ausüben. Insgesamt ist die altersabhängige 
Entwicklung der physischen Leistungsfähigkeit bezogen auf ältere Erwerbspersonen negativer als die Entwicklung der intellektuellen Leistungsfähigkeit. Aufgrund verschiedener Beobachtungen und Befragungen schätzt NAEGELE, daß ca. ein Drittel aller noch beschäftigten älteren Personen von Leistungseinbußen betroffen ist (vgl. NAEGELE, 1992, 86f.). Zur Schaffung der Voraussetzungen einer Ausweitung der Erwerbsbeteiligung älterer Personen sind Arbeitsaufgaben und Arbeitsplätze zukünftig zunehmend "so zu gestalten, daß sie auch bei gewandeltem bis eingeschränktem Leistungsvermögen produktive und sinnvolle Weiterbeschäftigung ermöglichen" (BÄCKER/NAEGELE, 1993c, 112).

\section{(2) Kenntnisse und Fertigkeiten}

Bezüglich des Niveaus der allgemeinen Schulausbildung kann festgestellt werden, daß die durchschnittliche Qualifikation der Erwerbstätigen bei jüngeren Altersgruppen höher ist (vgl. hierzu die folgende Abbildung).

\begin{tabular}{|c|c|c|}
\hline \multicolumn{3}{|c|}{ Allgemeine Schulabschlüsse (in \%) } \\
\hline & $\begin{array}{c}\text { 25-35jährige } \\
\text { Personen }\end{array}$ & 50-60jährigePersonen \\
\hline Volksschul-/Hauptschulabschluß & 34 & 71 \\
\hline AbschluB der polytechnischen Oberschule & 14 & 3 \\
\hline Realschul- oder gleichwertiger AbschluB & 25 & 15 \\
\hline Fachhochschul- oder Hochschulreife & 27 & 12 \\
\hline
\end{tabular}

Abb. 37: Verteilung der Schulabschlüsse (Quelle: Mikrozensus 1993 - freiwillige Angaben, STATISTISCHES BUNDESAMT, 1994c und eigene Zusammenfassung)

Auch die durchschnittliche berufliche Ausbildung ist bei jüngeren 'Generationen' höher als bei älteren. Zudem muß das Nachlassen der Aktualität des Ausbildungsstandes betont werden. Die Inhalte älterer Ausbildungen können in unterschiedlichem Maße an Relevanz verloren haben. Diese kohortenspezifischen Ausbildungsnachteile sprechen aus Sicht der Unternehmen für eine jugendzentrierte Personalpolitik, die darauf gerichtet ist, ältere Arbeitspersonen durch jüngere zu ersetzen (vgl. beispielsweise ROSENOW/NASCHOLD, 1994, 34).

Weiterhin kann festgestellt werden, daß ältere Arbeitspersonen unterdurchschnittlich häufig an Maßnahmen der beruflichen Weiterbildung teilnehmen. Die folgende Abbildung verweist darauf, daß nicht nur der durchschnittliche Umfang der Weiterbildung der älteren Erwerbspersonen geringer ist, sondern daß diese Gruppe in vergleichsweise geringem Maße an Weiterbildungsmaßnahmen teilnimmt. 


\begin{tabular}{|c|c|c|c|c|}
\hline $\begin{array}{c}\text { Jährlicher Zeitaufwand } \\
\text { für die berufliche } \\
\text { Weiterbildung in Stunden }\end{array}$ & \multicolumn{2}{|c|}{$\begin{array}{c}\text { pro Teilnehmer/in an } \\
\text { Weiterbildungsmaßnahmen }\end{array}$} & $\begin{array}{c}\text { pro Teilnehmer/in und Nicht- } \\
\text { teilnehmer/in an Weiter- } \\
\text { bildungsmaßnahmen }\end{array}$ \\
\cline { 2 - 5 } & alte Länder & neue Länder & alte Länder & neue Länder \\
\hline 19-34 Jahren & 195 & 261 & 48 & 76 \\
35-49 Jahren & 120 & 224 & 27 & 68 \\
50-64 Jahren & 85 & 145 & 9 & 18 \\
\hline
\end{tabular}

Abb. 38: Zeitaufwand für die berufliche Weiterbildung nach Alter und Gebiet je Teilnehmer/in und je Person der Gesamtgruppe (Quelle: Berichtssystem Weiterbildung, 1991 nach BERUFSBILDUNGSBERICHT, 1994, 126)

Insbesondere angesichts des rascher werdenden Verfalls des Wissens aufgrund der Nutzung neuer Techniken und Methoden, muß sich die Verteilung der Bildungsphasen im Altersverlauf ändern, so daß die Bildungsaktivitäten zukünftig gleichmäßiger über das Arbeitsleben verteilt und gerade Ältere stärker an Weiterbildungsmaßnahmen beteiligt werden (vgl. GIDION, 1992, 209ff.).

Versucht man aufgrund der derzeitigen Qualifikation auf das zukünftige Weiterbildungsverhalten Älterer zu schließen, so müssen verschiedene Einflußfaktoren beachtet werden:

- Der Alterseinfluß bezüglich der Weiterbildungsteilnahme wird überlagert vom Einfluß des Bildungsabschlusses oder der beruflichen Stellung. Aufgrund der Erhöhung des durchschnittlichen Ausbildungsniveaus kann der Einfluß des Bildungsabschlusses für die 'Beurteilung' der Weiterbildungsteilnahme der zukünftig älteren Erwerbstätigen allerdings an Bedeutung verlieren.

- Das Weiterbildungsinteresse und die Weiterbildungsbereitschaft der Älteren dürften zukünftig aufgrund höherer Bildungsabschlüsse, häufigerer Weiterbildungsmaßnahmen in früheren Phasen und der mit einer Verschiebung des Renteneintritts zukünftig verlängerten Nutzungsmöglichkeit der erworbenen Kenntnisse steigen.

- Neben den Eigenschaften und Lebenslagen der Arbeitspersonen wirken betriebliche Weiterbildungsmodelle und Laufbahnvorschriften sowie die Weiterbildungspolitik und Arbeitsmarktbedingungen auf die Weiterbildungsteilnahme.

- Qualifikatorische Nachteile Älterer hinsichtlich des Umgangs mit neuen Techniken dürften sich zukünftig abschwächen, da die zukünftigen älteren Erwerbspersonen diese Fähigkeit während ihres Berufslebens stärker eingeübt haben werden als heutige ältere Arbeitspersonen. Weiterhin dürften sich 
Techniken, die bisher noch begrenzt auf den Bereich der Arbeit Nutzung finden auch im privaten Lebensbereich verbreiten, so daß auch hierdurch der Umgang mit ihnen selbstverständlicher wird.

\section{(3) Verfügungsfreiheiten}

Auch hier muß vorangestellt werden, da $\beta$ die Verfügungsfreiheiten potentieller Arbeitspersonen abhängig von den persönlichen Lebensumständen sind und wesentlich durch außerberufliche Anforderungen und Rahmenbedingungen aber auch durch individuelle Interessen beeinflußt werden.

Hinsichtlich der zeitlichen Flexibilität älterer Arbeitspersonen kann insbesondere die Frage eines gleitenden Ruhestandes angesprochen werden. Mit dem Rentenreformgesetz 1992 wurden, im Interesse einer Verlängerung der Lebensarbeitszeit, die Möglichkeiten zum Bezug einer Teilrente im Falle der Ausübung einer (rentenanspruchssteigernden) Teilzeitbeschäftigung geregelt. Für eine breite Nutzung sollte die Teilzeitarbeit aus Sicht der Erwerbspersonen von einem möglichen Statusverlust befreit werden. Hierzu müßte die Teilzeitbeschäftigung der älteren Arbeitsperson am bisherigen Arbeitsplatz ermöglicht werden oder an einem Arbeitsplatz mit ähnlichen qualifikatorischen Anforderungen erfolgen.

Betrachtet man als Ausdruck räumlicher Mobilität den Wohnortwechsel über die Grenze von Kreisen hinweg, so läßt sich feststellen, daß die Mobilität mit zunehmendem Alter abnimmt. Jährlich wechseln drei Prozent der Gesamtbevölkerung ihren Wohnort, bei den 18 bis 30jährigen sind es acht bis zwölf Prozent und bei den über 50jährigen noch etwa ein Prozent (vgl. BUCHER, 1994, 6). Ähnliche Feststellungen lassen sich hinsichtlich der beruflichen Mobilität treffen, die mit dem Alter zurückgeht und deren gesamtwirtschaftliche Häufigkeit aufgrund der durchschnittlichen Alterung des Erwerbspersonenpotentials zukünftig deutlich abnehmen wird. Die regionale Mobilität konzentriert sich auf den Beginn der Erwerbsphase und geht mit dem Alter, beispielsweise aufgrund des Erwerbs von Hauseigentum und der mit der Betriebszugehörigkeit steigenden Arbeitsplatzsicherheit, zurück (vgl. GIDION, 1992). Andererseits wirkt der Trend zur Höherqualifizierung mobilitätsfördernd. So weisen Fachhochschul- und Hochschulabsolventen, z.B. zur Nutzung von Aufstiegschancen und aufgrund ihrer breiteren Einsetzbarkeit, eine höhere Mobilität auf als niedriger Qualifizierte. Die höhere Mobilität von Akademikern bleibt bis zu einem Alter von 55 Jahren hoch, während 
sie durchschnittlich ab einem Alter von 45 Jahren deutlich zurückgeht. Die mit dem Alter steigende Immobilität der Arbeitspersonen wird angesichts des steigenden Anteils der Älteren an der erwerbstätigen Bevölkerung zukünftig insbesondere dann zum Problem, wenn Jüngere dieses Mobilitätsdefizit nicht mehr im nötigen Maße ausgleichen können (vgl. GIDION/STEGMEIER, 1991).

\section{c) Auswirkungen auf die Erwerbståtigkeit ålterer Personen}

Die betrachteten Eigenschaften haben Auswirkungen auf die Erwerbstätigkeit von älteren Personen. So orientiert sich die betriebliche Beschäftigungspolitik besonders an solchen Werten, die eher Jüngeren zugeschrieben werden, wie Kraft, Mut, Risikobereitschaft, Ehrgeiz, Durchsetzungsvermögen, Flexibilität, Mobilität, Schnelligkeit und Belastbarkeit. Die Personalpolitik ist vornehmlich auf jüngere Nachwuchskräfte ausgerichtet. So vollziehen sich bis zum 35 . Lebensjahr beispielsweise drei Viertel der betrieblichen $\mathrm{Zu}$ - und Abgänge (vgl. VOLKHOLZ, $1992,9)$. Die Verjüngung der Belegschaft scheint vielen Unternehmen aufgrund der Nutzung neuer Technologien und zur Senkung der Lohnkosten sinnvoll. Auf Werte die eher Älteren zugeschrieben werden, wie Erfahrung, Weisheit, Weitsicht, Gelassenheit, Toleranz, Klarheit und Ausdauer, glaubt man bei der Mehrheit der Beschäftigten verzichten zu können.

Frühverrentungsmöglichkeiten werden besonders in großen und mittelgroßen Unternehmen sehr häufig genutzt. Diese Ausgliederungspraxis ist nach Untersuchungen des Wissenschaftszentrums Berlin allgemein verbreitet, im öffentlichen sowie im privaten Sektor, in unterschiedlichen Branchen und bezogen auf Erwerbstätige unterschiedlicher Qualifikation (vgl. ROSENOW/NASCHOLD, 1993, 147f. und 1994). Sie erscheint als sozial verträgliche personelle Anpassung im Sinne der jugendzentrierten Personalpolitik. Staat, Unternehmen und Beschäftigte unterstützten diese Frühverrentungspraxis bisher weitgehend übereinstimmend. Zukünftig gerät diese bisherige Handlungsweise allerdings sowohl auf makroökonomischer als auch auf mikroökonomischer Ebene zunehmend unter Veränderungsdruck. Entsprechende Stichworte sind: Altersstrukturveränderung des Erwerbspersonenpotentials, quantitative und qualitative Nachwuchsprobleme, Finanzierung der Sozialversicherung und Anstieg der Lohnnebenkosten (vgl. beispielsweise ROSENOW/NASCHOLD, 1993, 147). 
Die unterschiedlichen Formen und Alterszeitpunkte des Ausscheidens aus der Erwerbstätigkeit machen deutlich, daß fixierte Altersgrenzen willkürlich sind. Die vielfältigen interindividuellen Unterschiede zeigen, daß kein genereller Altersabbau stattfindet und nicht das Erreichen einer Regelaltersgrenze, sondern die individuelle Arbeitsfähigkeit und die individuelle Motivation zur Arbeit ausschlaggebend für das Ausscheiden aus der Erwerbstätigkeit sein sollten. Entsprechend hängt eine Verlängerung der Erwerbstätigkeit Älterer, neben dem angenommenen - derzeit nicht im erforderlichen Maß gegebenen - Vorhandensein von Arbeitsplätzen, von den gestellten Anforderungen, von den Eigenschaften, von den materiellen Bedürfnissen und gerade auch von der Bereitschaft der Älteren, länger erwerbstätig zu sein, ab. Zwischen den (äußeren) Notwendigkeiten bzw. vorgegebenen Regelungen und den (inneren) Vorstellungen bzw. Präferenzen besteht zudem ein interdependentes Verhältnis. So dienen die im Rentenrecht verankerten bisher unterschiedlichen Altersgrenzen für Männer und Frauen als Orientierungsdaten für das erwartete und das erwünschte Alter für den Übergang in den Ruhestand (vgl. STÖRTZBACH, 1992).

Angesichts der Geschwindigkeit des technisch-organisatorischen Wandels und der zukünftig zu erwartenden Änderungen der Arbeitsaufgaben sowie ihrer Anforderungen scheint eine altersbedingte Minderung der Fähigkeiten, die häufig zur Begründung des Ausscheidens älterer Arbeitspersonen verwendet wird, von geringer Bedeutung verglichen mit dem Veralten beruflicher Kenntnisse und Fertigkeiten. Diese Entwertung einmal erworbenen Wissens und die Notwendigkeit seiner kontinuierlichen Aktualisierung betreffen allerdings nicht nur Ältere und Frauen, die ihre Erwerbstätigkeit unterbrochen haben, sondern zunehmend auch jüngere und daher später qualifizierte Arbeitspersonen.

Vielfältige Neuerungen stellen Herausforderungen an die Anpassungs- und Lernfähigkeit der betroffenen Arbeitspersonen. Mit zunehmendem Alter fallen jedoch die Lern- und Anpassungsprozesse schwerer, zumal wenn früher erworbene Kenntnisse wieder verloren gegangen sind. Eine Studie des Ifo-Instituts kommt entsprechend zu dem Ergebnis, daß "die Annahme technologischer Neuerungen für ältere Menschen (insbesondere für diejenigen, die noch am Produktionsprozeß teilnehmen) mit höheren Kosten verbunden [ist], und dies sowohl auf der Ebene der individuellen Belastung als auch aus der betriebs- und gesamtwirtschaftlichen Perspektive" (KOCH, 1988, 81). Zudem kann mit dem Alter aufgrund des näher rückenden Renteneintritts die Zeitspanne abnehmen, während der sich die Investitionen in das Humankapital amortisieren müssen. 
Die Erhöhung des Anteils der Älteren an der Bevölkerung durch den demographischen Wandel kann gesellschaftlich einen Bedeutungszuwachs der Probleme und Bedürfnisse der Älteren und eine Veränderung derzeitiger Vorstellungen bewirken. $\mathrm{Zu}$ einer erfolgreichen Bewältigung des Wandels würde eine positivere Beurteilung von Erfahrung und Reife beitragen. Gelingt es beispielsweise durch die Eröffnung von Wahlmöglichkeiten zur Flexibilisierung der Altersgrenze die Lebensarbeitszeit zu verlängern, so stärkt dies die Unabhängigkeit älterer Personen, wirkt ihrem möglichen Ansehensverlust entgegen und erhöht die gesellschaftlichen Einflußmöglichkeiten dieser Bevölkerungsgruppe. Potentielle Konflikte zwischen verschiedenen Altersgruppen könnten gemildert, und der Generationenvertrag würde gestärkt werden. Außerdem können Maßnahmen zur Ermöglichung einer verlängerten Erwerbstätigkeit eine bessere Verteilung der Arbeit im Lebenslauf, eine Vergleichmäßigung der Humankapitalnutzung und eine Minderung der Arbeitsintensität während des gesamten Berufslebens bewirken. Andererseits können tarifvertragliche Regelungen, wie höhere Bezahlung, höherer Urlaubsanspruch und verlängerte Kündigungsfristen, die eine Absicherung älterer Arbeitnehmer zum Ziel haben, aber bisher die Weiterbeschäftigung und besonders die Neueinstellung Älterer erschwerten, infolge der Umsetzung der Erhöhung des gesetzlichen Renteneintrittsalters und zur Vermeidung von Arbeitslosigkeit während der nächsten Jahre verstärkt in Frage gestellt werden.

\section{Zwischenfazit}

Zur langfristigen gesellschaftlichen Ausschöpfung des Erwerbspersonenpotentials, zur Erleichterung einer 'gleichberechtigten' Teilhabe an den Möglichkeiten, produktiv tätig zu sein und damit zur Sicherung der individuellen Selbstbestimmung muß die berufsbezogene Entfaltung menschlicher Eigenschaften unabhängig von Alter und Geschlecht gefördert werden, indem die gegenseitige Anpassung von Mensch und Arbeit verbessert wird. Bezüglich der erforderlichen Anpassungsprozesse muß davor gewarnt werden, angesichts konjunktureller Schwankungen, struktureller Krisen und hoher Arbeitslosigkeit, Frauen und Ältere nur als Stille Reserve des Arbeitsmarktes insbesondere für einfachere Tätigkeiten zu betrachten. Denn die spezifischen Defizite an qualifizierten Arbeitskräften sind trotz der allgemein herrschenden Arbeitslosigkeit feststellbar. Außerdem setzt der zukünftig üblich und notwendig werdende Anstieg der Beteiligung von Frauen und Älteren an der Ausführung von Tätigkeiten mit höheren Anforderungen längere Qualifikationsund Einarbeitungsphasen voraus. Weiterhin dürfte die Anpassung der sozial 
vermittelten Einstellungen zur Wahl der Art und Dauer der Erwerbstätigkeit von Frauen und Älteren an die zukünftigen Erfordernisse eine längere Gewöhnungsbzw. Erfahrungszeit beanspruchen, da hierzu ein gesellschaftlicher Umdenkungsprozeß notwendig ist.

Die bisherige Angleichung der Berufsbiographie vieler Frauen an den für Männer üblichen Verlauf der Erwerbsarbeit, wie die erhöhte Erwerbsneigung, die Verlängerung der Betriebszugehörigkeit und die Verringerung der Unterbrechungszeiten, führte noch nicht zu einer entsprechenden Anpassung der beruflichen Entwicklungsmöglichkeiten von Arbeitnehmerinnen (vgl. ENGELBRECH, 1991a; ENGELBRECH/KRAFT, 1992). Die erforderlichen unternehmensbezogenen Maßnahmen für eine Erhöhung und Verbesserung der Erwerbsbeteiligung der Frauen müssen daher auf eine Anpassung der Arbeit an die Eigenschaften und Bedürfnissen der Frauen gerichtet sein. Aufgrund der bisherigen Betrachtungen werden in der folgenden Abbildung für die Gestaltung der Maßnahmen wesentliche Kennzeichen der Erwerbstätigkeit von Frauen zusammengefaßt.

\begin{tabular}{|l|l|}
\hline \multicolumn{2}{|c|}{ Wesentliche durchschnittliche arbeitsmarktbezogene } \\
Kennzeichen für die Erwerbstätigkeit von Frauen
\end{tabular}

Abb. 39: Arbeitsmarktbezogene Kennzeichen für die Erwerbstätigkeit von Frauen 
Zur Sicherung der Erwerbstätigkeit Älterer dürfen die Erwerbsquoten (immer jüngerer) älterer Arbeitspersonen langfristig nicht weiter fallen. Vielmehr müßte das durchschnittliche Renteneintrittsalter durch eine Verlängerung des individuellen Erwerbslebens wieder ansteigen. Die in den Unternehmen hierzu notwendigen Veränderungen der Arbeit müssen sich an den arbeitsmarktrelevanten Eigenschaften von Älteren orientieren, die hinsichtlich wesentlicher Ausprägungen und tendenzieller Änderungen nachfolgend zusammengefaßt werden (vgl. besonders auch hinsichtlich der Darstellungsart LAURIG, 1990, 65 und die dort angegebenen Autoren sowie NAEGELE, 1988, 38).

\begin{tabular}{|c|c|}
\hline \multicolumn{2}{|c|}{$\begin{array}{l}\text { Wesentliche durchschnittliche arbeitsmarktbezogene } \\
\text { Kennzeichen für die Erwerbstätigkeit von Älteren }\end{array}$} \\
\hline $\begin{array}{l}\text { Wettbewerbsnachteile } \\
\text { und negative } \\
\text { Entwicklungen }\end{array}$ & $\begin{array}{l}\text { - Nachlassen der körperlichen Leistungsfähigkeit } \\
\text { - Verringerung der Fähigkeiten der Sinnesorgane } \\
\text { - } \text { Anpasingerung der Wahrnehmungs- und } \\
\text { - Verminderung des Reaktionsvermögens und der } \\
\text { Informationsverarbeitungsgeschwindigkeit besonders bei } \\
\text { komplexen Aufgabenstellungen } \\
\text { - Verringerung der Abstraktionsfähigkeit } \\
\text { - Verringerung der Merkfähigkeit im Kurzzeitgedächtnis } \\
\text { - Verminderung der Aktualität und der Relevanz erworbener } \\
\text { Qualifikationen } \\
\text { - Verringerung der Anpassungs- und Umstellungsfähigkeit } \\
\text { - geringere Lernfähigkeit für abstrakte Zusammenhänge } \\
\text { geringere Weiterbildungsbeteiligung }\end{array}$ \\
\hline $\begin{array}{l}\text { Wettbewerbsvorteile } \\
\text { und positive } \\
\text { Entwicklungen }\end{array}$ & 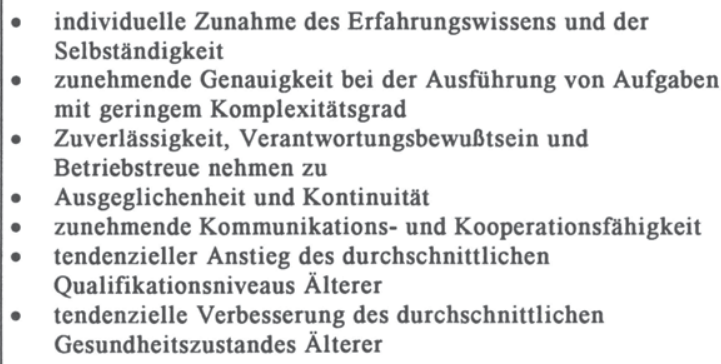 \\
\hline
\end{tabular}

Abb. 40: Arbeitsmarktbezogene Kennzeichen für die Erwerbstätigkeit von Älteren 


\section{B. Arbeitsaufgaben und Anforderungen}

Aufgrund äußerer Bedingungen und Einflüsse sowie ökonomischer Notwendigkeiten werden in den Unternehmen zur Erreichung der gesetzten Ziele technische und organisatorische Entscheidungen getroffen, die zu individuellen und sozialen arbeitspersonenbezogenen Auswirkungen führen. Die einzelnen Komponenten dieses Zusammenhangs werden wissenschaftlich in verschiedenen Disziplinen behandelt, von denen die wichtigsten in folgender Abbildung genannt werden. Die Forschungsergebnisse dieser Disziplinen sind in unterschiedlichem Maße miteinander verknüpft; sie betreffen subjekt- und objektbezogen Fragestellungen, die auch für die Ableitung von Maßnahmen zur Erhöhung der Erwerbsquoten von Frauen und Älteren relevant sind, so daß sie hier Beachtung finden müssen.

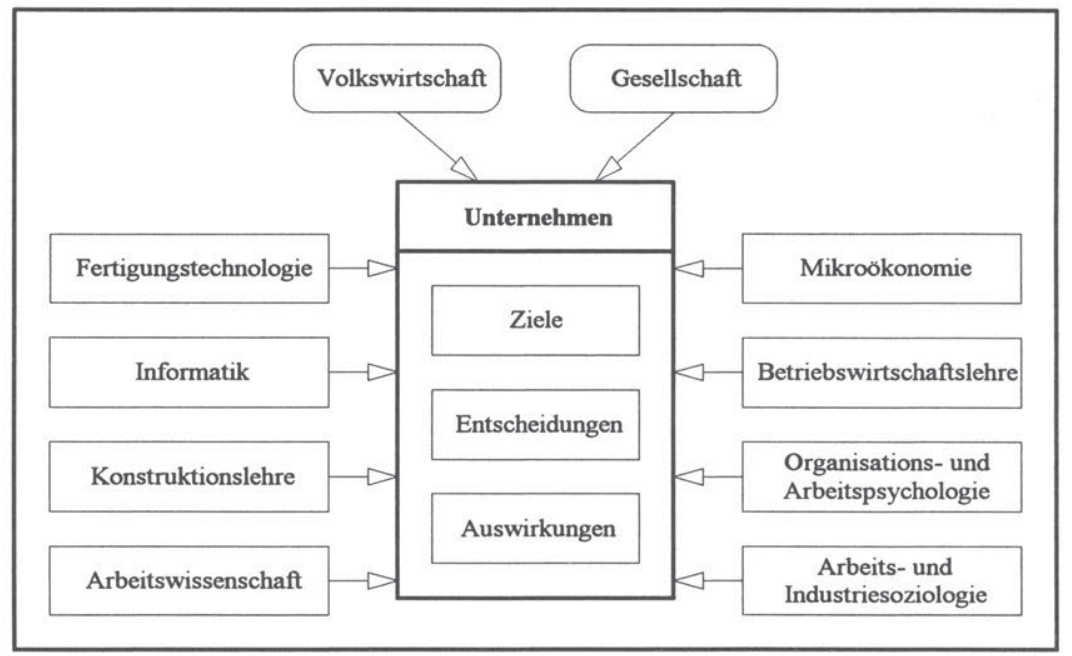

Abb. 41: Auf das Unternehmen einflußnehmende Disziplinen

Die Entscheidungen und Handlungsweisen in den Unternehmen orientieren sich an generellen Zielen, die sich z.B. auf Sicherheit bzw. Wachstum und insbesondere auf die Rentabilität beziehen. Diese generellen Ziele werden entsprechend der im einzelnen gestellten Anforderungen konkretisiert. Die Erreichung dieser Ziele erfordert die ständige Sicherung und Steigerung der Wettbewerbsfahigkeit der Unternehmen bzw. der angebotenen Güter und Dienstleistungen, so daß eine wesentliche Triebfeder der Entscheidungen und Handlungen in den Unternehmen die Konkurrenz durch potentielle Ersatzprodukte und -leistungen ist. Dieser 
Wettbewerb bleibt auch zukünftig erhalten bzw. wird sich angesichts der fortschreitenden Internationalisierung der Entwicklungsstandorte, der Produktionsstandorte und der Märkte sowie den sich ständig erweiternden Möglichkeiten der Informations- und Kommunikationstechniken noch verschärfen. Die in vielen Bereichen zu erkennende rasche Technikentwicklung führt in den Unternehmen zu häufiger werdenden Prozeß- und Produktneuerungen. Zudem steigen die Anforderungen der Beschaffungs- und Absatzmärkte, da die dort agierenden Unternehmen ihrerseits im Wettbewerb stehen. Wesentliche Einflußfaktoren auf die Unternehmen und sich hieraus ergebende Wettbewerbsmerkmale zeigt die folgende Abbildung (vgl. hierzu beispielsweise SIEMENS, 1990, 13ff. und SCHEER, 1990, 35ff.).

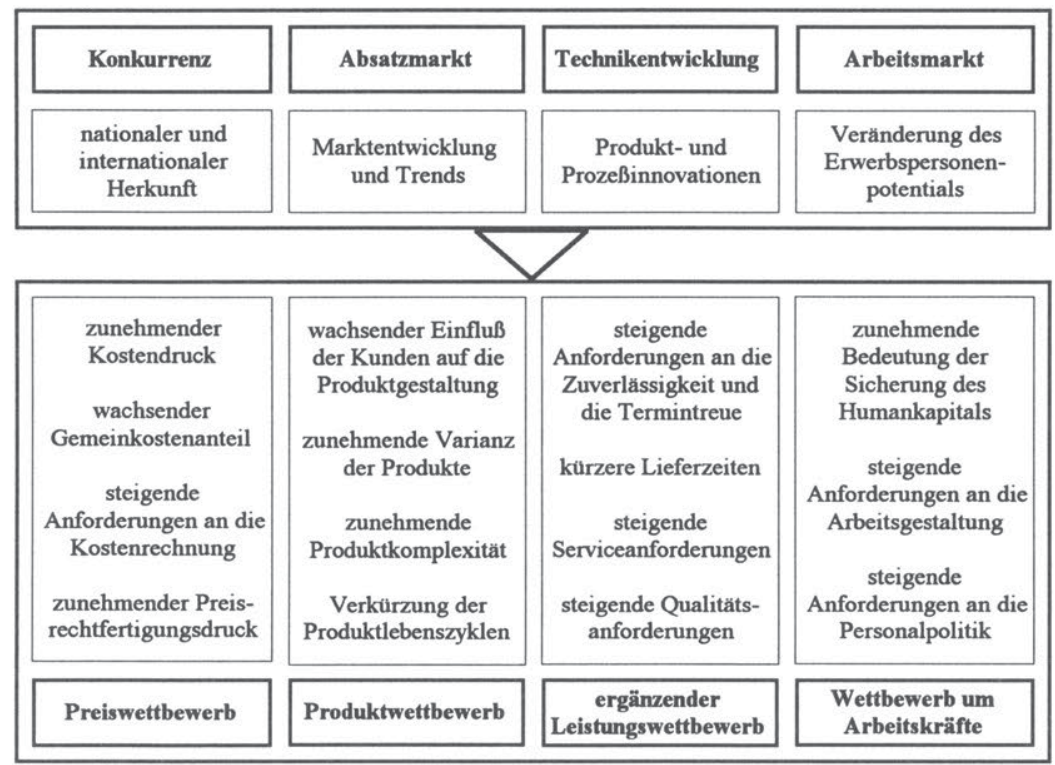

Abb. 42: Einflußfaktoren, Wettbewerbsmerkmale und Wettbewerbsdimensionen

Durch den auf die Preise, die Produkte und Leistungen wie insbesondere Qualität, Service, Zuverlässigkeit und Termintreue gerichteten Wettbewerb entsteht ein deutlicher Rationalisierungsdruck. Die zunehmende Komplexität und Dynamik der Einflußfaktoren erfordern flexible Unternehmen, die ihre Organisation, ihre Produktion, ihre Produkte und ihre Form der Techniknutzung den sich ändernden Anforderungen anpassen können. Der sich zukünftig verschärfende Wettbewerb um qualifizierte Arbeitskräfte wird angesichts der derzeitigen Arbeitsmarktsituation 
häufig ausgeblendet. Allerdings sollten seine besonderen Anforderungen frühzeitig erkannt und entsprechende Handlungsweisen entwickelt werden, um noch bestehende Handlungsspielräume für die Gestaltung und den Einsatz von Maßnahmen zur personellen Absicherung nutzen zu können. Zumal in den Unternehmen $\mathrm{zu}$ überprüfen ist, ob die jeweiligen betrieblichen personellen Ressourcen ausreichend sind, um moderne Produktions- und Organisationsmethoden zur Sicherung der Wettbewerbsfähigkeit umsetzen zu können.

Die Wettbewerbskomponenten stellen vielfältige Anforderungen, die wesentliche Zielsetzungen der Unternehmen bestimmen. Im Interesse der Wettbewerbsfähigkeit werden in den Unternehmen Formen der Umsetzung moderner Produktions- und Organisationsmethoden zur Verbesserung der Produktivität und der Wirtschaftlichkeit angestrebt. Sie orientieren sich hinsichtlich ihres Einsatzes und ihrer spezifischen Gestaltung neben dem übergeordneten Rentabilitätsziel an konkreteren Zielen, die neben den Kosten, insbesondere Produkte, Technologien und Mitarbeiter, d.h. alle Merkmale, die das Unternehmen zur effizienten Erfüllung der Bedürfnisse auf den Märkten befähigen, betreffen. In der folgenden Abbildung sind wesentliche mit dem Einsatz der Methoden verbundene und betriebsspezifisch zu gewichtende Erwartungen und Ziele zusammengestellt (vgl. beispielsweise CRONJÄGER, 1990, 135f.).

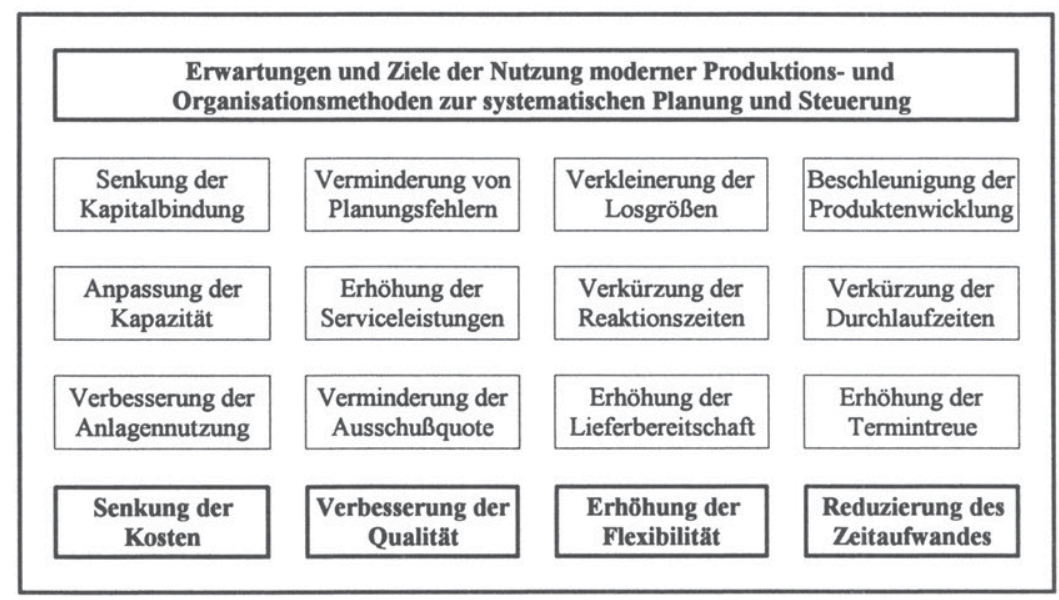

Abb. 43: Erwartungen und Ziele des Einsatzes moderner Methoden 
Diese Ziele sind auf die Steigerung der technisch-wirtschaftlichen Effizienz gerichtet. Sie müssen zukünftig insbesondere aufgrund der demographischen Entwicklung und der Einflüsse des Arbeitsmarktes um das Ziel der Bewahrung und Sicherung des Humankapitals ergänzt werden, zumal knappheitsbedingt die Kosten für fachlich qualifiziertes Personal steigen werden. Neben der Personalreduzierung und der Gewährleistung der erforderlichen Qualifikationsstruktur gewinnen 'Sozialziele', wie die Förderung der Akzeptanz, der Arbeitsmotivation und Arbeitszufriedenheit der Beschäftigten, die Minderung von Gesundheitsrisiken, die Schaffung von Qualifizierungsmöglichkeiten und Qualifizierungsanreizen aus wirtschaftlichen Gründen an Bedeutung. Humanisierungsziele, wie die Erreichung einer möglichst hohen Arbeitszufriedenheit, die Persönlichkeitsentfaltung oder die ganzheitliche Gestaltung von Arbeitsaufgaben werden somit aus Unternehmenssicht ein Mittel zur Sicherung des Humankapitals. Unter den bestehenden ökonomischen Bedingungen steigen damit die zukünftigen Umsetzungschancen der zur Humanisierung der Arbeit entwickelten Empfehlungen, da sie im Interesse der Sozialziele die technischen und wirtschaftlichen Ziele zunehmend ergänzen statt ihnen zu widersprechen. Anders ausgedrückt wird das traditionelle "technische Modell der Produktivität" durch ein "soziales Modell der Produktivität" ergänzt (NASCHOLD, 1987).

Moderne Produktions- und Organisationsmethoden, die zur Erreichung der angesprochenen Kosten-, Qualitäts-, Flexibilitäts- und Zeitziele zunehmend eingesetzt werden, interessieren hier, insofern sie sich auf die Bedingungen der Arbeitsausführung, die Art der Arbeitsaufgaben und die mit ihnen verbundenen Arbeitsanforderungen auswirken und somit auch das langfristige Bestehen des Unternehmens im Wettbewerb um Arbeitskräfte beeinflussen. Die Wahl bestimmter Produktions- und Organisationsmethoden ist eine vorgeordnete Strategieentscheidung (vgl. KÖTTER/VOLPERT, 1993, 131) aus der sich Arbeitsgestaltungsaufgaben ergeben. Ähnlich verweist NEUBERGER $(1985,79)$ darauf, daß jeder Technikwahl organisatorische Grundsatzentscheidungen vorausgehen und die Installation der Technik dann eine Reihe organisatorischer Folgeentscheidungen nach sich zieht. Zur Bestimmung der anforderungsbezogenen Auswirkungen, werden nachfolgend die Leitvorstellungen moderner Produktions- und Organisationsmethoden für die Fabrik und das Büro der Zukunft betrachtet. 


\section{Leitvorstellungen moderner Produktions- und Organisationsmethoden}

Die Produktions- und Organisationskonzepte richteten sich in der Vergangenheit vornehmlich auf die optimale Gestaltung und Auslastung der Betriebsmittel. Die Arbeitsproduktivität wurde kapazitäts- bzw. auslastungsorientiert durch stärkere Arbeitsteilung, verbesserte Maschinen und größere Massenproduktion erhöht. Im internationalen Vergleich blieben in Deutschland allerdings, insbesondere aufgrund der Bedeutung der Werkzeugmaschinenindustrie, handwerkliche Produktionsweisen trotz der sich verbreitenden Massenproduktion relativ wichtig (vgl. PIORE/SABEL, 1985; BENZ-OVERHAGE u.a., 1983). Heute und zukünftig geht es darum die Produktion (wieder) kundenspezifischer auszurichten. Somit steht der auftrags- und produktgerechte Produktionsdurchlauf im Vordergrund, so daß logistische Größen wie Bestände, Liefer- und Durchlaufzeiten markt- und materialfluß- bzw. ablauforientiert optimiert werden müssen, um die Anpassungsfähigkeit des Unternehmens zu sichern (vgl. beispielsweise WIENDAHL, 1991, 43ff. und $81 \mathrm{ff}$. sowie SCHEER, $1990,25 \mathrm{ff}$. und 162ff.).

Traditionell wurden entsprechend der tayloristischen Grundauffassung ingenieurwissenschaftliche Innovations- und Rationalisierungsformen genutzt. Die von Taylor entwickelte wissenschaftliche Betriebsführung beruhte insbesondere auf der Arbeitsteilung zur Vereinfachung der Tätigkeiten, auf der Verwissenschaftlichung im Sinne einer Bündelung des für die Arbeitsdurchführung notwendigen Wissens in Formeln und Gesetzen zur Standardisierung der Arbeitsprozesse sowie auf der Trennung planender und ausführender Tätigkeitsbestandteile. Als negative Auswirkungen dieser tayloristischen Arbeitsrationalisierung und fortschreitenden Automatisierung sind die besonders in industriesoziologischen Studien festgestellten Phänomene der Dequalifizierung, der einseitigen Belastung sowie der Demotivation, die sich in hohen Fehlzeiten und Fluktuationsraten niederschlagen, zu nennen (vgl. EULER, 1987). Daneben verhindert die Bürokratisierung der Planungs- und Verwaltungsaufgaben weitere Rationalisierungserfolge (vgl. GROB, 1994). Die dargestellten Wettbewerbsveränderungen und die hieraus abgeleiteten unternehmensbezogenen Erwartungen und Ziele erfordern andere Rationalisierungsmethoden, die sich in unterschiedlichem Maße von tayloristischen Vorstellungen unterscheiden (vgl. beispielsweise BECHTLE/LUTZ, 1989; HIRSCHKREINSEN u.a., 1990, 12ff. sowie BRÖDNER, 1985, 93ff. und 117ff.). Sie müssen insbesondere flexibilitäts- und innovationsfreundlicher sein und schwer planbare, langwierige Entwicklungs- und Produktionsprozesse sowie integrative, 
betriebsübergreifende Strukturen fördern, indem sie z.B. die Zentralisierung bzw. die fachliche (organisatorische), funktionale (nach Arbeitsfunktionen) und hierarchische Arbeitsteilung abbauen.

Seit einigen Jahren werden solche 'neuen' Formen der Rationalisierung diskutiert und umgesetzt, zumal die vorhandenen 'traditionellen' Rationalisierungspotentiale, zumindest in den Großunternehmen weitgehend ausgenutzt worden sind und gerade die Entwicklung der Kommunikations- und Informationstechniken neue Möglichkeiten bietet. Kennzeichnend für diese Rationalisierungsstrategien ist, daß sie sich nicht mehr auf einen einzelnen Arbeitsplatz und die Verbesserung einzelner Tätigkeiten oder Bearbeitungsstufen beziehen, sondern sich auf alle technischen und organisatorischen Prozesse innerhalb eines Betriebs und die Verknüpfung dieser Prozesse untereinander richten. Die Konzepte beziehen sich mit komplexen Lösungen auf das gesamte Unternehmen; der Fertigungsbereich wird weiterhin ebenso erfaßt, wie zunehmend Büro bzw. Verwaltung und die datentechnische Verknüpfung beider Bereiche. Die Rationalisierung greift zudem über die betrieblichen Grenzen hinweg, indem sie sich u.a. mit den Beziehungen zu Unternehmen des Liefer- und Distributionsbereichs befaßt. Diese unternehmensübergreifende Verknüpfung der Arbeitsabläufe wird besonders durch die fortschreitende Nutzung der Kommunikations- und Informationstechnik ermöglicht. Dabei trägt die zwischenbetriebliche Einflußnahme zur Vereinheitlichung der verwendeten Methoden und Techniken bei. Der Einfluß richtet sich von bedeutenden Anwendern bestimmter Produkte auf deren Hersteller und bezieht sich auf die Art der Umsetzung neuer Technologien (vgl. EULER, 1987, 5), oder er richtet sich von großen Unternehmen auf ihre Zulieferer und betrifft die einheitliche Bestimmung der zu nutzenden Technik.

Die Verbreitung dieser modernen Methoden, wie auch neuer Techniken zur Mechanisierung und Automatisierung, ist abhängig von der ökonomischen Situation der Unternehmen. Besonders in wirtschaftlich krisenhaften Situationen und angesichts der angesprochenen Wettbewerbsentwicklung wächst bei vielen beteiligten Gruppen der Wille zur Umgestaltung, so daß die Chancen für die Durchsetzung neuer Methoden, die Kostensenkung und Flexibilitätszuwachs versprechen, steigen. Andererseits erschweren äußere Instabilitäten und strukturkonservative Effekte der bisherigen tayloristischen Rationalisierungsweise die Umsetzung neuer Konzepte (vgl. BECHTLE/LUTZ, 1989, 67ff.). 
Unter der Bezeichnung "systemische Rationalisierung" werden diese umfassenden Konzepte insbesondere von Industriesoziologen (vgl. Studien des Instituts für sozialwissenschaftliche Forschung, München, wie beispielsweise HIRSCHKREINSEN u.a., 1990, 112f. oder Studien des Soziologischen Forschungsinstitutes Göttingen, wie BAETHGE/OBERBECK, 1986) erörtert. In der gewerkschaftlichen Diskussion werden unter diesem Stichwort besonders die Konsequenzen für die Möglichkeiten der betrieblichen Vertretung der Arbeitnehmerinteressen und die Gestaltung der gewerkschaftlichen Strategie thematisiert (vgl. beispielsweise ALTMANN/DÜLL, 1987; BREDEWEG/KOWOL, 1991; HELFERT, 1992). Weitere Formen der industriesoziologischen Einordnung moderner Produktionsund Organisationskonzepte (vgl. MINSSEN, 1993) erkennen einen "anthropozentrischen Entwicklungspfad" (vgl. BRÖDNER, 1985) oder "neue Produktionskonzepte" (vgl. KERN/SCHUMANN, 1984). Im folgenden soll allerdings nicht vertiefend auf die wissenschaftlichen Probleme der Einteilung und Zuordnung der Rationalisierungsformen eingegangen werden (vgl. für einen Überblick hierzu MINSSEN, 1993, 40ff.), vielmehr werden exemplarisch die Leitvorstellungen zweier wichtiger Produktions- und Organisationskonzepte betrachtet. Zunächst die Vorstellungen der Computer Integrated Manufacturing (CIM) und anschließend die der Lean Production. Die primären Gestaltungsempfehlungen dieser vielgestaltigen, anpassungsfähigen und betriebliche Anpassungsleistungen erfordernden Konzepte beziehen sich auf unterschiedliche Schwerpunkte: So richtet sich CIM besonders auf die technische Integration der Funktionen des Unternehmens, während die Lean Production die mitarbeiterbezogenen organisatorischen Voraussetzungen der zu erbringenden Leistungen betont. Dabei geht es nicht um eine umfassende Darstellung der beiden Konzepte oder um eine Abwägung ihrer jeweiligen wirtschaftlichen Vorteile, vielmehr sollen die jeweiligen Leitvorstellungen herausgearbeitet werden, um die aus ihrer zunehmenden Umsetzung erwachsenden Anforderungsänderungen einschätzen zu können. Dies ist notwendig, da die mit den Arbeitsaufgaben verbundenen Anforderungen zusammen mit den Eigenschaften der Arbeitspersonen, die gruppenspezifischen Möglichkeiten einer Erwerbstätigkeit nachzugehen und die unternehmensbezogenen Maßnahmen zur Verbesserung dieser Möglichkeiten bestimmen.

\section{a) CIM-Konzept}

Während der 80er Jahre konzentrierte sich die wissenschaftliche Diskussion auf die technischen Möglichkeiten der Rationalisierung, insbesondere auf computer- 
gestützte Arbeitsabläufe und ihre inner- bzw. überbetriebliche Verknüpfung. Demgegenüber wurden die Folgen für die einzelnen Arbeitspersonen und Fragen der Arbeitsorganisation, die noch in den 70er Jahren besonders thematisiert wurden, eher vernachlässigt. Die mit CIM verbundenen Vorstellungen betonten entsprechend zunächst die technisch-organisatorischen Aspekte und bemerkten hinsichtlich personeller Auswirkungen häufig lediglich die Bedeutung von Maßnahmen zur Information und zur Bedienungsschulung sowie die Notwendigkeit der Akzeptanz seitens der Mitarbeiter zur Sicherung der reibungslosen Einführung neuer Technologien. Zunehmend wird allerdings auch im CIM-Zusammenhang ausführlicher auf personalbezogene, organisatorisch-soziale Themenbereiche eingegangen und Fragen der Arbeitsorganisation, der Personalplanung und der Qualifikation (vgl. BULLINGER, 1992 und SCHEER, 1990, 63ff.) behandelt.

Die Leitvorstellungen von CIM beziehen sich auf die rechnerunterstützte Aufgabenerfüllung und die informationstechnische Verknüpfung insbesondere der Bereiche Konstruktion, Einkauf, Arbeitsplanung, Fertigung, Montage und Qualitätssicherung sowie Produktionsplanung und -steuerung. CIM betrifft somit das gesamte Unternehmen und zielt auf eine aus informationstechnischer Sicht bestmögliche Gestaltung der Geschäftsprozesse (vgl. WIENDAHL, 1991) und eine Erhöhung der Transparenz des betrieblichen Geschehens. Die Umsetzung des CIMKonzeptes richtet sich auf die Erhöhung der Leistungsfähigkeit des Unternehmens und zieht Veränderungen im Produktionsbereich sowie im Dienstleistungs- und Verwaltungsbereich nach sich. CIM soll zur Erreichung der genannten Kosten-, Qualitäts-, Flexibilitäts- und Zeitziele beitragen, d.h. Kosten oder Kundennutzung sollen nicht einseitig, sondern simultan verbessert werden (vgl. SCHEER, 1990, 5). Die rechnerunterstützte Integration und Vernetzung der Arbeitsabläufe wird besonders verbreitet in der metallverarbeitenden Industrie angestrebt (vgl. SCHULTZ-WILD u.a., 1989, 12f.). Eigens entwickelte Konzepte, die mit CAO (Computer Aided Office) oder CIO (Computer Integrated Office) abgekürzt werden, behandeln die Rechnerunterstützung im Bürobereich. Die Verknüpfung der Konzepte für die technischen und betriebswirtschaftlichen Bereiche der Unternehmen wird z.B. mit CAI (Computer Aided Industry) gemäß der Formel CAI = CIM + CAO oder mit CIB (Computer Integrated Business) gemäß der Formel CIB = CIM + CIO bezeichnet. In dieser Arbeit wird allerdings die Bezeichnung 'CIM' umfassend für die Gestaltungsvorstellungen im Produktions- und im Bürobereich benutzt, da sich zum einen CIM auf den Produktionsprozeß sowie u.a. die Funktionen Konstruktion, Einkauf, Arbeitsplanung, Materialwirtschaft bezieht und mithin auch Verwaltungs- und Bürotätigkeiten erfaßt und zum anderen die Leit- 
vorstellungen für beide Bereiche im wesentlichen gleich sind (vgl. CRONJÄGER, 1990, 17f.). Die Besonderheiten der Technisierung beider Bereiche und einige Entwicklungsstufen bis zu ihrer Verknüpfung werden nachfolgend betrachtet, um anschließend personalbezogene Auswirkungen zusammenfassen zu können.

\section{(1) Veränderungen in der Produktion}

Hinsichtlich der Computerunterstützung der Arbeit lassen sich verschiedene Entwicklungsstufen unterscheiden. Zunächst können einzelne Aufgaben oder Prozesse eines Bereichs automatisiert werden. Da die Rechnersteuerung in der Fertigung zu besonders vielfältig automatisierten Einrichtungen führte, soll dieser Bereich exemplarisch betrachtet werden. Die Abbildung zeigt die Einordnung automatisierter Fertigungseinrichtungen, die zunehmend an den innerbetrieblichen Informationsfluß angebunden werden, hinsichtlich ihrer Produktivität und Flexibilität.

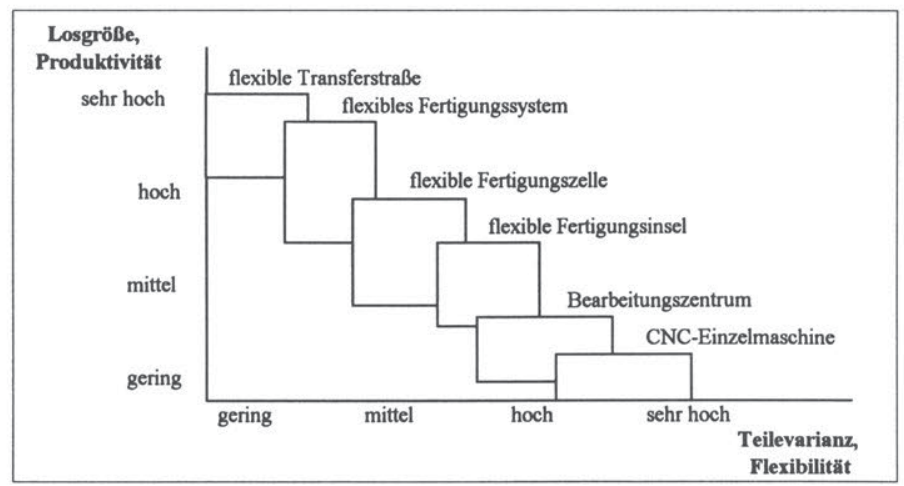

Abb. 44: Konzepte der automatisierten Fertigung (Quelle: CRONJÄGER, 1990, 78)

Auf der ersten Stufe der Computernutzung werden einzelne numerisch gesteuerte Werkzeugmaschinen eingesetzt. Diese CNC (Computerized Numerical Control)Maschinen führen jeweils ein einzelnes Bearbeitungsverfahren (z.B. Fräsen oder Drehen) aus. Werkzeuge und Werkstücke werden manuell gewechselt. Mit einem CNC-Bearbeitungszentrum können dagegen bereits mehrere Verfahren (z.B. Fräsen und Bohren) an einem Werkstück ausführt werden. Das Werkstück wird hierfür vom Maschinenbediener aufgespannt und automatisch gewechselt. Eine flexible Fertigungszelle ist zusätzlich mit Werkstückspeicher, Überwachungs- und Kontrolleinrichtungen ausgerüstet, so daß sie eine bestimmte Zeit ohne Bediener arbeiten kann. Die bedienerlose Schicht steigert die Produktivität in der Fertigung, 
die Maschinenlaufzeit wird unabhängiger von der Arbeitszeit der Beschäftigten. Flexible Fertigungssysteme fassen auf der nächsten Stufe unabhängig voneinander arbeitende Maschinen der zuvor genannten Fertigungstypen (u.a. ergänzt durch Fördersysteme und Handhabungsgeräte oder auch Fertigungsroboter) zusammen, indem die Steuerung der Werkzeugmaschinen zentralisiert wird. Die Bedienung erfordert qualifizierte Arbeitspersonen, die auch die Überwachungs- und Steuerfunktionen erfüllen müssen. Im Falle sehr hoher Losgrößen werden flexible Transferstraßen eingesetzt, die durch automatische Werkstücktransportsysteme gekennzeichnet sind, die die Maschinen nach dem Linienprinzip verknüpfen.

Eine veränderte Arbeitsorganisation findet sich im Rahmen der flexiblen Fertigung bei den besonders in der Klein- und Serienfertigung eingesetzten flexiblen (oder autonomen) Fertigungsinseln. Diese fassen alle Arbeitsplätze, die zur Bearbeitung ähnlich zu fertigender Werkstücke benötigt werden, räumlich und organisatorisch zusammen. Dies führt zum Abbau innerbetrieblicher Transport- und Lagerkosten und zu erweiterten Möglichkeiten einer absatzgesteuerten Produktion. Die Durchlaufzeit wird erheblich verringert, die Umlauf- und Lagerbestände werden abgebaut. Die Mitarbeiter der Fertigungsinsel führen alle direkten und möglichst viele indirekten Funktionen aus, von denen besonders die interne Disposition der Aufträge hervorzuheben ist. Diese Form der dezentralen Fertigungsfeinplanung unter Nutzung begrenzter Entscheidungsfreiheit (vgl. CRONJÄGER, 1990, 132f. und WIENDAHL, 1991, 101ff.) kann mit Hilfe eines vom unternehmensweiten Produktionsplanungs- und Steuerungssystem entkoppelten Systems in der Fertigung durchgeführt werden und entlastet damit zentrale Stellen, die nur noch eine Rückmeldung über komplett bearbeitete Aufträge erhalten. Die Mitarbeiter einer Fertigungsinsel sollen möglichst alle Funktionen erfüllen können und müssen daher entsprechend qualifiziert sein. Die Werkstückbearbeitung kann an Handarbeitsplätzen und an verschiedenartig automatisierten Maschinen erfolgen.

Da auch im weiteren der Untersuchung die Unterscheidung zwischen direkten und indirekten Funktionen im Bereich der Produktion angesprochen wird, werden in Abbildung 45 jeweils exemplarisch wesentliche Aufgaben genannt. Eine unterschiedliche Zuordnung ist möglich. Zur Steigerung der Produktivität können beispielsweise die für die computerunterstützte Fertigung wichtigen Programme zentral erstellt, verwaltet und zugeteilt werden. Von einem DNC (Distributed Numerical Control)-Leitrechner aus können, entsprechend geeignete CNCMaschinen vorausgesetzt, die Fertigungsteilsysteme zentral gesteuert sowie hinsichtlich des Fertigungsfortschritts und des Maschinenzustandes überwacht 
werden. Allerdings können DNC-Systeme auch "werkstattoffen" gestaltet werden, so daß die Programmierung durch die Facharbeiter dezentral erfolgen kann (vgl. beispielsweise SCHULTZ-WILD u.a., 1989, 190).

\begin{tabular}{|c|c|}
\hline direkte Funktionen & indirekte Funktionen \\
\hline $\begin{array}{l}\text { - Bearbeitung der Werkstücke } \\
\text { (z.B. an Handarbeitsplätzen, } \\
\text { konventionellen WZM, } \\
\text { CNC-Maschinen oder } \\
\text { Sondermaschinen) } \\
\text { - Rüsten der Maschinen, } \\
\text { - Be- und Entladen, } \\
\text { - Tätigkeiten der Steuerung, } \\
\text { - Überwachung und Kontrolle }\end{array}$ & $\begin{array}{l}\text { - Arbeitsplanung und -vorbereitung } \\
\text { - Produktions-, Fertigungs- und } \\
\text { Werkstattsteuerung } \\
\text { - Werkzeugverwaltung und -voreinstellung } \\
\text { - NC-Programmierung } \\
\text { - Materialflußsteuerung } \\
\text { - } \\
\text { - } \text { Instandhalaialtung } \\
\text { Qualitätskontrolle }\end{array}$ \\
\hline
\end{tabular}

Abb. 45: Direkte und indirekte Funktionen im Produktionsbereich

Die Vereinheitlichung der für den Fertigungsbereich genannten Typen der computergesteuerten Einrichtungen und die bereichsweite Verknüpfung der automatisierten Prozesse führen zu einheitlichen Bereichslösungen, v.a. Computer Aided Design (CAD), Planning (CAP), Manufacturing (CAM), Quality Assurance (CAQ), Service (CAS) sowie Produktionsplanung und -steuerung (PPS). Die Computernutzung innerhalb dieser Einzelsysteme entspricht noch weitgehend einer tayloristischen Strategie. Erst die bereichsübergreifende Verknüpfung und die (Re-) Dezentralisierung von Planungs- und Steuerungsteilaufgaben bedeuten eine Änderung der verfolgten Strategie in Richtung "systemischer" Strukturen (vgl. LUTZ/MOLDASCHL, 1989, 22ff.).

Das CIM-Konzept schließlich richtet sich auf die zentrale Steuerung, Abstimmung und Überwachung der automatisierten Bereiche, so daß die Informationsflüsse und Arbeitsabläufe beschleunigt und verbessert werden. Zur Umsetzung sollen die vorhandenen Bereichslösungen integriert werden. Diesbezüglich lassen sich verschiedene Integrationsmöglichkeiten und -stufen unterscheiden, die bis zur Funktionsintegration verschiedener miteinander kommunizierender Anwendungssysteme (eine Entscheidung in einem Anwendungssystem bewirkt automatische Abläufe in einem anderen Anwendungssystem) reichen (vgl. SCHEER, 1990, 147ff.). In einer bereits höheren Integrationsstufe ermöglicht z.B. die gemeinsame Nutzung einer Datenbasis, daß Arbeitspersonen verschiedener Bereiche verschiedene auftragsbezogene Funktionen gleichzeitig durchführen können. Die informationstechnische Rechnerintegration verkürzt daher die Durchlaufzeiten gegenüber der konventionellen Arbeitsabfolge. Die einzelnen Aufgaben können miteinander 
gekoppelt, einander begleitend und ergänzend erfüllt werden. Zudem wird zielgemäß die bereichsübergreifende Planung und Steuerung vereinfacht. Neben der Verkürzung der Durchlaufzeit soll besonders eine Erhöhung der Transparenz erreicht werden. Wesentliche Bestandteile des CIM-Systems und ihre Funktionen zeigt die folgende Abbildung, die der Empfehlung des Ausschusses für wirtschaftliche Fertigung entspricht.

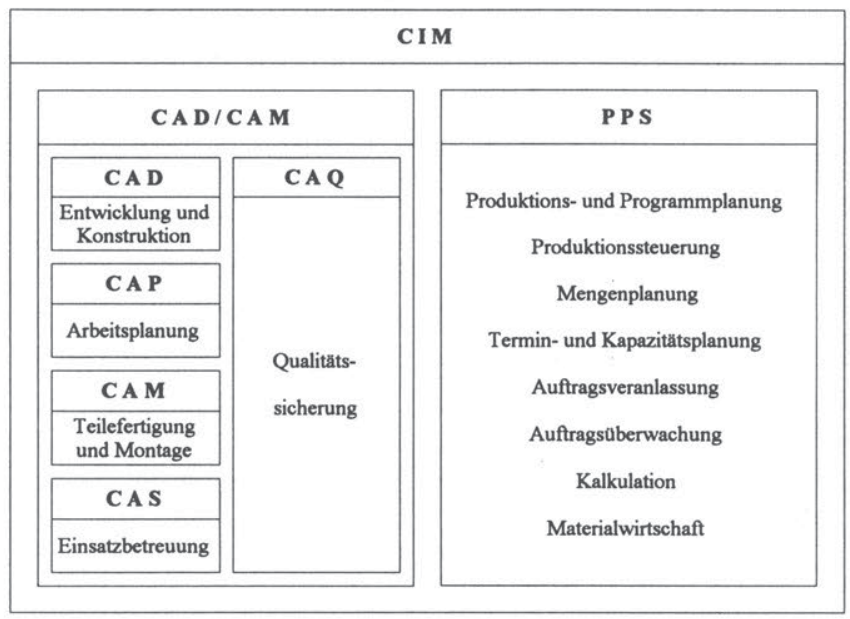

Abb. 46: Bestandteile eines CIM-Systems (in Anlehnung an CRONJÄGER, 1990, 9 und 112)

Die Umsetzung der im CIM-Konzept angestrebten Verknüpfung verschiedener Teilsysteme wirft allerdings Probleme auf, die z.B. die Beharrlichkeit von Insellösungen, die Hardware- und Herstellerabhängigkeit der Bereichslösungen und die Inkompatibilität der Datenkommunikation betreffen. Zur Lösung der Probleme die insbesondere mit der Software und der bisherigen Programmlogik zusammenhängen sollen Expertensysteme an Bedeutung gewinnen (vgl. LUTZ/MOLDASCHL, 1989). Expertensysteme sind Computerprogramme, die zur Bereitstellung und Verarbeitung von Wissen entwickelt werden und auf gespeichertem Wissen in Form von Fakten, Informationen über Verknüpfungsregeln und Wenn-dann-Regeln basieren. Diese Systeme stellen für komplexe Aufgaben mit einer Vielzahl zu beachtender Bedingungen Ergebnisse zur Verfügung, indem sie Antworten auf Anfragen geben, Diagnosen stellen und Entscheidungen vorschlagen bzw. treffen. Expertensysteme ergänzen bestehende Informationsverarbeitungssysteme (z.B. CAD, CAM, CAQ und PPS), in die sie zur Kommunikations-, Informations- und Prozeßunterstützung integriert werden können (vgl. WARNECKE, 1991, 37ff.). Sie können zur 
Verbesserung der betrieblichen Leistungsfähigkeit, zur Erhöhung der Wirtschaftlichkeit und zur Sicherung der Verfügbarkeit des Expertenwissens, das bisher nur knappe bzw. teure Spezialisten besaßen, eingesetzt werden (vgl. LUTZ/MOLDASCHL, 1989, 12ff.). So können Expertensysteme beispielsweise die Programmierung computergesteuerter Fertigungssysteme, die Fertigungssteuerung oder Instandhaltungsaufgaben unterstützen.

In der Praxis wird die Strategie der Vollintegration zunächst durch eine Integration von Insellösungen oder die Gestaltung einzelner Integrationspfade ersetzt. Beispielsweise erfolgt die Integration von Konstruktion und Arbeitsplanung durch die Integration von CAD- und CAM-Systemen. Aufgrund der Rechnersteuerung und -integration in Verbindung mit modernen Kommunikations- und Informationstechniken sind auch dezentrale Produktionsformen, die einen Teil der Arbeit örtlich bzw. organisatorisch aus dem Unternehmen herausnehmen, möglich. Für eine dezentrale Organisation bieten sich neben manchen Konstruktions-, Montage- und Prüftätigkeiten, besonders Dienstleistungs- und Verwaltungstätigkeiten an, die mit Hilfe der CAP und PPS-Systeme ausgeführt werden können. Der nächste Schritt zur Integration der Unternehmensorganisation richtet sich auf die Verknüpfung der Produktion mit der betriebswirtschaftlichen Planung und Verwaltung. Bevor einige Funktionen dieser Verknüpfung betrachtet werden, sollen zunächst Ansätze zur Umgestaltung des Bürobereichs dargestellt werden.

\section{(2) Veränderungen im Bürobereich}

Die Produktivitätssteigerungen der Vergangenheit wurden vor allem im Bereich der Produktion, insbesondere bei dem direkten Wertschöpfungsprozeß der Fertigung, erreicht (vgl. beispielsweise GROB, 1994, 84). Angesichts des vorhandenen Produktivitätssteigerungspotentials und der Entwicklung der Informations- und Kommunikationstechniken wird die Umgestaltung zukünftig in besonderem Maße den Bürobereich betreffen, d.h. die indirekt am Wertschöpfungsprozeß beteiligten Arbeiten der Planung, der Entwicklung, der Verwaltung und des Vertriebs.

Der Computereinsatz im Büro- und Verwaltungsbereich ist weitaus höher als in anderen Bereichen der Unternehmen und begann nach einer Umfrage des ISF München von 1986/87 in vielen Betrieben bereits Ende der 50er bzw. Anfang der 60er Jahre (vgl. SCHULTZ-WILD u.a., 1989). Der Einsatz computergestützter Techniken bewegte sich z.B. in der Maschinenbauindustrie nach dieser Erhebung je 
nach Funktion in Büro und Verwaltung zwischen $31 \%$ im Einkauf und $80 \%$ in der Finanz- und Geschäftsbuchhaltung, in der Fertigung dagegen zwischen $2 \%$ von Flexiblen Fertigungssystemen und $58 \%$ von CNC-Werkzeugmaschinen (die Zahlen sollen nur andeuten in welchem Größenverhältnis sich die Verbreitung der Computernutzung im Produktions- und Bürobereich bewegt, sie werden aufgrund ihrer Vergleichbarkeit verwendet, obwohl sie sich mittlerweile erhöht haben dürften). Im Vordergrund der Computernutzung standen zunächst die stark formalisierten Aufgaben der Finanz- und Geschäftsbuchhaltung sowie der Lohnund Gehaltsabrechnung, zunehmend werden nun auch alle übrigen Funktionen computergestützt erfüllt. Ähnlich wie für den Bereich der Fertigung betrachtet, wurde die für den Bürobereich typische Informationsverarbeitung technikorientiert behandelt, so daß sich isolierte bereichsbezogene Bearbeitungsformen verbreitet haben (vgl. ORTNER, 1991, 317ff.). Die zukünftig an Bedeutung gewinnenden Rationalisierungsanstrengungen richten sich auch im Bürobereich auf die Integration der betrieblichen Anwendungssysteme, vornehmlich durch eine gemeinsame, anwendungsübergreifende Datennutzung. Die Entwicklung der mikroelektronischen Datenverarbeitung und der Kommunikationstechnik ergibt eine Verlagerung von der punktuellen oder einzelfunktionsbezogenen zur systemischen Rationalisierung, die die Organisation, Steuerung und Durchführung der Arbeitsabläufe in einem Unternehmen und darüber hinaus "in einem Zug" neu gestaltet (vgl. BAETHGE/OBERBECK, 1986, 20ff.). Die Umgestaltung im Bürobereich unter Verwendung moderner Informations- und Kommunikationstechniken zielt (vgl. beispielsweise BAETHGE/OBERBECK, 1986, 62ff.) auf die

- Reduzierung von Personalkosten und Verwaltungsaufwand,

- Reduzierung von Routinetätigkeiten,

- Verbesserung der Verarbeitungsmöglichkeiten,

- Beschleunigung der Geschäftsabwicklung und Verkürzung der Durchlaufzeiten,

- Vermeidung von Bearbeitungsfehlern und Doppelarbeiten,

- Erhöhung der Transparenz,

- Verbesserung der Dokumentation und Kontrollmöglichkeiten,

- Verbesserung der Kalkulationsgrundlagen,

- Beschleunigung des Datenzugriffs und die

- Verbesserung der Aktualität der Information.

Die Rationalisierung soll die Informationsmenge für unternehmerische Planung, Entscheidung und Kontrolle erhöhen und insbesondere eine wirtschaftliche und schnelle Bereitstellung der benötigten Informationen ermöglichen. Neben den 
Kosten der Informationsverarbeitung interessiert daher auch im Bürobereich die Durchlaufzeit. Die Durchlaufzeit dient der Strukturierung der Büroarbeit und der prozeßorientierten Betrachtung der Leistungserstellung im Büro, sie setzt sich im wesentlichen aus der Bearbeitungszeit, der Transport-, Abstimmungs- sowie Kontrollzeit und vor allem der Liegezeit zusammen (vgl. ZANGL, 1985, 85ff.). Die Nutzung der Informationstechnik und die angestrebte Integration des Informationsflusses bzgl. der Absatz-, Bestands-, Produktions-, Materialbedarfs- und Beschaffungsplanung soll die Kosten senken und die Durchlaufzeit verkürzen. Die ganzheitliche Bearbeitung der Aufgaben und die Verringerung der Arbeitsteilung mindern Abstimmungsprobleme, der Zugriff auf Datenbanken erleichtert den Informationsbezug, der Übergang von der Verrichtungsorientierung auf die Objektorientierung baut mehrfaches Einarbeiten durch verschiedene Mitarbeiter ab und verkürzt die Liegezeit. Die Erreichung der Rationalisierungsziele erfordert in den Unternehmen Flexibilität hinsichtlich der Arbeitsgestaltung, der Ablauf- und Aufbauorganisation. In einer ersten Stufe kann die Computernutzung an einem einzelnen Arbeitsplatz erfolgen. Weiterhin können die Bildschirmarbeitsplätze elektronisch verknüpft sowie interne und externe Datenbanken und Expertensysteme genutzt werden. Eine solche Vernetzung der Computer kann dazu führen, daß Daten, Texte und Bilder intern und extern automatisiert übertragen werden. So können beispielsweise dezentrale Büros eingerichtet werden, die mit den Datenbanken und dem Informations- und Kommunikationsnetz der Zentrale verknüpft sind. Die organisatorische Umgestaltung bezieht sich damit auf den unternehmensinternen Zusammenhang der Arbeitsplätze und auf die Beziehungen zum Unternehmensumfeld. Die vielfältig sich entwickelnden Möglichkeiten der Hard- und Softwarenutzung führen auch im Bürobereich dazu, daß die (Informations-) Technik die Organisation der Arbeit nicht mehr vorbestimmt. Die Nutzung spezifisch angepaßter Informationstechnik ermöglicht es, organisatorische Strukturen hinsichtlich der Unternehmensziele und der Mitarbeiterbedürfnisse zu gestalten.

Die Automatisierung und interne Vernetzung von Abläufen im Verwaltungsbereich (z.B. von Rechnungswesen sowie Ein- und Verkauf) bereiten die zunehmende Verknüpfung der kaufmännischen Funktionen mit dem Produktionsbereich (z.B. über die Aufgaben der Produktionsplanung und der Materialwirtschaft) vor. Die schon hinsichtlich der Computernutzung in der Fabrik genannten PPS-Systeme unterstützen nicht nur die Arbeiten der Auftragsabstimmung, Produktionsplanung und Werkstattsteuerung, sondern auch die Materialbedarfsplanung und weitere materialwirtschaftliche, auftragsabwicklungsbezogene sowie kostenrechnerische 
Aufgaben. Die Trennung zwischen Fabrik und Büro, zwischen technischen und betriebswirtschaftlichen Aufgaben verliert sich damit immer weiter. Zur Verkürzung der Durchlaufzeiten und zur Senkung der Bestände wurden verschiedene Konzepte entwickelt, die auch im Rahmen der PPS-Systeme umzusetzen sind (vgl. CRONJÄGER, 1990, 125ff.). Hier können insbesondere das Just-In-Time-Konzept, das KANBAN-Prinzip und die belastungsorientierte Auftragsfreigabe genannt werden. Diese Konzepte basieren darauf, daß die Zulieferbeziehungen sowie die internen Produktions- und Auftragsfreigabebeziehungen vom Bringprinzip auf das Holprinzip umgestellt werden. So wie die betrachteten C-Techniken können PPSSysteme unterschiedlich genutzt werden, sie lassen sich als zentralistische Totalplanungssysteme oder als dezentral verfügbare Teilsysteme entwickeln und einsetzen (vgl. SCHULTZ-WILD u.a., 1989, 114). Die computergestützte Integration überschreitet schließlich die betrieblichen Grenzen und führt zur überbetrieblichen Vernetzung insbesondere mit Betrieben, die zum gleichen Unternehmen gehören, aber auch mit Kunden, Lieferanten und Dienstleistern.

Ergänzend muß auf die derzeitige 'Begrenztheit' der für das CIM-Konzept vorhandenen Technik und Software verwiesen werden. Schnittstellenprobleme bezogen auf unterschiedliche Hardwarekomponenten und Softwarestrukturen machen aufwendige spezifische Lösungen notwendig. Abteilungs- und bereichsspezifische Interessen und Arbeitsweisen erschweren eine einheitliche Umsetzung und sorgen für nicht-technische Schnittstellenprobleme (vgl. BAETHGE/ OBERBECK, 1986, 141ff.). Für die mit der vorliegenden Darstellung verfolgten Ziele sind allerdings die Entwicklungstendenzen von entscheidender Bedeutung und nicht die derzeitige betriebsspezifisch vollständige Umsetzbarkeit rechnerintegrierter Vorstellungen.

Die Auswirkungen dieser komplexen technisch-organisatorischen Änderungen sind, verglichen mit traditionellen, punktuell wirkenden Rationalisierungsmaßnahmen, nur schwer zu kalkulieren (vgl. beispielsweise SCHEER, 1990, 55ff. und $161 \mathrm{ff}$; SCHULTZ-WILD u.a., 1989, 92). Die Höhe der Kosten und Nutzen lassen sich im voraus schlecht bestimmen, zumal auch qualitative Effekte, wie die Erhöhung der Flexibilität, angestrebt werden. Tendenziell muß bei Umsetzung dieser kapitalintensiven Methoden mit einem steigenden Kapitalbedarf gerechnet werden, wenngleich eine Verkürzung der Durchlaufzeiten das erforderliche Umlaufkapital senkt. Der Fixkostenanteil wird sich voraussichtlich absolut und relativ erhöhen, der Anteil der Löhne an den Kosten kann sich nach einer Anpassungsphase verringern. Die Produktionskosten werden durch den Einsatz flexibler Fertigungs- 
einrichtungen zunehmend stärker von der Bearbeitungszeit als von der Höhe der gefertigten Stückzahl abhängig. In den Unternehmen sind zur Umsetzung der modernen Konzepte erhebliche Eigenleistungen mit vielfaltigen Investitionen in Technik und Humankapital notwendig, so da $\beta$ aufgrund der Beschränktheit der personellen und finanziellen Ressourcen eine stufenweise Einführung üblich und empfehlenswert ist.

\section{(3) Arbeitsaufgabenbezogene Auswirkungen}

Der integrierte Einsatz von Computersystemen wird zumeist technik- statt mitarbeiterorientiert betrachtet. So finden sich z.B. unter den in einer Erhebung ermittelten 18 Zielsetzungen des Technikeinsatzes personalbezogen nur das Ziel der Personaleinsparung (das von ca. $26 \%$ der Unternehmen, die eine CAD/CAMVernetzung realisiert haben genannt wird) und das Ziel der Humanisierung der Arbeit (das von ca. $8 \%$ dieser Unternehmen genannt wird). Die übrigen, z.T. weitaus häufiger genannten Ziele entsprechen den eingangs abgeleiteten Kosten-, Qualitäts-, Flexibilitäts- und Zeitzielen (vgl. SCHULTZ-WILD u.a., 1989, 87). Obwohl die Umsetzung des CIM-Konzepts demnach nicht ausdrücklich darauf gerichtet ist, werden die Arbeitsaufgaben und -bedingungen durch organisatorische Veränderungen beeinflußt. Die Aufbauorganisation wird besonders durch die Integration der Vorgänge und die Ablauforganisation durch die Integration der Daten verändert. Betrachtet man die dargestellte Entwicklung der CIM-Konzepte hinsichtlich ihrer Wirkungen auf die Arbeitsaufgaben, so kann festgehalten werden, daß sich die menschliche Tätigkeit tendenziell immer weiter von der direkten Bearbeitung der Werkstücke zu einer computervermittelten Einflußnahme verschiebt und Steuerungs- und Überwachungsaufgaben zunehmend zentralisiert und vereinheitlicht werden. Die Distanz der Arbeitsperson zum Produktionsprozeß wird zeitlich und sachlich immer größer, so daß beispielsweise die Arbeitszeit flexibler gestaltet werden kann. Die fortschreitende Automatisierung erweitert damit den Spielraum der Gestaltung der Betriebs- und Arbeitsorganisation.

Trotz der beschriebenen übergreifend wirkenden und insofern nicht-tayloristischen Ausrichtung der CIM-Rationalisierungsstrategie führt deren Umsetzung nicht zu einem generellen Abbau der (hierarchischen, fachlichen und funktionalen) Arbeitsteilung. Vielmehr können zumindest zwei oder drei Typen der Arbeitszuordnung und der Aufgabengestaltung unterschieden werden (vgl. SCHULTZ-WILD u.a., 1989, 169ff.; HIRSCH-KREINSEN u.a., 1990; LUTZ/MOLDASCHL, 1989, 
19ff.; SCHEER, 1990, 63ff.; BULLINGER, 1992, 42ff. und 96ff.). Hier werden exemplarisch die Gestaltungstypen Neo-Taylorismus, polarisierte Produktionsarbeit und qualifiziert homogene bzw. kooperative Produktionsarbeit und die damit zusammenhängende unterschiedliche Zuordnung dispositiver und ausführender Tätigkeiten betrachtet (vgl. SCHEER, 1990; BULLINGER, 1992; HIRSCHKREINSEN u.a., 1990). Eine neo-tayloristische Organisation der Arbeit behält eine hohe funktionale und fachliche Arbeitsteilung bei bzw. fördert diese zusätzlich, indem dispositive Planungs- und Steuerungsfunktionen (z.B. als werkstattexterne technische Arbeitsvorbereitung) rechnerunterstützt zentralisiert und indirekte Fertigungsaufgaben (z.B. Instandhaltung) spezialisierten Arbeitspersonen übertragen werden. Die im Bereich der direkten Fertigung auszuführenden Tätigkeiten werden ebenfalls arbeitsteilig insbesondere von Un- und Angelernten durchgeführt. Die 'Zwischenstufe' der polarisierten Produktionsarbeit führt die hohe fachliche und hierarchische Arbeitsteilung fort, während die funktionale Arbeitsteilung durch eine Verlagerung von Planungs- und Steuerungsaufgaben in den Fertigungsbereich (z.B. durch eine Auflösung von Arbeitsplätzen mit indirekten Aufgaben) abgebaut wird. Die dispositiven Tätigkeitsbestandteile werden allerdings nur einigen Mitgliedern der Fertigungsbelegschaft übertragen (z.B. zur Systemführung oder an Leitständen), so daß sich eine neue Form der Arbeitsteilung ergibt. Das Qualifikationsniveau dieser Arbeitsplätze steigt hierdurch deutlich und nähert sich dem der Ingenieure und Techniker. Die übrigen Tätigkeiten in der Fertigung werden weiterhin arbeitsteilig von Un- und Angelernten durchgeführt. Die qualifiziert homogene bzw. kooperative Produktionsarbeit ist dagegen durch eine niedrige funktionale und fachliche Arbeitsteilung gekennzeichnet. Dispositive und ausführende Funktionen werden in der Fertigung als ganzheitliche Tätigkeiten erfüllt. Die Tätigkeiten werden in wechselnder Zuordnung kooperativ von möglichst homogen qualifizierten Facharbeitern durchgeführt (z.B. Fertigungsinsel).

Die Gestaltungsmöglichkeiten bei der Umsetzung des CIM-Konzepts richten sich demnach auf eine technikorientierte Zentralisierung oder eine die menschlichen Qualifikationen nutzende Dezentralisierung, die Komponenten von stärker mitarbeiterorientierten Strategien enthält. Die Mehrheit der Betriebe behält arbeitsteilige, zentralisierte Strukturen bei, während eine starke Minderheit die Arbeitsteilung bereichs- und teilweise aufhebt und nur eine kleine Minderheit die Arbeitsteilung in der Fertigung oder im ganzen Betrieb in fachlicher, funktionaler und hierarchischer Hinsicht abbaut (vgl. SCHULTZ-WILD u.a., 1989, 194ff.). 
Die zukünftige Entwicklung der Techniknutzung wird auch von der Situation auf dem Arbeitsmarkt abhängen, da die Vorteile einer dezentralen Organisationsweise nur mit qualifiziertem Personal in ausreichender Zahl zu realisieren sind. Eine Verschärfung des Fachkräftemangels fördert tendenziell neo-tayloristische Strategien. Eine Antizipation der zu erwartenden arbeitsangebotsbezogenen Probleme kann zu problemverstärkenden Entscheidungen führen, da zentralistische Organisationsformen im geringeren Maße zur Entwicklung breiter fachlicher Qualifikationen und Kompetenzen beitragen.

CIM wird als Konzept vorgestellt, dessen Einführung Veränderungen der Organisations- und Personalstruktur unter dem Gesichtspunkt der Effizienz des Technologieeinsatzes erfordert (vgl. SCHEER, 1990, 22f.). Demnach wird eine Integration unternehmensspezifischer sozial-organisatorischer Gestaltungsgrundsätze und Mitarbeiterbedürfnisse ausgeblendet, obwohl die Abhängigkeit des CIM-Erfolgs von entsprechend qualifiziertem Personal allenthalben betont wird. Selbst bei der Behandlung personalbezogener Aufgaben wird nur auf die Veränderungen der Planungsmethoden und Organisationsstrukturen durch das CIM-Konzept eingegangen (vgl. WIENDAHL, 1991, 11f.). WIENDAHL unterscheidet hierfür die Anforderungen der Organisation und die Ansprüche der Mitarbeiter (das Unternehmen soll z.B. Entwicklungsmöglichkeiten schaffen, um die Mitarbeiter zu motivieren), vernachlässigt allerdings die wichtigen mitarbeiterbezogenen Ansprüche an die Organisation bzw. an das CIM-Konzept. Insgesamt sollte es "nicht um 'die' Auswirkungen 'der' Rechnerintegration, sondern um Veränderungen im Zusammenhang mit dem Einsatz von CIM-Techniken" gehen (SCHULTZ-WILD u.a., 1989, 170). Die eher technisch orientierte Behandlung der Rationalisierung im CIM-Konzept wird zunehmend durch mitarbeiterorientierte Elemente ergänzt. So soll CIM als technische Integration mit Formen der nicht-technischen Integration, insbesondere durch die Bildung von Arbeitsgruppen verknüpft werden. Tatsächlich wird diese "Gruppentechnologie" von CIM-Autoren zum Teil sogar als "neue möglich Form" zur Strukturierung der Arbeit bezeichnet (vgl. WIENDAHL, 1991, 159f.). Andere Autoren verweisen allerdings auch auf die mit der Umsetzung technikzentrierter Konzepte gemachten teuren Erfahrungen und beispielsweise auf die älteren Konzepte teilautonomer Arbeitsgruppen zur Durchführung ganzheitlicher Aufgaben (vgl. BULLINGER, 1992 und SCHULZ, 1990, 114). Weiterhin löst sich innerhalb des CIM-Konzepts die eine ausgeprägte Arbeitsteilung unterstützende Funktionsorientierung, so daß beispielsweise durch die Bildung von Fertigungsinseln ganzheitliche Aufgabenbereiche gebildet werden und die Prozeßorientierung an Bedeutung gewinnt. Solche mitarbeiterbezogenen Vorstellungen zur 
Entwicklung der Produktions- und Organisationsmethoden schlagen sich allerdings nicht nur in der CIM-Strategie, sondern stärker noch in anderen Konzepten nieder. Besonderen Einfluß auf diese vielfaltige Diskussion hatte und hat das Konzept der Lean Production, das die Arbeitspersonen als wichtigsten Erfolgsfaktor begreift.

\section{b) Lean Production}

\section{(1) Entwicklung und Ausrichtung des Konzeptes}

Als Lean Production bezeichnen die Autoren des Schlußberichtes der weltweiten Studie über grundlegende Veränderungen in der Automobilindustrie des Massachusetts Institute of Technologie (MIT) das bei japanischen Unternehmen, insbesondere bei Toyota, umgesetzte und als besonders effizient erkannte Arbeitsorganisationsmodell (WOMACK u.a., 1991). Für das vom MIT durchgeführte Forschungsprojekt "International Motor Vehicle Project" wurden in 14 Ländern über 5 Jahre hinweg Unternehmen der Automobilindustrie untersucht.

Kennzeichnend für die Lean Production sind 'schlanke' (vereinfachte, übersichtliche, klar gegliederte und verantwortete) Wertschöpfungsabläufe ohne Verschwendung oder Blindleistung und die integrative Verknüpfung unternehmensintern und -extern zu erbringender Aufgaben (vgl. auch zu folgendem WOMACK u.a., 1991). Durch die Lean Production sollen die Durchlaufzeit, der Raumbedarf, der Lagerbestand, der Aufwand für Werkzeuge und für Personal zur Kostensenkung reduziert und Produktivität, Flexibilität und Qualität verbessert werden. Die konsequente Rationalisierung richtet sich daher nicht nur auf die Senkung der Kosten, beispielsweise durch den Abbau von Stellen und Abteilungen, sondern auf die Umgestaltung der gesamten Unternehmensorganisation, sie erfaßt alle Unternehmensprozesse, die Produktentwicklung, Fertigung und Montage ebenso wie die Zulieferung, den Absatz und die Kundenbetreuung und wirkt sich bereichsspezifisch aus. Im folgenden stehen die grundlegenden Elemente der Lean Production im Vordergrund, die alle Bereiche des Unternehmens sowie ihre Verknüpfung betreffen und somit auch die Arbeit aller Beschäftigten beeinflussen.

Seit Veröffentlichung des zusammenfassenden MIT-Berichtes wurde die Bezeichnung Lean Production zum Synonym moderner Organisation und Rationalisierung. Anders als das technikorientierte und -basierte CIM-Konzept richtet sich die Lean Production auf die Nutzung mitarbeiterbezogener organisatorischer Maßnahmen zur 
Erreichung betriebswirtschaftlicher Ziele. Die Diskussion der Lean Production geht dabei nur wenig detailiert auf die tatsächlichen Zustände in japanischen Unternehmen, die dortigen Rahmenbedingungen, die negativen Auswirkungen und die Wandelbarkeit des Konzeptes ein. Der MIT-Bericht selbst und auch die durch ihn ausgelöste Diskussion können entsprechend kritisiert werden (vgl. JÜRGENS, 1993). Eine Übertragung und Anpassung der Lean Production erfordert die Überprüfung der Sichtweise wichtiger Kennzeichen und die Beachtung kultureller Eigenheiten (vgl. SCHERM/BISCHOFF, 1994) sowie nationaler, betrieblicher und arbeitsmarktbezogener Besonderheiten (vgl. MINSSEN, 1993; HOWALTD/KOPP, 1992).

Obwohl viele der Ideen, die im Rahmen der Diskussion der Lean Production behandelt werden, nicht neu sind, wird hier die Beschreibung dieses Konzeptes benutzt, um Leitvorstellungen für die Entwicklung der Arbeitsaufgaben zu gewinnen. Dies scheint insbesondere angesichts der Integration dieser Ideen in ein umfassendes Konzept gerechtfertigt. Weiterhin ist für die vorliegende mikroökonomisch ausgerichtete Arbeit von Vorteil, daß sich dieses Konzept im wesentlichen auf die Handlungsmöglichkeiten der Unternehmensleitung, im Sinne einer Umstrukturierung im Bereich der Unternehmen, bezieht (vgl. beispielsweise SUZAKI, 1994). Andere z.B. makroökonomische oder politische Faktoren (wie Lohnkostendifferenzen, unterschiedlich lange Arbeitszeiten, Industrie- und Strukturpolitik) die sich zwar ebenfalls auf die Wettbewerbsfähigkeit auswirken, aber außerhalb des Einflußbereichs der Unternehmensleitung liegen, werden nicht behandelt. Schließlich unterstreicht das Konzept der Lean Production die Bedeutung des Humankapitals für die Unternehmen. Das Leistungspotential der Arbeitspersonen soll stärker und umfassender genutzt werden, indem diese beispielsweise zur Verbesserung des Produktionsprozesses und der Produkte beitragen. Erwartet wird die Entwicklung vom technikzentrierten Taylorismus der Massenproduktion mit Arbeitsteilung und starren Hierarchien zu mitarbeiterzentrierten Lean-Konzepten mit Arbeitspersonen, die ihre Kreativität und ihr Mitdenken in Arbeitsgruppen einbringen, um die Arbeitsprozesse kontinuierlich $\mathrm{zu}$ verbessern und kundenorientiert zu produzieren. Die Lean Production soll die Vorteile der handwerklichen Fertigung und der Massenproduktion verbinden, die Stückkosten senken und die Qualität erheblich verbessern.

Die MIT-Studie gibt betriebswirtschaftliche Kennziffern an, die in einem weltweiten Vergleich von Montagewerken ermittelt wurden und z.B. die bezüglich der 
Produktivität (Montagestunden pro Fahrzeug) und der Qualität (Anteil fehlerbehafteter Fahrzeuge) vorteilhafte Situation japanischer Unternehmen darstellen.

\begin{tabular}{|l|c|c|c|c|}
\hline Leistung & $\begin{array}{c}\text { japanische } \\
\text { Werke in Japan }\end{array}$ & $\begin{array}{c}\text { japanische } \\
\text { Werke in USA }\end{array}$ & $\begin{array}{c}\text { amerikanische } \\
\text { Werke in USA }\end{array}$ & $\begin{array}{c}\text { europäische } \\
\text { Werke }\end{array}$ \\
\hline $\begin{array}{c}\text { Produktivität } \\
\text { (Stunden / Auto) }\end{array}$ & 16,8 & 21,2 & 25,1 & 36,2 \\
\hline $\begin{array}{c}\text { Qualität (Fehler } \\
\text { je 100 Autos) }\end{array}$ & 60,0 & 65,0 & 82,3 & 97,0 \\
\hline
\end{tabular}

Abb. 47: Leistungsmerkmale der Montagewerke, Großserienhersteller, 1989

(Durchschnitt der Fabriken je Region) (Quelle: WOMACK u.a., 1991, 97)

Die Produktivitäts- und Qualitätsdifferenzen werden mit Unterschieden hinsichtlich der Automatisierung, des Produktdesigns und der Produktkomplexität sowie der Arbeitsorganisation erklärt. Da nach den Ergebnissen der MIT-Studie die Art der Arbeitsorganisation etwa die Hälfte der Leistungsdifferenzen begründet, bildet die Einsatzweise des Produktionsfaktors Arbeit den Schwerpunkt des Konzepts der Lean Production. Die folgende Tabelle zeigt die bezüglich des Arbeitskräfteeinsatzes angegebenen Merkmale.

\begin{tabular}{|c|c|c|c|c|}
\hline $\begin{array}{c}\text { japanische } \\
\text { Arbeitskräfte }\end{array}$ & $\begin{array}{c}\text { japanische } \\
\text { Werke in USA }\end{array}$ & $\begin{array}{c}\text { amerikanische } \\
\text { Werke in USA }\end{array}$ & $\begin{array}{c}\text { europäische } \\
\text { Werke }\end{array}$ \\
\hline $\begin{array}{c}\text { \% der Arbeits- } \\
\text { kräfte in Teams }\end{array}$ & 69,3 & 71,3 & 17,3 & 0,6 \\
\hline $\begin{array}{c}\text { Job Rotation (0= } \\
\text { keine, 4=häufig) }\end{array}$ & 3,0 & 2,7 & 0,9 & 1,9 \\
\hline $\begin{array}{c}\text { Vorschläge / } \\
\text { Arbeitskraft }\end{array}$ & 61,6 & 1,4 & 0,4 & 0,4 \\
\hline $\begin{array}{c}\text { Anzahl der } \\
\text { Lohngruppen }\end{array}$ & 11,9 & 8,7 & 67,1 & 14,8 \\
\hline $\begin{array}{c}\text { Ausbildung neuer } \\
\text { Prod-Arbeiter (h) }\end{array}$ & 380,3 & 370,0 & 46,4 & 173,3 \\
\hline $\begin{array}{c}\text { Abwesenheit / } \\
\text { Arbeitskraft (\%) }\end{array}$ & 5,0 & 4,8 & 11,7 & 12,1 \\
\hline
\end{tabular}

Abb. 48: Merkmale des Arbeitskräfteeinsatzes der Montagewerke, Großserienhersteller, 1989

(Durchschnitt der Fabriken je Region) (Quelle: WOMACK u.a., 1991, 97)

Die drastischen Unterschiede der Merkmalswerte und die objektive Relevanz dieser spezifischen Daten sollen hier nicht detailiert untersucht werden, zumal die MITStudie kaum Hinweise zur Ermittlung der einzelnen Werte gibt. Wichtig allerdings ist, daß die aufgrund der empirischen Erhebungen abgeleiteten Vorstellungen der Lean Production zunehmend praktische Bedeutung gewinnen. Eine Umstrukturierung der 'westlichen' Industrie entsprechend den Grundsätzen der schlanken Produktion nach japanischem Vorbild scheint den MIT-Autoren unvermeidbar. Die Lean Production wird als das zukünftige Produktionskonzept bezeichnet, das 
ausgehend von der Automobilindustrie, in allen Industriebereichen bestimmend werden und noch praktizierte tayloristische Produktionsweisen verdrängen wird. Die Autoren betonen, daß die "fundamentalen Ideen der schlanken Produktion universal - überall und von jedem anwendbar" seien. Tatsächlich werden die Ideen in vielen Bereichen rezipiert. Die Umsetzung von Elementen des Lean-Konzeptes betrifft nicht nur die Automobilindustrie (vgl. neben WOMACK u.a., 1991 beispielsweise auch SHINGO, 1992; HOWALTD/KOPP, 1992; HOWALTD, 1994), sondern die gesamte Industrie (vgl. beispielsweise SCHULTETUS, 1993) und beispielsweise auch Unternehmen mit stärker administrativen Funktionen, wie Flughafenunternehmen (vgl. HÖLZEL, 1994).

Neben der Lean Production gibt es weitere Konzepte, die auf weitgehend gleichen Grundgedanken basieren und eine flexible, dezentralisierte, mitarbeiterorientierte Organisation empfehlen. Diese Organisationsformen werden z.B. chaotisch oder fraktal genannt (vgl. beispielsweise WARNECKE, 1992). Die Umsetzung dieser Konzepte dürfte zu ähnlichen Anforderungsverschiebungen führen wie die Lean Production. Weiterführungen der Grundgedanken der Lean Production (oder Lean Manufacturing) finden sich im Lean Management und ergeben über die Produktions- und Dienstleistungsunternehmen hinaus, unter den Stichworten Lean Government bzw. Lean Administration, Leitbilder für öffentliche Verwaltungen (vgl. KÜHNLEIN/WOHLFAHRT, 1994; STRUWE, 1995). Die allgemeinen Zielsetzungen sind in allen Bereichen ähnlich. Auch in Behörden und halbstaatlichen Organisationen soll durch leistungsorientierte Prozesse sowie die Erhöhung der organisatorischen und personellen Effizienz die Wirtschaftlichkeit und die Bürgerbzw. Kundennähe verbessert werden. Eine solche Entwicklung ist, aufgrund der komplementären Funktion der Leistungen der öffentlichen Verwaltung und Infrastruktur, auch im Interesse der Unternehmen (vgl. STEGER, 1994). Wissenschaftler verschiedener Disziplinen, besonders Betriebswirte sowie Automationsspezialisten, stimmen mit optimistischen Verbreitungsprognosen für die Ansätze der Lean Production überein. Sie betonen, ähnlich wie viele Arbeitgeber und Arbeitnehmervertreter, die Notwendigkeit von Veränderungen in Richtung einer schlanken Produktion. Dissens besteht allerdings bzgl. der empfehlenswerten und konkreten Ausgestaltung und Umsetzung des Konzeptes (vgl. beispielsweise die Beiträge in INSTITUT FÜR ANGEWANDTE ARBEITSWISSENSCHAFT, 1992 und 1994; EBERHARDT, 1993; HANS-BÖCKLER-STIFTUNG/INDUSTRIEGEWERKSCHAFT METALL, 1992). 


\section{(2) Umfeld-, unternehmensorganisations- und personalbezogene Vorstellungen}

Die umfassende Nutzung des Leistungspotentials der Arbeitspersonen zur Wertschöpfungsperfektionierung wird wesentlich durch die Bildung von Arbeitsteams, die dezentral Verantwortung übernehmen und sich um die ständige Verbesserung des übertragenen Arbeitsprozesses bemühen, erreicht. Die Gruppenarbeit, die Delegation von Verantwortung und der von den Arbeitspersonen getragene kontinuierliche Verbesserungsprozeß (vgl. IMAI, 1992) werden als wichtigste Prinzipien der Lean Production aufgefaßt. Weiterhin fühlt sich das Produktionsunternehmen mit den Unternehmen des Beschaffungs- und des Absatzbereichs dauerhaft verbunden, so $\mathrm{da} B$ an die Stellen austauschbarer Marktbeziehungen dauerhafte netzwerkartige Verbindungen treten. Im Gegensatz zur Rationalisierungsstrategie des Taylorismus, der für die standardisierte Massenproduktion die Bedeutung des Sachkapitals hervorhob und die Trennung von direkten und indirekten Tätigkeiten vorsah, betont die Lean Production die Bedeutung des Faktors Arbeit und begreift die verantwortlichen Beschäftigten als Quelle der Verbesserungen und der Rationalisierung des Arbeitsprozesses. Die Leitvorstellungen betreffen die Beziehungen des Unternehmens zu seinem Umfeld sowie die unternehmensinternen Strukturen und Abläufe des produzierenden und verwaltenden Bereichs. Die unternehmensbezogenen Leitvorstellungen können den abgeleiteten Kosten-, Qualitäts-, Flexibilitäts- und Zeitzielen zugeordnet werden. Aufgrund der mitarbeiterzentrierten Konzeption der Lean Production lassen sich zudem zahlreiche personalbezogene Vorstellungen bzw. Änderungserwartungen isolieren, die deutliche Auswirkungen auf die Arbeitsanforderungen haben dürften. Die folgende Zusammenstellung macht zudem deutlich, daß die Diskussion des Konzeptes der Lean Production viele selbständig entwickelte Vorschläge aufgreift und integriert.

Umfeldbezug

- Die Integration im Rahmen der Lean Production soll über die Unternehmensgrenzen hinaus erfolgen. Zulieferer und Händler bzw. Kunden werden nicht als austauschbaren Marktteilnehmer angesehen, sondern als sog. Wertschöpfungspartner, mit denen eine dauerhafte Zusammenarbeit anzustreben ist.

- Angesichts der Wettbewerbssituation ist das kundenorientierte Arbeiten aller Abteilungen des Unternehmens sowie der Zulieferer und Händler ein besonders anzustrebendes Ziel.

- Produktivităts- und Wirtschaftlichkeitsvergleiche entscheiden über eine mögliche Ausgliederung, d.h. den externen Bezug von bisher unternehmensintern erbrachten Leistungen.

- Die Entstandardisierung und die Verkürzung der Innovationszyklen sorgen für eine engere Kooperation zwischen Produzenten und Anwendern. Die Früherkennung von Chancen und Risiken soll durch eine kundennahe Produktentwicklung erreicht werden, die eine Verbesserung und Individualisierung des Angebots ermoglicht. 
Unternehmensorganisation

- Die Lean Production bezieht sich auf die systematische Neugestaltung der Organisation des gesamten Unternehmens, sie beschrănkt sich nicht auf einzelne Abteilungen, Arbeitsplătze oder Prozesse.

- Sie richtet sich auf den Abbau der Grenzen zwischen Funktionen und die geistige und răumliche Integration aller Arbeitspersonen und Aufgaben. Der Übergang von der Funktions- zur Prozeßorganisation soll bereichsübergreifende Zusammenarbeit fördern.

- Informationssysteme, Datenbanken sowie Expertensysteme sollen einen umfassenden Überblick und schnelle Reaktionen (auf unternehmensexterne und -interne Wünsche, Probleme und Vorschlăge) ermöglichen.

zur Senkung der Kosten:

- Das Unternehmen kann in autonome, überschaubare Wertschöpfungseinheiten zerlegt werden. Einzelne Abteilungen sind wie kleine Unternehmen zu führen, die sich gegenüber Konkurrenz 'von außen' durchsetzen müssen, so daß Marktmechanismen innerhalb der Unternehmen genutzt werden kőnnen und der Dienstleistungscharakter aller Funktionen betont wird.

- Abbau von Leistungen, die nicht der eigentlichen Wertschöpfung dienen.

- Eine absatzorientierte Fertigung und flußorientierte Fertigungsablaufe sollen die Kosten senken. Stichworte hierzu sind die pufferlose Fertigung, Just-In-Time-Systeme und die Synchronisierung der Produktion, Reduzierung der Zahl der Bauteile, Automatisierung von Routinetătigkeiten sowie die exakte Vorgabe von einfach beherrschbaren Arbeitsablăufen.

zur Verbesserung der Qualităt:

- Einführung eines stăndigen Verbesserungsprozesses unter Beteiligung aller Mitarbeiter zur permanenten Verbesserung des Arbeitsprozesses und der Erzeugnisse.

- Verankerung einer systematischen Qualitătsüberwachung und -kontrolle auf allen Entwicklungs- und Fertigungsstufen mit dem Ziel, Măngel und ihre Ursachen sofort aufzudecken und zu beseitigen, damit eine umfassende Fehlervermeidung und eine Annăherung an die perfekte (Null-Fehler-) Qualităt erreicht wird.

zur Erhơhung der Flexibilităt:

- Statt starrer Vorschriften und Vorgaben sollen allgemeine strategische Leitlinien entwickelt werden, die die Flexibilităt der Organisation sowie des Handelns erhöhen und es ermöglichen, auf Änderungen rasch zu reagieren.

- Eine flexible Automatisierung soll erreicht werden, indem entsprechende Anlagen in der Fertigung und Informationsverarbeitungssysteme in den Büros bei flexiblem Einsatz von Arbeitskrăften verwendet werden.

zur Reduzierung des Zeitaufwandes:

- Eine prozeBorientierte Organisation mit flacher Gliederung soll die funktionsorientierte Organisation mit stark hierarchischer Gliederung ersetzen. Der Abbau von Hierarchien und interner Bürokratie soll kurze Entscheidungswege ermoglichen und die Entscheidungsfindung beschleunigen. Die Dezentralisierung von Planungs- und Verwaltungsfunktionen soll dazu beitragen, daB Handlungskompetenz, Entscheidungskompetenz und Verantwortung integriert werden.

- Projektarbeit in Form von Teams deren Mitglieder aus unterschiedlichen Bereichen und Hierarchieebenen stammen soll zur Forderung von Produkt- und ProzeBinnovationen führen.

- Die innerbetriebliche Vernetzung (z.B. durch die Integration von Forschung/Entwicklung, Konstruktion und Fertigung zur parallelen Funktionserfüllung) soll die Entwicklungszeiten verkürzen, Mehrfacharbeit vermeiden und die frühzeitige Einbindung aller betroffenen Bereiche gewăhrleisten. 


\section{Personal}

- Die Lean Production strebt im Interesse der Wirtschaftlichkeit eine verbesserte Nutzung des Leistungspotentials der Mitarbeiter an. Entsprechendes Leitbild sind 'leistungsorientierte' Mitarbeiter mit hoher Arbeitsmotivation und einer starken Identifikation mit den betrieblichen Interessen.

- Zur Kostensenkung soll die Mitarbeiterzahl abgebaut werden.

- Durch die Integration von Planung, Entscheidung und Umsetzung sollen indirekte Aufgaben auf die Arbeitskräfte innerhalb des Wertschðpfungsprozesses übertragen werden. Die damit gekoppelte Delegation und Dezentralisierung der Verantwortung soll die Eigenverantwortung der einzelnen Mitarbeiter bzw. des Teams stărken sowie die Leistung und Arbeitsmotivation erhohen.

- Klar begrenzte und zum Teil erhöhte Einflußmöglichkeiten der Mitarbeiter sollen zur Verbesserung der Motivation und zur Entwicklung neuer Führungskräfte beitragen.

- Der Abbau von Hierarchiestufen, vor allem im Bereich des mittleren Managements, führt zur Verringerung der Aufstiegs- und Karrieremöglichkeiten. Dies bedeutet Änderungen der bisher ublichen (karriereorientierten) Motivation. Statt eines stetigen Aufstiegs sind hăufigere Aufgabenwechsel zu erwarten.

- Die betriebsinterne Kommunikation soll in Netzwerken persönlicher Beziehungen statt entsprechend der hierarchischen Organisation erfolgen. Die entsprechende Änderung des Führungsstils richtet sich auf ein kooperatives Führungsverhalten.

- Zur Bewaltigung der Aufgaben sollen Arbeitsteams gebildet werden. Jedes Mitglied der Gruppe soll sămtliche Arbeiten, die der Gruppe übertragen worden sind, erfüllen können.

- Die Flexibilităt des Arbeitseinsatzes soll durch variable Regelungen der Arbeitszeit nach Bedarf gesichert werden.

- Systeme zur Beurteilung, zur Personalbewertung (Leistung und Arbeitseinstellung) und zur Entgeltdifferenzierung (z.B. Prămienentlohnung und Zeitentlohnung mit persönlicher Leistungsbeurteilung) sollen die Mitarbeiter motivieren.

- Aufgrund der zunehmenden vielfaltigen Dezentralisierung sind neue Systeme und Methoden zur Messung und Kontrolle von Erfolg und Leistung einzuführen.

Abb. 49: Umfeld-, unternehmensorganisations- und personalbezogene

Vorstellungen des Lean-Konzeptes

Die Umsetzung dieser Vorstellungen erfordert erhebliche organisatorische und finanzielle Anstrengungen, außerdem ist je nach Element der Umstrukturierung mit verschieden langen Umstellungsphasen zu rechnen. Die Durchseizung der Elemente des Konzepts in den Unternehmen erfordert Beharrlichkeit und Geduld sowie die Fähigkeit die vielfältigen Schwierigkeiten, z.B. hinsichtlich einer Vorauskalkulation der zu erwartenden Kosten und Nutzen, auszugleichen.

Neben der angestrebten Verbesserung der Wettbewerbsfähigkeit, die zur Sicherung der Beschäftigung beiträgt, kann ein Effekt der Umsetzung des Lean-Konzepts die im geringeren Maße hervorgehobene Freisetzung von Mitarbeitern, beispielsweise des mittleren Managements, sein. Weiterhin dürfte die fortschreitende Automatisierung zu einer relativen Verringerung der Bedeutung der Arbeit in der industriellen Produktion führen und damit die relative Bedeutung der im geringeren Maße automatisierbaren Arbeit in den Bereichen Verwaltung, Handel und Dienstlei- 
stungen erhöhen. Jedoch bleibt die menschliche Arbeit auch im industriellen Bereich ein wichtiger Produktionsfaktor, denn die vielfältige Nutzung der Kreativität der Arbeitspersonen, beispielsweise zur Bewältigung technologischer und organisatorischer Änderungen, ist gerade ein Ziel der Entwicklung und Umsetzung neuer Produktions- und Organisationsmethoden. Die Auswirkungen der Umsetzung der Lean Production wie auch des CIM-Konzeptes auf die Beschäftigungshöhe sollen hier allerdings nicht eingeschätzt werden, zumal sich die Freisetzungs-, Kompensations- und Expansionseffekte ohnehin allenfalls auf der Makroebene saldieren lassen (vgl. RÜRUP/SCHNEIDER, 1992, 10) und unter mikroökonomischen Gesichtspunkten der qualitative Wandel der Arbeitsaufgaben relevanter erscheint.

Die bisherigen Betrachtungen zeigten die 'ganzheitliche', d.h. auf Technik und Mitarbeiter, Produktion und Verwaltung, das gesamte Unternehmen und das Umfeld usw. bezogene, Ausrichtung der modernen Methoden und Rationalisierungsansätze und betonten insbesondere die arbeitspersonenbezogenen Vorstellungen der Konzepte. Die behandelten Konzepte CIM und Lean Production stellen dabei keine klaren Alternativen dar, vielmehr dürften sie einander ergänzen, sich zukünftig gegenseitig beeinflussen und in der praktischen Umsetzung teilweise verschmelzen, so daß innerhalb der organisatorischen Gestaltung nach Vorstellungen der Lean Production eine zunehmende Computernutzung und flexible technische Integration gemäß dem CIM-Konzept möglich ist. In den Unternehmen wird die jeweilige Umsetzungsweise der Konzepte unterschiedlich sein, sie ist u.a. abhängig von der betrieblichen Ausgangslage, dem Unternehmensumfeld, der Zielsetzung sowie den finanziellen und personellen Möglichkeiten.

Exemplarisch kann auf den Einfluß der Unternehmensgröße eingegangen werden. In Einzel- und Kleinserienunternehmen ist die Beschäftigung fachlich qualifizierter Arbeitspersonen, die selbständig, kreativ und motiviert arbeiten, dominierend. Die arbeitsteilige Durchführung der mit niedrigen qualifikatorischen Anforderungen verbundenen Arbeitsaufgaben ist dagegen stärker in Großserienunternehmen verbreitet. In Abhängigkeit von der Unternehmensgröße kann entsprechend darauf verwiesen werden, daß der Abbau tayloristischer Arbeitsabläufe und indirekter Betriebsbereiche besonders in großen Unternehmen notwendig und zuküntig zu erwarten ist. Kleine und mittlere Unternehmen sind, bezogen auf die direkten Bereiche, dagegen vielfach bereits schlank organisiert und werden zur Erhöhung ihrer Produktivität zukünftig gerade auch die Leistung indirekter Betriebsbereiche zur Verbesserung der Arbeitsplanung und -verwaltung nutzen müssen (vgl. GROB, 1993, 1994). Diese unterschiedlichen Handlungsnotwendigkeiten beeinträchtigen 
allerdings nicht die wachsende Bedeutung der Zielsetzung, das Leistungspotential aller Arbeitspersonen stärker zu nutzen. Die zukünftigen Innovations- und Rationalisierungsprozesse besitzen daher zumindest hinsichtlich der beschriebenen grundlegenden Leitvorstellungen ausgeprägte Gemeinsamkeiten, so daß im weiteren Aussagen zu tendenziellen Veränderungen der mit den Arbeitsaufgaben verbundenen Anforderungen gemacht werden sollen.

\section{Auswirkungen der zunehmenden Umsetzung der Methoden auf die Tätigkeitsanforderungen}

In folgendem wird der Frage nachgegangen, wie sich die an die Erwerbstätigen gestellten Anforderungen aufgrund des zunehmenden Einsatzes moderner Produktions- und Organisationskonzepte tendenziell verändern werden. Die Auswirkungen sind Folge der fortschreitenden Technisierung bzw. Computerisierung der Arbeit und der allseits betonten Notwendigkeit, das Humankapital, d.h. das Arbeits- und Leistungsvermögen der Arbeitspersonen, stärker zu nutzen, um die eingangs abgeleiteten Effizienzziele zu erreichen und die Wettbewerbsfähigkeit der Unternehmen in einer sich immer rascher verändernden Umwelt zu sichern.

Die sich stetig entwickelnden modernen Produktions- und Organisationsmethoden verändern die Arbeitsaufgaben und die mit ihnen verbundenen Anforderungen an die Beschäftigten. Aus den dargestellten Vorstellungen der CIM- und Lean-Konzepte lassen sich für verschiedene Anforderungsdimensionen Entwicklungstendenzen ableiten. Hierbei sind einige Punkte zu beachten:

- Die Konzepte bieten vielseitige Gestaltungspotentiale, so daß die jeweilige konkrete Umsetzung über spezielle resultierende Auswirkungen entscheidet. Da sich zudem die branchen-, unternehmens- sowie abteilungsspezifischen Bedingungen unterscheiden, können nur tendenzielle Anforderungsauswirkungen angegeben werden. Die Betrachtung der in verschiedenen wissenschaftlichen Studien enthaltenen Prognosen hinsichtlich der zukünftigen Anforderungen macht deutlich, wie vielfältig und z.T. konträr die Sichtweisen sind. Hier werden tendenzielle Veränderungen relevant erscheinender Anforderungsdimensionen betrachtet und Aussagen zu besonders problematisch erscheinenden Entwicklungsmöglichkeiten betont. 
- Die Entwicklung der absoluten Höhe der Anforderungen läßt sich allenfalls durch die wiederholte Analyse konkreter Tätigkeiten ermitteln. Die hier angestellten Betrachtungen, die zu Aussagen mit breiterer Gültigkeit führen sollen und eine Einschätzung der relativen Veränderungen der Anforderungen und der Anforderungshöhen durch die Umsetzung der verschiedenen Konzepte anstreben, enthalten daher unvermeidliche Pauschalierungen. Die Betrachtungen müssen, ebenso wie zukünftige einzelfallbezogene empirische Prüfungen der durch die Umsetzung der Konzepte ausgelösten Anforderungsverschiebungen, technische und arbeitsorganisatorische Aspekte berücksichtigen.

- Die Behandlung der Arbeitssysteme in Abschnitt II. C. wird aufzeigen, inwiefern die zukünftigen Methoden die Chancen der Beschäftigung von Frauen und Älteren beeinflussen und insbesondere inwiefern sie diese Chancen zusätzlich verringern, indem sie z.B. die bereits vorhandenen Wettbewerbsnachteile der betrachteten Arbeitspersonengruppen noch verschärfen. Nachfolgend werden besonders die kritischen Anforderungsentwicklungen betrachtet, um zusätzlich entstehende, potentielle Probleme hinsichtlich der Erwerbstätigkeit von Frauen und Älteren und entsprechende Ansatzpunkte für Maßnahmen der Unternehmen aufdecken zu können. Sollten sich die Anforderungen positiver entwickeln als dargestellt wird, so ist dies um so günstiger für die zukünftig erreichbare Ausschöpfung des Erwerbspersonenpotentials.

Verfahren zur Analyse konkreter Tätigkeiten (wie das Arbeitswissenschaftliche Erhebungsverfahren zur Tätigkeitsanalyse, AET, ROHMERT/LANDAU, 1979 und das Tätigkeitsbewertungssystem, TBS, HACKER u.a. 1983) ermöglichen die Einstufung vielfalltiger Merkmale, die unter anderem die Anforderungen (im AET hinsichtlich der Bereiche Informationsaufnahme, Entscheidung, Handlung und im TBS besonders hinsichtlich der Informationsverarbeitung) differenziert erfassen. Für die hier anzustellenden Überlegungen ist eine allgemeiner einzuschätzende und weitaus gröbere Einteilung der Anforderungen zu verwenden. Die Anforderungsverschiebungen sollen in vier Dimensionen betrachtet werden, die sich an die Anforderungsarten des Genfer Schemas anlehnen. Im Vergleich zum Genfer Schema und den entsprechenden REFA-Anforderungsarten (vgl. beispielsweise REFA, 1989, 42ff.) wird allerdings für die geistigen und körperlichen Anforderungen auf die Unterscheidung zwischen (maximal benötigtem) Können und (durchschnittlicher) Belastung sowie auf die Behandlung der Umgebungseinflüsse (Klima, Beleuchtung, Unfallgefährdung), die durch die Umsetzung der dargestellten Methoden allenfalls in geringem Maße betroffenen werden, verzichtet. Ergänzend 
wird eine eigene Dimension zur Betrachtung psycho-sozialer Anforderungen gebildet.

Die Dimensionen

- Geistige Anforderungen

(Fachkenntnisse, Ausbildung, Erfahrung, Denkfähigkeit, Aufmerksamkeit)

- Körperliche Anforderungen

(Geschicklichkeit, Belastung der Muskeln, Sinne und Nerven)

- Verantwortung

(Arbeitsablauf, Betriebsmittel, Produkte, Sicherheit)

- Psycho-soziale Anforderungen

(Kommunikation, Kooperation, Arbeitsdruck, Komplexität)

werden durch die Nutzung moderner Produktions- und Organisationsmethoden in unterschiedlichem Maße betroffen. Im Vordergrund der Auswirkungen stehen die qualifikatorischen und die psycho-sozialen Anforderungen.

Die zukünftige Entwicklung der Anforderungen, im besonderen der geistigen bzw. qualifikatorischen Art läßt sich aufgrund empirischer Studien nicht eindeutig bestimmen, bzw. es lassen sich für kontroverse Einschätzungen scheinbar schlüssige empirische Belege angeben (vgl. BECHTLE/LUTZ, 1989, 14ff.). Die Entwicklungseinschätzung ist abhängig von der jeweiligen Interpretationsweise und ändert sich im zeitlichen Verlauf. Dieses Problem der Interpretation von Untersuchungsbefunden betrifft alle Anforderungsdimensionen und ebenso die später angesprochenen Beanspruchungsformen und kann als die "Historizität der Ergebnisse" bezeichnet werden (BAETHGE/OBERBECK, 1986, 57f.).

So entwickelten beispielsweise KERN/SCHUMANN in ihrer Studie von 1970 eine Polarisierungshypothese, die von einer Auflösung betrieblicher und fachspezifischer Qualifikationen ausgeht und zu einem geringen Anteil hochqualifizierter und einem großen Anteil niedrigqualifizierter Arbeitspersonen führt. Zusammenhängend mit der Strategie der Automatisierung und Arbeitsteilung in den siebziger Jahren wurden entsprechend Dequalifizierungstendenzen prognostiziert (KERN/ SCHUMANN, 1970). Der These der Polarisierung der Qualifikationsstruktur gerade im Bereich der Instandhaltung widersprachen beispielsweise die Ergebnisse einer Anfang der 80er Jahre in der Automobilindustrie, der Elektrotechnischen Industrie und im Maschinenbau durchgeführten Untersuchung über die Aus- 
wirkungen computergestützter Rationalisierung. Diese Untersuchung betonte den Differenzierungsproze $B$ betrieblicher Funktionen und die Tendenz zur Verbreitung abstrakter Qualifikationsanforderungen (vgl. BENZ-OVERHAGE u.a., 1983).

Mit ihrer 1984 erschienenen Studie, die besonders die Bereiche Automobilindustrie, Werkzeugmaschinenbau und chemische Industrie betraf, stellten KERN/ SCHUMANN die Frage nach dem Ende der Arbeitsteilung. Die Aufhebung der Arbeitsteilung würde zu einem Anstieg der qualifikatorischen Anforderungen führen. So wurden die Prozesse der "Reprofessionalisierung" und "Verberuflichung", die zu einem Bedeutungszuwachs qualitativ anspruchsvoller Tätigkeiten führen würden, betont (vgl. zur Diskussion der umstrittenen Studie von KERN/ SCHUMANN beispielsweise MALSCH/SELTZ, 1987). Die Autoren erkannten ein neues nicht-tayloristisches Produktions- und Rationalisierungsparadigma, das auf die Nutzung der Kompetenzen der Mitarbeiter zielt. Sie prognostizierten die Verbreitung einer ganzheitlichen Arbeits- und Aufgabengestaltung, die zur Qualifizierung der Arbeitspersonen führt, Polarisierungsmöglichkeiten abbaut und vielfältige gesellschaftliche sowie arbeitspolitische Modernisierungsmöglichkeiten bietet (vgl. KERN/SCHUMANN, 1984). Im Gegensatz hierzu sahen andere Autoren die Möglichkeit der Beibehaltung der tayloristischen Rationalisierungsstrategie, die bei der Nutzung moderner Techniken neue Potentiale der Aufgabenund Qualifikationspolarisierung offenlegt (vgl. HIRSCH-KREINSEN u.a., 1990, 172ff.). Aufgrund einer empirischen Analyse der Veränderung der Facharbeitertätigkeit durch die Einführung $\mathrm{CNC}$-gesteuerter Maschinen widersprachen BENDER/GRABL (1992) wiederum der Dequalifikationsthese. Die Autoren behandelten Möglichkeiten zur Bewahrung der Handlungsautonomie der Arbeiter und verwiesen auf die gewonnenen Erfahrungen und Kompetenzen, die sich aufgrund ihres Praxisbezugs, zugunsten der Anpassungsfahigkeit der Produktionsabläufe, nicht vollständig in Computerprogrammen zur Steuerung der Maschinen "verobjektivieren" lassen. Somit unterlaufen Facharbeiter einerseits aufgrund ihrer Erfahrung zentral vorgegebene Regeln der Arbeitsabläufe und erhöhen andererseits hierdurch die Anpassungsfähigkeit der Produktion und vermeiden Ausfälle. Theoretisches Grundlagenwissen wird somit durch praktisches Erfahrungswissen ergänzt (vgl. BENDER/GRAßL,1992).

In einer Folgestudie zu "Das Ende der Arbeitsteilung?" wurde neuerlich versucht repräsentative Aussagen zur Rationalisierung in den Bereichen Automobilindustrie, Werkzeugmaschinenbau und chemische Industrie zu treffen (vgl. SCHUMANN u.a., 1994). Nach Ansicht der Autoren erschwerte der Umbruchprozeß aufgrund der 
ökonomischen Krise, der Wiedervereinigung und des Zusammenbruchs der staatssozialistischen Gesellschaften die Umsetzung der Anfang der 80er Jahre erkannten "neuen Produktionskonzepte". Infolgedessen fand die Überwindung tayloristischer Prinzipen bezüglich des Einsatzes der Arbeitspersonen und die Reprofessionalisierung nur in Teilbereichen statt. Eine Verbreitung qualitativ hochwertiger Tätigkeiten der sog. "Systemregulierung", die zur Sicherung der Funktionsfähigkeit von Abläufen und Prozessen notwendig sind, wie Instandhaltung, Qualitätssicherung, Programmierung und Planung, fand sich nur in besonders automatisierten Teilbereichen. In arbeitsintensiven Bereichen werden zwar Grenzen zwischen Funktionen abgebaut, aber z.B. die Kurzzyklischkeit der Tätigkeiten beibehalten. Nach Feststellung der Autoren hat demnach der Paradigmenwechsel zwar begonnen, die Modernisierung schreitet allerdings nur zögernd voran (vgl. SCHUMANN u.a., 1994, 643ff.).

Die betrachteten Produktions- und Organisationskonzepte berücksichtigen die Folgen ihrer Umsetzung auf die Arbeitspersonen in zu geringem Maße und ein konsequenter Wechsel zu einem nicht-tayloristischen Paradigma ist nicht sichergestellt. Das CIM-Konzept ist trotz der auf das Gesamtsystem gerichteten Strategie, eher tayloristisch ausgerichtet, auch wenn es seit einigen Jahren mit Blick auf die Nutzung der Qualifikationen der Mitarbeiter weiterentwickelt wird. Die Lean Production ist schon eher nicht-tayloristisch, ganzheitlich sowie mitarbeiterorientiert und versucht die Kreativität, Kompetenz und Motivation der Arbeitspersonen auszuschöpfen. Allerdings sind z.B. die Autonomie der Beschäftigten und die Flexibilität der Arbeitsdurchführung eng begrenzt und es erscheint fraglich, ob der Hierarchieabbau verbreitet gelingen wird (vgl. SCHUMANN u.a., 1994, 17ff.). So sind die Folgen der Methodenumsetzung insbesondere für die qualifizierte Produktionsarbeit nicht eindeutig. Einerseits kann durch einen erfolgreichen Paradigmenwechsel das Ende tayloristischer Strukturen und die innovative, qualifikationsorientierte Gestaltung ganzheitlicher, dezentral durchzuführender Arbeitsaufgaben erreicht und somit zur Verbesserungen der Arbeitssituation beigetragen werden (vgl. beispielsweise KERN/SCHUMANN, 1984; BRÖDNER, 1985; MICKLER, 1981). Andererseits sind auch neo-tayloristische Strukturen denkbar, die auf Hierarchie und Arbeitsteilung gerichtet sind und die zumindest zum Teil dequalifizierend wirken bzw. bei denen ein höheres Qualifikationsniveau nicht mit einer niedrigeren Leistungsintensität einhergeht, so daß die Stabilität qualifizierter Produktionsarbeit fraglich wird (vgl. beispielsweise SCHUMANN u.a., 1994; HIRSCH-KREINSEN u.a., 1990; PRIES u.a., 1989 und 1990, 81ff.; SCHULTZ-WILD u.a., 1989). 
Diese insbesondere auf qualifikatorische bzw. nicht-körperliche Anforderungen bezogenen Feststellungen und Beispiele empirischer und theoretischer Studien machen deutlich, wie schwierig es ist, gültige Prognosen für den Anforderungswandel abzugeben (vgl. hierzu beispielsweise HELLER, 1993 sowie zu weiteren Studien und einer Systematisierung der vor allem industriesoziologischen Diskussion des Wandels der Produktionskonzepte und der Folgen für die Arbeit SCHUMANN u.a., 1994, 22ff.). Trotzdem werden aufgrund der behandelten Vorstellungen der Produktions- und Organisationsmethoden nachfolgend wesentliche Verschiebungen innerhalb der vier Anforderungsdimensionen dargestellt. Dabei konzentrieren sich die Überlegungen, wie bereits betont, auf potentielle Anforderungserhöhungen als arbeitsgestalterische Risiken der Umsetzung moderner Produktions- und Organisationsmethoden, die die Möglichkeiten der Erwerbstätigkeit von Frauen und Älteren gefährden.

\section{- Geistige Anforderungen}

Die geistigen Anforderungen werden durch technische und organisatorische Änderungen beeinflußt. Die qualifikatorischen Anforderungen steigen aufgrund der angewendeten Technik und mehr noch durch "die betrieblich-organisatorische Bewältigung dieser integrierten Systeme und die damit verbundenen individuellen, gruppen- und organisationsbezogenen Maßnahmen zu Organisations- und Personalentwicklung" (BULLINGER, 1992, 5). Der Einsatz computergestützter Techniken führt bezüglich der Qualifikationsanforderungen zu den deutlichsten Veränderungen im organisatorischen und personalwirtschaftlichen Bereich. So nannten in der bereits erwähnten ISF-Umfrage (Mehrfachnennungen waren möglich) $56 \%$ der Betriebe eine Steigerung der Qualifikationsanforderungen und $55 \%$ einen erhöhten Schulungsbedarf als Auswirkung der Computernutzung, nur $2,5 \%$ nannten eine Senkung als Auswirkung. Entsprechend erkannten $41 \%$ der Betriebe höhere und nur $4 \%$ geringere Leistungsanforderungen. Die Veränderungen der Arbeitsteilung sind weit weniger eindeutig, als die Steigerung der Qualifikationsanforderungen. So erkannten 27\% eine Verstärkung und 13\% einen Rückgang der Arbeitsteilung als Auswirkung. Diese Untersuchungsergebnisse widersprechen demnach der These einer generellen Reduzierung der Arbeitsteilung (vgl. SCHULTZ-WILD u.a., 1989, 162ff.). Nach dieser Untersuchung hängt die Erhöhung der Qualifikationsanforderungen eher mit der von ca. $55 \%$ der Unternehmen genannten Entlastung von Routinetätigkeiten zusammen. Die fortschreitende Nutzung der Technik für die Automatisierung, die Rechnersteuerung, die 
Rechnerintegration und die Vernetzung sorgt für einen Abbau von Maschinenbedien- und Routinetätigkeiten und für einen relativen Zuwachs der Tätigkeiten im Bereich der Steuerung, Planung, Kontrolle und Instandhaltung sowie konkret im Aufgabenbereich der Softwareentwicklung, Softwareanpassung und Rechnerprogrammierung. Aufgrund des zunehmenden Einsatzes computergestützter Techniken steigen die geistigen Anforderungen, beispielsweise hinsichtlich der Reaktionsschnelligkeit, Abstraktionsfähigkeit, Konzentrationsfähigkeit und Genauigkeit. So steigen beispielsweise durch die CNC-Steuerung von Werkzeugmaschinen und die damit verbundene Programmierung dreidimensionaler Werkzeugbewegungen die Anforderungen an das Vorstellungsvermögen. Die Verbreitung flexibler Fertigungssysteme stellt erhöhte qualifikatorische Anforderungen an die Arbeitspersonen, da die Bedienung, Steuerung und Instandhaltung dieser komplexen Systeme mechanische, elektrische, elektronische, informationstechnische und organisatorische Kenntnisse und Fertigkeiten erfordern. Im Bürobereich steigen aufgrund der Nutzung moderner Informations- und Kommunikationstechniken, die z.B. einen Zugriff verschiedener Stellen auf Datenbanken und die Netzwerkbildung ermöglichen, die Anforderungen an die Abstraktionsfähigkeit und die Fähigkeit zur sprachlichen Formalisierung. Der Rechnereinsatz verändert die Anforderungen an die Informationsaufnahme (v.a. visuell), die Informationsverarbeitung (Gedächtnis) und die Informationsabgabe (v.a. sprachlich).

BULLINGER (1992) unterteilt das erforderliche Wissen in die einander ergänzenden Komponenten theoretisches Wissen und Erfahrungswissen. Die erforderlichen theoretischen Kenntnisse steigen durch die CIM-Umsetzung bei jeder der angesprochenen Gestaltungsalternativen neo-tayloristischer, polarisierter und qualifizierter Produktionsarbeit. Unterschiede ergeben sich allerdings hinsichtlich der weiteren Bedeutung des Erfahrungswissens in Form manuell praktischer Kenntnisse und Fertigkeiten. Das Erfahrungswissen behält im Falle qualifizierter Produktionsarbeit seine Bedeutung und kann bei entsprechender Arbeitsorganisation und Techniknutzung auch erworben und erweitert werden. Die Sicherung des Erfahrungswissens ist wichtig für eine zuverlässige Praxis und den Aufbau eines Kenntnisreservoirs, das besonders bei Störfällen oder der Umsetzung von Innovationsvorhaben sowie als Flexibilitätsfaktor dringend benötigt wird. Diesbezüglich sind dezentrale Gestaltungsoptionen, die sich auf eine Nutzung der menschlichen Qualifikation richten erfolgsversprechender als zentralistische, technikzentrierte Varianten. Denn im Falle einer neo-tayloristischen Gestaltung verliert das Erfahrungswissen an Bedeutung. Die unterschiedlichen Auswirkungen 
verschiedener Gestaltungsvarianten auf die geistigen Anforderungen werden anhand nachfolgend dargestellter Entwicklungslinien exemplarisch verdeutlicht.

Die informationstechnische Vernetzung und die Verstetigung der unternehmensübergreifenden Beziehungen zwischen Herstellern und Anwendern sorgen für betriebsspezifische Auslegungen der Technik. Angesichts dieser Entwicklung wird sich nach BREDEWEG/KOWOL (1991) die Bedeutung des Erfahrungswissens der Facharbeiter für den Erfolg technischer Innovationen erhöhen, da die Anforderungen der Praxis der ingenieurwissenschaftlichen Technisierung vorauseilen und deren Grundlage bilden. Entsprechend läßt sich das Erfahrungswissen auch nicht einmalig erfassen und vergegenständlichen, vielmehr muß für eine erfolgreiche Technikentwicklung ein dauerhafter Wissensgenerierungsproze $B$ organisiert werden. Während der Arbeit an technischen Anlagen werden deren Defizite aufgedeckt, so daß weitere Innovationen erreicht werden können. Diese Teilhabe am Optimierungsprozeß stellt zusätzliche Anforderungen an die Qualifikation der Arbeitsperson.

Die Erfüllung von Planungs- und Steuerungsfunktionen in modernen, vernetzten technischen Systemen kann durch eine Integration von Expertensystemen zusätzliche Belastungskomponenten erhalten. Die Arbeit mit Expertensystemen erhöht die Anforderungen an die Abstraktionsfähigkeit und Interpretationsfähigkeit. Durch Expertensysteme kann es zu einer Polarisierung der Facharbeiter kommen; auf der einen Seite Arbeitspersonen, die den Vorgaben der Expertensysteme 'blind' folgen, auf der anderen Seite solche, die die Möglichkeiten der Systeme nutzen und die Wissensbasis weiterentwickeln (vgl. LUTZ/MOLDASCHL, 1989, 74). In arbeitsteiligen, zentralistischen, technozentristischen Konzepten werden Expertensysteme wegen ihrer experten-ersetzenden Wirkung eingesetzt, in nichttayloristischen Systemen mit partiell autonomen, kompetenten und selbstverantwortlichen Arbeitskräften kommen sie dagegen primär aufgrund ihrer expertenunterstützenden Wirkungen zum Einsatz. Die beiden Einsatzweisen haben unterschiedliche qualifikatorische Auswirkungen, besonders bei ihrem expertenersetzenden Einsatz und einer Überschätzung ihrer Leistungsfähigkeit kann es zu einer unterforderungsbedingten Erosion der Fähigkeiten kommen (vgl. LUTZ/MOLDASCHL, 1989, 27ff.).

Obwohl die qualifikatorischen Anforderungen nicht durch die Arbeitsteilung determiniert werden (vgl. SCHULTZ-WILD u.a., 1989, 162ff.), haben Unterschiede in der Arbeitsteilung deutliche Auswirkungen auf die Arbeitspersonen, da die 
Stärkung der Arbeitsteilung und Zentralisierung oder der Abbau funktionaler und hierarchischer Grenzen die Dispositionsmöglichkeiten, d.h. die Freiheitsgrade eigener Planung bestimmen. Eine zentralistische Techniknutzung schränkt die Gestaltungsspielräume und die Tätigkeitsspektren für die im direkten Wertschöpfungsprozeß Beschäftigten ein. Fortgesetzte tayloristische Strategien, die auf die Zerlegung der Tätigkeiten und die Trennung ausführender und dispositiver Teiltätigkeiten gerichtet sind, gefährden selbsttätige Qualifizierungsleistungen der Arbeitssysteme und erschweren somit die Anpassung an und von Innovationen und die Entwicklung vielseitiger Kompetenzen (vgl. HIRSCH-KREINSEN u.a., 1990, 167ff.). Demgegenüber ermöglichen dezentrale Entscheidungskompetenzen eine zeitliche und sachliche Optimierung im Interesse der Flexibilität und Produktivität sowie im Interesse der Gestaltung ganzheitlicher und qualifizierter Tätigkeiten im direkten Wertschöpfungsprozeß. Zur Sicherung der dezentralen Verfügbarkeit von Kompetenzen werden zudem insbesondere in Unternehmen, die eine nichttayloristische ("strukturinnovative") Rationalisierungsstrategie verfolgen, systematische Qualifizierungsmaßnahmen, die sich z.T. an den qualifikatorischen Voraussetzungen der Beschäftigten orientieren und zum Abbau der Tätigkeits- und Anforderungsprobleme beitragen, durchgeführt (vgl. HIRSCH-KREINSEN u.a., 1990, 103ff.).

Aufgrund der angestrebten organisatorischen Integration der Aufgabenbearbeitung und der Ausdehnung der Einsatzbereiche zur Erhöhung der Flexibilität, müssen die Arbeitspersonen breitere Aufgabengebiete überblicken und benötigen Kenntnisse über benachbarte Arbeitsbereiche und das Gesamtsystem. Die computergestützte Vernetzung erfordert zunehmend umfassendere Kenntnisse bzgl. der EDVAnwendung und -Organisation. Die fachlich-technischen Kenntnisse müssen durch das Zusammenhangswissen ergänzt werden, so daß sich die erforderlichen Kenntnisse vom Spezialistenwissen zum breiten Fachwissen entwickeln. Die Erweiterung der Arbeitsaufgaben um dispositive und planende Tätigkeitsinhalte, wie die Ergänzung der Fertigungstätigkeiten um indirekte Aufgaben der Qualitätssicherung, der Fertigungssteuerung, der Instandhaltung und der Verbesserung, sorgen zudem für eine Anstieg der quantitativen und qualitativen geistigen Anforderungen, da zusätzliche Tätigkeiten zu erfüllen sind, die auch zusätzliche Anforderungen an Aufmerksamkeit, Konzentrationsfähigkeit, Informationsverarbeitungsgeschwindigkeit und Problemlösefähigkeit stellen. In ähnlicher Weise dürfte die verstärkte Umsetzung des Prinzips der Gruppenarbeit wirken. Falls jedes Gruppenmitglied in der Lage sein soll, alle der Gruppe übertragenen Aufgaben zu erfüllen, erhöht sich die Breite der geistigen Anforderungen. Zudem stellen die 
gruppenintern zu übernehmenden Dispositionsaufgaben zusätzliche Anforderungen an die Planungs- und Abstimmungsfähigkeit.

Von niedrig qualifizierten 'Resttätigkeiten' abgesehen sorgen die Veränderungen der Aufgaben für einen Anstieg und eine Verbreiterung der geistigen Anforderungen. Die Mitarbeiter benötigen erhöhte und übergreifende Kenntnisse über Material, Arbeitsmittel, komplexe organisatorische sowie technische Konzepte und Verfahren, so daß neben der berufsfachlichen Qualifikation die Bedeutung der (informations-) technischen und organisatorischen Qualifikationen steigt. Die Lernfähigkeit der Arbeitspersonen muß gestärkt werden, so daß Fach-, Sozial- und Methodenkompetenz erworben und erhalten werden können. Weiterhin steigen insbesondere aufgrund der Tendenz zur Dezentralisierung die Anforderungen an die Motivation, die Eigenständigkeit, die Konzentrationsfähigkeit und die Entscheidungsfähigkeit. Die Qualifikation der Arbeitspersonen kann somit zum Bestimmungsmerkmal und zum Hemmnis der Umsetzung moderner Produktionsund Organisationsmethoden werden. Neben den erhöhten Anforderungen für die Durchführung der Aufgaben werden sich zusätzliche Anforderungen während der Innovationsprozesse ergeben. Die Erhöhung der Qualifikation der Mitarbeiter ist daher eine wichtige und möglichst früh $\mathrm{zu}$ beeinflussende Bedingung der Umsetzung moderner Methoden.

\section{- Körperliche Anforderungen}

Die fortdauernde Tendenz zur Mechanisierung und Automatisierung verspricht eine weitere Verringerung der Bedeutung schwerer dynamischer Muskelarbeit. Auch die einseitige dynamische Muskelarbeit kann durch die Automatisierung und die Verbreiterung der Tätigkeitsinhalte verringert werden. Andererseits kann eine Ausdehnung von Steuerungs-, Überwachungs- und Bildschirmtätigkeiten im Produktionsbereich und im Bürobereich zu einem Bedeutungszuwachs der einseitigen dynamischen Muskelarbeit führen. Außerdem stellen diese Tätigkeiten erhöhte Anforderungen an die Sinnesorgane und belasten aufgrund der visuellen Informationsaufnahme besonders die Augen. Die statische Muskelarbeit wird aufgrund der Zunahme der Bewegungsarmut und der Ausweitung der im Stehen ausgeführten Überwachungs- und Anlageführungstätigkeiten sowie der im Sitzen ausgeführten Computertätigkeiten an Bedeutung gewinnen. Sofern körperliche Anforderungen auf dem Erfahrungswissen basieren, kann auf dessen zuvor erwähnte differenzierte voraussichtliche Entwicklung verwiesen werden. Obwohl 
das entsprechende Wissen wie auch die manuell praktischen Kenntnisse und Fertigkeiten gerade für die Entwicklung und Anpassung technischer Innovationen wichtig bleiben, verlieren sie durch zentralistische, technikorientierte Konzepte an Bedeutung, so daß die Gefahr ihres Verlustes besteht.

Quantitativ und qualitativ geht die Bedeutung körperlicher Anforderungen zurück. Muskuläre und sensumotorische Fertigkeiten im Umgang mit Arbeitsgegenständen und Arbeitsmitteln verlieren aufgrund der abnehmenden manuellen Eingriffe in den Bearbeitungsprozeß gegenüber nicht-körperlichen bzw. informatorischen Arbeiten an Bedeutung (vgl. WIENDAHL, 1991, 148ff.; BULLINGER, 1992, 102). So kann insbesondere bezogen auf die Anwendung von CNC-Technik festgestellt werden, daß die körperlichen Anforderungen deutlich sinken, während nervliche Anforderungen steigen (vgl. FRÖHLICH/HILD, 1992).

\section{- Verantwortung}

Strategien, die auf eine zentrale Steuerung aller betrieblichen Abläufe zielen und ausschließlich die technischen Möglichkeiten der Verknüpfung und Rechnerunterstützung beachten, bewirken den Verlust dezentraler Verantwortung und vernachlässigen Vorteile der Nutzung des Wissens und der Kompetenz der im Wertschöpfungsprozeß tätigen Arbeitspersonen. Die gegenläufige Tendenz ist auf den Abbau des Personals der indirekten Bereiche und eine Aufgabendezentralisierung gerichtet. So zeigen besonders die flexiblen bzw. autonomen Fertigungsinseln an, daß die Arbeitsorganisation nicht durch die Technik determiniert ist. Aufgrund der Verknüpfung vielfältiger Funktionen wächst der für die eigene Aufgabenerfüllung relevante Arbeitsbereich, zumal angestrebt wird, daß alle im Arbeitssystem arbeitenden Personen alle Aufgaben ausführen können. Dezentrale Systeme und kooperative Modelle ermöglichen die Entfaltung von Qualifikationen und Kompetenzen. Die Einbeziehung der Mitarbeiter in Entscheidungen über Systemauswahl und Systemorganisation hat den Vorteil praxisgerechterer Entscheidungen; gleichzeitig wird die Verantwortung 'nach unten' delegiert. Das Ausmaß der Anforderung durch die Übertragung der Verantwortung ist wesentlich abhängig von der Einschätzung der möglichen Schadenshöhe, der Schadenswahrscheinlichkeit, der Komplexität und Beeinflußbarkeit der Abläufe oder Anlagen sowie der Kompetenz der Arbeitsperson. Aufgrund der Vielfältigkeit der Einflußfaktoren und besonders aufgrund der Abhängigkeit von der jeweiligen 
Arbeitsperson können nur wenige allgemeine Aussagen bezüglich einer möglichen Verschiebung der durchschnittlichen Verantwortung gemacht werden.

Der Abbau der Hierarchien, die Dezentralisierung, die Verlagerung der Kompetenzen, breitere Aufgabengebiete und erweiterte Zuständigkeiten tragen dazu bei, daß mehr Arbeitspersonen Verantwortung übernehmen müssen und die Anforderungen an die Fähigkeit, Verantwortung zu übernehmen, durchschnittlich steigen. Die Integration der Aufgabenbearbeitung, die Verdichtung und Technisierung der Arbeitsabläufe und die Vernetzung technischer Systeme sorgen dafür, daß Störungen vergleichsweise weitreichende und teure Folgen haben. Entsprechend wächst die Verantwortung der Arbeitspersonen Störungen zu vermeiden, so daß z.B. die Konzentrationsfähigkeit an Bedeutung gewinnt. Die Übernahme der Verantwortung, Störungen zu beheben, stellt zunehmende und vielfältige Anforderungen, die deutlich über die Fähigkeit zur Durchführung einer bestimmten Arbeitsaufgabe hinausgehen und das Verständnis der Funktionsweise, kreative Problemlösefähigkeiten, Reaktionsvermögen und allgemein die Fähigkeit, unter Zeitdruck arbeiten zu können, voraussetzen. Die Durchführung insbesondere hochtechnologisierter Tätigkeiten kann zudem eine Isolation der Arbeitspersonen bewirken, so daß die steigende Verantwortung, beispielsweise für zu überwachende automatisch ablaufende Prozesse und die fehlende Verfügbarkeit von Ansprechpartnern und potentiellen Helfern die psychischen Anforderungen deutlich erhöhen.

\section{- Psycho-soziale Anforderungen}

Auch diese Anforderungsdimension kann hinsichtlich der möglichen Einflüsse technischer und organisatorischer Veränderungen betrachtet werden. Die Einführung zunehmend komplexerer Techniken trägt zur Erhöhung der psychischen Anforderungen bei. So ergeben sich z.B. erhöhte psychische Anforderungen aus qualifikatorischen Problemen, da die Erkenntnis mit neuen Technologien am Arbeitsplatz nicht umgehen zu können, zu Frustrationen führt. Infolgedessen kann beispielsweise die Arbeitsmotivation sinken und die Technikfeindlichkeit steigen, so daß Möglichkeiten, die Erwerbstätigkeit 'frühzeitig' zu beenden, genutzt werden, um den gestellten Anforderungen auszuweichen. Eine effiziente Techniknutzung und Technikanpassung wird hierdurch behindert. Ein weiteres Problem besteht darin, $\mathrm{da} B$ die Kontrollierbarkeit der Arbeitsdurchführung und der Arbeitsergebnisse durch die Technisierung der Arbeit im Produktions- und insbesondere im Verwaltungsbereich zunimmt. Dies betrifft die Kontrolle durch Vorgesetzte sowie 
durch Abteilungen, deren Arbeitsaufgaben mit den eigenen verschränkt sind. Aufgrund der Einführung neuer EDV-Systeme ermittelten BAETHGE/OBERBECK (1986, 254ff.) ein bei den Angestellten verbreitetes Gefühl der Kontrolle bzw. der Kontrollierbarkeit, das tendenziell zur Auflösung von Dispositionsspielräumen und zur Aufgabe persönlicher Arbeitsstile führt. Die Autoren befürchten unter anderem durch den erhöhten Konzentrationsdruck steigende psychische Belastungen.

Aufgaben der Überwachung computergesteuerter Abläufe und automatischer Prozesse sind vielfach besonders belastend, da sich die Arbeitsperson einerseits passiv zu verhalten hat und sich andererseits auf unvorhersehbare Stör- und Ausnahmefälle konzentrieren muß. Komplexe Techniken erfordern gerade bei Störfällen eigenständige Handlungs- und Entscheidungsweisen, die hohe Anforderungen an die Kommunikationsfähigkeit, die Motivation und die Verantwortungsfähigkeit stellen (vgl. beispielsweise HIRSCH-KREINSEN u.a., 1990, 96 und LUTZ/MOLDASCHL, 1989). In diesen Sondersituationen müssen verantwortungsvoll Kompetenzen genutzt werden, die im Normalbetrieb durch die erhöhte Automatisierung und Rechnerstützung immer weniger geübt werden können. Die Technisierung und Integration der Abläufe führt zur Beschleunigung von Materialbzw. Informationsflüssen. Für die Arbeitspersonen entstehen z.B. durch Leistungsverdichtung und genauere Vorgaben für die Maschinenbedienung und Arbeitsweise Einschränkung der sachlichen und räumlichen Autonomie. Insgesamt werden hierdurch Streß und Zeitdruck zunehmen. Zudem können besonders in hochtechnisierten Anlagen Ohnmachtsgefühle und Gefühle der Abhängigkeit von Maschinen oder Experten sowie der Isolation entstehen.

Hinsichtlich des organisatorischen Wandels sind insbesondere die wachsenden psycho-sozialen Anforderungen hervorzuheben. So stellt die angestrebte Dezentralisierung von Gestaltungsspielräumen und Entscheidungsmöglichkeiten neben den Anforderungen an kognitive Fähigkeiten hohe Anforderungen an soziale Fähigkeiten, wie Team-, Kommunikationsfähigkeit. Ein zumindest partieller Abbau der Arbeitsteilung erhöht die Anforderungen an die Koordinationsfähigkeit, die Fähigkeit zur organisatorischen Abstimmung und die soziale Kompetenz (vgl. HIRSCH-KREINSEN u.a., 1990, 102 und $181 \mathrm{ff}$.). Die verstärkte Umsetzung der Gruppenarbeit stellt stärkere Anforderungen an die Fähigkeit zur Problem- und Konfliktlösung, zumal häufiger Konflikte mit Kollegen aufgrund gruppeninterner Disposition auftreten dürften (vgl. MINSSEN u.a., 1991). Gegenüber Einzelarbeitsplätzen steigen durch die Gruppenarbeit, durch die Integration der Arbeitsabläufe und auch durch die Umsetzung schwer planbarer, komplexer 
technisch-organisatorischer Änderungen die Anforderungen an die Kooperationsund Kommunikationsfähigkeit sowie die Flexibilität.

Facharbeiterorientierte Arbeitssysteme, die auf einen Abbau der Arbeitsteilung gerichtet sind, wie beispielsweise eine qualifizierte Gruppenarbeit, erfordern Kooperations- und Kommunikationsleistungen und sichern hierduch Qualifikationsmöglichkeiten, so daß es zu einem weiteren Abbau der Arbeitsteilung kommen kann. Während andererseits tayloristische auf Arbeitsteilung gerichtete Strategien den Kooperationsbedarf mindern, indem ausführende und dispositive Tätigkeiten getrennt und die Überlappung von Anforderungs- und Qualifikationsprofilen abgebaut werden (vgl. HIRSCH-KREINSEN u.a., 1990, 178ff.). Hierdurch schwinden Möglichkeiten der qualifikatorischen Anpassung und der Ausweitung der Kompetenzen, so daß sich die Arbeitsteilung in einem sich verstärkenden Kreislauf weiter erhöhen wird.

Die modernen Methoden sind auf eine verstärkte Nutzung der Arbeits- und Leistungspotentiale der Arbeitspersonen gerichtet. Entsprechend tragen eine hohe Arbeitsmotivation, ein hohes Engagement und die besonders starke Identifikation mit den betrieblichen Interessen wesentlich zur Steigerung der Produktivität bei. Wird das Gefühl dem Unternehmen in hohem Maße verpflichtet zu sein dominierend, so können hieraus Prozesse der (Selbst-) Überforderung, die einen nicht zu erfüllenden Leistungsanspruch der Arbeitspersonen an sich selbst darstellen, resultieren. Diese psychischen Anforderungen werden zudem durch die soziale Integration und die gegenseitige Verpflichtung der Arbeitspersonen sowie die Aufforderung dazu, Beiträge zur permanenten Verbesserung der Produktionsprozesse und der Produkte zu erbringen, verstärkt. Die Teilnahme am kontinuierlichen Verbesserungsproze $\beta$ durch die Entwicklung von Verbesserungsvorschlägen und ihre rasche Umsetzung unterstützen und dokumentieren die Bereitschaft der Beschäftigten, sich mit den Zielen des Unternehmens zu identifizieren. Ergänzend kann noch auf die erhöhten psychischen Anforderungen aufgrund verringerter Karrierechancen und veränderter beruflicher Positionen durch den Abbau von Hierarchien und indirekter Tätigkeitsbereiche, verschärfter Leistungskontrollen, erhöhter Flexibilitätsanforderungen sowie der Personalverknappung verwiesen werden. Zusammengefaßt steigen mit der zunehmenden Komplexität der Arbeitsaufgaben und der technischen Systeme die psycho-sozialen Anforderungen. Der Zeitdruck, die Bedeutung der internen und externen Kontakte und das erforderliche gegenseitige Verständnis nehmen zu. Die Arbeitsmotivation, die Konzentrationsleistungen, die Eigenverantwortung, das Verantwortungsbewußtsein, das Teamverhalten, die 
Kooperationsfähigkeit und die sozialen Qualifikation gewinnen quantitativ und qualitativ an Bedeutung.

\section{Zwischenfazit}

Der mit der Umsetzung moderner Methoden angestrebte Kostenabbau, beispielsweise durch die Verkürzung der Durchlaufzeiten und den Abbau von Puffern und Reserven und die ebenso angestrebte stärkere Nutzung des Leistungspotentials der Arbeitspersonen kann zur Leistungsverdichtung, zur Erhöhung der Arbeitsintensität und damit zur Erhöhung der Belastung führen. Flexible Produktions-, Organisations- und Arbeitsweisen erfordern neben flexiblen Fertigungsanlagen und Bürobzw. Verwaltungstechniken besonders auch flexible Arbeitspersonen mit technischem und organisatorischem Wissen, mit sozialen Kompetenzen, mit Kreativität und hoher Arbeitsmotivation. Der angestrebte flexiblere Arbeitseinsatz kann die Belastung zusätzlich erhöhen. Aufgrund der immer rascheren Änderungen der Umgebungsbedingungen und der häufigeren Innovationen wird die Zunahme der Höhe der Anforderungen von einer Erhöhung ihrer Dynamik begleitet.

In Abbildung 50 sind die wichtigsten anforderungsbezogenen Entwicklungstendenzen zusammengestellt. Sie können als arbeitsgestalterische Chancen und Risiken für die Beschäftigung von Personengruppen mit arbeitsmarktbezogenen Wettbewerbsnachteilen aufgefaßt werden.

Vielfältige technische und organisatorische Umsetzungsmöglichkeiten moderner Produktions- und Organisationsmethoden sorgen dafür, daß die zukünftige Realität nicht einheitlich und verbindlich determiniert ist und sich insofern auch die zukünftigen durchschnittlichen Anforderungen nicht eindeutig voraussehen lassen. Allerdings konnten ausgehend von den Leitvorstellungen voraussichtliche Anforderungsverschiebungen infolge der Umsetzung moderner Methoden aufgezeigt werden. Dabei wurden potentiell negativ erscheinende, zumeist anforderungserhöhende Effekte betont. Das bedeutet nicht, daß diese problematischen Entwicklungstendenzen unbeeinflußbar ablaufen und sich damit die Chancen für die Beschäftigung von Frauen und Älteren 'automatisch' verschlechtern werden. Aber gerade zur Verbesserung der zukünftigen Möglichkeiten der Frauen und Älteren erwerbstätig zu sein, müssen die Unternehmen verschiedene Bedingungen beachten und Maßnahmen ergreifen, die nachfolgend abgeleitet und beurteilt werden. 


\begin{tabular}{|c|c|}
\hline \multicolumn{2}{|c|}{$\begin{array}{l}\text { Wesentliche durchschnittliche anforderungsbezogene } \\
\text { Entwicklungstendenzen }\end{array}$} \\
\hline $\begin{array}{l}\text { Arbeitsgestalterische } \\
\text { Risiken }\end{array}$ & $\begin{array}{l}\text { - technische und organisatorische Änderungen nehmen } \\
\text { an Häufigkeit zu, der innovative Wandel wird zu einem } \\
\text { ständigen ProzeB } \\
\text { - Zunahme der Bedeutung technisch-, besonders } \\
\text { computervermittelter Arbeit } \\
\text { - zunehmende Distanz zur direkten Einwirkung } \\
\text { - differenzierte Entwicklung der Arbeitsteilung } \\
\text { - erhohte Anforderungen an Fach-, Sozial- und } \\
\text { Methodenkompetenz } \\
\text { - Verschiebung zu formal hoheren Ausbildungen } \\
\text { - zunehmende Anforderungen an das theoretische Wissen } \\
\text { und die organisatorischen und sozialen Kompetenzen } \\
\text { - zunehmende Anforderungen an die Abstraktionsfahigkeit } \\
\text { - } \text { differenzierte Entwicklung der Bedeutung des } \\
\text { Erfahrungswissens } \\
\text { - steigende Verantwortung } \\
\text { Erhøhung der psycho-sozialen Anforderungen, insbesondere } \\
\text { an die Kommunikations- und Kooperationsfahigkeit }\end{array}$ \\
\hline $\begin{array}{l}\text { Arbeitsgestalterische } \\
\text { Chancen }\end{array}$ & $\begin{array}{l}\text { die arbeitsorganisatorische Gestaltung wird, insbesondere } \\
\text { in automatisierten Bereichen, unabhăngiger von der } \\
\text { Techniknutzung } \\
\text { technische Entwicklungen erhøhen die } \\
\text { Anpassungsmoglichkeiten der Betriebs- und } \\
\text { Arbeitsorganisation } \\
\text { weiterer Abbau der Bedeutung schwerer körperlicher } \\
\text { Tătigkeiten u.a. durch die technisierungs- und } \\
\text { automatisierungsbedingte Abnahme direkter } \\
\text { Fertigungstătigkeiten }\end{array}$ \\
\hline
\end{tabular}

Abb. 50: Anforderungen als Einflubfaktor der Arbeitsgestaltung 


\section{Arbeitssysteme und Beanspruchungen}

Nachdem zuvor die Eigenschaften der Arbeitspersonen und die sich ändernden Anforderungen der Arbeitsaufgaben betrachtet wurden, behandelt dieser Abschnitt die Arbeitssysteme und die aus der Erwerbstätigkeit resultierenden Beanspruchungen. Hierzu werden die Anforderungsänderungen und die behandelten arbeitsmarktbezogenen Eigenschaften von Frauen und Älteren zusammengeführt und resultierende kritische Beanspruchungskomponenten hervorgehoben.

\section{Kennzeichen zukünftiger Arbeitssysteme}

Die Anforderungen der Arbeitsaufgaben und die Auswirkungen der Arbeitsumgebung führen, beeinflußt durch die individuellen Eigenschaften, Bedürfnisse sowie Handlungs- und Verarbeitungsweisen, zur Beanspruchung menschlicher Funktionen (vgl. ROHMERT, 1984). Die Beanspruchungen lassen sich theoretisch in physische und psychische Formen untergliedern. Beanspruchungen selbst lassen sich nicht messen, allerdings kann anhand psycho-physiologischer Größen auf sie geschlossen werden (vgl. BOKRANZ/LANDAU, 1991, 200ff.). Angemessene Beanspruchungen haben positive Folgen, die beispielsweise Lerneffekte, die Persönlichkeitsentwicklung, die Motivationszunahme, die Arbeitszufriedenheit und die allgemeine Zufriedenheit betreffen. Fehlbeanspruchungen in Form von Überforderungen oder Unterforderungen haben negative Folgen, wie übermäßigen Streß, physische bzw. psychische Schädigungen, Krankheit, Monotonie, Sättigung, psychische Ermüdung, Erschöpfung, Arbeitsunzufriedenheit und Angst. Außerdem beeinträchtigen Fehlbeanspruchungen die Produktivität der Arbeitspersonen und mindern die Akzeptanz der Beschäftigten für Entscheidungen der Unternehmensleitung. Die in den Arbeitssystemen erfolgende Nutzung persönlicher Eigenschaften zur Bewältigung der Arbeitsanforderungen kann geschlechts- bzw. altersspezifisch betrachtet werden. Darüber hinaus bestehen individuelle Unterschiede beispielsweise bezüglich der außerberuflichen Situation, der Motive, Ziele, Wahrnehmungsarten und Einstellungen.

Die Arbeitsaufgaben stellen zukünftig höhere Anforderungen an die Innovationsbereitschaft und -fähigkeit, die Flexibilität, die Qualifikation und die Motivation. Andererseits werden die Anteile solcher Arbeitspersonen an der Erwerbsbevölkerung steigen, die überdurchschnittliche außerberufliche Anforderungen zu bewältigen haben, die ihre Erwerbstätigkeit unterbrochen haben oder deren Berufsausbildung längere Zeit zurückliegt. Die Abstimmungsprobleme zwischen den 
Eigenschaften der Arbeitspersonen und den Anforderungen der Arbeitsaufgaben werden entsprechend weiter zunehmen. Im Interesse der Ausschöpfung des Erwerbspersonenpotentials muß die Arbeit so gestaltet werden, daß die Erwerbstätigkeit dauerhaft schädigungslos durchführbar ist und die Motivation zur Aufnahme bzw. Aufrechterhaltung einer Erwerbstätigkeit geweckt und gesichert wird. Die zukünftigen Probleme bei der Besetzung vorhandener Arbeitsplätze und bei der Übertragung von Arbeitsaufgaben auf Frauen und Ältere müssen durch arbeitssystembezogene Maßnahmen vermieden oder vermindert werden.

Bei der Gestaltung der Arbeitssysteme kommt es zur Funktionsteilung zwischen Arbeitspersonen und Maschinen, so daß tendenziell die einfacheren und routinisierbaren Tätigkeitsbestandteile den Maschinen und die komplexeren Aufgaben den Menschen zugeordnet werden. Die Bedingungen des Einsatzes der Personen am Arbeitsplatz hängen von der genutzten Technik sowie von organisatorischen und institutionellen Bedingungen ab. Der Prozeß der Gestaltung der Arbeitssysteme wird traditionell häufig in Stufenmodellen dargestellt (vgl. besonders das 6-StufenModell, REFA, 1987, 1991a,b,c). Die einzelnen Stufen werden als klar voneinander getrennte und nacheinander zu durchlaufende Phasen aufgefaßt, an deren Ende ein neu gestaltetes Arbeitssystem eingesetzt ist. Die modernen Produktions- und Organisationsmethoden beziehen sich allerdings in komplexer Weise auf das gesamte Unternehmen, so daß vielfältige und zeitversetzte Wechselwirkungen auftreten, die die Gestaltungsaufgabe zu einem langfristig orientierten, iterativen Prozeß machen (vgl. KÖTTER/VOLPERT, 1993). So müssen auch im Interesse einer Verbesserung der Ausschöpfung des Erwerbspersonenpotentials Bündel von Maßnahmen zur Beeinflussung der Arbeitssysteme umgesetzt werden. Die einzelnen Maßnahmen sind dabei unterschiedlich komplex und hängen hinsichtlich der zu erreichenden Wirkungen voneinander ab.

Die im Interesse der Wettbewerbsfähigkeit mit den modernen Produktions- und Organisationsmethoden angestrebte Erhöhung der Ausnutzung des Humankapitals kann für leistungsstarke, flexible und mobile Arbeitspersonen zu einer Verbesserung der Betätigungschancen führen. Andererseits werden bereits heute benachteiligte bzw. im geringeren Maße erwerbstätige Personengruppen, wie Frauen und Ältere, tendenziell zusätzlich benachteiligt. So führt beispielsweise die Umsetzung des Konzepts der Lean Production nicht mit der in der MIT-Studie suggerierten Sicherheit zur Entfaltung von Humanisierungswirkungen. Solche Wirkungen sind von der konkreten betriebsspezifischen Ausgestaltung der Arbeitsorganisation und von den individuellen Präferenzen der Arbeitspersonen abhängig (vgl. WENKER, 
1994). Zur Minderung der beanspruchungssteigernden Effekte der modernen Methoden müssen die Strukturen und Elemente der Konzepte an die Bedürfnisse der Arbeitspersonen angepaßt werden.

Der langfristige Erfolg der Einführung moderner Methoden erfordert neben einer angemessenen Einführungsstrategie insbesondere Maßnahmen der Arbeitsgestaltung und der Personalentwicklung zur gegenseitigen Anpassung der Anforderungen der Arbeitsaufgaben und der Eigenschaften der Arbeitspersonen. Durch entsprechende Handlungsweisen in den Unternehmen werden langfristig die Möglichkeit das vorhandene Arbeitskräftepotential auszunutzen, verbessert. Einerseits werden die Arbeitsanforderungen und damit die Nachfrage am Arbeitsmarkt und andererseits die Eigenschaften und damit das zur Verfügung stehende Arbeitskräfteangebot beeinflußt. Wirksame Maßnahmen bieten sich insbesondere aufgrund der bereits angesprochenen Zunahme der Gestaltungsmöglichkeiten, durch die fortschreitende Automatisierung, die Verbreitung der Informationstechnik und die Lockerung der sachlichen und zeitlichen Bindung menschlicher Arbeit an die unmittelbare Produktion. Die zu entwerfenden Strukturen der Arbeitswelt und die Veränderungen des Profils der Tätigkeitsanforderungen an bestehenden und neuen Arbeitsplätzen werden nicht mehr als technologisch determiniert angesehen (vgl. EULER, 1987, 5 und zu dem entsprechenden Paradigmenwechsel der Technikforschung LUTZ, 1990). Eher als neue Technologien und umgesetzte Techniken bestimmt die Arbeitsgestaltung die Struktur der Arbeitsaufgaben und der mit ihnen verbundenen Arbeitsanforderungen. Sofern die Probleme den betrieblichen Akteuren bewußt sind, besteht ein betriebspolitischer Handlungsspielraum zur Erreichung der erforderlichen Anpassung.

\section{Beanspruchungsentwicklung und Ableitung von Maßnahmen}

Arbeitsmarktbezogenes Ziel der Arbeitgeber ist es, aus dem gegebenen Erwerbspersonenpotential Personen zur Verrichtung der Arbeitsaufgaben zu beschäftigen, die den Arbeitsanforderungen gerecht werden. Hierzu müssen entsprechend qualifizierte Personen gefunden, Personen geeignet aus- bzw. weitergebildet und eingesetzt werden. In zunehmendem $\mathrm{Maße}$ muß den quantitativen und qualitativen Defiziten des Arbeitskräfteangebots begegnet werden, so daß die Förderung und Sicherung des zur Verfügung stehenden Humankapitals zum wichtigen unternehmenspolitischen Ziel wird. 
Unternehmensbezogene Maßnahmen zur Erhöhung der Erwerbsbeteiligung der verschiedenen Personengruppen müssen sich auf

- die Vermeidung von Fehlbeanspruchungen des Menschen durch die Arbeit,

- die Verbesserung der Eignung beschäftigter oder potentieller Arbeitspersonen und

- die Verbesserung der Möglichkeiten und die Erhöhung der Bereitschaft, erwerbstätig zu sein,

beziehen. Entsprechende Maßnahmen für Frauen und Ältere werden im folgenden abgeleitet.

\section{a) Maßnahmen für Frauen}

Die Betrachtung der erwerbstätigkeitsrelevanten Eigenschaften verdeutlichte, daß neben der Mehrfachbelastung und der damit zusammenhängenden geringeren zeitlichen Flexibilität die Unterbrechungen der beruflichen Tätigkeit und die daraus resultierenden Dequalifizierungsrisiken die wichtigsten arbeitsmarktbezogenen Wettbewerbsnachteile darstellen. Im Interesse der Erhöhung der Erwerbsbeteiligung der Frauen müssen daher Maßnahmen zur Verbesserung der Vereinbarkeit von Familie und Beruf und zur Verbesserung der Wiedereinstiegschancen ergriffen werden. Die Maßnahmen sollten darüber hinaus generell zur Verbesserung der beruflichen Chancen der Frauen beitragen.

Die notwendigen Maßnahmen richten sich auf eine Anpassung der Eigenschaften der Frauen an die Arbeit und auf eine Anpassung der Arbeit an die Bedürfnisse der Frauen und damit letztlich auf eine familiengerechte Gestaltung der Arbeit. Auch Maßnahmen für Männer können diesbezüglich zieladäquat sein, insofern sich hierdurch das quantitative und qualitative Arbeitskräfteangebot insgesamt erhöhen läßt. Unternehmen können, sofern sie daran einzelwirtschaftlich ein rechtzeitig wirksam werdendes Interesse entwickeln, durch die Gestaltung der Erwerbsarbeit die Rahmenbedingungen der Haus- und Familienarbeit beeinflussen. Unternehmensbezogene Maßnahmen (allein) können zwar nicht die gesellschaftlich vermittelte, traditionelle Arbeitsteilung ändern, aber sie können den beschäftigten Frauen helfen, Beruf sowie Haus- und Familienarbeit zu vereinbaren oder zumindest dazu beitragen, die Folgeprobleme einer Unterbrechung zu mindern. Geschlechtsunspezifische bzw. frauenfördernde betriebliche Arrangements können weiterhin dazu führen, daß Frauen mit geeigneten und zukünftig knapp werdenden Qualifikationen 
auf allen Niveaus der Unternehmen tätig werden und die Erwerbsquote der Frauen steigt. Die Maßnahmen müssen sich zudem an den technisch-organisatorischen Innovationen und den entsprechenden Veränderungen der Anforderungen orientieren.

Zur Verbesserung der Vereinbarkeit von Familie und Beruf und zur Verbesserung der Wiedereinstiegschancen können insbesondere arbeitszeitliche Maßnahmen beitragen, die eine Anpassung der individuellen Arbeitszeit an die jeweilige momentane Lebenssituation ermöglichen. Diesbezüglich soll die Teilzeitarbeit angesprochen werden. So suchen Frauen häufig eine vormittägliche Teilzeitarbeit, da ihre Kinder während dieser Zeit betreut sind, andererseits kann auch eine Beschäftigung in Abendschichten vorteilhaft sein. Teilzeitarbeit kann die Kontinuität der Erwerbstätigkeit der Frauen erhöhen und die Wiederaufnahme einer Erwerbstätigkeit erleichtern. Das Angebot an Teilzeittätigkeiten muß quantitativ und qualitativ erhöht werden. Teilzeitarbeit verkürzt die Unterbrechungsphase und wird nach ihr zum "Normalfall" (vgl. LAUTERBACH u.a. 1994). So ist mittlerweile die Hälfte aller Frauen, die ihre Erwerbstätigkeit unterbrochen haben nach ihrem Wiedereinstieg unterhalb der wöchentlichen Normalarbeitszeit beschäftigt (vgl. ENGELBRECH, 1991b). Teilzeitarbeit erhöht die Möglichkeiten der beruflichen Reintegration (vgl. SCHUPP, 1991) und ist nicht nur für den Wiedereinstieg, sondern als langfristige Beschäftigungsform zur Sicherung der individuellen Entscheidungs- und Handlungsmöglichkeiten sowie einer eigenständigen Altersversorgung hilfreich. Wirkungsvoller für die Erhöhung der weiblichen Erwerbsbeteiligung als die Teilzeitarbeit wäre allerdings die Vollzeitbeschäftigung statt oder nach der familienbedingten Unterbrechungsphase. Eine Maßnahme zur Flexibilisierung der Arbeitsanforderungen ist das Job-sharing, das Möglichkeiten zur Selbstorganisation bietet. Die Vereinbarkeit von Familie und Beruf wird erhöht, wenn beispielsweise im Falle der Krankheit von Kindern, die beruflichen Anforderungen vorübergehend vermindert werden können. Eine weitere Maßnahme zur Verbesserung der Vereinbarkeit von Erwerbstätigkeit und Familie betrifft die Flexibilisierung der Aufgabenbearbeitung durch die Möglichkeit der Heimarbeit. Zur Verbesserung der Wiedereinstiegschancen richten sich Maßnahmen zur Kontaktpflege auf den Erhalt beruflicher Qualifikationen, Einstellungen und unternehmensspezifischen Wissens. So können während der Unterbrechungsphase Urlaubsvertretungen oder Heimarbeiten übernommen und an Weiterbildungmaßnahmen teilgenommen werden. Zur Verbesserung der Wiedereinstiegschancen und zur Verbesserung der beruflichen Chancen der Frauen sind, gerade angesichts der raschen Entwicklung der Informations- und Kommunikationstechniken, Maßnahmen 
der betrieblichen Weiterbildung notwendig. Die Anwendung neuer Technologien betrifft die Arbeitsplätze von Frauen im gewerblichen Bereich, da sie dort häufig Tätigkeiten ausüben, die durch neue Technologien automatisiert werden können und im Bürobereich, da sie dort durch neue Technologien einer zunehmenden Qualifikationskonkurrenz ausgesetzt werden (vgl. BOLLE/SCHNEIDER, 1988, 131ff.). Während einer Unterbrechungsphase entwickeln sich die Anforderungen weiter, so daß Dequalifizierungsprozesse zu Schwierigkeiten beim Wiedereinstieg führen. Im Falle nicht ausreichender bzw. nicht rechtzeitiger Qualifizierung wird den Arbeitspersonen die Anpassungslast aufgebürdet. So können gerade bei der Einführung neuer Technologien und bei der Umsetzung moderner Konzepte, die auf die Nutzung der Leistungsbeiträge der Beschäftigten gerichtet sind, nur systematische Qualifizierungsmaßnahmen Überforderungen der Arbeitspersonen und Probleme im Produktionsablauf verhindern (vgl. BULLINGER, 1992, 65f.). Aus Qualifikationsdefiziten können Überforderung, Streß, gesundheitliche Gefährdungen, Motivations- und Produktivitätsverluste, Stör- und Ausfallrisiken entstehen. Vorhandene Qualifikationen ermöglichen den Arbeitspersonen dagegen die Bewältigung von Innovationen, mindern Akzeptanzprobleme und erhöhen die Laufzeiten bzw. die Auslastung der Maschinen.

Neben den Anstrengungen zur Erleichterung der Vereinbarkeit von Beruf und Familie, die auf eine Verringerung der Mehrfachbelastung von Frauen gerichtet sind, sollten Maßnahmen genutzt werden, die auch zu einer Veränderung der geschlechtsspezifischen Arbeitsteilung beitragen. In den Unternehmen können hierzu Unterbrechungsmöglichkeiten mit Rückkehrzusagen sowie sozialversicherungspflichtige Teilzeittätigkeiten für Männer und Frauen angeboten werden. Zur Verbesserung der beruflichen Chancen der Frauen werden Maßnahmen der Frauenförderung weiter an Bedeutung gewinnen, zumal sich die Forderungen nach Gleichberechtigung der Frauen in Wirtschaft und Verwaltung auch wegen ihres durchschnittlich gestiegenen Bildungsabschlusses verstärken werden. Private Unternehmen können sich der Erfüllung dieser Forderung immer weniger entziehen, sofern sie als Arbeitgeber für höher qualifizierte Frauen attraktiv bleiben wollen, zumal die entsprechenden Bedingungen im öffentlichen Dienst frauenfreundlicher sind. Eine individuelle Förderung von Frauen kann beispielsweise dazu beitragen, daß mehr Frauen Führungspositionen einnehmen. Erfahrungen hinsichtlich der Unterstützung der langfristigen Erwerbstätigkeit im öffentlichen Dienst durch Maßnahmen der Frauenförderung, wie Freistellungsregelungen mit Rückkehrgarantien und günstige, d.h. flexible Arbeitszeitregelungen, zeigen, daß entsprechende Maßnahmen erwerbsquotenerhöhend wirken können (vgl. beispielsweise 
LAUTERBACH u.a. 1994). Maßnahmen zur Frauenförderung in dem hier verwendeten Sinn beziehen sich inhaltlich demnach auch auf die übrigen genannten Maßnahmen, insbesondere der Weiterbildung und der Teilzeitbeschäftigung.

Die zunehmende Bedeutung sozialer Kompetenzen kann als positiver Faktor für die Beschäftigungschancen der Frauen gewertet werden, da Frauen häufig besondere soziale Kompetenzen zugeschrieben werden bzw. sie diese während der Bewältigung von Erziehungs- und Pflegetätigkeiten tatsächlich erworben haben. Aufgrund der sinkenden Bedeutung schwerer körperlicher Anforderungen werden hier keine Maßnahmen wegen der durchschnittlich geringeren körperlichen Leistungsfähigkeit von Frauen behandelt. Da der Anteil der Jugendlichen an der Bevölkerung abnimmt, wird die berufliche Ausbildung hier nicht ausführlich behandelt. Allerdings sollte im Bereich der Ausbildung die geschlechtsspezifische Segregation abgebaut werden, indem beispielsweise die Betriebe männliche und weibliche Jugendliche gemeinsam ausbilden. Nach einer vom IAB 1991 durchgeführten Betriebsbefragung ist dies nur in jedem zweiten Betrieb des kaufmännisch/verwaltenden Bereichs und jedem vierten des gewerblich/technischen Bereichs der Fall (vgl. ENGELBRECH/KRAFT, $1992,17)$.

Die abgeleiteten Maßnahmen können den genannten Ausrichtungen zugeordnet werden:

- Zur Verbesserung der Vereinbarkeit von Familie und Beruf

- Teilzeitarbeit

- Job-sharing

- Heimarbeit

- Zur Verbesserung der Wiedereinstiegschancen

- Teilzeitarbeit

- Kontaktpflege

- Weiterbildung

- Zur Verbesserung der beruflichen Chancen der Frauen

- Weiterbildung

- Unterbrechungsmöglichkeiten

Diese Maßnahmen zur Erhöhung der Erwerbsquoten von Frauen können so zusammengefaßt werden, daß Job-sharing im Sinne einer 'Partner-Teilzeitarbeit' in 
Verbindung mit Teilzeitarbeit als Arbeitszeitkonzepte behandelt wird; außerdem können Kontaktpflege und Unterbrechungsmöglichkeiten gemeinsam unter dem Stichwort der Frauenförderung betrachtet werden.

\section{b) Maßnahmen für Ältere}

Die mit der Umsetzung der behandelten Produktions- und Organisationsmethoden angestrebten Einsparungen führen dazu, daß die vorhandenen Leistungsfähigkeiten der Arbeitspersonen zur Sicherung der Flexibilität und zum Ausgleich abgebauter Reserven verstärkt ausgenutzt werden. Dies kann zur Leistungsverdichtung und damit zu einer erhöhten Beanspruchung der beschäftigten Personen führen. Weiterhin wächst mit der Ausrichtung auf leistungsstarke und hoch motivierte Mitarbeiter die Gefahr der Überforderung gerade älterer Arbeitspersonen.

Positive Eigenschaften und berufsbezogene Vorteile älterer Erwerbspersonen sind dagegen insbesondere die Berufserfahrung und die Erfahrung in der Bewältigung von Arbeitsanforderungen. Außerdem konnten ältere Arbeitspersonen zum Teil eine hohe Sozialkompetenz erwerben und besitzen durchschnittlich dem Unternehmen gegenüber eine hohe Betriebstreue. Die betriebsspezifische Qualifikation, das Erfahrungs- und Expertenwissen kann allerdings besonders durch den verstärkten Einsatz von Computern und die damit verbundene Aufgabe individueller Arbeitsweisen entwertet werden. Auch die Eigenschaft der Loyalität kann aufgrund der technisch verbesserten Transparenz und Kontrollmöglichkeiten an Relevanz verlieren (vgl. BAETHGE/OBERBECK, 1986, 41).

Berufsbezogene Nachteile Älterer bestehen in der Verschlechterung der Leistungsfähigkeit und der Abnahme der Flexibilität. Die beruflichen Qualifikationen werden formal vergleichsweise geringer sein als die der Jüngeren. Die erhöhte Beschäftigung Älterer erfordert daher Veränderungen der generierten Arbeitssysteme im Sinne einer Anpassung der Arbeitsaufgaben und -bedingungen an die Eigenschaften und Bedürfnisse älterer Personen sowie die Anpassung der Eigenschaften an die gestellten Anforderungen. Durch während des gesamten Erwerbslebens und damit altersübergreifend wirksam werdende Maßnahmen müssen Fehlbeanspruchungen vermieden werden, die andernfalls zur Ursache arbeitsmarktbezogener Wettbewerbsnachteile Älterer werden können. Arbeitsbedingte Erkrankungen, die auch zur Erwerbsunfähigkeit und zur Inanspruchnahme des Vorruhestandes führen können, müssen vermindert werden. Auftretende physische oder psychische arbeitsrelevante 
Schwierigkeiten müssen kompensiert und Qualifikationen erweitert werden. Insgesamt müssen die Möglichkeiten und die Motivation, über das mittlerweile übliche durchschnittliche Renteneintrittsalter hinaus erwerbstätig zu sein, verbessert werden. Die im folgenden exemplarisch dargestellten Kombinationen von Eigenschaften und Anforderungen führen zu Maßnahmen, die auf die Minderung von Fehlbeanspruchungen sowie die Erhöhung der Eignung und der Bereitschaft zur verlängerten Ausübung einer Erwerbstätigkeit gerichtet sind.

Die Aktualitätsverluste der Ausbildung Älterer, fehlende oder falsche Qualifikationen, stellen Gründe für berufliche Benachteiligungen dar. Gerade aufgrund der vielfältig wachsenden geistigen Anforderungen und der zunehmenden Bedeutung fachunabhängiger Qualifikationen wie Planungs-, Entscheidungs- und Methodenkompetenzen können Qualifikationsunterschiede zur Polarisierung der Beschäftigten führen. Zur Vermeidung altersspezifischer Segregationseffekte und zur Verbesserung der Motivation der Arbeitspersonen müssen Maßnahmen zur Anpassung der Qualifikationen ergriffen werden. Durch eine berufsbegleitende Fortbildung kann ein lebenslanges Lernen gefördert werden. Die regelmäßigen Maßnahmen der Weiterbildung zur Erhaltung und Anpassung der Qualifikation können durch zusätzliche Ausbildungsphasen ergänzt werden.

Das Nachlassen der körperlichen Leistungsfähigkeit und der Fähigkeit, sich gleichzeitig auf verschiedene Dinge zu konzentrieren, die abnehmende Geschwindigkeit und das Nachlassen des Reaktionsvermögens, der Abbau psychischer Belastbarkeit und die raschere Ermüdung legen verschiedene Anpassungsformen nahe. Eine Verringerung der zeitlichen Inanspruchnahme kann durch erweiterte Vereinbarungen von Teilzeitbeschäftigungen erreicht werden. Teilzeitarbeit kann dazu beitragen, qualifizierte Arbeitskräfte länger, d.h. über den Zeitpunkt des sonst erfolgenden Renteneintritts hinaus, im Unternehmen $\mathrm{zu}$ halten, indem die Beanspruchungssituation gemindert und die Motivation zur Erwerbstätigkeit verbessert wird. Teilzeitlösungen können ein gleitendes Ausscheiden im Sinne eines stufenweisen Ausstiegs aus dem Erwerbsleben ermöglichen und damit die Altersgrenzen flexibilisieren. Aufgrund der wachsenden psychischen Anforderungen und trotz des Rückgangs der körperlichen Anforderungen müssen für Ältere, die einen zunehmenden Teil der Arbeitspersonen stellen, Maßnahmen zur Vermeidung von Fehlbeanspruchungen ergriffen werden, da selbst bei gleicher Belastung und gleichem Arbeitsergebnis Ältere höher beansprucht werden, wenn ihre Fähigkeiten altersbedingt abnehmen (vgl. beispielsweise LAURIG, 1990, 71). Maßnahmen können in arbeitsplatzspezifischen Arbeitshilfen bestehen, die zum Ausgleich 
abnehmender körperlicher Leistungsfähigkeit, zur Erhöhung der Anpassungsfähigkeit und zur Erleichterung des Qualifikationserwerbs beitragen können. Weiterhin müssen zur Minderung der physischen und psychischen Beanspruchungen auch die Möglichkeiten der Beschäftigung an Schonarbeitsplätzen erweitert werden, um auf bestehende Beeinträchtigungen zu reagieren und die Frühverrentungszahlen zu senken. Solche Maßnahmen müssen insbesondere angesichts der wachsenden Bedeutung von anspruchsvollen Tätigkeiten, beispielsweise der Überwachung und der Systemregulierung, umgesetzt werden.

Infolge der Umsetzung und Nutzung moderner Methoden und Technologien stehen weniger die Beanspruchungen durch körperliche Belastungen als durch psychische Anforderungen im Vordergrund. Gerade die psychische Beanspruchung resultiert aus der objektiven Belastungssituation erst über den vermittelnden Prozeß der subjektiven Wahrnehmung und des jeweiligen Bewältigungsverhaltens. Neben der Belastung durch die auszuübende Tätigkeit, die Arbeitsumgebung und situative Faktoren wirken personale Faktoren auf die Beanspruchung (vgl. BRANDENBURG, 1987). Außer den bisher abgeleiteten unmittelbar wirkenden Maßnahmen müssen komplexer wirkende Gestaltungsweisen entworfen werden, die diese Zusammenhänge beachten und beispielsweise die Informations-, Kontroll- und Einflußmöglichkeiten, die soziale Unterstützung sowie die Motivation und Bewältigungsstrategie der Arbeitspersonen betreffen. Angesichts der durchschnittlich mit dem Alter zunehmenden Anpassungsschwierigkeiten und der Verringerung der Abstraktionsfähigkeit der Arbeitspersonen einerseits und der Zunahme der Innovationsrate und der Erhöhung der geistigen Anforderungen andererseits müssen die positiven Effekte der Ausübung einer entwicklungsförderlichen Tätigkeit beachtet werden.

Das aufgebaute Erfahrungswissen sollte im Interesse der Produktivität und zur Förderung der Erwerbstätigkeit Älterer verstärkt genutzt werden. Zur Sicherung der Beschäftigungsmöglichkeiten im Alter sollten zudem präventiv für alle Arbeitspersonen die Möglichkeiten zum Aufbau und zur Nutzung von Erfahrungswissen gesichert und erweitert werden. Hierzu müssen durch arbeitsgestalterische Maßnahmen Betätigungs-, Einfluß- und Entscheidungsmöglichkeiten gewährt werden. Allerdings muß diese Maßnahme gegen Tendenzen zur Entwertung beruflichen Wissens und beruflicher Erfahrung umgesetzt werden. Eine Arbeitsgestaltung und Technikentwicklung, die eine Abnahme der Bedeutung des Erfahrungswissens und eine einseitige Zunahme des theoretischen Wissens fördert, verschlechtert die Beanspruchungssituation Älterer, die häufiger über Erfahrungs- 
wissen und seltener über aktuelles theoretisches Wissen verfügen. Wird der Qualifikationsabbau allerdings durch umfassende und regelmäßige Weiterbildungsmaßnahmen vermieden, so kann das breite berufliche Fachwissen zur Erhöhung der Flexibilität und zur Anpassung von Innovationen genutzt werden. Den Arbeitspersonen müssen Möglichkeiten zur sozialen Teilhabe, zur Kontrolle der Arbeitsabläufe und zur individuellen zeitlichen bzw. mengenbezogenen Anpassung der Arbeitsleistung gegeben werden, so daß auch altersbedingte negative Veränderungen durch eine erhöhte berufliche Erfahrung kompensiert werden können.

Für die Entwicklung und Nutzung der Konıetenzen Älterer ist gerade auch die Gestaltung der an Bedeutung zunehmenden Gruppenarbeit wichtig, da hier besondere Probleme der Überforderung bestehen (vgl. beispielsweise VOLKHOLZ, 1992, 20f.). So stellt beispielsweise der Arbeitsaufgabenwechsel innerhalb der Gruppe hohe Anforderungen an die Anpassungsfähigkeit. Weiterhin kann der gruppenintern erzeugte Leistungsdruck zu Überforderungen der Arbeitspersonen führen. Die Gestaltung der Gruppenarbeit muß daher den wechselnden Einsatz frühzeitig fördern und andererseits Möglichkeiten zur Entkoppelung einzelner Arbeitsplätze bieten. Entsprechende Maßnahmen erfordern die erhöhte Beteiligung der Beschäftigten. Die in den modernen Produktions- und Organisationskonzepten betonte Aufforderung, ständig zur Verbesserung der Leistung und des Prozesses der Leistungserstellung beizutragen, kann zu psychischen Beanspruchungssteigerungen führen. Außerdem kann der kontinuierliche Verbesserungsprozeß Leistungsverdichtungen bewirken, die die Leistungsfähigkeit der Arbeitspersonen überschreiten.

Zur Vermeidung arbeitsbedingter Krankheiten müssen individuell und kollektiv wirkende Ansătze für einen langfristigen Gesundheitsschutz genutzt werden. Durch den Abbau überfordernd wirkender Belastungen für alle Altersgruppen und intensivierte arbeitsmedizinische Untersuchungen zur rechtzeitigen Anpassung der Anforderungen an Veränderungen des Leistungsvermögens, müssen Arbeitsaufgaben geschaffen werden, die nicht zu Fehlbeanspruchungen führen und zur Sicherung der langfristigen Bereitschaft, erwerbstätig zu sein, beitragen. Gleichzeitig müssen Trainingseffekte unterstützt und Ausgleichsbewegungen für körperlich einseitige Anforderungen ermöglicht werden.

Die zunehmende Relevanz sozialer Kompetenzen und die Verringerung der Bedeutung der körperlichen Kraft können sich günstig auf die Chancen der 
Erwerbstätigkeit Älterer auswirken, die während ihres Erwerbslebens Kommunikations- und Kooperationsfähigkeiten entwickeln konnten.

Die abgeleiteten Maßnahmen können zwei Ausrichtungen von Handlungsweisen zur Erhöhung der Erwerbsbeteiligung älterer Personen zugeordnet werden:

- Zur Verminderung von Fehlbeanspruchungen während des Erwerbslebens

- Erfahrungswissen

- Gruppenarbeit

- Arbeitsschutz

- Zur Verbesserung der Beschäftigungsmöglichkeiten Älterer

- Weiterbildung

- Teilzeitarbeit

- Arbeitshilfen

- Schonarbeitsplätze

- Erfahrungswissen

- Gruppenarbeit

Die Maßnahmen Arbeitshilfen und Schonarbeitsplätze sollen im weiteren miteinander verbunden behandelt werden.

\section{Unternehmensbezogene Maßnahmen im Überblick}

Nach ihrer gruppenspezifischen Ableitung können die Maßnahmen entsprechend der in Abschnitt I. E. entworfenen Systematik geordnet werden. Dabei können die Maßnahmen Erfahrungswissen und Gruppenarbeit sowie die gerade hinsichtlich der Erhöhung der Erwerbsquoten der Frauen als interessant erachtete Maßnahme der Heimarbeit systematisch dem Titel Aufgabenbearbeitung zugeordnet werden, auch wenn sie einzeln betrachtet werden sollen. Abbildung 51 zeigt die unternehmensbezogenen Maßnahmen zur Erhöhung der Erwerbsquoten von Frauen und Älteren in einer Übersicht.

Die Einteilung und Abgrenzung der Maßnahmen ist nicht trennscharf, denn die Wirkungsweisen der Maßnahmen überschneiden einander. Dies wird im besonderen deutlich beim Arbeitsschutz, der sich auf die Gestaltung der Arbeitsorganisation und der Arbeitsplätze beziehen kann. 


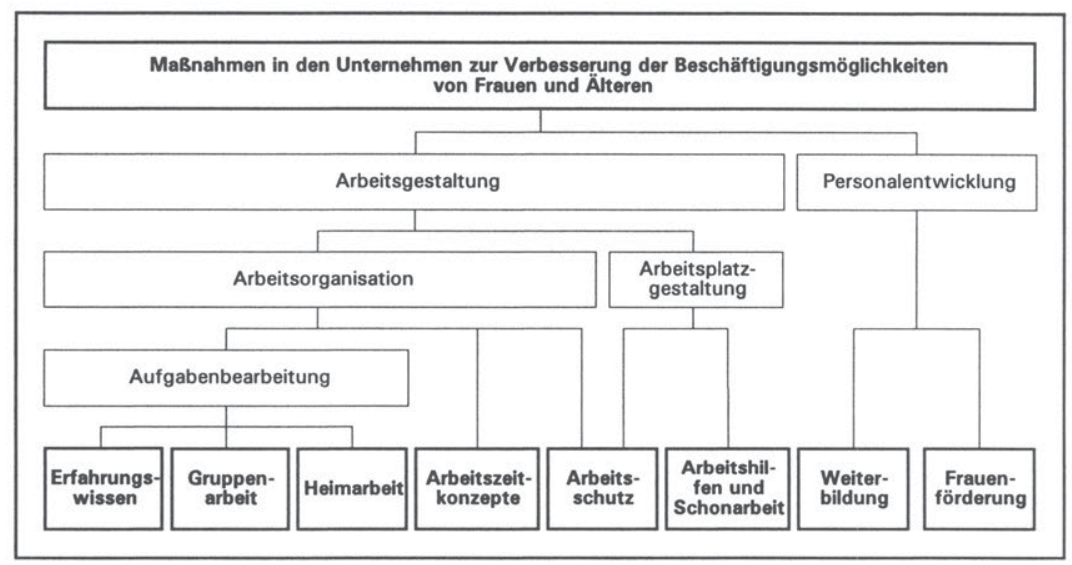

Abb. 51: Unternehmensbezogene Maßnahmen zur Erhöhung der Erwerbsbeteiligung

Die hier abgeleiteten Maßnahmen der Arbeitsorganisation, der Arbeitsplatzgestaltung und der Personalentwicklung finden ihre Ausrichtung anhand der behandelten Eigenschaften, Anforderungen und Beanspruchungen. Die Maßnahmen sind, orientiert an den Unternehmenszielen, inhaltlich näher zu beschreiben und zu beurteilen. Sie entsprechen den Handlungsspielräumen der Unternehmen und erscheinen insofern verbreitet anwendbar. Dabei müssen sie im Detail den Bedürfnissen der jeweils betroffenen Personen und den spezifischen betrieblichen Bedingungen angepaßt werden. Die integrierte Umsetzung dieser frauen- bzw. altersadäquaten Maßnahmen kann eine Umorientierung auf geschlechts- und altersindifferente Arbeitsplätze bewirken, die der Vergeudung vorhandenen Humankapitals und den zukünftigen Defiziten des Arbeitskräfteangebots entgegenwirkt. Indem die Maßnahmen die gegenseitige Anpassung von Arbeitspersonen und Arbeitsaufgaben ermöglichen, tragen sie außerdem zur Förderung der Nutzung moderner Produktions- und Organisationsmethoden bei.

Die im bisherigen Verlauf der Arbeit betrachteten Zusammenhänge sind arbeitssystembezogen in folgender Abbildung zusammenfassend dargestellt. 


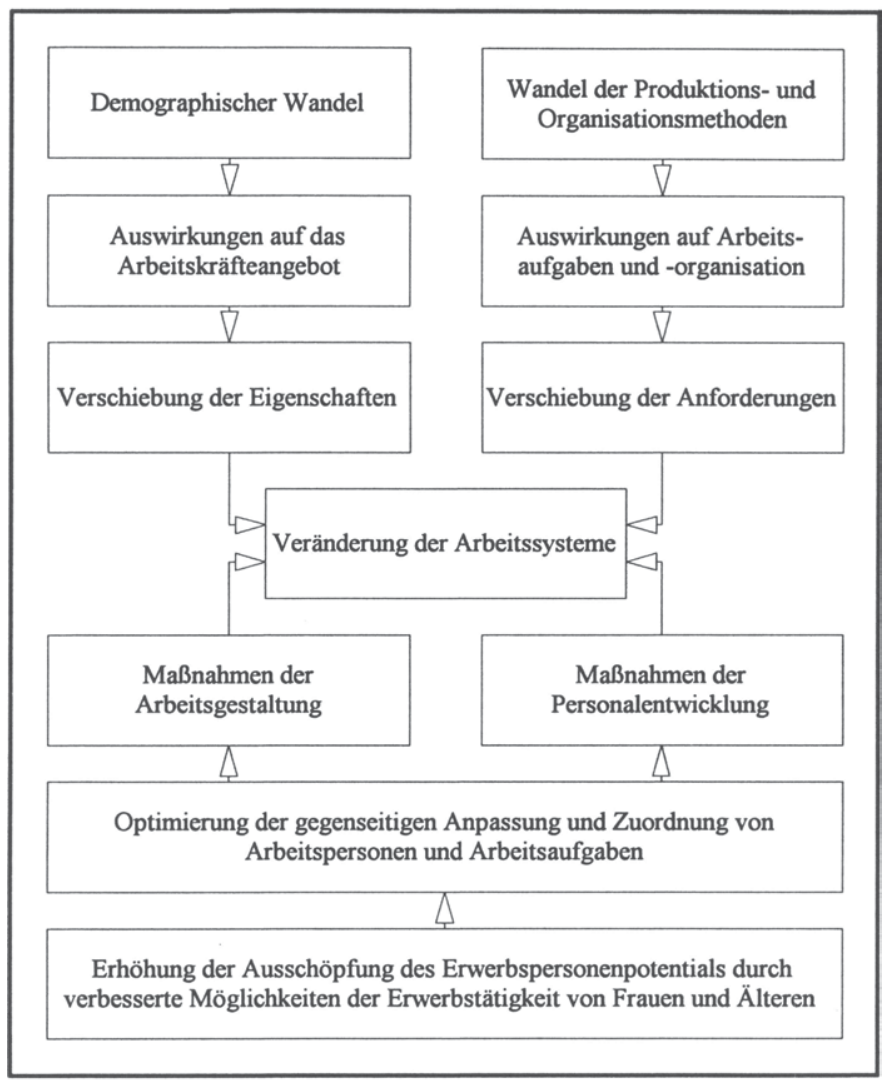

Abb. 52: Beeinflussung und Gestaltung der Arbeitssysteme 
Stefan Lorenz - 978-3-631-75027-8

Downloaded from PubFactory at 01/11/2019 09:06:41AM

via free access 


\section{Unternehmensbezogene Maßnahmen als mikroökonomische Lösungsbeiträge}

Zunächst werden im folgenden wesentliche Ziele, Motive und Handlungsmöglichkeiten der Arbeitgeber betrachtet, um das Feld unternehmerischer Handlungsweisen zur Bewältigung der beschriebenen Problemstellung zu beschreiben. Daneben werden Interessen der Arbeitnehmerseite und des Staates angesprochen, um Beeinflussungs- und Fördermöglichkeiten deutlich zu machen. Anschließend wird ein Raster zur Betrachtung der Maßnahmen erörtert, mit dem danach die zuvor abgeleiteten Maßnahmen insbesondere aus Sicht der Unternehmen beurteilt werden. Die Maßnahmen werden dann bezüglich der auf Frauen und Ältere bezogenen Optionen integriert betrachtet, bevor eine an den Problemen orientierte Strategie ihrer zeitlichen Umsetzung vorgestellt wird.

\section{A. Handlungsträger und Interessen}

Die konkrete Gestaltung und Entwicklung der Arbeit findet in den Unternehmen statt. Verschiedene Akteure mit unterschiedlichen Interessen, Vorstellungen und Strategien wirken auf diesen Prozeß ein. Insbesondere durch den demographischen Wandel und durch die Umsetzung der behandelten Organisations- und Produktionsmethoden, die auf eine stärkere Nutzung des Leistungsvermögens der Arbeitspersonen gerichtet sind, kann es zudem zu Veränderungen der Ausrichtung durchschnittlicher Interessen kommen. Diese Interessen beeinflussen ihrerseits die Umsetzungsmöglichkeiten der Methoden und der Maßnahmen zur Erhöhung der Erwerbsbeteiligung von Frauen und Älteren. Die Gestaltung, Auswahl, Einführung und Sicherung der Maßnahmen werden durch die Arbeitgeber, die Arbeitnehmer sowie ihre jeweiligen Vertreter bestimmt, so daß Kompromisse zwischen den verschiedenen Maßnahmenträgern notwendig werden; neue Instrumente und Verhandlungsformen müssen entwickelt werden. Dem Verhalten der an Entscheidungen innerhalb der Unternehmen beteiligten Gruppen der Arbeitgeberseite und der Arbeitnehmerseite liegen unterschiedliche Kriterienbündel zugrunde (vgl. zu den jeweiligen Handlungsbeiträgen ROSENOW/NASCHOLD, 1993, 148). Weitere Interessendivergenzen beispielsweise innerhalb des Managements oder zwischen Unternehmensleitung und Kapitalgebern finden vereinfachend hier keine Beachtung. 


\section{- Arbeitgeberseite}

Zur Einschätzung mikroökonomischer Handlungsweisen kann die Entscheidungssituation eines Unternehmens betrachtet werden, die durch folgende Fragen illustriert wird:

- Welche Maßnahmen können Unternehmen einzeln oder gemeinsam ergreifen, um die Deckung ihres Arbeitskräftebedarfs zu sichern?

- Mit welchen voraussichtlichen Nutzen und Kosten sind diese Maßnahmen verbunden?

- Wann und in welcher Reihenfolge sollten die Maßnahmen ergriffen werden?

Zur Bewältigung der Arbeitsaufgaben muß der in quantitativer und qualitativer Hinsicht zu beurteilende Produktionsfaktor Arbeit bereitgestellt werden. Ein Ziel der Arbeitgeber ist entsprechend die langfristige Sicherung des zur Verfügung stehenden quantitativen und qualitativen Arbeitskräfteangebots. Hierzu müssen die Arbeitgeber Maßnahmen ergreifen, die zum Teil auch den Interessen der Arbeitnehmer entsprechen. Für die Arbeitgeber ist von Bedeutung welche Auswirkungen die Arbeitnehmerinteressen und -handlungsweisen auf die Erreichung der Unternehmensziele haben. Arbeitnehmerinteressen können aus Sicht der Arbeitgeber in den Unternehmen zumindest insoweit erfült werden, als sie der Erreichung der Unternehmensziele nicht zuwiderlaufen.

Aus Arbeitgebersicht wird es zukünftig immer weniger eine eindeutig zu umreißende, technisch determinierte 'beste' Arbeitsgestaltung geben, vielmehr wird sich, aufgrund der Veränderung der Altersstruktur der Beschäftigten und der Verknappung der Nachwuchskräfte, der Zielkonflikt verstärken, einerseits die Leistungspotentiale der Arbeitspersonen möglichst gut auszuschöpfen und andererseits Arbeitskräfte langfristig zu motivieren und verfügbar $\mathrm{zu}$ halten. Entsprechend dieser beiden Zielsetzungen müssen arbeitsgestalterische Maßnahmen mit Hilfe der in Kapitel I genannten Kriterienbündel beurteilt werden. Die arbeitssystembezogenen Kriterien fanden Beachtung bei der Ableitung der Maßnahmen, die die Bedürfnisse der Arbeitnehmer berücksichtigen. Bei der nachfolgenden Beurteilung der Maßnahmen aus Sicht der Arbeitgeber werden primär die wirtschaftlichen Kriterien Verwendung finden.

Die Arbeitgeber werden auch zukünftig rationalerweise auf ihrer Entscheidungsgewalt bezüglich der Investitionen, der Arbeitsgestaltung und des Einsatzes der 
Arbeitspersonen bestehen. Gleichwohl ist es sinnvoll, und gerade mitarbeiterorientierten Produktions- und Organisationsmethoden angemessen, die Vorstellungen der Arbeitnehmerseite miteinzubeziehen.

\section{- Arbeitnehmerseite}

Wesentliche arbeitsbezogene Interessen der Arbeitspersonen betreffen die Verwertung der Arbeitsleistung (finanzielle Bedingungen, Aufstiegschancen, soziale Anerkennung, Aktivität, persönliche Identität), die Erhaltung der Nutzungschancen (Belastungsabbau, Qualifizierung, Sicherung der Handlungskompetenz) sowie die Gestaltung der Erwerbsarbeit (Zeitstrukturierung, Kooperation, soziale Kontakte, Verhaltens- und Entscheidungsspielräume, Minderung von Zwangssituationen) (vgl. FÜRSTENBERG, 1987, 67 und SEMMER/UDRIS, 1993, 134ff.). Die Arbeitspersonen sind vielfach daran interessiert, verwertbare Berufserfahrung zu sammeln sowie eine ihren Eigenschaften entsprechende und diese fördernde Tätigkeit durchzuführen. Die Interessen richten sich auf die persönliche Entfaltung, den Erhalt bzw. die Erweiterung individueller Spielräume und die Kompetenzsicherung und damit auf eine an ihren Bedürfnissen orientierte inhaltliche, zeitliche, räumliche, organisationale, soziale und finanzielle Arbeitsgestaltung, d.h. die Interessen richten sich zusammengefaßt auf die Humanisierung der Arbeit.

Entsprechend versuchen betriebliche Arbeitnehmervertretungen, die Arbeitsbedingungen $\mathrm{zu}$ verbessern und negative Rationalisierungsfolgen $\mathrm{zu}$ vermeiden. Hinsichtlich der Veränderung der Arbeitsorganisation kommen dem Betriebsrat Beteiligungsrechte zu (vgl. SIEBERT, 1993). Bei der Planung diesbezüglicher Maßnahmen muß der Arbeitgeber den Betriebsrat unterrichten, bei grundlegenden Änderungen der Arbeitsmethoden und der Betriebsorganisation müssen außerdem Beratungen mit dem Betriebsrat erfolgen. Dabei müssen die zukünftigen Arbeitsplätze und Arbeitsaufgaben ebenso dargestellt werden wie die zu erwartenden Anforderungen. Weiterhin gewährt das Betriebsverfassungsgesetz dem Betriebsrat Mitbestimmungsrechte hinsichtlich der Umsetzung vieler Einzelmaßnahmen, wie der Änderung der Arbeitszeit und der Entlohnungsart sowie der Durchführung grundlegender Weiterbildungsmaßnahmen. Zustimmungspflichtig nach dem Betriebsverfassungsgesetz sind die Versetzung und die Umgruppierung von Arbeitskräften; Kündigungen erfordern eine Anhörung des Betriebsrats. Eine Einflußnahme der Arbeitnehmervertreter auf die Arbeitsgestaltung ermöglicht das Betriebsverfassungsgesetz hinsichtlich Regelungen der Arbeitszeit und der Leistungskontrolle sowie hinsichtlich Belastungen, die den gesicherten 
arbeitswissenschaftlichen Erkenntnissen über die menschengerechte Gestaltung der Arbeit offensichtlich widersprechen. Dieses korrigierende Mitbestimmungsrecht hat in der Praxis allerdings relativ geringe Bedeutung, da die Voraussetzungen für seine Anwendung ('offensichtlicher' Widerspruch zu 'gesicherten' arbeitswissenschaftichen Erkenntnissen) sehr hoch sind (vgl. HALBACH u.a., 1994, $418 \mathrm{ff}$.). Zukünftig dürften sich die Schwierigkeiten einer Einflußnahme des Betriebsrates weiter vergrößern, da Betriebsräte gerade im Falle systemischer Rationalisierung kaum nachweisen können, daß eine Systemgestaltung den "gesicherten arbeitswissenchaftchen Erkenntnissen über die menschengerechte Gestaltung der Arbeit" widerspricht, so daß sie hierzu zumindest der Unterstützung der Gewerkschaften bedürfen (vgl. LINHART u.a., 1989, 138 und 143ff.; ALTMANN/DÜLL, 1987). $\mathrm{Da}$ angesichts der geschilderten überbetrieblich wirkenden Rationalisierungsformen, die auf die Verknüpfung verschiedener Unternehmen hinzielen, die konzeptionell betriebsbezogene Arbeitnehmerinteressenvertretung im Betriebsrat an EinfluB verliert, sollen auch gewerkschaftlche Interessen angesprochen werden.

Neben Lohn- und Gehaltsverhandlungen und der Abwehr von Gefährdungen der Arbeitnehmer müssen Gewerkschaften zunehmend daran interessiert sein, daß von Arbeitnehmerseite Einfluß auf die Entwicklung der Technik wie auch der Arbeitsgestaltung genommen wird. Die Wandlungen der Arbeitsaufgaben, der Rationalisierungsformen und der Interessen der Arbeitspersonen erfordern gewerkschaftliche Anpassungsleistungen. Die notwendigen Änderungen und Ergänzungen der traditionellen Tarif- und Betriebspolitik betreffen beispielsweise überbetriebliche Strategien und Koalitionsmöglichkeiten. Während im Falle der traditionell arbeitsplatz-, anlagen- oder abteilungsbezogenen Rationalisierung die Probleme und ihre Ursachen relativ leicht erkennbar sind, bestehen bei den modernen, komplexen, systemisch wirkenden Rationalisierungsstrategien erhebliche Schwierigkeiten für das gewerkschaftliche Handeln. Diese betreffen beispielsweise die Wahrnehmung von Problemen und ihren Ursachen, das rechtzeitige Erkennen der Folgewirkungen von Maßnahmen, die möglicherweise in anderen Unternehmensteilen oder auch in anderen Unternehmen eines Netzwerkes umgesetzt werden, die erforderliche Information und Sachkenntnis sowie die unklare Gegnerschaft bei Konflikten (vgl. $\mathrm{zu}$ den Schwierigkeiten der Arbeitnehmervertretung in internationalen Unternehmensnetzwerken SCHIENSTOCK, 1994, 264ff.). Seitens der Gewerkschaften wird daher eine erweiterte Mitbestimmung gefordert, die gewerkschaftliche Interessenvertreter in den gesamten Entwicklungsprozeß von Innovations- und Rationalisierungsmaßnahmen einbindet und somit die Möglichkeit ihrer Teilnahme an der Technikgestaltung ausbaut (vgl. HELFERT, 1992, 509f.). 
Aus Sicht der Gewerkschaften besteht allerdings das Problem, in nicht wirklich mitbestimmte Entscheidungsprozesse über betriebswirtschaftliche Fragen einbezogen zu werden, so daß die Arbeitnehmervertreter Mitverantwortung übernehmen, ohne sich der betriebswirtschaftlichen Entscheidungslogik entziehen zu können bzw. dieser wirksam andere Vorstellungen entgegensetzen zu können. Aufgrund der Vielfalt der erforderlichen Informationen und der Entwicklungsgeschwindigkeit dürfte es einzelnen Arbeitnehmervertretern nicht gelingen, das neueste Wissen unternehmensinterner oder -externer Experten auszugleichen (vgl. ALTMANN/DÜLL, 1987), vielmehr müssen angesichts der Komplexität der Gestaltungsaufgaben aus gewerkschaftlicher Sicht in höherem Maße Angestellte, insbesondere Ingenieure, die dieses Wissen einbringen können, zur betrieblichen Vertretung der Arbeitnehmerinteressen gewonnen werden. Weiterhin muß die Arbeitnehmervertretung anpassungsfähige Kriterien zur Bewertung von Maßnahmen entwickeln, um auf die Arbeitsgestaltung Einfluß nehmen zu können.

Eine überbetriebliche Vernetzung der Interessenvertretungen durch die Gewerkschaften kann zudem die Kooperations- und Integrationsbestrebungen in den Unternehmen "durch eine arbeitnehmerorientierte Perspektive .. ergänzen" (BREDEWEG/KOWOL, 1991, 424). Weitere arbeitnehmervertretungsbezogene Probleme ergeben sich aus der möglichen Entsolidarisierung aufgrund der Konkurrenz zwischen verschiedenen Gruppen der Belegschaft und der immer individuelleren Ausgestaltung der Arbeitsbeziehungen (beispielsweise durch die erhöhte Schaffung von Teilzeitarbeitsplätzen und Unterbrechungsmöglichkeiten).

Die zunehmenden Probleme der Vertretung der Arbeitnehmerinteressen sind auch für die Umsetzung moderner Produktions- und Organisationsmethoden sowie der Maßnahmen zur Erhöhung der Erwerbsbeteiligung von Frauen und Älteren von Bedeutung. Insofern sich hierdurch die Unsicherheit bzw. Unberechenbarkeit der Handlungsweise der Arbeitnehmerseite erhöht, müssen häufiger Konflikte und eine Minderung der Akzeptanz für Entscheidungen der Unternehmensleitung erwartet werden. Infolgedessen könnte sich die Motivation der Arbeitspersonen verringern, so daß die mit den modernen Produktions- und Organisationsmethoden angestrebten Ziele nur schwer erreicht werden können. Daher ist es auch aus Sicht der Arbeitgeber sinnvoll, daß die Beteiligung der Arbeitnehmer an Gestaltungsentscheidungen gefördert wird. Dies könnte durch die Erhöhung der betriebsbezogenen Information, Beratung und Mitwirkung erreicht werden. Allerdings bestehen aufgrund der besonderen Betriebsspezifität moderner Organisationsmethoden Schwierigkeiten hinsichtlich einer allgemeinen tarifvertraglichen oder einer 
gesetzlichen Regelung (vgl. HELFERT, 1992, 520), so daß hierzu einzelne Betriebsvereinbarungen genutzt werden müssen. Die Mitbestimmung am Arbeitsplatz kann besonders die Dispositionsfreiheit hinsichtlich der Arbeitsausführung sichern und mögliche Beiträge der Arbeitspersonen nutzen. Ergänzende beschäftigungssichernde Regelungen können in Betriebsvereinbarungen festgelegt werden und die Motivation, das Engagement und die Mitarbeit der Beschäftigten an den Gestaltungsaufgaben erhöhen.

Zu den Zielen, die im Interesse der Sicherung der Wettbewerbsfähigkeit der Unternehmen zur Steigerung der technisch-wirtschaftlichen Effizienz beitragen, kommt - wie bereits dargestellt - in zukünftig höherem Maße das Ziel der Sicherung des Humankapitals. Damit erweitert sich das Zielspektrum der Unternehmen derart, daß die gegensätzliche Position der Gewerkschaften scheinbar aufgesogen wird, da neben der Produktivitätssteigerung und der Entwicklung der Leistungsfähigkeit der Arbeitskräfte Maßnahmen zur Humanisierung an wirtschaftlicher Bedeutung gewinnen. Allerdings zeigen sich bei näherer Betrachtung genügend Konfliktpotentiale hinsichtlich der Maßnahmennutzung. Diese betreffen beispielsweise mögliche Rechtsansprüche auf Leistungen, die Auswahl der begünstigten Arbeitspersonen und weitere Mitbestimmungsmöglichkeiten.

\section{- Staat}

Es kann als Aufgaben der Politik aufgefaßt werden, gesellschaftliche Entwicklungen zu beobachten und nach dem Erkennen von Problemen und der Unzulänglichkeit der Märkte hierfür Lösungen bereitzustellen, politisch zu handeln, falls ein ausgeprägtes beispielsweise arbeitspolitisches Interesse an entsprechenden Lösungen besteht (vgl. LUTZ, 1990). Die unternehmensbezogenen Maßnahmen zur familienfreundlichen und altersgerechten Gestaltung der Arbeitswelt müssen von den Arbeitgebern bzw. von den Tarifvertragsparteien gemeinsam entwickelt und umgesetzt werden. Der Staat kann jedoch durch die Gestaltung der rechtlichen Rahmenbedingungen und die Gewährung entsprechender Leistungen Anreize für die Maßnahmenumsetzung geben. Bezüglich der hier betrachteten Problemstellung sollten die Anreize die Unternehmen im gesamtwirtschaftlichen Interesse vor allem dazu bewegen, rechtzeitig langfristig wirksame Maßnahmen zu ergreifen. Solche Anreize sind insbesondere deshalb erforderlich, da angesichts der derzeitigen, durch Arbeitslosigkeit geprägten Arbeitsmarktsituation aus einzelwirtschaftlicher Sicht Maßnahmen zur Erhöhung der Erwerbstätigkeit von Frauen und Älteren (noch) nicht dringend erforderlich erscheinen und da von den verzögert wirkenden 
Maßnahmen auch andere Unternehmen profitieren können, die keine entsprechenden 'Vorleistungen' erbringen.

Folgende Abbildung zeigt exemplarisch Anreizmechanismen, die zwischen den Gruppen von Akteuren des Arbeitsmarktes wirken können.

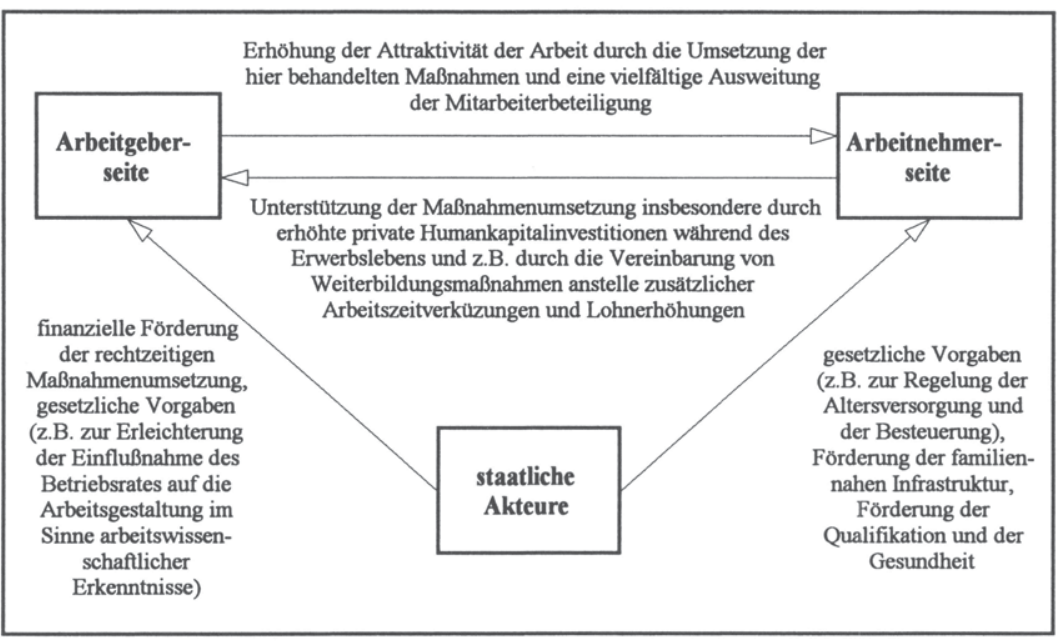

Abb. 53: Mikroökonomische und staatliche Anreizmechanismen

\section{B. Gestaltung und Beurteilung der Maßnahmen}

\section{Raster zur Betrachtung der Maßnahmen}

Die einzelnen Maßnahmen der Arbeitsgestaltung und Personalentwicklung werden jeweils nach den im folgenden erörterten drei Punkten betrachtet. Zunächst beziehen sich 'Gestaltungsformen' auf die Möglichkeiten einer Ausgestaltung der Maßnahmen. Die Gestaltungsform muß an den verschiedenen Interessen ausgerichtet sein und insbesondere grundsätzliche arbeitswissenschaftliche Vorstellungen beachten, damit die Maßnahmen zieladäquat wirken können. Im Interesse einer wirtschaftlichen Beurteilung der Maßnahmen werden weiterhin wesentlich 'betroffene Kosten und Nutzengrößen' zusammengestellt. Die 'Umsetzungsbedingungen' verweisen schließlich auf Umstände, die eine Umsetzung der jeweiligen Maßnahme fördern oder hemmen können. 


\section{a) Gestaltungsformen und -grundsătze}

Die abgeleiteten, zur Erhöhung der Erwerbsbeteiligung von Frauen und Älteren als notwendig erachteten Maßnahmen der Arbeitsgestaltung und Personalentwicklung müssen bei ihrer Umsetzung an die spezifischen Bedingungen in den Unternehmen und die jeweiligen Bedürfnisse der Arbeitspersonen angepaßt werden. Die Anpassung der Maßnahmen kann in der Praxis die Verwendung arbeitsanalytischer Verfahren erfordern und sollte sich am arbeitswissenschaftlichen Kenntnisstand orientieren. Zur Vorbereitung der Ausformung der Maßnahmen und ihrer späteren Einschätzung werden für die Gestaltung der Maßnahmen wesentliche arbeitswissenschaftliche Vorstellungen genannt, die die primär an Effizienzzielen orientierten Leitvorstellungen der CIM- und Lean Production Konzepte ergänzen (vgl. GROB, 1993 und 1994).

Ziel arbeitswissenschaftlicher Betrachtungen ist der möglichst produktive Einsatz der Arbeitspersonen unter Beachtung der persönlichen Leistungsgrenzen. Entsprechend werden wirtschaftliche und humane Ziele verfolgt und Humanisierung als "Partner" der Rationalisierung verstanden (ROHMERT, 1980, 66). Die Forschung richtet sich auf die Optimierung der Arbeitsprozesse unter Berücksichtigung der Bedürfnisse der betroffenen Arbeitspersonen sowie technologischer bzw. ökonomischer Anforderungen. Die effiziente und wirtschaftliche Nutzung vorhandener Potentiale soll unter humanen Arbeitsbedingungen erfolgen. Hierfür wird beispielsweise untersucht, wie sich betriebswirtschaftliche Rationalisierungen auf die Arbeitspersonen auswirken und wie negative Folgen der Arbeit überwunden werden können. Durch den Einsatz moderner Techniken kann der früher als gegeben angenommene technische Determinismus weitgehend überwunden werden. Formen variabler Arbeitsgestaltung sind zum Ausgleich spezieller Beanspruchungen möglich und aufgrund der sich verändernden Zusammensetzung des Arbeitspersonenpotentials zunehmend ökonomisch notwendig. Die zusammengestellten Vorstellungen richten sich primär auf die Gestaltung der Aufgaben oder eher auf die Entwicklung der Personen. 
Aufgaben- und personenbezogene Vorstellungen und Ziele

Primăr aufgabenbezogene Vorstellungen:

- Gestaltung menschengerechter Arbeitsplätze, Erhøhung der intrinsichen Motivation durch die Förderung einer Aufgabenorientierung (vgl. beispielsweise ULICH u.a., 1989, 24ff.) und Erhőhung der Einsatzflexibilităt der Arbeitspersonen.

- Mindcrung extremer Arbeitsteilung, Bildung ganzheitlicher Aufgaben, Gewăhrleistung von Rückmeldungen über den Arbeitsfortschritt und die Arbeitsqualităt.

- Vergrößerung von Freiheitsgraden und Sicherstellung möglichst hoher Autonomie hinsichtlich der Aufgabenbearbeitung; Bereitstellung der erforderlichen zeitlichen, sachlichen und personellen Ressourcen.

- Abbau monotoner und einseitig belastender Tătigkeiten, Erhöhung der Anforderungsvielfalt zur Minderung einseitiger Beanspruchungen.

- Abbau der strikten Zuordnung einer Arbeitsperson an einen Arbeitsplatz.

- Erweiterung der Arbeitsinhalte (job enlargement), Anreicherung der Arbeitsinhalte (job enrichment), Wechsel der Arbeitsinhalte (job rotation).

- Minderung der Arbeitsintensităt, Abbau des Zeitdrucks, Entkopplung der Arbeitsplătze vom Produktionstakt.

- Korrektive Arbeitsgestaltung zur Behebung erkannter Măngel, prăventive Arbeitsgestaltung zur Vermeidung von Schädigungen und Beeintrăchtigungen (Fehlbeanspruchungen) sowie prospektive Arbeitsgestaltung zur Schaffung von Möglichkeiten zur Persőnlichkeitsentwicklung (vgl. ULICH, 1991, 142ff. und 1993, 189ff.).

Primăr personenbezogene Vorstellungen:

- Flexibilităt kann als eine Aufgabe sozialer Gestaltung begriffen werden. Die Erhöhung der Flexibilităt wird arbeitsorganisatorisch durch die Schaffung von Handlungsspielräumen z.B. hinsichtlich der Arbeitsbereiche, Arbeitsfunktionen und Arbeitsbelastungen sowie hinsichtlich der Einteilung des zeitlichen Ablaufs und durch die begleitende Aktivierung der Arbeitspersonen erreicht (vgl. FÜRSTENBERG, 1987).

- Dispositionsmöglichkeiten bezüglich der Ausführung der Arbeitsaufgaben erlauben eine Anpassung der Belastungen (vgl. beispielsweise ROHMERT, 1984, 199). Eingerăumte Freiheitsgrade kőnnen dem Aufspüren individueller und effizienter Arbeitsweisen dienen. Inhaltlich komplexe Aufgaben mit vielfältigen Anforderungen sichern die geistige Beweglichkeit.

- Qualifikationsgerechter Einsatz der Arbeitspersonen zur Nutzung, zum Erhalt und zur Entwicklung der Qualifikation der Arbeitspersonen (vgl. beispielsweise LUCZAK, 1988). Aufbau, Sicherung und Nutzung von Erfahrungs- und Expertenwissen durch die Schaffung arbeitsimmanenter Lern- und Entwicklungsmöglichkeiten.

- Die Individualităt der Arbeitspersonen (ihrer Eigenschaften und Bedürfnisse) muB akzeptiert und genutzt werden, indem Formen der individuellen Leistungsentfaltung eroffnet werden. Eine differentielle Arbeitsgestaltung zur Bereitstellung verschieden komplexer Arbeitssysteme als Angebot alternativer Arbeitsstrukturen ermöglicht eine Anpassung an die Eigenschaften der Arbeitspersonen und die Forderung ihrer Entwicklung (vgl. ULICH, 1991, 189ff. und ZÜLCH/STARRINGER, 1984).

- Die Arbeitspersonen sollten - bei zugesicherten Korrekturmöglichkeiten - zwischen verschiedenen Arbeitsstrukturen wählen und je nach Alternativenangebot schrittweise wechseln können. So kann beispielsweise die Arbeitszeitflexibilisierung im Falle ausreichender Dispositionsrechte der Arbeitspersonen zur Erhshung der Attraktivităt der Arbeit beitragen, indem sie eine Anpassung der Erwerbstătigkeit an außerberufliche Anforderungen erleichtert.

- Förderung individueller und sozialer Verantwortung z.B. durch die Partizipation an betrieblichen Entscheidungen, durch die Verstărkung der Zusammenarbeit, durch die Förderung von Erkenntnissen über die Notwendigkeit einer differentiellen Beurteilung und Behandlung von Problemen und Arbeitspersonen und durch die Kontrolle eigener Arbeitsergebnisse. 
- Minderung von Kommunikationsproblemen; Erweiterung der Möglichkeiten einer sozialen
Interaktion beispielsweise durch kooperative Aufgabenbearbeitung; Förderung der
Kommunikation zwischen den Gruppen des Unternehmens.
Bildung von Teams und Gruppen gemäB den Notwendigkeiten des Arbeitsablaufs und unter
Beachtung der Neigungen der Arbeitspersonen; Einrichtung teilautonomer Arbeitsgruppen;
Gewährung grobtmöglicher Dispositionsfreiheit bei der Arbeitsausführung, weitgehende
Selbststeuerung, Nutzung z.B. von job rotation zum Ausgleich von Belastungen, zur
Erweiterung der Kompetenzen sowie der Kommunikations- und Kooperationsfahigkeiten
und -möglichkeiten.

Abb. 54: Grundlegende arbeitswissenschaftliche Vorstellungen und Ziele

Bei der Umsetzung der Methoden und Maßnahmen sollten die Unternehmen arbeitswissenschaftliche Vorstellungen und Vorgehensweisen zur Rationalisierung und zur gleichzeitigen Verbesserung der Arbeitsgestaltung berücksichtigen, um das Humankapital zu sichern und angemessen nutzen zu können. Diese Vorstellungen können die CIM- und Lean Production Konzepte ergänzen und beeinflussen, so daß die Arbeitssysteme an die Bedürfnisse der Arbeitspersonen angepaßt werden können. Bei der Behandlung der einzelnen Maßnahmen werden wichtige Gestaltungsformen und ihre Wirkungsweise auf die Erwerbstätigkeit der Frauen und Älteren angeführt. Daneben sind die Maßnahmen hinsichtlich der Unternehmensziele und der Einführung moderner Methoden zu betrachten.

\section{b) Wirtschaftliche Beurteilung}

Zur Beurteilung der Maßnahmen aus Sicht der Arbeitgeber müssen die technischwirtschaftlichen Ziele, das Ziel der Sicherung des Humankapitals und die betrachteten spezifischen Problemlagen Beachtung finden. Abbildung 55 unterstreicht die wechselseitige Abhängigkeit der relevanten Größen.

Nachdem die arbeitssystembezogenen Kriterien zur Ableitung der Maßnahmen dienten, werden die wirtschaftlichen Kriterien nunmehr zur Beurteilung der einzelnen Maßnahmen verwendet. Zur Vorbereitung müssen zunächst die Ziele und Möglichkeiten einer wirtschaftlichen Maßnahmenbeurteilung erörtert werden. Die Maßnahmen sind zur Minderung zukünftiger quantitativer und qualitativer Defizite des Arbeitskräfteangebots, d.h. zur Sicherung des Humankapitals, umzusetzen. Sie tragen demnach dazu bei, die personellen Voraussetzungen der mikro- und makroökonomischen Leistungsfähigkeit zu gewährleisten. Infolgedessen richtet sich die wirtschaftliche Einschätzung der einzelnen Maßnahmen darauf, die Wirkungen der Maßnahmenumsetzung festzuhalten, ohne auf eine Abwägung zwischen 
'Wirtschaftlichkeit' und 'Maßnahmenumsetzung' (bzw. 'Humankapitalsicherung') zu zielen. Denn die Maßnahmen erscheinen unabhängig davon notwendig, ob der Zusammenhang als Komplementarität, Indifferenz oder Konkurrenz aufzufassen ist (vgl. zu dieser Systematisierung des Zusammenhangs von Wirtschaftlichkeit und Humanität, die nur "eine begriffslogische Orientierung möglich" macht, KOUBEK, 1985, 1208ff.). Demnach wird es für die Maßnahmen zur Sicherung des Humankapitals auch keine Investitionsalternativen geben.

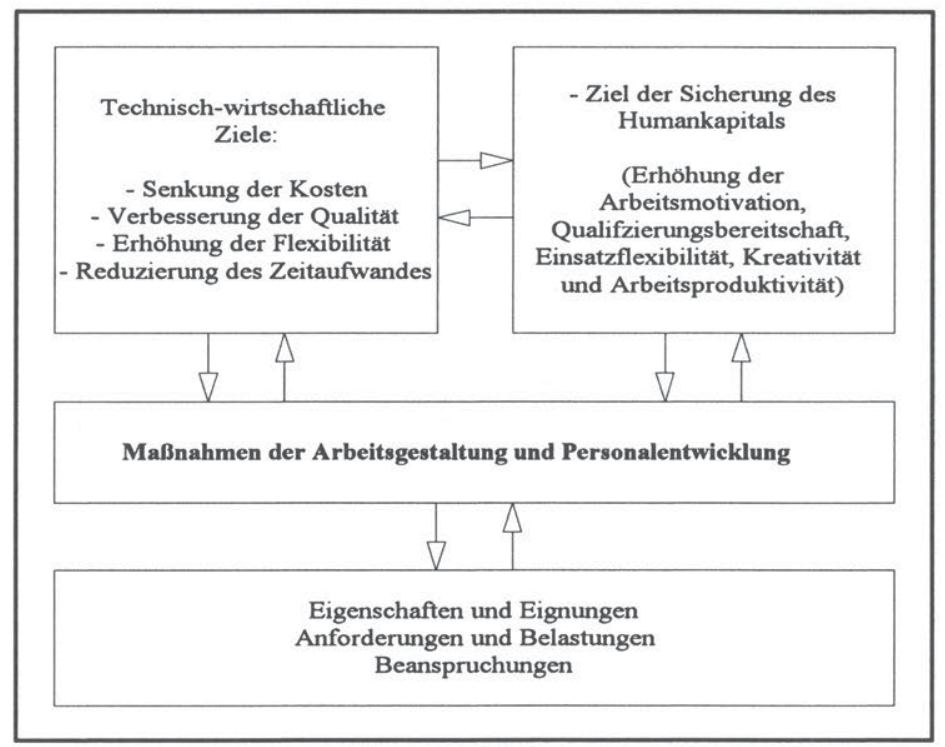

Abb. 55: Ziele und Bestimmungsfaktoren unternehmensbezogener Maßnahmen

Wirtschaftlichkeitsuntersuchungen sollen rationale Entscheidungen vorbereiten, indem sie das Verhältnis von entscheidungsrelevanten Kosten und Leistungen (bzw. Nutzen) betrachten. Die mit den zu erörternden Maßnahmen verbundenen Erwartungen sind vielfach allerdings nicht kurzfristig erreichbar, und die mehrjährigen Umsetzungs- und Wirkungsprozesse entziehen sich einer exakten betriebswirtschaftlichen Kalkulation. Neben den monetär quantifizierbaren Größen müssen regelmäßig auch monetär nicht quantifizierbare Faktoren Beachtung finden. Dies ist in besonderem Maße hinsichtlich der Beurteilung der Maßnahmen der Arbeitsgestaltung und Personalentwicklung der Fall. So lassen sich gerade die Nutzengrößen häufig nicht quantitativ bewerten. Verschiedene Nutzengrößen können in den einzelnen Betrieben allerdings durch indirekt beeinflußte Kosten- 
größen abgeschätzt werden, wie Qualitätsverbesserungen über die Veränderung der Nachbearbeitungs- oder Ausschußkosten und Flexibilitätserhöhungen über die Veränderung der fehlzeitenbedingten Kosten. Da die Maßnahmen sach- und personenbezogen sind, prägen sich zudem nicht nur die Einflüsse der Umgebung, die Unternehmensziele und die Interessen der Entscheidungsträger, sondern auch die Bedürfnisse der Arbeitspersonen - als 'Zielobjekte' der Maßnahmen - spezifisch und qualitativ unterschiedlich aus.

Die monetäre Abschätzung der Kosten und Nutzen ist damit nur begrenzt möglich, von vielen spezifischen Umständen abhängig und nicht interessenneutral vorzunehmen. Für eine umfassende Würdigung der Maßnahmen müßten außerdem neben den Effizienzkriterien auch 'strategische' Wirkungen hinsichtlich der verschiedenen Dimensionen der Wettbewerbsfähigkeit und des Unternehmenserfolgs beachtet werden. Häufig wird daher nur eine qualitative Einschätzung in Form einer Argumentenbilanz möglich sein, die neben den abzuschätzenden Kosten insbesondere Verweise auf plausible qualitative Vorteile enthält. Zur Beurteilung der einzelnen Maßnalımen werden in vorliegender Arbeit wesentlich betroffene Kostenarten und Leistungsgrößen zusammengestellt. Hierauf basierend wird für jede Maßnahme eine globale Beurteilung hinsichtlich der angesprochenen Ziele durchgeführt. Ergänzend ist zu bemerken, daß die Beurteilung nicht zur Auswahl einer Maßnahme aus gegebenen Alternativen erfolgt, da die Maßnahmen in einem, an den Unternehmenszielen und Beschäftigtenbedürfnissen ausgerichteten Bündel umzusetzen sind.

\section{c) Möglichkeiten und Bedingungen der Umsetzung}

Die beschriebenen Problemlagen und Interessenunterschiede der beteiligten Gruppen machen es erforderlich, daß die Maßnahmen sowohl an den Unternehmenszielen als auch an den Bedürfnissen der Arbeitspersonen ausgerichtet sein müssen, um wirkungsvoll umgesetzt werden zu können. Diesbezüglich ist auf die Notwendigkeit einer allseitigen Zusammenarbeit und Kompromißbereitschaft hinzuweisen. Die Handlungsspielräume innerhalb der Unternehmen sind dabei besonders aus Wettbewerbsgründen begrenzt.

Die Voraussetzungen für die erfolgreiche praktische Umsetzung der Maßnahmen sind teilweise sehr hoch. So hängt beispielsweise die Bereitschaft und Fähigkeit im späteren Erwerbsleben erfolgreich an Weiterbildungsmaßnahmen teilzunehmen vom 
erreichten Aus- und Weiterbildungsniveau und der Gewöhnung an lebenslanges Lernen ab. Weiterhin beeinflussen die Beanspruchungen während des gesamten Erwerbslebens die Möglichkeit, die Lebensarbeitszeit verlängern zu können. Demnach müssen die Maßnahmen früh genug ergriffen werden, um rechtzeitig wirksam zu werden. Die Vorlaufzeit der Maßnahmenwirkung wird neben der Erwerbsbiographie der Arbeitspersonen besonders von der jeweiligen Dauer des Wandels und der Gewöhnungsprozesse, von der Entscheidungsgeschwindigkeit innerhalb der Unternehmen sowie von der Einigungsgeschwindigkeit der Tarifpartner bestimmt.

Da die demographisch bedingten quantitativen Defizite langfristig auftreten und das Arbeitskräfteangebot erst in ca. 20 Jahren drastisch zurückgehen wird, erscheint einzelwirtschaftlich in einer kurz- bis mittelfristigen Betrachtung die Umsetzung der Maßnahmen möglicherweise als noch nicht notwendig. Aufgrund der langen Vorlaufzeit und der nur langfristigen Wirkungsentfaltung eines Teils der Maßnahmen ist die Verzögerung ihrer Umsetzung allerdings, zumindest aus gesamtwirtschaftlicher Sicht, als nachteilig zu bezeichnen. Staatliche Anreize für eine rechtzeitige Umsetzung der Maßnahmen zur Einrichtung alters- und frauengerechter Arbeitsplätze können zur Minderung der prognostizierten Defizite des Arbeitskräfteangebots beitragen, kontraproduktiv wirkende Handlungsweisen in den Unternehmen vermeiden helfen und zum gesamtwirtschaftlichen Nutzen verschiedene arbeitsmarktpolitische Zielsetzungen miteinander verbinden.

\section{Maßnahmen der Arbeitsgestaltung}

\section{a) Aufgabenbearbeitung}

\section{(1) Erfahrungswissen}

Diese Maßnahme bezieht sich darauf, daß die während des Erwerbslebens durchgeführte Arbeit und das während der Arbeitsdurchführung erworbene Erfahrungswissen die Möglichkeiten Älterer, erwerbstätig zu sein, in hohem Maße bestimmen. Die Förderung des Aufbaus und der Nutzung des beruflichen Erfahrungswissens richtet sich auf eine altersübergreifende Gestaltung der Aufgabenbearbeitung mit dem Ziel der Verbesserung der Möglichkeiten der Älteren, erwerbstätig zu sein. Die Umsetzung dieser Maßnahme soll dazu beitragen, daß das Erfahrungswissen und damit ein möglicher Kompetenzvorteil älterer 
Arbeitspersonen an Bedeutung gewinnt und zudem ihre Bereitschaft, länger zu arbeiten, unterstützt wird. Außer dieser Leistung im Sinne einer prospektiven Arbeitsgestaltung können präventive, fehlbeanspruchungsmindernde Wirkungen erzielt werden, indem während des Berufslebens Belastungswechsel und eine aufgabenadäquate Qualifizierung ermöglicht werden. Daneben kann ein betriebswirtschaftlich relevanter Effekt erreicht werden, da Arbeitspersonen mit Erfahrungswissen besonders geeignet sind, kritische Situationen (wie z.B. die Anpassung von Innovationen, die Optimierung von Prozessen sowie die Begrenzung und Behebung von Störfällen) zu bewältigen (vgl. ROSE, 1995).

Neben theoretischen Komponenten der beruflichen und fachlichen Grundqualifikation erfordert die Arbeitsdurchfuhrung in unterschiedlichem Maße die Beherrschung eines "spezifischen Handlungswissens, das durch praktische sinnliche Erfahrung und ganzheitliche Arbeitshandlungen erworben und eingesetzt wird. Es entsteht beim Vollzug von Handlungen und ist deshalb eine besondere Form praktischer Erfahrung, die sich am treffendsten als implizites Erfahrungswissen bezeichnen läßt" (ROSE, 1992a, 146). Dieses Erfahrungswissen betrifft die realen Abläufe in Produktion und Verwaltung und muß ebenso wie theoretisches Wissen erworben, entwickelt und erhalten werden. Insbesondere moderne Produktions- und Organisationsmethoden sind, angesichts häufiger organisatorisch-technischer Innovationen und der durch den Mitarbeitereinsatz zu erreichenden Effizienzziele, auf den Beitrag der Arbeitspersonen zur Flexibilisierung der Produktion und zur Sicherstellung der Qualität ausgerichtet. Das hierzu erforderliche Leistungs- und Arbeitspotential der Beschäftigten kann durch den Aufbau und die Nutzung des Erfahrungswissens erhöht werden.

Die Arbeitsaufgaben müssen in diesem Sinne 'entwicklungsförderlich' gestaltet sein (vgl. SCHUL, 1993, 31ff.). Dabei ordnet sich die Bedeutung der Entwicklungsförderlichkeit ein zwischen der Trainingswirksamkeit, die sich auf die maximale Ausbildung und Nutzung individueller Fähigkeiten und Fertigkeiten bezieht, und der Persönlichkeitsförderlichkeit, die die Möglichkeiten zur persönlichen Weiterentwicklung, die Arbeitsmotivation, die Einstellungen, die Bedürfnisse, die Leistungsbereitschaft, die Befähigung zur Verantwortungsübernahme und das Verhalten der Arbeitspersonen betrifft. Durch die Ausübung entwicklungsförderlich gestalteter Aufgaben werden Arbeitspersonen dazu befähigt und motiviert auch in der Spätphase der Erwerbstätigkeit, unter Nutzung des erworbenen Erfahrungswissens, anspruchsvolle Tätigkeiten auszuführen. Die Erreichung und Sicherung der Entwicklungsförderlichkeit der Arbeitsaufgaben muß angesichts des dargestellten 
Wandels der Arbeitsanforderungen und auch der Erwerbstätigenzusammensetzung zum Ziel der Arbeitgeber werden. Eine erfolgreiche Umsetzung dieser Maßnahme kann in Kombination mit Weiterbildungsanstrengungen die Abhängigkeit der Unternehmen vom Arbeitskräftenachwuchs entschärfen.

\section{- Gestaltungsformen}

Die Entwicklungen im Produktions- und im Verwaltungsbereich, insbesondere die Nutzung moderner Informations- und Kommunikationssysteme, lassen eine veränderte Arbeitsgestaltung zu. Arbeitsablauf, -ort und -zeit lassen sich flexibel und zum Teil ohne strikte Bindung an die Betriebszeiten, gestalten. Durch ablaufbezogene und zeitliche Flexibilisierung und örtliche Dezentralisierung können die Anforderungen der Erwerbsarbeit und individuelle Eigenschaften oder Präferenzen eher aneinander angepaßt werden. Eine solche differenzierend wirkende Entwicklung der Arbeit folgt der fortschreitenden Individualisierung der Gesellschaft und verstärkt diese zusätzlich.

Zur Gestaltung entwicklungsförderlicher Tätigkeiten sind verschiedene Empfehlungen zu beachten (vgl. ROSE, 1992a und 1992b). Die Zusammenführung vollständiger Handlungen zu einer Arbeitsaufgabe, die den Arbeitspersonen Freiheitsgrade hinsichtlich der Abfolge der einzelnen Tätigkeiten läßt, erleichtert den Aufbau umfassender Qualifikationen. Einfache und kurzzyklische Arbeitsgänge widersprechen dagegen dem Bedürfnis der Selbstentfaltung. So bedeutet eine ganzheitliche Aufgabenbearbeitung, daß die Arbeitspersonen Tätigkeiten der Planung, gegebenenfalls der Programmerstellung, der Ausführung, der Steuerung, der Überwachung und der Ergebnisbewertung sowie der Störungsanalyse und der Störungsbehebung ausüben. Hierdurch kann ein übergreifendes, viele Assoziationen ermöglichendes Erfahrungswissen aufgebaut werden. Freiheitsgrade können zur Erprobung alternativer Vorgehensweisen genutzt werden und tragen somit zur Sicherung und Erweiterung des Erfahrungswissens bei.

Qualifikationsfördernd gestaltete Arbeitsaufgaben bieten den Arbeitspersonen bei geringem Prozeßdruck gute Informations-, Beteiligungs- und Eingriffsmöglichkeiten. Die Nutzung von Computern zur Steuerung von Bearbeitungsprozessen und zur Steuerung und Überwachung ganzer Prozeßketten kann dadurch entwicklungsförderlicher gestaltet werden, daß den Arbeitspersonen direkte steuernde und regulierende Eingriffsmöglichkeiten zur Verfügung stehen. Hinsichtlich der verwendeten Programme sind vielfältige Simulationsmöglichkeiten zu schaffen. 
Weiterhin sollten Möglichkeiten zur direkten Informationsaufnahme, beispielsweise von Bearbeitungsgeräuschen und Abfragemöglichkeiten für interessierende Prozeßdaten gegeben werden (vgl. beispielsweise ROSE, 1992b, 25f.). Simulationsverfahren und Expertensysteme sind zur Unterstützung der Arbeitspersonen in Standardsituationen zu verwenden, ohne die Entwicklung des zur Sicherung der Innovationsfähigkeit und der Flexibilität notwendigen Erfahrungswissens zu behindern. Für die erfahrungsgeleitete Arbeit an NC-Maschinen sind in folgender Abbildung wesentliche Merkmale der notwendigen Technikentwicklung zusammengestellt.

\begin{tabular}{|c|c|c|}
\hline $\begin{array}{c}\text { Erhöhung } \\
\text { der } \\
\text { ProzeBtransparenz }\end{array}$ & $\begin{array}{l}\text { Verbesserung und } \\
\text { Vereinfachung des } \\
\text { Prozeßeingriffs }\end{array}$ & $\begin{array}{c}\text { Maschinennahe } \\
\text { Programmerstellung/ } \\
\text { Programmkorrektur }\end{array}$ \\
\hline $\begin{array}{l}\text { Konstruktive Maßnah- } \\
\text { men an den Werkzeug- } \\
\text { maschinen zur direkten } \\
\text { Wahrnehmung von Pro- } \\
\text { zeßäußerungen } \\
\text { Nutzung von Sensoren } \\
\text { auf der Grundlage neuer } \\
\text { analogischer Darstel- } \\
\text { lungsformen von Pro- } \\
\text { zeßäußerungen (optisch, } \\
\text { akustisch, taktil) } \\
\text {... }\end{array}$ & $\begin{array}{l}\text { Erweiterte Funktionen } \\
\text { des Handbedienpults } \\
\text { Nutzung manueller Ein- } \\
\text { gaben } \\
\text { On-Line-Speicherung } \\
\text { von Technologiewerten } \\
\text { Vereinfachtes Abfahren } \\
\text { von Programmabschnit- } \\
\text { ten z.B. nach Werk- } \\
\text { zeugkorrektur } \\
\text {... }\end{array}$ & $\begin{array}{l}\text { Einfache Nutzung von } \\
\text { Zyklen und Makros } \\
\text { - Teach-In-Verfahren } \\
\text { Verbesserung der Les- } \\
\text { barkeit von Program- } \\
\text { men } \\
\text { Vereinfachte Möglich- } \\
\text { keiten zur Programm- } \\
\text { korrektur } \\
\text {... }\end{array}$ \\
\hline
\end{tabular}

Abb. 56: Schwerpunkte technischer Entwicklungen für die Unterstützung erfahrungsgeleiteter Arbeit in der Werkstatt (Quelle: LENNARTZ, 1993, 75)

Die Förderung des Aufbaus und der Nutzung des Erfahrungswissens während der gesamten Erwerbstätigkeit muß weiterhin Überlegungen zum lebenslangen Lernen aufgreifen (vgl. beispielsweise KÖCHLING, 1992, 81). Eine Verflechtung von Qualifizierung und Arbeitstätigkeit ist ebenso zu fördern wie ein planmäßiger Wechsel zwischen Arbeits- und Lernphasen im Berufsverlauf. So könnte zukünftig eine umfassende und langfristige Berufslaufbahnplanung eine lebenslange Qualifizierung mit phasenweise gestalteten Arbeitszeiten und Arbeitsanforderungen verknüpfen, so daß die Möglichkeit und die Bereitschaft, länger erwerbstätig zu sein, gefördert werden (vgl. NAEGELE, 1994). Ergänzend unterstützt ein planmäßiger Wechsel der auszuführenden Tätigkeiten eine arbeitsplatzübergreifende und praxisgerechte Qualifizierung. Zudem müssen berufsbegleitende Weiterbildungsmaßnahmen theoretische Kenntnisse und umfassende Kompetenzen vermitteln, die die berufliche Qualifikation und das Erfahrungswissen berücksichtigen. Eine Zielgruppenansprache und Beratung können die individuelle und gruppen- 
spezifische Anpassung der Lerninhalte, die Klärung von Anwendungs- und persönlichen Entwicklungsperspektiven und die Lernmotivation unterstützen; eine entsprechende Schulung von Führungskräften ist hierfür zu empfehlen. Der Erhalt und die Entwicklung der Qualifikation stärken die Anpassungsfähigkeit und stabilisieren die Leistungsfähigkeit der Arbeitspersonen. Diese Effekte wirken sich insbesondere positiv auf die langfristige Nutzung der Humanressourcen und die Erwerbsmöglichkeiten in höheren Altersklassen aus.

Wesentlich für die Sicherung und Erweiterung des Erfahrungswissens ist der Austausch der Erfahrungen. Im Falle der Einzelarbeit sollten ausreichende inhaltliche Notwendigkeiten und zeitliche Möglichkeiten zur Kooperation zwischen den Arbeitspersonen gesichert werden. Die zunehmende Verbreitung der Gruppenarbeit kann organisatorisch zum Aufbau des Erfahrungswissens beitragen, indem die Erfahrungen, die auf mehrere Personen verteilt sind, individuell genutzt werden können und der mögliche interpersonelle Austausch von Erfahrungen die Aktualität und Relevanz des Wissens fördert. Die gegenseitige Unterstützung im Rahmen der Gruppenarbeit kann den Umfang der notwendigen Weiterbildungsmaßnahmen und auftretende psychische Beanspruchungen mindern. Andererseits erfordert eine gruppenorientierte Nutzung des Erfahrungswissens entsprechende Technikentwicklungen, beispielsweise hinsichtlich der Softwarenutzung, und organisatorische Regelungen, beispielsweise bezüglich der Aufgabenverteilung und der Verantwortungszuordnung (vgl. ROSE, 1992a, 145f;; 1993 und 1995).

Die Entwicklung des Erfahrungswissens und die Überwindung der Arbeitsteilung zur Nutzung der Vorteile dezentraler Organisationsweisen können einander fördern. Die Beibehaltung tayloristisch gestalteter, zentral kontrollierter Arbeitsaufgaben bietet den Arbeitspersonen demgegenüber zu geringe Kontroll- und Handlungsmöglichkeiten, um umfassendes Erfahrungswissen aufzubauen. Nachteilig in dieser Hinsicht sind sowohl technikorientierte Konzepte, die die Einflußnahme der Arbeitspersonen ersetzen wollen als auch mitarbeiterorientierte Konzepte, die die Leistungspotentiale der Beschäftigten gezielt, aber nur einseitig ausschöpfen. Eine nachteilige Arbeitssituation läßt sich exemplarisch für die an Bedeutung gewinnenden Überwachungsaufgaben beschreiben. Eine Tätigkeit, die fallweise (Störungen, Umstellungen, Reparaturen) hohe qualifikatorische Anforderungen stellt, läßt den Arbeitspersonen gleichzeitig im normalen Arbeitsablauf nur geringe Handlungs- und Entscheidungsspielräume. Die Arbeitspersonen können in diesem Fall durch die Arbeitsausführung nur in geringem Maße Handlungsweisen trainieren und Kompetenzen entwickeln, die sie dazu befähigen, auftretende Anforderungen durch 
erfahrungsgeleitetes, autonomes Handeln zu mindern. Die geringen Möglichkeiten zur Entwicklung und Einübung adäquater Handlungs- und Reaktionsweisen lassen fallweise überfordernde Beanspruchungen der Arbeitspersonen befürchten.

Negativ auf die Entwicklungsförderlichkeit der Tätigkeiten wirkt sich der Verlust von Erfahrungsmöglichkeiten durch den Abbau steuernder und regelnder Eingriffe aus. Durch das immer mehr zunehmende Einschieben von technischen Medien, symbolischen Informationen und abstrakten Vorgaben zwischen das Produktionsgeschehen und die menschlichen Sinne, kommt es zu einer Entsinnlichung der Tätigkeit und den Verlust konkreter Vorstellungen und praktischer Erfahrungen. Diese technische Mediatisierung der Arbeitsaufgaben (und auch der Beziehungen der Arbeitspersonen untereinander) resultiert wesentlich aus der vielfältigen Nutzung von Kommunikations- und Informationstechniken, beispielsweise in numerisch gesteuerten Verfahrensketten und in der Prozeßleittechnik. Am Beispiel der zu den 'konventionellen' Informationstechniken hinzukommenden Expertensysteme können die möglichen Wirkungen technischer Innovationen auf das Erfahrungswissen noch einmal dargestellt werden. Werden Expertensysteme eingeführt, um die Leistungen speziell qualifizierter Arbeitskräfte zu ersetzen, so lassen sich besondere qualifikationsbezogene Risiken und Probleme befürchten (vgl. LUTZ/MOLDASCHL, 1989). Da Expertensysteme bei automatisierten Prozeßabläufen beispielsweise für die Instandhaltung notwendige Entscheidungen und Diagnosen treffen (sollen), werden hierdurch menschliche Interventionsleistungen sowie Qualifizierungschancen und -anreize abgebaut. Das Erfahrungswissen, das zur Reaktionssicherheit in unvorhergesehenen Situationen beiträgt, wird durch die Anwendung der Expertensysteme abgebaut bzw. nicht mehr neu erworben, obwohl es als Ergänzung oder zur Korrektur der Leistungen der Expertensysteme weiterhin benötigt würde. Bei Störfällen und in Ausnahmesituationen müßten Qualifikationskomponenten eingesetzt werden, die durch die Einführung von Expertensystemen im Normalbetrieb nicht mehr erworben werden konnten.

Die positiv angesprochenen objektiven Bedingungen der Arbeitssituation können entwicklungs- und qualifikationsfördernd eingeschätzt werden. Allerdings sichern sie allein nicht die individuelle Wahrnehmung dieser Möglichkeiten durch die Arbeitsperson. Neben dem sozialen Klima und dem Führungsstil stellt besonders die Mitbestimmung der Arbeitspersonen am Arbeitsplatz, im Sinne einer Partizipation an Entscheidungen oder an der Entwicklung von arbeitsbezogenen Veränderungen, einen Beitrag zur Förderung der Nutzung der gegebenen Möglichkeiten dar. Der einzelne erlebt hierdurch in positiver Weise die Bedeutung der Arbeitstätigkeit, 
seiner Leistungsbeiträge sowie die Verbesserbarkeit und Beeinflußbarkeit der Arbeitssituation, so daß Qualifizierungsangebote verstärkt angenommen werden (vgl. FREI u.a., 1984, 110ff.).

\section{- Betroffene Kosten und Nutzengrößen}

Die Umsetzung dieser auf die Entwicklung und die Nutzung des Erfahrungswissens der Arbeitspersonen gerichteten Maßnahme betrifft verschiedene Kosten- und Nutzengrößen.

Kosten:

- Verzicht auf den Abbau der Beschäftigung qualifizierter Arbeitspersonen.

- Verzicht auf kurzfristig, beispielsweise durch umfassende Automatisierungsmaßnahmen, erreichbare Einsparungen.

- Tendenzielle Erhöhung der Lohnkosten, da durchschnittlich höhere Qualifikationen erforderlich werden und durch eine entsprechend ausgerichtete Entlohnung Anreize zum Erwerb von Qualifikationen gegeben werden können.

Nutzen:

- Wird das Erfahrungswissen der Arbeitspersonen genutzt, so kann durch einen Abbau qualitativer Unterforderung eine Verbesserung der Produktivität erreicht werden, ohne negativ zu bewertende Beanspruchungen zu erhöhen (vgl. beispielsweise ULICH, 1988, 122f.).

- Die ermöglichte Nutzung dezentral vorhandener Qualifikationen, Erfahrungen und Entscheidungskompetenzen leistet einen Beitrag zur Flexibilisierung der Arbeit. Hierdurch dürften weiterhin die Maschinennutzung, die Rüst- und Stillstandszeiten sowie die Durchlaufzeit positiv beeinflußt werden.

- Die zu erwartende Erhöhung der Einsatzflexibilität der Arbeitspersonen verbessert die Nutzung vorhandener Personalkapazitäten.

- Minderung der für Innovationen aufzuwendenden Kosten, da durch den Aufbau von Qualifikationsreserven die Anpassung und Nutzung neuer Techniken und Verfahren reibungsloser verlaufen können und die Motivation der Arbeitspersonen höher sein dürfte. 
Eine globale Beurteilung der in den Unternehmen zu erwartenden Auswirkungen der Umsetzung der verbesserten Möglichkeiten zur Entwicklung und Nutzung des Erfahrungswissens zeigt sich in der folgenden Abbildung.

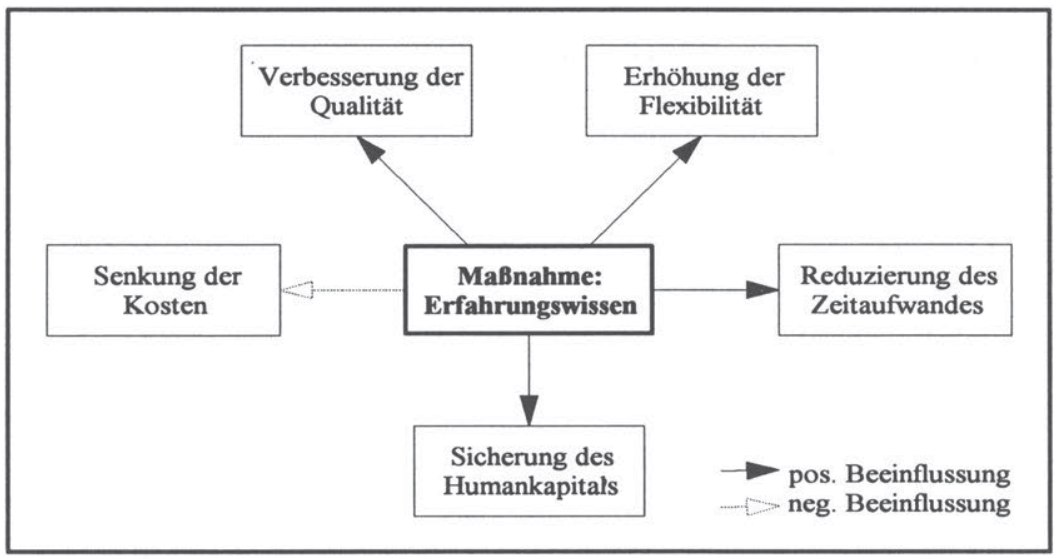

Abb. 57: Zielbeeinflussung durch die Umsetzung der Maßnahme Erfahrungswissen

Auch die übrigen Maßnahmen werden in dieser Form eingeschätzt werden. Die Einschätzung der Auswirkungen geht von der Umsetzung der für jede Maßnahme dargestellten Empfehlungen aus. Dabei ist die positive Beurteilung hinsichtlich des Ziels der Sicherung des Humankapitals selbstverständlich, da die Maßnahmen in vorliegender Arbeit zu diesem Zweck abgeleitet und gestaltet wurden. Eine detailiertere Bewertung der Beeinflussungsgrößen kann in den Unternehmen nur durch die Analyse konkreter Arbeitssysteme erfolgen. Die fast durchweg negative Einschätzung der Auswirkungen auf das Ziel der Kostensenkung bezieht sich auf die eher direkt und kurzfristig einschätzbaren Konsequenzen der Maßnahmenumsetzung. In einer langfristigen, umfassenden Betrachtung kann sich - in Abhängigkeit vom spezifischen Erfolg der Maßnahmen und von der individuellen Beschäftigungsdauer - auch bezüglich der Kostenwirkung ein günstigeres Bild ergeben.

\section{- Umsetzungsbedingungen}

Die dargestellten Leitvorstellungen insbesondere der Lean Production entsprechen den hier vorgeschlagenen Merkmalen einer erfahrungsbezogenen Aufgabenbearbeitung, sofern sie sich auf die Entwicklung und Ausschöpfung der Arbeits- und 
Leistungspotentiale beziehen. Allerdings muß sich die Gestaltung einer erfahrungsorientierten Aufgabenbearbeitung auf eine möglichst breite Nutzung und Entwicklung der Kompetenzen der Arbeitspersonen richten und autonomes Handeln auf allen Stufen der Beschäftigung fördern.

Während die Lean Production besonders auf eine Straffung der Arbeitsabläufe gerichtet ist, müssen zur Entwicklung des Erfahrungswissens Freiheitsgrade eingeräumt werden. Sofern Facharbeiter nicht entsprechend ihrer Ausbildung eingesetzt werden, kann der Wunsch nach einer besseren Nutzung bereits vorhandener Qualifikationen, d.h. nach einer qualifikationsadäquaten Beschäftigung, die Umstrukturierung im Sinne einer entwicklungsförderlichen Arbeit unterstützen. Der Abbau der Vergeudung von Humankapital geschieht im Interesse der Arbeitgeber, der Arbeitnehmer und im gesamtwirtschaftlichen Interesse. Angesichts der voraussichtlichen Arbeitsangebotsdefizite muß das Wissen der Arbeitspersonen kontinuierlich entwickelt werden. Hierzu trägt die angesprochene Gestaltung der Arbeit bei. Zur Einschätzung des Nutzens der erreichten Qualifizierung darf die individuell vorhandene Qualifikation der Arbeitspersonen immer weniger mit ihrer formalen Qualifikation gleichgesetzt werden, damit die Komponente des Erfahrungswissens genügend Beachtung finden kann.

Die Umsetzung der Maßnahme erleichtert die Überwindung tayloristischer Arbeitsstrukturen, indem sie einem Mangel qualifizierter Arbeitskräfte entgegenwirkt. Ein solcher Mangel würde dagegen - wie dargestellt - zu einer zukünftigen Durchsetzung neo-tayloristischer Rationalisierungsstrategien beitragen.

Zur Umsetzung der Maßnahme ist eine langfristige Personalplanung notwendig. Die Arbeitsgestaltung, Technikentwicklung und Personalentwicklung müssen integriert und möglichst früh in die Arbeitssystementwicklung einbezogen werden. Obwohl die unternehmensinterne altersübergreifende Gestaltung nicht durch äußere Einflußnahme bestimmt werden sollte, stellt eine ausgebaute staatliche Förderung der beruflichen Ausbildung, die Fortbildungsmaßnahmen unterstützt und Beratungsleistungen erbringt, einen Beitrag zur Schaffung der Voraussetzungen für die Umsetzung dieser Maßnahme dar. 


\section{(2) Gruppenarbeit}

Die Gruppen- bzw. Teamarbeit ist ein zentraler Punkt moderner Produktions- und Organisationskonzepte. Besonders die Diskussion der Lean Production führt dazu, daß die Idee der Gruppenarbeit als besondere Form der Aufgabenbearbeitung zunehmend an Bedeutung gewinnt. Zur Sicherung der zukünftigen Leistungsfähigkeit der Arbeitspersonen muß die Gruppenarbeit allerdings so gestaltet werden, daß Fehlbeanspruchungen vermindert und Entwicklungsmöglichkeiten gefördert werden. Eine beanspruchungsoptimierte Gestaltung der Aufgabenbearbeitung muß auf die Minderung des Arbeitsdrucks und die Verbesserung der Integration von nicht hochleistungsfähigen Personen in die Arbeitsgruppen gerichtet sein und zugleich die betrieblichen Möglichkeiten zur Schaffung von Einzelarbeitsplätzen berücksichtigen. Ein solches Verständnis der Gruppenarbeit weist über die Vorstellungen der Lean Production hinaus, indem ältere Konzeptionen zur Humanisierung der Arbeit aufgegriffen und zieladäquat weiterentwickelt werden.

\section{- Gestaltungsformen}

Der Begriff 'Gruppenarbeit' kann ganz unterschiedlich gestaltete Arbeitsweisen bezeichnen und aus unterschiedlichen Interessen eingeführt werden. Infolgedessen unterscheiden sich die Arbeitsanforderungen an die Arbeitspersonen, deren Beanspruchungen und die Möglichkeiten bestimmte Personengruppen langfristig zu beschäftigen. Gruppenarbeit in der Fertigung bedeutet beispielsweise bei Toyota Fließbandfertigung mit kurzen Taktzeiten, genaue Tätigkeitsvorgaben und job rotation hauptsächlich zur Erhöhung der Einsatzflexibilität; bei Volvo dagegen eine Form der Werkstattfertigung mit relativ hoher Gruppenautonomie (vgl. WENKER, 1994). Das in Deutschland vorhandene und besonders von schwedischen Erfahrungen beeinflußte Leitbild einer gruppenbezogenen Arbeitsgestaltung weicht beispielsweise dadurch von japanischen Vorstellungen $a b, d a ß$ eine größere Teilautonomie hinsichtlich der Arbeitsausführung gewährleistet werden soll. Insbesondere bezogen auf die Ebene der Facharbeiter wird ein möglichst qualifikationsgerechter Einsatz angestrebt. Die Beschäftigung in homogenen Teams und ein häufig wechselnder Arbeitseinsatz bei kurzzyklischen Aufgaben widersprechen der in Deutschland verbreiteten Ausbildungsstruktur und den verbreiteten beruflichen Erwartungen.

Da die Lean Production mit Blick auf Wettbewerbsvorteile Japans abgeleitet wurde, gibt folgende Abbildung in pointierter Weise 'japanische' (toyotistische, schlanke 
oder restriktive) und 'europäische' (human-zentrierte oder teil-/autonome) Vorstellungen hinsichtlich der Gruppenarbeit wieder (vgl. JÜRGENS, 1993; WEBER, 1994 und auch mit gesellschaftlichem Bezug ADAMI, 1994; ESSER/NAKAJIMA, 1994; HEIDENREICH, 1994).

\begin{tabular}{|c|c|}
\hline verbreitete 'japanische' Vorstellungen & verbreitete 'europäische' Vorstellungen \\
\hline $\begin{array}{l}\text { Gruppenarbeit wird im Interesse der Personal- } \\
\text { entwicklung zur Steigerung der Produktivität } \\
\text { und der Arbeitseffektivität verwendet. }\end{array}$ & $\begin{array}{l}\text { Gruppenarbeit ist das Ergebnis der Arbeitsge- } \\
\text { staltung im Interesse der Rationalisierung, der } \\
\text { Humanisierung und der Arbeitsattraktivität. }\end{array}$ \\
\hline $\begin{array}{l}\text { Prozeßorganisation } \\
\text { geringe Teilautonomie, Prozeßdruck z.B. } \\
\text { durch Just-In-Time-Organisation und Auf- } \\
\text { rechterhaltung der Taktbindung }\end{array}$ & $\begin{array}{l}\text { Aufgabenstrukturierung } \\
\text { hohe Teilautonomie durch Gestaltung des } \\
\text { Arbeitsablaufs und der Technik, Pufferbil- } \\
\text { dung, Entkopplung, Zeitsouveränität }\end{array}$ \\
\hline $\begin{array}{l}\text { standardisierte, repetitive Tätigkeit } \\
\text { Vereinheitlichung der Arbeitsvorgänge } \\
\text { Tätigkeiten auf gleichem Anforderungsniveau }\end{array}$ & $\begin{array}{l}\text { Handlungsspielräume, planerische Funktionen } \\
\text { individuelle Verantwortung, selbständiges } \\
\text { Handeln der Gruppenmitglieder }\end{array}$ \\
\hline $\begin{array}{l}\text { Freiheitsgrade und Gestaltungsraum bietet nur } \\
\text { die Beteiligung am Verbesserungsproze } B \text {. }\end{array}$ & $\begin{array}{l}\text { Selbstregulierung und Selbstorganisation bzgl. } \\
\text { der Auftragsreihenfolge und der Zeitplanung }\end{array}$ \\
\hline $\begin{array}{l}\text { Gruppendisziplin, Gruppe als Sanktions- } \\
\text { instanz, Selbstkontrolle }\end{array}$ & $\begin{array}{l}\text { Selbstdisziplin der Gruppenmitglieder, } \\
\text { Selbstbestimmung }\end{array}$ \\
\hline $\begin{array}{l}\text { Angelernte } \\
\text { qualifikatorisch homogene Teams, } \\
\text { Austauschbarkeit der Mitglieder }\end{array}$ & $\begin{array}{l}\text { Facharbeiter und Angelernte } \\
\text { gemischte Teams, unterschiedliche Ausbil- } \\
\text { dungen zur Nutzung von Synergieeffekten }\end{array}$ \\
\hline $\begin{array}{l}\text { Personalbewertungssystem beeinflußt Bonus- } \\
\text { zahlungen und Aufstieg; } \\
\text { das Verhalten in der Gruppe entscheidet über } \\
\text { Beförderung, Lohn und persönliche Ent- } \\
\text { wicklungsmöglichkeiten. }\end{array}$ & $\begin{array}{l}\text { Lohnsystem mit Anreizfunktion zur Verbesse- } \\
\text { rung der Motivation und der Arbeitsleistung } \\
\text { sowie zur Verringerung der Fehlzeiten; ange- } \\
\text { strebt wird eine möglichst hohe Akzeptanz der } \\
\text { Gruppenarbeit bei den Beschäftigten. }\end{array}$ \\
\hline $\begin{array}{l}\text { Beteiligungsmöglichkeiten primär zur Ver- } \\
\text { besserung der Abläufe und Produkte }\end{array}$ & $\begin{array}{l}\text { betriebliche und arbeitsplatzbezogene Mitbe- } \\
\text { stimmung }\end{array}$ \\
\hline
\end{tabular}

Abb. 58: Idealtypische Vorstellungen zur Gestaltung und Wirkung der Gruppenarbeit

Aufgrund der Mitarbeiterbezogenheit der Lean Production und der Betonung der Bedeutung der Gruppenarbeit kann die Annahme bzw. Hoffnung entstehen, daß mit der Umsetzung dieses Konzeptes sowohl wirtschaftliche als auch menschliche Bedürfnisse in einem neuen Maße erfüllt werden können (vgl. bereits WOMACK u.a., 1991; WENKER, 1994) und zukünftig höhere Qualität und niedrigere Kosten mit Arbeitsaufgaben einhergehen, die Humanisierungsforderungen besonders gut erfüllen. Bezogen auf die Gruppenarbeit kann allerdings anhand wesentlicher Grundsätze der arbeitswissenschaftlichen Arbeitsgestaltung dargestellt werden, daß zwar in der Lean Production der gleiche Begriff wie im Rahmen der Humani- 
sierungsforschung verwendet wird, sich die konkreten Formen der Arbeitsgestaltung jedoch unterscheiden. Die Lean Production konzentriert sich darauf, die Leistungspotentiale der Arbeitspersonen im Interesse der Wettbewerbsfähigkeit möglichst auszuschöpfen und beachtet in zu geringem Maße die Folgen für die Anforderungen an die Arbeitspersonen (vgl. HOWALDT/KOPP, 1992). Bevor näher auf die empfehlenswerten beanspruchungsmindernden Gestaltungsvorstellungen eingegangen wird, zeigt die folgende Gegenüberstellung die Idee der Gruppenarbeit idealtypisch in den betrachteten Konzepten der Produktions- und Organisationsmethoden und einer arbeitswissenschaftlich geprägten Vorstellung.

\begin{tabular}{|c|c|c|}
\hline \multicolumn{3}{|c|}{ Die Einordnung der Idee der Gruppenarbeit } \\
\hline CIM-Konzept & $\begin{array}{c}\text { Konzept der } \\
\text { Lean Production }\end{array}$ & $\begin{array}{c}\text { arbeitswissenschaftlicher } \\
\text { Ansatz }\end{array}$ \\
\hline technikorientiert & & $\begin{array}{l}\text { wirtschaftlich und human- } \\
\text { orientiert }\end{array}$ \\
\hline $\begin{array}{l}\text { Die Produktivitätssteige- } \\
\text { rungen sollen vor allem durch } \\
\text { den Einsatz technischer } \\
\text { Systeme und deren Ver- } \\
\text { knüpfung erreicht werden. } \\
\text { Zur Gewährleistung dieser } \\
\text { Produktivitätsgewinne werden } \\
\text { zunehmend gruppenbezogenen } \\
\text { Arbeitsstrukturen empfohlen. }\end{array}$ & $\begin{array}{l}\text { Die Produktivitătssteige- } \\
\text { rungen sollen vor allem durch } \\
\text { den Einsatz der Mitarbeiter } \\
\text { erreicht werden. Die gruppen- } \\
\text { bezogenen Arbeitsstrukturen } \\
\text { bewirken Produktivitäts- } \\
\text { gewinne durch die erhöhte } \\
\text { Ausnutzung der Leistungs- } \\
\text { făhigkeiten der Mitarbeiter. }\end{array}$ & $\begin{array}{l}\text { Die Arbeitspersonen sollen } \\
\text { entsprechend ihrer natürlichen } \\
\text { Grenzen so eingesetzt werden, } \\
\text { daß sich ihre Fähigkeiten voll } \\
\text { entfalten können und wirt- } \\
\text { schaftlich hieraus der höchste } \\
\text { Nutzen aus ihnen gezogen } \\
\text { werden kann. Gruppen- } \\
\text { bezogene Arbeitsstrukturen } \\
\text { tragen dazu bei, das Human- } \\
\text { kapital zu sichern. }\end{array}$ \\
\hline $\begin{array}{l}\text { Gruppenarbeit bedeutet für } \\
\text { das CIM-Konzept } \\
\text { - die Minderung der } \\
\text { Komplexität zentraler } \\
\text { Planungs-, Steuerungs- } \\
\text { und Entscheidungs- } \\
\text { prozesse, } \\
\text { die (Re-) Dezentrali- } \\
\text { sierung von Kompetenzen, } \\
\text { die Sicherung des } \\
\text { dezentralen Erwerbs von } \\
\text { Kompetenzen. }\end{array}$ & $\begin{array}{l}\text { Gruppenarbeit bedeutet für } \\
\text { das Konzept der Lean } \\
\text { Production } \\
\text { - die Dezentralisierung von } \\
\text { Planungs-, Steuerungs- } \\
\text { und Entscheidungs- } \\
\text { prozessen, } \\
\text { die Nutzung dezentral } \\
\text { vorhandener Kompetenzen } \\
\text { (im Verbesserungsprozeß), } \\
\text { Personalentwicklung und } \\
\text { Sanktionsinstanz. }\end{array}$ & $\begin{array}{l}\text { Teilautonome Gruppenarbeit } \\
\text { bedeutet } \\
\text { - eine grundlegende, auf } \\
\text { Kooperation angelegte } \\
\text { Änderung der Arbeits- } \\
\text { aufgabe, } \\
\text { eine menschengerechte } \\
\text { Form der Arbeitsge- } \\
\text { staltung, } \\
\text { - die aufgabenbereichsweite } \\
\text { Selbstbestimmung der } \\
\text { Gruppe. }\end{array}$ \\
\hline $\begin{array}{l}\text { Gruppenarbeit zur 'Wieder- } \\
\text { einbeziehung' der Mitarbeiter } \\
\text { und ihrer spezifischen Lei- } \\
\text { stungen. }\end{array}$ & $\begin{array}{l}\text { Gruppenarbeit zur } \text { intensiven } \\
\text { Nutzung der } \text { Leistungs- } \\
\text { potentiale der Mitarbeiter. }\end{array}$ & $\begin{array}{l}\text { Gruppenarbeit zur Berück- } \\
\text { sichtigung der Bedürfnisse der } \\
\text { Mitarbeiter. }\end{array}$ \\
\hline
\end{tabular}

Abb. 59: Einordnung der Gruppenarbeit in arbeits- bzw. produktionsund organisationsbezogene Vorstellungen 
Obwohl die Einführung der Gruppenarbeit neben dem primären Ziel der Rationalisierung der Unternehmen zum Teil auch das Ziel einer effizienten Integration älterer Arbeitspersonen verfolgt (vgl. FLÖTER/WENZEL, 1992), sind verschiedene negative Auswirkungen $\mathrm{zu}$ erwarten, falls die individuellen Bedürfnisse und sozialen Bedingungen bei der Gestaltung der Gruppenarbeit nicht genügend beachtet werden. Im Interesse der Ausschöpfung des Erwerbspersonenpotentials und insbesondere der Verlängerung der Lebensarbeitszeit müssen die möglichen hohen Beanspruchungen der Arbeitspersonen durch die Gruppenarbeit im Sinne der Lean-Konzepte nach japanischem Vorbild vermieden werden. Insgesamt bleibt daher auch für die Einführung der Gruppenarbeit in den Unternehmen die Aufgabe bestehen, eigene Arbeitsformen zu gestalten, statt vermeintlich ideale Vorbilder zu kopieren.

Durch die Einführung der Aufgabenbearbeitung im Sinne einer teilautonomen Gruppenarbeit sind verschiedene positive Auswirkungen zu erreichen (vgl. ULICH, 1991, 164ff., 188 und 378ff.), die sich auf die Arbeitspersonen sowie die Organisation und Produktivität der Unternehmen beziehen. Zur Erreichung dieser positiven Veränderungen ist es allerdings notwendig, verschiedene Voraussetzungen und Bedingungen zu erfüllen, die beispielsweise die Partizipation, die Qualifikation und die Lohnbemessung betreffen.

Die Gruppenarbeit sollte ohne starre interne Arbeitsteilung und mit erweiterten Dispositionsspielräumen durchgeführt werden, denn ohne eine Erhöhung der Autonomie und die Übertragung echter Entscheidungsbefugnisse auf die Gruppe gelangt die Aufgabenbearbeitung häufig nicht über Angelerntentätigkeiten hinaus (vgl. MINSSEN u.a., 1991) und es ergeben sich u.U. lediglich eine steigende Arbeitsintensität und eine Zunahme des gegenseitigen Leistungsdrucks, der sich besonders auf tatsächlich oder potentiell weniger leistungsfähige Arbeitspersonen auswirkt, deren Benachteiligung erhöht und zu Überforderungen führt.

Die Aufgabenrotation kann zur Verbesserung des betrieblichen Überblicks der Beschäftigten genutzt werden, die Kommunikation und das gegenseitige Verständnis fördern und insbesondere auch zu einem Abbau einseitiger Beanspruchungen führen. Eine ganzheitliche Problembearbeitung, die die weitgehende Selbststeuerung und interne Kooperation der Gruppe nutzt und zur Enthierarchisierung beiträgt, kann durch die erweiterten Handlungsspielräume der Arbeitspersonen beanspruchungsmindernd wirken. Zugleich können die 
interessanteren Arbeitsinhalte dazu führen, daß die Zunahme kognitiver und sozialer Anforderungen qualifikationsfördernd wirkt.

Die Gestaltung der Gruppenarbeit sollte auf einen qualifikationsgerechten Einsatz der Arbeitspersonen gerichtet sein. Die Facharbeiterorientierung darf nicht im Interesse kurzfristig größerer Einsatzflexibilität aufgegeben werden, zumal die Anforderungen an die Qualifikation aufgrund steigender Computerisierung und Automatisierung noch steigen werden. Die Umsetzung der Gruppenarbeit sollte auf eine Erweiterung und Anreicherung der Arbeitsaufgaben gerichtet sein und den Zeitdruck durch eine Entkopplung der Arbeitsgruppen mindern. Den Arbeitspersonen müssen ausreichende Handlungs- und Entscheidungsspielräume gegeben werden, deren Ausnutzung wiederum die Qualifikation der Beschäftigten erhöht.

Eine entsprechend gestaltete Gruppenarbeit ermöglicht eine Anpassung der Arbeit an den individuellen Arbeitsrhythmus, erleichtert die Qualifizierung durch vielfältige, kooperativ durchzuführende Tätigkeiten und führt zu einem Ausgleich der Belastungen durch Tätigkeitswechsel. Andererseits führen häufige Aufgabenwechsel, komplexere Aufgaben und erweiterte Entscheidungskompetenzen zu höheren Belastungen. Erhöhte Anforderungen können zur Selbstdisziplinierung und Selbstausbeutung der Gruppe führen, so daß insbesondere leistungsschwächere Arbeitspersonen unter Leistungsdruck gesetzt werden. Spannungen und Reibereien innerhalb der Gruppe bzw. zwischen den Gruppen beeinträchtigen die Leistung und bewirken zusätzliche Belastungen. Der Zwang zur gegenseitigen Rücksichtnahme und der gruppenbezogene Konformitätsdruck verdeutlichen persönliche Abhängigkeiten (vgl. NEUBERGER, 1985, 102ff.).

Zur Verbesserung der betrieblichen Integration Älterer, zur Vermittlung neueren Wissens und aktueller Ausbildungsbestandteile sowie zur Weitergabe und zum Erhalt des Erfahrungswissens sind zudem altersgemischte Gruppen sinnvoll. Ältere und jüngere Arbeitspersonen können hierdurch gewinnbringend zusammenarbeiten, so daß die jüngeren Arbeitspersonen körperlich anstrengendere oder Schnelligkeit und Reaktionsvermögen erfordernde Tätigkeiten übernehmen, während Ältere ihre Erfahrungen und ihr Durchhaltevermögen nutzen. Zur Vermeidung gruppeninterner Schwierigkeiten setzt die Bildung altersgemischter und (leistungs-) inhomogener Gruppen allerdings voraus, daß kein zu hoher Leistungs- und Zeitdruck besteht.

Gestalten Unternehmen zur Verbesserung der Wirtschaftlichkeit eine Form der Gruppenarbeit, die in der beschriebenen Weise Fehlbeanspruchungen vermeidet, so 
nähern sie sich damit tendenziell den Gruppenarbeitsformen, die mit dem Ziel der Humanisierung der Arbeit entworfen wurden. Auch manche Entwicklungen in der japanischen Industrie können derart interpretiert werden, daß sich auch die dortigen Unternehmen um menschengerechtere Gruppenarbeitsformen bemühen und damit zu einem 'revival' schwedischer Modelle beitragen können (vgl. JÜRGENS, 1993, 23).

\section{- Betroffene Kosten und Nutzengrößen}

Die (teilautonome) Gruppenarbeit, die die Bedürfnisse der Arbeitspersonen in einem hohen Maße berücksichtigt und sich damit insbesondere von der Gruppenarbeit im Sinne der Lean Production unterscheidet, beeinflußt verschiedene Kosten- und Nutzengrößen.

Kosten:

- Die empfohlene Minderung des Zeit- und Leistungsdrucks bzw. die geringere Arbeitsintensivierung führt zur Verringerung der mit anderen Formen der Gruppenarbeit erreichbaren Leistungserhöhungen. Die zur Minderung des Zeitdrucks notwendigen Materialpuffer erhöhen die Kapitalbindung.

- Die erweiterte Beteiligung der Beschäftigten und die Übertragung von Kompetenzen an die Gruppe können die gruppeninternen und gruppenexternen Abstimmungskosten erhöhen.

- Die Arbeitsaufgabenwechsel stellen erhöhte Anforderungen an die horizontale Mobilität und die Einsatzplanung.

- Im Falle eines innerbetrieblichen Wechsels erfordert gerade die teilautonome Gruppenarbeit von der Arbeitsperson zusätzlich zur Anpassung an die neue Tätigkeit die Integration in eine andere Arbeitsgruppe. Die Gruppenarbeit kann daher flexibilitäts- und mobilitätshemmend wirken.

- Die mit der Einführung der Gruppenarbeit und dem Abbau von Hierarchiestufen einhergehenden Einschränkungen der vertikalen Mobilität (Aufstiegsmöglichkeiten) können zu einer Minderung der Arbeitsmotivation führen.

Nutzen:

- Arbeiten, die Verantwortung verlangen und in der Gruppe durchgeführt werden, können die Motivation der Arbeitnehmer erhöhen und einen Abbau von Fehlzeiten und Fluktuationen bewirken. 
- Eine entsprechend den angesprochenen Punkten gestaltete Gruppenarbeit fördert die Qualifikation und Flexibilität der Beschäftigten und trägt zu einem Abbau von Arbeitsdruck und einseitigen Belastungen bei.

- Gruppenarbeit mit gut qualifizierten Arbeitspersonen kann Aufwendungen infolge unzureichender Abstimmung vermindern.

- Die Gruppenarbeit kann zur Verbesserung der Qualität, zur Erhöhung der Flexibilität, zur Integration von Funktionen und zu einem Abbau von Hierarchiestufen führen.

- Die Erhöhung der Einsatzflexibilität der Arbeitspersonen verringert die Abhängigkeit vom Arbeitsmarkt.

Eine globale Bewertung kommt zu einem Ergebnis, das in folgender Abbildung dargestellt ist. Dabei ist $\mathrm{zu}$ beachten, $\mathrm{daB}$ eine 'beanspruchungsoptimierte' Gruppenarbeit gegenüber einer Form der Gruppenarbeit, die einen höheren Arbeitsdruck akzeptiert, geringere zeitliche Einspareffekte erreichen dürfte.

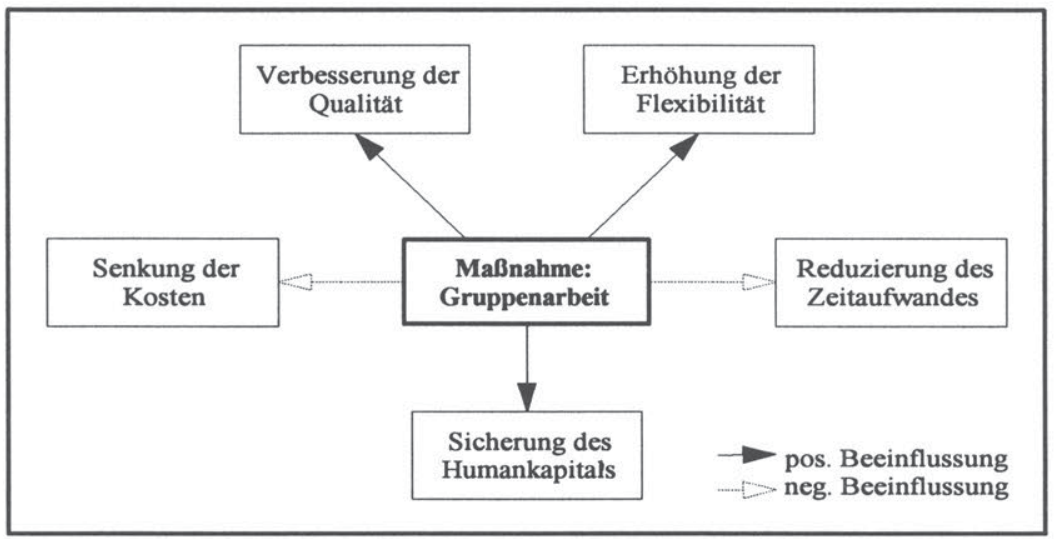

Abb. 60: Zielbeeinflussung durch die Umsetzung der Maßnahme Gruppenarbeit

\section{- Umsetzungsbedingungen}

Die Einführungsvoraussetzungen für die Gruppenarbeit haben sich aufgrund der Vorstellungen moderner Produktions- und Organisationsmethoden deutlich verbessert (vgl. ULICH, 1993). Allerdings sind verschiedenen Formen der Gruppenarbeit denkbar. $\mathrm{Zu}$ ihrer zieladäquaten Umsetzung müssen die genannten Humanisierungsaspekte beachtet werden. In die Prozesse der Gestaltung und 
Einführung der Gruppenarbeit sollten möglichst viele Mitarbeiter einbezogen werden, um die vorhandenen Erfahrungen zu nutzen und die individuellen Bedürfnisse zu berücksichtigen (vgl. die Darstellung eines problematischen Implementierungsprozesses in SCHWAGER/UDRIS, 1995).

Angesichts des relativ hohen und zukünftig weiter steigenden Ausbildungsniveaus und dem Ziel, das Arbeits- und Leistungspotential der Arbeitspersonen besser auszuschöpfen, erscheint die Gestaltung autonomer Gruppenarbeit als angemessene Form der Aufgabenbearbeitung (vgl. WEBER, 1994) Diese Form der Gruppenarbeit kann dazu beitragen, Fehlbeanspruchungen zu vermeiden und Kompetenzen zu entwickeln. Die gegenüber anderen Formen der Aufgabenbearbeitung höheren Kosten besitzen Investitionscharakter und erhöhen das dem Unternehmen zur Verfügung stehende Humanvermögen. Für eine erfolgreiche Umsetzung dieser Maßnahme sind Qualifizierungsmaßnahmen erforderlich, die sich insbesondere auf die Sicherstellung sozialer Kompetenzen zur Bewältigung gruppenarbeitsspezifischer Anforderungen und fachlicher Kompetenzen für die vorgesehenen Arbeitsplatzwechsel richten.

Trotz vielfaltig positiver Aspekte der Gruppenarbeit sollte bei ihrer Einführung in den Unternehmen darauf geachtet werden, daß Möglichkeiten der Einrichtung 'entkoppelter' Einzelarbeitsplätze erhalten bleiben, um bei der Einsatzbestimmung in differenzierender Weise auf die Bedürfnisse der Arbeitspersonen eingehen zu können.

\section{(3) Heimarbeit}

Die Maßnahme Heimarbeit bezieht sich insbesondere auf die Verbesserung der Möglichkeiten, berufliche und außerberufliche Tätigkeiten zu vereinbaren. Unter Heimarbeit werden hier verschiedene Formen der häuslichen Erwerbstätigkeit verstanden, während Heimarbeit nach dem Heimarbeitsgesetz eine bestimmte Rechtsform darstellt. Heimarbeit ist konzeptionell eine geschlechtsübergreifende Form der Aufgabenbearbeitung. Angesichts der bestehenden geschlechtlichen Arbeitsteilung dürfte sie allerdings vornehmlich die Vereinbarkeit von Beruf und Erziehung bzw. Pflege für Frauen erleichtern. Heimarbeit kann einerseits bereits erwerbstätigen Frauen bei der Bewältigung der verschiedenen Anforderungen helfen und andererseits das Arbeitspotential von Frauen, die ohne diese Tätigkeitsform nicht erwerbstätig wären, erschließen. Weiterhin kann diese Arbeitsform 
Dequalifizierungsprozesse vermeiden oder eine (Re-) Qualifizierung, beispielsweise während des Auslaufens einer Unterbrechungsphase, ermöglichen. Daneben kann diese Maßnahme, besonders als Teilzeit-Heimarbeit, dazu beitragen, die mit der Erwerbstätigkeit einhergehenden Anforderungen an die Eigenschaften der Arbeitspersonen anzupassen. So kann auch die berufliche Beanspruchung Älterer gemindert und deren verlängerte Erwerbstätigkeit gefördert werden.

\section{- Gestaltungsformen}

Während traditionelle Formen der Heimarbeit in der Vergangenheit deutlich an Bedeutung verloren haben, wächst seit einigen Jahren das Interesse an dezentralisierter Büroarbeit (vgl. SEBALDT, 1992; HEGNER u.a., 1989). Durch die Nutzung moderner Kommunikations- und Informationstechniken kann TeleHeimarbeit zukünftig für Angestelltentätigkeiten im Verwaltungs- und Bürobereich und für Tätigkeiten mit unterschiedlichen Qualifikationsanforderungen an Bedeutung gewinnen. Je nach Nutzung von Kommunikations- und Informationstechniken zur Verbindung eines Telearbeitsplatzes mit dem Unternehmen kann von On-line- oder Off-line-Telearbeit gesprochen werden. Aufgrund der technischen Entwicklung ist für die On-line-Telearbeit eine steigende Bedeutung zu erwarten.

Heimarbeit oder Erwerbsarbeit an dezentralen außerbetrieblichen Arbeitsstätten erweitert die zeitliche Gestaltungsfreiheit der Arbeitspersonen und bewirkt durch den Wegfall oder die Verkürzung der Anfahrt Zeit- und Kostenersparnisse. Gerade in strukturschwachen Gebieten können sich durch diese Arbeitsform die Möglichkeiten erwerbstätig zu sein, verbessern. Die vergrößerte Zeitsouveränität geht einher mit einer höheren Selbständigkeit. Zusammengenommen erfordert die Heimarbeit erhöhte Eigenmotivation, Disziplin, Selbstkontrolle und Eigeninitiative der Arbeitspersonen.

Die räumliche Dezentralisierung der Aufgabenbearbeitung in Form der Heimarbeit kann eine Verbindung von Wohnen und Arbeiten schaffen und die Bereiche Beruf und Haushalt/Familie verknüpfen. Der Wegfall der Wegezeiten erleichtert Frauen die Bewältigung der Mehrfachbelastung (vgl. HEGNER u.a., 1989). Neben dem räumlichen Aspekt kommt regelmäßig eine zeitliche Lockerung bzw. Entkopplung der Arbeit hinzu, die eine Anpassung der Arbeitszeit an außerberufliche Anforderungen ermöglicht. Insofern sollte insbesondere das Angebot an TeilzeitHeimarbeitsplätzen erhöht werden. Zur Erleichterung der zeitlichen Planbarkeit sollte das Arbeitsvolumen gleichmäßig oder zumindest ohne unvorhersehbare 
Schwankungen anfallen. Starre Terminvorgaben und die Möglichkeiten der einzelnen Arbeitsperson übersteigende Arbeitsaufgaben und Arbeitsmengen müssen vermieden werden.

Die Möglichkeit der Umsetzung der Maßnahme Heimarbeit ist abhängig von der Art der Arbeitsaufgabe. Geeignet sind solche Aufgaben, deren Ausführung keine direkten Kontakte zu Kollegen oder den betrieblichen Abläufen erfordern. Insofern sind insbesondere einzeln ausführbare, nicht personenbezogene und nicht mit direkten Funktionen im Produktionsbereich verbundene Tätigkeiten zur Heimarbeit geeignet. Aufgrund der sich zukünftig voraussichtlich weiter verbessernden Anbindungsmöglichkeiten werden zunächst besonders häufig computergestützte Tätigkeiten in dieser Form der Aufgabenbearbeitung durchgeführt werden. So besteht bei dem diesbezüglichen 'Vorreiter' IBM seit 1991 eine Betriebsvereinbarung über außerbetriebliche Arbeitsstätten, und eine Ausweitung dieser Arbeitsform ist angestrebt.

Je nach häuslicher Arbeitssituation können Probleme hinsichtlich des Arbeitsschutzes bestehen, da dessen Vorschriften und Empfehlungen nicht eingehalten werden und schwerlich überwacht werden können. Ungünstige Arbeitsbedingungen aufgrund ungeeigneter Arbeitsräume und fehlender Unterstützung werden zusätzlich durch das heimarbeitstypische Arbeitsprinzip, möglichst viel Arbeit zur gleichen Zeit durchzuführen, gefördert und durch den daraus entstehenden Arbeitsdruck verschärft (vgl. LUCZAK, 1993, 48). Eine Unterschätzung der dauerhaften Mehrfachbelastung, eine ständige Überforderung, ein Verschwinden der Grenze zwischen Arbeit und Freizeit sowie die tendenzielle Selbstausbeutung können zu erhöhten physischen und psychischen Beanspruchungen führen. Weiterhin schrumpft durch die räumliche Dezentralisierung das Ausmaß der Zusammenarbeit mit anderen Arbeitspersonen. Aufgrund des Wegfalls unmittelbarer persönlicher Kommunikation dürften häufig Rückmeldungen über die geleistete Arbeit fehlen. Regelmäßige Gesprächsrunden in den Unternehmen bieten eine Möglichkeit Kommunikationsdefizite zu mindern und Fragen der Arbeitsorganisation zu behandeln (vgl. HEGNER u.a., 1989, 214). 
- Betroffene Kosten und NutzengröBen

Kosten:

- Die Ausstattung und Wartung der (Tele-) Heimarbeitsplätze und die Anbindung an das Unternehmen verursachen hohe Kosten. Daneben ergeben sich ausbildungs- und betreuungsbedingte Kosten.

- Probleme bestehen insbesondere hinsichtlich der Überwachung der Arbeitsqualität und der Arbeitsdauer, der Kontrolle der Einhaltung von Terminen, der Vertraulichkeit und des Datenschutzes. Entsprechende Kontrollkosten beziehen sich auf die quantitative Erfassung der Arbeitsergebnisse und die Qualitätssicherung.

- Problematisch für die Arbeitspersonen und die betrieblichen Abläufe sind der mögliche Abbau persönlicher Kontakte und informeller Kommunikation sowie die Verlăngerung des Informationsflusses. Die Durchlaufzeiten können sich je nach der in Heimarbeit ausgeführten Tätigkeit verlängern. Zusätzlich Kosten für die Koordination der Arbeitsaufgaben entstehen bei der Einrichtung mehrerer Heimarbeitsplätze.

Nutzen:

- Neben den eingesparten Kosten der Arbeitszeitkontrolle und der Überstundenzuschläge sind langfristig Kostensenkungen für Büroflächen und Einrichtungsgegenstände denkbar.

- Heimarbeit trägt zur Erhöhung der Flexibilität und der Kundennähe bei. Durch Vereinbarung einer fallweisen Beschäftigung sind Personaleinsparungen und eine verbesserte Anpassung an Absatzschwankungen möglich.

- Die Individualisierung des Arbeitsverhältnisses kann die Identifikation der Arbeitspersonen mit dem Unternehmen und die Position des Unternehmens auf dem Arbeitsmarkt fördern.

- Die Motivation, die Kreativität und die Produktivität der Beschäftigten dürfte sich durch die individuellen Arbeitszeiten erhöhen, zumal Leerlauf- und Abwesenheitszeiten sowie Anfahrtswege vermindert werden.

- Darüber hinaus kann die Tătigkeit an Heimarbeitsplätzen gegenüber zentralen Arbeitsaufgaben erweitert und beispielsweise um Planungs- und Kontrollkomponenten bereichert sein. Die Arbeitsqualifikation der Beschäftigten dürfte sich hierdurch erhöhen. 
Eine globale Beurteilung der Maßnahme Heimarbeit erfolgt in der folgenden Abbildung. Bezogen auf das Kostenziel soll keine eindeutige Einschätzung gegeben werden, da den erhöhten Ausstattungskosten verschiedene Einsparmöglichkeiten gegenüberstehen, deren Realisierung allerdings von vielfältigen unternehmensspezifischen Bedingungen abhängt (vgl. BRANDES/BUTTLER, 1987). In Abhängigkeit vom Ausmaß der zur Einrichtung der Heimarbeitsplätze erforderlichen Aufwendungen wird eine periodenbezogene Mindestarbeitszeit notwendig sein, damit sich die Investitionen in Heimarbeitsplätze amortisieren können. Da die Koordinationskosten im Vergleich zu möglichen Produktivitätsgewinnen gerade bei niedriger qualifizierten Tätigkeiten geringer sind, dürfte sich die Heimarbeit besonders auf dieser Ebene ausweiten ( vgl. LENK, 1989).

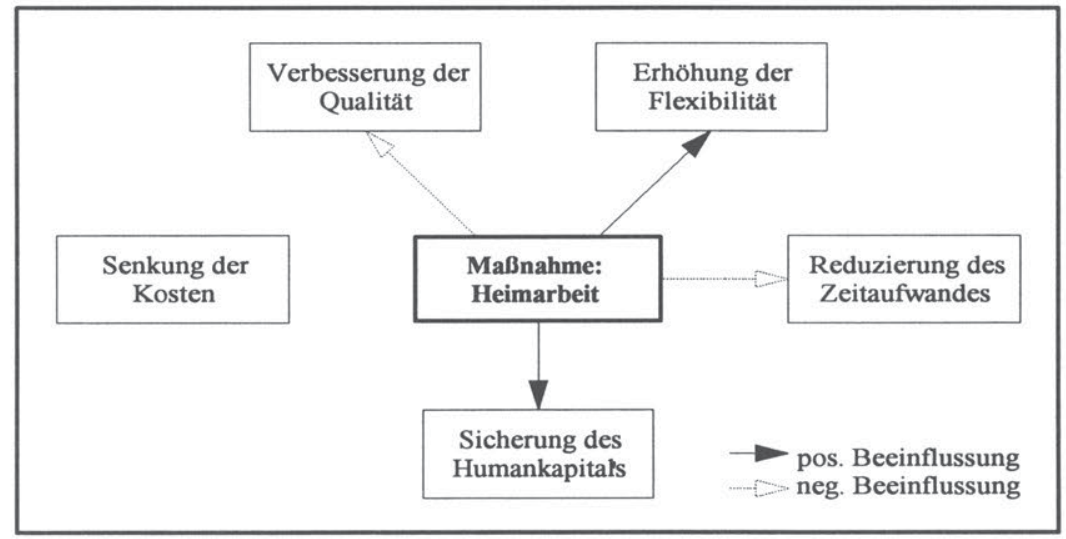

Abb. 61: Zielbeeinflussung durch die Umsetzung der Maßnahme Heimarbeit

\section{- Umsetzungsbedingungen}

Neben der prinzipiellen Bereitschaft zur Heimarbeit müssen die Arbeitspersonen ausreichende Motivation, Eigeninitiative und Selbstdisziplin besitzen. Zur Förderung dieser Eigenschaften, zur Minderung der sozialen Isolation und zur Vermeidung der gerade auch von Gewerkschaften befurchteten Entsolidarisierung der Beschäftigten könnten Satelliten- oder Nachbarschaftsbüros hilfreich sein (vgl. LENK, 1989, 205ff.). In diesen dezentral eingerichteten Büros können die Arbeitspersonen regelmäßig parallel oder zu bestimmten bzw. fallweise vereinbarten Zeiten zusammenkommen und kooperativ Tätigkeiten durchführen. 
Wesentlich für die Ausweitung der Heimarbeit, die Absicherung der Beschäftigten, den Erhalt der Einbindung der Arbeitspersonen in das Erwerbsleben und die Bindung vorhandenen Humankapitals an das Unternehmen ist die Beibehaltung des Arbeitnehmerstatus. Nachteilig für die breite Akzeptanz dieser Arbeitsform kann sich auswirken, daß die Arbeitspersonen, die in Heimarbeit beschäftigt sind, zur Randbelegschaft gerechnet werden, so daß Weiterbildungs- und Aufstiegschancen und eine befriedigende betriebliche Interessenvertretung fehlen. Entsprechend sollten zur Förderung der Heimarbeit die so beschäftigten Arbeitspersonen fachlich und sozial möglichst stark einbezogen werden.

Arbeitsorganisatorische Flexibilisierungsmaßnahmen wie die Heimarbeit können Arbeitszeitverkürzungen und vergleichsweise niedrige Löhne bewirken. Damit führt eine solche Beschäftigung aufgrund des an einem Normalarbeitsverhältnis orientierten Systems der gesetzlichen Rentenversicherung dazu, daß u.U. zu geringe Rentenansprüche erworben werden. Eine verbreitete Nutzung dieser Maßnahme erfordert daher von staatlicher Seite eine Anpassung des Rentensystems (vgl. RÜRUP/SCHNEIDER, 1992, 26ff.).

Zur Sicherung der möglichen Vorteile und zur Minderung der möglichen Nachteile der Heimarbeit könnte die Teilzeit-Heimarbeit mit einer innerbetrieblichen Teilzeitarbeit verknüpft werden, so daß Arbeitspersonen beispielsweise regelmäßig einer vormittäglichen Teilzeitarbeit nachgehen und bei Bedarf phasenweise zur Heimarbeit wechseln können.

\section{b) Arbeitszeitkonzepte}

In dem hier $\mathrm{zu}$ erörternden Zusammenhang richten sich arbeitszeitbezogene Überlegungen zum einen auf die Förderung der kontinuierlichen Beschäftigung von Frauen sowie ihre Reintegrationschancen nach einer Unterbrechungsphase und zum anderen auf die Verlängerung der Lebensarbeitszeit durch die Ermöglichung eines gleitenden Ausstiegs älterer Arbeitspersonen.

Die Arbeitszeit läßt sich in zwei Dimensionen beschreiben und gestalten:

- Die chronometrische Dimension betrifft die Dauer der Arbeitszeit und kann z.B. durch Überstunden, Kurzarbeit, Teilzeit und Job sharing beeinflußt werden.

- Die chronologische Dimension bezieht sich auf die Lage der Arbeitszeit und kann z.B. durch Schichtzeit und einfach gleitende Arbeitszeit gestaltet werden. 
Neben diesen Regelungen gibt es Arbeitszeitformen, wie die qualifiziert gleitende, die variable und die kapazitätsorientierte variable Arbeitszeit (KAPOVAZ) oder Kombinationen der zuvor genannten Modelle, die je nach Ausgestaltung beide der genannten Dimensionen der Arbeitszeit betreffen.

Traditionell ist die Arbeitszeit dadurch gekennzeichnet, daß sie pünktlich beginnt, gleichzeitig liegt, kollektiv geregelt ist und (z.B. mit Ausnahme der Schichtarbeit) einheitlich der Betriebslaufzeit entspricht. Von dieser 'Normalarbeitszeit' gibt es eine Bedeutungsverschiebung zur Flexibilisierung der Arbeitszeit. Die Flexibilisierung bezieht sich auf die Gestaltung der Arbeitszeiten der Beschäftigten und die Besetzungszeiten der Arbeitsplätze sowie die Betriebszeiten der Maschinen. Hierzu ist zu bemerken, daß die Normalarbeitszeit ein in der Realität nur begrenzt gültiges Orientierungsmuster ist, das insbesondere für Frauen zumeist nicht 'normal' ist und daß 'Flexibilisierung' ein sehr diffuser Begriff ist.

Für eine arbeitszeitlichen Gestaltung bestehen diverse Möglichkeiten, so kann beispielsweise die Tages-, Wochen-, Monats- oder Jahresarbeitszeit verkürzt werden. Statt festgelegter Tages- und Wochenarbeitszeiten können Monats- bzw. Jahresarbeitszeiten mit individuell schwankenden Zeiten der Unter- und Überarbeit vereinbart werden. Längere, über Jahre reichende Arbeitszeitvereinbarungen dürften besonders für hochqualifizierte Tätigkeiten an Bedeutung gewinnen. Zudem können Bildungsphasen vereinbart werden, so da $B$ arbeitsfreie Phasen, auch in Form unbezahlten Urlaubs, zur Aus- und Weiterbildung genutzt werden können. Daneben sind Gleitzeitregelungen, fixierte und flexible Teilzeitarbeit, Schichtzeit, Jobsharing (Arbeitsteilung), Teilzeitschichten, Block-Teilzeitarbeit, variable (arbeitsanfallorientierte) Arbeitszeit, und zahlreiche Kombinationsmöglichkeiten denkbar.

Im betrieblichen Interesse richtet sich die Arbeitszeitflexibilisierung als Bestandteil der tendenziellen Auflösung des Normalarbeitsverhältnisses besonders auf drei Zielsetzungen (vgl. HINRICHS, 1992, 321). Zunächst ermöglicht die Entkopplung von Arbeitszeiten und Betriebszeiten (chronologische Denormalisierung), daß die kapitalintensiven Produktionsanlagen durch eine Verlängerung der Betriebszeiten bzw. der Maschinenlaufzeiten wirtschaftlicher genutzt werden können. Dies ist gerade angesichts der gesunkenen Wochenarbeitszeit betriebswirtschaftlich sinnvoll, zumal so den erhöhten Lohnstückkosten mit einer Senkung der Kapitalstückkosten begegnet werden kann. Die Mehrfachbesetzung von Arbeitsplätzen kann somit zur Sicherung der Wettbewerbsfähigkeit der Unternehmen beitragen. Weiterhin kann 
die Arbeitszeitflexibilisierung dazu führen, daß die zu leistende Arbeitszeit gemä $\beta$ dem schwankenden Arbeitsanfall abgefordert wird (chronologische Diskontinuität oder Entstetigung). Schließlich erleichtern individuelle Regelungen der Arbeitszeit, $\mathrm{da} B$ Arbeitsaufgaben und Arbeitspersonen differenziert zusammengeführt werden (chronometrische Destandardisierung).

Im Unterschied $\mathrm{zu}$ den an den Unternehmensinteressen ausgerichteten Flexibilisierungszielen möchten die Beschäftigten ihre Zeitsouveränität durch eine Arbeitszeitflexibilisierung erhöhen. Hier ist besonders von Bedeutung, da $\beta$ eine Anpassung der Arbeitszeit an die Bedürfnisse der Mitarbeiter ermöglicht wird, so daß die Weiterbeschäftigung von Frauen und Älteren, die für ein Normalarbeitsverhältnis nicht mehr zur Verfügung stehen, erleichtert wird.

Im folgenden wird die Teilzeitarbeit, die die wichtigste Form der Arbeitszeitflexibilisierung darstellt, als Möglichkeit zur Verbesserung der Vereinbarkeit von Beruf und Familie und zur Realisierung einer gleitenden Pensionierung betrachtet. Die Erhöhung der Teilzeitarbeitsmöglichkeiten muß, um zieladäquat zu wirken, die Erwerbstätigkeit derjenigen Personen fördern, die ohne Teilzeitarbeit nicht erwerbstätig wären. Insofern müssen die Interessen von Frauen und Älteren an dieser Arbeitsform Beachtung finden.

\section{- Gestaltungsformen}

Gegenüber der tarifvertraglich vereinbarten Arbeitszeit der Vollzeitbeschäftigung versteht man unter Teilzeitarbeit eine verkürzte (Wochen-) Arbeitszeit, die nicht für alle Beschäftigten eines Unternehmens oder eines Betriebes gleichermaßen gilt. Neben der Verkürzung müssen die Lage der Arbeitszeit und die Dispositionsmöglichkeiten geregelt werden.

Die Entwicklung der Teilzeitarbeit zeigt ihre wachsende Bedeutung: 1960 waren ca. $4 \%, 1973$ ca. $9 \%$ und 1994 ca. $16 \%$ der Erwerbstätigen teilzeitbeschäftigt (vgl. STATISTISCHES BUNDESAMT, MIKROZENSUS; CORNELSEN, 1995). Wie bereits dargestellt ist die Teilzeitbeschäftigung geschlechtsspezifisch verteilt, so waren 1994 ein Drittel der erwerbstätigen Frauen, aber nur 2,8\% der erwerbstätigen Männer in Deutschland teilzeitbeschäftigt (vgl. CORNELSEN, 1995). Während der Anteil der Teilzeitarbeit an der Beschäftigung im Handel und Dienstleistungsbereich ca. $28 \%$ beträgt, erreicht er im Verarbeitenden Gewerbe lediglich ca. $8 \%$ (vgl. STATISTISCHES BUNDESAMT, MIKROZENSUS). Die 
voraussichtliche weitere Ausweitung des tertiellen Sektors wird die Bedeutung der Teilzeitarbeit weiter erhöhen. Allerdings ist zukünftig eine Erhöhung des Angebots an Teilzeitarbeitsplätzen im Bereich der Industrie notwendig, um diese Maßnahme zur Erhöhung der Erwerbsquoten von Frauen und Älteren nutzen zu können, zumal der Wunsch nach unterschiedlich langen Teilzeitbeschäftigungen weiter verbreitet ist als die bisher vereinbarte Teilzeitarbeit (vgl. DIW, 1994).

In verschiedenen Wirtschaftsbereichen wurde die Teilzeitarbeit tarifvertraglich geregelt. Allerdings wurden bisher noch keine rechtlichen Ansprüche auf Teilzeitarbeit oder die Rückkehr zur Vollzeitarbeit vereinbart. Familienfreundlichere Gestaltungsmöglichkeiten der Arbeitszeit (insbesondere die individuelle Arbeitszeitverkürzung und die Anpassung der Arbeitszeiten an die Öffnungszeiten von Kinderbetreuungseinrichtungen) wurden in Tarifverträgen der Cigaretten-, der Eisen- und Stahl- sowie der Papierverarbeitenden Industrie festgelegt (vgl. BDA, 1994).

Während der Wunsch nach Teilzeitarbeit bei den Männern häufig freizeit- oder bildungsorientiert ist, hängt er bei den Frauen insbesondere von der Lebenssituation ab. So sind fehlende Kinderbetreuungsmöglichkeiten und der Wunsch genügend Zeit für Kinder zu haben gerade für Frauen wichtige Motive, eine Teilzeitarbeit anzustreben, da diese Arbeitsform die Vereinbarkeit von Beruf und Familie erleichtern kann. Falls die Kinderbetreuung während der Arbeitszeit gesichert ist, kann Teilzeitarbeit insbesondere zu einer Erhöhung der Erwerbstätigkeit verheirateter Frauen beitragen. Folgende Abbildung gibt die Verteilung wesentlicher Gründe eines Teilzeitwunsches an, die im Rahmen des Soziooekonomischen Panels (SOEP) erfragt wurden.

\begin{tabular}{|l|c|c|c|}
\hline Angaben in \% & Insgesamt & Mănner & Frauen \\
\hline Erwerbspersonen mit Teilzeitwunsch & 27 & 10 & 51 \\
angegebene Gründe: & & & \\
& & & \\
1. Keine Kinderbetreuungsmöglichkeiten & 11 & 2 & 13 \\
2. Genügend Zeit für Kinder & 40 & 19 & 45 \\
3. Wegen Ehepartner & 25 & 20 & 27 \\
4. Andere familiäre Gründe & 19 & 9 & 21 \\
5. Einkommen reicht aus & 29 & 37 & 27 \\
6. Zu wenig Zeit für andere Dinge (Freizeit, & 53 & 70 & 48 \\
Ausbildung usw.) & & & \\
\hline
\end{tabular}

Abb. 62: Motive für den Wunsch nach Teilzeitarbeit

(Quelle: SCHULZE BUSCHOFF, 1994, nach SOEP-West 1990) 
Frauen wollen ihre Arbeitszeit häufiger und in größerem Umfang als Männer reduzieren. So wünschten nach den Ergebnissen des SOEP 1993 in Westdeutschland z.B. 16\% der Frauen und 5\% der Männer 30 Stunden pro Woche erwerbstätig sein; 42\% der Frauen und nur 5\% der Männer wollen wöchentlich unter 30 Stunden (hauptsächlich 20 Stunden) erwerbstätig sein (vgl. DIW, 1994). Insbesondere kann davon ausgegangen werden, daß zahlreiche Frauen nach der Geburt eines Kindes von der Vollzeit- zu einer Teilzeittätigkeit wechseln wollen. Eine phasenweise sozialversicherungspflichtige Teilzeitbeschäftigung kann eine Unterbrechungsphase vermeiden bzw. verkürzen und damit eine dauerhafte Erwerbsbeteiligung ermöglichen bzw. die Wiedereinstiegs-, Einkommens- und Aufstiegschancen verbessern, da Dequalifikationsprozesse vermieden werden sowie berufliche Kontakte und Einstellungen erhalten bleiben. Die Teilzeitbeschäftigung kann berufliche Benachteiligungen der Frauen insofern zumindest begrenzen und stellt eine wesentliche Möglichkeit zur Verknüpfung wichtiger Arbeits- und Lebensformen dar.

Die bisherigen Überlegungen zur Verbesserung der Vereinbarkeit von Beruf und Familie durch die Teilzeitarbeit der Frauen gehen implizit davon aus, daß Männer den größten Teil des Einkommens verdienen und vollzeitbeschäftigt bleiben (vgl. PFAU-EFFINGER/GEISSLER, 1992, 366f.). Andererseits kann es zukünftig zunehmend darum gehen auch das Lebens- und Erwerbsmodell der Männer zu verändern. In diesem Sinne kann eine Ausweitung der Teilzeitarbeit bei (Ehe-) Männern zieladäquat sein, wenn sich durch die Teilzeitarbeit beider Ehepartner die insgesamt angebotene Arbeitszeit erhöht. Wenn Männer und Frauen aufgrund der Teilzeitarbeit in der Lage sind, die Familien- und Haushaltsarbeiten neben ihrer beruflichen Tätigkeit zu bewältigen, kann das Nebeneinander von Familie und Beruf erleichtert werden und das quantitative und qualitative Arbeitsangebot gesichert und langfristig erhöht werden. Für eine solche partnerschaftliche Teilzeitarbeit müßten vermehrt Beschäftigungsmöglichkeiten im oberen Stundenbereich, d.h. knapp unterhalb der Vollarbeitszeit, angeboten werden, die besonders bei Männern auf ein größeres Interesse stoßen dürften (vgl. beispielsweise BÄCKER/STOLZWILLIG, 1995, 57; SCHULZE BUSCHOFF, 1994, 12). Eine höhere Akzeptanz der Teilzeitbeschäftigung für Männer, könnte sich zudem 'rückwirkend' positiv auf die beruflichen Perspektiven teilzeitbeschäftigter Frauen auswirken.

Teilzeitarbeit ist eine seit Jahrzehnten als Kompromißlösung zur Vereinbarung der Arbeit in Haushalt und Beruf diskutierte Form der Erwerbstätigkeit, die es Frauen ermöglicht, zum Lebensunterhalt und zur Erhöhung des Lebensstandards 
beizutragen und dem Streben nach Selbstverwirklichung im Beruf zu entsprechen. Die berufliche und betriebliche Integration der Teilzeitbeschäftigten und die Entwicklung der Teilzeitarbeit zu einer normalen Erscheinung erfordern die Aufhebung teilzeitbezogener Nachteile, wie geringere Chancen zur Selbstverwirklichung und die Marginalisierung der Teilzeitbeschäftigten in der Arbeitswelt (vgl. beispielsweise bereits FÜRSTENBERG, 1972, 155). Im Kontext vorliegender Arbeit gewinnt Teilzeitarbeit als Maßnahme neue Dimensionen, wenn die Unternehmen zur Humankapitalsicherung zukünftig in höherem Maße Teilzeitarbeitsplätze auf unterschiedlichen Qualifikationsstufen und für verschiedene Beschäftigtengruppen anbieten.

Eine solche quantitative und qualitative Ausweitung der Teilzeitarbeit eröffnet Möglichkeiten zur Anpassung von Arbeitsangebot und Arbeitsnachfrage. Gelingt es, die häufige Beschränkung auf niedrigqualifizierte Tätigkeiten zu überwinden und qualitativ verschiedenartige Arbeitssysteme mit Teilzeitarbeit zu verknüpfen sowie die Aufstiegschancen und Weiterbildungsmöglichkeiten der so Beschäftigten zu verbessern, so dürften die Wertschätzung der Teilzeitarbeit und das Identifikationsniveau der Teilzeitbeschäftigten steigen. Qualifizierte Teilzeitarbeiten sollten gerade angesichts der Höherqualifizierung der Frauen zunehmend angeboten werden. Ein vergrößertes und verbessertes Angebot an Teilzeittätigkeiten könnte dazu beitragen, daß weniger Frauen ihre Erwerbstätigkeit aufgeben und ein Teil der Stillen Reserve des Arbeitsmarktes zur Erhöhung der Erwerbsbeteiligung der Frauen aufgelöst wird. Eine Kombination von Teilzeitarbeit und Gleitzeit trägt zusätzlich zur Erleichterung der Vereinbarung von Familie und Beruf bei.

Älteren Personen ermöglicht die Teilzeitarbeit in den Ruhestand zu gleiten. Durch eine schrittweise Verkürzung der Arbeitszeit kann ein stufenweiser Ausstieg aus der Erwerbstätigkeit realisiert und eine Teilrente in Anspruch genommen werden, so $\mathrm{da} ß$ der rückläufige Arbeitsverdienst durch die steigende Rentenzahlung ausgeglichen wird. Bisher wird die von der gesetzlichen Rentenversicherung seit Anfang 1992 gebotene Möglichkeit des Bezugs einer Teilrente nur sehr selten und im wesentlichen nur in den alten Bundesländern in Anspruch genommen (vgl. OHSMANN/STOLZ, 1994, 417). Eine Voraussetzung für die stärkere Inanspruchnahme einer Teilrente ist ein höheres Angebot an Teilzeitarbeitsplätzen, das zukünftig zur Schaffung von Entscheidungsalternativen, zur Erhöhung der Flexibilität und zur Humanisierung des Arbeitslebens geschaffen werden sollte. 
Außerdem bieten flexible Formen des Übergangs in den Ruhestand den Arbeitgebern die Möglichkeiten, sich "einerseits eines Teils der Belegschaften, der relativ teuer ist und über obsolet werdende Kenntnisse verfügt, zu entledigen und andererseits Angestellte mit knapper werdenden wertvollen Fähigkeiten länger zu halten" (GUILLEMARD, 1993, 97).

Zur Förderung der Akzeptanz dieser Arbeitsform müssen teilzeitbedingte innerbetriebliche Statusverluste, die die Bereitschaft der älteren Arbeitspersonen zur Teilzeitarbeit einschränken, vermieden werden. Solche Statusverluste können allerdings durch die zunehmende Auflösung der Normalarbeitszeit ohnehin an Bedeutung verlieren (vgl. BÄCKER/NAEGELE, 1993b).

Weitere teilzeitbedingte Probleme der Beschäftigten sind ein überproportional verringertes Einkommen aufgrund der entfallenden Überstundenzuschläge sowie die relative Erhöhung der Wegezeiten und Fahrtkosten. Weiterhin wirken sich die Teilzeitbeschäftigungen bisher negativ auf das Pensionseinkommen, die Aufstiegschancen und die Weiterbildungsbeteiligung aus. Auch kann die Arbeitszeitflexibilisierung die zeitlichen Dispositionsräume einschränken und eine Erhöhung der Belastung durch unregelmäßige Arbeitszeiten bewirken. Die Dispositionsmöglichkeiten bei der Arbeitszeitgestaltung müssen entsprechend die Interessen der Arbeitspersonen berücksichtigen und eine gewisse Entscheidungsfreiheit zugestehen, damit die Erwerbstätigkeit von Frauen und Älteren tatsächlich erleichtert wird.

Falls bestimmte Tätigkeiten noch nicht für Teilzeitarbeit geeignet erscheinen, können sie arbeitsorganisatorisch geteilt werden. Dies kann vorteilhafte Effekte wie einen Abbau von Komplexităt und eine Erhöhung der Transparenz haben. Die Umsetzung der modernen Konzepte eröffnet Möglichkeiten zur zeitlichen Flexibilisierung, da tendenziell von der gleichförmigen, kontinuierlichen zu einer arbeitsanfallorientierten, diskontinuierlichen Arbeitsverteilung übergegangen wird. Weiterhin kann der Abbau der Hierarchien die Möglichkeiten zur Teilzeitarbeit erhöhen, da beispielsweise die unmittelbare Abhängigkeit von Weisungen anderer Arbeitsstellen sinkt. Zur Erreichung vielfältiger Flexibilisierungsziele und zur Ermöglichung der Teilbarkeit vieler Tätigkeiten ist die Zusammenarbeit von Vorgesetzten, Personalabteilung, Fachabteilungen und Betriebsrat erforderlich.

Ergänzend kann die organisatorische Arbeitsform des Job-sharing betrachtet werden, die eigene Möglichkeiten der 'selbstgesteuerten' Flexibilisierung zur 
Anpassung der Anforderungssituation bietet. Ähnlich wie Vollzeitkräfte bei Schichtarbeit einen Arbeitsplatz z.B. lückenlos besetzen, teilen sich beim Jobsharing mehrere Teilzeitbeschäftigte einen Vollzeitarbeitsplatz. Die Arbeitspersonen können die individuellen Arbeitszeiten in Absprache mit dem Vorgesetzten untereinander vereinbaren. Eine gegenseitige Vertretung ist möglich, muß aber abgesehen von dringenden betrieblichen Erfordernissen für den einzelnen Vertretungsfall geregelt werden (vgl. HALBACH u.a., 1994, 200).

Die eigenverantwortliche Abstimmung der Arbeitspersonen beim Job-sharing setzt erhöhte Kooperationsbereitschaft und -fähigkeit voraus, da Abstimmungsprobleme gelöst werden müssen und der Informationsfluß sicherzustellen ist. Da die zeitliche Belastung zwischen den Job-sharing-Partnern vereinbart werden kann, ermöglicht diese Arbeitsform flexiblere Reaktionsweisen auf außerberufliche Anforderungen oder auftretende Beeinträchtigungen. Diese Maßnahme kann demnach zur Verbesserung der Vereinbarkeit von Beruf und Familie, zur Anpassung an gesundheitliche Beeinträchtigungen und zum stufenweisen Ausscheiden genutzt werden. So könnte beispielsweise zur Erhöhung der Erwerbstätigkeit von Frauen mit Kindern eine individuelle Verlängerung des Jahresurlaubs auf die Dauer der Schulferien mit Job-sharing verknüpft werden, so daß die Arbeitsperson während der gesamten Dauer der Schulferien Urlaub hat.

\section{- Betroffene Kosten und Nutzengrößen}

Kosten:

- Die einmaligen und ständigen Einarbeitungskosten sind bei täglich verkürzten Arbeitszeiten höher. Es ergeben sich höhere Kosten der Eigeninformation sowie der Informationsweitergabe und der gegenseitigen Abstimmung.

- Durch die zeitliche Entkopplung der Anwesenheit der Arbeitspersonen entstehen Probleme hinsichtlich der Kommunikation. Die Teilzeitarbeit kann zu Reibungsverlusten und zur Verlängerung der Durchlaufzeiten beitragen.

- Die verglichen mit der Vollzeittätigkeit höhere Zahl der Beschäftigten verursacht zusätzliche Personalbeschaffungs-, Personalverwaltungs- und Betreuungskosten. Höhere Organisations- und Verwaltungskosten dürften allerdings durch den Einsatz von Computern und Personalzeitwirtschaftssystemen zunehmend an Bedeutung verlieren. 
- In den Arbeitsbereichen entsteht ein höherer Aufwand für Aufgaben der Organisation und der direkten Führung.

- Werden in Prozeßabläufe integrierte Einzelarbeitsplätze nur teilzeitig besetzt, müssen Puffer aufgebaut werden, die zu einer höheren Kapitalbindung führen. Außerdem steigen die Kosten zur flexiblen Einrichtung von Arbeitsplätzen, die mit verschiedenen Arbeitspersonen besetzt werden.

- Zusätzliche Kosten können durch gesetzliche Bestimmungen entstehen, in denen Teilzeitkräfte wie Vollzeitkräfte gezählt werden.

- Verdienen vollzeitbeschäftigte Arbeitspersonen über der Beitragsbemessungsgrenze, so erhöhen sich durch die 'ersatzweise' Beschäftigung von Teilzeitkräften, die unterhalb der Beitragsbemessungsgrenze entlohnt werden, die Arbeitgeberanteile zur Sozialversicherung.

Nutzen:

- Die Teilzeitbeschäftigung von Arbeitspersonen, die aus familiären, gesundheitlichen oder altersbedingten Gründen keine Vollzeittätigkeit mehr ausüben wollen oder können, trägt zur Sicherung des vorhandenen betriebsspezifischen Humankapitals bei. Das Arbeitskräftereservoir wird vergrößert und die Fluktuation verringert.

- Die Arbeitszufriedenheit, die Motivation, das Engagement und die Kreativität der Arbeitspersonen und damit auch die Ergebnisqualität können sich durch eine individuelle Arbeitszeitgestaltung und die Verbesserung der außerberuflichen Entfaltungsmöglichkeiten erhöhen. Während der verkürzten Arbeitszeit kann eine höhere Arbeitsintensität bewältigt werden. Die Arbeitsproduktivität kann insofern gesteigert werden.

- Teilzeitarbeit erleichtert eine Verlängerung der Betriebszeiten zur Verbesserung der Kapazitätsauslastung. Die mögliche höhere Flexibilität der Verfügbarkeit der Arbeitspersonen im Sinne der Anpassung der Arbeitszeit an den schwankenden Arbeitsanfall unterstützt die Verringerung von Leerlaufzeiten und die Verbesserung der Nutzung betrieblicher Kapazitäten.

- Teilzeitarbeit ermöglicht einen flexiblen Ausgleich tarifvertraglich vereinbarter Arbeitszeitverkürzung. Eventuell notwendige Entlassungen können vermieden werden. Fehlzeiten können durch Teilzeitarbeit leichter überbrückt werden. Durch flexible Arbeitszeiten kann Mehrarbeit (mit den entsprechenden Zuschlägen) verringert werden.

- Umfangreiche und vielfältige Teilzeitangebote verbessern die Position des Unternehmens am Arbeitsmarkt. 
Die Gegenüberstellung der Kosten- und Nutzengrößen macht deutlich, daß die teilzeitbedingten Kosten besonders bei Arbeitsaufgaben auftreten, die höhere Qualifikations- und Kommunikationsanforderungen stellen, während bei niedriger qualifizierten Tätigkeiten die Kostenvorteile an Bedeutung gewinnen. Neben traditionellen Vorbehalten dürfte dies ein wesentlicher Grund für die bisherige Beschränkung der Teilzeitarbeit auf geringer qualifizierte Tätigkeiten sein (vgl. BÄCKER/STOLZ-WILLIG, 1995, 58). So steht der Ausweitung höher qualifizierter Teilzeittätigkeiten beispielsweise entgegen, $\mathrm{da} B$ die durch Weiterbildungsmaßnahmen erworbenen Qualifikationen nur zeitlich verkürzt genutzt werden können.

Eine globale Beurteilung der Arbeitszeitkonzepte ist in folgender Abbildung wiedergegeben.

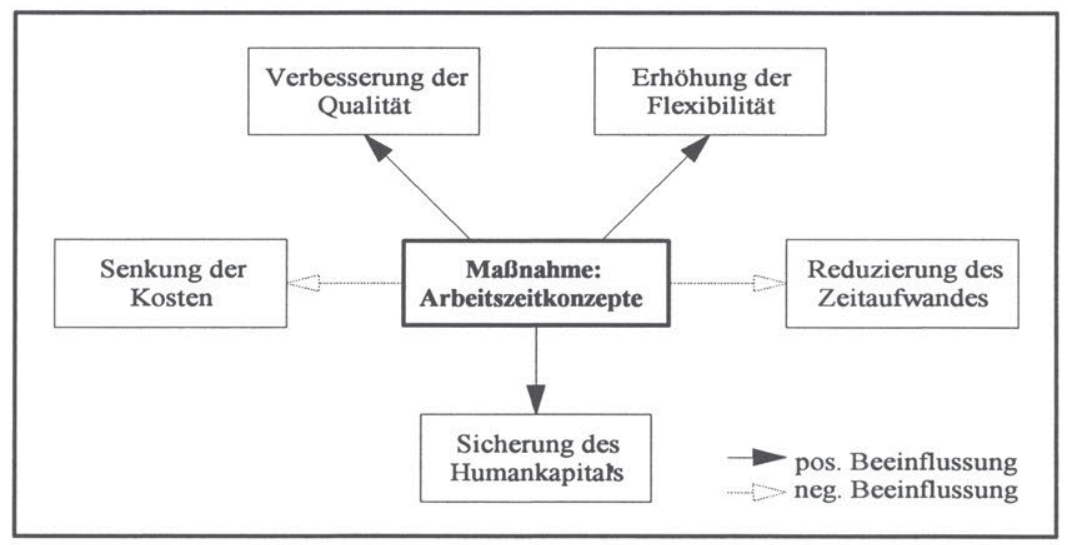

Abb. 63: Zielbeeinflussung durch die Umsetzung der Arbeitszeitkonzepte

\section{- Umsetzungsbedingungen}

Die kurz- und mittelfristig rationale Nutzung der Arbeitszeitverkürzung als ergänzendes Mittel zur Verminderung von Arbeitslosigkeit muß zukünftig langfristig reversibel vereinbart werden, um eine flexible Anpassung an sich ändernde Rahmenbedingungen zu ermöglichen. Entsprechend dieser Perspektive läßt sich die Teilzeitbeschäftigung verschieden nutzen, zunächst noch zur Vermeidung von Entlassungen und zum Ausgleich von Arbeitszeitverkürzungen und 
langfristig zur Ausschöpfung des Arbeitsangebots und zur Erhöhung der insgesamt geleisteten Arbeitszeit.

Vereinfacht richten sich die arbeitszeitbezogenen Interessen der Arbeitgeber auf die Flexibilität der Arbeitssysteme sowie des Arbeitskräftereservoirs, die Rationalisierungwirkungen, die Ausdehnung der Betriebslaufzeiten, den Abbau einschränkender Vereinbarungen und die Erleichterung der Abstimmung von Arbeitseinsatz, Arbeitsanforderungen und Produktionsablauf. Die Gewinnung zusätzlicher Arbeitskräfte durch das Angebot von Teilzeitarbeitsplätzen hat derzeit nur nachgeordnete Bedeutung, wie exemplarisch die Ergebnisse einer Befragung von 4.000 mittelständischen westdeutschen Unternehmen im Herbst 1994 zeigen.

\begin{tabular}{|l|c|c|c|}
\hline \multicolumn{1}{|c|}{$\begin{array}{c}\text { Angaben in \% } \\
\text { mit } \\
\text { Mehrfachnennungen }\end{array}$} & $\begin{array}{c}\text { Belastungsspitzen } \\
\text { abbauen }\end{array}$ & $\begin{array}{c}\text { erfahrene } \\
\text { Arbeitskræufte } \\
\text { halten }\end{array}$ & $\begin{array}{c}\text { neue Arbeitskrafte } \\
\text { einstellen }\end{array}$ \\
\hline Verarbeitendes Gewerbe & 60 & 39 & 20 \\
Baugewerbe & 65 & 35 & 15 \\
Handel & 67 & 44 & 15 \\
Dienstleistungsgewerbe & 58 & 50 & 34 \\
\hline
\end{tabular}

Abb. 64: Anteile mittelstăndischer Unternehmen verschiedener Branchen, die Teilzeitstellen aus den angegebenen Gründen anbieten (Quelle: Creditreform nach INSTITUT DER DEUTSCHEN WIRTSCHAFT, 1994)

Die arbeitszeitbezogenen Interessen der Arbeitnehmer und ihrer Vertreter beziehen sich demgegenüber auf die Zeitsouveränität und den Abbau zeitlicher $Z$ wänge, die Vereinbarung individueller Dispositionsrechte, die Wahlfreiheit der einzelnen Arbeitsperson, Verkürzungs- und Umkehrbarkeitsrechte, die Kontrolle durch die betriebliche Interessenvertretung, die tarifrechtliche und soziale Absicherung, die Beschäftigungsgarantie für den angestammten oder einen gleichwertigen Arbeitsplatz und die Erhöhung der Beschäftigung (vgl. beispielsweise BÄCKER/NAEGELE, 1993b, 246f.).

Die zukünftigen Regelungen müssen sich an beiden Interessenlagen orientieren. Sie müssen den betrieblichen Notwendigkeiten entsprechen und beispielsweise eine kostengünstige Nutzung der Anlagen ermöglichen sowie die individuellen Bedürfnisse der Arbeitspersonen beachten und damit die Rekrutierung und Bindung qualifizierter Arbeitspersonen unterstützen. Zur Vermeidung kontraproduktiver Auswirkungen muß hierbei jede Leistungsverdichtung, die zur Erhöhung der Gefahr von Fehlbeanspruchungen führen kann, vermieden werden. Zudem sollten 
Mindestarbeitszeiten eingehalten werden, um ungeschützte Beschäftigungsverhältnisse zu vermeiden und beispielsweise den Anteil der Wegezeiten nicht zu hoch werden zu lassen.

Damit sich die Arbeitszeitflexibilisierung nicht negativ auf die übrigen Sozialziele, wie die Förderung der Attraktivität der Erwerbstätigkeit und der Motivation der Arbeitspersonen sowie die Vermeidung gesundheitlicher Beeinträchtigungen, auswirkt, muß die Arbeitszeitgestaltung auf den Lebensrhythmus der Arbeitspersonen und das soziale Umfeld Rücksicht nehmen. In Familien können andererseits allgemein als ungünstig einzustufende Arbeitszeitlagen (am frühen Morgen, am späten Abend, in der Nacht, am Wochenende) eines Ehepartners die Vereinbarkeit von Familie mit der Erwerbstätigkeit beider Elternteile erleichtern.

Entscheidend für eine breite Nutzung dieser Maßnahme sind die Handlungsweisen der Arbeitgeber. Bestehende Vorbehalte und organisatorische Schwierigkeiten hinsichtlich des Angebots von qualifizierten Teilzeittätigkeiten müssen überwunden werden. Andererseits ist es notwendig, daß die Unternehmen die angesprochenen Nutzengrößen ausschöpfen können. Hierzu müssen sich die Arbeitspersonen den betrieblichen Anforderungen anpassen. Insbesondere darf sich die Bereitschaft zur Teilzeitarbeit nicht nur auf den Vormittag richten. Berücksichtigt man die außerberuflichen Anforderungen der Arbeitspersonen so erscheint es sinnvoll, daß gerade Ältere nachmittags beschäftigt werden, während Frauen verstärkt vormittags erwerbstätig sein können. Daneben sollten zukünftig auch andere Teilzeitmodelle als Halbtagsarbeit Verbreitung finden. Flexibilität ist somit auf beiden Seiten notwendig und - wie die Diskussion arbeitszeitlicher Regelungen zeigt - in Teilbereichen bereits gegeben.

Die Verbreitung der Teilzeitarbeit für Männer im Interesse ihrer stärkeren Beteiligung an den Haushalts- und Erziehungsarbeiten scheint als Strategie zur gesamtwirtschaftlich wirksam werdenden Unterstützung der Erwerbstätigkeit von Frauen allerdings auch zukünftig wenig aussichtsreich. Denn Männer interessieren sich aus anderen als haushalts- und familienbezogenen Gründen für Teilzeitarbeit und der Wechsel eines Arbeitnehmers von der Vollzeit zur Teilzeitarbeit entspricht nicht dem Interesse des jeweiligen Arbeitgebers, da der einzelne Arbeitgeber nicht direkt von einem evtl. gesteigerten Gesamtarbeitsangebot des Paares profitiert, falls nicht beide Partner im gleichen Unternehmen beschäftigt sind. 


\section{c) Arbeitsschutz}

Während sich der soziale Arbeitsschutz auf den zeitlichen Schutz und Vorschriften für besonders schutzbedürftige Personengruppen, wie Jugendliche, Mütter und Schwerbehinderte bezieht, richtet sich der technische Arbeitsschutz beispielsweise auf die Anlagen- und Gerätesicherheit, die Organisation der betrieblichen Arbeitssicherheit und den Schutz vor schädlichen Einwirkungen am Arbeitsplatz (z.B. durch Chemikalien oder Lärm). Im folgenden werden einzelne Aspekte des technischen Arbeitsschutzes hinsichtlich des Ziels einer erhöhten Ausschöpfung des Erwerbspersonenpotentials betrachtet. Diese Maßnahme wird nicht alters- oder geschlechtsspezifisch behandelt, da "Arbeitsschutz für Ältere nicht anders als Arbeitsschutz für Jüngere, Männer wie Frauen" (MÜLLER, 1994, 2) ist.

Der Arbeitgeber ist verpflichtet, den Arbeitnehmer möglichst weitgehend vor gesundheitlichen Gefährdungen bei der Arbeit und durch die Arbeit zu schützen. Außer den vielfältigen nationalen rechtlichen Vorgaben zum Arbeitsschutz gewinnen zunehmend die EG-Richtlinien zum Binnenmarkt (Artikel 100a) und zum Arbeitsschutz (Artikel 118a) an Bedeutung (vgl. HALBACH u.a., 1994, 469ff.; BIENECK/RÜCKERT, 1994). Neben den traditionellen Aufgaben der Unfallverhütung und der Vermeidung von Berufskrankheiten gehören auch die Förderung der menschengerechten Gestaltung der Arbeit und die Erhaltung der menschlichen Arbeitskraft zu den Aufgaben des Arbeitsschutzes. Der Arbeitsschutz richtet sich daher auf einen präventiven Schutz und zunehmend auch auf die Förderung der Gesundheit der Arbeitspersonen (vgl. RICHTER u.a., 1995), so daß sich das Aufgabengebiet, von den Ausführungsbedingungen ausgehend, auf immer weitere Felder der Arbeitsgestaltung ausdehnt.

Angesichts dieser erweiterten Auffassung betonen die folgenden Überlegungen die Bedeutung des Arbeitsschutzes zur Prävention im Sinne der Vermeidung von Fehlbeanspruchungen und betrachten nicht die Aspekte der Sicherheit und Unfallhäufigkeit, die im Einzelfall auch gegenläufige Gestaltungsempfehlungen nahelegen können (vgl. RICHTER u.a., 1995, 8). Darüber hinaus führt eine konsequente Umsetzung der genannten Zielsetzungen des Arbeitsschutzes dazu, daß neben einer präventiven Arbeitsgestaltung Maßnahmen zur prospektiven Arbeitsgestaltung im Sinne der Förderung der Gesundheit ergriffen werden (vgl. ULICH, 1991, 142ff.). 


\section{- Gestaltungsformen}

Arbeits- und Gesundheitsschutz tragen durch den Erhalt der Eigenschaften und die Minderung der Belastungen dazu bei, daß Fehlbeanspruchungen, die auch die Arbeitssicherheit gefährden können, vermieden werden und die Arbeitsaufgaben während des gesamten Arbeitslebens ausgeführt werden können. Arbeitsschutz ist eine ständige Aufgabe zur Sicherung des eigenschaftsadäquaten Einsatzes der Arbeitspersonen.

Ein deutlicher am Individuum orientierter Arbeitsschutz muß präventiv und prospektiv wirken. Ziel sollte nicht nur die Vermeidung gesundheitsschädigender Einflüsse der Arbeit sein, sondern die Entwicklung gesundheitsfördernder Arbeit. Hierzu muß der Arbeitsschutz in die Planung, Konzeption und Umsetzung neuer Produktions- und Organisationsmethoden integriert werden.

Die folgenden Punkte sind für eine solche Form des Arbeits- und Gesundheitsschutzes wesentlich:

- Einstellungsuntersuchungen dürfen nicht nur körperliche, sondern müssen zunehmend auch die psychische Belastbarkeit feststellen.

- Regelmäßige, tätigkeitsbezogene arbeitsmedizinische Untersuchungen müssen die Einstellungsuntersuchungen ergänzen, um die Tauglichkeit der Arbeitspersonen (soweit möglich ohne negative Folgen für die Beschäftigten) zu überprüfen und um potentielle Beeinträchtigungen der Gesundheit der Arbeitspersonen, z.B. durch physische oder psychische Überforderungen, aufzudecken.

- Vermeidung arbeitsbedingter gesundheitlicher Gefährdungen und Beeinträchtigungen durch Früherkennungsuntersuchungen und Maßnahmen zum individuellen Gesundheitsschutz.

- Durchführung regelmäßiger Arbeitsanalysen, insbesondere zur Feststellung konkreter Belastungs- und Beanspruchungsverschiebungen infolge des Einsatzes neuer Produktions- und Organisationsmethoden.

- Wichtig ist die Analyse tätigkeits- und gesundheitsbezogener Daten zum Erkennen von Risiken und Gesundheitsschäden sowie zur Entdeckung unternehmens- bzw. tätigkeitsspezifischer Zusammenhänge. Solche Zusammenhänge können z.B. zwischen Eigenschaften der Arbeitspersonen (wie die Art der individuellen Erfahrung und die Dauer der Betriebszugehörigkeit) und der Arbeitsunfähigkeit von Arbeitskräften bestehen. 
- Arbeitsplatzbezogene Betrachtung und Beratung in arbeitsmedizinischer, arbeitswissenschaftlicher, organisatorischer und ergonomischer Hinsicht. Abbau von Überforderungen und Ausgleich von Unterforderungen.

- Arbeitsschutzspezifische (gesundheitliche und sicherheitsbezogene) Schulungen. Beispielsweise müssen Arbeitsmediziner hinsichtlich der physiologischen sowie psycho-sozialen Wirkungen der Alterung geschult werden.

- Weiterhin muß die Zusammenarbeit von arbeitsmedizinischen Diensten, Krankenkassen, Berufsgenossenschaften und staatlicher Stellen zur Prävention arbeitsbedingter Krankheiten gefördert werden.

Zur Gestaltung einer entwicklungsförderlichen Arbeit, die Fehlbeanspruchungen vermeidet, muß die Arbeit anforderungsverschiedene Bestandteile enthalten, ohne durch zu viele Tătigkeiten überfordernd zu wirken. Neben dem Einsatz moderner Techniken ist arbeitsorganisatorisch ein Wechsel der Belastungen anzustreben, um monotone, einseitige und hohe körperliche Belastungen zu vermeiden. Solche Belastungswechsel führen beispielsweise zur Begrenzung der Bildschirmarbeit, zur Verbesserung der innerbetrieblichen Kommunikation und zur Minderung körperlich einseitiger Belastungen. Die Verringerung des körperlichen Verschleißes erfordert insbesondere den weiteren Abbau des Hebens und Tragens schwerer Lasten sowie ungünstiger und belastender Zwangshaltungen, wie Überkopfarbeit, Bücken, Hocken oder Knien. Weiterhin müssen die Belastungen durch Lärm und gefährdende Stoffe Beachtung finden.

Bezogen auf die Beanspruchungsfolgen geistiger Arbeit sollte die Arbeit mehr als nur eine Bearbeitungsfunktion, einen mittleren Kommunikations- und Kooperationsbestandteil (von täglich bis zu zwei Stunden) und eine mittlere Anzahl von inhaltlichen Freiheitsgraden (bzgl. der Bestimmung der Arbeitsabfolge, der zu nutzenden Arbeitsmittel und der einzuschlagenden Arbeitswege) enthalten, um negative Beanspruchungsfolgen wie Monotonie und Sättigung zu mindern (vgl. RICHTER u.a., 1995).

Unabhängig von der Möglichkeit und der Art des Nachweises sind gesundheitliche Beschwerden eine Ursache für die Beendigung der Erwerbstätigkeit. Insofern sie zukünftig durch arbeitsgestalterische Maßnahmen zu mindern sind, sollten Unternehmen auch diese Möglichkeit nutzen, um insbesondere ältere Arbeitspersonen länger beschäftigen zu können. Zur Entwicklung konkreter technischer und organisatorischer Verbesserungsmöglichkeiten muß auf die Beteiligung der betroffenen Arbeitspersonen zurückgegriffen werden. 


\section{- Betroffene Kosten und Nutzengrößen}

Arbeitsschutz kann zur Erfüllung rechtlicher Vorschriften im Interesse der Beschäftigten und im Interesse der Wirtschaftlichkeit des Unternehmens erfolgen.

Kosten:

- Die Ausweitung des Arbeitsschutzes zieht zusätzliche Personalkosten für Werkärzte, Arbeitsmediziner, Ergonomen nach sich.

- Die erweiterten und häufigeren Untersuchungen der Arbeitspersonen, die geforderten Analysen der Arbeitstätigkeiten und die schulungsbedingte Arbeitsausfalle verursachen erhöhte Kosten.

- Die Geschwindigkeit und Flexibilität der Handlungsweisen der Arbeitspersonen können durch die Beachtung vielfaltiger Empfehlungen des Arbeitsschutzes verringert werden.

Nutzen:

- Maßnahmen des Arbeitsschutzes tragen zur Minderung des vorzeitigen Ausscheidens der Arbeitspersonen und zur Sicherung des vorhandenen Humankapitals bei.

- Die Verringerung der Fluktuation der Beschäftigten senkt weiterhin die Kosten der Einarbeitung neuer Arbeitspersonen.

- Neben dem Erhalt der Leistungsfähigkeit der Arbeitssysteme ist die Förderung der Motivation der Beschäftigten ein positiver Effekt des Arbeitsschutzes. Die Identifikation der Arbeitspersonen mit dem Unternehmen und die Arbeitszufriedenheit werden gefördert. Die Position des Unternehmens am Arbeitsmarkt verbessert sich.

- Arbeitsschutz reduziert die Fehlzeiten und Arbeitsausfallkosten sowie die Kosten krankheitsbedingter Vertretungen (z.B. während Erkrankungsphasen und Kuren der beschäftigten Arbeitspersonen).

Eine globale Beurteilung der Maßnahme Arbeitsschutz verdeutlicht folgende Abbildung. Bezüglich des Ziels der Verbesserung der Qualität läßt sich keine eindeutige Wirkung der Maßnahmenumsetzung einschätzen. 


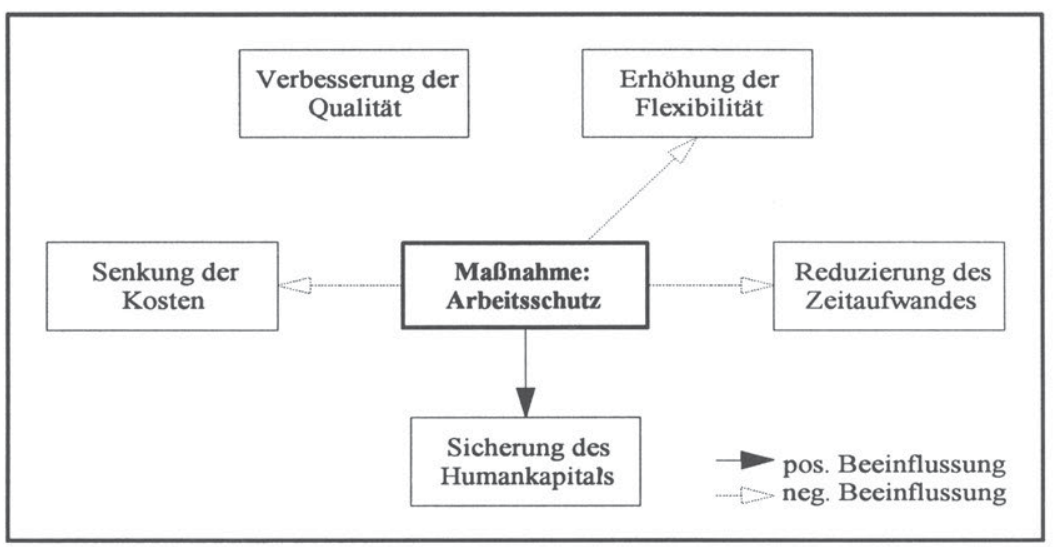

Abb. 65: Zielbeeinflussung durch die Umsetzung der Maßnahme Arbeitsschutz

\section{- Umsetzungsbedingungen}

Trotz der Entschärfung vieler gesundheitlicher Gefahren am Arbeitsplatz (vgl. FRUHMANN, 1993) müssen gerade zur Erhöhung der Erwerbsbeteiligung älterer Personen auch zukünftig arbeitsschutzbezogene Probleme erörtert und konkrete Maßnahmen entworfen werden. Insbesondere müssen die zunehmenden geistigen und psycho-sozialen Anforderungen beachtet werden. Ein erfolgreicher Arbeitsund Gesundheitsschutz erfordert die Zusammenarbeit verschiedener betrieblicher Stellen, die Unterstützung des Betriebsrates zur Erhöhung der Akzeptanz und die Bereitschaft der Arbeitspersonen zur Teilnahme an Untersuchungen und zur Erarbeitung von Verbesserungsmöglichkeiten. Hierzu können auf einer arbeitsplatznahen Ebene ergänzend zu überbetrieblichen Arbeitsschutzinstitutionen und innerbetrieblichen Arbeitsschutzinstanzen Gesundheitszirkel eingeführt werden (vgl. VON FERBER/VON FERBER, 1991; SLESINA/BROEKMANN, 1992). Gesundheitszirkel nutzen das belastungs- und beanspruchungsbezogene Erfahrungswissen der Arbeitspersonen für die Entwicklung von Änderungsvorschlägen. Ihre Konzeption entspricht der tendenziellen Ausweitung der Gruppenarbeit und unterstützt die Mitbestimmung der Beschäftigten am Arbeitsplatz. 


\section{d) Arbeitshilfen und Schonarbeit}

Während eine korrektive Arbeitsgestaltung erkannte gestalterische Mängel nachträglich korrigiert bzw. bereits gegebene Gesundheitsschädigungen ausgleicht, trägt die präventive Arbeitsgestaltung zur Vermeidung von Gesundheitsschädigungen und Beeinträchtigungen bei (vgl. ULICH, 1991, 144). Entsprechend muß eine präventiv wirkende und gesundheitsschützende ergonomische Arbeitsplatzgestaltung in anthropometrischer, physiologischer, bewegungstechnischer sowie sicherheits- und informationstechnischer Hinsicht angestrebt werden, um insbesondere eine Verlängerung der durchschnittlichen Lebensarbeitszeit realisieren zu können.

Im folgenden werden präventiv wirkende Arbeitshilfen zur Vermeidung arbeitsbedingter Krankheiten und Beschwerden, die zu einer späteren Frühverrentung führen können, sowie die korrektiv wirkende Einrichtung von Schonarbeitsplätzen behandelt. Die Betrachtung von Arbeitshilfen und Schonarbeit erfolgt gemeinsam in einem Abschnitt, da wirksame und über das gesamte Arbeitsleben wirkende Arbeitshilfen die Notwendigkeit der gesonderten Einrichtung von Schonarbeitsplätzen verringern können.

Weiterhin können Maßnahmen zur Gestaltung von Arbeitsplätzen wirtschaftlich beurteilt werden (vgl. beispielsweise PICOT u.a., 1985 und SEIDEL/ SCHUMACHER, 1985), während die Gestaltung von Schonarbeit als Reaktion auf Leistungsminderungen eine gerade in sozialer Hinsicht notwendige Maßnahme darstellt, die innerhalb dieses Abschnitts zwar angesprochen, aber einer ökonomischen Abwägung entzogen werden soll.

\section{- Gestaltungsformen für Arbeitshilfen}

Die Arbeitsplatzgestaltung und der Entwurf von Arbeitshilfen beziehen sich beispielsweise auf

- den Arbeitsablauf und die Gestaltung von Ein- oder Ausgabeformen,

- die Arbeitsmittel und die Verstellmöglichkeiten von Tischen, Stühlen, Ablageflächen, Arbeitsgeräten, z.B. zur Vermeidung von $Z$ wangshaltungen,

- das Arbeitsumfeld und die Beeinflussung der Einwirkungen durch Klima, Licht, Lärm, Staub und Farbgebung sowie

- die Arbeits- und Sozialräume hinsichtlich ihrer Gestaltung, Einrichtung und Nutzung. 
Ähnlich wie die Maßnahme Arbeitsschutz ist auch die Gestaltung von Arbeitshilfen nicht geschlechts- oder altersspezifisch zu konzipieren, sofern es um die Vermeidung arbeitsbedingter Beeinträchtigungen geht. Andererseits müssen bei der Arbeitsplatzgestaltung die individuellen Eigenschaften der Arbeitspersonen berücksichtigt werden, die geschlechts- oder altersspezifisch sein können.

So müssen beispielsweise hinsichtlich der räumlichen Gestaltung Unterschiede der Körperabmessungen beachtet werden, indem eine genügend große Verstellbarkeit und Anpaßbarkeit ermöglicht wird. Besondere arbeitsplatzgestalterische Maßnahmen sind gegen spezifische Arbeitsbelastungen, wie monotone, hohe sensorische und motorische Belastungen durch repetitive oder taktgebundene Tätigkeiten notwendig. Ungünstige Bedingungen, Konstruktionen und Anordnungen können dadurch entdeckt werden, daß ältere Personen Schwierigkeiten damit haben, da ihre Toleranz negativen Einflüssen gegenüber zumeist geringer ist als die der Jüngeren. In diesem Sinn sind Ältere, beispielsweise gegenüber körperlichen Fehlhaltungen, sensibler. Eine bessere Gestaltung der Arbeitsmittel oder der Bedienungsweise von Maschinen nutzt allerdings nicht nur den Älteren sondern erleichtert auch jüngerer Personen die Durchführung der Arbeit. Die Arbeitsaufgaben können zudem sicherer und weniger fehleranfällig gelöst werden.

Angesichts der besonderen Bedeutung der Nutzung technischer Innovationen und der damit auftretenden arbeitsgestalterischen Anforderungen kann auf das Beispiel der Hard- und Software-Ergonomie eingegangen werden.

Die Software-Ergonomie ist ein Forschungs- und Entwicklungsbereich, der die technikzentrierte Betrachtung (insbesondere der Informatik) überschreitet und Aufgaben der Arbeitsgestaltung betrifft. Zur Gestaltung der Software müssen individuelle, technische, aufgabenspezifische und organisatorische Gegebenheiten beachtet werden, so daß jede Gestaltungsentscheidung in vielfältiger Weise von diesen Gegebenheiten abhängt (vgl. z.B. MAAß, 1993). Die Erfüllung technischer Anforderungen wird zunächst vorausgesetzt, denn wichtiger als Funktionen und technische Leistungsfähigkeiten sind die Faktoren Erlernbarkeit, Bedienbarkeit und Betreuungsaufwand. Durch Maßnahmen der Software-Ergonomie können die sehr hohen Kosten computerbezogener, unproduktiver Tätigkeiten, wie Fehlbedienungen und Lösen von Bedienungsproblemen, vermindert werden (vgl. beispielsweise KLOTZ, 105ff.). Die Software-Ergonomie richtet sich auf eine aufgaben- und benutzergerechte Gestaltung der Software zur Optimierung computernutzender 
Arbeitssysteme (vgl. speziell zur Computernutzung durch ältere Arbeitspersonen STAUFER, 1992). Gestaltungskriterien für die Software betreffen insbesondere die folgenden Punkte:

- Die sog. Mensch-Rechner-Kommunikation bzw. die Dialogführung an den Kommunikationsschnittstellen muß praxisnah gestaltet werden.

- Die Benutzeroberflächen sollten möglichst einheitlich sein. Die Programme müssen, beispielsweise durch die Vereinfachung von Befehlen sowie durch eine wirkungsvolle Bedienerführung, angepaßte Erklärungen und Hilfsfunktionen, selbsterklärend und leicht erlernbar sein.

- Die Software sollte kurze Antwortzeiten ermöglichen und Vorabinformationen über die Dauer zeitintensiver Verarbeitungsprozesse geben. Daneben müssen jederzeit Informationen über den Systemstatus bereitgestellt und Abbruchmöglichkeiten sowie Rückstellmöglichkeiten geboten werden.

- Test- und Simmulationssysteme tragen zur Erhöhung der Benutzungssicherheit bei. Eine ausreichende Signalgebung muß zudem die Fehlererkennung und Fehlerbewältigung erleichtern.

Von Interesse ist auch die Unterstützung von Gruppenarbeit durch sog. Groupware, die sich nicht auf individuelle Benutzer richtet, sondern auf deren organisatorische Einbindung und eine gruppengerechte Gestaltung der Systeme zielt, indem insbesondere Fragen der Kommunikation und Kooperation zwischen den Gruppenmitgliedern behandelt werden.

Die Hardware-Ergonomie bezieht sich vor allem auf die Gestaltung von Maschinenund Bedienelementen und im EDV-Bereich auf die Rechnerausstattung. Gestaltungskriterien für Hardware können insbesondere in folgenden Anforderungen gesehen werden:

- Die Bedienung der Rechner und Anlagen muß auch unter Zeitdruck fehlerfrei erfolgen können.

- Die Geräte müssen wartungsarm sein.

- Die Geräte müssen über eine ausreichend hohe Speicherkapazität verfügen und kurze Antwortzeiten ermöglichen.

- Die Auswahl und Gestaltung der Ein- und Ausgabegeräte muß sich an den Benutzerbedürfnissen orientieren, so daß beispielsweise hochwertige blend- und flimmerfreie Bildschirme benutzt werden. 
Insgesamt sollte sich die Gestaltung und Nutzungsweise der Hard- und Software sowie der Technik insgesamt am Erfahrungswissen der Arbeitspersonen orientieren und dieses nutzen, so daß Facharbeiter - wie beispielsweise mit der Werkstattprogrammierung der Maschinen realisiert - den Arbeitsablauf bestimmen und flexibel auf Probleme reagieren können. Auf diese Weise können durch erfahrungsbezogene Technikanwendungen tayloristische Arbeitsformen überwunden werden (vgl. beispielsweise KLOTZ, 108f.).

\section{- Gestaltungsformen für Schonarbeitsplätze}

Eine Erhöhung der durchschnittlichen Lebensarbeitszeit erfordert auch die Weiterbeschäftigung leistungsgeminderter Arbeitspersonen. Die bisher entsprechend angebotenen Arbeitsplätze betreffen zumeist einfache Tätigkeiten (vgl. HOFFMANN, 1993). So bieten beispielsweise die Aufgaben von Pförtnern und Boten, Arbeiten im Magazin und Lager sowie Tätigkeiten der leichten Kontrolle, der Reinigung, Wartung, Pflege, und Registratur Weiterbeschäftigungsmöglichkeiten (vgl. NAEGELE, 1992, 90 und die dort angeführte Literatur). Durch den Wegfall vieler einfacher Arbeitsaufgaben einerseits und die zukünftige Erhöhung des Anteils älterer Arbeitspersonen, die altersbedingt häufiger leistungsgemindert sein dürften, werden die Weiterbeschäftigungschancen tendenziell sinken. Daher müssen verstärkt Arbeitsplätze identifiziert und eingerichtet werden, an denen Arbeitspersonen mit Schädigungen bzw. Beeinträchtigungen beschäftigt werden können.

Obwohl die Einrichtung von Schonarbeitsplätzen eine Art Defensivmaßnahme darstellt, die lediglich korrektiv wirkende ergonomische Verbesserungen zum Ausgleich beschäftigungsbedingter bzw. altersbedingter Verschlechterungen körperlicher Funktionen umsetzt, ist sie ein notwendiger Beitrag zur Verlängerung der Lebensarbeitszeit. Schonarbeitsplätze müssen zunehmend als 'normale' Arbeitsplätze aufgefaßt werden.

Können Arbeitspersonen mit eingeschränkter Leistungsfähigkeit an ihren bisherigen Arbeitsplätzen nicht weiter beschäftigt werden, sind Möglichkeiten des innerbetrieblichen Arbeitsplatzwechsels und der ergonomischen Arbeitsplatzumgestaltung zur Berücksichtigung der Bedürfnisse vermindert anpassungsfähiger Arbeitspersonen zu nutzen. Innerhalb der Unternehmen ist eine entsprechende 
Arbeitsplatzüberprüfung nach allgemeinen Gestaltungsregeln sinnvoll. Drei Gruppen von Arbeitsplätzen lassen sich unterscheiden:

- Die Arbeitsplätze entsprechen den Anforderungen an einen Schonarbeitsplatz. In diesem Fall können die betroffenen Arbeitspersonen an ihren Arbeitsplätzen verbleiben.

- Die Arbeitsplätze können als Schonarbeitsplätze gestaltet werden. Nach arbeitsgestalterischen Eingriffen können die betroffenen Arbeitspersonen weiterhin an diesen Arbeitsplätzen beschäftigt werden.

- Die Arbeitsplätze können nicht zu Schonarbeitsplätzen umgestaltet werden. Die Einsatzbestimmung der Arbeitspersonen muß geändert und ein Wechsel der Arbeitsplätze ermöglicht werden.

Schonarbeitsplätze können insbesondere durch folgende Punkte gekennzeichnet sein:

- Minderung physischer und/oder psychischer Belastungen,

- Abbau von Verantwortung,

- Abbau von übermäßigem Leistungsdruck,

- Abbau von Zeitdruck, Termindruck und Arbeitstempo (keine Vorgabe von Arbeitszeiten oder Stückzahlen, kein Prozeßdruck),

- Einzelarbeitsplätze, die eine individuelle Arbeitsgestaltung ermöglichen und der Arbeitsperson Wahlmöglichkeiten hinsichtlich der Arbeitsdurchführung bieten,

- Verlängerung der Pausen- und Erholungszeiten,

- Normalschicht,

- Wechsel der Arbeitsinhalte zur Vermeidung einseitiger Belastung.

Die Art der übertragenen Tätigkeiten bestimmt sich nach den Eigenschaften der Arbeitsperson und ihrer verbliebenen Leistungsfähigkeit. Die Umrüstung des Arbeitsplatzes kann beispielsweise in der Ausrüstung des Arbeitsplatzes mit technischen Hilfsmitteln, wie Halte-, Hebe-, Transportvorrichtungen, bestehen. Die Maßnahmen der Arbeitsgestaltung und der Personalentwicklung müssen im Einzelfall durch medizinische und physiotherapeutische Maßnahmen ergänzt werden. Wie bereits betont ist die Gestaltung von Schonarbeitsplätzen nicht als vorbeugende, sondern eher als ergänzende Maßnahme im Falle individueller Beeinträchtigungen $\mathrm{zu}$ betrachten. Problematisch sind Schonarbeitsplätze besonders, falls sie für die Arbeitspersonen eine berufliche Herabsetzung, einen geringeren Verdienst oder einen betrieblichen Ansehensverlust bedeuten. 
- Betroffene Kosten und Nutzengrößen

Für die präventive Gestaltung von Arbeitshilfen lassen sich die folgenden wesentlichen Kosten- und Nutzengrößen anführen.

Kosten:

- Die Einrichtung von Arbeitshilfen führt zur Erhöhung der Investitionen und des Aufwandes.

- Eine individuelle Umgestaltung und Betreuung läßt zusätzliche Kosten entstehen.

- Zugunsten einer menschengerechten und gesundheitsschützenden Arbeitsplatzgestaltung muß u.U. auf sonstige $\mathrm{zu}$ erreichende technisch-organisatorische Vorteile verzichtet werden.

Nutzen:

- Arbeitshilfen können zur Erhöhung der Produktivität und zur Minderung des vorzeitigen Ausscheidens der Arbeitspersonen beitragen.

- Die Einarbeitung im Falle einer Neueinstellung oder einer Umsetzung kann beschleunigt und der Qualifizierungsaufwand gesenkt werden. Entsprechend steigt die Flexibilität des Einsatzes der Arbeitspersonen.

- Minderung der Ermüdung, Verringerung von Streß und Überforderung.

- Erhöhung der Motivation, der Arbeitszufriedenheit und der Akzeptanz. Stärkung und Nutzung des persönlichen Engagements der Arbeitskräfte.

- Arbeitshilfen können eine raschere Durchführung der Tätigkeiten bewirken und die Fehlerhäufigkeit senken, so daß sich die Qualität erhöht.

Eine globale Beurteilung der Arbeitshilfen läßt sich gemäß der folgenden Abbildung erstellen. Die Einrichtung von Schonarbeitsplätzen soll nicht in dieser Weise beurteilt werden, da sie im Einzelfall aufgrund der individuellen Bedürfnisse der Arbeitsperson insbesondere unter sozialen Gesichtspunkten unabdingbar sein kann. 


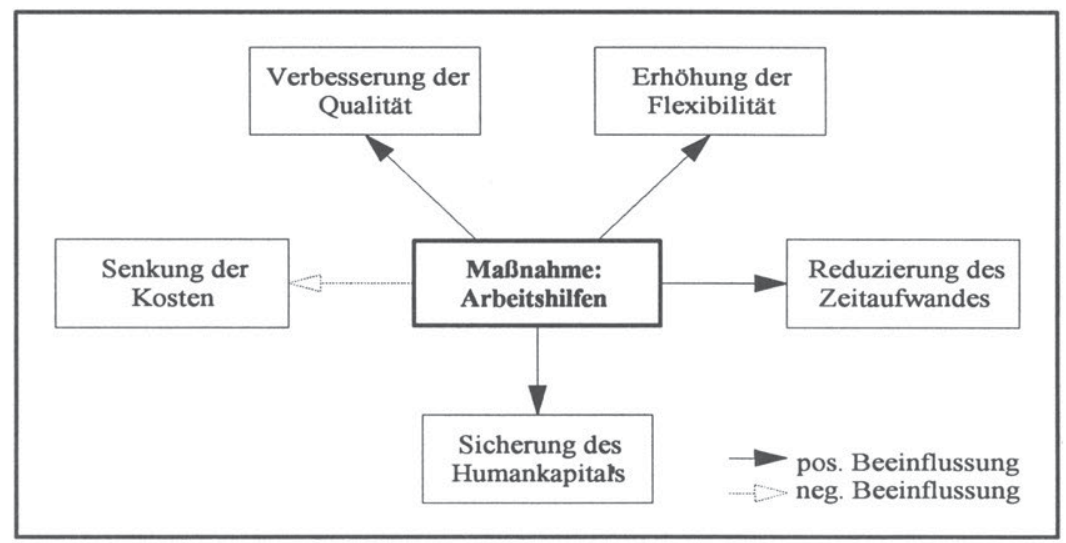

Abb. 66: Zielbeeinflussung durch die Umsetzung der Maßnahme Arbeitshilfen

\section{- Umsetzungsbedingungen}

Aufwendige Maßnahmen der Arbeitsplatzgestaltung sind eher in Großbetrieben als in Klein- und Mittelbetrieben durchführbar. Aufgrund der größeren Zahl der Arbeitsplätze finden sich in Großbetrieben zudem eher geeignete Möglichkeiten zur Beschäftigung spezifisch leistungsgeminderter Personen. Allerdings kann gerade in kleineren Unternehmen die betriebsspezifische Erfahrung der Arbeitspersonen hilfreich sein, so daß leistungsgeminderte Arbeitspersonen eher an ihrem (entsprechend umgestalteten) Arbeitsplatz bleiben können.

Neben der Arbeitsperson und ihren Vorgesetzten müssen verschiedene unternehmensinterne (v.a. Werksarzt, Personalabteilung und Betriebsrat) und unternehmensexterne Stellen (v.a. behandelnder Arzt, Berufsgenossenschaft und Krankenkasse) zusammenarbeiten, um Schonarbeitsplätze individuell zu gestalten. Durch die Gestaltung anspruchsvoller aber schonender Arbeitsaufgaben muß der Erhalt des Status und des Ansehens der Arbeitspersonen gesichert werden.

Die Arbeitspersonen müssen zur Mitwirkung bei der individuellen (Um-) Gestaltung des Arbeitsplatzes, zum Abbau gesundheitsschädigender Verhaltensweisen und gegebenenfalls zum Arbeitsplatzwechsel bereit sein. Allerdings kann gerade die Umsetzung auf einen Schonarbeitsplatz selbst psychisch stark belastend wirken. 
Staatliche Stellen können die Identifizierung und Einrichtung entsprechender Arbeitsplätze durch Finanzmittel und Beratungsleistungen sowie die stärkere Beteiligung der Arbeitgeber an den Kosten arbeitsbedingter Krankheiten fördern. Die Tarifpartner können durch den Abbau von finanziellen Bestandsschutzregelungen die Weiterbeschäftigungschancen erhöhen.

\section{Maßnahmen der Personalentwicklung}

Die Maßnahmen der Personalentwicklung müssen ebenso wie die der Arbeitsgestaltung im Anspruchsfeld der individuellen Bedürfnisse der Arbeitspersonen und der unternehmerischen Ziele gestaltet werden. Zentrale Aufgaben der Personalentwicklung beziehen sich auf die Qualifikation und Motivation der Arbeitspersonen, die das Kreativitäts- und Innovationspotential der Unternehmen darstellen. Im weiteren werden Maßnahmen zur Weiterbildung und zur betrieblichen Frauenförderung betrachtet.

\section{a) Weiterbildung}

Bezüglich der Einflußfaktoren auf die zukünftige betriebliche Weiterbildung lassen sich die folgenden Punkte hervorheben:

- Demographisch bedingt geht der zur Verfügung stehende Nachwuchs, wie beispielsweise die Zahl der Absolventen von Ausbildungen des dualen Systems, zurück. Entsprechend verringert sich der Zustrom aktuell qualifizierter Arbeitskräfte.

- Die Umsetzung neuer Produktions- und Organisationsmethoden und technische Innovationen bewirken die Integration der Arbeitsabläufe und fördern komplexere Arbeitsaufgaben mit höheren und häufiger wechselnden Anforderungen. Daher werden in höherem Maße als bislang aktuelle und umfassende Qualifikationen erforderlich.

- Es besteht eine Wechselwirkung zwischen der Beschäftigung qualifizierter Arbeitspersonen und den Möglichkeiten der Nutzung bzw. Anpassung neuer Methoden sowie der Gestaltung anspruchsvoller Arbeitsaufgaben.

Die Anpassung der Qualifikation der Beschäftigten, die besonders wegen der Verkürzung der Produktlebenszeit, der Verbreitung der Gruppenarbeit sowie der rascher fortschreitenden technischen Entwicklung, der Automatisierung, Integration 
und Dezentralisierung häufiger notwendig wird, läßt sich immer weniger durch informelle und fallweise Weiterbildungsmaßnahmen erreichen. Statt dessen gewinnt eine kontinuierliche Qualifikationsentwicklung an Bedeutung. $\mathrm{Da}$ keine ausreichenden staatliche Maßnahmen in diesem Bereich zur Verfügung stehen, ist eine weitere Ausweitung der innerbetrieblichen Weiterbildung notwendig. Besonders kleine und mittlere Unternehmen müssen die Qualifikation ihrer Mitarbeiter erhalten und steigern, da sie im Wettbewerb um Nachwuchskräfte tendenziell größeren Unternehmen unterliegen werden.

Eine Verringerung des Anstiegs der erforderlichen Weiterbildungsmaßnahmen kann durch die Umsetzung der Maßnahme zur Verbesserung des Aufbaus und der Nutzung des Erfahrungswissens erreicht werden. Sind während der Arbeitsdurchführung bessere Möglichkeiten zum Erwerb vielseitiger Kompetenzen gegeben, so sinkt der Weiterbildungsbedarf. Aufgrund der zunehmenden Höherqualifizierung dürfte sich das Lerninteresse stärker verselbständigen, so daß zukünftig Lernchancen während der Arbeit häufiger gefordert und besser genutzt werden. Allerdings kann der zunehmenden Bedeutung des theoretischen Wissens nicht durch bloße Stärkung und Eröffnung von Lernchancen während der Arbeitsdurchführung begegnet werden (vgl. beispielsweise BAETHGE, 1992).

\section{- Gestaltungsformen}

Die betriebliche Weiterbildung hat je nach Gestaltungsform unterschiedliche Wirkungen. So können kurzfristige Anpassungsqualifizierungen beispielsweise ein Mittel zur Bewältigung der durch technisch-organisatorische Umstellungen veränderten Anforderungen sein. Andererseits erfordert die Erreichung der Akzeptanz und der kreativen Unterstützung der Arbeitspersonen eine längerfristige und umfassendere Weiterbildung. Während eine betriebs- bzw. anlagenspezifische Weiterbildung das Ziel hat, den Bestand qualifizierter Arbeitnehmer zu sichern und Abwanderungen $\mathrm{zu}$ vermeiden, tragen Weiterbildungsmaßnahmen, die breitere Kompetenzen vermitteln, zur Erhöhung der Flexibilität des Einsatzes der Arbeitspersonen bei. Differenzierte und flexible Formen von Weiterbildungsmaßnahmen erlauben die Anpassung der Qualifikationen an die innerbetrieblichen Gegebenheiten.

Betriebliche Qualifizierungsmaßnahmen können beispielsweise der Ergänzung der beruflichen Grundbildung und der praktischen Einarbeitung dienen, sie können Förder- und Aufbaulehrgänge darstellen oder die Beteiligung der Arbeitspersonen 
bei der Einführung neuer Techniken und Methoden vorbereiten und fördern. Dabei sollte z.B. die Einarbeitung am Arbeitsplatz außerhalb der normalen Tätigkeit erfolgen, um der Qualifizierung genügend Spielraum zu geben und um verschiedene Vorgehensweisen erproben zu können ohne den normalen Betriebsablauf zu stören.

Obwohl das Lernen am Arbeitsplatz (vgl. MÜNCH, 1990), gerade auch bei Gruppenarbeit, als Form der Dezentralisierung der Weiterbildung zur Nutzung von Selbststeuerungs- und Synergieeffekten und als Möglichkeit das Erfahrungswissen aufzubauen und anzuwenden, unterstützt werden sollte, reicht es nicht aus, um einen ausreichenden Qualifizierungsstand zu sichern. Zur Minderung des Fehlerrisikos und der Anlaufzeit technisch-organisatorischer Innovationen, zur Begrenzung der Beanspruchung der Arbeitspersonen sowie zur Sicherung ihrer Anpassungsfähigkeit sind wiederholte und kontinuierliche Weiterbildungsmaßnahmen erforderlich, die sich zudem nicht nur am aktuellen Bedarf orientieren, sondern Entwicklungsmöglichkeiten eröffnen müssen. Hierzu können Veranstaltungen der betrieblichen und überbetrieblichen Weiterbildung genutzt werden, die beispielsweise nach Art der Weiterbildungsform, Ausbildungsort und Ausbilder unterschiedlich organisiert und miteinander kombiniert werden können.

Aus Sicht der Arbeitgeber wird die Weiterbildung zur Aktualisierung und Erweiterung der Qualifikationen der Beschäftigten im Interesse der Verbesserung der Wettbewerbsfähigkeit des Unternehmens, zur Erhöhung der Leistungsfähigkeit, der Arbeitszufriedenheit, der arbeitsbezogenen Motivation, der Sicherheit des Umgangs mit neuen Techniken sowie zur Stärkung des Selbstwertgefühls der Beschäftigten durchgeführt. Die weiterbildungsbezogenen Interessen der Arbeitspersonen richten sich auf den Erwerb von Qualifikationen, die der Verbesserung oder Absicherung der beruflichen Position dienen bzw. auf dem Arbeitsmarkt verwertbar sind.

Die Beteiligung an Weiterbildungsmaßnahmen ist bisher äußerst ungleichmäßig auf die Beschäftigten verteilt. Wichtige Faktoren für die Weiterbildungsteilnahme sind insbesondere das Lebensalter, das Geschlecht, die Schul- und Berufsausbildung sowie die berufliche Stellung der Arbeitsperson, wie folgende Abbildung zeigt. 


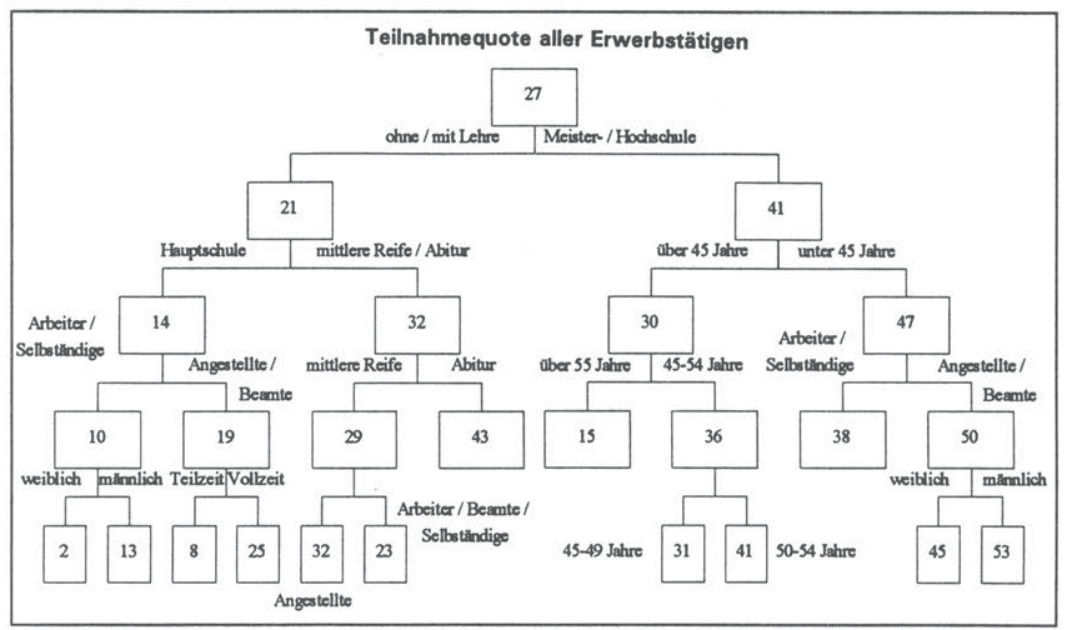

Abb. 67: Prozentuale Teilnahmequoten verschiedener Gruppen von Erwerbstätigen an Maßnahmen der beruflichen Weiterbildung in den alten Bundesländern - Ergebnisse einer Kontrastgruppenanalyse (Berichtssystem Weiterbildung, BSW 1991), (Quelle: STRAKA/KUWAN, 1994)

Durch die unterschiedlich hohe Beteiligung verschiedener Personengruppen an der Weiterbildung werden tendenziell qualifikatorisch Benachteiligte zusätzlich benachteiligt. So artikulieren gerade Höherqualifizierte den höchsten Weiterbildungsbedarf. Die Bildungsunterschiede nehmen entsprechend im Lebensverlauf durchschnittlich zu (vgl. beispielsweise MAYER, 1992, 530f. und zu Selektionsund Segmentationseffekten BAETHGE, 1992, 317).

Die zukünftige Weiterbildung muß zur Erhöhung der Erwerbsbeteiligung der Frauen und Älteren gerade die geschlechts- und altersbezogene Diskriminierung vermeiden. Die Qualifizierung sollte im Gegenteil gerade Frauen und Ältere unterstützen, eine umfassende und längerfristig orientierte Qualifizierung ermöglichen und positive Anreize für eine ausgeweitete Erwerbstätigkeit von Frauen und Älteren geben. Hierzu muß die Weiterbildung an den Interessen und Bedürfnissen der Arbeitspersonen anknüpfen und Wünsche bzw. Erwartungen bezüglich des zukünftigen beruflichen Einsatzes beachten. Darüber hinaus muß die Konzentration der Weiterbildungsteilnahme auf die ersten Berufsjahre aufgehoben werden, um Bildung gerade zur Kompensation von zeitlich bedingten Qualifikationsverlusten und alterungsbedingten Fähigkeits- und Funktionsverlusten zu nutzen (vgl. auch für zusätzliche Bedeutungsdimensionen von Bildung und Arbeit 
im Alter beispielsweise MAYER, 1992). Die Maßnahmen müssen von dem bisherigen Arbeitseinsatz ausgehen und neben der Qualifikation auch die persönliche Entwicklung fördern, um das Leistungs- und Arbeitspotential der Arbeitspersonen ausschöpfen zu können und die Bereitschaft zur Erwerbstätigkeit zu unterstützen.

Hinsichtlich der Ausweitung der Frauenerwerbsbeteiligung kann die Weiterbildung insbesondere zur Verbesserung der Aufstiegschancen, zur Erhöhung der Erwerbsmotivation und zur Förderung der Wiederbeschäftigungschancen nach einer Unterbrechungsphase beitragen. Die Weiterbildungsmaßnahmen müssen die verbreiteten erwerbsbiographischen Brüche und die erziehungs- bzw. pflegebedingten Anforderungen berücksichtigen. Insbesondere ist die Teilnahme an Weiterbildungsmaßnahmen während der Unterbrechungsphase zu fördern. Wiedereinstiegshilfen, die nach der Unterbrechungsphase die berufliche Orientierung, beispielsweise durch Praktika, Informationsangebote und kurze einweisende Qualifizierungen, ermöglichen und die Aktualisierung der Qualifikationen gerade im naturwissenschaftlichen Bereich und in technischer Hinsicht erleichtern, sind individuell zu konzipieren (vgl. BUSCH/KOCH, 1990). Daneben können Weiterbildungsmaßnahmen die Motivation der Frauen zur Überwindung der geschlechtsspezifischen Arbeitsteilung fördern. Hierzu muß insbesondere die Aufstiegsweiterbildung für Frauen ausgeweitet werden. Organisatorisch wünschenswert ist die Weiterbildung am Arbeitsort während bzw. direkt nach Ende der Arbeitszeit mit begleitend angebotenen Kinderbetreuungsmöglichkeiten.

Bezüglich einer verlängerten Erwerbstätigkeit der Älteren müssen besonders mögliche Aktualitäts- und Relevanzverluste der vorhandenen Qualifikationen beachtet werden. Die bisher dominierende technisch-fachliche Anpassungsqualifizierung wird zukünftig nicht ausreichen, um die Aktualität der Qualifikation der Beschäftigten so zu sichern, daß Ältere im notwendigen Maße befähigt werden ihren Beruf länger auszuüben. Eine altersgerechte Ausgestaltung der Qualifizierung muß sich an den im Lebenslauf gewonnenen Fertigkeiten und Kenntnissen sowie den gemachten Erfahrungen orientieren. Neben einer altersübergreifenden kontinuierlichen Weiterbildung, die die Voraussetzung für den Qualifikationserhalt und die Möglichkeit der Verlängerung der Erwerbstätigkeit schafft, müssen besonders im Falle umfassender Anforderungsumstellungen zusätzliche Aus-bildungsphasen während der Erwerbstätigkeit erwogen werden. Neben der Weiterbildung kann eine stärker ausgedehnte Qualifizierungsphase etwa zwischen dem 40. und 50. 
Lebensjahr notwendig werden, um der Arbeitslosigkeit aufgrund veränderter Qualifikationsanforderungen bzw. geringerer Qualifikation mittlerer und älterer Jahrgänge zu begegnen (vgl. VOLKHOLZ, 1992, 11 und 16) und um zukünftige Arbeitsangebotsdefizite zu mindern.

Die Planung von betrieblichen Qualifizierungsmaßnahmen (vgl. SCHULZ, 1990, 132ff.) setzt die Bestimmung der an einer Arbeitsstelle durchzuführenden Tätigkeiten, eine Stellenbeschreibung und die Beschreibung der gestellten Anforderungen voraus. Bei den Beschäftigten muß der Ausbildungsstand erfaßt werden. Durch einen Vergleich von Qualifikationsanforderungen und der vorhandenen Qualifikationsstruktur müssen Qualifizierungsdefizite und der Ausbildungsbedarf ermittelt werden. Im weiteren sind die unternehmensintern und unternehmensextern angebotenen Ausbildungsmöglichkeiten und die vorhandenen finanziellen und personellen Ressourcen festzustellen, bevor - unter Beteiligung der betroffenen Mitarbeiter - Ausbildungspläne erstellt werden. Schließlich müssen die Aus- und Weiterbildungsmaßnahmen geplant, durchgeführt und kontrolliert werden.

Inhaltlich muß angesichts der Veränderungen der qualifikatorischen Anforderungen die fachbezogene $\mathrm{Ab}$ - und Eingrenzung zunehmend überwunden werden. Auch die Grenze zwischen kaufmännischen und gewerblich-technischen Qualifikationskomponenten wird, besonders durch den fortschreitenden Einsatz der Informationstechnik, immer fließender (vgl. beispielsweise NOVAK, 1993). Dementsprechend muß ein breites Grundverständnis für technische und organisatorische Zusammenhänge vermittelt und ein Einverständnis mit der Notwendigkeit permanenten Lernens erwirkt werden. Hierzu ist die Vermittlung einer übergreifenden, orientierenden Qualifikation notwendig. Erforderlich sind beispielsweise Kenntnisse über die Informatik, die Produktionsverfahren, die Funktionsweise technischer Anlagen und Prozesse sowie die komplexen Zusammenhänge im organisatorischen Gefüge des Unternehmens. Eine solche Verbreiterung der Bildung trägt dazu bei, Zusammenarbeit auch bei individuell hoher Spezialisierung zu erleichtern, da Probleme nicht einseitig (vermeintlich allkompetent) betrachtet werden.

Erst durch übergreifendes Wissen (vgl. beispielsweise REFA, 1987, 37ff.) können die Arbeitspersonen den erweiterten Aufgabenbereich überschauen, so daß die Arbeitsaufgaben mit Blick auf den Zusammenhang im gesamten Arbeitsprozeß durchgeführt und die Vernetzung der Probleme erkannt werden können. Hinsichtlich der Gruppenarbeit unterstützt dieses Wissen die Kommunikations- und 
Kooperationsfähigkeit sowie die Problem- und Konfliktlösungsfähigkeit. Weiterhin werden die Delegation und Übernahme von Verantwortung, der selbstorganisatorische Personaleinsatz, die flexible Aufgabenverteilung und Auftragsdisposition sowie notwendige Qualifikationsanpassungen erleichtert.

Die Schulungen müssen ebenso wie die betriebliche Praxis theoretisches Wissen und Erfahrungswissen gleichermaßen berücksichtigen (vgl. auch zu folgendem beispielsweise BULLINGER, 1992, 65ff. und 170). Die Weiterbildungsmaßnahmen müssen neben betriebs- und anlagenspezifischen Kenntnissen ganzheitliche, übergreifende und umfassende Kenntnisse vermitteln, die zudem regelmäßig aktualisiert und angepaßt werden müssen. Es gibt zahlreiche Ansätze die zu vermittelnden Kenntnisse und Kompetenzen zu systematisieren. Hier sollen vier Gruppen dargestellt werden, die zusammen die Grundlage für die Entwicklung der Handlungskompetenz der Arbeitspersonen bilden.

1. Die Fachkompetenz bezieht sich auf das erforderliche arbeitsprozeßspezifische Wissen. Dieses Wissen ist wegen der angestrebten Integration und gegenseitigen Verknüpfung der Aufgaben zunehmend bereichsübergreifend. Neben der berufsfachlichen Qualifikation und dem Wissen über die Technologie müssen die systematischen Zusammenhänge bekannt sein, um die Verantwortungsfähigkeit zu gewährleisten. Neben der grundlegenden fachlichen Qualifikation erhöhen und verbreitern sich die betriebs- und anlagen- bzw. systemspezifischen Qualifikationen. Zudem müssen Grundlagenkenntnisse der Informations- und Kommunikationstechnik vorhanden sein.

2. Die Methodenkompetenz betrifft die Abstraktions-, Informationsselektions-, Planungs- und Entscheidungsfähigkeit, die Problemlösungskompetenz und die organisatorische Qualifizierung, die zur Verbesserung der Kommunikation mit den Beschäftigten anderer Abteilungen oder Bereiche notwendig ist. Sie ist besonders zur Übernahme ergänzender oder neuer (z.B. dispositiver) Aufgaben erforderlich.

3. Die Sozialkompetenz beinhaltet die Kommunikations-, Kooperations- und Kontaktfähigkeit, die Fähigkeit zur Zusammenarbeit in der Gruppe, zwischen Abteilungen und über Bereichsgrenzen hinweg, zur Gruppenarbeit und zur Konfliktlösung. Außerdem kann sie die Artikulationsfähigkeit und die Fähigkeit, komplizierte Sachverhalte verständlich aufbereitet darzustellen, umfassen.

4. Die Lernkompetenz beeinflußt die Motivation, die Lernbereitschaft, das Interesse, das Engagement sowie die Entwicklung der Fähigkeiten und Fertigkeiten. Weiterhin trägt sie zur Integration der übrigen Kompetenzen bei. 
Die Fachkompetenz kann durch arbeitsplatz-, verrichtungs- und betriebsmittelbezogene Schulungen, die das erforderliche Faktenwissen und die selbständige, flexible Handlungskompetenz vermitteln, erworben werden. Die Methoden- und Sozialkompetenz werden besonders durch eine Vermittlung von Kommunikationund Arbeitstechniken (z.B. in Lerngruppen) erweitert. Der zukünftige Qualifizierungsbedarf richtet sich insbesondere auf die Entwicklung der extrafunktionalen oder Schlüsselqualifikationen, wie Denkvermögen, Verantwortung, Sorgfalt, Flexibilität, Fähigkeit zur Transformation des Wissens, Kommunikations- und Informationsselektionsfähigkeit.

Qualifizierungsmaßnahmen sollten flexibel angeboten werden, so daß eine zeitliche und inhaltliche Anpassung an die betrieblichen und individuellen Anforderungen, Gegebenheiten und Bedürfnisse möglich ist. Sie sollten ausreichend lange und terminlich günstig, aufgrund der physiologischen Leistungsbereitschaft vornehmlich am Vormittag, stattfinden. Fehlbeanspruchungen im Sinne von Über- oder Unterforderungen, Leistungsdruck und Selektionsmechanismen sind bei der Gestaltung der Weiterbildungsmaßnahmen zu vermeiden. Zur Vorbereitung und Unterstützung des technisch-organisatorischen Wandels ist mit Blick auf lernungewohnte und lernentwöhnte Arbeitspersonen eine arbeitsprozeßnahe Qualifizierung anzustreben, die an vorhandenen Erfahrungen und Vorgehensweisen anknüpft (vgl. ein entsprechendes Konzept für die betrieblich ausgerichtete Qualifizierung in Mittelbetrieben WITZGALL/WÖCHERL, 1989).

Ergänzend kann die innerbetriebliche Personalrotation eine umfassendere Qualifizierung der Arbeitspersonen unterstützen und die berufliche Flexibilität erhalten (vgl. beispielsweise NAEGELE, 1994). Neben dem betriebsinternen Arbeitsplatzwechsel sind diverse wechselnde Möglichkeiten des Einsatzes denkbar, wie der Austausch von Arbeitspersonen zwischen zusammenarbeitenden Unternehmen zum Zwecke der Qualifikation und zur Sicherung und Intensivierung der Beziehungen. Die Institutionalisierung solcher personalbezogener Kooperationsformen könnte die Entwicklung umfassender Kompetenzen und die Umsetzung moderner Organisationsformen fördern. Allerdings läßt sich feststellen, daß Maßnahmen, wie job rotation, Qualitätszirkel, Austauschprogramme und Lernstatt, durchschnittlich relativ selten und etwas verbreitet nur in Großunternehmen angewendet werden. Nach einer Erhebung des Statistischen Bundesamtes und des Bundesinstituts für Berufsbildung von 1993 werden diese Qualifizierungsmöglichkeiten nicht einmal von $5 \%$ der befragten Unternehmen genutzt (vgl. SCHMIDT/HOGREVE, 1994). 


\section{- Betroffene Kosten und Nutzengrößen}

Kosten:

Die Kosten der beruflichen Weiterbildung werden von den Unternehmen, dem Staat, der Bundesanstalt für Arbeit und den teilnehmenden Personen getragen. Die aus Unternehmenssicht betroffenen Kosten lassen sich in zwei Gruppen teilen:

- Direkte Kosten der Bildungsmaßnahmen, wie Honorare, Teilnahmegebühren, Lehrgangsgebühren, Sach-, Reise-, Aufenthalts- und Betreuungskosten.

- Indirekte Kosten der Bildungsmaßnahme, wie Personalkosten während der Weiterbildung (ausfallende Arbeitsleistung und evtl. auch personeller Mehraufwand für Vertretungen und Überstunden während der Schulung sowie für Lernund Übungsphasen nach der Schulung), Entwicklungskosten, Organisationskosten und Verwaltungskosten.

Nach einer Erhebung in 1.450 repräsentativ ausgewählten Betrieben (ohne Landwirtschaft, Freie Berufe und Organisationen ohne Erwerbscharakter) des Instituts der deutschen Wirtschaft, betrugen 1992 die direkten Weiterbildungskosten (Sachausgaben, Honorar für Lehrkräfte, Lehrgangsgebühren) durchschnittlich $771 \mathrm{DM}$ und die indirekten Weiterbildungskosten (Lohnfortzahlung während der Weiterbildung) durchschnittlich 1.153 DM je Mitarbeiter, so daß zusammen $1.924 \mathrm{DM}$ je Mitarbeiter für die Weiterbildung aufgewendet wurden (vgl. WEIß, 1994). Deutliche Unterschiede zwischen den Unternehmen zeigten sich in Abhängigkeit von der Betriebsgröße, da die durchschnittlichen Aufwendungen je Mitarbeiter tendenziell mit zunehmender Betriebsgröße sinken. Neben unterschiedlich hohen Weiterbildungsaktivitäten dürften hierfür besonders unterschiedliche Organisationsarten verantwortlich sein. So können Großbetriebe z.B. eher kostengünstige interne Lehrveranstaltungen durchführen (vgl. WEIß, 1994 und SCHMIDT/HOGREVE, 1994).

Die Unternehmen könnten versuchen, zukünftig einen größeren Teil der Weiterbildungsaufwendungen auf die Beschäftigten zu verlagern. Dies kann dadurch geschehen, daß die Arbeitspersonen die direkten Kosten häufiger teilweise oder vollständig selbst tragen und die indirekten Kosten der Weiterbildung dadurch gesenkt werden, daß die Maßnahmen außerhalb der entlohnten Arbeitszeit stattfinden. Außerdem können zukünftige tarifvertragliche Arbeitszeitverkürzungen durch weiterbildungsbezogene Vereinbarungen ersetzt bzw. ergänzt werden. 
Parallel zu der Unternehmensumfrage des Instituts der deutschen Wirtschaft kann eine repräsentative Untersuchung des Bundesinstituts für Berufsbildung betrachtet werden. 1992 wurden 1.607 Weiterbildungsteilnehmer im Alter von 19 bis 64 Jahren nach den Kosten ihrer betrieblichen und privaten beruflichen Weiterbildung während eines Jahreszeitraums (1. Oktober 1991 bis 30. September 1992) befragt. Dabei ergab sich, daß die Weiterbildungsteilnehmer durchschnittlich 1.001 DM aufgewendet haben. Zusätzlich wurden durchschnittlich 544 DM erstattet, so daß insgesamt je Teilnehmer durchschnittlich 1.555 DM Weiterbildungskosten ermittelt wurden. Die von den Teilnehmern eingesetzte Freizeit von durchschnittlich 316 Stunden pro Jahr (besonders für Unterrichtszeit, Fahrzeit, Vor- und Nachbereitungszeit) wurde dabei nicht berücksichtigt. Bei der betrieblichen Weiterbildung sind den Teilnehmern zumeist keine oder nur geringe Kosten entstanden (vgl. BERUFSBILDUNGSBERICHT, 1994, 135).

- Zur Förderung des Weiterbildungsengagements muß sich die Entlohnung stärker an der vorhandenen, durch Aus- und Weiterbildung erreichten Qualifikation orientieren und eine höhere Einsatzflexibilität der Arbeitspersonen honorieren. Die Ausweitung der Weiterbildungsmaßnahmen führt dementsprechend zum Anstieg der Personalkosten.

Nutzen:

- Weiterbildungsmaßnahmen ermöglichen die Sicherung und Weiterentwicklung des vorhandenen Humankapitals.

- Gezielte Weiterbildungsmaßnahmen können zu einer raschen Verbesserung der Einsetzbarkeit bestimmter Arbeitspersonen führen.

- Breite Weiterbildungsmaßnahmen für die Beschäftigten tragen zur Erhöhung der personellen und technischen Einsatzflexibilität und zur Schaffung von Qualifikationsreserven bei.

- Die Handlungsflexibilität der Arbeitspersonen und die Qualität der Arbeitsleistung werden erhöht.

- Überforderungs- und Streßsituationen, die aufgrund unzureichender Qualifikation auftreten, können vermindert werden.

- Weiterbildungsmaßnahmen unterstützen die Verbesserung der Arbeitsproduktivität sowie die Erhöhung der Arbeitsmotivation und der Arbeitszufriedenheit. 
Eine globale Beurteilung der Maßnahme Weiterbildung ergibt sich entsprechend folgender Abbildung. Die Beeinflussung des Ziels 'Reduzierung des Zeitaufwandes' wird hier nicht eindeutig eingeschätzt, da einerseits die Durchführung von Qualifikationsmaßnahmen (Arbeits-) Zeit benötigt und abwesenheitsbedingte Verzögerungen auftreten können, und andererseits durch die verbesserte Qualifikation der Beschăftigten zeitliche Einsparungen erwartet werden können.

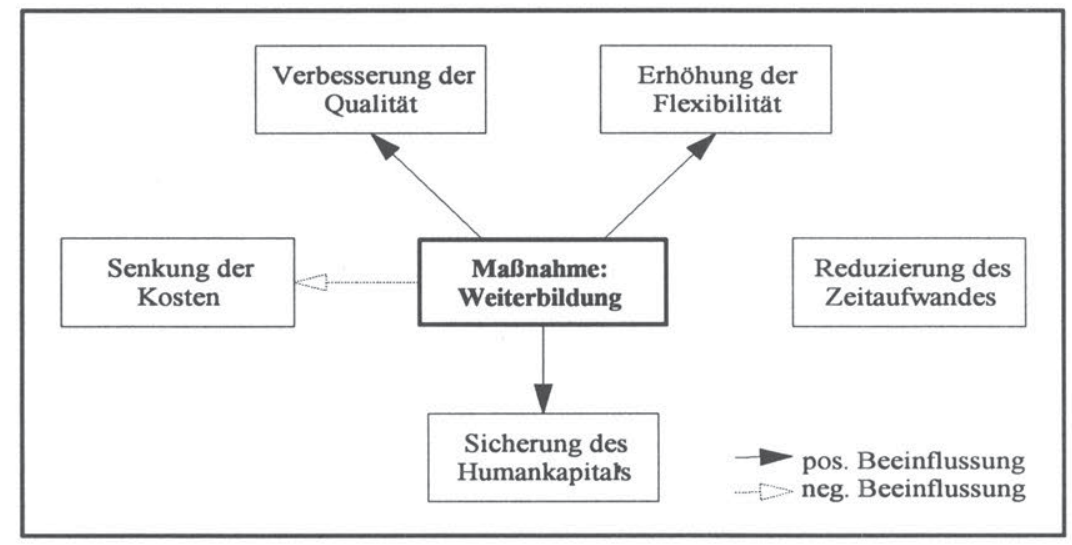

Abb. 68: Zielbeeinflussung durch die Umsetzung der Maßnahme Weiterbildung

Die für die Amortisation der Investitionen in das Humankapital Älterer zur Verfügung stehende Zeit scheint kürzer zu sein als bei jüngeren Arbeitspersonen, deren potentielle Beschäftigungsdauer länger ist. Dieses Argument gegen eine stärkere Beteiligung Älterer an Weiterbildungsmaßnahmen verliert allerdings aufgrund verschiedener Entwicklungstendenzen zukünftig an Bedeutung (vgl. beispielsweise STAUFER, 1992, 155). So sorgt die zunehmende Geschwindigkeit des sich vollziehenden Wandels dafür, daß sich die Halbwertszeit des Wissens verringert und damit die Amortisationszeit von Bildungsinvestitionen für alle Arbeitspersonen verkürzt. Weiterhin sollte die mit dem Alter zunehmende Betriebstreue bzw. der häufigere Beschäftigungs- oder Unternehmenswechsel jüngerer Arbeitspersonen beachtet werden. Außerdem können die Unternehmen angesichts voraussichtlicher Defizite des Arbeitskräfteangebots das Investitionsrisiko eingehen, da die Weiterbildungsmaßnahmen selbst eine Verschiebung des Renteneintritts erleichtern und sich die Nutzungszeit somit verlängern läßt.

Bei der Beurteilung der Weiterbildungs- und Ausbildungsmaßnahmen muß die Reflexivität der Anforderungen und der Qualifikationen Beachtung finden. Die 
vorhandenen Qualifikationen sind Bestimmungsfaktor der Entwicklung und Gestaltung der Arbeit. Breite Qualifikationen sind notwendig, um neue Techniken und besonders um neue Organisationsmethoden zur Erhöhung der Produktivität nutzen zu können. Umfassende Qualifikationen helfen Folgeprobleme von Innovationen $\mathrm{zu}$ vermeiden, Anpassungsleistungen $\mathrm{zu}$ erbringen und evtl. notwendige Umorientierungen zu erkennen und nutzen zu können. Qualifizierungsmaßnahmen tragen zur Minderung der Fehlerhäufigkeit, zur Erhöhung der Problemlösungsfähigkeit, zur Verbesserung der Anlagennutzung, zur Sicherung der Systemverfügbarkeit, zu einer Verringerung der Inanspruchnahme externer Serviceleistungen und damit zur Erhöhung der Wirtschaftlichkeit bei. Weiterbildungsmaßnahmen sind zur Qualifikationsanpassung bzw. zum Aufbau und zur Verbesserung des qualifikatorischen Anpassungspotentials notwendig. Weiterbildung sollte in diesem Sinn genutzt werden, um längerfristig Humanressourcen aufzubauen bzw. zu erhalten. Diese Maßnahme ist ein wirkungsvoller Beitrag zu einer präventiv wirkenden Personalentwicklung, die technische, betriebliche, arbeitsgestalterische und personalwirtschaftliche Prozesse als Einheit betrachtet (vgl. SCHULTZ-WILD u.a., 1989, 229ff.).

\section{- Umsetzungsbedingungen}

Die tendenzielle Höherqualifizierung des Erwerbspersonenpotentials kann zu einer durchschnittlichen Erhöhung des Lerninteresses, des Durchhaltevermögens und der Motivation beitragen, so da $\beta$ die Bereitschaft der Arbeitspersonen, kontinuierlich an Weiterbildungsmaßnahmen teilzunehmen, zukünftig steigen dürfte.

Die wachsende Bedeutung des theoretischen Wissens erfordert in zunehmenden Maße Weiterbildungsmaßnahmen außerhalb der Arbeitsdurchführung. Insbesondere in kleineren Unternehmen wird hinsichtlich der Teilnahme an betrieblichen und überbetrieblichen Maßnahmen die fehlende Abkömmlichkeit der Arbeitspersonen zum Problem (vgl. beispielsweise CRAMER/KRAMER, 1990). Die Freistellungsmöglichkeiten für Fachkräfte werden durch die voraussichtlichen Arbeitsangebotsdefizite zusätzlich eingeschränkt.

Maßnahmen der Weiterbildung müssen systematisch geplant und mit allen beteiligten Gruppen und Personen langfristig abgestimmt sein, so daß sich gerade hinsichtlich technisch-organisatorischer Veränderungen die Investitionen in das Sachkapital und in das Humankapital zeitlich und problembezogen ergänzen. Die 
Weiterbildung kann zukünftig stärker in die Mitbestimmung einbezogen werden, um somit einen Konsens zwischen Unternehmensleitung und Betriebsrat bzgl. der Weiterbildungsmaßnahmen zu finden und ihre breite Umsetzung zu fördern. Neben der Weiterbildung während der Arbeitszeit können Qualifizierungsmaßnahmen durch Aufgabenwechsel oder unternehmensinterne Arbeitsplatzwechsel und während arbeitsfreier Phasen vereinbart werden.

Die zur Nutzung moderner Techniken und Methoden unerläßliche Qualifizierung der Arbeitspersonen läßt einen 'sorgsamen' Umgang mit den Beschäftigten aus Arbeitgebersicht wirtschaftlich sinnvoll erscheinen, so daß sich aus der Notwendigkeit der Qualifizierung und dem Interesse an der Sicherung und Verwendung getroffener Humankapitalinvestitionen zusätzliche Anreize für die Durchführung der übrigen Maßnahmen ergeben, die dazu beitragen die Beschäftigten an das Unternehmen zu binden.

\section{b) Frauenförderung}

Frauenförderung bezieht sich auf die Förderung der beruflichen Chancengleichheit und kann zugunsten einer kontinuierlichen Beschäftigung und zur Erleichterung der Reintegration wirken. Die hier betrachtete Maßnahme richtet sich insbesondere auf die Verbesserung der Vereinbarkeit von Familie und Beruf und den Erhalt des Humankapitals während einer Unterbrechungsphase. Hierzu werden Komponenten der übrigen Maßnahmen, wie der Teilzeitarbeit und der Weiterbildung genutzt. Die Aussagen zu den jeweiligen Maßnahmen behalten daher Geltung. Gleichwohl sollen einige ergänzende Anmerkungen gemacht werden.

\section{- Gestaltungsformen}

Die in verschiedenen Unternehmen anzutreffenden Maßnahmen zur Verbesserung der Vereinbarkeit der Erwerbstätigkeit mit Arbeiten der Pflege oder der Kindererziehung richten sich, auch wenn sie zumeist nicht geschlechtsspezifisch gestaltet sind, auf die Erleichterung der Erwerbstätigkeit von Frauen. Qualifizierte Frauen sollen hierdurch an das Unternehmen gebunden werden. Beispiele fördernder Maßnahmen in Großunternehmen sind in folgender Abbildung zusammengestellt (vgl. KÖSTERS/LUECKEL, 1994 und HOFFMANN/LEESON, 1994, 15ff.). 


\section{AEG}

Familienpause

für Mitarbeiterinnen

Pflegeurlaub für Mitarbeiter/innen
Wiedereinstellungszusage nach einer befristeten Unterbrechungsphase zur Kindesbetreuung.

Zur Pflege einer nahestehenden Person wird ein maximal 12monatiger Pflegeurlaub gewährt.

\section{BASF}

Es bestehen Möglichkeiten der Teilzeitbeschäftigung.

Zur Pflege von Angehörigen kann die Erwerbstätigkeit seit 1990 bis zu zwei Jahren unterbrochen werden.

Bayer

Familie und Beruf $\quad$ Eine Gesamtbetriebsvereinbarung seit 1987 (Überarbeitung 1992) gibt den Mitarbeitern eine Wiedereinstellungszusage nach einer bis zu siebenjährigen Unterbrechungsphase zur Kindeserziehung, bei der Teilnahme an Fortbildungsmaßnahmen. Unterstützung der Kinderbetreuung.

Teilzeitarbeit Eine Gesamtbetriebsvereinbarung zur Teilzeitarbeit besteht seit 1987.

Betreuungspause Eine befristete Freistellung oder Teilzeitarbeit zur Betreuung erkrankter Familienangehöriger ist geplant.

Bayerische Vereinsbank

Beruf und Familie

Eine Betriebsvereinbarung ermöglicht individuelle Teilzeitverträge, die Wiedereinstellung nach einer Familienpause und regelt einen Vermittlungsservice für die Betreuung von Kindern.

Boston Consulting Group

Unterbrechungsphase

Unter der Bezeichnung "leave of absence" wird es den Mitarbeitern ermöglicht nach abgeschlossenen Projekten zeitweise zu pausieren und anschließend ihre Erwerbstätigkeit ohne Nachteile wieder aufzunehmen.

Commerzbank

Erziehungsurlaub

Im Rahmen eines sog. "Comeback"-Programms wird mindestens drei Jahre lang beschäftigten Mitarbeiter/innen, an deren Rückkehr Interesse besteht, die Wiederbeschäftigung an ihrem Arbeitsplatz nach einem dreijährigen Erziehungsurlaub angeboten.

Teilzeitarbeit Der Ausbau von Teilzeitmodellen ist beabsichtigt.

Degussa

Familienförderung Insbesondere werden Wiedereinstellungsgarantien gegeben und Kinderzulagen gewährt.

Deutsche Bank

Chancengleichheit, Beruf Die Betriebsvereinbarung von 1990 regelt eine Verlängerung des und Familie

Erziehungsurlaubs, individuelle Wiedereinstellungszusagen und einen Anspruch auf einen Teilzeitarbeitsplatz.

Hewlett Packard

job sharing

Es bestehen Beschäftigungsmodelle für den Erziehungsurlaub und eine Kindertagesstätte.

Hauptsächlich für Frauen bereits seit 1983 geregelt.

IBM Deutschland

Die Zahl der Heimarbeitsplätze soll ausgeweitet werden.

Kaufhof Holding

Es bestehen innovative Arbeitszeitmodelle für die mittlere Führungsebene und die Mitarbeiter in Verkauf und Hauptverwaltung. 


\begin{tabular}{|c|c|}
\hline Mercedes & $\begin{array}{l}\text { Es bestehen Möglichkeiten der Teilzeitbeschäftigung. } \\
\text { Möglichkeiten der Unterbrechung der Erwerbstätigkeit bis zu } \\
\text { einem Jahr zur Pflege sind seit } 1992 \text { vereinbart. Falls die } \\
\text { Erwerbstätigkeit danach nicht wieder aufgenommen wird, bleibt } \\
\text { die Arbeitskraft bis zu drei Jahren auf einer 'Reserveliste'. }\end{array}$ \\
\hline Nestlé Deutschland & $\begin{array}{l}\text { Es bestehen Regelungen zur Frauen- und Familienförderung, } \\
\text { Teilzeitarbeit und Wiedereingliederung. }\end{array}$ \\
\hline $\begin{array}{l}\mathbf{R}+\mathbf{V} \text { Versicherung } \\
\text { Elternurlaub }\end{array}$ & $\begin{array}{l}\text { Ein maximal siebenjähriger Elternurlaub mit Wiedereinstellungs- } \\
\text { garantie wird gewährt. }\end{array}$ \\
\hline $\begin{array}{l}\text { RWE Energie } \\
\text { Erziehungsurlaub }\end{array}$ & $\begin{array}{l}\text { Eine Betriebsvereinbarung regelt eine Verlängerung des gesetzlich } \\
\text { bestimmten Erziehungsurlaubs auf vier Jahre, während der die } \\
\text { Vergütung garantiert und die kostenlose Teilnahme an } \\
\text { Weiterbildungsmaßnahmen ermöglicht wird. } \\
\text { Möglichkeiten der Teilzeitbeschäftigung werden angeboten. }\end{array}$ \\
\hline Siemens & $\begin{array}{l}\text { Flexible Arbeitszeiten und job-sharing sind möglich. } \\
\text { Zur Pflege kann die Erwerbstätigkeit bis zu einem Jahr (bei } \\
\text { zugesicherter Rückkehrmöglichkeit) unterbrochen werden. }\end{array}$ \\
\hline $\begin{array}{l}\text { VEBA } \\
\text { Familienpause } \\
\text { für Mitarbeiter/innen }\end{array}$ & $\begin{array}{l}\text { Während der Familienpause wird durch die Teilnahme an } \\
\text { Weiterbildungsmaßnahmen und Aushilfstătigkeiten die Qualifi- } \\
\text { kation erhalten. }\end{array}$ \\
\hline $\begin{array}{l}\text { VW } \\
\text { qualitative Tarifpolitik } \\
\text { bzgl. der Arbeits- } \\
\text { gestaltung } \\
\text { Arbeitszeitverkürzung } \\
\text { ohne vollen Lohnaus- } \\
\text { gleich }\end{array}$ & $\begin{array}{l}\text { Tarifvertrag zur sozialen Sicherung der Arbeitnehmer bei } \\
\text { technischen und organisatorischen Änderungen für die } \\
\text { Volkswagen AG von } 1987 \text {. } \\
\text { Tarifvertrag zwischen VW und der IG Metall, Bezirksleitung } \\
\text { Hannover von } 1993 \text {. }\end{array}$ \\
\hline
\end{tabular}

Abb. 69: Beispiele für Vereinbarungen, die der Frauenförderung dienen können

Eine Vielzahl der Maßnahmen ist erst in den letzten Jahren in Kraft getreten. Insofern sind die spezifischen Wirkungen noch nicht vergleichend festzustellen. In einem Großunternehmen wie der Bayer AG (vgl. BAUSCH-WEIRAUCH, 1992) richten sich die Maßnahmen der Frauen- und Familienförderung zur Herstellung der Chancengleichheit an Frauen und an Männer. Mehr als die Hälfte der Mitarbeiterinnen, die ein Kind bekommen, nutzt die Möglichkeit für eine begrenzte Zeit aus dem Erwerbsleben auszuscheiden. Bis Ende 1991 haben ca. 800 Personen ihre Erwerbstätigkeit unterbrochen, darunter befanden sich allerdings nur 13 Männer. Auch leitende Mitarbeiterinnen nutzen hauptsächlich aufgrund des zu erwartenden Wissensverlustes die Unterbrechungsmöglichkeit so gut wie nicht. Mehr als die Hälfte der Teilnehmerinnen pausieren länger als drei Jahre und beabsichtigen die 
sieben Jahre Berufspause auszuschöpfen, so daß Unsicherheiten bezüglich des Rückkehrverhaltens bestehen.

Der Anteil der Unternehmen, die Regelungen zur Frauenförderung und zur Familienpause vereinbart haben bzw. planen oder ausbauen, steigt mit der Zahl der Beschäftigten an. Andererseits dürften auch in Klein- und Mittelbetrieben individuelle Absprachen Regelungen ermöglichen, die eine Vereinbarung von Familie und Beruf zum Ziel haben. Eine Fixierung beispielsweise in Betriebsvereinbarungen stellt Klein- und Mittelbetrieben vor Schwierigkeiten, da starre und für alle Arbeitspersonen gleichermaßen beanspruchbare Regelungen die personelle Flexibilität hier stärker einschränken, als in Großunternehmen (vgl. REICHLING, 1992).

Besonders in größeren Unternehmen kann das zeitliche Nacheinander von Familie und beruflicher Tätigkeit durch eine Berufsunterbrechung erleichtert werden. Eine solche Unterbrechung entspricht den gesellschaftlichen Vorstellungen und den Wünschen vieler Frauen. Trotz der gleichzeitig vereinbarten Erleichterung der Berufsrückkehr ist diese Erwerbspause gesamtwirtschaftlich betrachtet allerdings ungünstig für die Nutzung des Leistungspotentials der Frauen. Denn das vorhandene Humanvermögen wird dem Arbeitsmarkt entzogen und Dequalifizierungsprozesse sind zu erwarten. Zudem kann eine völlige Aufgabe der Erwerbstätigkeit vorbereitet werden. Auch einzelwirtschaftlich sind diese Vereinbarungen für die Unternehmen belastend, da die Rückkehrgarantien die Entscheidungsspielräume des Unternehmens einschränken und die Personalplanung erschweren. Andererseits wird durch die familienbedingte Unterbrechungsphase der Frauen die Nutzung der Leistungspotentiale der Männer erleichtert, da diese von der Familien- und Hausarbeit entlastet werden. Insgesamt scheinen Unterbrechungsphasen aus Unternehmenssicht akzeptabel, sofern sie nicht zu lange dauern und die Qualifikationen und berufsbezogenen Einstellungen erhalten bleiben.

Mit der Einführung des Erziehungsurlaubs, eines Erziehungsgeldes und der Anerkennung von Erziehungszeiten bei der Rentenversicherung und im Arbeitsförderungsrecht (Hilfen für Berufsrückkehrerinnen) wurde von staatlicher Seite damit begonnen, der Haus- und Familienarbeit gesellschaftliche Aufmerksamkeit und Absicherung zu widmen. Diese Regelungen unterstützen allerdings tendenziell die traditionelle Rollenverteilung (vgl. DIW, 1992). Demgegenüber könnten eine eigenständige soziale Sicherung und eine deutlichere gesellschaftliche Anerkennung der Hausarbeit bzw. der Kindererziehung dazu beitragen, daß sich die 
Beteiligung der Männer an diesen Tätigkeiten erhöht und damit die Möglichkeiten der Frauen einer Erwerbstätigkeit nachzugehen vergrößert werden.

Da Unterbrechungsphasen häufig zu einer Abwertung des Humankapitals führen, die Erwerbskarrieren von Frauen beeinträchtigen, Einkommenseinbußen nach sich ziehen und angesichts steigender Scheidungsraten Versorgungsrisiken vergrößern, sollten auch für Frauen dauerhafte und kontinuierliche Erwerbstätigkeiten ermöglicht werden. Statt eine vorübergehende Unterbrechung der Erwerbstätigkeit zu ermöglichen, müßte die längerfristige Vereinbarkeit von Erziehungstätigkeiten oder Pflegeleistungen und Beruf gesichert werden. Als Unterstützungsmaßnahmen bieten sich finanzielle Hilfen, kurzfristig hilfreiche Informationsprogramme, dauerhafte Beratungs- und Unterstützungsleistungen sowie besonders die Vereinbarung flexibler Arbeitszeitformen an (vgl. HOFFMANN/LEESON, 1994, 12f.).

Daneben kann auch die betriebliche Einrichtung oder Förderung von Kindergärten und Kindertagesstätten unterstützend wirken. So können die Unternehmen organisatorische bzw. finanzielle Unterstützung bei der Suche und Vereinbarung von Kinderbetreuungsformen, z.B. durch Tagesmütter, bieten. Damit können Unternehmen zur Minderung der außerberuflichen Anforderungen beitragen. Hinsichtlich einer Bewertung solcher Maßnahmen kommt es auf die jeweilige Ausgestaltung der Betreuungsform, die Trägerschaft und regionalen Rahmenbedingungen an. Verglichen mit betriebseigenen Betreuungseinrichtungen kann die Unterstützung von Kinderbetreuungseinrichtungen in fremder Trägerschaft, die es erlaubt eine bestimmte Anzahl von Betreuungsplätzen zu belegen (Belegkindertagesstätten), für viele Unternehmen als kostengünstigere Lösung bezeichnet werden (vgl. SCHWARZ/VOGEL, 1992, 244f.), da die Investitionshöhe geringer sein dürfte und eine Anpassung an den jeweiligen Betreuungsbedarf leichter möglich ist. Nachteilig ist, daß die Betriebszeiten der Belegkindertagesstätten nicht unbedingt an den Betriebszeiten orientiert sind und solche Fördermaßnahmen eher nur für größere Unternehmen umsetzbar sind, falls sich nicht mehrere Unternehmen zur Umsetzung dieser Maßnahme zusammenschließen.

Maßnahmen zur Kontaktpflege können zur Verbesserung der Rückkehrchancen, zur Erleichterung der Reintegration und zur Verbesserung der Aufstiegschancen beitragen. Im einzelnen werden hierzu während einer Unterbrechungsphase Vertretungen bei Krankheit oder Urlaub durchgeführt und Weiterbildungsmaßnahmen absolviert. Die Weiterbildungmaßnahmen müssen kontinuierlich auch 
während der Unterbrechungsphase und zu besonderen Anlässen, wie die Rückkehr in den Beruf, genutzt werden, so daß Frauen nach der Unterbrechungsphase (re-) qualifiziert werden und gleichwertige Tätigkeiten wie vor der Erwerbspause übernehmen können.

\section{- Betroffene Kosten und Nutzengrößen}

Kosten:

- Das in der Ausbildung und während der Erwerbstätigkeit gebildete Humanvermögen kann zumindest für die Dauer der Unterbrechungsphase nicht genutzt werden und verliert an Wert.

- Es entstehen Verwaltungs- und Betreuungskosten.

- Das Unternehmen geht mit der Vereinbarung von Unterbrechungsphasen u.U. langfristige personelle Bindungen ein, obwohl die Rückkehr der Arbeitspersonen unsicher bleibt.

- Je nach Gestaltung der Frauenförderung entstehen beispielsweise Weiterbildungskosten oder teilzeitbedingte Kosten.

Nutzen:

- Der flexible Einsatz betriebserfahrener Arbeitskräfte beispielsweise für Krankheits- oder Urlaubsvertretungen und nach Unterbrechungsphasen wird erleichtert.

- Das vorhandene betriebsspezifische Humankapital wird gesichert und an das Unternehmen gebunden. Die Fluktuation der Beschäftigten und die Einarbeitungskosten werden gemindert.

- Die Attraktivität des Unternehmens am Arbeitsmarkt wird erhöht und die Rekrutierungsmöglichkeiten werden verbessert.

- Die Umsetzung der an den Bedürfnissen der Arbeitspersonen orientierten Maßnahmen dürfte sich positiv auf die Loyalität und die Arbeitsmotivation der Beschäftigten auswirken.

Folgende Abbildung bietet eine globale Beurteilung der Maßnahme Frauenförderung. Eine Auswirkung hinsichtlich des Ziels der Qualitätsverbesserung läßt sich für diese Maßnahme nicht einschätzen, da hierfür noch spezifischere Einflußfaktoren relevant erscheinen als bezüglich der übrigen Ziele. Der Bezug der Maßnahme zur 
Qualität der geleisteten Arbeit ist darüber hinaus relativ lose, da die Frauenförderung zumindest teilweise während Unterbrechungsphasen wirkt, so daß in dieser Situation häufig unbekannt ist, ob und wann wieder einer Erwerbstätigkeit nachgegangen werden wird.

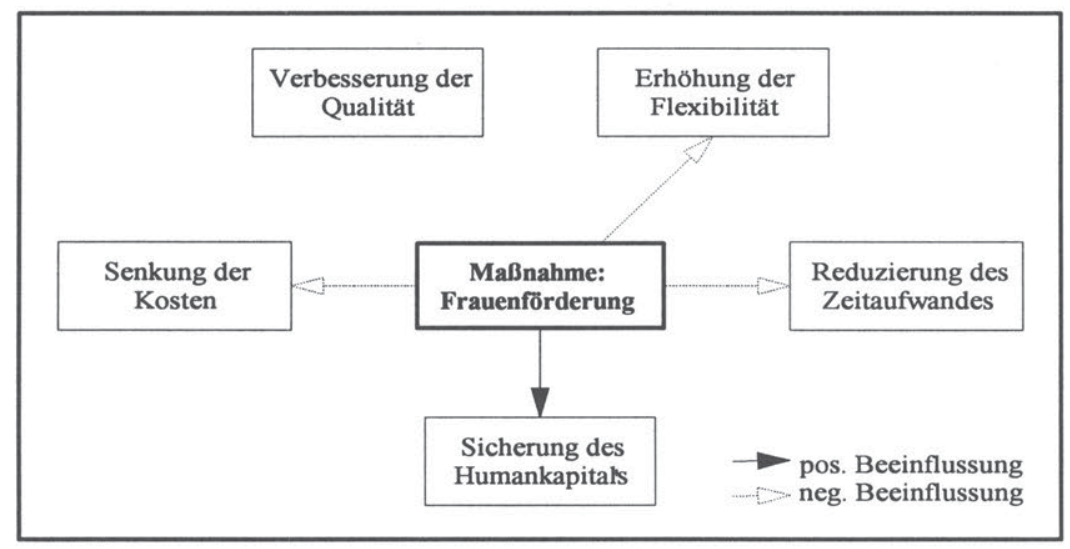

Abb. 70: Zielbeeinflussung durch die Umsetzung der Maßnahme Frauenförderung

\section{- Umsetzungsbedingungen}

Werden die erwähnten Maßnahmen für Frauen und Männer gleichermaßen angeboten, so bieten die Unternehmen ihren Beschäftigten damit Möglichkeiten die geschlechtsspezifische Aufgabenteilung zu überwinden. Auch wenn es kein Unternehmensziel ist, die gesellschaftlich verbreitete geschlechtsspezifische Arbeitsteilung und Lebensauffassung $\mathrm{zu}$ ändern, bieten die Maßnahmen Möglichkeiten hierzu. Die bisher geringe praktische Relevanz der geschlechtsindifferenten Vereinbarungen für Veränderungen von Einstellungen und Handlungsweisen könnte durch entsprechende Anreize gesteigert werden.

Aus Sicht der Unternehmen sind allerdings Investitionen in das Humankapital der Frauen, aufgrund der häufigeren Berufsunterbrechungsphasen mit einem größeren Risiko behaftet, und dieses Investitionsrisiko wird durch die Verbesserung von Unterbrechungsmöglichkeiten zusätzlich erhöht. Weiterhin ist anzunehmen, daß Männer auch zukünftig nicht in einem so hohen Maße Erziehungsurlaub nehmen werden, als daß hierdurch wesentliche Veränderungen in der geschlechtsspezifischen Arbeitsteilung ausgelöst würden, zumal kein entsprechendes Interesse 
der Unternehmen vorausgesetzt werden kann. Daher erscheinen verbesserte Angebote zur Rückkehr auf den Arbeitsplatz als ein wichtigeres Mittel zur Erhöhung der Erwerbsbeteiligung der Frauen.

Ergänzend soll angemerkt werden, daß es entsprechend der behandelten Optionen zukünftig nicht nur eine Frauenförderung sondern auch eine ausdrückliche und spezifische Förderung der Beschäftigung älterer Arbeitspersonen geben sollte, die sich beispielsweise mit der Umgestaltung der Lebensarbeitszeit und den anderen hier erörterten Maßnahmen befassen müßte.

\section{Integrierende Gesamtbetrachtung der behandelten Optionen}

Wie bereits bei der Herausarbeitung der spezifischen Problemlagen deutlich wurde, wirkt sich die Entwicklung der Produktions- und Organisationsmethoden auf die Möglichkeiten der Erwerbstätigkeit Älterer nachteiliger aus als auf die der Frauen. Während zur Erhöhung der Frauenerwerbsbeteiligung primär an den außerberuflichen Anforderungen orientierte Maßnahmen zu empfehlen sind, richten sich die Maßnahmen zur Erhöhung der Erwerbsquoten Älterer vor allem auf eine altersübergreifende Gestaltung der Arbeitsplätze zur Minderung beruflicher Fehlbeanspruchungen und zur Förderung der Möglichkeiten, verlängert erwerbstätig zu sein.

Von korrektiven Maßnahmen zum Ausgleich bestehender Beeinträchtigungen abgesehen, erfordert eine höhere Erwerbsbeteiligung Älterer die Umsetzung erst langfristig wirksam werdender arbeitsgestalterischer Maßnahmen. Veränderte Formen der Aufgabenbearbeitung oder des Arbeitsschutzes zur Förderung des Erfahrungswissens und zur Minderung von Fehlbeanspruchungen müssen früh im Arbeitsleben einsetzen und dauerhaft während des Erwerbslebens beachtet werden, damit ihre positiven Auswirkungen in der Spätphase der Erwerbstätigkeit zur Verlängerung der Lebensarbeitszeit beitragen können.

Die Maßnahmen zur Steigerung der Erwerbsbeteiligung der Frauen können dagegen relativ kurzfristig wirken, insbesondere indem sie Unterbrechungsphasen vermeiden, verkürzen oder deren negative Folgen vermindern. Langfristig tragen Maßnahmen zur quantitativen und qualitativen Förderung der Erwerbstätigkeit der Frauen auch zur erhöhten Ausschöpfung des Erwerbspersonenpotential der Älteren bei, da 
Frauen, deren Erwerbstätigkeit in der frühen und mittleren Phase ihres Erwerbslebens unterstützt wurde, auch in der Spätphase bessere Möglichkeiten der Erwerbsbeteiligung und eine höhere Erwerbsmotivation haben dürften.

Nur eine gebündelte Umsetzung der betrachteten Maßnahmen ermöglicht eine Realisierung der behandelten Optionen zur Sicherung des Humankapitals. Je nach konkreter Situation des Unternehmens und der beschäftigten Arbeitspersonen stehen hierbei andere Maßnahmen im Vordergrund, so daß sich verschieden ausgerichtete Maßnahmenbündel ergeben. Die Maßnahmenumsetzung muß unternehmensspezifisch in die Anpassungs- und Einführungsprozesse moderner Produktions- und Organisationsmethoden integriert werden. Damit können negative arbeitspersonenbezogene Folgen vermindert und positive qualifikations- und flexibilitätserhöhende Effekte unterstützt werden. Zur Einordnung der gestalterischen Chancen und Risiken werden im folgenden wesentliche Widersprüche bzw. Zielkonflikte, Kostenrelationen und erreichbare Personenpotentiale aufgezeigt.

\section{Widersprüche und Zielkonflikte}

Hinsichtlich der Umsetzung der Maßnahmen und der Realisierung der Optionen bestehen verschiedene Widersprüche und Zielkonflikte:

- Der Verbesserung der Vereinbarkeit von Beruf, Haushalt und Familie für weibliche Arbeitspersonen stehen neben den Anforderungen und Bedingungen der beruflichen Tätigkeiten auch die Einstellungen und Erwartungen vieler Männer entgegen. Werden Maßnahmen im Interesse der Erhöhung der Frauenerwerbsbeteiligung umgesetzt, so werden damit männliche arbeitsmarktbezogene Wettbewerbsvorteile und Privilegien abgebaut. Gelingt eine Erhöhung der Erwerbsbeteiligung der Frauen, so sind Männer davon im beruflichen und im außerberuflichen Bereich betroffen. Neben der verbesserten Wettbewerbsfähigkeit der Frauen auf dem Arbeitsmarkt werden die durchschnittlichen Entlastungsleistungen seitens der Frauen zurückgehen, so daß sich Männer in geringerem Maße ausschließlich auf ihre Erwerbstätigkeit konzentrieren können. Allerdings verliert der Beruf in der Einstellung vieler Männer (ihr Anteil wächst in niedrigeren Altersgruppen) seine zentrale Stellung (vgl. STRÜMPEL, 1988), so $\mathrm{da} \beta$ die Haus- und Familienarbeit für sie an Bedeutung gewinnen kann und Maßnahmen zur Erreichung der beruflichen Chancengleichheit der Frauen zukünftig erfolgreicher umgesetzt werden können. 
- Eine Erleichterung der Vereinbarkeit von Familie und Beruf, beispielsweise durch vielfältigere bzw. flexiblere Arbeitszeitvarianten und bessere Kinderbetreuungsmöglichkeiten, hat zur Folge, daß sich für verheiratete und alleinerziehende Frauen bessere Möglichkeiten, einer Erwerbstätigkeit nachzugehen, ergeben. Eine solche Verbesserung der arbeitsplatzbezogenen Wettbewerbssituation von Frauen sorgt dafür, $\mathrm{da} B$ sie sich verstärkt gegenüber älteren Arbeitspersonen durchsetzen können, so daß deren Beschäftigungschancen hierdurch tendenziell verschlechtert werden.

- Eine erhöhte Förderung der Erwerbstätigkeit der Älteren kann den Spielraum für die Nachwuchsförderung einschränken. Eine Verlängerung der Beschäftigung älterer Personen in qualifizierten Positionen verzögert für jüngere Arbeitspersonen die Aufstiegsmöglichkeiten, die zudem durch den angestrebten Abbau von Hierarchien und die Dezentralisierung von Handlungs- und Entscheidungskompetenzen vermindert werden.

- Bezüglich der zum Aufbau und zur Nutzung von Erfahrungswissen empfohlenen nicht-tayloristische Gestaltung der Arbeitsplätze kann betont werden, daß diese nicht unabhängig von den betroffenen Arbeitspersonen als ausschließlich positiv einzuschätzen ist. Denn die auf viele Arbeitsplätze ausgedehnte Aufhebung der Arbeitsteilung und Dezentralisierung der Kompetenzen verschärfen den beispielsweise durch die Automatisierung und den Strukturwandel geprägten Trend zum Wegfall einfacher Tätigkeiten und mindern die Arbeitsmarktchancen niedrig qualifizierter bzw. qualifizierbarer Personen.

- Die mögliche Ausweitung der Heimarbeit kann aufgrund der geringeren Betreuung der Arbeitspersonen und der fehlenden direkten Arbeitsplatzgestaltung dazu führen, das arbeitsschutzbezogene Probleme entstehen und Fehlbeanspruchungen die langfristige Nutzung des Arbeitspotentials der beschäftigten Personen gefährden. Andererseits kann die Erhöhung des Angebots an Teilzeitarbeit die Bedeutung der Heimarbeit schmälern.

- Eine Erhöhung der Erwerbsquoten von Frauen verschiedener Altersklassen kann im Falle des Fortbestandes der für viele erwerbstätige Frauen typischen Mehrfachbelastung zu einer Verschlechterung des durchschnittlichen Gesundheitszustand zukünftig älterer (weiblicher) Arbeitspersonen führen. Allerdings fehlen die für eine Prognose erforderlichen Daten und Analysen über den Gesundheitszustand und die Arbeitsschutzbedingungen (vgl. MÜLLER, 1994). 
Neben solchen Inkompatibilitäten bleiben trotz der erreichbaren und für die einzelnen Maßnahmen aufgezeigten Wirkungsweisen und Nutzengrößen Probleme bezüglich der Umsetzungsbereitschaft in den Unternehmen bestehen. Die verbreitete Umsetzung der Maßnahmen und die Realisierung der Optionen werden hierdurch gefährdet.

\section{Kostenrelationen}

Die Höhe der Kosten, die mit der Umsetzung der Maßnahmen und mit einer erhöhten Beschäftigung von Frauen und Älteren verbunden sind, werden sich zukünftig allenfalls im konkreten Fall einschätzen lassen. Sie sind abhängig von der Form der Institutionalisierung der Maßnahmen und müssen im Zusammenhang aller Kosten der Produktionsfaktoren unter Berücksichtigung der vielfältigen Produktions- und Marktbedingungen beurteilt werden.

Aus Sicht der Unternehmen kann betont werden, daß die Umsetzung komplexer und erst langfristig wirksam werdender Maßnahmen ein höheres Investitionsrisiko birgt als die Nutzung spezifisch und direkt wirkender Maßnahmen. Je länger die Amortisationszeit der Maßnahmen ist, desto größer ist die Gefahr der Abwerbung und Abwanderung der Beschäftigten. Der angestrebte Nutzen von Maßnahmen, die in langfristiger Wirkungsweise eine Verbesserung der Erwerbschancen anstreben, ist beim Ausscheiden der Arbeitsperson nicht realisierbar.

Die Herausbildung des Erfahrungswissens der Arbeitspersonen, die beanspruchungsoptimierte Gestaltung der Gruppenarbeit und die präventive Ausrichtung des Arbeitsschutzes beziehen sich auf das gesamte Erwerbsleben, um besonders die Erwerbsquoten der Älteren zu erhöhen. Das Investitionsrisiko läßt sich bei der Umsetzung dieser Maßnahmen schwerer begrenzen als dies für andere Maßnahmen möglich ist. So lassen sich eine Ausweitung der Teilzeitarbeit, der Heimarbeit sowie der Frauenförderung und die Konzeption von Arbeitshilfen kurzfristiger und damit auch mit geringerer Unsicherheit umsetzen. Bei abgrenzbaren spezifischen Weiterbildungsmaßnahmen kann es sogar gelingen, die Beschäftigten an den direkten Kosten der Maßnahmen zu beteiligen, so daß das maßnahmenbezogene Risiko der Unternehmen verringert wird. Unter diesem kosten- und risikobestimmten Gesichtspunkt ist eine kurzfristig erfolgreiche Förderung der Frauenbeschäftigung aus Unternehmenssicht eher anzustreben als eine Investition in die Verlängerung der Beschäftigung Älterer. Die Umsetzung der Maßnahmen zur Erhöhung der 
Erwerbsbeteiligung Älterer sollte demzufolge durch betrieblich wirksam werdende Anreize und den Abbau bestehender Hemmnisse gefördert werden. In diesem Sinn sollte die Entlohnungsstruktur verändert werden, um den Anstieg der Kosten der Beschäftigung Älterer zu verringern.

Entlohnungsformen sollen im allgemeinen die Erreichung der Kosten-, Qualitäts-, Flexibilitäts- und Zeitziele sowie die Befriedigung der an die Arbeitspersonen gerichteten Ansprüche auf Qualifizierungsbereitschaft, Flexibilität und Arbeitsmotivation fördern. Die Entlohnungsformen müssen den arbeitsorganisatorischen Änderungen, beispielsweise der Hierarchiegefüge und der Form der Aufgabenbearbeitung, angepaßt werden.

Versucht man arbeitsmarktbezogene Wettbewerbsnachteile Älterer zu mindern, so muß sich die Entlohnung zukünftig stärker an der erforderlichen und vorhandenen ausbildungs-, fortbildungs- und erfahrungsbedingten Qualifikation orientieren. Noch vorhandene Vorstellungen einer Senioritätsentlohnung und einer vom Alter bzw. vom Zeitablauf abhängigen Beförderungsweise müssen abgebaut werden. Das Problem der Senioritätsentlohnung betrifft die bei der Beschäftigung Älterer durchschnittlich höheren Lohnkosten. Diese müssen aus Arbeitgebersicht besonders dann als unangemessen hoch angesehen werden, sofern von einer altersbedingten Produktivitätsminderung, beispielsweise aufgrund länger zurückliegender Ausbildungen, ausgegangen wird. Jedenfalls stellt die mit dem Alter ansteigende Entlohnung, zumindest solange andere Arbeitskräfte verfügbar sind, für die Unternehmen einen Anreiz dar, ältere Arbeitspersonen auszugliedern. Ein Abbau der Senioritätsentlohnung könnte dazu beitragen, daß sich die Möglichkeiten Älterer länger erwerbstätig zu bleiben, verbessern.

Ein solcher Abbau altersbegründeter Lohn- und Gehaltsunterschiede ist nicht nur tarifvertraglich möglich, sondern könnte auch über eine Anpassung der (zusätzlich zum Tarifentgelt gezahlten) Zuschläge erfolgen, beispielsweise dadurch, daß bestimmte Zuschläge nur an jüngere Arbeitspersonen gezahlt werden. Eine Flexibilisierung der Entlohnung wäre auch in Abhängigkeit von der individuellen Leistung, von der Erreichung bestimmter Ziele und vom Unternehmenserfolg möglich. Alternativ könnten staatliche Stellen altersabhängige Vorteile ausgleichen, indem den Unternehmen beispielsweise die Kosten eines höheren Urlaubsanspruchs älterer Arbeitspersonen erstattet werden. Für die Beurteilung der individuellen Leistung sollten solche Kriterien ausgewählt werden, die bezüglich ihrer möglichen Auswirkungen auf die Beanspruchungssituation als ungefährdend angesehen werden 
können, so daß statt der Größen Bearbeitungszeit oder Stückzahl beispielsweise das Arbeitsverhalten, die Kommunikations- und Kooperationsbereitschaft, die Weiterbildungsbereitschaft oder auch die Initiative der Arbeitsperson honoriert werden.

Andererseits könnten die Unternehmen älteren Personen einen Anreiz bieten, länger erwerbstätig zu bleiben, indem die betrieblichen Alterssicherungssysteme (die durch Tarifverträge oder Betriebsvereinbarungen geregelt sind) entsprechend gestaltet werden. So könnte das Renteneinstiegsalter wie auch die Rentenhöhe in Abhängigkeit vom Alter beim Ausscheiden aus der Erwerbstätigkeit variiert werden.

Eigene Entlohnungsfragen stellen sich bezüglich der Gruppenarbeit. Die Produktivitätsfortschritte, die durch die Einführung der Gruppenarbeit erreicht werden sollen, beruhen wesentlich auf leistungsbezogenen Entgeltdifferenzierungen. Die Höhe des Arbeitsentgelts ist abhängig von den Anforderungen der Arbeitsaufgabe und der Leistung der einzelnen Arbeitsperson oder der Arbeitsgruppe. Besonders werden Formen des Prämienlohns diskutiert (vgl. beispielsweise EYER, 1993). Da verschiedene Produktions- und Prämienziele einander widersprechen, kann nur eine Kombination mehrerer Prämien funktional wirken. Die Prämien können sich beispielsweise auf die Kosteneinsparung, das Qualitätsniveau sowie die Termin- und Vorgabezeiteinhaltung richten. Zur Unterstützung der CIM-Umsetzung und der Teamarbeit kann eine Entlohnungskombination vorgeschlagen werden, die als Grundlohn einen Zeitlohn, verschiedene anforderungsabhängige Gruppenprämien und nach beherrschtem Tätigkeitsspektrum und Arbeitsverhalten bemessene personenbezogene Zulagen enthält (vgl. BULLINGER, 1992, 32ff.). Gerade die am Können ausgerichteten personenbezogenen Zulagen können die Bereitschaft zur Qualifizierung und zum Arbeitsplatzwechsel und die Einsatzflexibilität unterstützen. Verhaltensbezogene Zulagen können gleichzeitig beispielsweise die Kooperationsbereitschaft, die Selbständigkeit und den Arbeitseinsatz der Beschäftigten fördern.

Die Entgeltdifferenzierung entsprechend der Leistung der Arbeitsgruppe und der individuellen Leistungsbeiträge kann aufgrund des Gruppendrucks, der Konkurrenz zwischen den Gruppen und gruppenintern zwischen den Beschäftigten zu höheren Beanspruchungen der Arbeitspersonen beitragen. Dies kann zu Überforderungen der Leistungsschwächeren führen. Insbesondere im Interesse älterer Arbeitspersonen sollten Entlohnungsformen, die Leistungsdruck erzeugen, vermieden werden. Dabei sind der zeitliche bzw. prozeßabhängige Druck ebenso zu beachten 
wie ein möglicher sozialer Druck. Herrscht zwischen den Gruppenmitgliedern ein Leistungsgefälle, so kann die Gruppenprämie eine Beschäftigung leistungsschwächerer Arbeitspersonen erschweren, da diese überfordert oder aus der Gruppe gedrängt werden können.

\section{Potentialabschätzung}

Die quantitative Wirkung der Umsetzung der mikroökonomisch orientierten Maßnahmen auf das makroökonomische Arbeitsangebot läßt sich nur schwer abschätzen. Die Prognosemöglichkeiten für die Zahl der von den einzelnen Maßnahmen potentiell betroffenen Personen werden durch die angesprochene Vielfalt arbeitsbezogener Entwicklungslinien und die interdependente Wirkungsweise der Maßnahmen beeinträchtigt. Außerdem sind - ähnlich wie bezüglich der zukünftigen Defizite betont - auch für die hier einzuschätzenden Wirkungen der Maßnahmenumsetzung die beschriebenen qualitativen Komponenten häufig von größerer Bedeutung als die quantitativen Auswirkungen auf das gesamtwirtschaftliche Arbeitsangebot.

Zukünftige Änderungen der arbeitsmarktlichen Situation, wie eine langfristige Senkung der Arbeitslosigkeit und eine teilweise Auflösung der Stillen Reserve, werden auch die Einstellung von Arbeitgebern und potentiellen Arbeitspersonen verändern. Wenn diese schwer abschätzbaren, dynamischen Prozesse durch eine Form der Arbeitsgestaltung ergänzt werden, die die individuellen Bedürfnisse der Arbeitspersonen berücksichtigt, so kann es zu einer sich in positiver Weise verstärkenden Ausweitung der Erwerbsquoten kommen, die die voraussehbaren Defizite mindert.

Die Betrachtung der Potentiale erfolgt arbeitsangebotsbezogen und betrifft die Frage, wieviele Personen bei Umsetzung der behandelten Maßnahmen zusätzlich erwerbstätig werden können, d.h. in welchem Ausmaß sich das Arbeitsangebot erhöhen läßt. Für die Abschätzung wird angenommen, daß nachfrageseitig keine Restriktionen bestehen.

Aussagen zur möglichen quantitativen Auswirkung der Maßnahmenumsetzung lassen sich aufgrund kombinierter Betrachtungen des Status-quo und der prognostizierten Situation unter Berücksichtigung der voraussichtlichen Änderungen wesentlicher Einflußfaktoren gewinnen. 


\section{a) Erhöhung der Erwerbsquote der Frauen}

Die Abschätzung der möglichen Ausweitung der Erwerbsbeteiligung der Frauen wird durch eine Betrachtung der einzelnen Maßnahmen hinsichtlich ihrer jeweiligen Quantifizierbarkeit vorbereitet. Hier werden die Maßnahmen behandelt, die sich auf die Erhöhung der Erwerbsquoten von Frauen jüngerer und mittlerer Altersklassen richten. Danach wird die Dimension der möglichen Ausweitung der Erwerbstätigkeit älterer Frauen und Männer betrachtet.

Die Datenbasis zur Maßnahme Heimarbeit ist sehr unsicher, da bereits die Zahl bestehender Heimarbeitsverhältnisse unbekannt ist. Dies begründet sich dadurch, daß diese Beschäftigungsform zwischen den Erwerbsformen Selbständigkeit und abhängiger Tätigkeit anzuordnen ist und sie statistisch entsprechend unterschiedlich zugerechnet wird. Außerdem läßt sie sich nicht über die Sozialversicherungsstatistik identifizieren, und z.B. auch den Gewerkschaften fehlen Informationen über die Häufigkeit der Heimarbeit ihrer Mitglieder (vgl. BRANDES/BUTTLER, 1987, 76; LENK, 1989, 44ff.). Die Maßnahme Heimarbeit ist in der dargestellten Weise geeignet, die Vereinbarkeit von Familie und Beruf und die Wiedereinstiegschancen nach einer Unterbrechungsphase zu verbessern. Allerdings kann davon ausgegangen werden, daß Heimarbeit, trotz der voraussichtlichen Ausweitung dieser Arbeitsform, lediglich ergänzend zur Erhöhung der Erwerbsquoten von Frauen beitragen kann.

Von größerer Bedeutung dürfte die Maßnahme Arbeitszeitkonzepte und insbesondere die Ausweitung der Teilzeitarbeit sein. Nach Hochrechnungen auf Basis des SOEP 1993 bevorzugen gerade Frauen der Stillen Reserve, die nicht als arbeitssuchend registriert sind, aber erwerbstätig sein möchten eine Teilzeitbeschäftigung. Ca. 700.000 Frauen würden diese Beschäftigungsform sofort oder innerhalb eines Jahres und über 1,5 Millionen in zwei bis fünf Jahren oder später wählen (vgl. DIW, 1994, 619ff.). Anhand dieser Umfrageergebnisse läßt sich schätzen, welche Auswirkungen die mögliche Ausweitung der Erwerbsbeteiligung von teilzeitbeschäftigten Frauen auf die insgesamt zusătzlich geleistete Zahl der Arbeitsstunden je Zeitperiode hat. Aufgrund der genannten Hochrechnungen soll angenommen werden, daß jährlich ca. 500.000 Frauen der Stillen Reserve eine Teilzeitbeschäftigung mit durchschnittlich 20 Wochenstunden annehmen würden, falls ein entsprechendes Angebot an Arbeitsplätzen vorliegen würde. Damit wird deutlich, daß die Umsetzung dieser Maßnahme die Erwerbsbeteiligung der Frauen in einem relevanten $\mathrm{Maß}$ steigern könnte. Bereits ohne zusätzliche motivierende 
Anstrengungen seitens der Unternehmen könnte die Teilzeitarbeit eine Ausweitung des Arbeitskräfteangebots in Höhe von ca. 250.000 Vollzeitkräften bewirken. Allerdings sind solche Überschlagsrechnungen mit einer großen Unsicherheit behaftet, da die möglichen Auswirkungen einer erleichterten Teilzeiterwerbstätigkeit äußerst vielfältig sind. Beispielsweise können bisher nichterwerbstätige aber auch bisher erwerbstätige Personen zur Teilzeitarbeit wechseln, so daß der resultierende zusätzlich geleistete Arbeitsstundensaldo von Bedeutung ist. Ein erweitertes Teilzeitangebot für qualifikatorisch anspruchsvolle Tätigkeiten würde das Interesse an dieser Arbeitsform erhöhen. Trotz aller Unwägbarkeiten kann festgehalten werden, daß die Teilzeitarbeit einen wesentlichen Beitrag zur Verbesserung der Vereinbarkeit der Anforderungen von Familie und Beruf und damit zur Erhöhung der Erwerbsbeteiligung der Frauen leisten kann.

Weiterbildungsmaßnahmen, die sich auf bereits beschäftigte Personen und auf arbeitslose und nicht erwerbstätige Personen beziehen können, bestimmen entscheidend die qualitative Zusammensetzung des zukünftigen Erwerbspersonenpotentials. Die direkten quantitativen Auswirkungen dürften demgegenüber gering sein. Allerdings schafft die breite Umsetzung dieser Maßnahmen die Voraussetzungen für eine rasche und erfolgreiche Reintegration von Frauen nach einer Unterbrechungsphase und erhöht die Erwerbsmotivation potentieller Arbeitnehmerinnen.

Die Maßnahme Frauenförderung trägt durch die Verknüpfung von Unterbrechungsphasen mit Rückkehrzusagen zur Erleichterung der Vereinbarkeit von Beruf und Familie bei. Kontaktpflegemaßnahmen unterstützen die Wiedereingliederungschancen nach einer Erwerbspause und fördern den Erhalt der Qualifikationen.

Die Maßnahmen können in Anlehnung an die Form ihrer Ableitung entsprechend der beiden wichtigsten arbeitsmarktbezogenen Wettbewerbsnachteile der Frauen in zwei Gruppen eingeteilt werden. Die eine Gruppe richtet sich insbesondere auf die Erleichterung der Vereinbarkeit beruflicher und familiärer Anforderungen und die zweite Gruppe zielt darauf die Wiedereingliederungschancen von Frauen nach einer Unterbrechungsphase zu erhöhen. 


\begin{tabular}{|l|l|}
\hline $\begin{array}{l}\text { Gruppen arbeitsmarktbezogener } \\
\text { Wettbewerbsnachteile }\end{array}$ & $\begin{array}{l}\text { Maßnahme zur Förderung der Erwerbs- } \\
\text { beteiligung von Frauen jüngerer und } \\
\text { mittlerer Altersklassen }\end{array}$ \\
\hline $\begin{array}{l}\text { Schwierigkeiten der Vereinbarkeit } \\
\text { von Beruf und Familie }\end{array}$ & $\begin{array}{l}\text { - Arbeitszeitkonzepte } \\
\text { - Unterbrechungsmöglichkeiten }\end{array}$ \\
\hline Schwierigkeiten der Reintegration & $\begin{array}{l}\text { - Weiterbildung } \\
\text { - Kontaktpflege }\end{array}$ \\
\hline
\end{tabular}

Abb. 71: Bildung zweier Maßnahmenbündel zur Erhöhung der Erwerbsbeteiligung von Frauen

In einer überschlägigen Rechnung soll ermittelt werden, in welcher Dimension die Zahl erwerbstätiger Frauen durch die Umsetzung der genannten Maßnahmen zukünftig erhöht werden kann. Hierzu werden zunächst Zahlen zur Bevölkerung und zur Erwerbstätigkeit von Frauen ohne Kinder und mit Kindern sowie jeweils von Frauen und Männern insgesamt zusammengestellt (vgl. STATISTISCHES BUNDESAMT, MIKROZENSUS). Für die hier vorgenommene Abschätzung wird angenommen, daß sich durch die Umsetzung der Maßnahmen die Differenzen der Erwerbsquoten des Jahres 1993 zwischen Frauen ohne Kinder und Männern sowie zwischen Frauen mit Kindern und Frauen ohne Kinder bis zum Jahr 2010 halbieren lassen. Daraus ergeben sich die angegebenen überschlägigen Erwerbsquoten. Unter Beibehaltung der Gruppenanteile von 1993 ergibt sich eine Erhöhung der Erwerbsquote für Frauen insgesamt um 8,6\%-Punkte auf 73,6\%.

\begin{tabular}{|c|l|c|c|c|c|}
\hline $\begin{array}{l}\text { im Alter von 25 bis } \\
\text { unter 55 Jahren } \\
\text { - Deutschland - }\end{array}$ & Männer & $\begin{array}{c}\text { Frauen } \\
\text { insgesamt }\end{array}$ & $\begin{array}{c}\text { Frauen ohne } \\
\text { Kinder }\end{array}$ & $\begin{array}{c}\text { Frauen mit } \\
\text { Kindern }\end{array}$ \\
\hline 1993 & $\begin{array}{l}\text { Bevölkerung } \\
\text { Erwerbspersonen } \\
\text { Erwerbsquote }\end{array}$ & $\begin{array}{c}17.177 .000 \\
94 \%\end{array}$ & $\begin{array}{c}17.605 .000 \\
11.511 .000 \\
65 \%\end{array}$ & $\begin{array}{c}6.949 .000 \\
75 \%\end{array}$ & $\begin{array}{c}10.956 .000 \\
7.515 .000 \\
59 \%\end{array}$ \\
\hline & & $73,6 \%$ & $84,5 \%$ & $67 \%$ \\
\hline
\end{tabular}

Abb. 72: Erwerbstătigkeit von Mănnern und Frauen im Alter von 25 bis 55 Jahren im Jahre 1993

Überträgt man diese Erhöhung der Erwerbsquote der Frauen auf die zukünftigen Stände der Bevölkerung in Deutschland (unveröffentlichte Daten zur Variante 2 der 
achten koordinierten Bevölkerungsvorausberechnung des Statistischen Bundesamtes und der Statistischen Landesämter, SOMMER, 1994), so ergibt sich ein jährlich erhöhtes Arbeitsangebot, wie es in folgender Abbildung angegeben ist.

\begin{tabular}{|c|c|c|}
\hline $\begin{array}{c}\text { Erhöhung der } \\
\text { Erwerbsquote um 8,6\% }\end{array}$ & $\begin{array}{c}\text { Frauen im Alter von 25 bis } \\
\text { unter 55 Jahren }\end{array}$ & $\begin{array}{c}\text { geschätzte zusätzliche Zahl } \\
\text { der Erwerbspersonen }\end{array}$ \\
\hline $\mathbf{2 0 1 0}$ & 17.055 .100 & $\mathbf{1 . 4 6 7 . 0 0 0}$ \\
\hline 2020 & 15.212 .500 & $\mathbf{1 . 3 0 8 . 0 0 0}$ \\
\hline $\mathbf{2 0 3 0}$ & 13.522 .500 & $\mathbf{1 . 1 6 3 . 0 0 0}$ \\
\hline $\mathbf{2 0 4 0}$ & 12.479 .000 & $\mathbf{1 . 0 7 3 . 0 0 0}$ \\
\hline
\end{tabular}

Abb. 73: Geschätzte zukünftig erreichbare Ausweitung der Zahl der erwerbstätigen Frauen

Der geschätzte Beitrag der prozentual konstant angenommenen Ausweitung der Erwerbstätigkeit der Frauen geht in den Jahren 2010 bis 2040 von jährlich knapp 1,5 Millionen Personen auf ca. eine Million Personen zurück. In diesem Rückgang spiegelt sich die demographische Entwicklung wider. In folgender Abbildung ist die geschätzte Bedeutung der behandelten Maßnahmen zur Erhöhung der Erwerbsquoten von Frauen graphisch dargestellt.

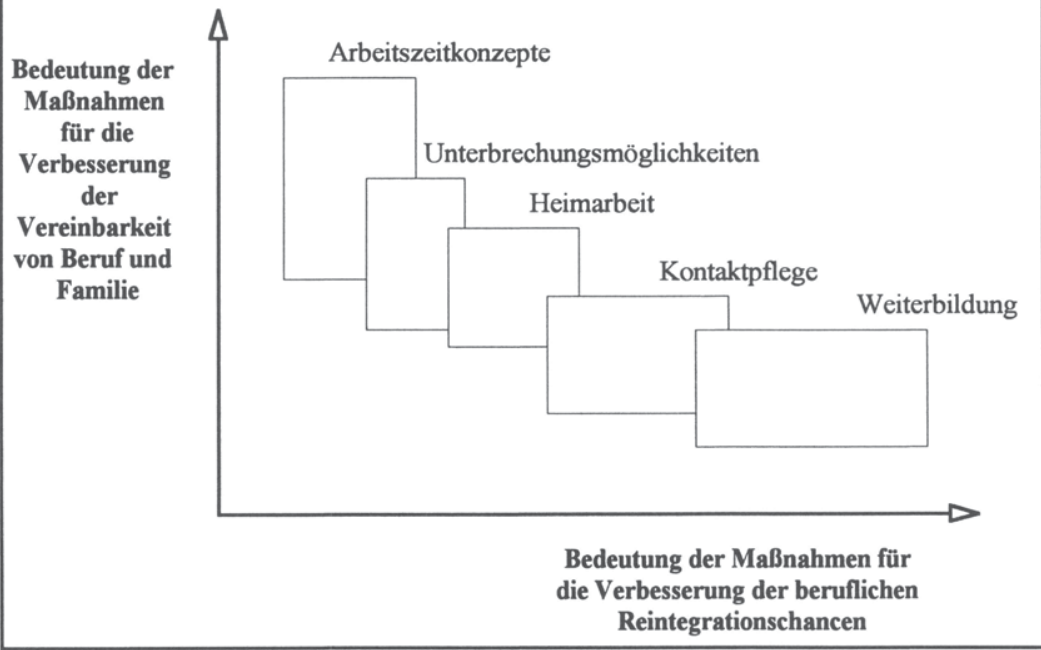

Abb. 74: Beiträge der Maßnahmen zur Ausweitung der Erwerbsbeteiligung von Frauen jüngerer und mittlerer Altersklassen 


\section{b) Erhöhung der Erwerbsquote ålterer Personen}

Zur Abschätzung des zu aktivierenden Erwerbspersonenpotentials der Älteren können die Zahlen des Rentenzugangs betrachtet werden. Unter der vereinfachenden Annahme, daß der Rentenzugang dem Austritt aus der Erwerbstätigkeit entspricht (Differenzen, die beispielsweise aufgrund freiwilliger und unfreiwilliger Unterbrechungsphasen auftreten, werden hier vernachlässigt), können - wie ähnlich bereits bei der Ableitung der Maßnahmen in Kapitel II geschehen - dem Grund des Rentenzugangs Gruppen der behandelten Maßnahmen zugeordnet werden, die zu einer zeitlichen Verschiebung des Rentenzugangs beitragen können.

Dem Rentenzugang wegen verminderter Erwerbsfähigkeit entsprechen Maßnahmen, die sich auf den Erhalt der Gesundheit beziehen, Fehlbeanspruchungen abbauen und Leistungsminderungen ausgleichen. Auf den Rentenzugang aus Altersgründen richten sich Maßnahmen, die insbesondere qualifikations- und motivationsfördernde Wirkungsweisen besitzen und somit den Ausstieg aus der Erwerbstätigkeit verschieben können.

\begin{tabular}{|l|l|}
\hline Grund des Rentenzugangs & $\begin{array}{l}\text { Maßnahme zur Verschiebung } \\
\text { des Rentenzugangs }\end{array}$ \\
\hline $\begin{array}{l}\text { Rentenzugang wegen verminderter } \\
\text { Erwerbsfähigkeit }\end{array}$ & $\begin{array}{l}\text { - Arbeitsschutz } \\
\text { - Arbeitshilfen und Schonarbeit } \\
\text { - Arbeitszeitkonzepte } \\
\text { - Erfahrungswissen }\end{array}$ \\
\hline Rentenzugang wegen des Alters & $\begin{array}{l}\text { - Eruppenarbeit } \\
\text { - Weiterbildung }\end{array}$ \\
& $\begin{array}{l}\text { - Aruppenarbeit } \\
\text { Arbeitszeitkonzepte }\end{array}$ \\
\hline
\end{tabular}

Abb. 75: Bildung zweier Maßnahmenbündel zur Erhöhung der Erwerbsbeteiligung Älterer

Im Jahr 1993 waren in Deutschland 208.810 der 662.025 Zugänge an Versichertenrenten bei den über 50 bis unter 65 Jahre alten Personen Renten wegen verminderter Erwerbsfähigkeit (vgl. VDR, 1993). Dies entspricht einem Anteil an den Rentenzugängen von $32 \%$. Der Zugang an Versichertenrenten wegen Alters betraf 1993 in dieser Altersgruppe 453.215 Personen (68\%). In folgender Tabelle sind Zahlen zum Rentenzugang zur gesetzlichen Rentenversicherung (vgl. VDR, 1993) und Daten zur Bevölkerung und zur Erwerbstätigkeit (vgl. STATISTISCHES 
BUNDESAMT, MIKROZENSUS) zusammengefaßt für zwei Altersgruppen dargestellt.

\begin{tabular}{|c|c|c|c|}
\hline & $\begin{array}{l}\text { Männer und Frauen } \\
\text { - Deutschland - }\end{array}$ & $\begin{array}{c}\text { im Alter von } \\
50 \text { bis unter } \\
60 \text { Jahren }\end{array}$ & $\begin{array}{c}\text { im Alter von } \\
60 \text { bis unter } \\
65 \text { Jahren }\end{array}$ \\
\hline \multirow[t]{2}{*}{1993} & $\begin{array}{l}\text { Bevölkerung } \\
\text { Erwerbspersonen } \\
\text { Erwerbsquote }\end{array}$ & $\begin{array}{l}11.840 .000 \\
8.259 .000 \\
70 \%\end{array}$ & $\begin{array}{c}4.493 .000 \\
887.000 \\
20 \%\end{array}$ \\
\hline & $\begin{array}{l}\text { Rentenzugang } \\
\text { wegen verminderter Erwerbsfähigkeit } \\
\text { wegen Alters }\end{array}$ & $\begin{array}{c}178.028 \\
-\end{array}$ & $\begin{array}{c}30.782 \\
453.215\end{array}$ \\
\hline
\end{tabular}

Abb. 76: Erwerbstätigkeit und Rentenzugang Älterer im Jahre 1993

Überträgt man das Verhältnis der Rentenzugänge dieser Altersgruppe auf die vorausberechneten zukünftigen Stände der Bevölkerung in Deutschland (unveröffentlichte Daten zur Variante 2 der achten koordinierten Bevölkerungsvorausberechnung des Statistischen Bundesamtes und der Statistischen Landesämter, SOMMER, 1994) und behält alle übrigen Faktoren konstant, so ergeben sich folgende Schätzwerte bezüglich des Rentenzugangs.

\begin{tabular}{|c|c|c|c|c|}
\hline & $\begin{array}{l}\text { Männer und Frauen } \\
\text { - Deutschland - }\end{array}$ & $\begin{array}{c}\text { im Alter } \\
\text { von } 50 \text { bis } \\
\text { unter } \\
60 \text { Jahren }\end{array}$ & $\begin{array}{c}\text { im Alter } \\
\text { von } 60 \text { bis } \\
\text { unter } \\
65 \text { Jahren }\end{array}$ & Summe \\
\hline 2000 & $\begin{array}{l}\text { Rentenzugang } \\
\text { wegen verminderter Erwerbsfähigkeit } \\
\text { wegen Alters }\end{array}$ & $\begin{array}{c}151.000 \\
-\end{array}$ & $\begin{array}{c}39.000 \\
578.000\end{array}$ & $\begin{array}{l}190.000 \\
578.000\end{array}$ \\
\hline 2010 & $\begin{array}{l}\text { Rentenzugang } \\
\text { wegen verminderter Erwerbsfähigkeit } \\
\text { wegen Alters }\end{array}$ & $\begin{array}{c}181.000 \\
-\end{array}$ & $\begin{array}{c}32.000 \\
476.000\end{array}$ & $\begin{array}{l}213.000 \\
476.000\end{array}$ \\
\hline 2020 & $\begin{array}{l}\text { Rentenzugang } \\
\text { wegen verminderter Erwerbsfähigkeit } \\
\text { wegen Alters }\end{array}$ & $\begin{array}{c}211.000 \\
-\end{array}$ & $\begin{array}{c}41.000 \\
604.000 \\
\end{array}$ & $\begin{array}{l}252.000 \\
604.000 \\
\end{array}$ \\
\hline 2030 & $\begin{array}{l}\text { Rentenzugang } \\
\text { wegen verminderter Erwerbsfähigkeit } \\
\text { wegen Alters }\end{array}$ & $\begin{array}{c}150.000 \\
-\end{array}$ & $\begin{array}{c}44.000 \\
647.000\end{array}$ & $\begin{array}{l}194.000 \\
647.000 \\
\end{array}$ \\
\hline 2040 & $\begin{array}{l}\text { Rentenzugang } \\
\text { wegen verminderter Erwerbsfähigkeit } \\
\text { wegen Alters }\end{array}$ & $\begin{array}{c}158.000 \\
-\end{array}$ & $\begin{array}{c}32.000 \\
465.000\end{array}$ & $\begin{array}{l}190.000 \\
465.000\end{array}$ \\
\hline
\end{tabular}

Abb. 77: Geschätzte zukünftige jăhrliche Rentenzugănge Älterer unter Beibehaltung der Erwerbsquoten und Rentenzugangsanteile von 1993 
In einer überschlägigen Rechnung soll angenommen werden, daß es bis 2010/2020 gelingt den durchschnittlichen Rentenzugang wegen verminderter Erwerbsfähigkeit in der Altersklasse der 50- bis 60jährigen um zwei/drei Jahre und in der Altersklasse der 60- bis 65jährigen um ein/zwei Jahr/e sowie den Rentenzugang aus Altersgründen ebenfalls um ein/zwei Jahr/e zu verschieben. Für die Jahre bis 2040 wird die gleiche zeitliche Verschiebung des Renteneintritts wie 2020 angenommen. Folgende Tabelle zeigt die quantitativen Auswirkungen.

\begin{tabular}{|c|c|c|c|c|}
\hline & $\begin{array}{l}\text { Männer und Frauen } \\
\text { - Deutschland - } \\
\text { potentielle jährliche Minderung des ... }\end{array}$ & $\begin{array}{c}\text { im Alter } \\
\text { von } \\
50 \text { bis unter } \\
60 \text { Jahren }\end{array}$ & $\begin{array}{l}\text { im Alter } \\
\text { von } \\
60 \text { bis unter } \\
65 \text { Jahren }\end{array}$ & Summe \\
\hline 2010 & $\begin{array}{l}\text { Rentenzugangs } \\
\text { wegen verminderter Erwerbsfähigkeit } \\
\text { wegen Alters }\end{array}$ & $\begin{array}{c}362.000 \\
- \\
\end{array}$ & $\begin{array}{r}32.000 \\
476.000 \\
\end{array}$ & $\begin{array}{l}394.000 \\
476.000 \\
\end{array}$ \\
\hline & Summe 2010 & 362.000 & 508.000 & 870.000 \\
\hline 2020 & $\begin{array}{l}\text { Rentenzugangs } \\
\text { wegen verminderter Erwerbsfähigkeit } \\
\text { wegen Alters }\end{array}$ & $\begin{array}{c}633.000 \\
- \\
\end{array}$ & $\begin{array}{c}82.000 \\
1.208 .000 \\
\end{array}$ & $\begin{array}{c}715.000 \\
1.208 .000 \\
\end{array}$ \\
\hline & Summe 2020 & 633.000 & 1.290 .000 & 1.923 .000 \\
\hline 2030 & $\begin{array}{l}\text { Rentenzugangs } \\
\text { wegen verminderter Erwerbsfähigkeit } \\
\text { wegen Alters } \\
\end{array}$ & $\begin{array}{c}450.000 \\
- \\
\end{array}$ & $\begin{array}{c}88.000 \\
1.294 .000 \\
\end{array}$ & $\begin{array}{c}538.000 \\
1.294 .000 \\
\end{array}$ \\
\hline & Summe 2010 & 450.000 & 1.382 .000 & 1.832 .000 \\
\hline 2040 & $\begin{array}{l}\text { Rentenzugangs } \\
\text { wegen verminderter Erwerbsfähigkeit } \\
\text { wegen Alters }\end{array}$ & $\begin{array}{c}474.000 \\
- \\
\end{array}$ & $\begin{array}{r}64.000 \\
930.000 \\
\end{array}$ & $\begin{array}{r}538.000 \\
930.000 \\
\end{array}$ \\
\hline & Summe 2020 & 474.000 & 994.000 & 1.468 .000 \\
\hline
\end{tabular}

Abb. 78: Geschätzte zukünftig mögliche Erhöhung der Zahl älterer Erwerbspersonen

Diese Überschlagsrechnung verdeutlicht die Dimension der betroffenen Personenzahl. Gelingt durch die Umsetzung der behandelten unternehmensbezogenen Maßnahmen eine solche Verschiebung des Renteneintritts, so erhöhen sich damit die Erwerbsquoten älterer Personen erheblich, wie folgende Abbildung zeigt. Die zum Vergleich angegebenen Erwerbsquoten von 1970 und 1993 machen deutlich, daß die Annahmen zur möglichen Verschiebung des Renteneintrittsalters (gerade auch unter Einbeziehung der seit $1970 \mathrm{zu}$ verzeichnenden Zunahme der Frauenerwerbstätigkeit) nicht $\mathrm{zu}$ hoch angesetzt sind. Aufgrund des weiteren Anstiegs der Erwerbsbeteiligung der Frauen dürften die langfristig in den höheren Altersgruppen erreichbaren Erwerbsquoten sogar erkennbar höher liegen, so daß diese Option einen größeren Beitrag zum Ausgleich möglicher zukünftiger Defizite leisten kann. 


\begin{tabular}{|c|c|c|}
\hline $\begin{array}{c}\text { Erwerbsquoten } \\
\text { (in \%) }\end{array}$ & $\begin{array}{c}\text { Alter von 50 bis } \\
\text { unter 60 Jahren }\end{array}$ & $\begin{array}{c}\text { Alter von 60 bis } \\
\text { unter 65 Jahren }\end{array}$ \\
\hline 1970 & 62 & 45 \\
\hline 1993 & 70 & 20 \\
\hline 2010 & 73 & 31 \\
\hline 2020 bis 2040 & 75 & 42 \\
\hline
\end{tabular}

Abb. 79: Geschătzte zukünftig erreichbare Erwerbsquoten Älterer

Nach diesen Berechnungen beziehen sich die Maßnahmen zur Verschiebung des Rentenzugangs wegen verminderter Erwerbsfähigkeit im Jahr 2010 mindestens auf eine jährlich zusätzliche Erwerbspersonenzahl von ca. 394.000. Im Jahr 2020 sind 715.000 sowie 2030 und 2040 jeweils 538.000 Personen zusätzlich erwerbstätig. Besonders die Maßnahmen Arbeitsschutz und Arbeitshilfen aber auch die Maßnahmen Erfahrungswissen und gegebenenfalls Gruppenarbeit tragen zur Vermeidung von Fehlbeanspruchungen und damit von Minderungen der Erwerbsfähigkeit bei. Die Maßnahmen Teilzeit und Schonarbeit können darüber hinaus die Erwerbstätigkeit trotz vorhandener Minderungen der Erwerbsfähigkeit ermöglichen.

Das Maßnahmenbündel zur Verschiebung des Rentenzugangs aus Altersgründen richtet sich im Jahr 2010 auf eine jährliche Ausweitung von ca. 476.000, 2020 auf 1.208.000, 2030 auf 1.294 .000 und 2040 auf 930.000 Personen. Die Maßnahmen Erfahrungswissen, Weiterbildung und gegebenenfalls Gruppenarbeit sowie die Möglichkeit der Teilzeitarbeit können aufgrund ihrer positiven Auswirkungen auf die Qualifikation und die Motivation der Arbeitspersonen den Ausstieg aus der Erwerbstätigkeit verzögern. Darüber hinaus können sie wesentlich die voraussichtlichen qualitativen Defizite verringern.

Die beiden Maßnahmenbündel zur Erhöhung der Erwerbstätigkeit der Älteren können nach dieser Schätzung zur Ausweitung des Angebots auf dem Arbeitsmarkt um knapp 900.000 Personen im Jahr 2010, um knapp 2 Millionen Personen in den Jahren 2020 und 2030 und um knapp 1,5 Millionen Personen im Jahr 2040 beitragen. Folgende Abbildung zeigt die relative Bedeutung der behandelten Maßnahmen zur Erhöhung der Erwerbstätigkeit älterer Personen. 


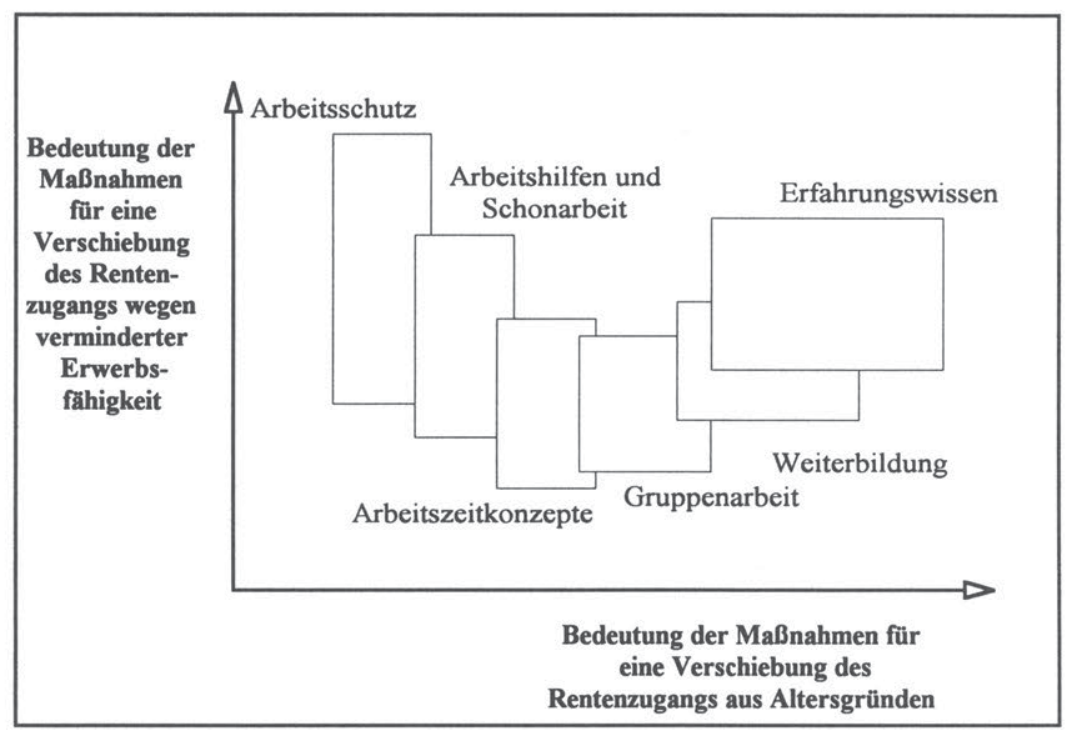

Abb. 80: Beiträge der Maßnahmen zur Ausweitung der Erwerbsbeteiligung Älterer

\section{c) Auswirkungen auf die gesetzliche Rentenversicherung}

Im folgenden wird geschätzt in welchem Maße sich die Ausschöpfung der aufgezeigten Potentiale verändernd auf die Entwicklung der finanziellen Situation der gesetzlichen Rentenversicherung auswirken kann. Hierzu soll in Anlehnung an Überlegungen und Berechnungen des Verbandes Deutscher Rentenversicherungsträger (vgl. BARTH u.a., 1994, 248ff.) die Effektivlastquote betrachtet werden. Diese Kenngröße ergibt sich aus der Division der geschätzten Zahl an Rentenempfängern durch die geschätzte Zahl der Beitragszahler. Bereits die gegenwärtige Zahl der Rentenempfänger muß geschätzt werden, da nur die Zahl der Rentenfälle aber nicht die Doppelrenten registriert werden. Etwa $85 \%$ der 60 Jahre alten und älteren Personen sind Rentner (vgl. BARTH u.a., 1994, 250). Diese grobe Schätzung wird für die hier durchgeführten überschlägigen Rechnungen übernommen, obwohl insbesondere der Rentenzugang wegen verminderter Erwerbsfähigkeit der Personen unter 60 Jahren nicht erfaßt wird und die Effektivlastquote infolgedessen durchgängig etwas zu günstig dargestellt wird. Nach den Daten zur Erwerbstätigkeit von 1993 kann weiterhin angenommen werden, daß ca. $61 \%$ der Personen im Alter von 20 bis unter 60 Jahren und ca. $11 \%$ der Personen im Alter 
von 60 bis unter 65 Jahren als Arbeiter und Angestellte und somit als Beitragszahler aktiv sind (vgl. STATISTISCHES BUNDESAMT, MIKROZENSUS).

Die folgende Abbildung zeigt welche effektiven Belastungen sich im Falle der Beibehaltung dieser Anteile von Erwerbstätigen und Rentnern für die vorausberechneten zukünftigen Stände der Bevölkerung in Deutschland (unter Verwendung unveröffentlichter Daten zur Variante 2 der achten koordinierten Bevölkerungsvorausberechnung des Statistischen Bundesamtes und der Statistischen Landesämter, SOMMER, 1994) ergeben. Weiterhin wird berechnet wie sich die möglich erscheinende Erhöhung der Erwerbsquoten von Frauen und Älteren auf die zukünftige Zahl von Erwerbstätigen und Rentnern auswirken kann. Die Zahl zusätzlich erwerbstätiger Frauen und älterer Personen erhöht die Erwerbstätigenzahl. Die Zahl der zusätzlich erwerbstätigen Älteren mindert zudem die Zahl der Rentner. Die schließlich berechneten veränderten Effektivlastquoten machen im Vergleich zur fortgeschriebenen effektiven Belastungen deutlich, in welcher Größenordnung die Umsetzung der behandelten Maßnahmen zur Verbesserung der zukünftigen Finanzierungssituation der gesetzlichen Rentenversicherung beitragen kann.

\begin{tabular}{|c|c|c|c|c|c|c|c|c|c|}
\hline \multirow{3}{*}{$\begin{array}{c}\text { Ende } \\
\text { des } \\
\text { Jahres }\end{array}$} & \multicolumn{4}{|c|}{$\begin{array}{c}\text { zukünftige Situation aufgrund } \\
\text { der Fortschreibung der } \\
\text { Verhältnisse von } 1993 \\
\end{array}$} & \multicolumn{2}{|c|}{$\begin{array}{c}\text { geschätztes } \\
\text { Potential zus. } \\
\text { Erwerbspersonen }\end{array}$} & \multicolumn{3}{|c|}{$\begin{array}{c}\text { veränderte zukünftige } \\
\text { Situation }\end{array}$} \\
\hline & $\begin{array}{c}\text { Alten- } \\
\text { quotient }\end{array}$ & $\begin{array}{c}\text { Erwerbs } \\
\text {-tatige } \\
\end{array}$ & Rentner & $\begin{array}{l}\text { Effektiv- } \\
\text { lastquote } \\
\end{array}$ & Frauen & Ältere & $\begin{array}{l}\text { Erwerbs } \\
\text {-tatige }\end{array}$ & Rentner & $\begin{array}{l}\text { Effektiv- } \\
\text { lastquote }\end{array}$ \\
\hline & in $\%$ & in 1.000 & in 1.000 & in $\%$ & in 1.000 & in 1.000 & in 1.000 & in 1.000 & in $\%$ \\
\hline 1993 & 35,0 & $29.348,7$ & $14.095,3$ & 48 & - & - & $29.348,7$ & $14.095,3$ & 48 \\
\hline 2010 & 44,1 & $29.268,4$ & $17.679,2$ & 60 & 1.467 & 870 & $31.605,4$ & $16.809,2$ & 53 \\
\hline 2020 & 51,7 & $27.716,5$ & $19.478,0$ & 70 & 1.308 & 1.923 & $30.947,5$ & $17.555,0$ & 57 \\
\hline 2030 & 67,8 & $24.111,8$ & $22.119,9$ & 92 & 1.163 & 1.832 & $27.106,8$ & $20.287,9$ & 75 \\
\hline 2040 & 67,8 & $22.599,3$ & $20.864,4$ & 92 & 1.073 & 1.468 & $25.140,3$ & $19.396,4$ & 77 \\
\hline
\end{tabular}

Abb. 81: Veränderung des zukünftigen Belastungsanstiegs durch die Ausschöpfung der aufgezeigten Potentiale 
Aufgrund der Fortschreibung der Situation von 1993 beträgt der Belastungsanstieg von 1993 bis 2040 gemessen mit dem Anstieg des Altersquotienten 94\% und gemessen mit dem Anstieg der Effektivlastquote 92\%. Die veränderte Effektivlastquote steigt im betrachteten Zeitraum um $60 \%$. Gelingt die Realisierung der auf die Erhöhung der Erwerbstätigkeit von Frauen und Älteren gerichteten Optionen in dem hier angenommenen $\mathrm{Maße}$, so wird der voraussichtliche Anstieg der effektiven Belastung deutlich, nämlich um rund ein Drittel, vermindert.

$\mathrm{Zu}$ dieser Verbesserung der zukünftigen Situation trägt die Ausweitung der Erwerbstätigkeit von Frauen der mittleren Altersklassen ca. ein Viertel bei, während die Erhöhung der Erwerbsquoten der Älteren dreiviertel der Minderung des Anstiegs der Effektivlastquote bewirkt. Eine Verschiebung des Renteneintritts ist unter dem Gesichtspunkt der Verbesserung der Finanzierungssituation der gesetzlichen Rentenversicherung prinzipiell die wirkungsvollere Maßnahme, da Personen, die länger erwerbstätig sind, die Zahl der Beitragszahler erhöhen und gleichzeitig die Zahl der Rentenempfänger senken. Eine frühzeitige Ausweitung der Erwerbstätigkeit der Frauen erhöht zudem die Häufigkeit eines späteren Rentenanspruch, so daß sich der Rentnerquotient bzw. die betrachtete Effektivlastquote nach Austritt der Frauen aus der Erwerbstätigkeit verschlechtert.

Aufgrund der in Quotienten ausgedrückten Ergebnisse der Bevölkerungsvorausberechnung und der fortgeschriebenen Effektivlastquote, könnte man vermuten, daß der Proze $\beta$ der demographischen Alterung im Jahr 2040 seinen Höhepunkt überschritten habe und sich beispielsweise bis 2050 die Situation verbessern würde. Betrachtet man allerdings die Entwicklung der Zahl der Personen der verschiedenen Altersgruppen vor 2040 detailierter, so wird deutlich, daß auch zwischen 2030 und 2040 die vorausberechnete Zahl der unter 20 Jahre alten Personen sowie der 20 bis 40 jährigen zurückgegangen ist (-10,5\% sowie $-9 \%)$. Gerade die Personen dieser Altersgruppen können zur gesellschaftlichen Entlastung der Erwerbstätigen bzw. zur Senkung des Durchschnittsalters der Bevölkerung beitragen, da sie den Nachwuchs der Erwerbstätigen darstellen bzw. üblicherweise während dieser Altersphase Kinder bekommen. Da sich die Bevölkerung insgesamt lediglich um $6,5 \%$ verringert, wird sich der gesellschaftliche Alterungsprozeß voraussichtlich auch nach 2040 fortsetzen.

Für ein Andauern des Prozesses der gesellschaftlichen Alterung sprechen außerdem Überlegungen, die einen Rückgang der Sterblichkeit über die Annahmen der Bevölkerungsvorausberechnung hinaus, das heißt nach 2000 in den alten 
Bundesländern und nach 2030 in den neuen Bundesländern, einbeziehen und hiermit eine fortgesetzte und stärkere Alterung der Bevölkerung berechnen (vgl. BOMSDORF, 1994).

\section{Zeitliche Aspekte angesichts der demographischen Entwicklung}

Die Ausschöpfung der Erwerbspersonenpotentiale zur Verbesserung der zukünftigen Situation erfordert neben der Umsetzung der behandelten Maßnahmen zur Erweiterung der Möglichkeiten von Frauen und Älteren erwerbstätig zu sein, die Bereitschaft der Arbeitspersonen zur Aufrechterhaltung und zur Verlängerung ihrer Erwerbstätigkeit. Eine Voraussetzung der Akzeptanz auf Seiten der betroffenen Arbeitspersonen, der Arbeitgeber und des übrigen sozialen Umfeldes ist die rechtzeitige Einübung der Nutzung flexibler Gestaltungselemente. Die frühzeitige Umsetzung der Maßnahmen ermöglicht vielfältige Gewöhnungsprozesse und erhöht langfristig die breite Wirksamkeit der Maßnahmen.

Die rechtzeitige Umsetzung der Maßnahmen begleitend, muß in den einzelnen Unternehmen die Attraktivität der Arbeit erhöht werden, um die Erwerbsmotivation zu fördern. Diesbezügliche Anstrengungen tragen zur Sicherung der arbeitsmarktbezogenen Wettbewerbsfähigkeit der Unternehmen bei.

Außer dem demographischen Wandel und den Änderungen der Produktions- und Organisationsverfahren erfordern auch die gesellschaftlichen Entwicklungslinien, die als Wertewandel bezeichnet werden, Veränderungen der Arbeit. Wertewandel beschreibt die seit der ersten Hälfte der 60er Jahre zu verzeichnende Bedeutungsverschiebung von Pflicht- und Akzeptanzwerten, wie Pflichterfüllung, Fleiß, Gehorsam und Pünktlichkeit, zu Selbstentfaltungswerten, wie Emanzipation, Autonomie, Eigenständigkeit und Partizipation (vgl. beispielsweise KLAGES, 1984, 18 und 1993). Neben den Bereichen Konsum und Freizeit (vgl. SZALLIES/ WISWEDE, 1991) ist die Arbeit durch den Wertewandel betroffen. Die Arbeit dient nicht mehr nur zur Existenzsicherung, sondern zur Selbstverwirklichung und zur Erlangung von Anerkennung, Freude sowie Zufriedenheit. Zur Sicherung des Arbeitskräfteangebots und zur Nutzung der aufgezeigten Potentiale ist entsprechend eine gestalterische Anpassung der Arbeit an die Wertewelt der Arbeitspersonen hilfreich (vgl. beispielsweise bezogen auf die Gruppen junge Akademiker und Führungskräfte VON ROSENSTIEL, 1989, 23; KOLLAR/ 
STENGEL, 1990). Die Arbeitsmotivation und die Bereitschaft im Alter länger zu arbeiten kann dadurch gefördert werden, daß für die Arbeitspersonen ein Gleichgewicht zwischen Arbeit und Freizeit erreichbar wird (vgl. SPIEß u.a., 1992).

Die Befriedigung arbeitsinhaltlicher Interessen, die dezentrale Übertragung von Verantwortung, die Sicherung bzw. Ausweitung von Dispositionsmöglichkeiten sowie die Gewährung von Teilautonomie hinsichtlich der Ausführung der Arbeit bilden Motivatoren einer zukunftsorientierten Arbeitsorganisation (vgl. JÜRGENS, 1993, 21f.). Neben den gezeigten Vorteilen eines ganzheitlichen Aufgabenzuschnitts sowie des Angebots von Möglichkeiten zur Kompetenzsteigerung und zur Flexibilisierung können erweiterte Formen der Mitbestimmung die Motivation der Arbeitsperson und ihre Identifikation mit der Arbeit erhöhen. So sollten beispielsweise bei der Einführung moderner Methoden die Beschäftigten die Neugestaltung des individuellen Arbeits- und Verantwortungsbereichs mitbestimmen können. Durch Formen der Mitarbeiterbeteiligung, kooperative Arbeitsformen, den Aufbau und Erhalt sozialer Kontakte und eine teamorientierte Führung können eine Annäherung der Merkmale des beruflichen und außerberuflichen Bereichs bewirkt und damit die Motivation der Beschäftigten erhöht werden. Die Umsetzung der genannten Punkte, die vielfach den seitens der Gewerkschaften geforderten Gestaltungsgrundsätzen entgegenkommen (vgl. beispielsweise BREDEWEG/ KOWOL, 1991, 425f.), entspricht dem Interesse der Arbeitnehmer und der Arbeitgeber an der Sicherung des vorhandenen Humankapitals.

Die Umsetzung der Maßnahmen sind als Eingriffe in traditionelle Strukturen anzusehen, die frühzeitige Planungs- und Entscheidungsprozesse erfordern. Die Arbeitspersonen müssen sich an die Veränderungen lange bestehender Strukturen und Einstellungen gewöhnen. Die erforderlichen Änderungen zur Vermeidung der zukünftigen Defizite lassen sich entsprechend nur mittel- oder langfristig erreichen (vgl. beispielsweise FÜRSTENBERG, 1987, 68). Zur Sicherung der Akzeptanz und der Motivation der Arbeitspersonen sind frühzeitig Überlegungen unter Abwägung der unterschiedlichen Interessen und der Beteiligung aller Interessengruppen anzustellen.

In der Diskussion muß neben den unterschiedlichen Fristen des Wirksamwerdens der Maßnahmen besonders die voraussichtliche Entwicklung der Arbeitsmarktsituation beachtet werden. So stellt sich die Frage, wie bei herrschender Arbeitslosigkeit Maßnahmen zur Vermeidung bzw. Minderung zukünftiger Arbeitskräfteangebotsdefizite umgesetzt werden können. Die Frage richtet sich auf 
eine Strategie, die es erlaubt, die Beiträge zur Lösung der divergierenden Problemstellungen planerisch zu integrieren. Entsprechend der Ziele der Unternehmen und unter Beachtung der Bedürfnisse der Arbeitspersonen kann eine sinnvolle Folge der Förderung, Gestaltung und Nutzung der Maßnahmen empfohlen werden.

Ohne eine punktgenaue Prognose der Entwicklung zu beabsichtigen, können die demographisch bedingte zukünftige Arbeitsmarktentwicklung und die mit der Maßnahmenumsetzung anvisierten Altersgruppen von Arbeitspersonen grob abgeschätzt werden.

Beabsichtigt beispielsweise ein Unternehmen aufgrund einer sehr langfristigen Personalplanung, nach dem Jahr 2015 verstärkt ältere Erwerbspersonen (in einem Alter bis 65 Jahre) $\mathrm{zu}$ beschäftigen, so ergeben sich hieraus verschiedene Konsequenzen. So kann das Alter der Arbeitspersonen begrenzt werden, auf die die in der Zeit vor 2015 umzusetzenden Maßnahmen wirken müssen, um die zukünftigen Möglichkeiten dieser Kohorte bis zu einem Alter von 65 Jahre erwerbstätig zu sein, zu erhöhen.

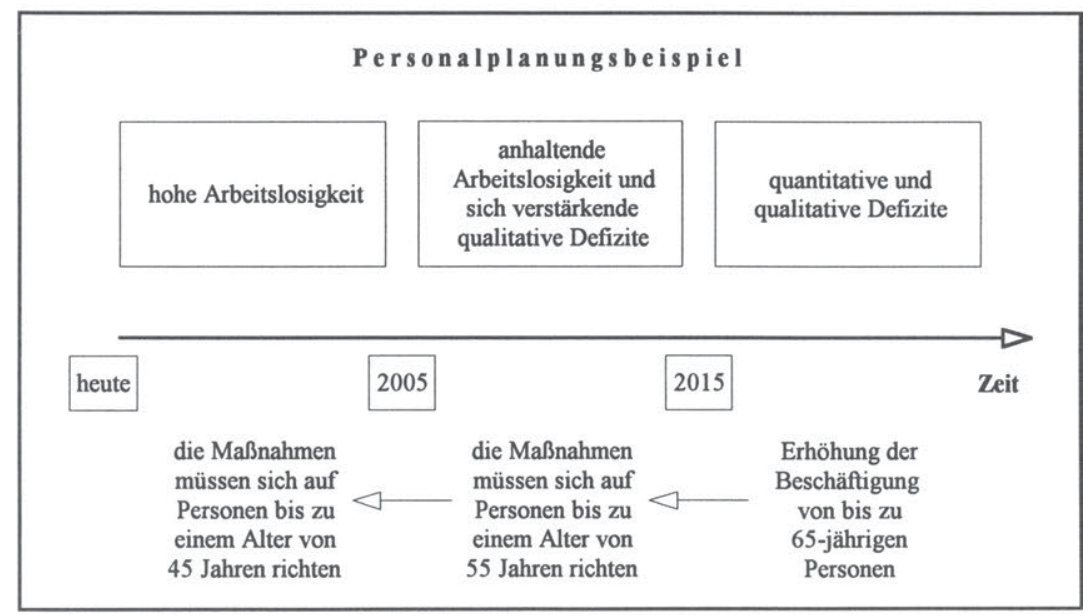

Abb. 82: Möglichkeit einer zeitlichen Systematisierung

Dieses Beispiel illustriert die Notwendigkeit, zur Sicherung des Humankapitals zunächst langfristig wirkende Maßnahmen für jüngere und später kurzfristig wirkende Maßnahmen für ältere Erwerbspersonengruppen umzusetzen. Entsprechend läßt sich eine sinnvolle Strategie der Maßnahmenumsetzung 
vorschlagen. Allerdings bestehen vielfältige Abhängigkeiten zwischen den $M a ß n a h m e n$, so daß nur eine grobe Schrittfolge angegeben werden kann. In einem speziellen Unternehmen müssen ergänzend beispielsweise die Situation auf dem für das Unternehmen relevanten Arbeitsmarkt, die arbeitsmarktbezogene Wettbewerbsposition, der gegenwärtige und zukünftige Personalbedarf sowie die gegebene und zukünftige Altersstruktur der Belegschaft beachtet werden.

- Zunächst können Maßnahmen zur Förderung der Erwerbstätigkeit von Frauen qualitative Defizite des Arbeitskräfteangebots ausgleichen und langfristig auch quantitativ zur Erhöhung der Beschäftigung Älterer wirken. Unterbrechungsmöglichkeiten sollten kombiniert mit Maßnahmen der Kontaktpflege, die zum Erhalt betriebs- und berufsspezifischer Kenntnisse sowie Einstellungen während einer Unterbrechungsphase beitragen, gewährt werden. Hierdurch können Dequalifizierungsprozesse und ein endgültiger Ausstieg aus der Erwerbstätigkeit vermieden werden. Maßnahmen wie Teilzeitangebote, Job-sharing und Heimarbeit können mit der Zeit in wachsendem Maße Unterbrechungsphasen verkürzen oder vermeiden, um die Zahl der zur Verfügung stehenden Arbeitskräfte zu erhöhen.

- Weiterhin müssen prospektiv wirkende Maßnahmen zum Aufbau des Erfahrungswissens und zur Nutzung der Leistungspotentiale der Arbeitspersonen sowie präventiv wirkende Maßnahmen des Arbeitsschutzes und der Arbeitsplatzgestaltung zur Vermeidung von Fehlbeanspruchungen umgesetzt werden. Falls die Umsetzung dieser Maßnahmen auf verschiedene Altersgruppen unterschiedlich ausgerichtet werden soll und kann, z.B. durch eine unterschiedliche Beteiligung an der Gruppenarbeit, sollten sie sich zunächst auf jüngere Altersgruppen konzentrieren und sich mit der Zeit über das Ausscheiden der Älteren auf die gesamte Belegschaft ausdehnen. Die Maßnahmen können langfristig zum Ausgleich quantitativer und qualitativer Defizite des Arbeitskräfteangebots beitragen.

- Später können für Arbeitspersonen, bei denen sich beispielsweise aufgrund des technisch-organisatorischen Wandels Qualifikationsnachteile abzeichnen, zusätzliche betriebliche Ausbildungsphasen angeboten werden. So kann insbesondere die Aktualität der Qualifikation von älteren Beschäftigten erhalten werden. 
- Schließlich müssen zum Ausgleich vorhandener Defizite kurzfristig wirksame Maßnahmen ergriffen werden. So können Teilzeitarbeitsplätze für Ältere und korrektive Maßnahmen wie Schonarbeitsplätze zur Vermeidung von Frühverrentungen bzw. zur Verlängerung der Lebensarbeitszeit mit späterem Rentenbezug beitragen. Allerdings erfordert die Umkehr des Trends zu einem früheren Berufsaustritt Gewöhnungsprozesse, so $\mathrm{da} \beta$ mit der Umsetzung dieser Maßnahmen nicht zu spät begonnen werden darf, um eine ausreichende Wirkung zu erzielen.

- Kontinuierliche Weiterbildungsmaßnahmen während des gesamten Erwerbslebens sind für alle Arbeitspersonen anzustreben. Sie können sich zunächst besonders auf Frauen (und gegebenenfalls auch Männer) während und nach einer Unterbrechungsphase konzentrieren und sich dann immer stärker auch auf ältere Arbeitspersonengruppen ausdehnen.

Entsprechend dieser Strategie der Maßnahmenumsetzung kann erwartet werden, daß sich zunächst die Beschäftigungschancen jüngerer Frauen verbessern werden. Die Chancen älterer Arbeitspersonen, die aufgrund ihrer niedrigen Qualifikation nicht zum Ausgleich qualitativer Defizite benötigt werden, bleiben auf mittlere Sicht durchschnittlich unverändert. Die Heraufsetzung der Altersgrenzen birgt somit zumindest mittelfristig die Gefahr einer Erhöhung der Arbeitslosigkeit älterer Personen, die arbeitsmarktbezogene Nachteile aufweisen (vgl. BÄCKER/ NAEGELE, 1993b, 236f.). Erst für Arbeitspersonen, die heute in der ersten Hälfte ihres Arbeitslebens stehen, werden die Möglichkeiten im Alter einer Erwerbstätigkeit nachgehen zu können, durchschnittlich erkennbar besser sein. Die Umsetzung der Maßnahmen, die sich auf eine Minderung von Fehlbeanspruchungen und eine Vermeidung von Dequalifikationsprozessen beziehen, schaffen die Voraussetzungen für die Verlängerung der Berufsausübung. Die Maßnahmen beziehen sich auf alle Erwerbstätigen und können im Falle ihrer verbreiteten Umsetzung in beschriebener Weise zur Veränderung der Arbeit beitragen.

Die folgende Abbildung stellt eine voraussichtliche Abfolge der Maßnahmennutzung dar, nennt die Stufen der Auswirkungen der Maßnahmenumsetzung und skizziert die Entwicklung der hierdurch vermeidbaren Defizite. 


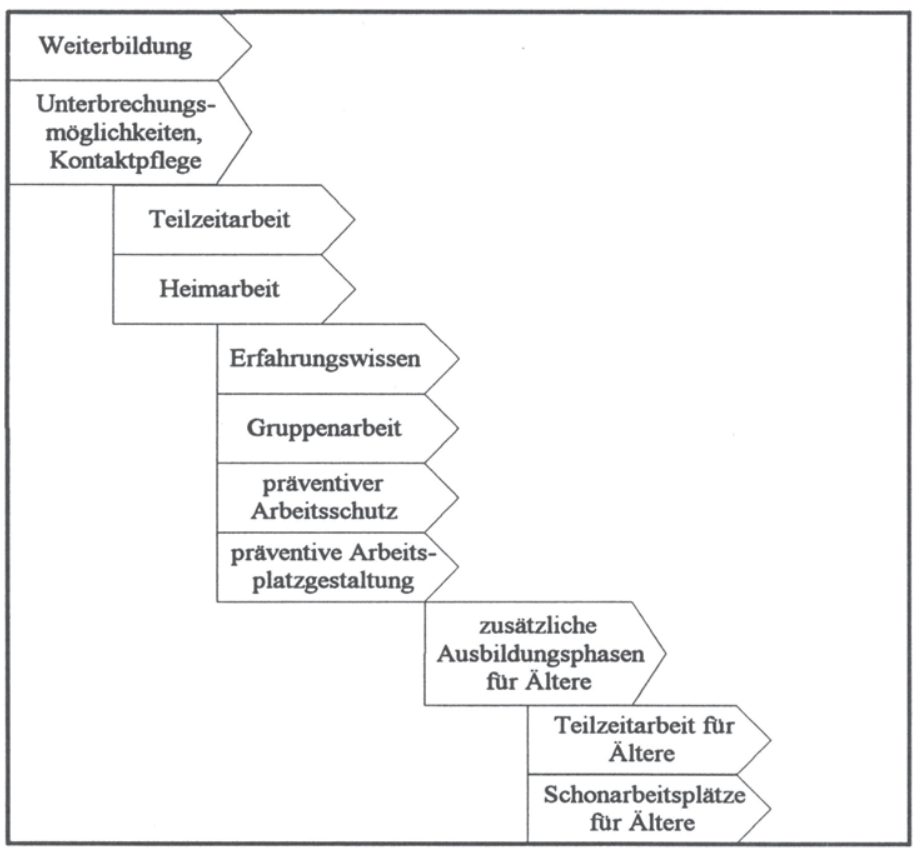

Wirkungsstufen der Maßnahmenumsetzung:

Erhalt der Erhöhung der Erweiterung der Erhöhung der Qualifikation Erwerbsquoten Qualifikationen Erwerbsquoten

Entlastung des der Frauen der Älteren

Arbeitsmarktes Abbau von Fehlbeanspruchungen

Maßnahmenwirkung im zeitlichen Verlauf:

Vermeidung sich vergrößernder Defizite qualitativer und quantitativer Art

Abb. 83: Zeitliche Einordnung der Maßnahmenumsetzung und ihrer Wirkungen 


\section{Schlußbetrachtung}

Der Arbeitsmarkt sowie das Bildungs- und Ausbildungssystem sind zukünftig, besonders angesichts der demographischen Entwicklung mit ihren Auswirkungen auf die Zusammensetzung des Arbeitskräfteangebots und der raschen qualitativen Veränderung der Arbeitskräftenachfrage, immer weniger dazu in der Lage, ein gut qualifiziertes und leistungsfahiges Arbeitskräfteangebot in ausreichendem Maße sicherzustellen, um die sich abzeichnenden Arbeitsaufgaben durchzuführen.

Zum Ausgleich der in Kapitel I dargestellten zukünftigen arbeitsmarktbezogenen Anpassungsdefizite, zur Sicherung des zur Verfügung stehenden Humankapitals und zur Verringerung seiner Vergeudung wird eine veränderte Gestaltung der Arbeit immer notwendiger. So müssen die Unternehmen dazu beitragen, eine höhere Erwerbsbeteiligung von Frauen und Älteren zu ermöglichen. Für die Verbesserung der gegenseitigen Anpassung von Arbeit und Arbeitspersonen ist die breite Nutzung der in vorliegender Arbeit betrachteten unternehmensbezogenen Maßnahmen zu empfehlen.

Begegnen die Handlungsträger des Arbeitsmarktes den voraussichtlichen Problemlagen rechtzeitig mit konstruktiv wirkenden Maßnahmebündeln, so dürfte dies eine Umsetzung von bereits in anderen Zusammenhängen lange diskutierten, aber relativ selten verwirklichten Ansätzen bedeuten. Die bisherigen Umsetzungsdefizite von Maßnahmen zur 'Humanisierung des Arbeitslebens', zur 'Flexibilisierung der Altersgrenzen' oder zur 'Förderung der Chancengleichheit', die auch bei der Erörterung der Eigenschaften und arbeitsmarktbezogenen Wettbewerbsnachteile von Frauen und Älteren deutlich geworden sind, können zukünftig vermindert werden, indem entsprechende Maßnahmen zur Sicherung des Humankapitals zunehmend Bestandteile umfassender betrieblicher Veränderungsprozesse werden. Zumal die diskutierten Strategien zur Verbesserung der Wettbewerbsfähigkeit der Unternehmen einerseits und zur Humanisierung der Arbeit - im Sinne der Erhöhung der Entwicklungsförderlichkeit und der Minderung arbeitsbedingter Schädigungen - andererseits, in geringerem Maße als früher ideologisch oder gesellschaftspolitisch geprägt sind.

Angesichts dieser möglichen Integration vielfältiger Konzepte und Ideen kann der demographische und organisatorisch-technische Wandel als Chance zur gesellschaftlichen Entwicklung betrachtet werden. Allerdings kann es ohne Änderungen der makro- und mikroökonomischen Handlungs- und Gestaltungsweisen zu einer 
Beeinträchtigung der wirtschaftlichen Entwicklung kommen und vorhandene Benachteiligungen können, beispielsweise infolge der gesetzlichen Erhöhung des Renteneintrittsalters ohne entsprechende Verbesserungen der Erwerbschancen Älterer, zusätzlich verschärft werden.

Die in Kapitel II abgeleiteten arbeitsgestalterischen und personalentwicklungsbezogenen Maßnahmen mindern zukünftige Personalengpässe und sichern Humankapitalpotentiale, auf die die Unternehmen zur wirtschaftlichen Entwicklung sowie zur Einführung und Nutzung moderner Produktions- und Organisationsmethoden zukünftig immer weniger verzichten können. Die Auswirkungen der Umsetzung der Maßnahmen wurden in Kapitel III erwerbstätigkeitsbezogen betrachtet und beurteilt.

Die Maßnahmen müssen zu integrierten Teilen der strategischen Unternehmensplanung und -ausrichtung werden und bewegen sich im Spannungsfeld der Ziele einer optimierten Techniknutzung, Organisationsgestaltung und Mitarbeiterentwicklung. Die erfolgreiche Anpassung und Umsetzung der Maßnahmen in den Unternehmen erfordern eine langfristige Planung der Arbeitsgestaltung und der Personalentwicklung und die vielfältige Berücksichtigung mikro- und makroökonomischer Einflußfaktoren. Neben den Eigenschaften und Bedürfnissen der Arbeitspersonen und den Zielen und Handlungsweisen der Unternehmen müssen auch gesellschaftlich vorherrschende Einstellungen und Erwartungen beachtet werden. Entsprechend muß in den Unternehmen ein hohes $\mathrm{Ma} \beta$ an Motivation und Kooperation aufgewendet werden, damit die Maßnahmen, die zu einer Flexibilisierung und Individualisierung der Arbeitsleistung beitragen, effizient umgesetzt werden können. Aus Sicht der Unternehmen werden im Interesse einer möglichst hohen einzelwirtschaftlichen Flexibilität der Maßnahmengestaltung individuellvertragliche Lösungen bevorzugt werden. Andererseits können entsprechend variabel gestaltete kollektive Vereinbarungen vereinfachend wirken und eine breite rechtzeitige Maßnahmenumsetzung fördern.

Noch einmal soll festgehalten werden, wie unterschiedlich sich die Arbeitsmarktsituation einerseits kurz- bis mittelfristig und andererseits langfristig darstellt und wie unterschiedlich, abhängig von der zeitlichen Perspektive, rationale einzelwirtschaftliche Entscheidungen seitens der Unternehmen ausfallen. Während langfristig aufgrund der demographischen Entwicklung Nachwuchsmangel auftreten wird, muß zumindest mittelfristig noch mit hoher Arbeitslosigkeit gerechnet werden. Entsprechend muß zunächst die Arbeitslosigkeit mit flexiblen und 
reversiblen Maßnahmen bekämpft werden. Gleichzeitig müssen die quantitativen und qualitativen Voraussetzungen für die Verminderung zukünftiger Arbeitskräfteangebotsdefizite geschaffen werden. Die Maßnahmen zur Vermeidung von Dequalifikationsprozessen und arbeitsbedingten Krankheiten und zur Förderung der Motivation zur Erwerbstätigkeit sind hierfür zu nutzen. Für eine langfristig höhere Ausschöpfung des Erwerbspersonenpotentials müssen die Unternehmen allerdings auch die übrigen betrachteten Maßnahmen zur Erhöhung der Erwerbsbeteiligung von Frauen und Älteren rechtzeitig ergreifen, denn falls sie damit zu lange zögern, gefährden sie ihre Wettbewerbsfähigkeit und vermindern die Wirkkraft der Maßnahmen. Da im internationalen Vergleich die Maßnahmen zur Lösung der betrachteten demographisch bedingten Probleme in Deutschland früh entwickelt und umgesetzt werden müssen, kann nur begrenzt auf Erfahrungen zurückgegriffen werden. Daher sind Fehlentwicklungen in höherem Maße zu erwarten als in anderen Industrieländern, in denen diese Probleme später auftreten werden. Demgegenüber können die Erfahrungsprozesse hinsichtlich der Entwicklung und Nutzung der Maßnahmen zur gegenseitigen Anpassung von Arbeit und Arbeitspersonen die Wettbewerbsfähigkeit der inländischen Unternehmen langfristig stärken. Hierfür sprechen die Überlegungen $\mathrm{zu}$ mitarbeiterorientierten Organisations- und Produktionsmethoden sowie das Verständnis, daß Rationalisierung und Humanisierung einander ergänzende Partner sind.

Die Erwerbsbeteiligung der Frauen dürfte sich zukünftig weiter erhöhen. Jedoch müssen mit dem Anstieg der Erwerbsquoten in zunehmendem Maße unterstützende und fördernde Maßnahmen umgesetzt werden. Die quantitative und qualitative Ausweitung der Teilzeitarbeitsangebote und der Möglichkeiten zum Job-sharing können ebenso wie die verstärkte Nutzung von Heimarbeit zu einer Verbesserung der Vereinbarkeit von Familie und Beruf beitragen. Dabei ist allerdings die angesprochene Verstetigung der Mehrfachbelastung der Frauen durch Haushalt, Familie und Beruf zu beachten. Zur Erleichterung des Wiedereinstiegs nach einer beruflichen Unterbrechungsphase sind insbesondere Maßnahmen zur Kontaktpflege durch Arbeitsvertretungen und durch die Teilnahme an Weiterbildungsmaßnahmen während der Erwerbspause zu nutzen. Eine Verbesserung der Weiterbildungsbeteiligung der Frauen und die flexible Regelung von Unterbrechungsmöglichkeiten in Kombination mit Kontaktpflegemaßnahmen können die beruflichen Chancen der Frauen verbessern.

Die Umsetzung der erörterten Maßnahmen wird eine Erhöhung und qualitative Verbesserung der Erwerbsbeteiligung der Frauen bewirken, so daß damit indirekt 
auch die geschlechtsspezifische Arbeitsteilung beeinflußt wird. Andere u.U. direktere Beiträge zur Aufhebung der geschlechtsspezifischen Arbeitsteilung sind allerdings von unternehmensbezogenen Maßnahmen in nennenswerter Höhe nicht zu erwarten. So liegen beispielsweise die Verminderung der beruflichen Belastung oder die Erhöhung der Teilzeitarbeit der (Ehe-) Männer zugunsten einer möglichen Entlastung der Frauen bei der Durchführung von erziehungs- und pflegebezogenen Aufgaben, ebensowenig im Interesse der Unternehmen, wie die geschlechtsindifferente Nutzung von Unterbrechungsmöglichkeiten. Denn aus Sicht eines Unternehmens würden hierdurch personalbezogene Planungsunsicherheiten auf die Beschäftigung männlicher Arbeitspersonen ausgedehnt und damit insgesamt erhöht werden. Außerdem dürfte der mögliche Nutzen in Form einer überkompensierenden Ausweitung des Arbeitsangebots der (Ehe-) Frauen in der Regel nicht direkt dem Unternehmen zugute kommen, dessen männliche Arbeitnehmer in geringerem Maße zu Verfügung stehen.

Allerdings wird auch die weitere Erhöhung der Erwerbsbeteiligung von Frauen mittlerer Altersklassen die voraussichtlichen Defizite nicht vollständig ausgleichen können. Aufgrund der demographischen Veränderung der Altersstruktur müssen langfristig in den Unternehmen häufiger ältere Frauen und Männer beschäftigt werden. Durch die Umsetzung moderner Produktions- und Organisationsmethoden verstärken sich jedoch gerade die arbeitsmarktbezogenen Wettbewerbsnachteile dieser Personengruppe, so daß die entwickelten unternehmensbezogenen Maßnahmen zur Ausweitung der Erwerbstätigkeit Älterer an Bedeutung gewinnen werden. Kontinuierliche berufsbegleitende Lernprozesse und die Ausweitung der Weiterbildungsbeteiligung älterer Arbeitspersonen tragen zur Minderung der Aktualitätsverluste ihrer Qualifikation und damit zur Verbesserung der Beschäftigungschancen Älterer bei. Altersübergreifende, beanspruchungsmindernde, arbeitsorganisatorische Gestaltungsmaßnahmen können ebenso wie erweiterte Formen eines individuellen und präventiv wirksam werdenden Arbeitsschutzes Abbauprozesse mindern und Kompetenzen stärken, so daß eine Verringerung von Frühverrentungen und eine Verbesserung der Eigenschaften älterer Arbeitspersonen erreicht werden können. Korrektive Maßnahmen der Arbeitsplatzgestaltung wie spezielle Arbeitshilfen und Schonarbeitsplätze können zudem die Beschäftigungsmöglichkeiten leistungsgewandelter Arbeitspersonen erhöhen.

Trotz der gegenwärtig bestehenden Schwierigkeiten Älterer, eine Erwerbstätigkeit fortzuführen bzw. wiederaufzunehmen, darf bei der Gestaltung unternehmensbezogener Maßnahmen nicht vergessen werden, daß besonders zur Erhöhung der 
Erwerbsbeteiligung älterer Personen nicht nur die Beseitigung bzw. Minderung vorhandener arbeitsmarktbezogener Wettbewerbsnachteile bedeutsam ist, sondern daß die Motivation zu einer Verlängerung der Erwerbstätigkeit gestärkt werden muß. Zumal die Vermögenssituation zukünftig Älterer dazu führen kann, daß ein zunehmender Teil dieser Personengruppe aus wirtschaftlichen Gründen, trotz der gesetzlich angestrebten Erhöhung des Renteneintrittsalters, kein Interesse an der Verlängerung der Lebensarbeitszeit haben wird. Daher müssen die Unternehmen die Attraktivität der Arbeitsaufgaben und damit der Berufstätigkeit fördern. Eine Erhöhung des durchschnittlichen Renteneintrittsalters ist mithin von interdependenten Präferenzen und $Z$ wängen abhängig. Mit prospektiver Wirkungsweise sind zur Erhöhung der Attraktivität und zur Ausweitung der Möglichkeiten älterer Arbeitspersonen, erwerbstätig zu sein neben den Maßnahmen der Personalentwicklung besonders solche arbeitsorganisatorische Maßnahmen zu nutzen, die zur Entwicklungsförderlichkeit der Arbeit beitragen. So unterstützt die altersübergreifende Förderung der Entwicklung und der Anwendungsmöglichkeiten von Erfahrungswissen einen spezifischen Kompetenzvorteil Älterer und erleichtert die Integration altersverschiedener Beschäftigtengruppen.

Darüber hinaus konnte gezeigt werden, daß eine entwicklungsförderliche Arbeitsgestaltung die personalbezogenen Möglichkeiten zum Abbau tayloristisch gestalteter Arbeitsaufgaben erweitert und damit die Einführung moderner Produktions- und Organisationsmethoden sowie die Nutzung ihrer Vorteile erleichtert. Die einzelwirtschaftliche Umsetzung der behandelten Maßnahmen wird jedoch die Beschäftigungschancen Älterer erst langfristig erhöhen, da die meisten Maßnahmen zur Erhöhung der Erwerbsbeteiligung Älterer eine lange Vorlaufzeit bis zur Entfaltung ihrer Wirkung besitzen. Außerdem dürfte sich die tendenzielle Jugendzentriertheit der Personalpolitik angesichts der andauernden Arbeitslosigkeit nur langsam ändern. Bevor es zu einer deutlichen Erhöhung der Erwerbsquoten Älterer kommen wird, werden die Unternehmen versuchen, das Frauenerwerbspotential auszuschöpfen.

Die folgende Abbildung gibt einen Überblick darüber, wie die Auswirkungen einer Umsetzung der dargestellten Maßnahmen auf die Erreichung wesentlicher Unternehmensziele eingeschätzt werden können. Unterschieden werden eher positiv $(+)$ oder negativ (-) und nicht eindeutig (+/-) einzuschätzende Beeinflussungsweisen. 


\begin{tabular}{|c|c|c|c|c|c|}
\hline $\begin{array}{r}\text { Ziele } \\
\text { Maßnahmen }\end{array}$ & $\begin{array}{c}\text { Senkung } \\
\text { der } \\
\text { Kosten }\end{array}$ & $\begin{array}{c}\text { Verbesserung } \\
\text { der } \\
\text { Qualität }\end{array}$ & $\begin{array}{c}\begin{array}{c}\text { Erhöhung } \\
\text { der }\end{array} \\
\text { Flexibilität }\end{array}$ & $\begin{array}{c}\text { Reduzierung } \\
\text { des Zeit- } \\
\text { aufwandes }\end{array}$ & $\begin{array}{c}\text { Sicherung } \\
\text { des Human- } \\
\text { kapitals }\end{array}$ \\
\hline $\begin{array}{l}\text { Erfahrungs- } \\
\text { wissen }\end{array}$ & - & + & + & + & + \\
\hline $\begin{array}{l}\text { Gruppen- } \\
\text { arbeit }\end{array}$ & - & + & + & - & + \\
\hline Heimarbeit & $+/-$ & - & + & - & + \\
\hline $\begin{array}{l}\text { Arbeitszeit- } \\
\text { konzepte }\end{array}$ & - & + & + & - & + \\
\hline Arbeitsschutz & - & $+/-$ & - & - & + \\
\hline Arbeitshilfen & - & + & + & + & + \\
\hline $\begin{array}{l}\text { Weiter- } \\
\text { bildung }\end{array}$ & - & $\begin{array}{l}1 \\
+\end{array}$ & + & $+/-$ & + \\
\hline $\begin{array}{l}\text { Frauen- } \\
\text { förderung }\end{array}$ & - & $+/-$ & - & - & + \\
\hline
\end{tabular}

Abb. 84: Zusammenfassende Einschätzung der Beeinflussung wesentlicher Unternehmensziele durch die Umsetzung der Arbeitsgestaltungs- und Personalentwicklungsmaßnahmen

Die zunehmende Differenzierung, Pluralisierung, Segmentierung und Flexibilisierung in vielen Bereichen der Gesellschaft führen dazu, daß die 'Normalbiographie' und damit auch das Normalarbeitsverhältnis an Bedeutung verliert und sich die Wahlmöglichkeiten hinsichtlich der Lebensweise und des Lebensverlaufs, auch durch die Erhöhung des durchschnittlichen Bildungsniveaus, tendenziell erweitern. Diese Entwicklung wird zusätzlich durch die Umsetzung der Maßnahmen, die zur inter- und intrapersonellen Flexibilisierung der Arbeitsverhältnisse beitragen, verstärkt. Zukünftig müssen sich die Handlungsträger in den Unternehmen bemühen, die Tendenz zur Auflösung der Normalarbeitsverhältnisse noch stärker zur Erreichung der Unternehmensziele zu nutzen, indem flexibilisierende Regelungen vereinbart werden, wie es bereits in Ansätzen gerade bezüglich der Arbeitszeit geschieht. Dabei muß die Flexibilisierung und Destandardisierung auch die individuellen Interessen der Beschäftigten berücksichtigen und verschiedene Entscheidungsmöglichkeiten eröffnen. Für eine wirkungsvolle dezentrale Gestaltung in den Unternehmen sollte weiterhin die betriebliche Interessenvertretung der Beschäftigten einbezogen werden. 
Durch eine Minderung der Konflikte zwischen beruflichen und außerberuflichen Tätigkeiten und Verpflichtungen muß die Kompatibilität dieser beiden Dimensionen verbessert werden. So können beispielsweise durch Maßnahmen einer differentiellen Arbeitsgestaltung Arbeitsanforderungen und individuelle Eigenschaften einander angepaßt werden. Weiterhin kann durch eine Erhöhung der Beteiligung der Beschäftigten an Entscheidungen und/oder am Kapital der Unternehmen die Bereitschaft zur Weiterbildung, zur Übernahme von Verantwortung und zur Unterstützung notwendiger Innovationsprozesse gefördert werden. Eine stärkere Partizipation der Arbeitspersonen erscheint gerade angesichts der Ausrichtung moderner Produktions- und Organisationsmethoden mikroökonomisch sinnvoll. Sie fördert die Arbeitsmotivation und die Bereitschaft zur Identifikation mit den betrieblichen Interessen und entspricht zudem der Tendenz zur Höherqualifizierung. Die Förderung der Mitbestimmung und Mitgestaltung der Arbeitspersonen muß durch eine entsprechende Anerkennung ihrer Leistungen und beispielsweise durch Vereinbarungen zur Gewährleistung größtmöglicher Arbeitsplatzsicherheit ergänzt werden.

Aufgrund der Analyse der gegenwärtigen und der voraussichtlichen zukünftigen Erwerbssituationen der Arbeitspersonen und ihrer Eigenschaften, der Arbeitsaufgaben und ihrer Anforderungen sowie der Arbeitssysteme und der sich ergebenden Beanspruchungen wurden in der vorliegenden Arbeit in typisierender Weise arbeitsgestalterische und personalentwicklungsbezogene Maßnahmen abgeleitet und beurteilt. Es konnte verdeutlicht werden, daß eine Integration vieler Maßnahmen in moderne Produktions- und Organisationsmethoden möglich und auch ökonomisch sinnvoll ist. Die Maßnahmen sollten in den Unternehmen Umsetzung finden, um mikroökonomisch und arbeitswissenschaftlich betrachtet das Humankapital zu sichern und die menschliche Leistungsabgabe im Arbeitsproze $B$ im langfristigen Interesse der Arbeitgeber und der Arbeitnehmer zu optimieren und, um makroökonomisch betrachtet, Humankapitalvergeudungen $\mathrm{zu}$ mindern und das zukünftige Erwerbspersonenpotential verbessert ausschöpfen zu können. Die notwendige unternehmensspezifische Anpassung der Maßnahmen erfordert die Beachtung der jeweiligen technischen, organisatorischen und personellen Bedingungen sowie die ergänzende Analyse konkreter Arbeitssysteme. 
Stefan Lorenz - 978-3-631-75027-8

Downloaded from PubFactory at 01/11/2019 09:06:41AM

via free access 


\section{Literaturverzeichnis}

Adami, Norbert (1994), Die Gruppe und der Einzelne - zu einigen Grundstrukturen der japanischen Gesellschaft, in: ESSER/KOBAYASHI, 1994, S. 50-60

Altmann, Norbert / Düll, Klaus (1987), Rationalisierung und neue Verhandlungsprobleme im Betrieb, in: WSI Mitteilungen 5/1987, S. 261-269

Amann, Anton (1993), Der Übergang von der Erwerbstătigkeit in den Ruhestand, in: OTTO, 1993, S. $69-83$

Băcker, Gerhard (1993), Im Übergang vom Erwerbsleben in den Ruhestand, in: Aus Politik und Zeitgeschichte, Beilage zur Wochenzeitung Das Parlament, B 44/93, S. 16-26

Băcker, Gerhard / Naegele, Gerhard (1993a), Geht die Entberuflichung des Alters zu Ende? Perspektiven einer Neuorganisation der Alterserwerbsarbeit, in: NAEGELE/TEWS, 1993, S. 135-157

Băcker, Gerhard / Naegele, Gerhard (1993b), Erwerbsarbeit und Ruhestand in einer alternden Gesellschaft, Demographischer Wandel, Arbeitsmarktentwicklung und Arbeitszeitpolitik für Ältere, in: SEIFERT, 1993, S. 218-248

Băcker, Gerhard / Naegele, Gerhard (1993c), Alternde Gesellschaft und Erwerbsarbeit im Alter. Anforderungen an Beschätigungssicherung und -forderung, in: KLOSE, 1993, S. 95120

Bäcker, Gerhard / Stolz-Willig, Brigitte (1995), Mehr Teilzeitarbeit - aber wie? Zur Diskussion über Förderung und soziale Absicherung optionaler Arbeitszeiten, in: Sozialer Forschritt, 3/95, S. 54-64

Baethge, Martin (1992), Die vielfaltigen Widersprüche der beruflichen Weiterbildung, in: WSI Mitteilungen, 6/1992, S. 313-321

Baethge, Martin / Oberbeck, Herbert (1986), Zukunft der Angestellten, Neue Technologien und berufliche Perspektiven in Büro und Verwaltung, Campus Verlag, Frankfurt/Main, New York

Baltes, Paul B. / Baltes, Margret M. (1992), Gerontologie: Begriff, Herausforderung und Brennpunkte, in: BALTES/MITTELSTRAB, 1992, S. 1-34

Baltes, Paul B. / MittelstraB, Jürgen (Hrsg.) (1992), Zukunft des Alterns und gesellschaftliche Entwicklung, Akademie der Wissenschaften zu Berlin, Forschungsbericht 5, Walter de Gruyter, Berlin, New York

Barth, Siegrun / Hain, Winfried / Müller, Horst-Wolf (1994), Langfristige demographische Bestimmungsfaktoren der Rentenfinanzen - Eine Vorausrechnung mit dem neuen Rentenmodell 1993, in: Deutsche Rentenversicherung 3-4/94, S. 228-258

Baus, Magdalena (1991), Emanzipation, in: LISSNER u.a., 1991, Spalten 212-221

Bausch-Weirauch, Helgard (1992), Frauen- und Familienförderung in einem Großunternehmen - Ein Erfahrungsbericht, in: SCHWARZ, 1992, S. 247-255 
BDA (Bundesvereinigung der Deutschen Arbeitgeberverb̆̊nde) (Hrsg.) (1994), Modernes Arbeitszeitmanagement - Mit mehr Teilzeitarbeit mehr Flexibilităt schaffen, Köln

Bechtle, Günter / Lutz, Burkart (1989), Die Unbestimmtheit post-tayloristischer Rationalisierungsstrategie und die ungewisse Zukunft industrieller Arbeit - Überlegungen zur Begründung eines Forschungsprogramms, in: DÜLL/LUTZ, 1989, S. 9-91

Beckmann, Petra / Engelbrech, Gerhard (Hrsg.) (1994), Arbeitsmarkt für Frauen 2000 - Ein Schritt vor oder ein Schritt zurück? Kompendium zur Erwerbstătigkeit der Frauen, Beiträge zur Arbeitsmarkt- und Berufsforschung 179, IAB, Nürnberg

Bender, Christiane / Grabl, Hans (1992), Erfahrung und Kompetenz - die Grenzen der Technisierung der Arbeitsplătze in der industriellen Fertigung, in: Soziale Welt, Jg. 43, Heft 3, S. 301-312

Benz-Overhage, Karin / Brumlop, Eva / Freyberg, Thomas von / Papadimitriou, Zissis (1983), Computergestützte Produktion: Fallstudien in ausgewăhlten Industriebetrieben, (Schriftenreihe Humanisierung des Arbeitslebens; Bd. 43), Campus Verlag, Frankfurt/Main, New York

Berufsbildungsbericht (1994), Bundesministerium für Bildung und Wissenschaft, Bock Verlag, Bad Honnef, Grundlagen und Perspektiven für Bildung und Wissenschaft; 40

Bieneck, Hans-Jürgen / Rückert, Anette (1994), Neue Herausforderungen für die Arbeitswissenschaft - Konsequenzen aus den EG-Richtlinien, in: Zeitschrift für Arbeitswissenschaft 48 (20 NF) 1994/1, S. 1-4

Birg, Herwig (1989), Die demographische Zeitenwende, in: Spektrum der Wissenschaft, Januar, S. $40-49$

Bokranz, Rainer / Landau, Kurt (1991), Einführung in die Arbeitswissenschaft, Analyse und Gestaltung von Arbeitssystemen, Ulmer, Stuttgart

Bolle, Michael / Schneider, Ellen-Ruth in Zusammenarbeit mit Angela Ehrmann (1988), Neue Technologien und neue Qualifikationsanforderungen für Frauen, Beitrăge zur Sozialokonomik der Arbeit, Bd. 14, Minerva-Publikation, München

Bomsdorf, Eckart (1994), Alternative Modellrechnungen der ălteren Bevolkerung Deutschlands bis zum Jahr 2050, Josef Eul Verlag, Bergisch Gladbach, Koln

Boos-Nũnning, Ursula (1990), Einwanderung ohne Einwanderungsentscheidung: Auslăndische Familien in der Bundesrepublik Deutschland, in: Aus Politik und Zeitgeschichte, Beilage zur Wochenzeitung Das Parlament, B 23-24/90, S. 16-25

Born, Claudia (1994), Plădoyer für einen Perspektivenwechsel in der Betrachtung der Frauenarbeit, in: BECKMANN/ENGELBRECH, 1994, S. 209-228

Borneff, Joachim (Hrsg.) (1973), Arbeitsmedizin in Vorlesungen, F.K. Schattauer Verlag, Stuttgart, New York

Brandenburg, U. (1987), Auswirkungen neuer Technologien auf die Gesundheit der Arbeitnehmer, in: ErgoMed, 11. Jahrgang (1987) 6, S. 146-156 
Brandenburg, U. / Grimm, H.-G. / Marschall, B. (1989), Ausländische Arbeitnehmer, in: KONIETZKO/DUPUIS, 1989, Abschnitt III-6.4

Brandes, Wolfgang / Buttler, Friedrich (1987), Alte und neue Heimarbeit, Eine arbeitsökonomische Interpretation, in: Soziale Welt, Jg. 38, Heft 1, S. 74-91

Bredeweg, Udo / Kowol, Uli (1991), Systemische Rationalisierung und Technikgestaltung, in: WSI Mitteilungen 7/1991, S. 419-427

Brődner, Peter (1985), Fabrik 2000: Alternative Entwicklungspfade in die Zukunft der Fabrik (Wissenschaftszentrum Berlin, Internationales Institut für Vergleichende Gesellschaftsforschung, Arbeitspolitik), Ed. Sigma Bohn, 3. Aufl. 1986

Bucher, Hansjörg (1994), Die răumliche Dimension der Alterung, Bundesforschungsanstalt für Landeskunde und Raumordnung, Enquete-Kommission Demographischer Wandel, Kommissionsdrucksache 262 vom 02.05.1994

Bucher, Hansjörg / Kocks, Martina (1991), Aus- und Übersiedler und alternde Bevőlkerung, Wird die "ergraute Gesellschaft" nicht kommen? Informationen zur Raumentwicklung, Heft $3 / 4$, S. $111-122$

Bullinger, Hans-Jörg (Band-Hrsg.) (1992), Personalentwicklung und Qualifikation, SpringerVerlag, Berlin, Heidelberg, New York, Verlag TÜV Rheinland, Köln (CIM-Fachmann)

Bullinger, Hans-Jőrg / Volkholz, Volker / Betzl, Konrad / Köchling, Annegret / Risch, Wolfram (Hrsg.) (1993), Alter und Erwerbsarbeit der Zukunft, Arbeit und Technik bei verănderten Alters- und Belegschaftsstrukturen, KongreB vom 3. und 4. November 1992 in Berlin, Springer-Verlag, Berlin, Heidelberg, New York

Busch, Carola / Koch, Christiane (1990), Frauen in der betrieblichen Weiterbildung, in: SCHLAFFKE/WEIB, S. 287-310

Buslei, Hermann (1995), Vergleich langfristiger Bevölkerungsvorausberechnungen für Deutschland, Dokumentation Nr 95-01, ZEW Zentrum für Europăische Wirtschaftsforschung GmbH, Mannheim

Buttler, Günter (1993), Deutschlands Wirtschaft braucht die Einwanderer, in: KLOSE, 1993, S. $51-64$

Coleman, David A. (1992), Does Europe needs immigrants? Population and work force projections, in: International Migration Review, 2, 413-461, Center for Migration Studies, New York

Cornelsen, Carsten (1995), Erste Ergebnisse des Mikrozensus April 1994, in: Wirtschaft und Statistik, Heft 4, S. 279-286

Cramer, Georg / Kramer, Beate (1990), Probleme der Weiterbildung in Klein- und Mittelbetrieben des Handwerks, in: SCHLAFFKE/WEIB, S. 79-98

Cronjăger, Ludolf (Band-Hrsg.) (1990), Bausteine für die Fabrik der Zukunft, SpringerVerlag, Berlin, Heidelberg, New York, Verlag TÜV Rheinland, Köln (CIM-Fachmann) 
Cullen, Kevin / Clarkin, Nadia (1994), Working and caring, Opportunities and social issues raised by technologies, Work Research Centre Ltd., Working Paper No.: WP/94/31/EN, European Foundation for the Improvement of Living and Working Conditions, Dublin

Dale, Angela / Joshi, Heather (1992), The Economic and Social Status of British Women, in: SCHWARZ, 1992, S. 97-121

Diekmann, Andreas (1994), Hat das steigende Ehescheidungsrisiko das berufliche Engagement von Frauen gefordert? In: Soziale Welt, Jg. 45, Heft 1, S. 83-97

Dinkel, Reiner (1992), Demographische Alterung: Ein Überblick unter besonderer Berücksichtigung der Mortalitatsentwicklung, in: BALTES/MITTELSTRAB, 1992, S. 62-93

Dinkel, Reiner / Lebok, Uwe (1993), Könnten durch Zuwanderung die Alterung der Bevolkerung und die daraus resultierenden Zusatzlasten der Sozialen Sicherung aufgehalten oder abgemildert werden? In: Deutsche Rentenversicherung 6/93, S. 388-400

DIW (1994), Ist Teilzeitarbeit der richtige Weg? Arbeitszeitprăferenzen in West- und Ostdeutschland, Deutsches Institut für Wirtschaftsforschung, Wochenbericht des DIW, Nr. 35, S. 618-626

DIW (1992), Unterbrochene Erwerbsverlaufe von Frauen mit Kindern, Traditionelles familienpolitisches Leitbild fragwürdig, Deutsches Institut für Wirtschaftsforschung, Wochenbericht des DIW, Nr. 19, S. 249-257

Düll, Klaus / Lutz, Burkart (1989), Technikentwicklung und Arbeitsteilung im internationalen Vergleich, Fünf Aufsătze zur Zukunft industrieller Arbeit, Campus Verlag, Frankfurt/Main, New York, (Forschungsberichte aus dem Institut für Sozialwissenschaftliche Forschung e.V., ISF, München)

Eberhardt, Hermann (Hrsg.) (1993), Lean Production, Gruppenarbeit, Dokumentation zum 7. Arbeitswissenschaftlichen Forum ' 92 am 22. Oktober 1992 in Bad Homburg, Blaue Reihe von HESSEN METALL Nr. 9/93, Frankfurt am Main

Engelbrech, Gerhard (1987), Erwerbsverhalten und Berufsverlauf von Frauen. Ergebnisse neuerer Untersuchungen im Überblick, in: Mitteilungen aus der Arbeitsmarkt- und Berufsforschung, 2/87, S. 181-196

Engelbrech, Gerhard (1991a), Berufsausbildung, Berufseinstieg und Berufsverlauf von Frauen. Empirische Befunde zur Erklărung beruflicher Segregation, in: Mitteilungen aus der Arbeitsmarkt- und Berufsforschung, 3/91, S. 531-558

Engelbrech, Gerhard (1991b), Frauenspezifische Restriktionen des Arbeitsmarktes Situationsbericht und Erklărungsansătze zu Phasen des Berufsverlaufs anhand von IABErgebnissen, in: MAYER u.a., 1991, S. 91-118

Engelbrech, Gerhard (1992a), Berufliche Segregation - Erklărungsansătze und empirische Befunde -, in: WSI Mitteilungen, 4/92, S. 187-194 
Engelbrech, Gerhard (1992b), Die Situation der Frauen in den neuen Bundesländern beim Übergang zur Marktwirtschaft: Individualisierung arbeitsmarkt- und sozialpolitischer Problemlagen oder gesellschaftliche Herausforderung? In: SCHWARZ, 1992, S. 45-63

Engelbrech, Gerhard / Kraft, Hermine (1992), Sind Frauen das zukünftige Innovationspotential? Gegenwărtige Hemmnisse und berufliche Möglichkeiten von Frauen Ergebnisse einer Betriebsbefragung, in: Mitteilungen aus der Arbeitsmarkt- und Berufsforschung, 1/92, S. 13-26

Enquete-Kommission (1994), Zwischenbericht der Enquete-Kommission Demographischer Wandel, Herausforderungen unserer älter werdenden Gesellschaft an den einzelnen und die Politik, Deutscher Bundestag, Drucksache 12/7876, Bonn

Esser, Martin / Kobayashi, Kaoru (Hrsg.) (1994), Kaishain, Personalmanagement in Japan, Verlag für Angewandte Psychologie, Gobttingen

Esser, Martin / Nakajima, Fuyuki (1994), Prinzipien japanischen Personalmanagements, in: ESSER/KOBAYASHI, 1994, S. 139-158

Estor, Marita (1991), Frauenberufe, in: LISSNER u.a., 1991, Spalten 314-322

Euler, Hanns Peter (1987), Soziale Auswirkungen neuer Technologien in der Produktion, in: Zeitschrift für Arbeitswissenschaft 41 (13 NF) 1987/1, S. 1-6

Eurostat (Hrsg.) (1993), Schnellberichte Bevőlkerung und soziale Bedingungen, 10, Frauen in der Europäischen Gemeinschaft, Luxemburg

Eurostat (Hrsg.) (1994), Begegnung in Zahlen, Ein statistisches Portrăt der Europäischen Union, Brüssel, Luxemburg

Eyer, Eckhard (1993), Anforderungs- und leistungsgerechte Entlohnung in teilautonomen Arbeitsgruppen, in: angewandte Arbeitswissenschaft (1993), Nr. 135, S. 1-22

Ferber, Christian von / Ferber, Liselotte von (1991), Gesundheitszirkel - eine Strategie zur Gesundheitsförderung am Arbeitsplatz, in: Sozialer Fortschritt 12/91, S. 293-298

Flőter, R. I Wenzel, Bernhard (1992), Produktivităt durch Arbeitsorganisation am Beispiel der Adam Opel AG, Gemeinsames Arbeiten jüngerer und alterer Mitarbeiter, Unterlagen zu einem Referat anläblich des Kongresses "Erwerbsarbeit der Zukunft", Arbeit und Technik bei verănderten Alters- und Belegschaftsstrukturen, am 3. und 4.11.1992 in Berlin

Franz, Wolfgang (1994), Arbeitsokonomik, Springer Verlag, Berlin, Heidelberg, New York, Tokyo, zweite, verbesserte Auflage

Frei, Felix / Duell, Werner / Baitsch, Christof (1984), Arbeit und Kompetenzentwicklung, Theoretische Konzepte zur Psychologie arbeitsimmanenter Qualifizierung, (Schriften zur Arbeitspsychologie; Nr. 39), Verlag Hans Huber, Bern, Stuttgart, Wien

Frőhlich, Dieter / Hild, Paul (1992), Qualitative Beschäftigungswirkungen neuer Informationstechniken: Qualifikationseinsatz und Arbeitssituation beim Umgang mit der CNC-Technik, in: WSI Mitteilungen 10/1992, S. 675-681 
Fruhmann, G. (1993), "Arbeitsmedizinische Aspekte und Bedingungen des Arbeitsschutzes bei der Beschăftigung ălterer Arbeitnehmer", Stellungnahme zur öffentlichen Anhörung am 14. Januar 1994 in Bonn, Enquete-Kommission Demographischer Wandel, Kommissionsdrucksache 182 vom 04.01.1994

Fürstenberg, Friedrich (1972), Teilzeitarbeit für Frauen, in: Arbeit und Leistung, 6/1972, S. 154-157

Fürstenberg, Friedrich (1987), Flexible Gestaltung industrieller Arbeitsbedingungen, in: Zeitschrift für Arbeitswissenschaft 41 (13 NF) 1987/2, S. 65-68

Gaugler, Eduard (1988), Das Normalarbeitsverhăltnis in betriebswirtschaftlicher Sicht, in: Besters, Hans (Hrsg.), Auflösung des Normalarbeitsverhăltnisses?, (Gespräche der List-Ges. e.V.; N.F., Bd. 11), Nomos Verl.-Ges., Baden-Baden, S. 12-28

Gidion, Gerd (1992), Zukunft der Frauen in Familie und Erwerbstătigkeit, Betriebliche Folgen soziodemographischer Verănderungen und Frauenerwerbsbeteiligung, in: SCHWARZ, 1992, S. $205-223$

Gidion, Gerd / Stegmeier, Thomas (1991), Alter und Mobilităt: Szenarien zu regionaler Mobilităt von Beschăftigten, Auszug aus dem Bericht "Betriebliche Personalentwicklungen Folgen soziodemographischer Verănderungen", Fraunhofer-Institut für Arbeitswirtschaft und Organisation, Stuttgart, zugleich: Enquete-Kommission Demographischer Wandel, Kommissionsdrucksache 30 vom 16.02.1993

Gőckenjan, Gerd (1993), Alter - Ruhestand - Generationsvertrag? Zum Altersdiskurs aus historisch-struktureller Perspektive, in: Aus Politik und Zeitgeschichte, Beilage zur Wochenzeitung Das Parlament, B 17/93, S. 3-10

Grob, Robert (1993), Auf die Plătze ... fertig ... lean! Gefahren einer Modewelle, in: Arbeitgeber, 13/14, 45, 1993, S. 491-494

Grob, Robert (1994), "Lean" und REFA - Gegensătze oder sinnvolle Ergănzung? In: Zeitschrift für Arbeitswissenschaft 48 (20 NF) 1994/2, S. 83-89

Grütz, Jens / Lankes, Fidelis / Tautz, Roland / Roppel, Ullrich (1993), Modellrechnung zum Erwerbspersonenpotential und zur Arbeitsmarktbilanz bis zum Jahre 2030, in: Deutsche Rentenversicherung 7/93, S. 449-462

Guillemard, Anne-Marie (1993), Ältere Menschen auf dem Arbeitsmarkt, in: Alber, Jens / Guillemard, Anne-Marie / Walker, Alan, Ältere Menschen in Europa: Wirtschafts- und Sozialpolitik, Bericht der europåischen Beobachtergruppe, Kommission der Europäischen Gemeinschaften, Generaldirektion V, Beschäfigung, Arbeitsbeziehungen und Soziale Angelegenheiten, S. 68-105

Gusy, Christoph (1994), Möglichkeiten und Grenzen eines effektiven und flexiblen Einwanderungsrechts, in: forum demographie und politik (Hrsg.: Hans-Ulrich Klose), Heft 5, S. $156-171$ 
Hacker, Winfried / Iwanowa, Anna / Richter, Peter (1983), Tătigkeitsbewertungssystem TBS - Handanweisung und Merkmale, Psychodiagnostisches Zentrum, Berlin

Hagedorn, Jobst (1992), Ein völlig überholtes Auslaufmodell, in: Arbeitgeber 8/44, S. 274-275

Halbach, Günter / Paland, Norbert / Schwedes, Rolf / Wlotzke, Otfried (1994), Übersicht über das Arbeitsrecht, herausgegeben vom Bundesministerium für Arbeit und Sozialordnung, Bonn, 5., neubearbeitete und erweiterte Aufl.

Hans-Bøckler-Stiftung / Industriegewerkschaft Metall (Hrsg.) (1992), Lean Production, Kern einer neuen Unternehmenskultur und einer innovativen und sozialen Arbeitsorganisation?; Gewerkschaftliche Auseinandersetzung mit einem Managementkonzept, Dialogtagung der Hans-Böckler-Stiftung und der Industriegewerkschaft Metall, Frankfurt/M., 30. Juni und 1. Juli 1992, Nomos Verlagsgesellschaft, Baden-Baden

Hegner, Friedhart / Klocke-Kramer, Marianne / Lakemann, Ulrich / Schlegelmilch, Cordia (1989), Dezentrale Arbeitsplătze, Eine empirische Untersuchung neuer Erwerbs- und Familienformen, Campus Verlag, Frankfurt/Main, New York

Heidenreich, Martin (1994), Gruppenarbeit zwischen Toyotismus und Humanisierung, Eine international vergleichende Perspektive, in: Soziale Welt, Jg. 45, 1994, Heft 1

Helfert, Mario (1992), Betriebsverfassung, neue Rationalisierungsformen, lean production, in: WSI Mitteilungen 8/1992, S. 505-521

Heller, Waltraud (1993), Neue Technologien erfordern neue Qualifikationen der Mitarbeiter oder auch nicht, in: Zeitschrift für Personalforschung, 1/93, S. 134-153

Hinrichs, Karl (1992), Zur Zukunft der Arbeitszeitflexibilisierung, Arbeitnehmerpräferenzen, betriebliche Interessen und Beschäftigungsauswirkungen, in: Soziale Welt, Jg. 43, Heft 3, S. 313-330

Hirsch-Kreinsen, Hartmut / Schultz-Wild, Rainer / Kohler, Christoph / Behr, Marhild von (1990), Einstieg in die rechnerintegrierte Produktion: Alternative Entwicklungspfade der Industriearbeit im Maschinenbau, Campus Verlag, Frankfurt/Main, New York, (Forschungsberichte aus dem Institut für Sozialwissenschaftliche Forschung e.V., ISF, München)

Hoem, Britta (1992), The Way to the Gender-Segregated Swedish Labour Market, in: SCHWARZ, 1992, S. 77-95

Hoets, Anna (1993), Förderung sozialer Kompetenzen als Aufgabe der Personalentwicklung Instrument der Anpassung, insbesondere für Frauen? In: Zeitschrift für Personalforschung, 1/93, S. 115-133

Hof, Bernd (1992), Arbeitskräftebedarf der Wirtschaft, Arbeitsmarktchancen für Zuwanderer, in: Forschungsinstitut der Friedrich-Ebert-Stiftung, Abteilung Arbeits- und Sozialforschung (Hrsg.), Zuwanderungspolitik der Zukunft, Bonn, S. 7-22 
Hof, Bernd (1993), Bevolkerungsentwicklung, Zuwanderung, Arbeitsmarkt - Zukunftsszenarien über die 12 EG-Staaten -, in: forum demographie und politik (Hrsg.: Hans-Ulrich Klose), Heft 4, S. 63-90

Hof, Bernd (1994), Beschaftigungsschwelle und Wachstum - was besagt die Empirie? In: ifo Studien, 40. Jahrgang, 1994/4, S. 127-144, (Kurzfassung in iwd, Nr 13, 1994, S. 4-5)

Hoffmann, Edeltraud (1993), Zur Beschaftigung alterer Arbeitnehmer in Westdeutschland Qualitative und quantitative Aspekte, in: Mitteilungen aus der Arbeitsmarkt- und Berufsforschung, 3/93, S. 313-335

Hoffmann, Marina / Leeson, George (1994), Eldercare and employment: Workplace policies and initiatives to support workers who are carers, DansAge Foundation, Kopenhagen

Hohn, Charlotte / Hullen, Gert (1993), Bestimmungsgründe der Alterung der Bevőlkerung in Deutschland - Gestern, heute und morgen, in: OTTO, 1993, S. 3-39

Holling, Heinz / Liepmann, Detlev (1993), Personalentwicklung, in: SCHULER, 1993, S. 385316

Holst, Elke / Schupp, Jürgen (1994), Perspektiven der Erwerbsbeteiligung von Frauen im vereinten Deutschland, in: BECKMANN/ENGELBRECH, 1994, S. 140-174

Holst, Elke / Schupp, Jürgen (1995), Erwerbsbeteiligung und Erwerbsorientierung von Frauen nach der Wende, in: Sozialer Fortschritt 1/95, S. 5-8

Holzel, Manfred (1994), "Vom Verwalter zum Dienstleister« - Qualifikationsanforderungen an den Lean Administrator, in: STEGER, 1994, S. 211-220

Howaltd, Jürgen (1994), KVP-Aktivităten in Deutschland. Eine Befragung von Betriebsrăten der Automobil- und Automobilzulieferindustrie, in: Arbeit, Jg. 3, Heft 4, 1994, S. 320-330

Howaltd, Jürgen / Kopp, Ralf (1992), lean production = mean production? Lean Production und Arbeitsbedingungen in der Automobilindustrie, in: Arbeit, Jg. 1, Heft 3, 1992, S. $233-$ 245

Imai, Masaaki (1992), Kaizen, Der Schlüssel zum Erfolg der Japaner im Wettbewerb, Ullstein, Frankfurt/Main, 3. Aufl. 1993

Institut der deutschen Wirtschaft (Hrsg.) (1994), Dokumentation, Akademikerbeschätigung, Ein Fünftel unter Niveau, iwd, Nr 30, S. 4-5, Deutscher Instituts-Verlag, Koln

Institut für angewandte Arbeitswissenschaft (Brsg.) (1992), Lean Production, Idee - Konzept - Erfahrungen in Deutschland, Erweiterte Dokumentation der IfaA-Fachtagung am 18.3.1992 in Stuttgart, Schriftenreihe des IfaA, Band 27, Wirtschaftsverlag Bachem, Koln

Institut für angewandte Arbeitswissenschaft (Hrsg.) (1994), Lean Production II, Erfahrungen und Erfolge in der $M+$ E-Industrie, Schriftenreihe des IfaA, Band 29, Wirtschaftsverlag Bachem, Koln

Institut der deutschen Wirtschaft (Hrsg) (1994), Teilungsprobleme, Informationsdienst der deutschen Wirtschaft, iwd, Nr 51/52, 1994, S. 1 
Jagoda, Bernhard (1993), Mittelfristige Arbeitsmarktentwicklung und -probleme, in: Sozialer Fortschritt, Heft 8, S. 175-177

Jani-Le Bris, Hannelore (1994), Berufstătigkeit und Pflege: Entwicklungen am Arbeitsplatz für pflegende Angehörige alter Menschen und Behinderter, Grundlagenpapier, Internationale Konferenz, Europăische Stiftung zur Verbesserung der Lebens- und Arbeitsbedingungen, Bundesministerium für Familie und Senioren, Bonn

Jürgens, Ulrich (1993), Mythos und Realităt von Lean Production in Japan - eine kritische Auseinandersetzung mit den Ergebnissen der MIT-Studie, in: Fortschrittliche Betriebsführung und Industrial Engineering, 42 (1993) 1, S. 18-23

Kern, Horst / Schumann, Michael (1970), Industriearbeit und Arbeiterbewußtsein, Eine empirische Untersuchung über den Einfluß der aktuellen technischen Entwicklung auf die industrielle Arbeit und das Arbeiterbewußtsein, Studienreihe des Soziologischen Forschungsinstituts Gottingen (SOFI), 2 Bănde, Studienausgabe, Frankfurt, 3. unverănderte Aufl. 1974

Kern, Horst / Schumann, Michael (1984), Das Ende der Arbeitsteilung? Rationalisierung in der industriellen Produktion: Bestandsaufnahme, Trendbestimmung, Verlag C.H. Beck, München, 2. Aufl. 1985

Klages, Helmut (1984), Wertorientierungen im Wandel, Rückblick, Gegenwartsanalyse, Prognosen, Campus Verlag, Frankfurt/Main, New York

Klages, Helmut (1993), Wertewandel in Deutschland der 90er Jahre, in: Rosenstiel, Lutz von (Hrsg.), Wertewandel: Herausforderungen für die Unternehmenspolitik in den 90er Jahren, Schăffer-Poeschel, Stuttgart, 2., überarb. Aufl., S. 1-15

Klauder, Wolfgang (1992a), Wirtschaftliche und gesellschaftliche Bedeutung der Frauenerwerbstătigkeit heute und morgen, in: SCHWARZ, 1992, S. 13-44

Klauder, Wolfgang (1992b), Ausreichend Mitarbeiter für Tätigkeiten von morgen? Europäisches Arbeitskräftepotential und demographiebedingte Engpässe des Arbeitsmarktes, Vortrag auf der Plenarveranstaltung des Kongresses "Erwerbsarbeit der Zukunft: Arbeit und Technik bei verănderten Alters- und Belegschaftsstrukturen" des Bundesministers für Forschung und Technologie und des Bundesministers für Arbeit und Sozialordnung am 3. November 1992 in Berlin

Klauder, Wolfgang (1992c), Die Arbeitswelt der Zukunft, in: Ehses, Susanne / Nobis, EvaMaria (Hrsg.) (1992), Zukunft der Arbeit - Arbeit der Zukunft, Symposium Oeconomicum, Lit Verlag, Münster, Hamburg, S. 14-37

Kleinbeck, U. (1993), Beanspruchung durch Fließbandarbeit, Akkordarbeit, Gruppenarbeit, in: KONIETZKO/DUPUIS, 1989, Abschnitt III-3.2.2

Klose, Hans-Ulrich (Hrsg.) (1993), Antworten auf den demopraphischen Wandel, Bund-Verlag, Köln 
Klotz, Ulrich (1994), Objektorientierung - ein facettenreiches Leitbild verbindet Flexibilität mit humaner Arbeitsgestaltung, in: Zeitschrift für Arbeitswissenschaft 48 (20 NF) 1994/2, S. 99 112

Knauth, Bettina (1992), Frauenerwerbsbeteiligung in den Staaten der Europäischen Gemeinschaft, in: SCHWARZ, 1992, S. 265-323

Knauth, Bettina (1993), Ältere Menschen in der Europäischen Gemeinschaft - Fakten und Probleme, in: OTTO, 1993, S. 219-235

Koch, Heleni (1988), Bevőlkerung und Neuerungsaktivităt: Auswirkungen demographischer Faktoren auf Invention und Innovation, Ifo-Studien zur Bevölkerungsökonomie; 5, IfoInstitut für Wirtschaftsforschung, München

Köchling, Annegret (1992), Arbeitsplătze der Zukunft, in: forum demographie und politik (Hrsg.: Hans-Ulrich Klose), Heft 2, S. 60-81

Kohli, Martin (1992), Altern in soziologischer Perspektive, in: BALTES/MITTELSTRAB, 1992, S. 231-259

Kollar, Rudolf / Stengel, Martin (1990), Sind Berufsorientierungen und organisationale Identifikation Chimären der Forschung? Zur Verhaltensrelevanz berufsbezogener Orientierungen und Einstellungen bei Führungskraften, in: Zeitschrift für Arbeits- und Organisationspsychologie 34 (N.F. 8) 2, S. 74-84

Konietzko, Johannes (1989), Die Gefăhrdung der Frau am Arbeitsplatz, in: KONIETZKO/DUPUIS, 1989, Abschnitt III-6.1

Konietzko, Johannes / Dupuis, H. (Hrsg.) (1989), Handbuch der Arbeitsmedizin: Arbeitsphysiologie, Arbeitspathologie, Prăvention, Loseblatt-Ausgabe, ecomed Verlagsgesellschaft, Landsberg, München, Zürich

Kðsters, Andreas / Lueckel, Gordon (1994), Die hundert besten Arbeitgeber, Campus Verlag, Frankfurt/Main, New York

Kötter, Wolfgang / Volpert, Walter (1993), Arbeitsgestaltung als Arbeitsaufgabe - ein arbeitspsychologischer Beitrag zu einer Theorie der Gestaltung von Arbeit und Technik, in: Zeitschrift für Arbeitswissenschaft 47 (19 NF) 1993/3, S. 129-140

Koubek, Norbert (1985), Wirtschaftlichkeit, in: OTT/BOLDT, 1985, S. 1205-1222

Krūger, Helga (Hrsg.) (1992), Frauen und Bildung, Wege der Aneignung und Verwertung von Qualifikationen in weiblichen Erwerbsbiographien, Bollert, KT-Verlag, Bielefeld

Kühnlein, Gertrud / Wohlfahrt, Norbert (1994), Lean administration / lean government - ein neues Leitbild für die öffentlichen Verwaltungen? In: Arbeit, Heft 1, Jg. 3 (1994), S. 3-18

Lang, Klaus (1993), Gewerkschaftliche Arbeitszeitpolitik - 35-Stunden-Woche und was danach? In: SEIFERT, 1993, S. 249-270

Lang, Oliver (1994), Die Einkommens- und Vermögensverhăltnisse künftiger Altengenerationen in Deutschland, Expertise im Auftrag der Enquete-Kommission "Demographischer Wandel", Deutscher Bundestag, Bonn 
Laurig, Wolfgang (1990), Grundzüge der Ergonomie: Erkenntnisse und Prinzipien, REFA, Verband für Arbeitsstudien und Betriebsorganisation e.V., Beuth, Berlin, 3., vollst. überarb. Aufl.

Lauterbach, Wolfgang (1991), Erwerbsmuster von Frauen, Entwicklungen und Veränderungen seit Beginn dieses Jahrhunderts, in: MAYER u.a., 1991, S. 23-56

Lauterbach, Wolfgang / Huinink, Johannes / Becker, Rolf (1994), Erwerbsbeteiligung und Berufschancen von Frauen, Theoretische Ansătze, methodische Verfahren und empirische Ergebnisse aus der Lebensverlaufperspektive, in: BECKMANN/ENGELBRECH, 1994, S. 175-208

Lehr, Ursula (1988), Kompetenz im Alter - Beiträge aus gerontologischer Forschung und Praxis, Auszug aus: Kompetenz im Alter, Beiträge zur III. Gerontologischen Woche, Heidelberg, 2.5.-6.5.1988, Peutinger-Institut für angewandte Wissenschaften, S. 1-14, zugleich: Enquete-Kommission Demographischer Wandel, Kommissionsdrucksache 51 vom 08.03.1993

Lenk, Thomas (1989), Telearbeit - Moglichkeiten und Grenzen einer telekommunikativen Dezentralisierung von betrieblichen Arbeitsplătzen, Duncker u. Humblot, Berlin

Lennartz, Klaus Dieter (1993), Die Bedeutung des Erfahrungswissens in der NCVerfahrenskette, in: BULLINGER, u.a., 1993, S. 73-77

Linhart, Danièle / Düll, Klaus / Bechtle, Günter (1989), Neue Technologien und industrielle Beziehungen im Betrieb - Erfahrungen aus der Bundesrepublik Deutschland und Frankreich, in: DÜLL/LUTZ, 1989, S. 93-159

Lissner, Anneliese / Süssmuth, Rita / Walter, Karin (Hrsg.) (1991), Frauenlexikon. Wirklichkeit und Wünsche von Frauen, Herder, Freiburg

Löwenberg, Herwig (1988), Frauen in der beruflichen Weiterbildung, in: BOLLE/SCHNEIDER, 1988, S. $185-211$

Luczak, Holger (1988), Arbeitsgestalterische - insbesondere informationstechnische - Aspekte neuer Technologien, in: ZINK, 1988, S. 159-197

Luczak, Holger (1993), Arbeitswissenschaft, Springer, Berlin, Heidelberg

Lutz, Burkart (1990), Technikforschung und Technologiepolitik: Förderstrategische Konsequenzen eines wissenschaftlichen Paradigmenwechsels, in: WSI Mitteilungen 10/1990, S. 614-622

Lutz, Burkart / Moldaschl, Manfred (1989), Expertensysteme und industrielle Facharbeit: ein Gutachten über denkbare qualifikatorische Auswirkungen von Expertensystemen in der fertigenden Industrie; erstellt im Auftrag der Enquete-Kommission "Technikfolgenabschătzung und -bewertung" des Deutschen Bundestages, Campus Verlag, Frankfurt/Main, New York, (Forschungsberichte aus dem Institut für Sozialwissenschaftliche Forschung e.V., ISF, München) 
Maab, Susanne (1993), Software-Ergonomie. Benutzer- und aufgabenorientierte Systemgestaltung, in: Informatik-Spektrum, Bd. 16, H. 4, S. 191 - 205

Maier, Friederike (1992), Weiterbildung und Frauenerwerbstătigkeit, in: WSI Mitteilungen 6/1992, S. $372-379$

Malsch, Thomas / Seltz, Rüdiger (Hrsg.) (1987), Die neuen Produktionskonzepte auf dem Prufstand: Beitrăge zur Entwicklung der Industriearbeit, Edition Sigma Rainer Bohn Verlag, Berlin

Mayer, Karl Ulrich (1992), Bildung und Arbeit in einer alternden Bevölkerung, in: BALTES/MITTELSTRAB, 1992, S. 518-543

Mayer, Karl Ulrich / Allmendinger, Jutta / Huinink, Johannes (Hrsg.) (1991), Vom Regen in die Traufe, Frauen zwischen Familie und Beruf, Reihe Lebensverlaufe und gesellschaftlicher Wandel, Campus Verlag, Frankfurt/Main, New York

Mickler, Otfried (1981), Facharbeit im Wandel: Rationalisierung im industriellen ProduktionsprozeB, Campus Verlag, Frankfurt/Main, New York (Studienreihe des Soziologischen Forschungsinstitutes Göttingen, SOFI)

Miegel, Meinhard / Wahl, Stefanie (1993), Das Ende des Individualismus: Die Kultur des Westens zerstơrt sich selbst, Verlag Bonn Aktuell im Verlag Moderne Industrie, München, Landsberg/Lech

Minssen, Heiner (1993), Lean Production - Herausforderung für die Industriesoziologie, in: Arbeit, Jg. 2, Heft 1, 1993, S. 36-52

Minssen, Heiner / Howaldt, Jürgen / Kopp, Ralf (1991), Gruppenarbeit in der Automobilindustrie - Das Beispiel Opel Bochum -, in: WSI Mitteilungen 7/1991, S. 434-441

Müler, Rainer (1994), "Arbeitsmedizinische Aspekte und Bedingungen des Arbeitsschutzes bei der Beschaftigung alterer Arbeitnehmer", Stellungnahme zur Offentlichen Anhörung der Enquete-Kommission des Deutschen Bundestages "Demographischer Wandel" am 14. Januar 1994 in Bonn, Enquete-Kommission Demographischer Wandel, Kommissionsdrucksache 177 vom 15.12.1993

Mäller-Daehn, Sigrid / Fooken, Insa (1992), Besondere Belange der Situation von Frauen im Alter, Expertise für die Altenberichtskommission des Bundesministers für Familie und Senioren, Juni 1992, Bonn, zugleich: Enquete-Kommission Demographischer Wandel, Kommissionsdrucksache 20 vom 10.02.1993

Münch, Joachim (1990), Lernen am Arbeitsplatz - Bedeutung innerhalb der betrieblichen Weiterbildung, in: SCHLAFFKE/WEIB, S. 141-176

Myrdal, Alva / Klein, Viola (1971), Die Doppelrolle der Frau in der Familie und Beruf, Kiepenheuer \& Witsch, Koln

Naegele, Gerhard (1988), Zur Zukunft der alteren Arbeitnehmer in einer verănderten Arbeitslandschaft - Neue Chancen oder Risiken? In: Sozialer Fortschrittt, Jg. 37, 1988, Heft $2 / 3$, S. $33-45$ 
Naegele, Gerhard (1992), Zwischen Arbeit und Rente, Maro-Verlag, Augsburg, (Beitrăge zur Sozialpolitik-Forschung; Bd. 9)

Naegele, Gerhard (1994), Demographische und strukturelle Verănderungen in der Arbeitswelt Neue Herausforderungen an berufliche Fort- und Weiterbildung, Enquete-Kommission Demographischer Wandel, Kommissionsdrucksache 242 vom 23.04.1994

Naegele, Gerhard / Tews, Hans Peter (1993), Lebenslagen im Strukturwandel des Alters, Alternde Gesellschaft - Folgen für die Politik, Westdeutscher Verlag, Opladen

Naschold, Frieder (1987), Organisationsentwicklung und technische Innovation, in: Zeitschrift für Arbeitswissenschaft 41 (13 NF) 1987/4, S. 193-195

Nerge, Sonja / Stahmann, Martina (1991), Mit Seidentuch und ohne Schlips - Frauen im Management: eine empirische Untersuchung ihrer Lebens- und Arbeitsbedingungen, Verlag Peter Lang, Frankfurt am Main, Bern, New York, Paris (Europăische Hochschulschriften: Reihe 23, Soziologie; Bd. 216)

Neuberger, Oswald (1985), Arbeit: Begriff - Gestaltung - Motivation - Zufriedenheit, (Basistexte Personalwesen; Bd. 5), Ferdinand Enke Verlag, Stuttgart

Novak, Hermann (1993), Die Herausforderung der betrieblichen Berufsbildung durch neue Informationstechnologien, Ein Bericht aus der Modellversuchspraxis, in: Arbeit, Jg. 2, Heft 1,1993 , S. $20-35$

Ohsmann, Sabine / Stolz, Ulrich (1994), Alte und neue Bundeslănder im Vergleich, Der Rentenzugang der BfA 1993, in: Die Angestelltenversicherung, 11/94, S. 409-417

Olbrich, Erhard (1988), Kompetentes Verhalten älterer Menschen - Epochale Aspekte, Auszug aus: Kompetenz im Alter, Beitrăge zur III. Gerontologischen Woche, Heidelberg, 2.5.6.5.1988, Peutinger-Institut für angewandte Wissenschaften, S. 32-61, zugleich: EnqueteKommission Demographischer Wandel, Kommissionsdrucksache 52 vom 08.03.1993

Ortner, Erich (1991), Informationsmanagement. Wie es entstand, was es ist und wohin es sich entwickelt, in: Informatik-Spektrum, Bd. 14, H. 6, S. 315-327

Ott, Alfred E. (1980), Auswirkungen demographischer Entwicklungen auf die sektorale Wirtschaftsstruktur, in: Ministerium für Wirtschaft, Mittelstand und Verkehr BadenWürttemberg (Hrsg.), Auswirkungen der Bevolkerungsentwicklung auf Wirtschafts- und Regionalstruktur, Stuttgart, S. 48-70

Ott, Erich / Boldt, Alfred (1985), Handbuch zur Humanisierung der Arbeit, Bd. I und II. (Herausgegeben von der Bundesanstalt für Arbeitsschutz, Dortmund), Wirtschaftsverlag NW, Verlag für neue Wissenschaft $\mathrm{GmbH}$, Bremerhaven

Otto, Johannes (Hrsg.) (1993), Die älter werdende Gesellschaft, Deutsche Gesellschaft für Bevollkerungswissenschaft, 27. Arbeitstagung vom 25. bis 27. Februar 1993 in Bad Homburg v.d. Hohe, (Materialien zur Bevolkerungswissenschaft, Heft 80, herausgegeben vom Bundesinstitut für Bevőlkerungsforschung, Wiesbaden)

Pfarr, Heide (1991), Mădchen-/Frauenschutz, in: Lissner u.a., 1991, Spalten 672-678 
Pfau-Effinger, Birgit / Geissler, Birgit (1992), Institutionelle und sozio-kulturelle Kontextbedingungen der Entscheidung verheirateter Frauen für Teilzeitarbeit, Ein Beitrag zu einer Soziologie des Erwerbsverhaltens, in: Mitteilungen aus der Arbeitsmarkt- und Berufsforschung, 3/92, S. 358-370

Picot, Arnold / Reichwald, Ralf / Behrbohm, Peter (1985), Vier-Ebenen-Modell der Wirtschaftlichkeitsbeurteilung, (Menschengerechte Arbeitsplätze sind wirtschaftlich), Schriftenreihe Wirtschaftlichkeitsberechnung, Rationalisierung-Kuratorium der Deutschen Wirtschaft (RKW), Eschborn

Piore, Michael J. / Sabel, Charles F. (1985), Das Ende der Massenproduktion, Studie über die Requalifizierung der Arbeit und die Rückkehr der Ókonomie in die Gesellschaft, Wagenbach, Berlin

Plantenga, Janneke (1992), Women and Work in the Netherlands - Some notes about female labour force participation and the nature of the welfare state -, in: SCHWARZ, 1992, S. $123-$ 132

Pries, Ludger / Schmidt, Rudi / Trinczek, Rainer (Hrsg.) (1989), Trends betrieblicher Produktionsmodernisierung. Chancen und Risiken für Industriearbeit, Expertenberichte aus sieben Branchen, Westdeutscher Verlag, Opladen, Wiesbaden

Pries, Ludger / Schmidt, Rudi / Trinczek, Rainer (1990), Entwicklungspfade von Industriearbeit: Chancen und Risiken betrieblicher Produktionsmodernisierung, Westdeutscher Verlag, Opladen, (Sozialverträgliche Technikgestaltung Bd. 7,2. Hrsg.: Der Minister für Arbeit, Gesundheit und Soziales des Landes Nordrhein-Westfalen)

Prognos AG, von Rothkirch, Christoph / Weidig, Inge / Barth, Hans / Eckerle, Konrad / Masuhr, Klaus / Wolff, Heimfried (1985), Die Zukunft der Arbeitslandschaft: Zum Arbeitskräftebedarf nach Umfang und Tătigkeiten bis zum Jahre 2000, Beiträge zur Arbeitsmarkt- und Berufsforschung 94.1 (Textband), 94.2 (Anlageband), IAB, Nürnberg

Prognos AG, Hofer, Peter / Weidig, Inge / Wolff, Heimfried (1989), Arbeitslandschaft bis 2010 nach Umfang und Tătigkeitsprofilen, Beitrăge zur Arbeitsmarkt- und Berufsforschung 131.1 (Textband), 131.2 (Anlageband), IAB, Nürnberg

Prognos AG, Eckerle, Konrad / Franzen, Detlef / Rommerskirchen, Stefan / Schilling, Karl (EDV) / Weidig, Inge / Wirz-Bergmann, Christel / Wolff, Heimfried (1990), Die Arbeitsmărkte im EG-Binnenmarkt bis zum Jahr 2000, Beitrăge zur Arbeitsmarkt- und Berufsforschung 138.1 (Textband), 138.2 (Anlageband), IAB, Nürnberg

REFA (1985a), Methodenlehre der Organisation für Verwaltung und Dienstleistung, Teil 1. Grundlagen, REFA, Verband für Arbeitsstudien und Betriebsorganisation e.V., Carl Hanser, München

REFA (1985b), Methodenlehre des Arbeitsstudiums, Teil 3. Kostenrechnung, Arbeitsgestaltung, REFA, Verband für Arbeitsstudien und Betriebsorganisation e.V., Carl Hanser, München, 7. Auflage 
REFA (1987), Methodenlehre der Betriebsorganisation, Planung und Gestaltung komplexer Produktionssysteme, REFA, Verband für Arbeitsstudien und Betriebsorganisation e.V., Carl Hanser, München

REFA (1989), Methodenlehre der Betriebsorganisation, Anforderungsermittlung (Arbeitsbewertung), REFA, Verband für Arbeitsstudien und Betriebsorganisation e.V., Carl Hanser, München, Verănderter Nachdruck der 1. Aufl.

REFA (1991a), Methodenlehre der Betriebsorganisation, Grundlagen der Arbeitsgestaltung, REFA, Verband für Arbeitsstudien und Betriebsorganisation e.V., Carl Hanser, München

REFA (1991b), Methodenlehre der Betriebsorganisation, Arbeitsgestaltung im Bürobereich, REFA, Verband für Arbeitsstudien und Betriebsorganisation e.V., Carl Hanser, München

REFA (1991c), Methodenlehre der Betriebsorganisation, Arbeitsgestaltung in der Produktion, REFA, Verband für Arbeitsstudien und Betriebsorganisation e.V., Carl Hanser, München

Reichling, Joachim (1992), Frauenförderung in Klein- und Mittelbetrieben, in: SCHWARZ, 1992, S. 257-263

Rettke, Ursula unter Mitarbeit von Susanne Kretzer (1992), Analyse der Übergangsproblematik junger Frauen beim Übergang vom Allgemeinbildenden in das Berufsbildende und in das Beschăftigungssystem, in: KRÜGER, 1992, S. 87-142

Richter, Gabriele / Weißgerber, Barbara / Fritsche, Birgit (1995), Arbeitsinhaltsgestaltung zur Förderung von Arbeitssicherheit und Gesundheit, in: Zeitschrift für Arbeitswissenschaft 49 (121 NF) 1995/1, S. $1-9$

Rohmert, Walter (1972), Aufgaben und Inhalt der Arbeitswissenschaft, in: Die berufsbildende Schule 24, Heft 1, S. 3-14

Rohmert, Walter (1980), Humanisierung der Arbeit durch Ergonomie? In: Zeitschrift für Arbeitswissenschaft 34 (6 NF) 1980/2, S. 65-69

Rohmert, Walter (1984), Das Belastungs-Beanspruchungs-Konzept, in: Zeitschrift für Arbeitswissenschaft 38 (10 NF) 1984/4, S. 193-200

Rohmert, Walter / Landau, Kurt (1979), Das Arbeitswissenschaftliche Erhebungsverfahren zur Tătigkeitsanalyse (AET), Handbuch mit Merkmalheft, Verlag Hans Huber, Bern, Stuttgart, Wien

Rose, Helmuth (1992a), Erfahrungsgeleitete Arbeit als Innovationskonzept für Arbeitsgestaltung und Technikentwicklung, in: Zeitschrift für Arbeitswissenschaft 46 (18 NF) $1992 / 3$, S. $145-149$

Rose, Helmuth (1992b), Erfahrungsgeleitete Arbeit als Fokus für Arbeitsgestaltung und Technikentwicklung, in: Zeitschrift für Arbeits- und Organisationspsychologie 36 (N. F. 10) 1, S. 22-29

Rose, Helmuth (1993), Die Bedeutung des Erfahrungswissens bei automatischer Prozeßsteuerung, in: BULLINGER, u.a., 1993, S. 68-72 
Rose, Helmuth (1995), Erfahrungsgeleitete Arbeit und Kooperation als Leistungspotentiale gruppenorientierter Produktionsstrukturen, Neue Formen der Arbeitsorganisation aus dem Blickwinkel der CeA-Forschung, in: Arbeit, Jg. 4, Heft 2, 1995, S. 170-184

Rosenow, Joachim / Naschold, Frieder (1993), Ältere Arbeitnehmer - Produktivitătspotential oder personalwirtschaftliche Dispositionsmasse? In: Sozialer Fortschritt 6-7, S. 146-152

Rosenow, Joachim / Naschold, Frieder (1994), Die Regulierung von Altersgrenzen, Strategien von Unternehmen und die Politik des Staates, unter Mitarbeit von Maria Oppen und Gert Wagner, (Hrsg. vom Wissenschaftszentrum Berlin für Sozialforschung, Abteilung: Regulierung von Arbeit), Edition Sigma, Berlin

Rosenstiel, Lutz von (1989), Selektions- und Sozialisationseffekt beim Übergang vom Bildungsins Beschăftigungssystem, in: Zeitschrift für Arbeits- und Organisationspsychologie 33 (N.F. 7) 1, S. 21-32

Rothkirch, Christoph von (1993), Langfristige Perspektiven der Arbeitsmarktentwicklung bis 2010, in: Sozialer Fortschritt, Heft 8, S. 178-183

Rupprecht, R. / Olbrich, Erhard / Gunzelmann, Th. / Oswald, W.-D. (1991), Erhaltung und Förderung von Kompetenz im hőheren Lebensalter, in: Beitrăge zur Gerontologie und Altenarbeit, Expertisen zum ersten Teilbericht der Sachverständigenkommission zur Erstellung des ersten Altenberichts der Bundesregierung, herausgegeben vom Deutschen Zentrum für Altersfragen e.V., Berlin, S. 235-300

Rürup, Bert / Schneider, Hilmar (1992), Herausforderungen an den Wohlfahrtsstaat im strukturellen Wandel, in: Hujer, Reinhard / Schneider, Hilmar / Zapf, Wolfgang (Hrsg.), Herausforderungen an den Wohlfahrtsstaat im strukturellen Wandel, Reihe "Wirtschaftswissenschaften«, Band 23, Campus Verlag, Frankfurt/Main, New York

Rürup, Bert / Sesselmeier, Werner (1989), Anforderungen an die heutige und zukünftige Arbeitsmarkt- und Beschăftigungspolitik - Mesoőkonomische Auswege aus den beschaftigungspolitischen Blockaden, in: Buttler, Friedrich / Kühlewind, Gerhard (Hrsg.), Erwerbstătigkeit und Generationsvertrag - Perspektiven bis 2030 -, Beitrăge zur Arbeitsmarkt- und Berufsforschung 130, IAB, Nünberg, S. 146-159

Rürup, Bert / Sesselmeier, Werner (1993a), Schrumpfende und alternde deutsche Bevolkerung. Arbeitsmarktpolitische Perspektiven und Optionen, in: KLOSE, 1993, S. 27-50

Rürup, Bert / Sesselmeier, Werner (1993b), Einwanderung: Die wirtschaftliche Perspektive, in: Balke, Friedrich / Habermas, Rebekka / Nanz, Patrizia / Sillem, Peter (Hrsg.), Schwierige Fremdheit - Über Integration und Ausgrenzung in Einwanderungsländern, Fischer, Frankfurt am Main, S. 285-304

Rürup, Bert / Sesselmeier, Werner (1993c), Die demographische Entwicklung Deutschlands: Risiken, Chancen, politische Optionen, in: Aus Politik und Zeitgeschichte, Beilage zur Wochenzeitung Das Parlament, B 44/93, S. 3-15 
Rürup, Bert / Sesselmeier, Werner (1994), Zu den wichtigsten Auswirkungen von Einwanderung auf Arbeitsmarkt und Sozialversicherungen, in: forum demographie und politik (Hrsg.: Hans-Ulrich Klose), Heft 5, S. 64-89

Scheer, August-Wilhelm (Band-Hrsg.) (1990), CIM-Strategie als Teil der Unternehmensstrategie, Springer-Verlag, Berlin, Heidelberg, New York, Verlag TÜV Rheinland, Koln (CIM-Fachmann)

Scherm, Martin / Bischoff, Patrick R. (1994), Lean Management - stereotype Sichtweisen japanischer Unternehmensphănomene, in: ESSER/KOBAYASHI, 1994, S. 100-107

Schienstock, Gert (1994), Globale Konzerne: Netzwerkstrukturen, Organisationsstrategien und Arbeitsbeziehungen, in: Arbeit, Jg. 3, Heft 3, 1994, S. 254-269

Schlaffke, Winfried /WeiB, Reinhold (Hrsg.) (1990), Tendenzen betrieblicher Weiterbildung, Aufgaben für Forschung und Praxis, Deutscher Instituts-Verlag, Köln

Schmăhl, Winfried (1993), Zur Einkommenssituation im Alter - Daten, Fragen, Hypothesen Vorinformation zum Vortrag wăhrend der Sitzung der Enquete-Kommission Demographischer Wandel am 28.4.1993, Deutscher Bundestag, Bonn, Kommissionsdrucksache 89 vom 23.04.1993

Schmid, Josef / Chruscz, Detlef (1994), Demographische Untersuchung zur Frage eines Einwanderungsbedarfs in der Europäischen Union, in: forum demographie und politik (Hrsg.: Hans-Ulrich Klose), Heft 5, S. 18-45

Schmidt, Bernd / Hogreve, Holger (1994), Erhebung zur beruflichen Weiterbildung in Unternehmen im Rahmen des EG-Aktionsprogramms FORCE, in: Wirtschaft und Statistik, Heft 4, S. 247-258

Schmidtke, Heidrun / Schwitzer, Klaus-Peter (1993), Lebensbedingungen und Handlungsintensionen alterer Menschen im Zuge des Transformationsprozesses in den neuen Lădern, Sozialwissenschaftliches Forschungszentrum Berlin-Brandenburg, KSPW, Kommission für die Erforschung des sozialen und politischen Wandels in den neuen Bundesländern e.V., Halle, Graue Reihe, Nr. 601, zugleich: Enquete-Kommission Demographischer Wandel, Kommissionsdrucksache 117 vom 08.06.1993

Schneider, Ulrike (1992), Privater Verbrauch im demographischen Reifungsprozeß, in: forum demographie und politik (Hrsg.: Hans-Ulrich Klose), Heft 2, S. 82-108

Schul, Sebastian (1993), Arbeitswissenschaftliche Beurteilung und Gestaltung von Arbeitssituationen für mehrfachbehinderte junge Menschen in ihrer beruflichen Bildung, Verlag Peter Lang, Frankfurt am Main, Berlin, Bern, New York, Paris, Wien, (Europăische Hochschulschriften: Reihe 11, Pädagogik; Bd. 542)

Schuler, Heinz (Hrsg.) (1993), Lehrbuch Organisationspsychologie, Verlag Hans Huber, Bern, Gottingen, Toronto, Seattle

Schultetus, Wolfgang (1993), Japan, Auch im Maschinenbau vorn? In: Arbeitgeber 5/45, S. 167-171 
Schultz-Wild, Rainer / Nuber, Christoph / Rehberg, Frank / Schmierl, Klaus (1989), An der Schwelle zu CIM: Verbreitung, Strategien und Auswirkungen, unter Mitarbeit von Marhild von Behr, Hartmut Hirsch-Kreinsen und Cristoph Kohler, RKW-Verlag, Eschborn, Verlag TÜV Rheinland, Köln

Schulz, Erika (1993), Bevőlkerungsentwicklung in Deutschland bis zum Jahr 2010 mit Ausblick auf 2040, in: Wochenbericht des DIW, Nr. 29, S. 393-404

Schulz, Erika / Kirner, Ellen (1994), Arbeitskrafteangebot von Frauen in Deutschland bis zum Jahr 2010, Empirische Ergebnisse und Ansătze zu einer kohortenspezifischen Projektion, in: BECKMANN/ENGELBRECH, 1994, S. 73-96

Schulz, H. (Band-Hrsg.) (1990), CIM-Planung und -Einführung, Springer-Verlag, Berlin, Heidelberg, New York, Verlag TÜV Rheinland, Köln (CIM-Fachmann)

Schulz, Reiner (1991), Zeitbudgetstrukturen erwerbstătiger Frauen, in: Zeitschrift für Bevollkerungswissenschaft, Jg. 17, Heft 3, S. 227-250

Schulze Buschoff, Karin (1994), Arbeitszeitpråferenzen, Basisdaten für eine bedürfnisgerechte Arbeitszeitgestaltung, Arbeitsgruppe Sozialberichterstattung, Wissenschaftszentrum Berlin für Sozialforschung (WZB), P 94-102, Mărz 1994, Berlin

Schumann, Michael / Baethge-Kinsky, Volker / Kuhlmann, Martin / Kurz, Constanze / Neumann, Uwe (1994), Trendreport Rationalisierung: Automobilindustrie, Werkzeugmaschinenbau, chemische Industrie, Ed. Sigma Bohn, Berlin

Schupp, Jürgen (1991), Teilzeitarbeit als Moglichkeit der beruflichen (Re-)Integration, in: MAYER u.a., 1991, S. 207-232

Schwager, Thomas / Udris, Ivars (1995), Der mühsame Weg zur Gruppenarbeit, Eine qualitative Rekonstruktion eines betrieblichen Reorganisationsprozesses, in: Arbeit, Jg. 4, Heft 2, 1995, S. 121-141

Schwarz, Karl (1990), Die Bildungsabschlüsse der Frauen und ihre Bedeutung für den Arbeitsmarkt, die EheschlieBung und die Familienbildung, in: Zeitschrift für Bevolkerungswissenschaft, Jg. 15, 4/1990, S. 361-382

Schwarz, Karl (Hrsg.) (1992), Frauenerwerbstătigkeit - Demographische, soziologische, okonomische und familienpolitische Aspekte -, Deutsche Gesellschaft für Bevölkerungswissenschaft, 26. Arbeitstagung vom 19. bis 21. Februar 1992 in Gosen bei Berlin, (Materialien zur Bevolkerungswissenschaft, Heft 77, herausgegeben vom Bundesinstitut für Bevőlkerungsforschung, Wiesbaden)

Schwarz. Thomas / Vogel, Claudia (1992), Betriebliche Sozialpolitik im Spannungsfeld von Familie und Erwerbstătigkeit, in: SCHWARZ, 1992, S. 241-245

Schweres, Manfred (1980), Strukturelemente einer integrativen Arbeitswissenschaft, in: Zeitschrift für Arbeitswissenschaft 34 (6 NF) 1980/1, S. 1-12 
Sebaldt, Martin (1992), Heimarbeit und Politik, Zur Bedeutung dezentraler Arbeitsplätze im deutschen Wirtschaftsleben und zur Entstehungsgeschichte des Heimarbeitsgesetzes vom 14.3.51, in: Sozialer Fortschritt, Jg. 41, 1992, Heft 8, S. 183-187

Seidel, Eberhard / Schumacher, L. (1985), Betriebsökonomische Effizienzindikatoren, Dokumentation erweiterter Wirtschaftlichkeitsverfahren, (Menschengerechte Arbeitsplătze sind wirtschaftlich), Schriftenreihe Wirtschaftlichkeitsberechnung, RationalisierungKuratorium der Deutschen Wirtschaft (RKW), Eschborn

Seifert, Hartmut (Hrsg.) (1993), Jenseits der Normalarbeitszeit, Perspektiven für eine bedürfnisgerechtere Arbeitszeitgestaltung, Bund-Verlag, Köln

Semmer, Norbert / Udris, Ivars (1993), Bedeutung und Wirkung von Arbeit, in: SCHULER, 1993, S. 133-165

Shingo, Shigeo (1992), Das Erfolgsgeheimnis der Toyota-Produktion, Verlag Moderne Industrie, Landsberg/Lech, 2. Aufl. 1993

Siebert, Helmut (1993), Lean Production und Arbeitsrecht, in: Fortschrittliche Betriebsführung und Industrial Engineering, 42 (1993) 2, S. 85 - 88

Siegmund, Jan (1994), Bevőlkerungsrückgang, Alterung und technischer Fortschritt, Dresdner wirtschaftswissenschaftliche Beitrăge: Reihe Volkswirtschaftslehre), Schăffer-Poeschel, Stuttgart

Siemens-Aktiengesellschaft (1990), CIM: A Management Perspective, Siemens-Aktienges., (Abt. Verl.), Berlin, München

Slesina, Wolfgang / Broekmann, Manfred (1992), Gesundheitszirkel zur Verstărkung des Gesundheitsschutzes im Betrieb, in: Arbeit, Jg. 1, Heft 2, 1992, S. 166-186

Sommer, Bettina (1992), Entwicklung der Bevölkerung bis 2030, Ergebnis der siebten koordinierten Bevollkerungsvorausberechnung, in: Wirtschaft und Statistik, Heft 4, S. $217-$ 222

Sommer, Bettina (1994), Entwicklung der Bevolkerung bis 2040, Ergebnis der achten koordinierten Bevőlkerungsvorausberechnung, in: Wirtschaft und Statistik, Heft 7, S. $497-$ 503

Sonntag, Karlheinz / Heun, Dieter / Schaper, Niclas (1992), Der Leitfaden zur qualitativen Personalplanung bei technisch organisatorischen Innovationen (LPI), in: Zeitschrift für Arbeitswissenschaft 46 (18 NF) 1992/1, S. 51-54

SpieB, Erika / Kaschube, Jürgen / Nerdinger, Friedemann W. / Rosenstiel, Lutz von (1992), Das Erleben von Arbeit und Freizeit nach Eintritt in den Beruf - Eine qualitative Studie bei Jungakademikern, in: Zeitschrift für Arbeits- und Organisationspsychologie 36 (N.F. 10) 2 , S. 77-83

Standfest, Erich (1995), Erste Ergebnisse aus dem PROGNOS-Gutachten, in: Deutsche Rentenversicherung 1/95, S. 1-18

Statistisches Bundesamt (Hrsg.) (1991), Haushalte und Familien, Fachserie 1, Reihe 3 
Statistisches Bundesamt (Brsg.) (1993), Bildung und Kultur, Fachserie 11, Reihe 3, Berufliche Bildung 1992

Statistisches Bundesamt (Hrsg.) (1994a), Im Blickpunkt: Ältere Menschen in der Europăischen Gemeinschaft

Statistisches Bundesamt (Hrsg.) (1994b), Wo bleibt die Zeit? Die Zeitverwendung der Bevolkerung in Deutschland, Herausgegeben vom Bundesministerium für Familie und Senioren und dem Statistischen Bundesamt

Statistisches Bundesamt (Hrsg.) (1994c), Bildung im Zahlenspiegel

Statistisches Bundesamt (Hrsg.) (Jahrbuch, div. Jahre), Statistisches Jahrbuch für die Bundesrepublik Deutschland

Statistisches Bundesamt (Hrsg.) (Mikrozensus, div. Jahre), Bevolkerung und Erwerbstătigkeit, Fachserie 1, Reihe 4.1.1

Staufer, Michael (1992), Ältere Beschăftigte und technischer Wandel, Computereinsatz im Büro, MaBnahmen betrieblicher Unterstützung, Hogrefe, Verlag für Psychologie, Gơttingen

Steger, Ulrich (1994), Lean-Administration: Die Krise der offentlichen Verwaltung als Chance, Campus Verlag, Frankfurt/Main, New York, (Schriftenreihe / Haniel-Stiftung; Bd. 7)

Steinmann, Gunter (1993), Zusammenhang zwischen Alterungsproze $B$ und Einwanderung, Expertise im Auftrag der Enquete-Kommission "Demographischer Wandel", Deutscher Bundestag, Bonn

Stortzbach, Bernd (1992), Übergang in eine neue Lebensphase - Erwartungen für das Leben im Alter, in: Zeitschrift für Bevolkerungswissenschaft, Harald Boldt Verlag, Jg. 18, 3/1992, S. 291-311

Straka, Gerald A. (1990), Alte Menschen und junge Medien, Einstellungsmuster zu neuen Kommunikationstechniken, in: Neue Zürcher Zeitung vom 27. Juni 1990, S. $65 \mathrm{f}$.

Straka, Gerald A. / Fabian, Thomas / Will, Jorg (1990), "Berufsverbot" mit 65 ? Pladoyer für flexible Altersgrenzen bei der Erwerbstătigkeit und eine aktive Bildungspolitik für die zweite Lebenshălfte, in: Psychologie und Gesellschaftskritik, 14. Jahrgang Heft 4

Straka, Gerald A. / Kuwan, H. (1994), EinfluBfaktoren auf die berufliche Fort- und Weiterbildung von Erwerbstătigen unter besonderer Berücksichtigung des Lebensalters, in: ABEL, J. (Hrsg.) (1994), Berufliche Weiterbildung und neue Technologien, Waxmann, Münster

Strümpel, Burkhard (1988), Arbeit - neue Werte, alte Strukturen, in: Rapin, Hildegard (Hrsg.) (1988), Frauenforschung und Hausarbeit, (Campus: Forschung; Bd. 575), (Reihe "Stiftung Der Private Haushalt"; Bd. 4), Campus Verlag, Frankfurt/Main, New York, S. 25-41

Struwe, Jochen (1995), Lean Administration und Verwaltungscontrolling, Das Instrumentarium, in: Aus Politik und Zeitgeschichte, Beilage zur Wochenzeitung Das Parlament, B 5/95, S. 20-32 
Suzaki, Kiyoshi (1994), Die ungenutzten Potentiale: Neues Management im Produktionsbetrieb, Hanser, München, Wien

Szallies, Rüdiger / Wiswede, Günter (Hrsg.) (1991), Wertewandel und Konsum: Fakten, Perspektiven und Szenarien für Markt und Marketing, Verlag Moderne Industrie, Landsberg/Lech

Tessaring, Manfred (1991), Tendenzen des Qualifikationsbedarfs in der Bundesrepublik Deutschland bis zum Jahre 2010. Implikationen der IAB/Prognos-Projektion 1989 für die Qualifikationsstruktur der Arbeitsplătze in Westdeutschland, in: Mitteilungen aus der Arbeitsmarkt- und Berufsforschung 1/91, S. 45-62

Tessaring, Manfred (1993), Das duale System der Berufsausbildung in Deutschland: Attraktivităt und Beschăftigungsperspektiven, in: Mitteilungen aus der Arbeitsmarkt- und Berufsforschung 2/93, S. 131-161

Thon, Manfred (1991), Perspektiven des Erwerbspersonenpotentials in Gesamtdeutschland bis zum Jahre 2030, in: Mitteilungen aus der Arbeitsmarkt- und Berufsforschung 4/91, S. 706712

Ulich, Eberhard (1988), Arbeits- und organisationspsychologische Aspekte neuer Technologien, in: ZINK, 1988, S. 117-141

Ulich, Eberhard (1991), Arbeitspsychologie, Verlag der Fachvereine, Zürich; Poeschel, Stuttgart

Ulich, Eberhard (1993), Gestaltung von Arbeitstătigkeiten, in: SCHULER, 1993, S. 189-208

Ulich, Eberhard / Conrad-Betschart, Hanspeter / Baitsch, Christof (1989), Arbeitsform mit Zukunft: ganzheitlich-flexibel statt arbeitsteilig, Grundlagen und 7 Fallstudien aus der Maschinenindustrie, Peter Lang, Bern, Frankfurt am Main, Nex York, Paris

Ulmer, Hans-Volkhart (1985), Physiologische Grundlagen menschlicher Arbeit, in: Reichel, Gerhard (Hrsg.), Grundlagen der Arbeitsmedizin, Kohlhammer, Stuttgart, Berlin, Köln, Mainz

VDI (1989), Sozialvertrăgliche Gestaltung von Automatisierungsvorhaben, VDIHandlungsempfehlung, Verein Deutscher Ingenieure, Düsseldorf

VDI, REFA (1983), Lexikon der Produktionsplanung und -steuerung, VDI-Gesellschaft Produktionstechnik (ADB), REFA, Verband für Arbeitsstudien und Betriebsorganisation e.V., VDI-Verlag, Düsseldorf, 3. Auflage

VDR (1993), VDR STATISTIK, Bd. 109 - Rentenzugang 1993, Verband Deutscher Rentenversicherungstrăger

Vogel, Dita (1994), Sozialpolitische Integration als zuwanderungspolitisches Steuerungsinstrument, in: forum demographie und politik (Hrsg.: Hans-Ulrich Klose), Heft 5 , S. $132-155$

Volkholz, Volker (1992), Erwerbsarbeit der Zukunft, Vorschlag für eine Diskussion nicht nur in den Sozialwissenschaften, in: Arbeit, Jg. 1, Heft 1, 1992, S. 5-24 
Warnecke, Günter (Band-Hrsg.) (1991), Expertensysteme in CIM, Springer-Verlag, Berlin, Heidelberg, New York, Verlag TÜV Rheinland, Köln (CIM-Fachmann)

Warnecke, Hans J. (1992), Die Fraktale Fabrik: Revolution der Unternehmenskultur, SpringerVerlag, Berlin, Heidelberg

Weber, Wolfgang G. (1994), Autonome und restriktive Gruppenarbeit in der Produktion, Anmerkungen zu einer arbeitspsychologischen Unterscheidung, in: Zeitschrift für Arbeitswissenschaft 48 (20 NF) 1994/3, S. 147-156

WeiB, Reinhold (1994), Weiterbildungskosten 1992, in: Hauptabteilung Bildung und Gesellschaftswissenschaft des Instituts der Deutschen Wirtschaft (Hrsg.), Informationen zur beruflichen Bildung, Ein Kompendium der Berufspolitik und -praxis, Register 10 Bildungsokonomie/Bildungskosten, Blatt 12, Deutscher Instituts-Verlag, Koln

Wendt, Hartmut (1992), Frauenalltag in der ehemaligen DDR, in: SCHWARZ, 1992, S. 171188

Wenker, Harald (1994), Ist von neuen Konzepten der Arbeitsorganisation im Rahmen von "Lean Production" ein Beitrag zur Humanisierung der Arbeit zu erwarten? In: Sozialer Fortschritt, Jg. 43, 1994, Heft 1, S. 5-7

Werner, Heinz (1990), Die Freizügigkeit der Arbeitskrăfte und der EG-Binnenmarkt Konsequenzen für den Arbeitsmarkt? In: Buttler, Friedrich / Walwei, Ulrich / Werner, Heinz (Hrsg.), Arbeits- und Sozialraum im Europăischen Binnenmarkt, Beitrăge zur Arbeitsmarktund Berufsforschung 129, IAB, Nürnberg, S. 113-123

Wiendahl, Hans-Peter (Band-Hrsg.) (1991), Analyse und Neuordnung der Fabrik, SpringerVerlag, Berlin, Heidelberg, New York, Verlag TÜV Rheinland, Köln (CIM-Fachmann)

Witzgall, Elmar / Wocherl, Hans (1989), Qualifizierung in der mittelbetrieblichen Fertigung Grundprobleme und ein neues Losungskonzept, in: Zeitschrift für Arbeitswissenschaft 43 (15 NF) $1989 / 4$, S. 203-206

Wobbe, Gerd (1980), Die Integration organisationstheoretischer Überlegungen in das System der Arbeitsgestaltung, in: Zeitschrift für Arbeitswissenschaft 34 (6 NF) 1980/1, S. 13-20

Womack, James P. / Jones, Daniel T. / Roos, Daniel (1991), Die zweite Revolution in der Automobilindustrie: Konsequenzen aus der weltweiten Studie aus dem Massachusetts Institute of Technology, Campus Verlag, Frankfurt/Main, New York, 4. Aufl. 1992

Zangl, Hans (1985), Durchlaufzeiten im Büro, Prozeßorganisation und Aufgabenintegration als effizienter Weg zur Rationalisierung der Büroarbeit mit neuen Bürokommunikationstechniken, (Bd. 3 der Schriftenreihe Mensch und Arbeit im technischorganisatorischen Wandel, herausgegeben von Rainer Marr und Ralf Reichwald), Erich Schmidt Verlag, Berlin; 2., überarbeitete Auflage 1987

Zink, Klaus J. (Hrsg.) (1988), Arbeitswissenschaft und neue Technologien, Dokumentation zur Herbstkonferenz 1986 der Gesellschaft für Arbeitswissenschaft an der Universităt Kaiserslautern, Rationalisierungs-Kuratorium der Deutschen Wirtschaft (RKW) 
Zülch, Gert (1992a), Ansătze und Defizite einer arbeitsorganisatorischen Methodenlehre - Teil 1, Bezugsrahmen der Arbeitsorganisation, in: Zeitschrift für Arbeitswissenschaft 46 (18 NF) 1992/3, S. 133-138

Zülch, Gert (1992b), Ansătze und Defizite einer arbeitsorganisatorischen Methodenlehre - Teil 2, Beitrăge zur arbeitsorganisatorischen Methodenlehre, in: Zeitschrift für Arbeitswissenschaft 46 (18 NF) 1992/4, S. 243-249

Zülch, Gert / Starringer, Manfred (1984), Differentielle Arbeitsgestaltung in Fertigungen für elektronische Flachbaugruppen, in: Zeitschrift für Arbeitswissenschaft 38 (10 NF) 1984/4, S. 211-216 


\section{SOZALÖKONOMISCHE SCHRIFTEN}

Herausgegeben von Professor Dr. Dr. h.c. Bert Rürup

Band 1 Marietta Jass: Erfolgskontrolle des Abwasserabgabengesetzes. Ein Konzept zur Erfassung der Gesetzeswirkungen verbunden mit einer empirischen Untersuchung in der $\mathrm{Pa}$ pierindustrie. 1990.

Band 2 Frank Schulz-Nieswandt: Stationäre Altenpflege und "Pflegenotstand" in der Bundesrepublik Deutschland. 1990.

Band 3 Helmut Böhme, Alois Peressin (Hrsg.): Sozialraum Europa. Die soziale Dimension des Europăischen Binnenmarktes. 1990.

Band 4 Stephan Ruß: Telekommunikation als Standortfaktor für Klein- und Mittelbetriebe. Telekommunikative Entwicklungstendenzen und regionale Wirtschaftspolitik am Beispiel Hessen. 1991.

Band 5 Reinhard Grünewald: Tertiărisierungsdefizite im Industrieland Bundesrepublik Deutschland. Nachweis und politische Konsequenzen. 1992.

Band 6 Bert Rürup, Uwe H. Schneider (Hrsg.): Umwelt und Technik in den Europäischen Gemeinschaften. Teil I: Die grenzüberschreitende Entsorgung von Abfällen. Bearbeitet von: Thomas Kemmler, Thomas Steinbacher. 1993.

Band 7 Mihai Nedelea: Erfordernisse und Möglichkeiten einer wachstumsorientierten Steuerpolitik in Rumänien. Dargestellt am Beispiel der Textil- und Bekleidungsindustrie. 1995.

Band 8 Andreas Schade: Ganzjăhrige Beschăftigung in der Bauwirtschaft - Eine Wirkungsanalyse. Analyse und Ansätze für eine Reform der Winterbauförderung. 1995.

Band 9 Frank Schulz-Nieswandt: Ökonomik der Transformation als wirtschafts- und gesellschaftspolitisches Problem. Eine Einführung aus wirtschaftsanthropologischer Sicht. 1996.

Band 10 Werner Sesselmeier / Roland Klopfleisch / Martin Setzer: Mehr Beschäftigung durch eine Negative Einkommensteuer. Zur beschăftigungspolitischen Effektivităt und Effizienz eines integrierten Steuer- und Transfersystems. 1996.

Band 11 Sylvia Liebler: Der Einfluß der Unabhăngigkeit von Notenbanken auf die Stabilităt des Geldwertes. 1996.

Band 12 Werner Sesselmeier: Einkommenstransfers als Instrumente der Beschäftigungspolitik. Negative Einkommensteuer und Lohnsubventionen im Lichte modemer Arbeitsmarkttheorien und der Neuen Institutionenökonomik. 1997.

Band 13 Stefan Lorenz: Der Zusammenhang von Arbeitsgestaltung und Erwerbsleben unter besonderer Berücksichtigung der Erwerbstătigkeiten von Frauen und Älteren. 1997. 